June $1997 \bullet$ NREL/SR-540-23143 • DE97050873 • UC Category: 1504

\title{
Determination of Alternative Fuels Combustion Products: Phase 2 Final Report
}

\author{
Kevin A. Whitney \\ Southwest Research Institute
}

NREL Technical Monitors: Brent Bailey and Michelle Bergin

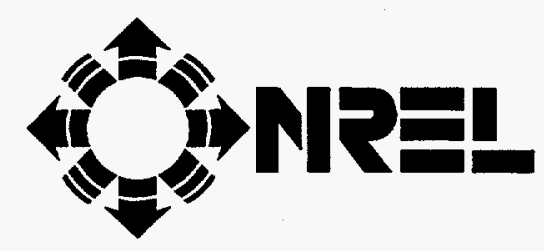

National Renewable Energy Laboratory

1617 Cole Boulevard

Golden, Colorado 80401-3393

A national laboratory of the

U.S. Department of Energy

Managed by the Midwest Research Institute

For the U.S. Department of Energy

Under Contract No. DE-AC36-83CH10093

Prepared under Subcontract Number YAW-3-13253-01

June 1997 
This publication was reproduced from the best available camera-teady copy submitted by the subcontractor and received no editorial review at NREL.

\section{NOTICE}

This report was prepared as an account of work sponsored by an agency of the United States govemment. Neither the United States government nor any agency thereof, nor any of their employees, makes any warranty, express or implied, or assumes any legal liability or responsibility for the accuracy, completeness, or usefulness of any infomation, apparatus, product, or process disclosed, or represents that its use would not infringe privately owned rights. Reference herein to any specific commercial product, process, or service by trade name, trademark, manufacturer, or otherwise does not necessariiy consitute or imply its endorsement, recommendation, or favoring by the United States government or any agency thereof. The views and opinions of authors expressed herein do not necessarily state or reflect those of the United States government or any agency thereof.

Available to DOE and DOE contractors from:

Office of Scientific and Technical Information (OSTI)

P.O. Box 62

Oak Ridge, TN 37831

Prices available by calling (423) $576-8401$

Available to the public from:

National Technical Information Service (NTIS)

U.S. Department of Commerce

5285 Port Royal Road

Springfield, VA 22161

(703) $487-4650$ 


\section{DISCLAMMER}

Portions of this document may be illegible in electronic image products. Images are produced from the best available original document. 



\section{Foreword}

This report covers the second year of a continuing program conducted for the National Renewable Energy Laboratory (NREL) by the Department of Emissions Research of Southwest Research Institute (SwRI). This program was authorized by NREL Subcontract No. YAW-313253-01. Midwest Research Institute operates and manages NREL for the United States Department of Energy under Prime Contract No. DE-AC36-83CH10093. This program began on October 1, 1993 and is currently scheduled to continue through August 20,1997. Testing covered in this report was conducted from March 1995 through February 1996. The initial project was based on SwRI Proposal 08-14326 to NREL. The tasks covered in this report are based on SwRI Proposal 08-17314. The overall program was identified within SwRI as Project 08-6068. The NREL technical monitors for this program were Mr. Brent Bailey and Mr. Chris Colucci of NREL's Center for Transportation Technologies and Systems in Golden, Colorado. The SwRI project manager was Dr. Lawrence R. Smith, and the project leader was Mr. Kevin A. Whitney. Individual task leaders included Mr. Matthew S. Newkirk, Mr. Patrick M. Merritt, and Mr. E. Robert Fanick. Mr. Danny Terrazas, laboratory supervisor, was responsible for emissions testing. 



\begin{abstract}
This report describes the laboratory efforts to accomplish four independent tasks: (1) speciation of hydrocarbon exhaust emissions from a light-duty vehicle operated over the chassis dynamometer portion of the light-duty FTP after modifications for operation on butane and butane blends; (2) evaluation of NREL's Variable Conductance Vacuum Insulated Catalytic Converter Test Article 4 for the reduction of cold-start FTP exhaust emissions after extended soak periods for a Ford FFV Taurus operating on E85; (3) support of UDRI in an attempt to define correlations between engine-out combustion products identified by SwRI during chassis dynamometer testing, and those found during flow tube reactor experiments conducted by UDRI; and (4) characterization of small-diameter particulate matter from a Ford Taurus FFV operating in a simulated fuel-rich failure mode on CNG, LPG, M85, E85, and reformulated gasoline.
\end{abstract}




\section{Table of Contents}

Page

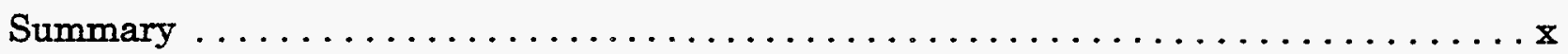

I. Introduction $\ldots \ldots \ldots \ldots \ldots \ldots \ldots \ldots \ldots \ldots \ldots \ldots \ldots \ldots \ldots$

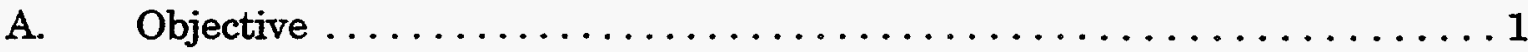

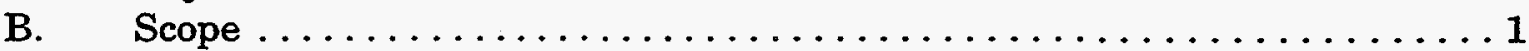

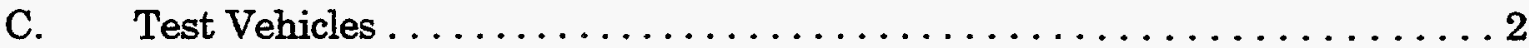

D. Test Fuels . . . . . . . . . . . . . . . . . . . . . 2

E. Test Procedures $\ldots \ldots \ldots \ldots \ldots \ldots \ldots \ldots \ldots \ldots \ldots \ldots \ldots \ldots \ldots$

F. Emissions Sampling and Analysis Procedures $\ldots \ldots \ldots \ldots \ldots \ldots \ldots$

II. Task 1 - Hydrocarbon Speciation of Emissions from a Vehicle

Operating on Butane-Blended Fuels . . . . . . . . . . . . . . . 6

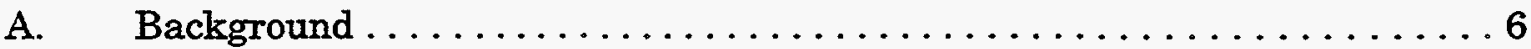

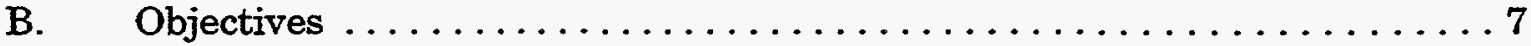

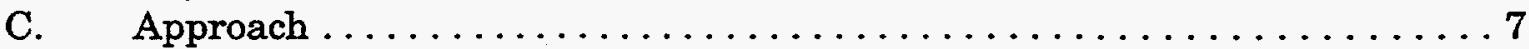

D. Vehicle Description and Conversion $\ldots \ldots \ldots \ldots \ldots \ldots \ldots \ldots \ldots$

E. Test Fuels . . . . . . . . . . . . . . . . . . . . . . . . 9

F. Emission Test Procedures $\ldots \ldots \ldots \ldots \ldots \ldots \ldots \ldots \ldots \ldots \ldots$

G. Emission Test Results $\ldots \ldots \ldots \ldots \ldots \ldots \ldots \ldots \ldots \ldots \ldots \ldots$

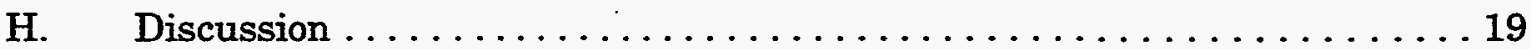

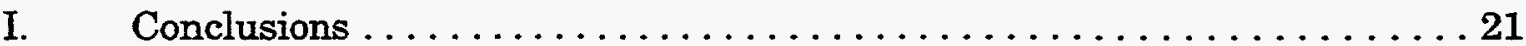

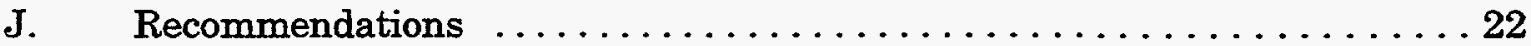

III. Task 2 - Evaluation of a Vacuum Insulated Catalyst in Reducing Cold-Start Exhaust Emissions $\ldots \ldots \ldots \ldots \ldots \ldots \ldots \ldots \ldots \ldots$

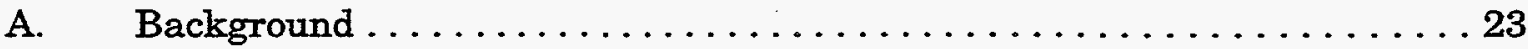

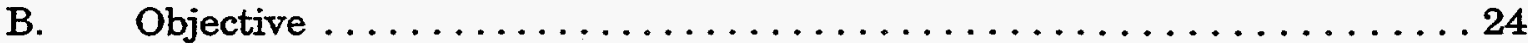

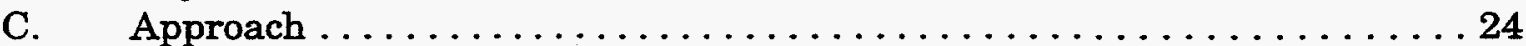

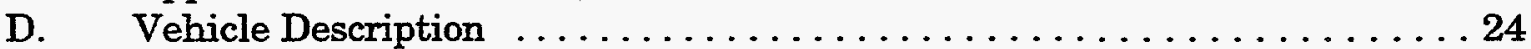

E. Test Fuels . . . . . . . . . . . . . . . . . . . . . 25

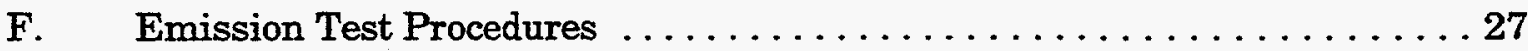

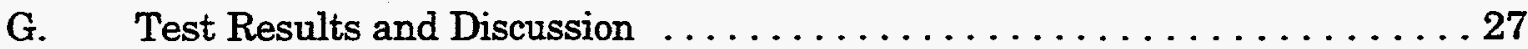

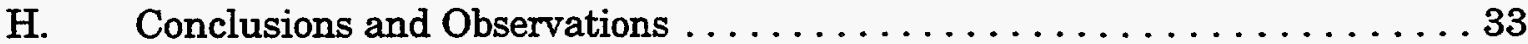

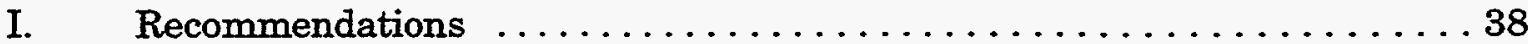




\section{Table of Contents Concluded}

$\underline{\text { Page }}$

IV. Task 3 - Technical Support Studies Performed by the University of Dayton Research Institute

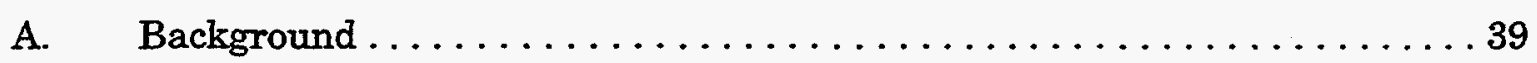

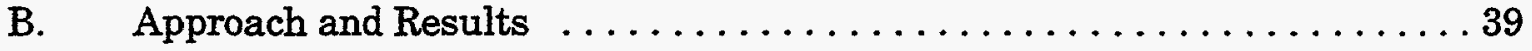

V. Task 4 - Characterization of Exhaust Particulate Emissions from

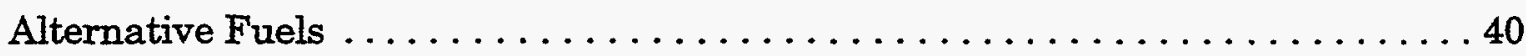

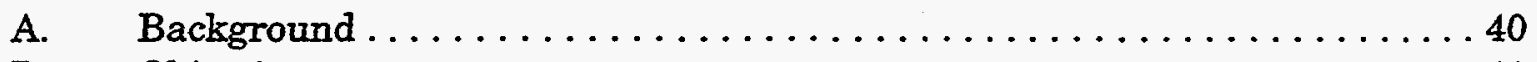

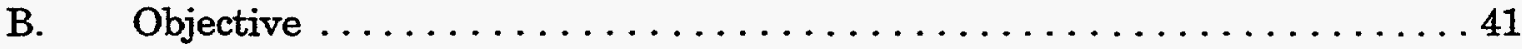

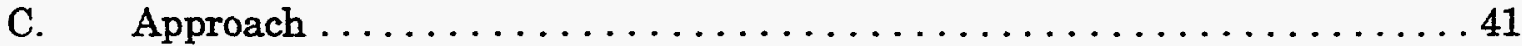

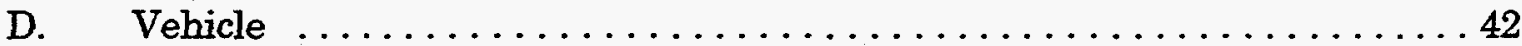

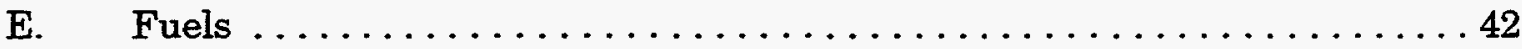

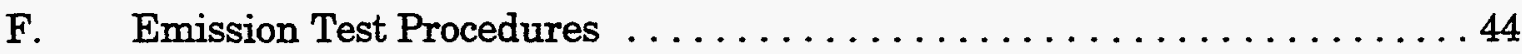

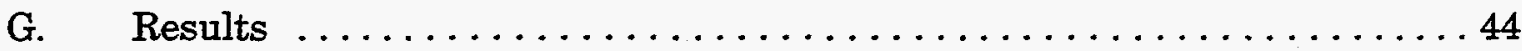

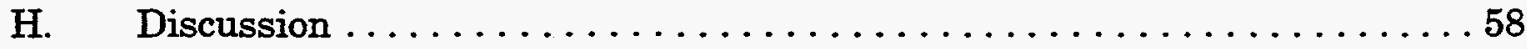

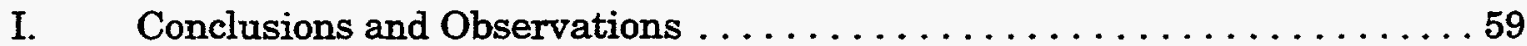

J. Recommendations ............................... 60

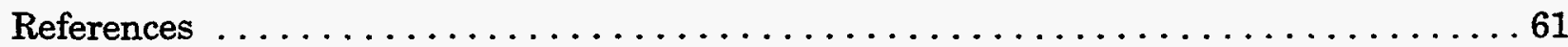

Appendices

A - Detailed Computer Printouts from Task 1 FTPs

B - Detailed Speciation Data from Task 1 FTPs

C - Detailed Computer Printouts from Task 2 FTPs

D - Detailed Computer Printouts from Task 4 FTPs 


\section{List of Figures}

\section{Figure}

Page

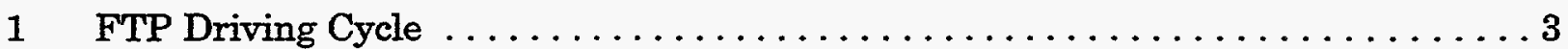

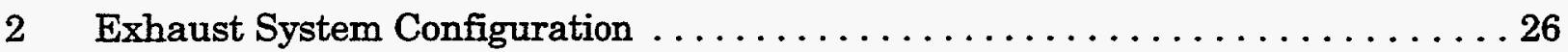

3 Insulated Catalyst Cooldown Temperature Profile $\ldots \ldots \ldots \ldots \ldots \ldots$

4 Taurus FFV with Insulated Catalyst, Summary of FTP Emissions . . . . . . . . 30

5 Comparison of Uninsulated and Insulated Catalyst Temperatures . . . . . . . 32

6 Taurus FFV with Insulated Catalyst, FTP FID HC after Catalyst . . . . . . . . . . . . . . . . . . . . . 34

7 Taurus FFV with Insulated Catalyst, FTP CO after Catalyst . . . . . . . . 35

8 Taurus FFV with Insulated Catalyst, FTP NO after Catalyst $\ldots \ldots \ldots \ldots \ldots \ldots . \ldots 36$

9 Temperature of TA-CC4 During Soak $\ldots \ldots \ldots \ldots \ldots \ldots \ldots \ldots \ldots$

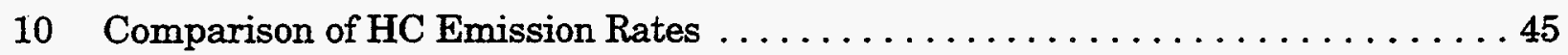

11 Comparison of CO Emission Rates $\ldots \ldots \ldots \ldots \ldots \ldots \ldots \ldots \ldots \ldots \ldots \ldots \ldots \ldots$

12 Comparison of $\mathrm{NO}_{\mathrm{x}}$ Emission Rates $\ldots \ldots \ldots \ldots \ldots \ldots \ldots \ldots \ldots \ldots$

13 Comparison of Total Particulate Emission Rates $\ldots \ldots \ldots \ldots \ldots \ldots \ldots \ldots$

14 Comparison of Average Fuel Economy Values . . . . . . . . . . . . . . . 49

15 Particle Size Distribution of Laboratory Ambient and CVS Background Particulate .............................. 52

16 Particle Size Distribution by Fuel $\ldots \ldots \ldots \ldots \ldots \ldots \ldots \ldots \ldots \ldots \ldots \ldots \ldots \ldots$

17 Equivalent Aerodynamic Diameter Particle Size Distribution . . . . . . . . . 55

18 Particle Size Distribution Data for Each Fuel $\ldots \ldots \ldots \ldots \ldots \ldots \ldots \ldots$ 


\section{List of Tables}

Table

Page

1 Summary of FTP Driving Schedule $\ldots \ldots \ldots \ldots \ldots \ldots \ldots \ldots \ldots \ldots$

2 Vehicle Description $\ldots \ldots \ldots \ldots \ldots \ldots \ldots \ldots \ldots \ldots \ldots \ldots \ldots \ldots \ldots \ldots$

3 Summary of RF-A and CP2 Fuel Properties $\ldots \ldots \ldots \ldots \ldots \ldots \ldots$

4 Summary of Alternative Fuel Compositions $\ldots \ldots \ldots \ldots \ldots \ldots \ldots$

5 Summary of Pre- and Post-Conversion Average Regulated Emissions . . . . . . 11

6 Summary of Average Regulated Emissions Data . . . . . . . . . . . . . . . 12

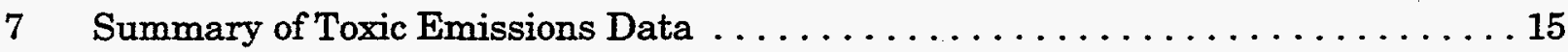

8 Summary of Specific Reactivity and Adjusted NMOG Emissions . . . . . . 17

9 Summary of Major HC Contributors to FTP Potential Ozone $\ldots \ldots \ldots \ldots$

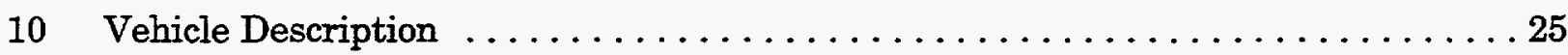

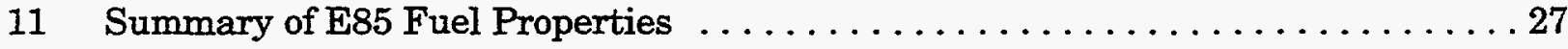

12 FTP Exhaust Emissions from Ford FFV Equipped with TA-CC4 . . . . . . . 29

13 Fuel Analysis for Liquid Fuels $\ldots \ldots \ldots \ldots \ldots \ldots \ldots \ldots \ldots \ldots$

14 Fuel Gas Analysis Summary $\ldots \ldots \ldots \ldots \ldots \ldots \ldots \ldots \ldots \ldots \ldots \ldots \ldots$

15 Summary of Composite FTP Regulated Emissions $\ldots \ldots \ldots \ldots \ldots \ldots \ldots \ldots$

16 Composite Emission Results for Alcohols, Aldehydes, and Ketones ........51

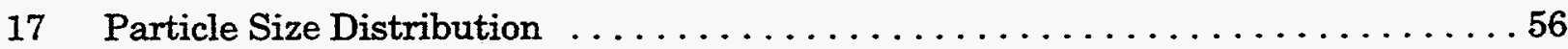




\section{Acronyms and Abbreviations}

pL.

um

$\mathrm{ADP}$

cfm

CFR

$\mathrm{CH}_{4}$

CNG

$\mathrm{CO}$

$\mathrm{CO}_{2}$

CP2

CRC

CVS

DNPH

E85

ECU

EGO

EGR

EP

EPA

FFV

FID

FTP

$\mathrm{g} / \mathrm{mi}$

gal

GC

h

$\mathrm{HC}$

HFET

HPLC

IBP

L

LEV

LNG

LPG

M85

MAP

$\mathrm{mi}$

$\min$

MIR

$\mathrm{mL}$

$\mathrm{mm}$

MON

MPFI

MTBE

NAAQS

NDIR microliter

micron

Adaptive Digital Processor

cubic foot per minute

Code of Federal Regulations

Methane

Compressed Natural Gas

Carbon Monoxide

Carbon Dioxide

California Phase 2

Coordinating Research Council

Constant Volume Sampler

2,4-dinitrophenylhydrazine

$85 \%$ denatured ethanol and $15 \%$ gasoline

Engine Control Unit

Exhaust Gas Oxygen

Exhaust Gas Recirculation

End Point

Environmental Protection Agency

Flexible Fuel Vehicle

Flame Ionization Detector

Federal Test Procedure

grams per mile

gallon

Gas Chromatograph

hour

Hydrocarbons

Highway Fuel Economy Test

High Performance Liquid Chromatograph

Initial Boiling Point

liter

Low Emission Vehicle

Liquefied Natural Gas

Liquefied Petroleum Gas

85\% methanol and $15 \%$ gasoline

Manifold Absolute Pressure

mile

minute

Maximum Incremental Reactivity

milliliter

millimeter

Motor Octane Number

Multi-Point Fuel Injection

methyl tertiary-butyl ether

National Ambient Air Quality Standard

Non-Dispersive Infrared 


\section{Acronyms and Abbreviations Concluded}

$\begin{array}{ll}\text { NMOG } & \text { Non-Methane Organic Gas } \\ \text { NO } & \text { Oxides of Nitrogen } \\ \text { NREL } & \text { National Renewable Energy Laboratory } \\ \mathrm{O}_{3} & \text { Ozone } \\ \text { OEM } & \text { Original Equipment Manufacturer } \\ \text { OFP } & \text { Ozone Forming Potential } \\ \text { OMHCE } & \text { Organic Material Hydrocarbon Equivalent } \\ \text { OMNMHCE } & \text { Organic Material Non-Methane Hydrocarbon Equivalent } \\ \text { PCM } & \text { Phase Change Material } \\ \text { ppm } & \text { parts per million } \\ \text { psi } & \text { pounds per square inch } \\ \text { RAF } & \text { Reactivity Adjustment Factor } \\ \text { RF-A } & \text { Industry Average Gasoline } \\ \text { RFG } & \text { Reformulated Gasoline } \\ \text { RON } & \text { Research Octane Number } \\ \text { RVP } & \text { Reid Vapor Pressure } \\ \text { S } & \text { second } \\ \text { SAE } & \text { Society of Automotive Engineers } \\ \text { scfm } & \text { standard cubic feet per minute } \\ \text { SwRI } & \text { Southwest Research Institute } \\ \text { TA-CC4 } & \text { Test Article 4 } \\ \text { THC } & \text { Total Hydrocarbons } \\ \text { TLEV } & \text { Transitional Low Emission Vehicle } \\ \text { UDDS } & \text { Urban Dynamometer Driving Schedule } \\ \text { UDRI } & \text { University of Dayton Research Institute } \\ \text { UEGO } & \text { Universal Exhaust Gas Oxygen } \\ \text { ULEV } & \text { Ultra-Low Emission Vehicle } \\ \text { uv } & \text { ultraviolet } \\ \text { VOC } & \text { Volatile Organic Compound }\end{array}$




\section{Summary}

Four separate tasks were conducted under the second year of this program to determine combustion products from vehicles operating on alternative fuels: (1) speciation of hydrocarbon exhaust emissions from a light-duty vehicle modified to operate on butane and butane blends; (2) evaluation of NREL's Variable Conductance Vacuum Insulated Catalytic Converter (TA-CC4) for the reduction of cold-start FTP exhaust emissions from a Ford FFV Taurus operating on E85 after extended soak periods; (3) support of UDRI in an attempt to define correlations between engine-out combustion products identified by SwRI during chassis dynamometer testing, and those found during flow tube reactor experiments conducted by UDRI; and (4) characterization of small-diameter particulate matter from a Ford Taurus FFV operating in a simulated fuel-rich failure mode on CNG, LPG, M85, E85, and reformulated gasoline.

Under Task 1, "Hydrocarbon Speciation of Emissions from a Vehicle Operating on Butane-Blended Fuels," regulated exhaust emissions, speciated hydrocarbon emissions, ozoneforming potentials, and specific reactivity factors were determined for 11 test fuels. These fuels included California Phase 2 gasoline, industry-average gasoline (RF-A), 100\% n-propane, $100 \% \mathrm{n}$-butane, and a variety of butane blends. In all, 18 exhaust emission tests were conducted on a light-duty vehicle (equipped with a gaseous-fuel conversion kit) using the chassis dynamometer portion of the FTP. Some of the findings are outlined below.

- Optimum post-conversion emissions performance appeared to be achieved with the vehicle operating on $100 \%$ propane. The next best performance was achieved with the $50 \%$ n-butane $/ 50 \%$ propane blend.

- All of the alternative fuels allowed a converted California TLEV to potentially meet ULEV NMOG and $\mathrm{NO}_{\mathrm{x}}$ standards at the mileage tested. All fuels except a $100 \% \mathrm{n}-$ butane fuel and a $90 \% \mathrm{n}$-butane/10\% pentane fuel blend met the ULEV CO standard.

- In general, for all alternative fuels tested, more than three-fourths of the calculated total FTP potential ozone came from the cold-start segment of the FTP test. Primary ozone hydrocarbon precursors were unburned fuel components and two partial combustion products, ethylene and propylene.

Under Task 2, "Evaluation of a Vacuum Insulated Catalyst in Reducing Cold-Start Exhaust Emissions," the effectiveness of NREL's Vacuum Insulated Catalytic Converter (TACC4) in reducing cold-start FTP exhaust emissions after extended soak periods was evaluated. Regulated exhaust emissions and estimated NMOG emissions from a light-duty vehicle (operating on a blend of $85 \%$ denatured ethanol and $15 \%$ gasoline and equipped with the TACC4 test article) were determined utilizing the chassis dynamometer portion of the FTP. Five different combinations of vehicle preconditioning type and soak period duration were evaluated. Some of the findings are summarized below.

- Compared to a similar but uninsulated catalyst, reductions of more than $90 \%$ were observed for $\mathrm{HC}$ and $\mathrm{CO}$ emissions, and $75 \%$ for $\mathrm{NO}_{\mathrm{x}}$ emissions for tests 
conducted following extended vehicle preconditioning and soak sequences (18and 24-hour soak periods).

- Results following an extended vehicle preconditioning sequence and a 36-hour soak showed reductions of more $70 \%$ from baseline levels for $\mathrm{HC}, \mathrm{CO}$, and $\mathrm{NO}_{\mathrm{x}}$ exhaust emissions.

- After conducting a standard vehicle prep and a 36-hour soak, exhaust emissions were found to be approximately $30 \%, 20 \%$, and $55 \%$ lower for the $\mathrm{HC}, \mathrm{CO}$, and $\mathrm{NO}_{\mathrm{x}}$ emissions, respectively, compared to baseline levels. In addition, THC and $\mathrm{CO}$ levels were approximately $20 \%$ less than those from testing with a similar, but uninsulated, catalyst configuration while $\mathrm{NO}_{\mathrm{x}}$ levels were similar.

- As expected, most of the emission reductions were achieved in the first few minutes of the cold-start phase of the FTP.

Under Task 3, "Technical Support of the University of Dayton Research Institute," SwRI provided technical support to UDRI in its attempt to define correlations between engineout combustion products identified by SwRI during chassis dynamometer testing, and those found by UDRI during flow tube reactor experiments.

Under Task 4, "Characterization of Exhaust Particulate from Alternative Fuels," particulate and regulated gaseous emissions were characterized from a flexible fuel vehicle operating on five different test fuels. These fuels included Federal reformulated gasoline; M85 - $85 \%$ methanol and $15 \%$ gasoline; E85 - 85\% ethanol denatured with 5\% gasoline, and $15 \%$ gasoline; LPG meeting HD-5 specifications; and industry-average CNG. The vehicle was operated fuel-rich to simulate a vehicle failure mode for the increased production of particulate matter. Particulate emissions were characterized by total mass and particle size. Ten exhaust emission evaluations were performed using the chassis dynamometer portion of the FTP for light-duty vehicles. Some of the findings are outlined below.

- Even while operating at rich fuel/air equivalence ratios, total particulate mass did not exceed the 1996 light-duty vehicle particulate standard for any of the fuels. In addition, particulate emissions while running rich on LPG and CNG were similar to those measured during the baseline run on gasoline.

- While operating fuel-rich, the trend for total particulate mass was $L P G \cong C N G$ $<\mathrm{E} 85 \cong \mathrm{M} 85<\mathrm{RFG}$.

- Approximately one-third of all particulate produced was $<0.2 \mu \mathrm{m}$ while operating on RFG, M85, E85, and CNG. While operating on LPG, nearly twothirds of all particulate was $<0.2 \mu m$.

- Alcohol fuels produced particle size distributions in relation to the soot-forming ability of the specific alcohol in a flame.

- Gaseous fuels produced a lower percentage of larger particles than the other fuels. 
- Particle agglomeration appeared to be one of the mechanisms for larger particle formation; smaller particles agglomerate to form different equivalent aerodynamic diameter ranges depending on the fuel. Predominant particle agglomeration ranges by fuel were:

RFG - greater than $1 \mu \mathrm{m}$ (44\% of total)

M85 - broad range from 0.7 to greater than $3 \mu \mathrm{m}$ (44\% of total)

E85 - 1 to $2 \mu \mathrm{m}$ (36\% of total)

LPG - greater than $3 \mu \mathrm{m}$ ( $12 \%$ of total)

CNG - greater than $1 \mu \mathrm{m}$ ( $43 \%$ of total). 


\section{Introduction}

In recent years, both government and industry have increased their efforts to develop and advance technology that allows an effective use of alternative transportation fuels, including LPG, CNG, ethanol, methanol, and butane. However, concerns continue about the potential impact of these fuels on air quality. For alternative fuels to be viable candidates for replacing conventional fuels, it must be demonstrated that their impact on air quality will be no worse than that of existing fuels, and preferably they should show characteristics that will improve air quality. To make this determination, it is necessary to identify the exhaust components that may be generated from alternative fuels and compare them to those from conventional fuels, to investigate new applications of alternative fuel blends, and to evaluate novel aftertreatment systems for use with alternative fuel vehicles.

\section{A. Objective}

The objectives of the four separate tasks in this project were: (1) speciation of hydrocarbon exhaust emissions from a light-duty vehicle modified to operate on butane and butane blends; (2) evaluation of NREL's Variable Conductance Vacuum Insulated Catalytic Converter TA-CC4 for the reduction of cold-start FTP exhaust emissions from a Ford FFV Taurus operating on E85 after extended soak periods; (3) support of UDRI in an attempt to define correlations between engine-out combustion products identified by SwRI during chassis dynamometer testing, and those found during flow tube reactor experiments conducted by UDRI; and (4) characterization of small-diameter particulate matter from a Ford Taurus FFV operating in a simulated fuel-rich failure mode on CNG, LPG, M85, E85, and reformulated gasoline.

\section{B. Scope}

The four tasks conducted under this program were fully independent, and will be dealt with in detail in the following sections of this report. A brief overview of each task is given below.

1. Task 1 - Hydrocarbon Speciation of Emissions from a Vehicle Operating on Butane-Blended Fuels

Under this task, which was jointly funded by ARCO Products Company, NREL, and SwRI's Department of Emissions Research, regulated exhaust emissions, speciated hydrocarbon emissions, ozone-forming potentials, and specific reactivity factors were determined for 11 test fuels. These fuels included California Phase 2 gasoline, industry average gasoline (RF-A), $100 \%$ n-propane, $100 \%$ n-butane, and a variety of butane blends. In all, 18 exhaust emissions tests were conducted on a light-duty vehicle, equipped with a gaseous-fuel conversion kit, using the chassis dynamometer portion of the FTP.

\section{Task 2 - Evaluation of a Vacuum Insulated Catalyst in Reducing Cold- Start Exhaust Emissions}

The effectiveness of NREL's Vacuum Insulated Catalytic Converter TA-CC4 in reducing cold-start exhaust emissions after extended soak periods was evaluated in this task. 
Regulated exhaust emissions and estimated NMOG emissions from a light-duty vehicle, operating on a blend of $85 \%$ denatured ethanol and $15 \%$ gasoline and equipped with TA-CC4, were determined utilizing the chassis dynamometer portion of the FTP. Five different combinations of vehicle preconditioning cycles and soak period durations were evaluated.

\section{Task 3 - Technical Support of the UDRI}

Under this task, SwRI provided technical support to UDRI in an attempt to define correlations between engine-out combustion products identified by SwRI during chassis dynamometer testing, and those found during flow tube reactor experiments conducted by UDRI.

\section{Task 4 - Characterization of Exhaust Particulate from Alternative Fuels}

Particulate and regulated gaseous emissions were characterized under this task for a flexible fuel vehicle operating on five different test fuels. These fuels included RFG, M85, E85, LPG meeting HD-5 specifications, and industry-average CNG. The vehicle was operated fuel-rich to simulate a vehicle failure mode for the increased production of particulate matter. Particulate emissions were characterized by total mass and particle size. Ten exhaust emission evaluations were performed using the chassis dynamometer portion of the FTP for light-duty vehicles.

\section{Test Vehicles}

A detailed description of the test vehicles used in Tasks 1,2, and 4 may be found in the appropriate task sections of this report.

\section{Test Fuels}

Details of the specific fuels used in each task of this project can be found in the corresponding task sections of this report.

\section{E. Test Procedures}

\section{Driving Cycle}

All exhaust emissions were evaluated using the chassis dynamometer portion of the FTP for light-duty vehicles as specified in the Code of Federal Regulations, Title 40, Part 86, Subpart B. The FTP utilizes the UDDS, which is 1372 in duration. The UDDS is divided into a 505-second segment and an 867-second segment. An FTP is composed of a 505 coldstart transient portion (Bag 1) and an 867 cold stabilized portion (Bag 2), followed by a 10-min soak and then a 505 hot-start transient portion (Bag 3). A summary of the cycle duration, driving distance, and average speed is given in Table 1. The FTP driving schedule with the cold and hot test segments identified is given in Figure 1. 
Table 1. Summary of FTP Driving Schedule

\begin{tabular}{|l|c|c|c|}
\hline \multicolumn{1}{|c|}{ Segment } & $\begin{array}{c}\text { Duration, } \\
\text { seconds }\end{array}$ & $\begin{array}{c}\text { Distance, } \\
\text { miles }\end{array}$ & $\begin{array}{c}\text { Average Speed, } \\
\text { miles/hr }\end{array}$ \\
\hline \hline Transient phase & 505 & 3.60 & 25.7 \\
\hline Stabilized phase & 867 & 3.90 & 16.2 \\
\hline UDDS & 1372 & 7.50 & 19.7 \\
\hline
\end{tabular}
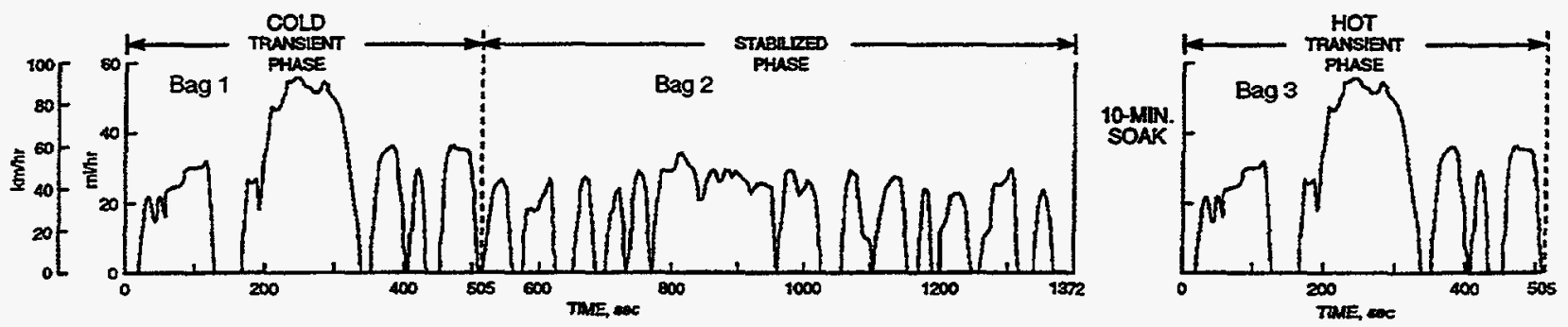

Figure 1. FTP Driving Cycle

\section{Chassis Dynamometer and CVS}

A Clayton Model ECE-50 passenger car dynamometer with a direct drive variable inertia flywheel system was used for all testing. The inertia weight simulates equivalent weights of vehicles from $1,000 \mathrm{lb}$ to $4,875 \mathrm{lb}$ in 125 - $\mathrm{lb}$ increments. A nominal 10 inch diameter by 16-ft long full-flow exhaust dilution tunnel was used in conjunction with a CVS and a positive displacement pump. This unit has a nominal capacity of $325 \mathrm{scfm}$. The filter box at the entrance of the dilution tunnel was equipped with an MSA UItraTM filter to remove small particles, a charcoal filter to absorb background HC, and a MSA Dustfoe ${ }^{\mathrm{TM}}$ Space Filter as a backup filter to collect any particles that pass through the first two filters. The average temperature in the dilution tunnel at the particulate sampling zone was $110^{\circ} \mathrm{F}$, and did not exceed $125^{\circ} \mathrm{F}$ during testing. The vehicle hood was maintained fully open during all cycles and was closed during the soak periods. A cooling fan of 5,000 cfm capacity was used in front of the test vehicle to provide air flow during all tests. During soak periods, the fan was turned off.

\section{F. Emissions Sampling and Analysis Procedures}

\section{Regulated Gaseous Emissions}

THC or OMHCE, $\mathrm{CO}, \mathrm{NO}_{x}$, and $\mathrm{CO}_{2}$ emissions were quantified in a manner consistent with EPA protocols for light-duty emissions testing as given in the Code of Federal Regulations, Title 40, Part 86, Subpart B. $\mathrm{HC}, \mathrm{CO}, \mathrm{NO}_{x}$, and $\mathrm{CO}_{2}$ were sampled using proportional exhaust gas samples collected in Tedlar bags. HC were measured using an FID. $\mathrm{CO}$ and $\mathrm{CO}_{2}$ were determined using NDIR instruments. $\mathrm{NO}_{\mathrm{x}}$ was measured using a chemiluminescent instrument. Wet absorption techniques were employed to collect methanol, ethanol, and aldehydes for the determination of OMHCE. These techniques are discussed in more detail below. 
Methane levels were determined using proportional exhaust gas samples collected in Tedlar bags. A GC equipped with an FID was utilized in accordance with the SAE J1151 procedure to analyze the samples. The GC system was equipped with a packed column to resolve methane from other hydrocarbons in the sample. Samples were introduced into a $5-\mathrm{mL}$ sample loop via a diaphragm pump. For analysis, the valve was switched to the inject position and the helium carrier gas swept the sample from the loop toward the detector through a $61 \mathrm{~cm} \times 0.3 \mathrm{~cm}$ Porapak $\mathrm{N}$ column in series with a $122 \mathrm{~cm} \times 0.3 \mathrm{~cm}$ molecular sieve $13 \mathrm{X}$ column. As soon as the methane peak passed into the molecular sieve column, the helium flow was reversed through the Porapak $N$ column to vent. Peak areas were compared to an external calibration standard.

\section{Particulate Emissions}

Total particulate mass was measured using a 47-mm fluorocarbon-coated glass fiber filter, which collected a proportional exhaust sample from the dilution tunnel. Filters were conditioned and weighed in accordance with the appropriate sections of the CFR for lightduty vehicles. ${ }^{(1)}$ Particle size distribution measurements were performed with a Sierra Series 220 In-Stack Cascade Impactor using a 0.375-in. internal-diameter isokinetic sampling nozzle. ${ }^{(2)}$ Stages 3 through 8 were used to collect particulate mass in equivalent aerodynamic diameter cut-off ranges from about $0.2 \mu \mathrm{m}$ to greater than $3 \mu \mathrm{m}$. The particles were collected on stainless steel substrates. This system uses a patented radial-slot impactor design with four radial rectangular slots $90^{\circ}$ apart. The slots between adjacent stages were $45^{\circ}$ apart. The last stage was followed by a fluorocarbon-coated glass fiber filter to collect particles below $0.2 \mu \mathrm{m}$.

\section{Hydrocarbon Speciation Procedures}

NMOG emissions were determined by hydrocarbon speciation. Analytical procedures for conducting the hydrocarbon speciation $\left(C_{1}\right.$ to $C_{12}$ hydrocarbons, aldehydes and ketones, and alcohols) were similar to the CRC Auto/Oil Phase II methods. With these methods, exhaust samples are analyzed for the presence of more than 190 different exhaust species. The sum of the masses of non-methane species is equivalent to the NMOG emissions rate. Three GC procedures and one HPLC procedure were used to identify and quantify specific compounds. A brief description of these procedures is given below.

\section{a. $\mathbf{C}_{1}-\mathrm{C}_{4}$ Species}

The first GC procedure allowed the separation and determination of exhaust concentrations of $\mathrm{C}_{1}-\mathrm{C}_{4}$ individual hydrocarbon species, including methane; ethane; ethylene; acetylene; propane; propylene; propadiene; butane; trans-2-butene; 1-butene; 2methylpropene; 2,2-dimethylpropane; propyne; 1,3-butadiene; 2-methylpropane; 1-butyne; and cis-2-butene. Bag samples were analyzed using a gas chromatograph equipped with an FID. The gas chromatograph system utilized a Hewlett-Packard Model 5890 Series II GC with an FID, two pneumatically operated and electrically controlled valves, and two analytical columns. The carrier gas was helium. An external multiple component standard in zero air was used to quantify the results. Detection limits for the procedure were on the order of 5 $\mathrm{ppbC}$ in dilute exhaust for all compounds. 


\section{b. $\quad \mathbf{C}_{5}-\mathbf{C}_{12}$ Species}

The second GC procedure provided separation and exhaust concentrations for more than $100 \mathrm{C}_{5}-\mathrm{C}_{12}$ individual $\mathrm{HC}$ compounds. Bag samples were analyzed using a gas chromatograph equipped with an FID. The GC system utilized a Hewlett-Packard Model 5890 Series II GC with an FID, a pneumatically operated and electrically controlled valve, and an analytical column. The carrier gas was helium. An external multiple component standard in zero air was used to quantify the results. Detection limits for the procedure were on the order of $10 \mathrm{ppbC}$ in dilute exhaust for all compounds.

\section{c. Benzene and Toluene}

The third GC procedure used a separate system configured similarly to those mentioned above to determine individual concentrations of benzene and toluene according to the CRC Auto/Oil Phase II Protocol.

\section{d. Aldehydes and Ketones}

An HPLC procedure was utilized for the analysis of aldehydes and ketones. Samples were collected by bubbling dilute exhaust at a nominal flowrate of $4 \mathrm{~L} / \mathrm{min}$ through chilled glass impingers containing an acetonitrile solution of 2,4-DNPH and perchloric acid. For analysis, a portion of the acetonitrile solution was injected into a liquid chromatograph equipped with a UV detector. External standards of the aldehyde and ketone DNPH derivatives were used to quantify the results. The aldehydes and ketones measured were: formaldehyde, acetaldehyde, acrolein, acetone, propionaldehyde, crotonaldehyde, isobutyraldehyde/methylethylketone (not resolved from each other during normal operating conditions, and so reported together), benzaldehyde, and hexanaldehyde. Detection limits for this procedure were on the order of $0.005 \mathrm{ppm}$ aldehyde or ketone in dilute exhaust.

\section{Alcohols}

The collection of methanol and ethanol in exhaust was accomplished by bubbling exhaust through glass impingers. Each impinger contained $25 \mathrm{~mL}$ of deionized water maintained at ice-bath temperature. Exhaust samples were collected continuously during test cycles at a nominal flow rate of $4 \mathrm{~L} / \mathrm{min}$ through a Teflon sample line held at $102^{\circ} \mathrm{C}\left(215^{\circ} \mathrm{F}\right)$. For analysis, a 1- $\mu \mathrm{L}$ portion of the sample was injected into the GC equipped with an FID and an analytical column. The analytical column was a $0.53-\mathrm{mm} \times 30-\mathrm{m}$ capillary column with a 1- $\mu \mathrm{m}$ film of DB-WAX as the stationary phase. The GC carrier gas was helium at a column head pressure of approximately $4 \mathrm{psi}$. The column oven temperature was maintained at $70^{\circ} \mathrm{C}$ for $1 \mathrm{~min}$, then ramped to $110^{\circ} \mathrm{C}$ at $10^{\circ} / \mathrm{min}$, and held at $110^{\circ} \mathrm{C}$ for $5 \mathrm{~min}$. External standards in deionized water were used to quantify the results. Detection limits for this procedure were on the order of $0.06 \mathrm{ppm}$ in dilute exhaust. 


\section{Task 1- Hydrocarbon Speciation of Emissions from a Vehicle Operating on Butane-Blended Fuels}

\section{A. Background}

Butane has been an important gasoline blending component for many years. With a road octane number of 92 and a high blending vapor pressure, butane has been used to upgrade the octane of gasoline blends and to aid in wintertime cold starting. Because of RFG requirements for reduced maximums in fuel vapor pressure, industry has had to remove increasing amounts of butane from the gasoline pool. However, butane has many of the attributes of propane, and tends to be in slight oversupply in the United States. Thus, butane appears to be an attractive candidate for use as an alternative fuel.

LPG currently is the most widely used alternative fuel in the United States. The primary constituent of LPG used for this purpose is propane. Propane has many attributes that make it an attractive vehicular fuel. Because it is a single relatively simple species, engines and aftertreatment systems can be designed to burn it cleanly. It also reacts very slowly in the atmosphere so it tends to form less ground-level ozone in cities. Propane is also stored on board as a liquid at ambient temperature in a pressurized system; therefore, by definition, it produces no evaporative emissions. As a liquid, it has a good volumetric energy content, improving vehicle range in comparison to many of the other alternative fuels.

However, barriers exist that may inhibit the growth of propane as a motor fuel. There are other developed, mature markets for propane such as its use as a heating fuel or as a chemical feedstock. Propane is produced as a side product from natural gas production or petroleum refinery processing. Additional demand could create shortages and/or price increases, because it is unlikely that either of these two sources would be ramped up solely to produce more propane. Although vehicle range is good, it remains three-fourths that of conventional gasoline vehicles. These constraints have limited LPG vehicle implementation.

A thoughtful consideration of the use of butane as a motor fuel shows that it should have many of the positive attributes of propane. Butane is also a single, relatively simple chemical species, so theoretically, engines and exhaust catalysts could be optimized for low emissions with its use. It also has relatively low reactivity in the atmosphere, and like propane, would be stored on board as a pressurized liquid. Consequently, a butane-fueled vehicle should also have no evaporative emissions. Butane has a greater volumetric energy content than propane, so vehicle range could be extended over that of propane. Because butane storage pressure is lower than that of propane, there is an opportunity to use the same equipment for delivery and refueling of butane as is currently used for propane.

Butane tends to be in slight oversupply on a yearly basis in the United States, and this excess should grow larger as gasoline volatility is reduced. The imbalance is projected to equal as much as 100,000 barrels per day this year and increase by $20 \%$ by the year $2000 .^{(3)}$ Although butane is used as a chemical feedstock, it has not been used extensively as a heating fuel as has propane. Like propane, butane production is associated with natural gas production and petroleum refining. However, because of current configurations for refining reformulated gasoline, it is easier to increase the amount of butane from the refinery stream than it is for propane. This flexibility could allow butane supply to more easily balance demand than propane. 


\section{B. Objectives}

In order to investigate the use of butane as a vehicular fuel, ARCO Products Company (ARCO), NREL, and SwRI jointly conducted a project with the following objectives: (1) determine if a current technology vehicle could be properly operated on butane or on butaneblended fuels using currently available LPG conversion kit technology; (2) determine regulated FTP emissions from a "converted" vehicle operating on gasoline, LPG, and butane; (3) determine regulated FTP emissions from the conversion vehicle operating on various butane blends that could potentially be distributed as real world fuels; (4) conduct detailed hydrocarbon speciation analyses on FTP-generated exhaust from the converted vehicle operating on each fuel, to allow calculation of fuel-specific OFPs using MIRs adopted by $\mathrm{CARB}^{(4)}$; and (5) use the calculated OFPs to determine a specific reactivity and RAF for each fuel tested ${ }^{(4)}$.

\section{Approach}

The vehicle selected for this program was a 1994 Pontiac Grand Am, California emission-certified to be a TLEV. This vehicle was chosen because of its widespread distribution, its availability, and because it was representative of current technology. Prior to converting the vehicle to operate on butane, it was subjected to 1,000 miles of operation over the Modified Durability Driving Schedule to provide a limited amount of vehicle break-in and to stabilize the catalyst. Following mileage accumulation, emission tests were conducted using CP2 gasoline to ensure proper vehicle operation and to obtain baseline emission data prior to vebicle conversion.

Next, the vehicle was converted by IMPCO Technologies, Inc. to operate in a dual-fuel mode using gasoline or butane/LPG. Baseline emission tests were again conducted using California Phase 2 gasoline to ensure that the vehicle was operating in the same manner as before the conversion. In addition, emissions tests were conducted using RF-A gasoline, a fuel used by CARB as the reference point for determining RAFs. Subsequently, vehicle emission tests were conducted using $100 \% \mathrm{n}$-propane, and without any fuel control system adjustments, 100\% n-butane. These emissions data were then used as a comparison to reactivity data currently available from CARB for LPG fuel and for CP2. In addition, data were also used to determine if vehicles operating on propane could be operated on butane (without modification or adjustment of any kind) and still provide acceptable emissions. Following these initial emission tests, the converted vehicle was operated on a variety of butane blends, which included mixtures with propane, other $\mathrm{C}_{4}$ components, and pentane. During the course of testing, the vehicle was again tested on CP2 and 100\% n-butane to ensure that no drift in emission levels had occurred. To ensure that the vehicle adaptive learning system was stable, an extended vehicle prep cycle was run after each fuel change. This extended prep cycle was similar to that used in the CRC Auto/Oil Research Program.

\section{Vehicle Description and Conversion}

The vehicle used for testing in this program was a 1994 Pontiac Grand Am equipped with a California emissions system, and certified to be a TLEV. A more detailed description of the vehicle is provided in Table 2. 
Table 2. Vehicle Description

\begin{tabular}{||l|l||}
\hline \multicolumn{1}{|c|}{ Item } & \multicolumn{1}{|c|}{ Configuration of Pontiac Grand Am } \\
\hline \hline Model year & 1994 \\
\hline Body style & 4-door sedan \\
\hline Transmission & Automatic-4 \\
\hline VIN & 1 G2NE1538RM542537 \\
\hline Tires & P185/75 R14 \\
\hline Accessories & $\begin{array}{l}\text { Power locks, steering, brakes, air } \\
\text { conditioning }\end{array}$ \\
\hline Engine family & R1G2.3V7G2EA \\
\hline Engine displacement & $2.3 \mathrm{~L}$ (138 CID) \\
\hline Number of cylinders & 4 \\
\hline Fuel system & MPFI \\
\hline $\begin{array}{l}\text { Ignition system } \\
\text { Emission control system }\end{array}$ & $\begin{array}{l}\text { Underbody three-way catalyst, EGR, } \\
\text { knock sensor }\end{array}$ \\
\hline $\begin{array}{l}\text { Chassis dynamometer: } \\
\text { Inertia setting } \\
\text { Road load @ 50 mph }\end{array}$ & $\begin{array}{l}1530 \mathrm{~kg}(3375 \mathrm{lb}) \\
4.9 \mathrm{~kW} \text { (6.6 hp) }\end{array}$ \\
\hline \hline
\end{tabular}

The vehicle was converted to operate on butane for this project by IMPCO Technologies, Inc. using commercially available equipment. Conversion equipment included an ADP controller and a carburetion system consisting of a carburetor and converter. The IMPCO $\mathrm{ADP}$ is a closed-loop fuel controller designed for use with LPG, CNG, or LNG. It incorporates an engine MAP sensor and engine speed input. Feedback information from the EGO sensor is used to update the digital block learn memory. This strategy is similar to fuel control strategies used by OEMs. The carburetor is an air valve design that utilizes a relatively constant pressure drop to draw fuel into the system. The air metering device (or mixer) is selfcontained (i.e., it requires no idle vacuum line to the intake manifold). The converter is a combined two-stage regulator and vaporizer that uses engine coolant circulated through a heat exchanger to assist fuel vaporization ${ }^{(5)}$. During emission testing using the IMPCO system, the alternative fuels were supplied as liquids from an external tank to the vaporizer. To maintain sufficient fuel pressure, a heating blanket was used to elevate the tank temperature. 


\section{E. Test Fuels}

Eleven fuels were used in this project. Two of the fuels were gasolines: CP2 and RF-A (see Table 3). The remaining nine fuels were either single components (propane or butane), or were butane blends prepared to cover a broad range of LPG that could potentially be marketed. These nine fuels were nominally $100 \% \mathrm{n}$-butane, $100 \%$ propane, $90 \% \mathrm{n}$-butane/10\% isobutane, $90 \% \mathrm{n}$-butane/10\% mixed butenes (roughly equal amounts of isobutylene, 1-butene, cis-2-butene, and trans-2-butene), 60\% n-butane/40\% isobutane, $80 \%$ n-butane/20\% propane, $90 \%$ n-butane $/ 10 \%$ pentane, $50 \%$ n-butane $/ 50 \%$ propane, and $20 \%$ n-butane $/ 80 \%$ propane. A summary of the composition of each of these fuels is given in Table 4.

\section{F. Emission Test Procedures}

The Pontiac Grand Am was evaluated using the chassis dynamometer portion of the FTP for light-duty vehicles. A Clayton Model ECE-50 passenger car dynamometer in conjunction with a positive displacement pump constant volume sampler was used for all testing. THC, $\mathrm{CO}, \mathrm{NO}_{\mathrm{x}}$, and $\mathrm{CO}_{2}$ emissions were collected as dilute exhaust in Tedlar gas sample bags, in a manner consistent with EPA protocols for light-duty emission testing. Fuel economy was determined by carbon balance. Hydrocarbon speciation analysis and determination of OFP and specific reactivities were conducted in a manner consistent with Auto/Oil Phase 2 procedures and CARB methodologies. A more detailed description of these procedures is presented in Sections I.E and I.F.

\section{G. Emission Test Results}

\section{Baseline Emissions}

To establish baseline FTP emissions prior to converting the vehicle to operate in a dual-fuel mode (i.e., either gasoline or alternative fuels) and to ensure that the vehicle was operating properly after conversion and throughout the project, duplicate FTPs on CP2 and $100 \% \mathrm{n}$-butane were conducted. Complete hydrocarbon speciation was conducted for one of the duplicate tests. Table 5 contains a summary of average bag 1 , bag 2 , bag 3 , and composite FTP emissions. Included in this summary are values representing the average of the duplicate test points for THC by FID, CO, $\mathrm{NO}_{\mathrm{x}}$, and fuel economy. Values given for NMOG emissions were determined for one of the two tests (i.e., not an average) using hydrocarbon speciation data up to $\mathrm{C}_{12}$. Based on data presented in Table 5, the conversion did not appear to significantly change vehicle emissions or performance. Furthermore, emission test results for CP2 run during the project appeared similar to the pre-conversion and post-conversion results, suggesting that the vehicle performance remained consistent throughout the project. 
Table 3. Summary of RF-A and CP2 Fuel Properties

\begin{tabular}{|l|c|c|}
\hline \multicolumn{1}{|c|}{ Fuel Property } & RF-A & CP2 \\
\hline RVP, psi & 9.0 & 6.8 \\
\hline HC Composition, vol \% & & \\
Aromatics & 32.9 & 26.6 \\
Olefins & 8.3 & 5.3 \\
Saturates & 58.8 & 68.1 \\
\hline MON & 83.7 & 88.1 \\
\hline RON & 92.9 & 97.5 \\
\hline Distillation temp., ${ }^{\circ}$ F & & \\
IBP & 98 & 90 \\
10\% & 131 & 137 \\
$50 \%$ & 219 & 208 \\
90\% & 329 & 292 \\
EP & 414 & 380 \\
\hline Oxygenate, vol \% & 0.0 & $11.2^{\mathrm{a}}$ \\
\hline Benzene, vol \% & 1.4 & 0.8 \\
\hline Sulfur, ppm & 348 & 39 \\
\hline Specific gravity & 0.7461 & 0.7385 \\
\hline Carbon, wt. \% & 86.4 & 84.3 \\
Hydrogen, wt. \% & 13.6 & 13.7 \\
Oxygen, wt. \% & 0.0 & 2.0 \\
\hline \hline a MTBE & \multicolumn{2}{|l|}{} \\
\hline
\end{tabular}

Table 4. Summary of Alternative Fuel Compositions

\begin{tabular}{||l|c|c|c|c||}
\hline \multirow{2}{*}{ Nominal Fuel Blend } & \multicolumn{4}{c|}{ Analysis Results, mole percent } \\
\cline { 2 - 5 } & Propane & n-Butane & Isobutane & Other \\
\hline \hline $100 \%$ n-butane & nd & 100.0 & nd & nd \\
\hline $100 \%$ propane & 100.0 & nd & nd & nd \\
\hline $90 \%$ n-butane/10\% isobutane & nd & 90.3 & 9.7 & nd \\
\hline $90 \%$ n-butane/10\% mixed butenes & nd & 89.3 & nd & $10.7^{\text {a }}$ \\
\hline $60 \%$ n-butane/40\% isobutane & 0.1 & 60.9 & 39.0 & nd \\
\hline $80 \%$ n-butane/20\% propane & 22.2 & 77.8 & nd & nd \\
\hline $90 \%$ n-butane/10\% pentane & nd & 91.2 & nd & $8.8^{b}$ \\
\hline $50 \%$ n-butane/50\% propane & 53.4 & 46.6 & nd & nd \\
\hline $20 \%$ n-butane/80\% propane & 82.1 & 17.9 & nd & nd \\
\hline $\begin{array}{l}\text { nd - none detected } \\
\text { a 2.6\% isobutylene, 2.6\% 1-butene, 2.8\% cis-2-butene, 2.7\% trans-2-butene }\end{array}$ \\
\hline \begin{tabular}{l} 
bn-pentane \\
\hline
\end{tabular}
\end{tabular}


Table 5. Summary of Pre- and Post-Conversion Average Regulated Emissions

\begin{tabular}{|c|c|c|c|c|c|c|}
\hline \multirow[b]{2}{*}{ Fuel } & \multirow{2}{*}{$\begin{array}{l}\text { Test } \\
\text { Cycle } \\
\end{array}$} & \multicolumn{4}{|c|}{ Average Emissions, $\mathrm{g} / \mathrm{mi}$} & \multirow{2}{*}{$\begin{array}{c}\text { Fuel } \\
\text { Economy } \\
\text { mi/gal }\end{array}$} \\
\hline & & THC & NMOG $^{\mathbf{a}}$ & co & $\mathrm{NO}_{x}$ & \\
\hline $\begin{array}{c}\mathrm{CP2} \\
\text { (pre-conversion) }\end{array}$ & $\begin{array}{l}\text { Bag } 1 \\
\text { Bag } 2 \\
\text { Bag } 3 \\
\text { FTP } \\
\end{array}$ & $\begin{array}{l}0.273 \\
0.008 \\
0.053 \\
0.075 \\
\end{array}$ & $\begin{array}{l}0.227 \\
0.007 \\
0.038 \\
0.061 \\
\end{array}$ & $\begin{array}{l}2.795 \\
0.246 \\
0.736 \\
0.910 \\
\end{array}$ & $\begin{array}{l}0.200 \\
0.002 \\
0.017 \\
0.047 \\
\end{array}$ & $\begin{array}{l}21.87 \\
20.78 \\
24.27 \\
21.89 \\
\end{array}$ \\
\hline $\begin{array}{c}\text { CP2 } \\
\text { (post-conversion) }\end{array}$ & $\begin{array}{l}\text { Bag } 1 \\
\text { Bag } 2 \\
\text { Bag } 3 \\
\text { FTP } \\
\end{array}$ & $\begin{array}{l}0.248 \\
0.011 \\
0.043 \\
0.069 \\
\end{array}$ & $\begin{array}{l}0.196 \\
0.009 \\
0.025 \\
0.052 \\
\end{array}$ & $\begin{array}{l}2.438 \\
0.109 \\
0.380 \\
0.668 \\
\end{array}$ & $\begin{array}{l}0.275 \\
0.016 \\
0.022 \\
0.072 \\
\end{array}$ & $\begin{array}{l}21.16 \\
20.81 \\
24.27 \\
21.76 \\
\end{array}$ \\
\hline $\begin{array}{c}\text { CP2 } \\
\text { (during testing) }\end{array}$ & $\begin{array}{l}\text { Bag } 1 \\
\text { Bag } 2 \\
\text { Bag } 3 \\
\text { FIP }\end{array}$ & $\begin{array}{l}0.238 \\
0.005 \\
0.036 \\
0.062 \\
\end{array}$ & $\begin{array}{l}0.194 \\
0.004 \\
0.023 \\
0.049\end{array}$ & $\begin{array}{l}2.711 \\
0.085 \\
0.453 \\
0.734\end{array}$ & $\begin{array}{l}0.239 \\
0.025 \\
0.027 \\
0.070\end{array}$ & $\begin{array}{l}21.70 \\
21.09 \\
24.79 \\
22.14\end{array}$ \\
\hline
\end{tabular}

\section{Candidate Fuel Emissions}

Following vehicle conversion and testing using CP2, duplicate FTP tests were conducted using each of the remaining ten fuels with the OEM catalytic converters still installed on the vehicle. In addition, duplicate emission tests were also conducted without the catalysts to determine engine-out emissions using $100 \% \mathrm{n}$-butane, $100 \%$ propane, $50 \% \mathrm{n}$ butane $/ 50 \%$ propane, and CP2. Complete hydrocarbon speciation was conducted for one of each of the duplicate tests. Table 6 contains a summary of average bag 1 , bag 2 , bag 3 , and composite FTP emissions for each configuration and fuel tested following vehicle conversion. Individual FTP regulated emission test results for all emission tests conducted during this project are given in Appendix A.

Several observations can be made based on an analysis of the regulated emissions data (summarized in Table 6) with the exhaust system in the OEM configuration (i.e., with catalysts). FTP THC emission rates for all fuels tested (including CP2 and RF-A) ranged between about 0.06 and $0.08 \mathrm{~g} / \mathrm{mi}$. FTP NMOG emissions for CP2 $(0.052 \mathrm{~g} / \mathrm{mi})$ were slightly lower than those for RF-A $(0.054 \mathrm{~g} / \mathrm{mi})$, but both were still equal to or lower than several of the alternative fuels before adjustment for reactivity was taken into account. For the alternative fuels, FTP NMOG emissions (non-reactivity adjusted) were lowest for the 100\% propane $(0.042 \mathrm{~g} / \mathrm{mi})$ and highest for the $100 \% \mathrm{n}$-butane $(0.066 \mathrm{~g} / \mathrm{mi})$. FTP CO emissions were lowest for the $100 \%$ propane fuel $(0.319 \mathrm{~g} / \mathrm{mi})$ and were highest for the $90 \% \mathrm{n}$-butane $/ 10 \% \mathrm{n}$ pentane blend (2.052 g/mi). FTP $\mathrm{NO}_{\mathrm{x}}$ emissions for all of the alternative fuels except $100 \%$ propane ranged between 0.011 and $0.017 \mathrm{~g} / \mathrm{mi}$. However, the FTP $\mathrm{NO}_{\mathrm{x}}$ emissions for the $100 \%$ propane fuel $(0.047 \mathrm{~g} / \mathrm{mi})$ remained lower than the $\mathrm{NO}_{\mathrm{x}}$ emissions for $\mathrm{CP} 2(0.072 \mathrm{~g} / \mathrm{mi})$ and $\mathrm{RF}$ $A(0.071 \mathrm{~g} / \mathrm{mi})$.

Based on the Auto/Oil method of calculating equivalence ratio ${ }^{(6)}$, it appears that the converted vehicle was operating slightly rich of stoichiometric for all of the alternative fuels except $100 \%$ propane. This observation is consistent with the higher CO emissions 
Table 6. Summary of Average Regulated Emissions Data

\begin{tabular}{|c|c|c|c|c|c|c|}
\hline \multirow[b]{2}{*}{ Fuel } & \multirow{2}{*}{$\begin{array}{l}\text { Test } \\
\text { Cycle }\end{array}$} & \multicolumn{4}{|c|}{ Average Emissions, $\mathrm{g} / \mathrm{mi}$} & \multirow{2}{*}{$\begin{array}{c}\text { Fuel } \\
\text { Economy } \\
\text { mi/gal }\end{array}$} \\
\hline & & THC & $\mathrm{NMOG}^{\mathrm{a}}$ & $\mathrm{CO}$ & $\mathrm{NO}_{\mathbf{x}}$ & \\
\hline $\begin{array}{c}\mathrm{CP} 2 \\
\text { (after conversion) }\end{array}$ & $\begin{array}{l}\text { Bag } 1 \\
\mathrm{Bag} 2 \\
\mathrm{Bag} 3 \\
\text { FTP }\end{array}$ & $\begin{array}{l}0.248 \\
0.011 \\
0.043 \\
\mathbf{0 . 0 6 9} \\
\end{array}$ & $\begin{array}{l}0.196 \\
0.009 \\
0.025 \\
0.052 \\
\end{array}$ & $\begin{array}{l}2.438 \\
0.109 \\
0.380 \\
0.668 \\
\end{array}$ & $\begin{array}{l}0.275 \\
0.016 \\
0.022 \\
\mathbf{0 . 0 7 2} \\
\end{array}$ & $\begin{array}{l}21.16 \\
20.81 \\
24.27 \\
21.76 \\
\end{array}$ \\
\hline RF-A & $\begin{array}{l}\text { Bag } 1 \\
\text { Bag } 2 \\
\text { Bag 3 } \\
\text { FTP }\end{array}$ & $\begin{array}{l}0.261 \\
0.010 \\
0.045 \\
0.072\end{array}$ & $\begin{array}{l}0.209 \\
0.006 \\
0.031 \\
0.054\end{array}$ & $\begin{array}{l}2.333 \\
0.185 \\
0.507 \\
0.721\end{array}$ & $\begin{array}{l}0.304 \\
0.003 \\
0.023 \\
0.071\end{array}$ & $\begin{array}{l}21.84 \\
21.01 \\
24.52 \\
22.06\end{array}$ \\
\hline $100 \%$ n-Butane & $\begin{array}{l}\text { Bag } 1 \\
\text { Bag } 2 \\
\text { Bag } 3 \\
\text { FTP }\end{array}$ & $\begin{array}{l}0.273 \\
0.009 \\
0.074 \\
0.081\end{array}$ & $\begin{array}{l}0.251 \\
0.002 \\
0.044 \\
\mathbf{0 . 0 6 5}\end{array}$ & $\begin{array}{l}4.237 \\
0.695 \\
2.015 \\
1.787 \\
\end{array}$ & $\begin{array}{l}0.052 \\
0.002 \\
0.002 \\
0.012\end{array}$ & $\begin{array}{l}17.43 \\
16.54 \\
19.91 \\
17.56\end{array}$ \\
\hline $100 \%$ Propane & $\begin{array}{l}\text { Bag } 1 \\
\text { Bag } 2 \\
\text { Bag 3 } \\
\text { FTP }\end{array}$ & $\begin{array}{l}0.221 \\
0.009 \\
\underline{0.025} \\
0.057\end{array}$ & $\begin{array}{l}0.174 \\
0.004 \\
0.012 \\
0.042\end{array}$ & $\begin{array}{l}0.800 \\
0.166 \\
\underline{0.248} \\
0.319\end{array}$ & $\begin{array}{l}0.098 \\
0.015 \\
\underline{0.066} \\
0.047\end{array}$ & $\begin{array}{l}16.45 \\
15.49 \\
18.85 \\
16.52\end{array}$ \\
\hline $\begin{array}{l}90 \% \text { n-Butane } \\
10 \% \text { lsobutane }\end{array}$ & $\begin{array}{l}\text { Bag } 1 \\
\text { Bag } 2 \\
\text { Bag } 3 \\
\text { FTP }\end{array}$ & $\begin{array}{l}0.260 \\
0.007 \\
\underline{0.049} \\
\mathbf{0 . 0 7 1}\end{array}$ & $\begin{array}{l}0.181 \\
0.002 \\
0.018 \\
0.044\end{array}$ & $\begin{array}{l}4.603 \\
0.724 \\
1.239 \\
1.661\end{array}$ & $\begin{array}{l}0.032 \\
0.001 \\
0.009 \\
0.010\end{array}$ & $\begin{array}{l}17.81 \\
16.68 \\
20.58 \\
17.86\end{array}$ \\
\hline $\begin{array}{c}90 \% \text { n-Butane } \\
10 \% \text { Mixed Butenes }\end{array}$ & $\begin{array}{l}\text { Bag } 1 \\
\text { Bag } 2 \\
\text { Bag } 3 \\
\text { FTP }\end{array}$ & $\begin{array}{l}0.233 \\
0.008 \\
0.053 \\
0.067 \\
\end{array}$ & $\begin{array}{l}0.174 \\
0.003 \\
0.031 \\
0.045 \\
\end{array}$ & $\begin{array}{l}3.838 \\
0.707 \\
1.462 \\
1.547 \\
\end{array}$ & $\begin{array}{l}0.049 \\
0.002 \\
0.001 \\
\mathbf{0 . 0 1 1} \\
\end{array}$ & $\begin{array}{l}17.95 \\
17.26 \\
20.39 \\
18.17 \\
\end{array}$ \\
\hline $\begin{array}{l}60 \% \text { n-Butane } \\
40 \% \text { Isobutane }\end{array}$ & $\begin{array}{l}\text { Bag 1 } \\
\text { Bag } 2 \\
\text { Bag 3 } \\
\text { FTP } \\
\end{array}$ & $\begin{array}{l}0.242 \\
0.020 \\
0.059 \\
0.077 \\
\end{array}$ & $\begin{array}{l}0.198 \\
0.007 \\
\underline{0.033} \\
\mathbf{0 . 0 5 4} \\
\end{array}$ & $\begin{array}{l}4.051 \\
0.739 \\
1.321 \\
1.583 \\
\end{array}$ & $\begin{array}{l}0.045 \\
0.000 \\
0.002 \\
0.010 \\
\end{array}$ & $\begin{array}{l}17.38 \\
16.50 \\
20.06 \\
17.56 \\
\end{array}$ \\
\hline $\begin{array}{l}80 \% \text { n-Butane } \\
20 \% \text { Propane }\end{array}$ & $\begin{array}{l}\text { Bag } 1 \\
\text { Bag } 2 \\
\text { Bag } 3 \\
\text { FTP }\end{array}$ & $\begin{array}{l}0.224 \\
0.006 \\
0.075 \\
0.071\end{array}$ & $\begin{array}{l}0.207 \\
0.002 \\
0.052 \\
0.058\end{array}$ & $\begin{array}{l}3.093 \\
0.433 \\
1.467 \\
1.272 \\
\end{array}$ & $\begin{array}{l}0.051 \\
0.003 \\
0.004 \\
\mathbf{0 . 0 1 3} \\
\end{array}$ & $\begin{array}{l}17.51 \\
16.57 \\
20.16 \\
17.65\end{array}$ \\
\hline $\begin{array}{l}90 \% \text { n-Butane } \\
10 \% \text { n-Pentane }\end{array}$ & $\begin{array}{l}\text { Bag } 1 \\
\text { Bag } 2 \\
\text { Bag 3 } \\
\text { FTP } \\
\end{array}$ & $\begin{array}{l}0.270 \\
0.007 \\
0.066 \\
0.078 \\
\end{array}$ & $\begin{array}{l}0.194 \\
0.005 \\
0.030 \\
0.051 \\
\end{array}$ & $\begin{array}{l}5.683 \\
0.730 \\
\underline{1.800} \\
2.052 \\
\end{array}$ & $\begin{array}{l}0.045 \\
0.015 \\
0.017 \\
0.017 \\
\end{array}$ & $\begin{array}{l}17.80 \\
16.94 \\
20.80 \\
18.03 \\
\end{array}$ \\
\hline
\end{tabular}


Table 6 (Concluded). Summary of Average Regulated Emissions Data

\begin{tabular}{|c|c|c|c|c|c|c|}
\hline \multirow[b]{2}{*}{ Fuel } & \multirow{2}{*}{$\begin{array}{l}\text { Test } \\
\text { Cycle }\end{array}$} & \multicolumn{4}{|c|}{ Average Emissions, $g / \mathrm{mi}$} & \multirow{2}{*}{$\begin{array}{c}\text { Fuel } \\
\text { Economy } \\
\text { mi/gal }\end{array}$} \\
\hline & & THC & NMOG $^{\mathrm{a}}$ & Co & $\mathrm{NO}_{x}$ & \\
\hline $\begin{array}{l}50 \% \text { n-Butane } \\
50 \% \text { Propane }\end{array}$ & \begin{tabular}{|l} 
Bag 1 \\
$\mathrm{Bag} 2$ \\
$\mathrm{Bag} 3$ \\
FTP \\
\end{tabular} & $\begin{array}{l}0.227 \\
0.004 \\
0.056 \\
0.065 \\
\end{array}$ & $\begin{array}{l}0.182 \\
0.004 \\
0.036 \\
0.050 \\
\end{array}$ & $\begin{array}{l}1.241 \\
0.350 \\
0.769 \\
0.651 \\
\end{array}$ & $\begin{array}{l}0.068 \\
0.004 \\
0.005 \\
0.017 \\
\end{array}$ & $\begin{array}{l}16.91 \\
15.82 \\
19.63 \\
16.97 \\
\end{array}$ \\
\hline $\begin{array}{l}20 \% \text { n-Butane } \\
80 \% \text { Propane }\end{array}$ & \begin{tabular}{|l} 
Bag 1 \\
Bag 2 \\
Bag 3 \\
FTP
\end{tabular} & $\begin{array}{l}0.205 \\
0.007 \\
0.039 \\
\mathbf{0 . 0 5 8}\end{array}$ & $\begin{array}{l}0.197 \\
0.005 \\
0.018 \\
0.049\end{array}$ & $\begin{array}{l}1.278 \\
0.365 \\
0.591 \\
0.619\end{array}$ & $\begin{array}{l}0.088 \\
0.021 \\
\underline{0.030} \\
0.036\end{array}$ & $\begin{array}{l}16.17 \\
15.32 \\
18.69 \\
16.33 \\
\end{array}$ \\
\hline $\begin{array}{c}100 \% \text { n-Butane } \\
\text { (second set of runs) }\end{array}$ & $\begin{array}{l}\text { Bag } 1 \\
\text { Bag } 2 \\
\text { Bag } 3 \\
\text { FTP }\end{array}$ & $\begin{array}{l}0.257 \\
0.003 \\
0.028 \\
0.072\end{array}$ & $\begin{array}{l}0.184 \\
0.001 \\
0.030 \\
0.047 \\
\end{array}$ & $\begin{array}{l}5.401 \\
0.697 \\
1.757 \\
1.984 \\
\end{array}$ & $\begin{array}{l}0.041 \\
0.006 \\
0.015 \\
0.016 \\
\end{array}$ & $\begin{array}{l}17.64 \\
16.84 \\
20.52 \\
17.91 \\
\end{array}$ \\
\hline \multicolumn{7}{|c|}{ Engine-out (no catalyst) Emissions } \\
\hline $100 \%$ n-Butane & $\begin{array}{l}\text { Bag } 1 \\
\text { Bag } 2 \\
\text { Bag } 3 \\
\text { FTP } \\
\end{array}$ & $\begin{array}{l}1.244 \\
1.082 \\
0.975 \\
1.086 \\
\end{array}$ & $\begin{array}{l}1.274 \\
1.066 \\
0.942 \\
1.075 \\
\end{array}$ & $\begin{array}{l}16.021 \\
11.413 \\
12.300 \\
12.623 \\
\end{array}$ & $\begin{array}{l}4.541 \\
3.680 \\
4.403 \\
4.061 \\
\end{array}$ & $\begin{array}{l}17.45 \\
16.83 \\
20.41 \\
17.85 \\
\end{array}$ \\
\hline $100 \%$ Propane & $\begin{array}{l}\text { Bag } 1 \\
\text { Bag } 2 \\
\text { Bag } 3 \\
\text { FTP } \\
\end{array}$ & $\begin{array}{l}1.015 \\
0.942 \\
0.795 \\
\mathbf{0 . 9 1 5} \\
\end{array}$ & $\begin{array}{l}0.969 \\
0.915 \\
\underline{0.761} \\
\mathbf{0 . 8 8 4} \\
\end{array}$ & $\begin{array}{l}7.946 \\
7.803 \\
\mathbf{8 . 0 2 2} \\
\mathbf{7 . 8 8 2} \\
\end{array}$ & $\begin{array}{r}4.331 \\
3.672 \\
4.223 \\
3.958 \\
\end{array}$ & $\begin{array}{l}16.56 \\
15.46 \\
18.17 \\
16.37 \\
\end{array}$ \\
\hline $\begin{array}{l}50 \% \text { n-Butane } \\
50 \% \text { Propane }\end{array}$ & $\begin{array}{l}\text { Bag } 1 \\
\text { Bag } 2 \\
\text { Bag } 3 \\
\text { FTP }\end{array}$ & $\begin{array}{l}1.067 \\
1.025 \\
0.836 \\
0.951\end{array}$ & $\begin{array}{l}1.070 \\
0.971 \\
0.834 \\
0.954\end{array}$ & $\begin{array}{l}9.662 \\
9.187 \\
9.280 \\
9.281\end{array}$ & $\begin{array}{l}4.316 \\
3.707 \\
4.210 \\
3.961\end{array}$ & $\begin{array}{l}17.47 \\
16.51 \\
19.96 \\
17.56\end{array}$ \\
\hline
\end{tabular}

measured for all other alternative fuels compared to $100 \%$ propane. However, an inspection of the results indicates that rich operation accounts for only some of the extremely low $\mathrm{NO}_{\mathrm{x}}$ results observed for the higher concentration butane fuels. While equivalence ratio accounts for nearly all of the variation in $\mathrm{CO}$ emissions for these fuels, $\mathrm{NO}_{\mathbf{x}}$ emissions are correlated much less strongly.

It also appears that the volatility of the alternative fuels may have played a role in bag 1 and bag 3 FTP THC emissions. In general, as fuel volatility increased, start-up emissions decreased. This observation is logical because the IMPCO alternative fuel management system uses engine coolant to vaporize the fuel. Less volatile fuels would, therefore, be less likely to fully vaporize during the start-up phases of the FTP when the engine coolant is not as hot. Because catalyst light-off times should not vary significantly for similar fuels, this fuel volatility hypothesis could partially explain the greater amount of unburned fuel emissions found in bags 1 and 3 with the lower volatility fuels. 
As expected, fuel economy of each of the alternative fuels was related to its respective liquid density and heat of combustion (energy content). The average FTP fuel economy for the $100 \% \mathrm{n}$-butane fuel (17.56 mi/gal) was $81 \%$ that of CP2 (21.76 mi/gal). Fuel economy for the $100 \%$ propane fuel $(16.52 \mathrm{mi} / \mathrm{gal})$ was $76 \%$ that of CP2. All of the other alternative fuels had FTP fuel economies within this range.

\section{Toxic Emissions}

The Clean Air Act Amendments of 1990 define four toxics of importance for gasoline vehicles. They are 1,3-butadiene, benzene, formaldehyde, and acetaldehyde. Table 7 provides bag 1 , bag 2 , bag 3 , and composite FTP emission rates for these compounds for each fuel tested. The last column of Table 7 is the summed total of all toxics emitted for each fuel.

None of the butane fuels produced higher FTP levels of 1,3-butadiene, including the $90 \% \mathrm{n}$-butane $10 \%$ mixed butenes blend, than the gasolines. The $90 \% / 10 \%$ mixed butenes blend did produce slightly higher bag 1 emissions of 1,3 -butadiene $(0.4 \mathrm{mg} / \mathrm{mi})$ than the other alternative fuels $(0.2 \mathrm{mg} / \mathrm{mi}$ or less), but no significant amounts of 1,3 -butadiene were detected in bags 2 or 3 . For the gasoline fuels, FTP emissions of 1,3-butadiene were three to four times those of the $90 \%$ n-butane/10\% mixed butenes blend.

As might be expected, FTP benzene emissions for the alternative fuels were very low (0.1 to $0.3 \mathrm{mg} / \mathrm{mi}$ ) compared to benzene emissions for CP2 or RF-A (2.4 and $3.7 \mathrm{mg} / \mathrm{mi}$, respectively). Formaldehyde and acetaldehyde emissions were similar for all of the gasoline and alternative fuels tested in the OEM exhaust configuration. Engine-out formaldehyde emissions, however, were high for the alternative fuels, indicating that it is a significant combustion product. On average, FTP total toxics were roughly three to four times higher for the gasoline fuels compared to the alternative fuels. It is worth noting that the levels of toxic emissions observed during this study were extremely low for all fuels evaluated, including the gasolines, when the vehicle was in the OEM exhaust configuration.

\section{Speciated Hydrocarbon Emissions}

Each hydrocarbon speciation conducted in this project resulted in a five-page table of individual organics emitted, from methane through $\mathrm{C}_{12}$ compounds (see Appendix B). For gasolines, many of the 190 compounds analyzed were detected at measurable levels. For the alternative fuels, comparatively few compounds were found. To allow for a comparison of emissions on the basis of OFP, CARB has published a list of MIRs for a number of VOCs, defined as grams of ozone per gram of specific VOC emitted.(4) These MIRs were either estimated by CARB or derived from smog chamber experiments based on Los Angeles atmospheric conditions. Each MIR can be multiplied by the mass emission rate of that compound to yield potential mass of ozone $\left(\mathrm{O}_{3}\right)$ formed. The summation of these products yields the OFP for a specific fuel (i.e., $\mathrm{g} \mathrm{O}_{3} / \mathrm{mi}=\sum\left(\mathrm{VOC}_{\mathrm{i}} \times \mathrm{MIR}_{\mathrm{i}}\right)$ ). Both the masses of the individual organics and the potential masses of ozone formed were summed over all the compounds emitted during each test.

Values for NMOG, calculated ozone formed, specific reactivity, RAFs, and reactivity-adjusted NMOG for each fuel combination are summarized in Table 8. RAFs and reactivity-adjusted NMOG emissions have not been given for tests conducted without a 
Table 7. Summary of Toxic Emissions Data

\begin{tabular}{|c|c|c|c|c|c|}
\hline \multirow[b]{2}{*}{ Fuel } & \multirow[b]{2}{*}{$\begin{array}{l}\text { Test } \\
\text { Cycle }\end{array}$} & \multicolumn{4}{|c|}{ Emissions, $\mathrm{mg} / \mathrm{mi}$} \\
\hline & & $\begin{array}{c}\text { 1,3- } \\
\text { Butadiene }\end{array}$ & Benzene & $\begin{array}{c}\text { Form- } \\
\text { aldehyde }\end{array}$ & $\begin{array}{c}\text { Acet- } \\
\text { aldehyde }\end{array}$ \\
\hline $\begin{array}{c}\mathrm{CP2} \\
\text { (after conversion) }\end{array}$ & $\begin{array}{l}\text { Bag } 1 \\
\text { Bag } 2 \\
\text { Bag } 3 \\
\text { FTP }\end{array}$ & $\begin{array}{l}1.4 \\
0.0 \\
0.0 \\
0.3\end{array}$ & $\begin{array}{l}7.8 \\
0.2 \\
\frac{2.5}{2.4}\end{array}$ & $\begin{array}{l}2.0 \\
0.0 \\
0.0 \\
0.4\end{array}$ & $\begin{array}{l}0.7 \\
0.0 \\
0.0 \\
0.2\end{array}$ \\
\hline RF-A & $\begin{array}{l}\text { Bag } 1 \\
\text { Bag } 2 \\
\text { Bag } 3 \\
\text { FTP }\end{array}$ & $\begin{array}{l}1.6 \\
0.0 \\
0.0 \\
0.4\end{array}$ & $\begin{array}{l}13.4 \\
0.0 \\
3.3 \\
3.7\end{array}$ & $\begin{array}{l}1.7 \\
0.0 \\
0.0 \\
0.4\end{array}$ & $\begin{array}{l}0.9 \\
0.0 \\
0.0 \\
0.2\end{array}$ \\
\hline $100 \%$ n-Butane & $\begin{array}{l}\text { Bag } 1 \\
\text { Bag } 2 \\
\text { Bag } 3 \\
\text { FTP }\end{array}$ & $\begin{array}{l}0.2 \\
0.0 \\
\underline{0.0} \\
0.0\end{array}$ & $\begin{array}{l}0.5 \\
0.0 \\
0.2 \\
0.1\end{array}$ & $\begin{array}{l}2.1 \\
0.0 \\
0.4 \\
0.6\end{array}$ & $\begin{array}{l}0.6 \\
0.0 \\
\underline{0.0} \\
\mathbf{0 . 1}\end{array}$ \\
\hline 100\% Propane & $\begin{array}{l}\text { Bag } 1 \\
\text { Bag } 2 \\
\text { Bag } 3 \\
\text { FTP }\end{array}$ & $\begin{array}{l}0.0 \\
0.0 \\
0.0 \\
0.0\end{array}$ & $\begin{array}{l}0.3 \\
0.2 \\
0.2 \\
0.2\end{array}$ & $\begin{array}{l}1.3 \\
0.0 \\
0.0 \\
0.3\end{array}$ & $\begin{array}{l}0.8 \\
0.0 \\
0.0 \\
0.2\end{array}$ \\
\hline $\begin{array}{l}90 \% \text { n-Butane } \\
10 \% \text { Isobutane }\end{array}$ & $\begin{array}{l}\text { Bag } 1 \\
\text { Bag } 2 \\
\text { Bag } 3 \\
\text { FTP }\end{array}$ & $\begin{array}{l}0.1 \\
0.0 \\
0.0 \\
0.0\end{array}$ & $\begin{array}{l}0.2 \\
0.1 \\
0.0 \\
0.1\end{array}$ & $\begin{array}{l}1.5 \\
0.0 \\
0.1 \\
0.3\end{array}$ & $\begin{array}{l}0.3 \\
0.0 \\
\underline{0.0} \\
\mathbf{0 . 1}\end{array}$ \\
\hline $\begin{array}{c}90 \% \text { n-Butane } \\
10 \% \text { Mixed Butenes }\end{array}$ & $\begin{array}{l}\text { Bag } 1 \\
\text { Bag } 2 \\
\text { Bag } 3 \\
\text { FTP }\end{array}$ & $\begin{array}{l}0.4 \\
0.0 \\
0.0 \\
0.1\end{array}$ & $\begin{array}{l}0.3 \\
0.3 \\
0.4 \\
0.3\end{array}$ & $\begin{array}{l}1.8 \\
0.0 \\
\underline{0.1} \\
\mathbf{0 . 4}\end{array}$ & $\begin{array}{l}0.6 \\
0.0 \\
0.0 \\
0.1\end{array}$ \\
\hline $\begin{array}{l}60 \% \text { n-Butane } \\
40 \% \text { Isobutane }\end{array}$ & $\begin{array}{l}\text { Bag } 1 \\
\text { Bag } 2 \\
\text { Bag } 3 \\
\text { FTP }\end{array}$ & $\begin{array}{l}0.0 \\
0.0 \\
0.0 \\
0.0\end{array}$ & $\begin{array}{l}0.2 \\
0.3 \\
0.3 \\
0.3\end{array}$ & $\begin{array}{l}1.7 \\
0.2 \\
0.2 \\
0.5 \\
\end{array}$ & $\begin{array}{l}0.5 \\
0.0 \\
0.0 \\
\mathbf{0 . 1}\end{array}$ \\
\hline $\begin{array}{l}80 \% \text { n-Butane } \\
20 \% \text { Propane }\end{array}$ & $\begin{array}{l}\text { Bag } 1 \\
\text { Bag } 2 \\
\text { Bag } 3 \\
\text { FTP }\end{array}$ & $\begin{array}{l}0.0 \\
0.0 \\
0.0 \\
0.0\end{array}$ & $\begin{array}{l}0.2 \\
0.4 \\
0.3 \\
0.3\end{array}$ & $\begin{array}{l}1.7 \\
0.0 \\
0.2 \\
0.4\end{array}$ & $\begin{array}{l}0.8 \\
0.0 \\
0.0 \\
0.2\end{array}$ \\
\hline $\begin{array}{l}90 \% \text { n-Butane } \\
10 \% \text { n-Pentane }\end{array}$ & $\begin{array}{l}\text { Bag } 1 \\
\text { Bag } 2 \\
\text { Bag } 3 \\
\text { FTP }\end{array}$ & $\begin{array}{l}0.1 \\
0.0 \\
0.0 \\
0.0\end{array}$ & $\begin{array}{l}0.5 \\
0.2 \\
0.1 \\
0.3\end{array}$ & $\begin{array}{l}1.6 \\
0.3 \\
0.4 \\
0.6 \\
\end{array}$ & $\begin{array}{l}0.7 \\
0.0 \\
0.0 \\
\mathbf{0 . 1}\end{array}$ \\
\hline
\end{tabular}


Table 7 (Concluded). Summary of Toxic Emissions Data

\begin{tabular}{|c|c|c|c|c|c|}
\hline \multirow[b]{2}{*}{ Fuel } & \multirow[b]{2}{*}{$\begin{array}{l}\text { Test } \\
\text { Cycle }\end{array}$} & \multicolumn{4}{|c|}{ Emissions, $\mathrm{mg} / \mathrm{mi}$} \\
\hline & & $\begin{array}{c}\text { 1,3- } \\
\text { Butadiene }\end{array}$ & Benzene & $\begin{array}{l}\text { Form- } \\
\text { aldehyde }\end{array}$ & $\begin{array}{c}\text { Acet- } \\
\text { aldehyde }\end{array}$ \\
\hline $\begin{array}{l}50 \% \text { n-Butane } \\
50 \% \text { Propane }\end{array}$ & $\begin{array}{l}\text { Bag } 1 \\
\text { Bag } 2 \\
\text { Bag } 3 \\
\text { FTP }\end{array}$ & $\begin{array}{l}0.1 \\
0.0 \\
0.0 \\
0.0\end{array}$ & $\begin{array}{l}0.3 \\
0.3 \\
0.1 \\
0.2 \\
\end{array}$ & $\begin{array}{l}1.6 \\
0.2 \\
0.4 \\
0.6\end{array}$ & $\begin{array}{l}1.1 \\
0.7 \\
0.0 \\
0.6\end{array}$ \\
\hline $\begin{array}{l}20 \% \text { n-Butane } \\
80 \% \text { Propane }\end{array}$ & $\begin{array}{l}\text { Bag } 1 \\
\text { Bag } 2 \\
\text { Bag } 3 \\
\text { FTP }\end{array}$ & $\begin{array}{l}0.1 \\
0.0 \\
0.0 \\
0.0\end{array}$ & $\begin{array}{l}0.2 \\
0.2 \\
0.1 \\
0.2\end{array}$ & $\begin{array}{l}0.7 \\
0.2 \\
0.2 \\
0.3\end{array}$ & $\begin{array}{l}0.7 \\
0.0 \\
0.0 \\
0.2\end{array}$ \\
\hline $\begin{array}{c}100 \% \mathrm{n} \text {-Butane } \\
\text { (second set of runs) }\end{array}$ & $\begin{array}{l}\text { Bag } 1 \\
\text { Bag } 2 \\
\text { Bag } 3 \\
\text { FTP }\end{array}$ & $\begin{array}{l}0.1 \\
0.0 \\
0.0 \\
0.0\end{array}$ & $\begin{array}{l}0.2 \\
0.0 \\
0.0 \\
0.0\end{array}$ & $\begin{array}{l}1.4 \\
0.1 \\
0.1 \\
0.4\end{array}$ & $\begin{array}{l}0.7 \\
0.1 \\
0.0 \\
0.2\end{array}$ \\
\hline \multicolumn{6}{|c|}{ Engine-out (no catalyst) Emissions } \\
\hline $100 \%$ n-Butane & $\begin{array}{l}\text { Bag } 1 \\
\text { Bag } 2 \\
\text { Bag } 3 \\
\text { FTP }\end{array}$ & $\begin{array}{l}1.9 \\
2.0 \\
1.4 \\
1.8\end{array}$ & $\begin{array}{l}0.7 \\
0.6 \\
1.2 \\
0.8\end{array}$ & $\begin{array}{l}39.0 \\
44.8 \\
37.1 \\
41.4\end{array}$ & $\begin{array}{l}9.1 \\
9.6 \\
8.0 \\
9.1\end{array}$ \\
\hline $100 \%$ Propane & $\begin{array}{l}\text { Bag } 1 \\
\text { Bag } 2 \\
\text { Bag } 3 \\
\text { FTP }\end{array}$ & $\begin{array}{l}0.9 \\
0.8 \\
0.9 \\
0.8\end{array}$ & $\begin{array}{l}0.3 \\
0.6 \\
0.3 \\
0.4\end{array}$ & $\begin{array}{l}16.6 \\
21.7 \\
21.7 \\
20.7\end{array}$ & $\begin{array}{l}7.6 \\
8.0 \\
6.1 \\
7.4\end{array}$ \\
\hline $\begin{array}{l}50 \% \text { n-Butane } \\
50 \% \text { Propane }\end{array}$ & $\begin{array}{l}\text { Bag } 1 \\
\text { Bag } 2 \\
\text { Bag } 3 \\
\text { FTP }\end{array}$ & $\begin{array}{l}1.4 \\
1.3 \\
1.2 \\
1.3 \\
\end{array}$ & $\begin{array}{l}0.6 \\
0.3 \\
0.5 \\
0.4 \\
\end{array}$ & $\begin{array}{l}36.6 \\
41.8 \\
33.2 \\
38.4 \\
\end{array}$ & $\begin{array}{l}8.2 \\
8.3 \\
6.7 \\
7.9 \\
\end{array}$ \\
\hline
\end{tabular}


Table 8. Summary of Specific Reactivity and Adjusted NMOG Emissions

\begin{tabular}{|c|c|c|c|c|c|c|}
\hline Fuel & $\begin{array}{l}\text { Test } \\
\text { Cycle }\end{array}$ & $\begin{array}{c}\text { NMOG, } \\
\text { g/mi }\end{array}$ & $\begin{array}{c}\text { Potential } \\
\text { Ozone }\left(\mathrm{O}_{3}\right), \\
\mathrm{g} / \mathrm{mi}\end{array}$ & $\begin{array}{c}\text { Specific } \\
\text { Reactivity, } \\
\text { g O } \text { O }_{3} / \mathrm{g} \text { NMOG }\end{array}$ & $\begin{array}{l}\text { FTP } \\
\text { RAF }\end{array}$ & $\begin{array}{c}\text { Reactivity } \\
\text { Adjusted } \\
\text { NMOG, } \\
\text { g/mi }\end{array}$ \\
\hline $\begin{array}{c}\mathrm{CP} 2 \\
\text { (after conversion) }\end{array}$ & $\begin{array}{l}\text { Bag } 1 \\
\text { Bag 2 } \\
\text { Bag 3 } \\
\text { FTP }\end{array}$ & $\begin{array}{l}0.194 \\
0.009 \\
0.025 \\
0.052 \\
\end{array}$ & $\begin{array}{l}0.72 \\
0.02 \\
0.05 \\
\mathbf{0 . 1 7} \\
\end{array}$ & $\begin{array}{l}3.72 \\
1.87 \\
2.19 \\
3.35 \\
\end{array}$ & $0.98^{\mathrm{a}}$ & 0.051 \\
\hline RF-A & $\begin{array}{l}\text { Bag } 1 \\
\text { Bag 2 } \\
\text { Bag 3 } \\
\text { FTP }\end{array}$ & $\begin{array}{l}0.206 \\
0.006 \\
0.031 \\
0.054\end{array}$ & $\begin{array}{l}0.80 \\
0.01 \\
0.08 \\
0.20\end{array}$ & $\begin{array}{l}3.87 \\
2.24 \\
2.74 \\
3.59\end{array}$ & $1.05^{\mathrm{a}}$ & 0.057 \\
\hline $100 \%$ n-Butane & $\begin{array}{l}\text { Bag } 1 \\
\text { Bag 2 } \\
\text { Bag 3 } \\
\text { FTP }\end{array}$ & $\begin{array}{l}0.251 \\
0.002 \\
0.044 \\
0.065\end{array}$ & $\begin{array}{l}0.48 \\
0.00 \\
0.09 \\
0.13 \\
\end{array}$ & $\begin{array}{l}1.89 \\
2.00 \\
2.10 \\
1.93 \\
\end{array}$ & $0.62^{b}$ & 0.040 \\
\hline $100 \%$ Propane & $\begin{array}{l}\text { Bag } 1 \\
\text { Bag } 2 \\
\text { Bag 3 } \\
\text { FTP }\end{array}$ & $\begin{array}{l}0.174 \\
0.004 \\
0.012 \\
0.042\end{array}$ & $\begin{array}{l}0.23 \\
0.01 \\
0.01 \\
0.05\end{array}$ & $\begin{array}{l}1.30 \\
1.80 \\
0.95 \\
1.29\end{array}$ & $0.41^{b}$ & 0.017 \\
\hline $\begin{array}{l}90 \% \text { n-Butane } \\
10 \% \text { Isobutane }\end{array}$ & $\begin{array}{l}\text { Bag } 1 \\
\text { Bag } 2 \\
\text { Bag 3 } \\
\text { FTP }\end{array}$ & $\begin{array}{l}0.181 \\
0.002 \\
0.018 \\
0.044 \\
\end{array}$ & $\begin{array}{l}0.37 \\
0.00 \\
0.03 \\
0.09 \\
\end{array}$ & $\begin{array}{l}2.06 \\
2.48 \\
1.70 \\
2.03 \\
\end{array}$ & $0.65^{b}$ & 0.029 \\
\hline $\begin{array}{c}90 \% \text { n-Butane } \\
10 \% \text { Mixed Butenes }\end{array}$ & $\begin{array}{l}\text { Bag } 1 \\
\text { Bag } 2 \\
\text { Bag } 3 \\
\text { FTP }\end{array}$ & $\begin{array}{l}0.174 \\
0.003 \\
0.031 \\
0.045\end{array}$ & $\begin{array}{l}0.44 \\
0.00 \\
0.06 \\
0.11\end{array}$ & $\begin{array}{l}2.52 \\
1.77 \\
1.97 \\
2.40\end{array}$ & $0.77^{b}$ & 0.035 \\
\hline $\begin{array}{l}60 \% \text { n-Butane } \\
40 \% \text { Isobutane }\end{array}$ & \begin{tabular}{|l} 
Bag 1 \\
Bag 2 \\
Bag 3 \\
FTP \\
\end{tabular} & $\begin{array}{l}0.198 \\
0.007 \\
0.033 \\
0.054 \\
\end{array}$ & $\begin{array}{l}0.41 \\
0.01 \\
0.06 \\
0.11 \\
\end{array}$ & $\begin{array}{l}2.07 \\
1.85 \\
1.71 \\
1.99 \\
\end{array}$ & $0.64^{\mathrm{b}}$ & 0.034 \\
\hline $\begin{array}{l}80 \% \text { n-Butane } \\
20 \% \text { Propane }\end{array}$ & $\begin{array}{l}\text { Bag 1 } \\
\text { Bag 2 } \\
\text { Bag 3 } \\
\text { FTP }\end{array}$ & $\begin{array}{l}0.207 \\
0.002 \\
0.052 \\
\mathbf{0 . 0 5 8} \\
\end{array}$ & $\begin{array}{l}0.34 \\
0.00 \\
0.09 \\
\mathbf{0 . 1 0} \\
\end{array}$ & $\begin{array}{l}1.65 \\
2.01 \\
1.66 \\
1.66 \\
\end{array}$ & $0.53^{b}$ & 0.031 \\
\hline $\begin{array}{c}90 \% \text { n-Butane } \\
10 \% \text { n-Pentane }\end{array}$ & $\begin{array}{l}\text { Bag 1 } \\
\text { Bag 2 } \\
\text { Bag 3 } \\
\text { FTP }\end{array}$ & $\begin{array}{l}0.194 \\
0.005 \\
0.030 \\
0.051 \\
\end{array}$ & $\begin{array}{l}0.41 \\
0.01 \\
\underline{0.05} \\
\mathbf{0 . 1 1} \\
\end{array}$ & $\begin{array}{l}2.12 \\
2.59 \\
1.67 \\
2.07 \\
\end{array}$ & $0.66^{\mathrm{b}}$ & 0.034 \\
\hline
\end{tabular}


Table 8 (Concluded). Summary of Specific Reactivity and Adjusted NMOG Emissions

\begin{tabular}{|c|c|c|c|c|c|c|}
\hline Fuel & $\begin{array}{l}\text { Test } \\
\text { Cycle } \\
\end{array}$ & $\begin{array}{c}\text { NMOG, } \\
\text { g/mi }\end{array}$ & $\begin{array}{c}\text { Potential } \\
\text { Ozone }\left(\mathrm{O}_{3}\right) \text {, } \\
\mathrm{g} / \mathrm{mi}\end{array}$ & $\begin{array}{c}\text { Specific } \\
\text { Reactivity, } \\
\text { g O } \mathrm{O}_{3} / \mathrm{g} \text { NMOG }\end{array}$ & $\begin{array}{l}\text { FTP } \\
\text { RAF } \\
\end{array}$ & $\begin{array}{c}\text { Reactivity } \\
\text { Adjusted } \\
\text { NMOG, } \\
\text { g/mi }\end{array}$ \\
\hline $\begin{array}{l}50 \% \text { n-Butane } \\
50 \% \text { Propane }\end{array}$ & $\begin{array}{l}\text { Bag 1 } \\
\text { Bag 2 } \\
\text { Bag 3 } \\
\text { FTP }\end{array}$ & $\begin{array}{l}0.182 \\
0.004 \\
0.036 \\
0.050\end{array}$ & $\begin{array}{l}0.27 \\
0.01 \\
0.04 \\
0.07\end{array}$ & $\begin{array}{l}1.48 \\
3.23 \\
1.06 \\
1.47\end{array}$ & $0.47^{b}$ & 0.024 \\
\hline $\begin{array}{l}20 \% \text { n-Butane } \\
80 \% \text { Propane }\end{array}$ & $\begin{array}{l}\text { Bag 1 } \\
\text { Bag 2 } \\
\text { Bag 3 } \\
\text { FTP }\end{array}$ & $\begin{array}{l}0.197 \\
0.005 \\
0.018 \\
0.049\end{array}$ & $\begin{array}{l}0.27 \\
0.00 \\
0.02 \\
0.06\end{array}$ & $\begin{array}{l}1.35 \\
0.98 \\
0.89 \\
1.29\end{array}$ & $0.41^{b}$ & 0.020 \\
\hline $\begin{array}{c}100 \% \text { n-Butane } \\
\text { (second set of runs) }\end{array}$ & $\begin{array}{l}\text { Bag 1 } \\
\text { Bag 2 } \\
\text { Bag 3 } \\
\text { FTP } \\
\end{array}$ & $\begin{array}{l}0.184 \\
0.001 \\
0.030 \\
0.047 \\
\end{array}$ & $\begin{array}{l}0.39 \\
0.00 \\
0.06 \\
0.10 \\
\end{array}$ & $\begin{array}{l}2.12 \\
2.88 \\
1.89 \\
2.09 \\
\end{array}$ & $0.67^{b}$ & 0.031 \\
\hline \multicolumn{7}{|c|}{ Engine-out (no catalyst) Emissions } \\
\hline $100 \%$ n-Butane & $\begin{array}{l}\text { Bag } 1 \\
\text { Bag } 2 \\
\text { Bag 3 } \\
\text { FTP }\end{array}$ & $\begin{array}{l}1.274 \\
1.066 \\
0.942 \\
1.075 \\
\end{array}$ & $\begin{array}{l}3.91 \\
3.64 \\
2.95 \\
3.50 \\
\end{array}$ & $\begin{array}{l}3.07 \\
3.41 \\
3.13 \\
3.26 \\
\end{array}$ & $\mathbf{n} / \mathbf{a}$ & $\mathbf{n} / \mathbf{a}$ \\
\hline $100 \%$ Propane & $\begin{array}{l}\text { Bag 1 } \\
\text { Bag 2 } \\
\text { Bag 3 } \\
\text { FTP } \\
\end{array}$ & $\begin{array}{l}0.969 \\
0.915 \\
0.761 \\
0.884 \\
\end{array}$ & $\begin{array}{l}2.28 \\
2.42 \\
1.97 \\
2.26 \\
\end{array}$ & $\begin{array}{l}2.35 \\
2.64 \\
2.59 \\
2.56 \\
\end{array}$ & $\mathbf{n} / \mathbf{a}$ & n/a \\
\hline $\begin{array}{l}50 \% \text { n-Butane } \\
50 \% \text { Propane }\end{array}$ & $\begin{array}{l}\text { Bag 1 } \\
\text { Bag 2 } \\
\text { Bag 3 } \\
\text { FTP } \\
\end{array}$ & $\begin{array}{l}1.070 \\
0.971 \\
0.834 \\
0.954 \\
\end{array}$ & $\begin{array}{l}2.78 \\
2.87 \\
2.41 \\
2.72 \\
\end{array}$ & $\begin{array}{l}2.60 \\
2.95 \\
2.89 \\
2.85 \\
\end{array}$ & $\mathbf{n} / \mathbf{a}$ & $\mathbf{n} / \mathbf{a}$ \\
\hline $\begin{array}{l}\text { RAFs for gasoline } \\
\text { verage specific re } \\
\text { RAFs for alternati } \\
\text { EV/ULEV average }\end{array}$ & $\begin{array}{l}\text { ere c } \\
\text { vity } 0 \\
\text { uels }\end{array}$ & $\begin{array}{l}\text { lated } \\
2 \mathrm{~g} \mathrm{C} \\
\text { calc } \\
\text { ctivity }\end{array}$ & $\begin{array}{l}\text { dividing FT } \\
\text { NMOG. } \\
\text { ed by divid } \\
3.13 \text { g } 0.0\end{array}$ & $\begin{array}{l}\text { ecific reacti } \\
\text { TP specific } \\
\text { OG. }\end{array}$ & $\overline{C A}$ & $\begin{array}{l}\text { EV } \\
\text { RB }\end{array}$ \\
\hline
\end{tabular}

catalyst because no baseline "industry average" gasoline tests were speciated (without catalyst) to allow calculation of these values. "Specific reactivity" is defined as the ratio of mass of $\mathrm{O}_{3}$ formation potential to mass of NMOG in the total exhaust. The CARB TLEV average specific reactivity of $3.42 \mathrm{~g} \mathrm{O}_{3} / \mathrm{g} \mathrm{NMOG}$ was then used as a basis for normalizing all gasoline data and determining RAFs. For the alternative fuels, however, with the application of a RAF, the vehicle could potentially be certified to LEV or ULEV standards. Therefore, the LEV/ULEV specific reactivity of $3.13 \mathrm{~g} \mathrm{O}_{3} / \mathrm{g}$ NMOG was used to calculate RAFs for the 
alternative fuels. This method of calculation is consistent with that used by CARB. ${ }^{(3)}$ RAFs for each fuel were then multiplied by the respective NMOG emission rates to give reactivityadjusted NMOG emission rates. The overriding result shown in Table 8 is that the resulting $\mathrm{O}_{3}$ formation potential (and hence specific reactivity) did depend strongly on the fuel.

An analysis of the data contained in Table 8 shows that for this TLEV vehicle all of the alternative fuels gave reactivity-adjusted FTP NMOG emission rates less than the $0.040 \mathrm{~g} / \mathrm{mi}$ CARB ULEV 50,000-mile emission standard when the vehicle was equipped with the OEM catalyst system. The FTP RAF for all of the alternative fuels ranged from a low of 0.41 for $100 \%$ propane to a high of 0.77 for the $90 \%$-butane/10\% mixed butenes blend. In general, about three-fourths or more of the NMOG mass emissions and potential ozone came from bag 1, primarily from unburned and partially-burned fuel. Propane has an assigned MIR

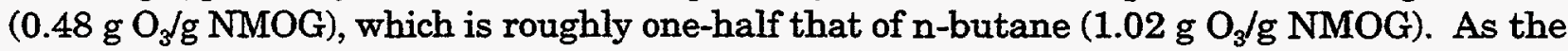
concentration of propane in the fuel blends increases, the RAF decreases. Specifically, even though the bag 1 NMOG emission rates for all of the alternative fuels were similar, reactivityadjusted NMOG emissions were lower for the fuels containing propane.

For the engine-out tests, FTP NMOG, potential ozone and specific reactivity were highest for $100 \%$ n-butane, followed by $50 \%$ n-butane $/ 50 \%$ propane, and $100 \%$ propane, respectively. This trend is the same as was observed for tailpipe emissions using these fuels and implies that catalytic reductions were, in general, proportional and based on engine-out concentrations of specific emission products. These observations make sense as the predominant emission products from these three fuels are similar (ethylene, propylene, and unburned fuel components).

\section{H. Discussion}

Based on results of hydrocarbon speciation data contained in Appendix B, it appears that approximately $80 \%$ of the potential ozone formed from combustion of CP2 and RF-A is derived from olefins and aromatics. Approximately $12 \%$ to $16 \%$ of the potential ozone is derived from paraffins. In contrast, aromatics account for less than $2 \%$ of the potential ozone for the $100 \% \mathrm{n}$-butane and $100 \%$ propane fuels. Most of the potential ozone from combustion of these fuels is derived from a relatively small emission rate of alkenes and a larger amount of alkanes (primarily in bag 1 , and to a lesser extent bag 3 ). In addition, it can be seen that for CP2 and RF-A, the potential ozone formed comes from compounds of all molecular sizes, from $\mathrm{C}_{1}$ through $\mathrm{C}_{12}$. For the $100 \% \mathrm{n}$-butane fuel, almost all of the potential ozone is derived from $\mathrm{C}_{2}, \mathrm{C}_{3}$, and $\mathrm{C}_{4}$ compounds (mostly ethylene, propylene, and n-butane). For the $100 \%$ propane fuel, the majority of the potential ozone comes from $\mathrm{C}_{2}$ and $\mathrm{C}_{3}$ compounds (ethylene, propylene, and propane).

Table 9 provides a summary of major HC contributors to FTP potential ozone. In general, from these data, it is evident that the majority of the potential ozone for all of the alternative fuels is derived from ethylene, propylene, and unburned fuel emissions, and that these species are found primarily in bag 1 , and to a lesser extent bag 3 (refer to Table 8 ). 
Table 9. Summary of Major HC Contributors to FTP Potential Ozone

\begin{tabular}{|c|c|c|c|c|c|c|c|c|c|}
\hline \multirow[b]{2}{*}{$\begin{array}{l}\text { Hydrocarbon } \\
\text { Species }\end{array}$} & \multicolumn{9}{|c|}{ Weight Percent of Potential FTP Ozone Formed } \\
\hline & $100 \mathrm{NB}$ & $100 \mathrm{P}$ & $\begin{array}{l}90 \mathrm{NB} / \\
10 \mathrm{IB}\end{array}$ & $\begin{array}{l}\text { 9ONBI } \\
\text { 10BE }\end{array}$ & $\begin{array}{l}60 \mathrm{NB} / \\
401 \mathrm{~B}\end{array}$ & $\begin{array}{l}\text { 80NBI } \\
20 \mathrm{P}\end{array}$ & $\begin{array}{l}90 \mathrm{NB} / \\
10 \mathrm{NP}\end{array}$ & $\begin{array}{l}\text { 50NB/ } \\
50 \mathrm{P}\end{array}$ & $\begin{array}{l}\text { 20NB } / \\
80 \mathrm{P}\end{array}$ \\
\hline Ethane & 0.32 & 0.28 & 0.32 & 0.28 & 0.28 & 0.90 & 0.42 & 0.34 & 0.19 \\
\hline Ethylene & 29.83 & 27.86 & 31.68 & 25.14 & 23.15 & 32.85 & 30.60 & 26.94 & 29.39 \\
\hline Propane & 0.05 & 28.04 & 0.05 & 0.04 & 0.04 & 8.69 & 0.12 & 18.04 & 25.68 \\
\hline Propylene & 14.88 & 18.25 & 17.50 & 14.09 & 16.78 & 17.03 & 15.47 & 16.57 & 19.97 \\
\hline Acetylene & 0.47 & 0.27 & 1.37 & 0.53 & 0.57 & 0.37 & 0.73 & 0.32 & 0.32 \\
\hline n-butane & 41.17 & 1.83 & 32.32 & 28.78 & 21.79 & 27.39 & 30.25 & 16.51 & 13.29 \\
\hline trans-2-Butene & 1.17 & 0.22 & 0.54 & 5.51 & 0.84 & 0.49 & 0.88 & 0.34 & 0.24 \\
\hline 1-Butene & 1.39 & 0.65 & 1.32 & 4.98 & 1.24 & 1.44 & 1.38 & 0.72 & 0.78 \\
\hline Isobutylene & 0.49 & 0.10 & 1.05 & 2.91 & 2.92 & 0.35 & 0.15 & 0.00 & 0.18 \\
\hline 1,3-Butadiene & 0.29 & 0.18 & 0.30 & 0.72 & 0.00 & 0.23 & 0.29 & 0.20 & 0.27 \\
\hline Isobutane & 0.00 & 0.37 & 3.47 & 0.01 & 18.56 & 0.11 & 0.07 & 0.07 & 0.05 \\
\hline cis-2-Butene & 1.07 & 0.00 & 0.00 & 7.61 & 0.19 & 0.00 & 0.13 & 0.00 & 0.00 \\
\hline Benzene & 0.05 & 0.19 & 0.05 & 0.12 & 0.11 & 0.15 & 0.11 & 0.13 & 0.11 \\
\hline Formaldehyde & 3.38 & 4.26 & 2.73 & 2.65 & 3.49 & 2.88 & 3.81 & 5.46 & 3.37 \\
\hline Acetaldehyde & 0.57 & 1.77 & 0.39 & 0.66 & 0.55 & 0.93 & 0.74 & 4.33 & 1.44 \\
\hline n-Pentane & 0.07 & 0.45 & 0.11 & 0.17 & 0.23 & 0.15 & 3.67 & 0.05 & 0.06 \\
\hline $\begin{array}{l}\text { Total for all } \\
\text { Selected Species }\end{array}$ & 95.18 & 84.71 & 93.18 & 94.19 & 90.75 & 94.00 & 88.82 & 90.02 & 95.34 \\
\hline \multicolumn{10}{|c|}{$\begin{array}{l}\text { Fuels: } \\
\text { 100NB - } 100 \% \text { n-Butane } \\
\text { 100P - } 100 \% \text { Propane } \\
90 \mathrm{NB} / 10 \mathrm{~B}-90 \% \text { n-Butane } / 10 \% \text { Isobutane } \\
90 \mathrm{NB} / 10 \mathrm{BE}-90 \% \text { n-Butane } / 10 \% \text { Mixed Butenes } \\
60 \mathrm{NB} / 40 \mathrm{~B}-60 \% \text { n-Butane } / 40 \% \text { Isobutane } \\
80 \mathrm{NB} / 20 \mathrm{P}-80 \% \text { n-Butane/20\% Propane } \\
90 \mathrm{NB} / 10 \mathrm{NP}-90 \% \text { n-Butane } / 10 \% \text { n-Pentane } \\
50 \mathrm{NB} / 50 \mathrm{P}-50 \% \text { n-Butane } / 50 \% \text { Propane } \\
\text { 20NB/80P - } 20 \% \text { n-Butane/80\% Propane } \\
\end{array}$} \\
\hline
\end{tabular}




\section{Conclusions}

Although the data presented in this report are representative of only a single current technology vehicle, the following conclusions have been derived from the results.

- Because of conversion system calibration, the optimum post-conversion emissions performance appeared to be achieved with the vehicle operating on $100 \%$ propane. The next best performance was achieved with the $50 \% \mathrm{n}$ butane/50\% propane blend. This observation is encouraging because it suggests that operators of LPG vehicles could potentially use this blend, without modification to their vehicles, and see no detrimental effects on emissions and potential ozone.

- In general, for all of the alternative fuels tested, more than three-fourths of the total FTP potential ozone came from bag 1 . The primary ozone HC precursors were unburned fuel, ethylene, and propylene.

- Overall, volumetric fuel economy for the butane fuels was approximately $81 \%$ of CP2. The fuel economy for the $100 \%$ propane fuel was $76 \%$ of CP2. The fuel economy for the $50 \%$ n-butane/50\% propane blend was $78 \%$ of CP2.

- All of the alternative fuels allowed the converted California TLEV to potentially meet ULEV NMOG and $\mathrm{NO}_{\mathrm{x}}$ standards at the mileage tested. All but the $100 \%$ n-butane and $90 \%$-butane $10 \%$ pentane alternative fuels met the ULEV CO standard. It is thought, however, that the conversion system calibration could be optimized slightly to allow all the alternative fuels to meet all California ULEV emission standards.

- Although the conversion system was optimized for $100 \%$ propane, it provided better reactivity-adjusted NMOG and $\mathrm{NO}_{\mathrm{x}}$ emission performance for all of the alternative fuels (without adjustment) evaluated in this study than with the gasolines. The highest $\mathrm{NO}_{x}$ result for the alternative fuels was seen with the $100 \%$ propane fuel.

- The primary incomplete (engine-out) hydrocarbon combustion products of $100 \%$ n-butane are methane, ethylene, and propylene. They are produced primarily during the cold-start phase of the FTP.

- It is thought that additional exhaust aftertreatment (i.e., utilizing a close coupled catalyst) would provide bag 1 and bag $3 \mathrm{HC}$ emission reductions that could, in turn, reduce the OFP and the FTP RAF adjusted NMOG for all the alternative fuels tested. 


\section{J. Recommendations}

Although this project produced a relatively consistent data set, it should by no means be considered representative of the performance of all alternative fuel conversion systems or vehicles. There is a great deal more research that must be conducted using other vehicles, including those that have undergone significant mileage accumulation, different conversion technologies, etc., before broad conclusions can be made. When using the data developed for this report, care must be taken not to overgeneralize the results. Once a sufficient database has been developed, it will be possible to make more general conclusions related to specific reactivities and OFPs for butane and butane-blended alternative fuels.

The results presented here indicate areas for future study. More vehicles and realworld (i.e., on-road) conditions should be tested to delineate emissions benefits in fleet operations. 


\section{Task 2 - Evaluation of a Vacuum Insulated Catalyst in Reducing Cold-Start Exhaust Emissions}

\section{A. Background}

Currently, $70 \%$ to $90 \%$ of the HC and CO emissions from the EPA certification test for light-duty vehicles are produced in the first few minutes of the vehicle cold-start. During this period, the engine is operating very rich to maintain driveability. However, during this period, the catalyst is not at its operating temperature and can not effectively control exhaust emissions. To achieve future emission standards, the catalytic converter must be immediately effective after the engine is started. When operating a vehicle on E85, catalyst light-off is a further problem because the heat of combustion for ethanol is much lower than that of gasoline. This leads to lower exhaust temperatures, which lengthens the time required for the catalyst to reach light-off temperature. Further, the lower volatility of E85 may dictate additional enrichment during the initial cold start.

Cold-start emissions can be reduced by several methods. One method is to electrically preheat the catalytic converter. This approach has been developed to the point where suppliers are introducing systems to the automotive industry. Great strides have also been made with other technologies, such as HC traps, gas-fired catalyst pre-heaters, and closecoupled catalysts, in reducing cold-start emissions. Of these, close-coupled catalysts are favored because they are not as mechanically complex as the other approaches. Yet, it is likely that even these catalysts will need a temperature boost to perform adequately in low ambient temperature conditions.

Beginning with 1996 production, $100 \%$ of OEM production vehicles must comply with the EPA cold $\left(20^{\circ} \mathrm{F}\right) \mathrm{CO}$ emissions standards. That standard requires all light-duty vehicles and light-duty trucks weighing less than $3750 \mathrm{lb}$ to emit less than $10.0 \mathrm{~g} / \mathrm{mile} \mathrm{CO}$ at $20^{\circ} \mathrm{F}^{(7)}$ In addition, California has specified 100\% compliance for all 1996 model year light-duty vehicles for standards issued for $\mathrm{CO}, \mathrm{NO}_{\mathrm{x}}, \mathrm{NMOG}$, and formaldehyde measured at $50^{\circ} \mathrm{F}^{(8)}$.

The EPA has shown in the "Baltimore Study"(10) that average trip patterns in urban areas are quite short, 2.5 to 4.9 miles. Forty percent of all soak periods were between $10 \mathrm{~min}$ and $2 \mathrm{~h}$. In addition, the study indicated that only $30 \%$ of all in-use starts occur with the catalyst hot enough for prompt light-off. Thus, technology to effectively combat emissions from in-use vehicles should accommodate both short trips and frequent re-starts after soak periods longer than $10 \mathrm{~min}$.

The approach followed by researchers at NREL to answer these challenges was to store waste exhaust energy, and to use that energy to maintain the vehicle catalytic converter at elevated temperatures for future cold-engine starting. Of the various approaches to reducing cold-start emissions, storage of energy that would be otherwise wasted holds the most appeal. No additional energy input is required for such a system, and its operation is passive. To this end, NREL fabricated a Vacuum Insulated Catalytic Converter (TA-CC4) for evaluations over the chassis dynamometer portion of the FTP for light-duty vehicles. The testing of TA-CC4 complemented other work performed at SwRI under NREL Subcontract No. YAW-3-12243-01, "Development of a Dedicated Ethanol Utra-Low Emission Vehicle (ULEV)." 
Energy storage in TA-CC4 is accomplished with PCM and vacuum insulation. During the FTP vehicle emissions test, the catalyst bed temperature of the typical vehicle often exceeds $600^{\circ} \mathrm{C}$, well above the $350^{\circ} \mathrm{C}$ range required for catalyst light-off. This thermal energy can be stored and later used to provide nearly instantaneous catalyst light-off. PCMs absorb substantial amounts of thermal energy by virtue of their heat of fusion during the transition from a solid phase to a liquid phase. This stored energy is later released isothermally as the material solidifies. Harnessing this latent heat characteristic allows much greater energy storage density. Coupling this high-density heat storage medium with the efficiency of vacuum insulation yields a device that can maintain the catalyst bed at elevated temperatures for more than $36 \mathrm{~h}$.

Vacuum insulation coupled with PCM has been successfully demonstrated in an automotive application by Schatz, in a system to store heat that is subsequently dumped to the engine coolant. ${ }^{(11)}$ The system is currently being offered by Volkswagen in Germany as a dealer-installed option. Furthermore, Ivanov and co-workers reported using PCM heat storage for comfort heating in electric vehicles. ${ }^{(12)}$ Both of these applications operate at much lower temperatures than those found in automotive exhaust systems, however.

\section{B. Objective}

The objective of this task was to evaluate the effectiveness of NREL's Vacuum Insulated Catalytic Converter TA-CC4 in reducing cold-start exhaust emissions after extended soak periods when using ethanol-based fuel. In particular, evaluation of the effects of the test article in overcoming the inherently lower exhaust temperatures from ethanol was desired. TA-CC4 was evaluated over five FTPs with varying vehicle preconditioning sequences and soak periods.

\section{Approach}

As received, the insulated catalyst had not been exposed to vehicle exhaust. Before emissions evaluations, it was installed in an engine dynamometer test cell for break-in. Following break-in, the catalyst was installed on a Ford FFV Taurus. To establish a baseline, an FTP was conducted with the catalyst at room temperature prior to testing. Complete melting of the internal PCM in TA-CC4 was desired, so an extended vehicle preconditioning sequence was devised. After the extended preconditioning sequence, the insulated catalyst was evaluated over the FTP following either an 18-, 24-, or 36-h soak period. An additional test was conducted following a standard vehicle preconditioning sequence and a $36-\mathrm{h}$ soak.

\section{Vehicle Description}

The vehicle used in this evaluation was a 1993 Ford FFV Taurus. This vehicle is designed to operate satisfactorily on gasoline and gasoline-methanol blends. A description of the vehicle is given in Table 10 . 
Table 10. Vehicle Description

\begin{tabular}{||l|l||}
\hline \multicolumn{1}{|c|}{ Item } & \multicolumn{1}{c|}{ Configuration of Ford Taurus FFV } \\
\hline \hline Model year & 1993 \\
\hline Body style & 4-door sedan \\
\hline Transmission & Automatic-4 \\
\hline VIN & 1FALP5218P6281001 \\
\hline Tires & P205/65 R15 \\
\hline Accessories & $\begin{array}{l}\text { Power locks, windows, steering, brakes, } \\
\text { air conditioning }\end{array}$ \\
\hline Engine family & PFM3.0V5FFFC3 \\
\hline Engine displacement & 3.0 L \\
\hline Number of cylinders & 6 \\
\hline Fuel system & SFI \\
\hline Ignition system & Electronic \\
\hline Emission control system & $\begin{array}{l}\text { Insulated underbody three-way catalyst, } \\
\text { TA-CC4, heated oxygen sensor, EGR }\end{array}$ \\
\hline $\begin{array}{l}\text { Chassis Dynamometer: } \\
\text { Inertia Setting } \\
\text { Road Load @ 50 mph }\end{array}$ & $\begin{array}{l}3500 \text { lb } \\
6.8 \text { hp }\end{array}$ \\
\hline \hline
\end{tabular}

The test vehicle was fitted with an exhaust system modified to accommodate the insulated catalyst. Because of space constraints under the vehicle and the large diameter of the catalyst, it was installed much further downstream in the exhaust system than the OEM catalysts. Figure 2 shows the OEM and experimental exhaust system configurations. The twin OEM catalysts both reside approximately $14 \mathrm{in.} \mathrm{downstream} \mathrm{of} \mathrm{the} \mathrm{exhaust} \mathrm{manifold}$ flange. In the case of the experimental exhaust system, blank pipes were installed where the OEM catalysts were removed, and the insulated catalyst was installed at the termination of the Y-pipe. This placed the face of the insulated catalyst approximately $37 \mathrm{in}$. from the exhaust manifold flange on one side of the exhaust system, and $58 \mathrm{in}$. downstream of the manifold flange on the other side. In an effort to compensate for the heat loss, the exhaust system was wrapped with insulated fiber tape from the exhaust manifold flange to the termination of the Y-pipe.

\section{E. Test Fuels}

For these evaluations, the vehicle was operated on E85. A summary of properties for this fuel is given in Table 11. 

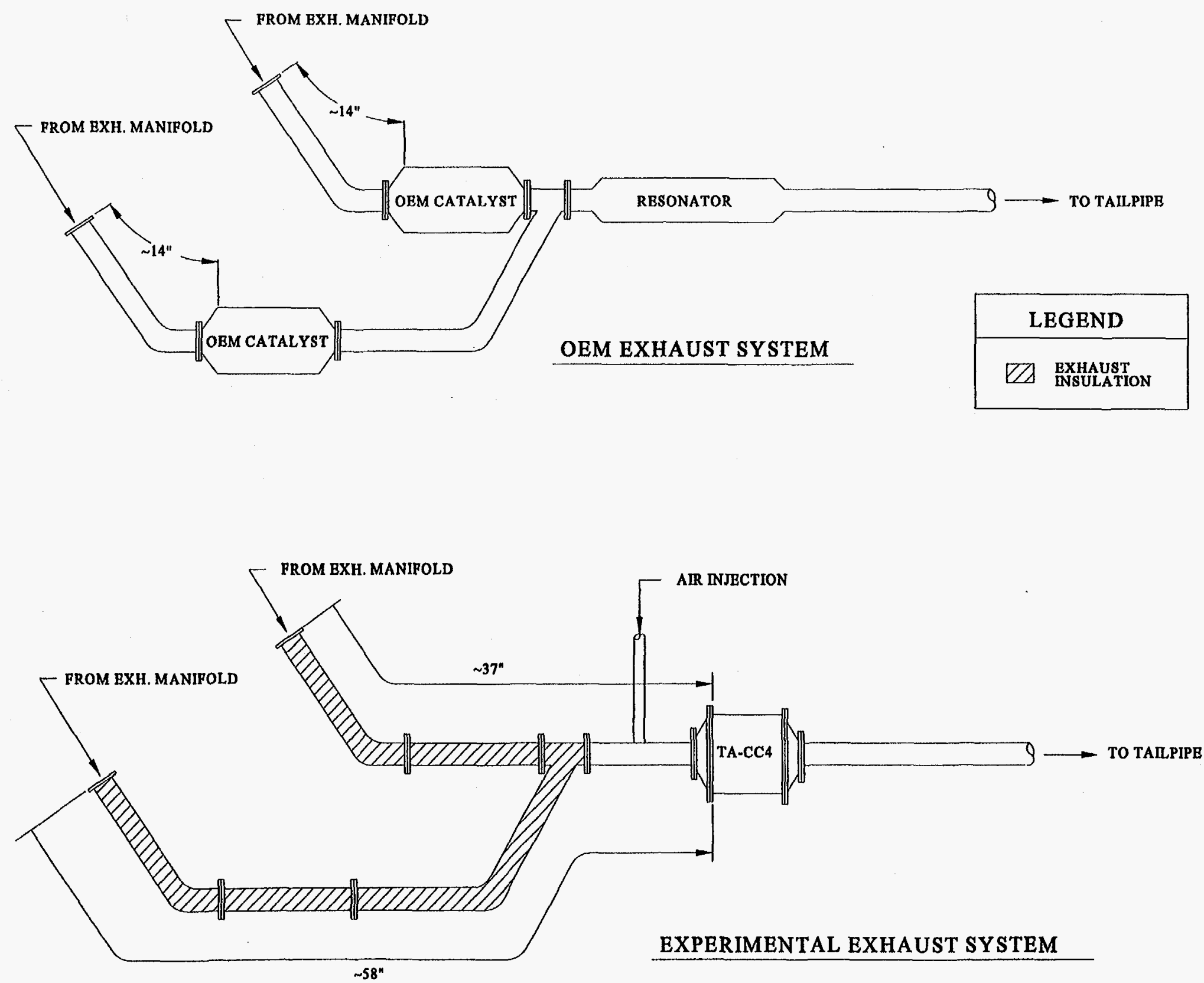

Figure 2. Exhaust System Conflguration 
Table 11. Summary of E85 Fuel Properties

\begin{tabular}{||l|c|}
\hline \multicolumn{1}{|c|}{ Fuel Property } & E85 \\
\hline RVP, psi & 6.76 \\
\hline Specific gravity & 0.7795 \\
\hline Carbon, wt. \% & 61.20 \\
Hydrogen, wt. \% & 13.21 \\
Oxygen, wt. \% & 25.59 \\
\hline
\end{tabular}

\section{F. Emission Test Procedures}

The insulated catalyst was evaluated using the chassis dynamometer portion of the FTP for light-duty vehicles. Complete melting of the internal PCM in TA-CC4 was desired, so an extended vehicle prep sequence was devised. This sequence consisted of the standard UDDS, followed by three highway fuel economy test schedules (HFETs). This extended prep sequence was used for each test except the baseline test and one of the 36-h soak tests, which used a standard preconditioning sequence. A Clayton Model ECE-50 passenger car dynamometer in conjunction with a positive displacement pump constant volume sampler was used for all testing. THC, methane, $\mathrm{CO}, \mathrm{NO}_{x}$, and $\mathrm{CO}_{2}$ emissions were collected as dilute exhaust in Tedlar gas sample bags. Aldehydes and ketones, and ethanol were sampled using wet adsorption techniques. All sampling was conducted in a manner consistent with EPA protocols for light-duty emissions testing. A detailed description of these procedures is presented in Sections I.E. and I.F. NMOG was estimated using NMHC from the gasoline portion of the fuel as measured by an FID rather than by HC speciation. Gasoline-derived NMHC was determined by measuring HC with a FID calibrated on propane, then correcting the results for the removal of methane and ethanol.

\section{G. Test Results and Discussion}

\section{Catalyst Break-In and Cool-Down}

Before emissions evaluations were conducted, TA-CC4 was installed in an engine dynamometer test cell for catalyst break-in. The catalyst was broken in for $24 \mathrm{~h}$ using a Ford 460 in. ${ }^{3}$ V-8 engine running on gasoline. Engine parameters were set to achieve $500^{\circ}$ $\pm 50^{\circ} \mathrm{C}$ at the thermocouple located between the intumescent mat and the steel wall which are positioned between the catalyst brick and the container for the PCM. As shown in Figure 3, temperature measurements recorded during the cool-down period after break-in correspond very closely to those temperatures observed by NREL during bench-testing at its facilities.

\section{Exhaust Emissions Testing}

Weighted exhaust emissions results from the five FTPs are given in Table 12 and Figure 4. Detailed computer printouts of these tests can be found in Appendix C. For these tests, supplemental air was injected into the exhaust system prior to the insulated catalyst during the first $100 \mathrm{~s}$ of the cold-start phase (Bag 1), and for $15 \mathrm{~s}$ at the beginning of 

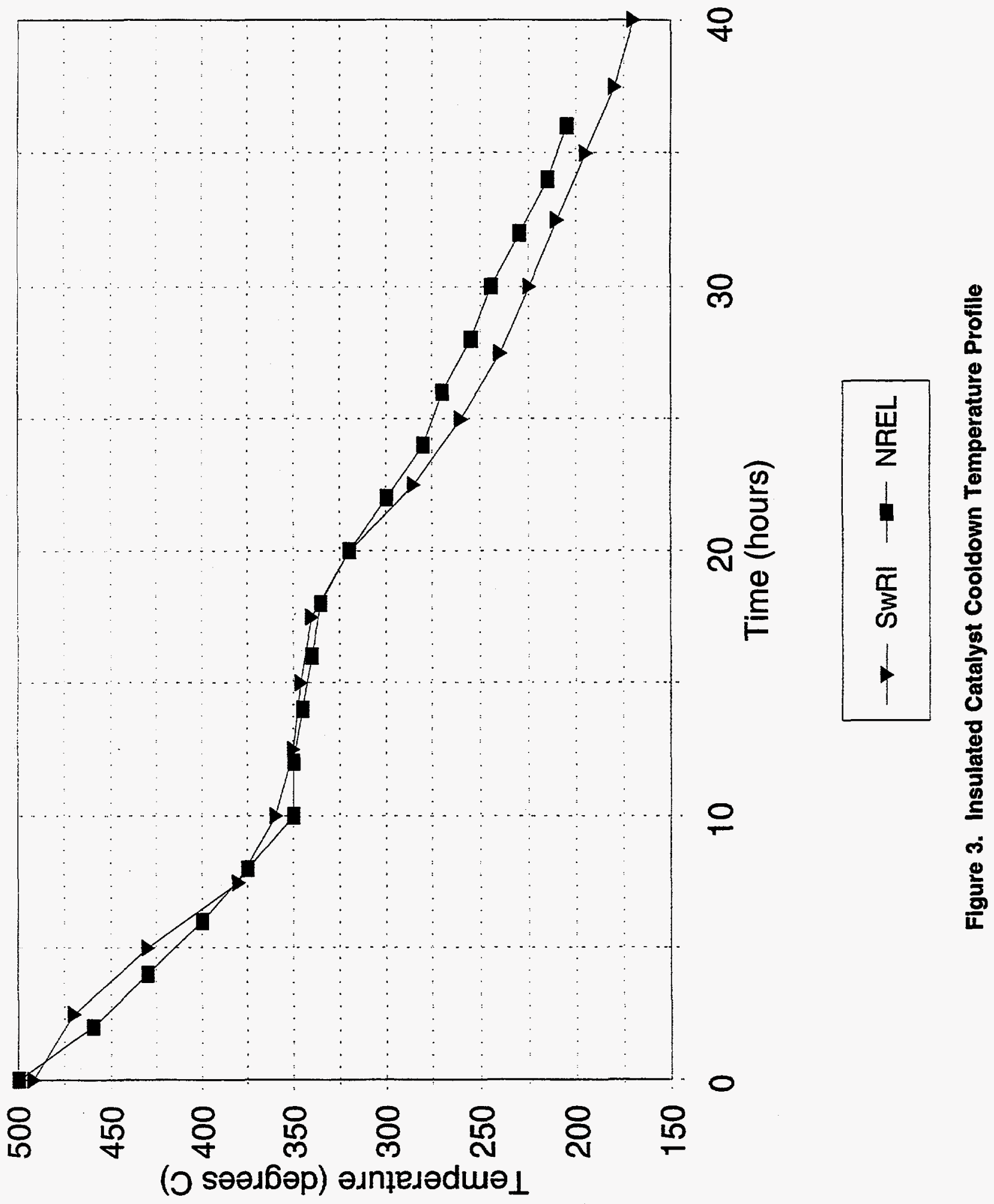
Table 12. FTP Exhaust Emissions from Ford FFV Equipped with TA-CC4

\begin{tabular}{|c|c|c|c|c|c|c|}
\hline Test Condition & $\begin{array}{c}\text { Uninsulated } \\
\text { Catalyst } \\
\text { Baseline }^{\mathrm{g}} \\
\end{array}$ & Baseline & $\begin{array}{l}\text { 18-h } \\
\text { Soak }\end{array}$ & $\begin{array}{l}\text { 24-h } \\
\text { Soak }\end{array}$ & $\begin{array}{l}\text { 36-h } \\
\text { Soak }\end{array}$ & $\begin{array}{l}\text { 36-h } \\
\text { Soak }\end{array}$ \\
\hline $\begin{array}{c}\text { Vehicle Preconditioning } \\
\text { Sequence }\end{array}$ & UDDS & UDDS & $\begin{array}{l}\text { UDDS + } 3 \\
\text { HFETs }\end{array}$ & $\begin{array}{l}\text { UDDS + } 3 \\
\text { HFETs }\end{array}$ & $\begin{array}{l}\text { UDDS + } 3 \\
\text { HFETs }\end{array}$ & UDDS \\
\hline $\begin{array}{c}\text { Catalyst Temperature at } \\
\text { Start of Test }\left({ }^{\circ} \mathrm{C}\right)\end{array}$ & 22 & 25 & 290 & 234 & 175 & 118 \\
\hline Test Number & $\begin{array}{c}\text { E-80- } \\
\text { CATA\&INS }\end{array}$ & $\begin{array}{l}\text { TA4-E85- } \\
\text { BASE }\end{array}$ & $\begin{array}{l}\text { TA4-E85- } \\
\text { 18HR }\end{array}$ & $\begin{array}{l}\text { TA4-E85- } \\
\text { 24HR }\end{array}$ & $\begin{array}{l}\text { TA4-E85- } \\
\text { 36HR }\end{array}$ & $\begin{array}{l}\text { TA4-E85- } \\
\text { 36HRC }\end{array}$ \\
\hline $\mathrm{THC}^{\mathrm{a}}(\mathrm{g} / \mathrm{mi})$ & 0.313 & 0.393 & 0.040 & 0.034 & 0.088 & 0.258 \\
\hline $\mathrm{CO}(\mathrm{g} / \mathrm{mi})$ & 1.75 & 1.71 & 0.125 & 0.124 & 0.465 & 1.38 \\
\hline $\mathrm{NO}_{x}(\mathrm{~g} / \mathrm{mi})$ & 0.068 & 0.177 & 0.044 & 0.044 & 0.049 & 0.078 \\
\hline $\mathrm{CH}_{4}(\mathrm{~g} / \mathrm{mi})$ & 0.043 & 0.080 & 0.032 & 0.026 & 0.029 & 0.031 \\
\hline $\mathrm{NMHC}^{\mathrm{b}}(\mathrm{g} / \mathrm{mi})$ & 0.073 & 0.115 & 0.006 & 0.005 & 0.037 & 0.080 \\
\hline Total Carbonyls ${ }^{\complement}(\mathrm{g} / \mathrm{mi})$ & 0.04 & 0.045 & 0.003 & 0.002 & 0.013 & 0.034 \\
\hline Alcohols ${ }^{d}(\mathrm{~g} / \mathrm{mi})$ & 0.156 & 0.153 & ND & ND & 0.010 & 0.113 \\
\hline Estimated $\mathrm{NMOG}^{\mathrm{e}}(\mathrm{g} / \mathrm{mi})$ & 0.27 & 0.314 & 0.009 & 0.008 & 0.059 & 0.227 \\
\hline Est NMOG $\times \operatorname{RAF}^{\prime}(\mathrm{g} / \mathrm{mi})$ & 0.181 & 0.210 & 0.006 & 0.005 & 0.040 & 0.152 \\
\hline Formaldehyde $(\mathrm{mg} / \mathrm{mi})$ & 8.67 & 6.29 & 2.28 & 1.73 & 1.64 & 3.31 \\
\hline Acetaldehyde (mg/mi) & 30.4 & 37.8 & 0.159 & 0.214 & 10.1 & 28.8 \\
\hline \multicolumn{7}{|c|}{$\begin{array}{l}{ }^{2} \text { THC = NMOG + CH4 } \\
\text { Gasoline derived NMHC = FIDHC - }\left(\mathrm{CH}_{4} \times \text { FIDRCH4) - (Ethanol } \times \text { FIDRETH); }\right. \\
\text { FIDHC = HC measured with FID calibrated on propane; } \\
\text { FIDRCH4 = FID response factor for methane; FIDRETH = FID response factor for ethanol } \\
\text { ' Summation of all measured aldehydes and ketones including: formaldehyde, acetaldehyde, acrolein, acetone, } \\
\text { propionaldehyde, crotonaldehyde, isobutyraidehyde + methyl ethyl ketone, and hexanaldehyde } \\
\text { Methanol and ethanol } \\
\text { MMOG = NMHC + Carbonyls + Alcohols } \\
\text { N RAF = } 0.67 \text { as measured by Kroll at Volkswagen (SAE 932676) } \\
\text { g Represents testing with different catalyst system installed, using a similar catalyst formulation. } \\
\text { ND = not detected above limit of ouantitation }\end{array}$} \\
\hline
\end{tabular}




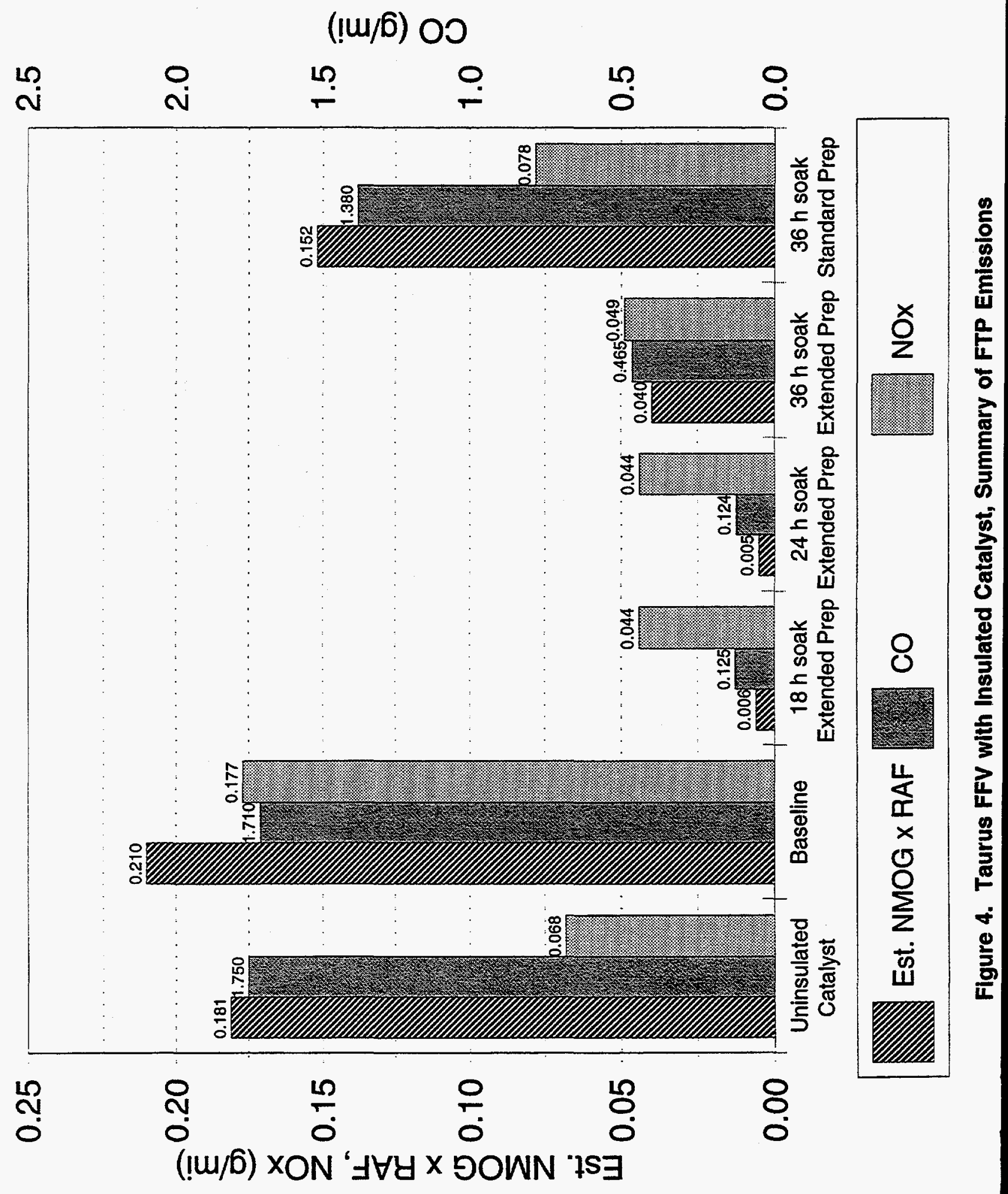


hot-start phase (Bag 3). The nominal flow rate was $5 \mathrm{ft}^{3} / \mathrm{min}$. Supplemental air provided sufficient oxygen levels in the exhaust stream so unburned fuel and $\mathrm{CO}$ would be more effectively oxidized in the catalyst. Air injection is necessary because the catalyst is at operating temperatures when the engine is first started and is in open loop operation (running rich). Air injection is not effective in conventional vehicles because the catalyst has not reached light-off temperature while the engine is in open loop operation.

For comparison purposes, results from a previous test conducted on this vehicle (E80-CATA\&INS) are also presented in Table 12. This test was conducted in September 1994, as part of Subcontract No. YAW-3-12243-01, "Development of a Dedicated Ethanol Ultra-Low Emission Vehicle (ULEV)." The catalytic converter used during this test was the same size, and had the same formulation as TA-CC4 but was insulated. Similar break-in procedures were conducted on both catalysts, and both catalysts were located in the same position under the vehicle.

Data from the baseline test with TA-CC4 (TA4-E85-BASE) show the performance of the system without the initial temperature assist from the vacuum insulation and heat storage via PCM. Following a standard vehicle preconditioning sequence and a soaking period of nearly $43 \mathrm{~h}$, the catalyst had not cooled to ambient temperature. Therefore, dry filtered shop air was blown through the exhaust system until the PCM and catalyst reached ambient temperature.

A comparison of exhaust emissions from the previously tested uninsulated catalyst (E80-CATA\&INS) and the baseline test with TA-CC4 (TA4-E85-BASE) shows slightly higher $\mathrm{THC}$ and $\mathrm{NO}_{\mathrm{x}}$ exhaust emissions with the insulated catalyst. CO levels were similar between the two tests. These slightly higher exhaust emissions with TA-CC4 are likely due to heat being conducted away from the catalyst brick into the PCM, lengthening the time it takes for the catalyst to reach light-off temperature. Higher than normal emissions result from this situation, which represents the case in which a vehicle would sit until all stored heat energy was depleted. This phenomenon is illustrated in Figure 5, which gives catalyst front brick temperature and exhaust outlet temperature for the uninsulated catalyst and the baseline test with the insulated catalyst (TA-CC4). Although the front face temperature of the catalyst is similar during both tests, the temperature of the exhaust exiting the insulated catalyst is substantially lower. However, EPA estimates that approximately $98 \%$ of all trips occur within $36 \mathrm{~h}$ of the previous trip. ${ }^{(13)}$ Therefore, most common driving patterns will result in the catalyst being at elevated temperatures at the next cold start, minimizing the heat sink effect. Careful analysis of both the effect that this cooling has on the exhaust emissions and the frequency with which this situation is likely to occur is necessary to determine whether use of such device will impart any net improvement in exhaust emissions from the vehicle under normal use.

Tests conducted after 18- and 24-h soak periods produced similar results, and demonstrated the effectiveness of the insulated catalyst in reducing exhaust emissions after an overnight soak. Reductions of more than $90 \%$ were observed for $\mathrm{HC}$ and $\mathrm{CO}$ emissions, while $\mathrm{NO}_{\mathrm{x}}$ emissions were reduced approximately $75 \%$. Results following an extended vehicle preconditioning sequence and a $36-\mathrm{h}$ soak show reductions of more than $80 \%$ from baseline levels for $\mathrm{HC}, \mathrm{CO}$, and $\mathrm{NO}_{\mathrm{x}}$ exhaust emissions. For emissions certification testing, a vehicle may soak up to $36 \mathrm{~h}$ following a single UDDS prep. Test TA4-E85-36HRC was to demonstrate the system performance at the maximum soak period following a standard prep with the PCM 


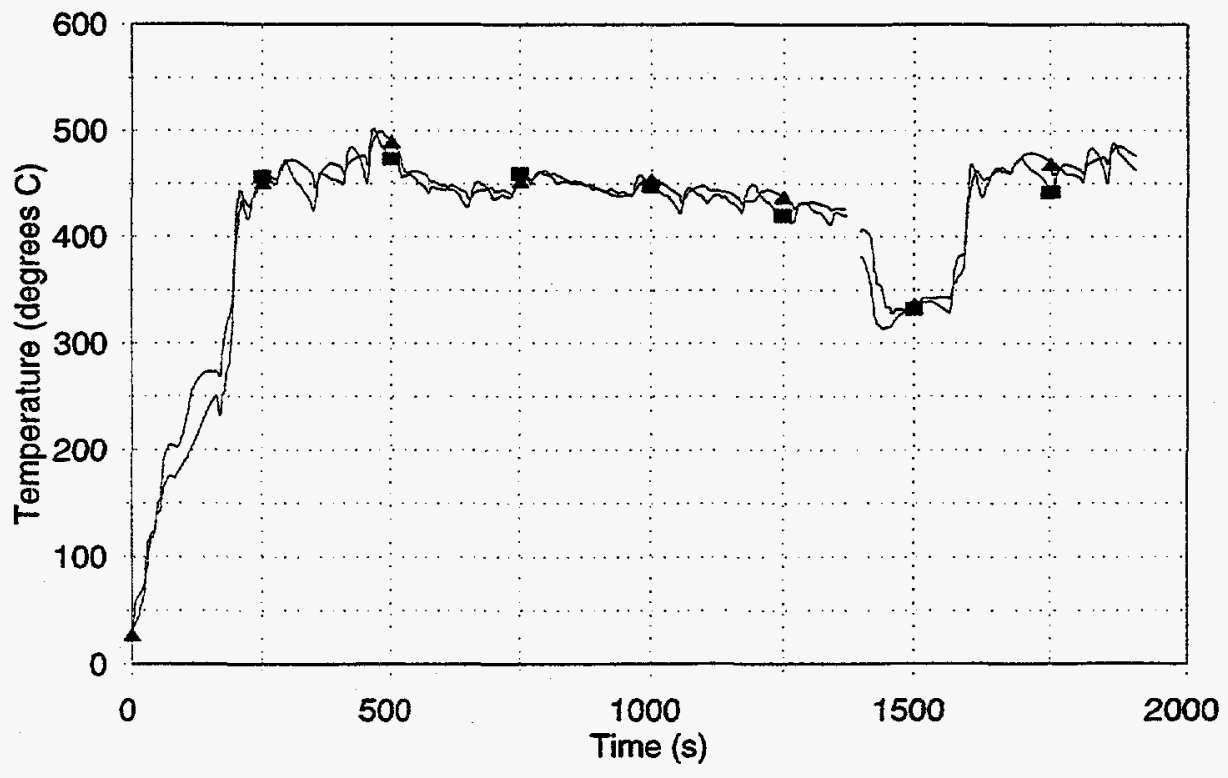

$\rightarrow-$ Uninsulated Catalyst $\rightarrow$ Insulated Catalyst

a. Catalyst Front Face Temperatures

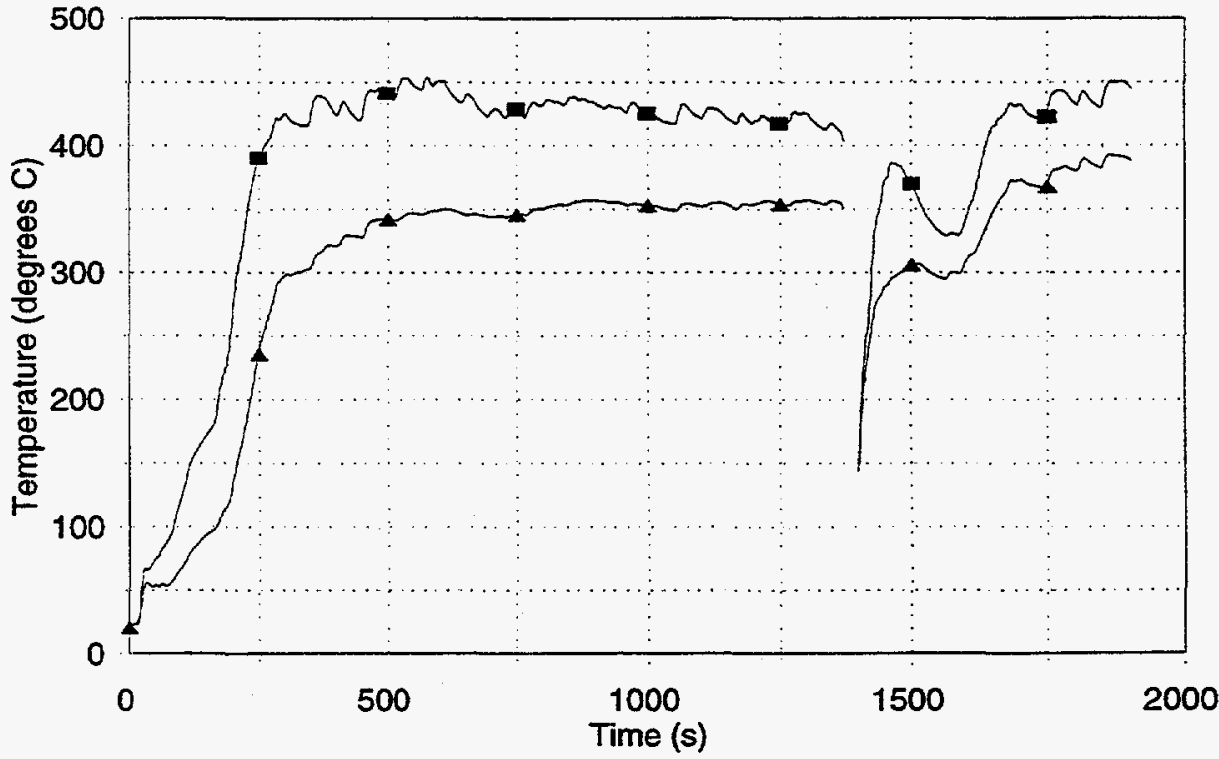

$\rightarrow$ Uninsulated Catalyst $\rightarrow$ Insulated Catalyst

b. Catalyst Outlet Temperatures

Figure 5. Comparison of Uninsulated and Insulated Catalyst Temperatures 
initially at ambient temperature (i.e., no extended prep sequence to ensure full melt of the PCM). This test showed reductions of approximately $30 \%, 20 \%$, and $55 \%$ in $\mathrm{HC}, \mathrm{CO}$, and $\mathrm{NO}_{\mathrm{x}}$ emissions, respectively, compared to baseline levels. In addition, THC and CO levels were approximately $20 \%$ less than those from testing with the uninsulated catalyst, while $\mathrm{NO}_{x}$ levels were similar.

Most of the emissions reductions were achieved in the first few minutes of the cold-start phase of the FTP. Figures 6 through 8 show continuous raw exhaust emissions concentrations measured after the insulated catalyst for the first $140 \mathrm{~s}$ of the FTP, for tests conducted following an extended vehicle preconditioning sequence and 24 - and $36-\mathrm{h}$ soaks, and for the test conducted following a standard prep and a 36-h soak. Exhaust emissions levels were similar for all tests following the first few minutes of operation.

Figure 9 shows representative temperature profiles of the front of the catalyst during soak following standard and extended vehicle preps. Other tests conducted with extended or standard preconditioning sequences showed similar results. Figure 9 illustrates the differences between starting with the PCM melted and with it only partially melted. The tests in which the PCM was fully melted clearly show the isothermal period in which the PCM was fusing between the 5- and 10-h marks, thus prolonging the time at elevated temperature. Figure 9 also shows the temperature gains made with the extended preconditioning sequence compared to the standard vehicle prep. Also note the rise in temperature during the first hour following a standard prep as heat from the catalyst brick is transferred to the PCM.

\section{H. Conclusions and Observations}

A number of conclusions can be drawn from the results of this testing:

- Compared with an uninsulated catalyst of similar configuration, baseline tests (no stored energy) with TA-CC4 show slightly higher $\mathrm{THC}$ and $\mathrm{NO}_{\mathrm{x}}$ exhaust emissions, with similar $\mathrm{CO}$ levels. These slightly higher exhaust emissions are likely due to heat being conducted away from the catalyst brick into the PCM.

- Reductions from baseline of more than $90 \%$ were observed for HC and CO emissions, and $75 \%$ for $\mathrm{NO}_{\mathrm{x}}$ emissions for tests conducted following an extended vehicle preconditioning sequence and 18 - and 24 -h soak periods.

- Results following an extended vehicle preconditioning sequence and a 36-h soak show reductions of more than $70 \%$ from baseline levels for $\mathrm{HC}, \mathrm{CO}$, and $\mathrm{NO}_{\mathrm{x}}$ exhaust emissions.

- After conducting a standard vehicle prep and a 36-h soak, exhaust emissions were approximately $30 \%, 20 \%$, and $55 \%$ lower for $\mathrm{HC}, \mathrm{CO}$, and $\mathrm{NO}_{\mathrm{x}}$ emissions, respectively, compared to baseline levels. In addition, THC and CO levels were approximately $20 \%$ less than those from testing with the uninsulated catalyst, while $\mathrm{NO}_{\mathrm{x}}$ levels were similar.

- Most of the emissions reductions were achieved in the first few minutes of the cold-start phase of the FTP. 


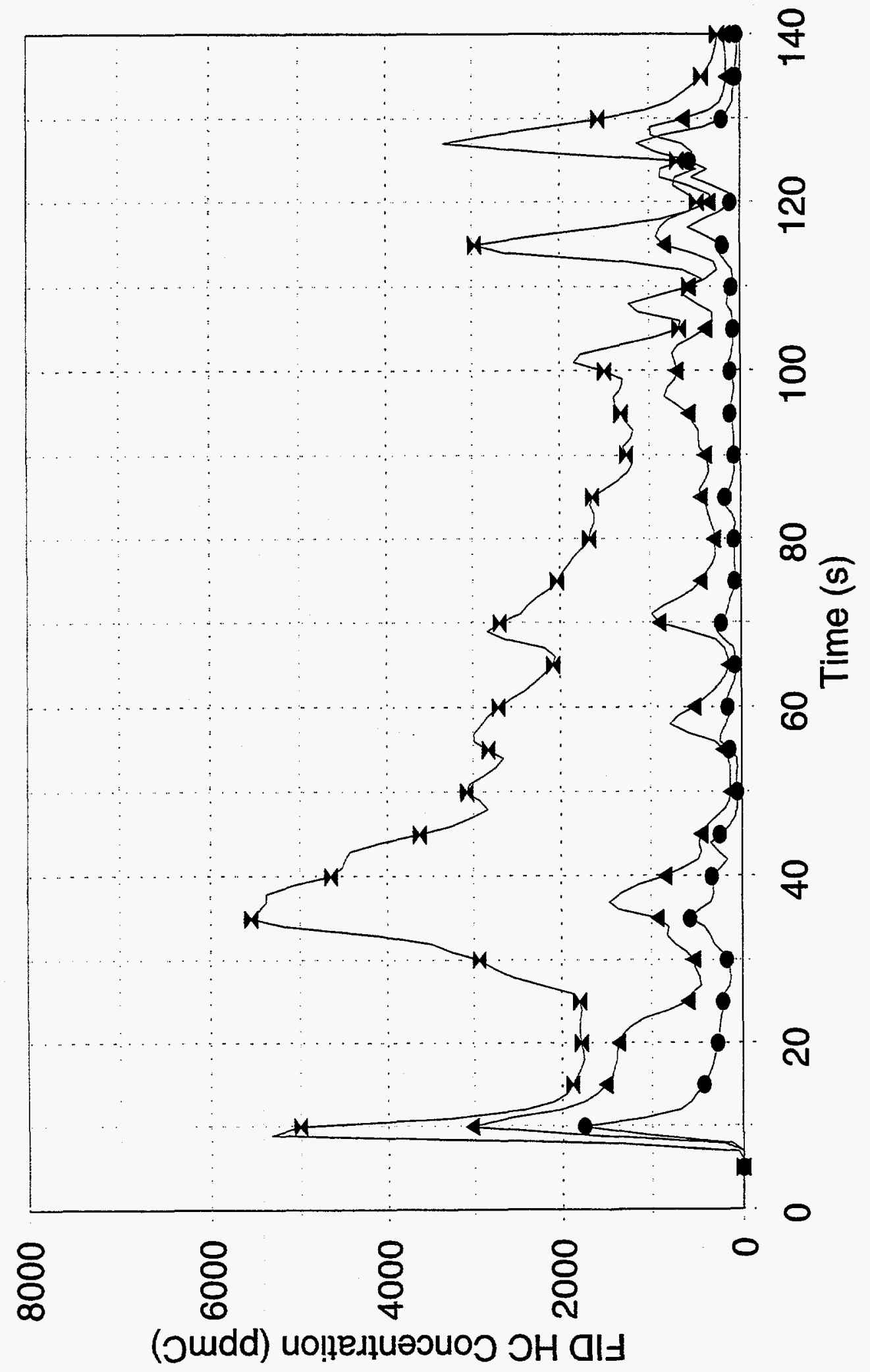



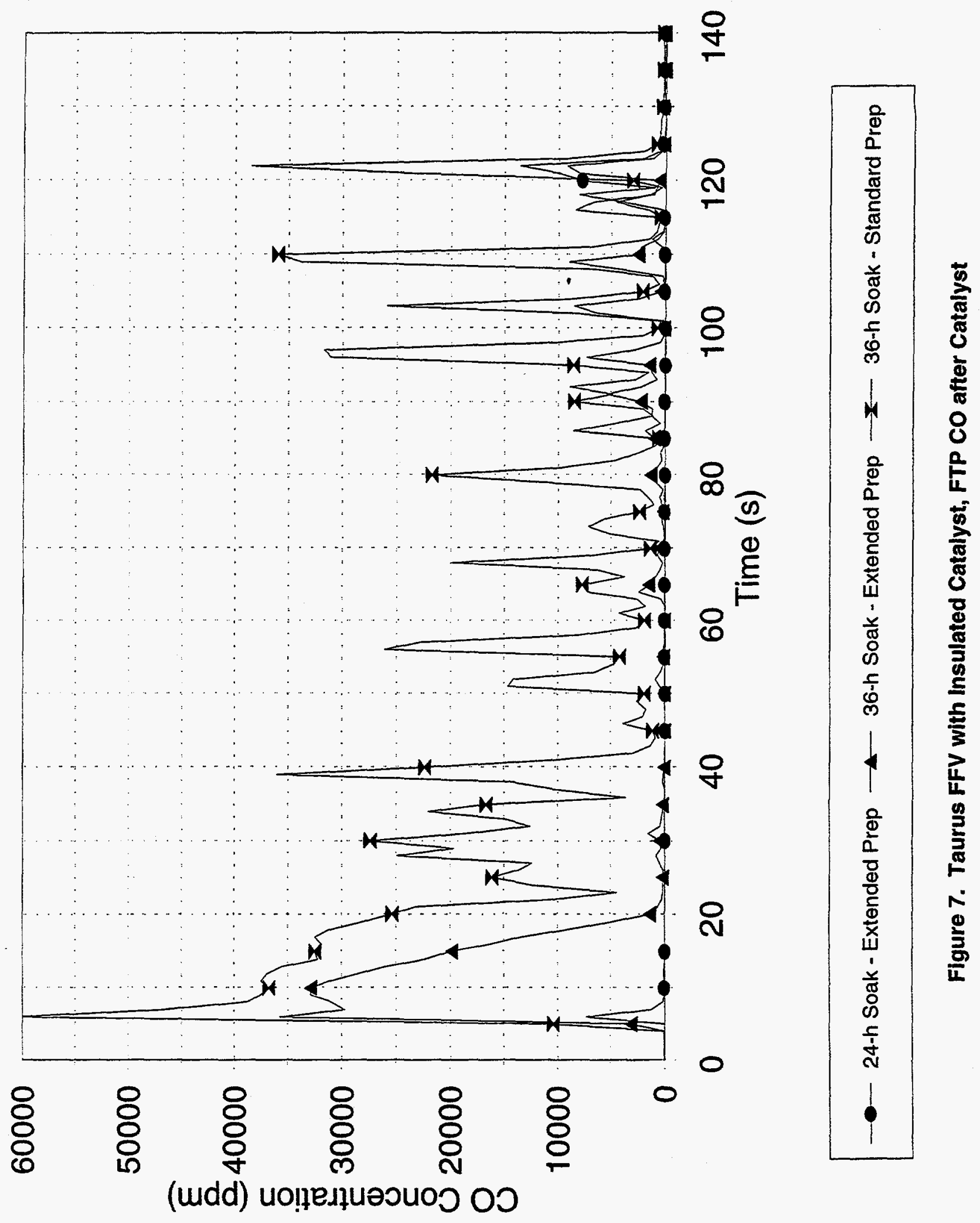

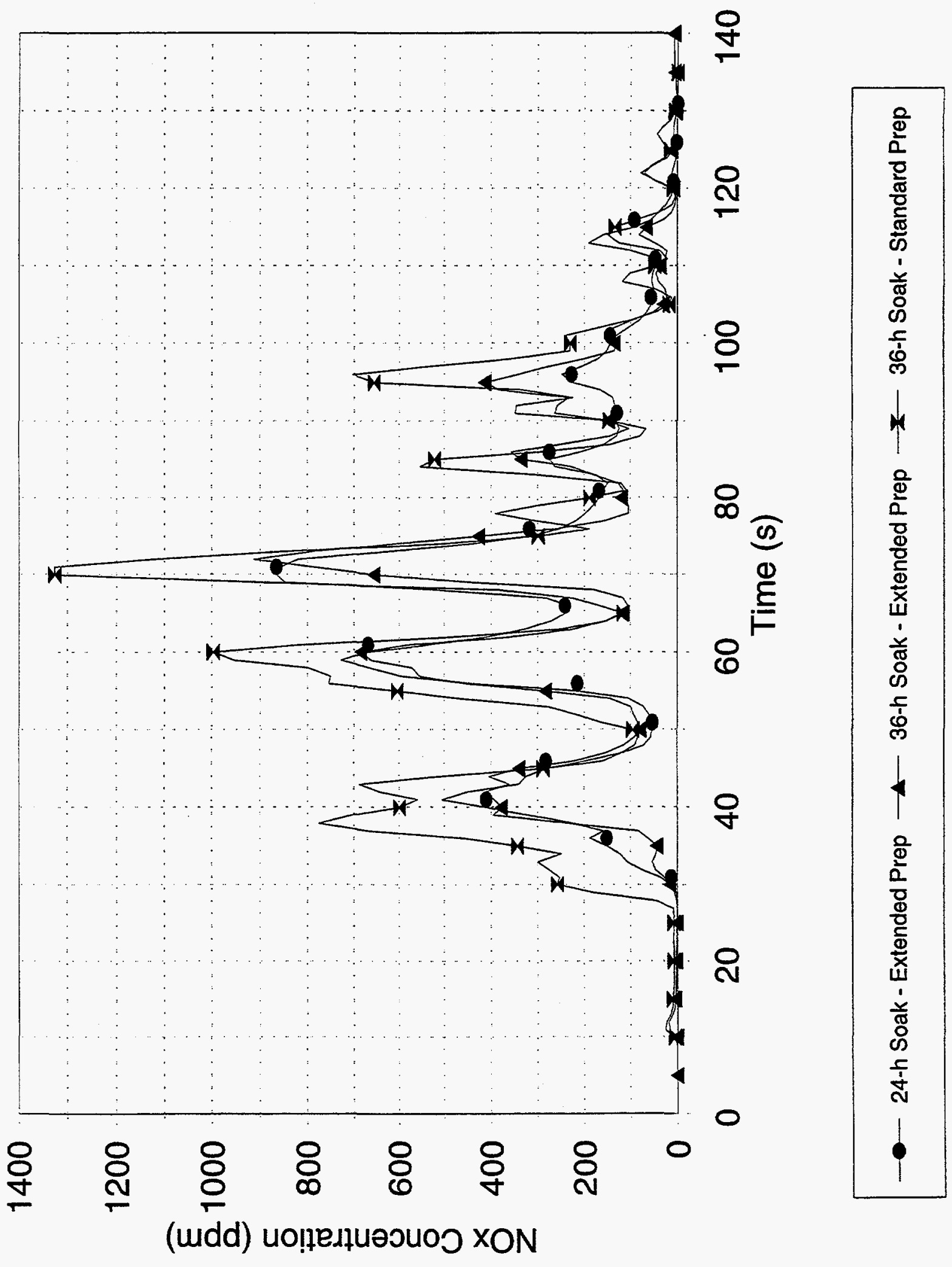

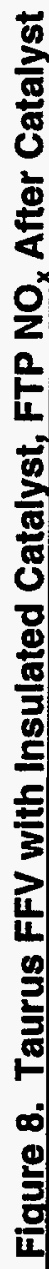



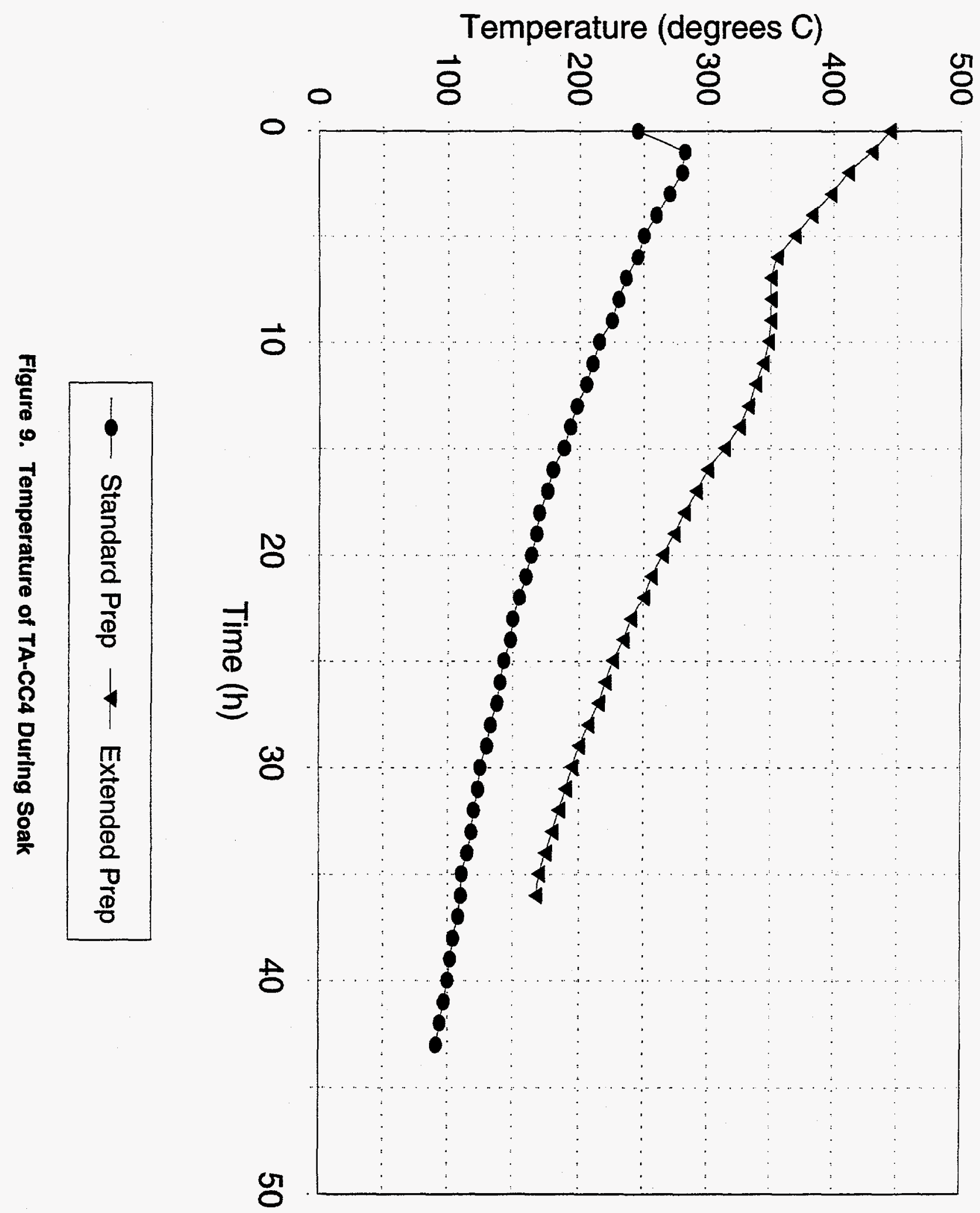


\section{Recommendations}

Further design improvements to hasten the full melt of the PCM would likely improve the net utility of the device, although the effects of the heat sink properties under certain circumstances can be detrimental. In its present state, a 38-mile extended prep sequence consisting of an UDDS followed by three HFETs was required to achieve full melt of the PCM. For the device to be fully effective, it would need to achieve full melt within the average in-use trip length, which was shown earlier to be on the order of 2.5 to 4.9 miles.

Heat storage capacity does not come without a weight penalty. Along with alternate configurations to accommodate the constricted space allotted to exhaust aftertreatment systems, weight is a big concern for automakers. As the system is refined, it would be expected that each of these design criteria would be further addressed.

Many studies of heat storage through PCM indicate that there is degradation of performance after a certain number of cycles, which varies with the particular PCM. Degradation of PCM performance can also be brought about by impurities, so not only will manufacturing practices need to be tightly controlled, but the PCM containment structure will have to exclude contamination as well as allow for expansion and contraction. Furthermore, material compatibility issues will have to be taken into account. Whether the PCM is a inorganic salt eutectic or a eutectic metal, interaction of the molten material with the container can be a daunting technical challenge. Failure of the PCM containment could lead to catastrophic results. Again, design for long-term durability will need to address these two concerns.

In selecting a PCM, not only will the above performance criteria need to be addressed, but the lifecycle environmental impact of the selected material must be carefully evaluated. As with any new technology, environmental consequences of manufacture, use, and re-use or disposal must be studied and factored into the material selection process. 


\section{A. Background}

UDRI has been conducting experiments with a flow reactor to determine alterative fuel combustion products. These studies are being conducted for NREL under Subcontract HZ 111208-1. One objective of this study is to compare results from flow reactor studies with data generated from engine and vehicle tests. SwRI conducted similar studies to determine alternative fuel combustion products in the first year of this program using vehicles operating on a chassis dynamometer.

\section{B. Approach and Results}

SwRI provided technical support to the UDRI in its effort to define correlations between engine-out combustion products identified by SwRI during chassis dynamometer testing, and those found during flow tube reactor experiments conducted at UDRI. Support included consultations concerning data previously provided by $S w R I$, providing fuel samples used in the first year of the SwRI program to UDRI for use in flow reactor experiments, and providing analyses of the fuels used at SwRI. When fuel samples were not available, SwRI staff provided fuel compositions to UDRI. In addition, SwRI staff reviewed data and reports generated by UDRI. UDRI will report the results of this correlation effort. 


\section{Task 4 - Characterization of Exhaust Particulate Emissions from Alternative Fuels}

\section{A. Background}

Particulate emissions have normally been a concern for diesel engines, but smoky and ill-smelling exhaust from spark-ignited engines was mentioned in the literature as early as 1910. ${ }^{(14)}$ At that time, the New York City Department of Health adopted a resolution regarding the discharge of smoke from motor vehicles within the city. This resolution suggests that people were becoming aware of a growing problem. Almost 60 years later in 1968, HC and $\mathrm{CO}$ emissions from light-duty vehicles were first regulated by EPA, but this action did not include a particulate emission standard. Particulate emissions for light-duty diesels were first regulated starting in 1982 , at $0.37 \mathrm{~g} / \mathrm{km}(0.60 \mathrm{~g} / \mathrm{mi})$. More recently, particulate emissions from all light-duty vehicles operating on gasoline, methanol, CNG, and LPG have been regulated for the first time. The EPA 1994 Tier 1 standards require that a minimum of $40 \%$ of a manufacturer's sales meet a $0.05 \mathrm{~g} / \mathrm{km}(0.08 \mathrm{~g} / \mathrm{mi})$ intermediate useful life standard and a 0.06 $\mathrm{g} / \mathrm{km}(0.10 \mathrm{~g} / \mathrm{mi})$ full useful life standard. In $1995,80 \%$ of the manufacturer's sales had to meet these standards; in 1996, $100 \%$ had to meet the standards. ${ }^{(15)}$

Because of demonstrated adverse health effects, particulate matter was designated a "criteria pollutant" under the Clean Air Act of 1970. The current 24-h NAAQS for $\mathrm{PM}_{10}$ is 150 $\mu \mathrm{g} / \mathrm{m}^{3}$, and California is considering a more stringent $50 \mu \mathrm{g} / \mathrm{m}^{3}$ standard. The term $\mathrm{PM}_{10}$ is defined as the portion of the total suspended particulate matter with an equivalent aerodynamic diameter of less than $10 \mu \mathrm{m}$. Particles in this size range are considered respirable, and have properties of biological importance because they are able to adsorb chemicals, and because of their specific compositions (such as containing asbestos). These properties have resulted in regulations for this particle size. More recently, particles in the smaller end of the $\mathrm{PM}_{10}$ size range have also been considered separately for their health effects and for air quality standards. Smaller particles in the range less than $2.5 \mu \mathrm{m}$, called $\mathbf{P M}_{2.5}$ or fine particulate matter, have unique properties in terms of selectively penetrating deep into the lungs. The uptake of fine particulate matter is about 70\%, which is higher than for larger particles. ${ }^{(16)}$ One major difference between these two particle sizes is the deposition of the larger particles from gravitational settling within several hours, whereas fine particles may remain suspended for days. With the pending changes to the NAAQS to include a standard for $\mathrm{PM}_{2.5}$, the contribution of particulate mass from all sources will be important.

The number and size of particles in engine exhaust are a function of many variables. The 1994 EPA definition of particulate is any mass collected on a fluorocarbon-coated glass fiber or fluorocarbon-based (membrane) filter, from a dilute exhaust stream, at a sample zone temperature less than $52^{\circ} \mathrm{C}\left(125^{\circ} \mathrm{F}\right) .^{(1)}$ For a given engine, the particle size and number depend on where the measurement is taken in the exhaust plume. This dependency on location is the result of the temperature, dilution, and "age" of the particles. As engine exhaust cools, the heavier gaseous HC begin to condense into aerosols, and the particles tend to agglomerate.

Particle size can be defined in several ways based on different assumptions about properties of the particles. If exhaust particulate was all perfect spheres, the particle size 
could be defined as the diameter of the sphere. Unfortunately, particles from combustion sources have highly irregular shapes. Several definitions of particle size result from these irregular shapes:

\begin{tabular}{ll}
- & Aerodynamic diameter \\
- & Geometric size \\
- & Stokes' diameter \\
- & Electrical mobility diameter \\
\hline
\end{tabular}

The definition used in this study is the equivalent aerodynamic diameter, which is defined as the diameter of a unit density sphere $(1 \mathrm{~g} / \mathrm{cc}$ ) having the same settling speed in air as the measured particle. Because particles may have many different physical sizes, shapes, and densities, the equivalent aerodynamic diameter indicates how the particle will behave in the environment in terms of particle transport. Sizing devices such as cascade impactors use aerodynamic principles to size particles. In an impactor, a sample containing particles with a mixture of shapes and densities is fractionated and collected according to aerodynamic characteristics. Particles that are aerodynamically equivalent in size to the unit density spheres that would collect on a specific stage during calibration under similar conditions are collected. As the sample stream passes through stages in the impactor with apertures of decreasing width or diameter, flow is accelerated and progressively smaller particles collect on impaction plates. ${ }^{(2)}$ The mass collected on each stage indicates the percentage of particles within a specific equivalent aerodynamic diameter range.

\section{B. Objective}

Particulate and regulated gaseous emissions were characterized for a 1994 Ford Taurus FFV operating on five fuels. The five fuels included RFG, M85, E85, LPG meeting HD-5 specifications, and industry-average CNG. Particulate emissions were characterized by total mass and particle size. The regulated gaseous emissions included: either THC and NMHC, or OMHCE and OMNMHCE; CO; and $\mathrm{NO}_{\mathbf{x}}$.

\section{Approach}

Experiments to evaluate the particulate emissions from five different fuels involved:

- Establishing a baseline condition with RFG under stoichiometric operating conditions

- Installing duplicate wide-range oxygen sensors and associated control circuitry to allow rich operation on liquid fuels (RFG, M85, and E85)

- Installing kits for operation on LPG and CNG

- Performing two repetitive cold-start FTP tests with each of the five fuels to collect gaseous and particulate emissions.

To collect sufficient particulate for mass determination, samples were collected on the same filters over the duration of duplicate FTP tests performed on consecutive days. 


\section{Vehicle}

A 1994 Ford Taurus FFV with a 3.0 L V-6 engine, a three-way catalyst, exhaust gas recirculation, and about 20,000 odometer miles was used for the testing. This vehicle conformed to EPA and CARB regulations applicable to 1994 model year vehicles introduced into commerce solely for sale in California, and has been certified to meet the CARB TLEV standards.

The vehicle was operated fuel-rich to simulate a failure mode for the increased production of particulate matter. No other changes were made to the vehicle calibration while operating on RFG, M85, or E85. For operation on CNG and LPG, the vehicle was equipped with an appropriate conversion kit for each of the gaseous fuels. Both kits were carbureted systems, and were obtained from IMPCO Technologies, Inc. These kits utilized feedback signals from the OEM EGO sensor to regulate a fuel control valve, maintaining fuel/air stoichiometry.

To simulate a fuel-rich failure mode while operating on all five fuels, the EGO sensors were replaced with UEGO sensors, which were capable of operating throughout the range of fuel/air equivalence ratios required by this program. The UEGO signals were passed through a compactor circuit to generate a switching output similar to that of an EGO sensor. A compactor output was connected to the fuel control system's EGO sensor inputs. By using a tunable compactor switch point, the closed loop controller was able to operate the vehicle at a rich fuel/air equivalence ratio.

For each fuel, the fuel control system was tuned to a rich fuel/air equivalence ratio set point by operating the vehicle on a chassis dynamometer at a steady-state condition of $80 \mathrm{kph}$ $(50 \mathrm{mph})$ and $5.1 \mathrm{~kW}(6.8 \mathrm{hp})$ actual road load. Nominal fuel/air equivalence ratio at this condition was set at approximately 1.1 for all fuels. Vehicle performance and driveability were satisfactory over the FTP (no significant stumbling or hesitation) for all fuels at this mixture setting.

It should be noted that the goal of this study was to determine the feasibility of measuring particulate emissions from alternative fuels. Rich vehicle operation was chosen to increase the chance of collecting a measurable mass of particulate from each of the fuels. Due to the nature of this feasibility study, tests were not conducted on the alternative fuels during stoichiometric operation.

\section{E. Fuels}

The five fuels included RFG, M85, E85, LPG meeting HD-5 specifications, and industry-average CNG. ${ }^{17}$ ) Industry-average gasoline (RF-A) was used as the blending agent and denaturant when preparing the M85 and E85 alcohol blends. The denatured ethanol was prepared with 5\% RF-A gasoline and 95\% ethanol. RFG and LPG were purchased from commercial suppliers, while the E85, M85, and CNG were blended in house. The HD-5 specifications for LPG included less than $5 \%$ propylene and greater than $90 \%$ propane. Table 13 lists the fuel properties for the three liquid fuels, and Table 14 presents the fuel gas analysis for the two gaseous fuels. 
Table 13. Fuel Analysis for Liquid Fuels

\begin{tabular}{|l|c|c|c|}
\hline \multicolumn{1}{|c|}{ Property } & RFG & M85 & E85 \\
\hline \hline \multicolumn{2}{|c|}{ Carbon, Hydrogen, and Oxygen, wt\% } \\
\hline Carbon & 84.37 & 43.06 & 61.20 \\
\hline Hydrogen & 13.24 & 12.72 & 13.21 \\
\hline Oxygen & 2.39 & 44.22 & 25.59 \\
\hline \hline & Other Properties & \\
\hline RVP & 7.12 & 8.05 & 6.76 \\
\hline Specific gravity & 0.7538 & 0.7935 & 0.7795 \\
\hline API gravity & 56.2 & 46.8 & 50.0 \\
\hline
\end{tabular}

Table 14. Fuel Gas Analysis Summary

\begin{tabular}{|c|c|c|}
\hline \multirow{2}{*}{ Component } & \multicolumn{2}{|c|}{ Compound Concentration, mole \% } \\
\cline { 2 - 3 } & CNG & LPG \\
\hline Carbon dioxide & 0.00 & 0.00 \\
\hline Nitrogen & 3.72 & 0.00 \\
\hline Methane & 90.13 & 0.00 \\
\hline Ethane & 4.04 & 3.00 \\
\hline Propane & 2.12 & 96.40 \\
\hline Propylene & 0.00 & 0.30 \\
\hline Isobutane & 0.00 & 0.28 \\
\hline Nomal butane & 0.00 & 0.02 \\
\hline Pentane + & 0.00 & 0.00 \\
\hline Fuel fraction nitrogen & 0.642 & 0.687 \\
\hline Fuel fraction hydrogen & 0.712 & 0.817 \\
\hline Energy equivalent fuel density, $\mathrm{kg}$ L & 0.229 & 0.183 \\
\hline Fun carbon & & 0.059 \\
\hline
\end{tabular}




\section{F. Emission Test Procedures}

The Ford Taurus FFV was evaluated using the chassis dynamometer portion of the FTP for light-duty vehicles. In addition, the vehicle was preconditioned using three consecutive UDDS cycles to minimize the effects of the fuel changes on the ECU's adaptive learning and to help condition the dilution tunnel to minimize particulate carryover between fuels. A Clayton Model ECE-50 passenger car dynamometer, in conjunction with a positive displacement pump constant volume sampler, was used for all testing. $\mathrm{THC}, \mathrm{CO}, \mathrm{NO}_{\mathrm{x}}$, and $\mathrm{CO}_{2}$ emissions were collected as dilute exhaust in Tedlar gas sample bags, in a manner consistent with EPA protocols for light-duty emission testing. Fuel economy was determined by carbon balance. Wet absorption techniques were employed to collect methanol, ethanol, and aldehydes and ketones for the determination of OMHCE. A detailed description of these procedures is presented in Sections I.E. and I.F.

Total particulate mass was measured using a 47-mm fluorocarbon-coated glass fiber filter that collected a portion of the sample from the dilution tunnel. Particle size distribution measurements were performed with an in-stack cascade impactor. To collect sufficient particulate for mass determination, samples were collected on the same filters over the duration of duplicate FTP tests performed on consecutive days. Particulate filters and impactor stages were stored in a temperature- and humidity-controlled weighing chamber between tests. This chamber is maintained at $75^{\circ} \mathrm{F} \pm 10^{\circ} \mathrm{F}$ and a relative humidity of $58 \% \pm$ $10 \%$.

\section{G. Results}

\section{Gaseous Emissions}

Table 15 summarizes the FTP composite emissions for each fuel, and Figures 10 through 14 illustrate the average regulated emissions and fuel economy for each fuel. Detailed printouts of individual test results are given in Appendix D. With the vehicle operating at stoichiometry on RFG, the emission rates were well below the 1994 EPA Tier 1 standards for pollutants. The fuel economy for the gaseous fuels was based on an energy equivalent fuel density of $5.360 \mathrm{lb} / \mathrm{gal}$ for CNG and $5.729 \mathrm{lb} / \mathrm{gal}$ for LPG.

In the following tables and figures, fuel code RFG-Base indicates that the vehicle was tested at stoichiometric operating condition, and fuel codes RFG-1 and RFG-3 were repeat tests using RFG with the vehicle at a rich fuel/air equivalence ratio. Fuel code CNG-1 was tested at a lean fuel/air equivalence ratio because the emissions test results indicated that the vehicle, operating with the wide-range oxygen sensors and associated control circuitry, was actually adjusted to allow lean operation. The system, during this initial test with CNG, was not functioning as expected; so an additional test was performed (fuel code CNG-2) at a rich fuel/air equivalence ratio. When the fuel code RFG appears in tables and figures, it represents the average results for tests RFG-1 and RFG-3. When CNG appears, it represents the results for Test CNG-2. 


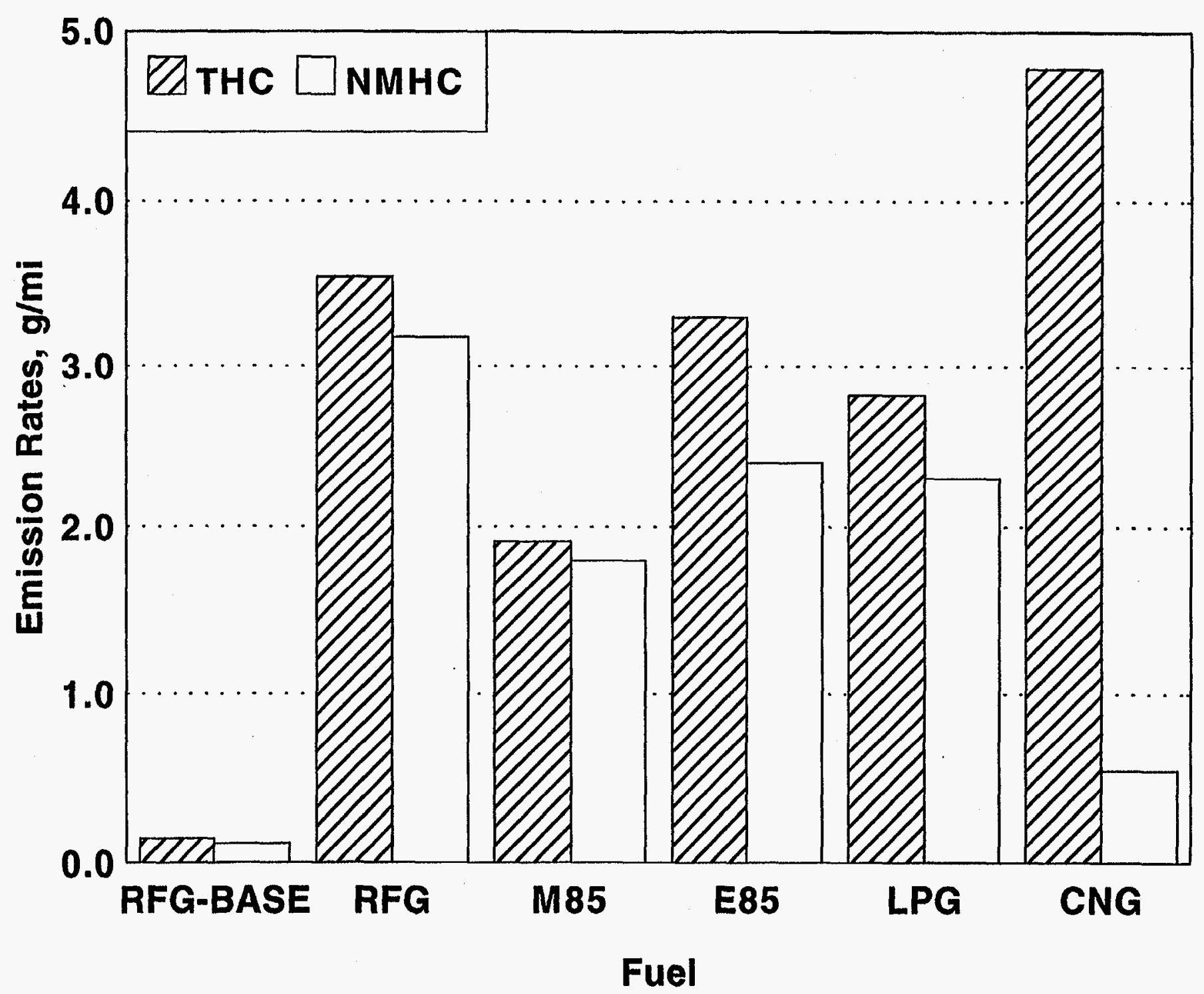

Figure 10. Comparison of HC Emission Rates 


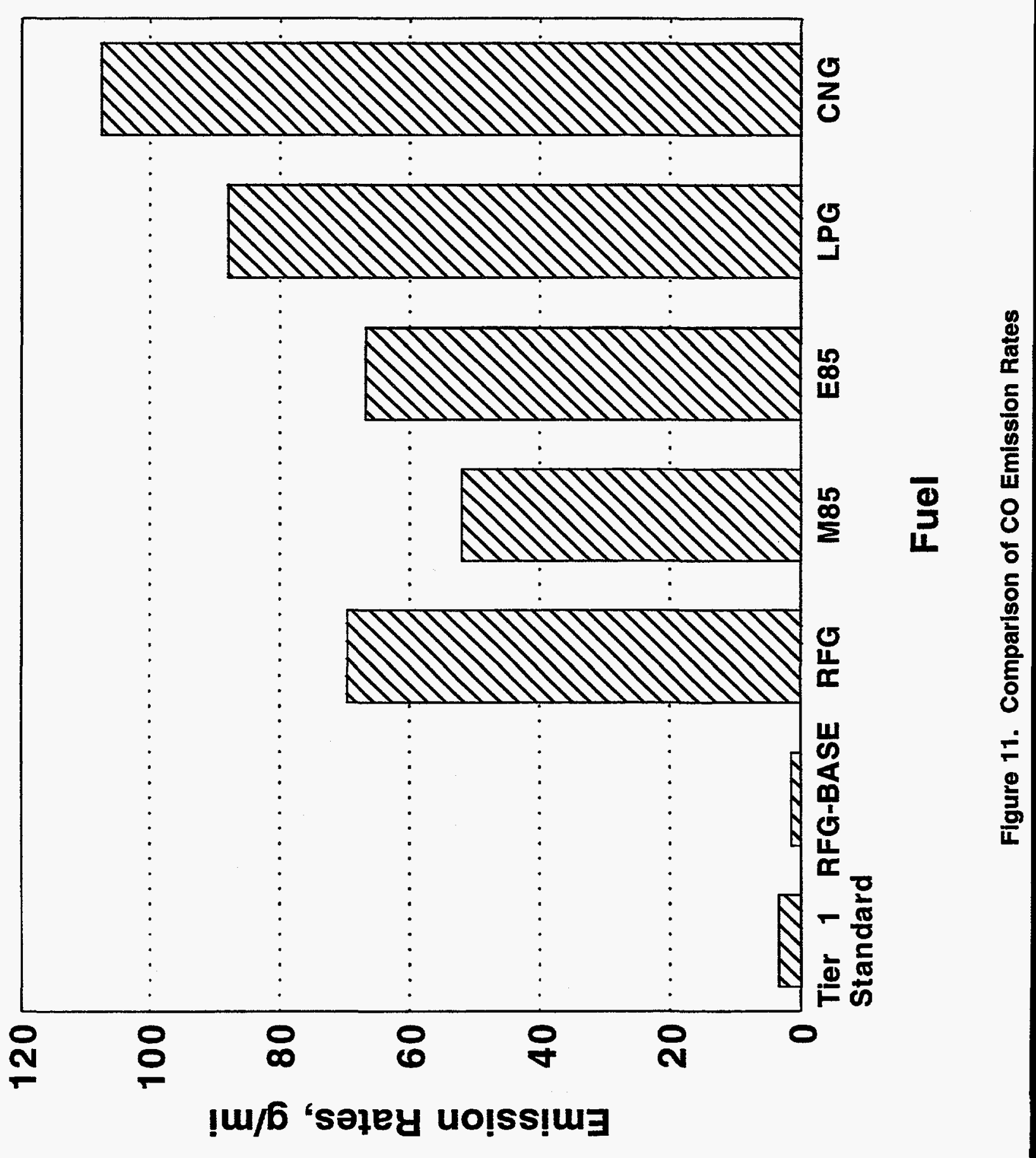




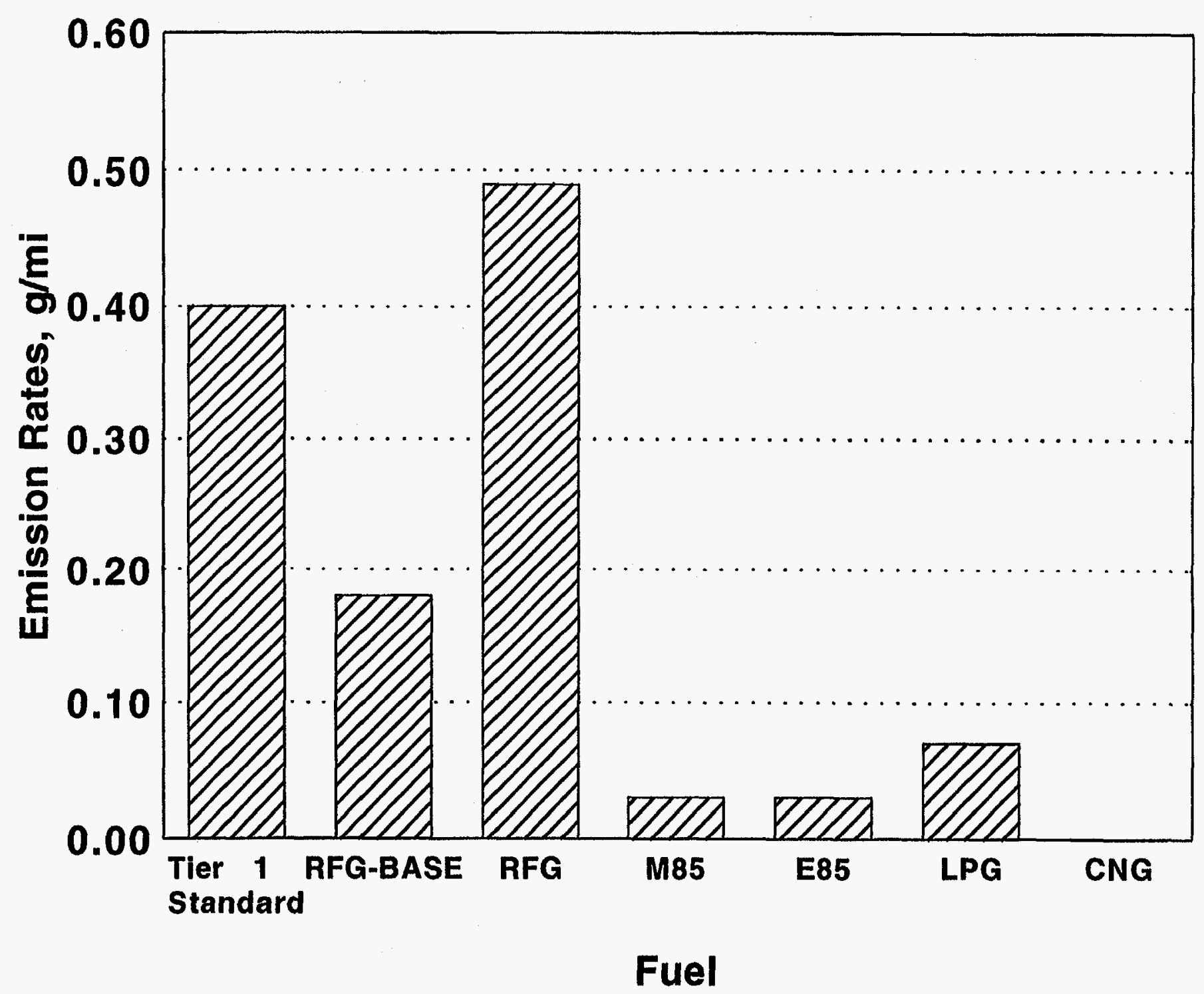

Figure 12. Comparison of $\mathrm{NO}_{x}$ Emission Rates 


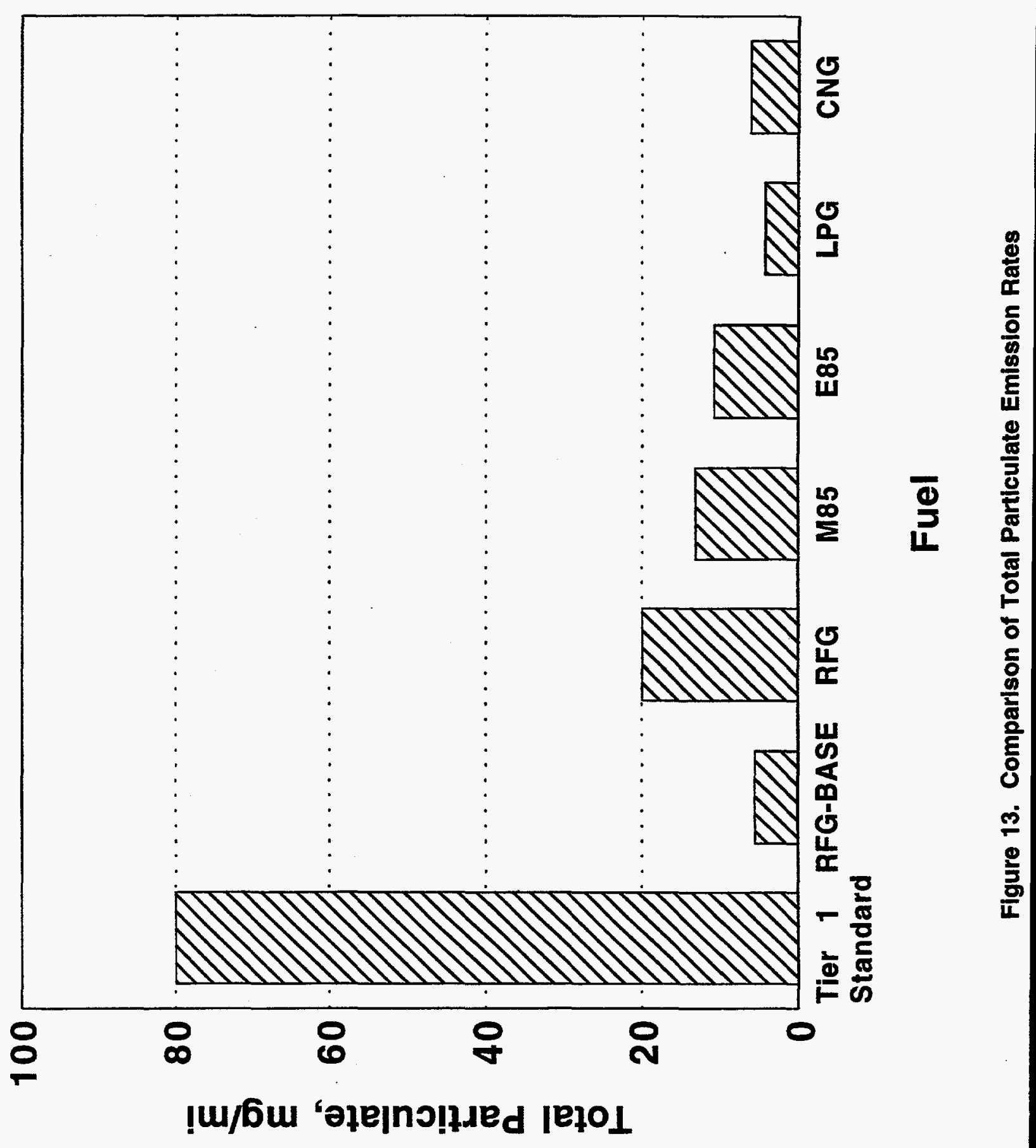




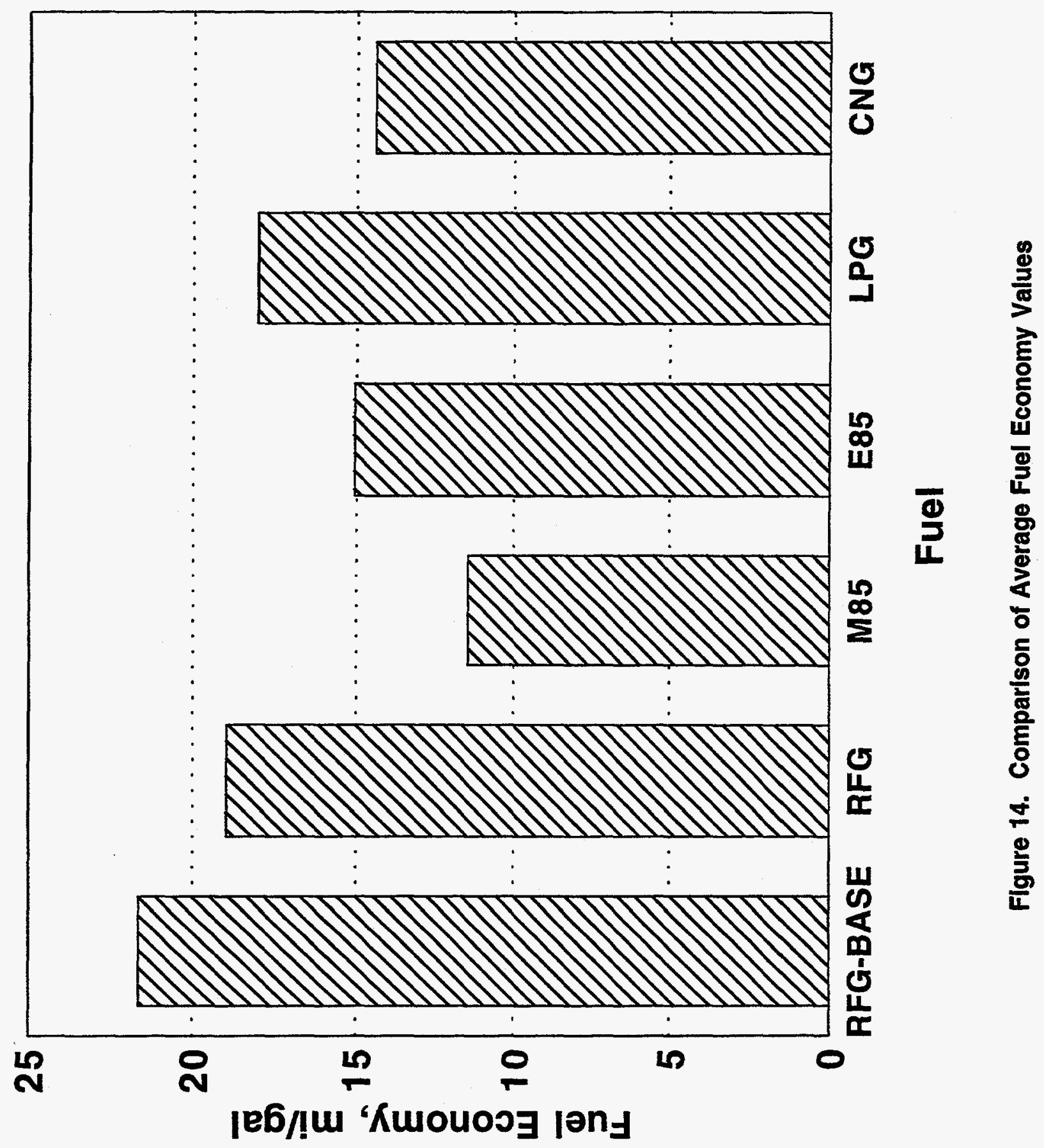


Table 15. Summary of Composite FTP Regulated Emissions

\begin{tabular}{|c|c|c|c|c|c|c|}
\hline \multirow[b]{2}{*}{ Fuel } & \multicolumn{4}{|c|}{ Emissions Results, $\mathbf{g} / \mathrm{mi}^{\mathrm{a}}$} & \multirow{2}{*}{$\begin{array}{c}\text { Particulate, } \\
\mathrm{mg} / \mathrm{mi}\end{array}$} & \multirow{2}{*}{$\begin{array}{c}\text { Fuel economy, } \\
\mathrm{mi} / \mathrm{gal}\end{array}$} \\
\hline & $\mathrm{THC}^{6}$ & NMHC ${ }^{\circ}$ & co & $\mathrm{NO}_{\mathrm{x}}$ & & \\
\hline Tier 1 standard & 0.41 & 0.25 & 3.4 & 0.4 & 80 & $N A^{d}$ \\
\hline RFG-base ${ }^{e}$ & 0.14 & 0.11 & 1.44 & 0.18 & 5.5 & 21.67 \\
\hline RFG & 3.55 & 3.18 & 69.45 & 0.49 & 19.9 & 18.98 \\
\hline M85 & 1.91 & 1.80 & 51.93 & 0.03 & 13.1 & 11.48 \\
\hline E85 & 3.31 & 2.40 & 66.63 & 0.03 & 10.7 & 15.07 \\
\hline $\mathrm{LPG}$ & 2.82 & 2.30 & 87.90 & 0.07 & 4.2 & $18.03^{\prime}$ \\
\hline $\mathrm{CNG}$ & 4.78 & 0.54 & 107.57 & 0.00 & 6.1 & $14.44^{9}$ \\
\hline \multicolumn{7}{|c|}{$\begin{array}{l}\text { Numbers in parentheses in terms of g/mi; conversion factor }-\mathrm{g} / \mathrm{km} \times 1.609 \mathrm{~km} / \mathrm{mi}=\mathrm{g} / \mathrm{mi} \\
\text { THC for gasoline, CNG, and LPG; and OMHCE for methanol and ethanol } \\
\text { NMHC for gasoline, CNG, and LPG; and OMNMHCE for methanol and ethanol } \\
\text { NA-Not applicable } \\
\text { Nehicle operating at stoichiometry; all others rich operation } \\
\text { Energy equivalent fuel density of } 5.729 \mathrm{lb} / \mathrm{gal} \text { for LPG } \\
\text { Energy equivalent fuel density of } 5.360 \mathrm{lb} / \mathrm{gal} \text { for CNG }\end{array}$} \\
\hline
\end{tabular}

Baseline THC, $\mathrm{CO}$, and $\mathrm{NO}_{\mathrm{x}}$ exhaust emission rates on RFG correlate with historical data from this vehicle, and with measurements from other Taurus FFVs. When the vehicle was operated on RFG at a rich fuel air equivalence ratio, THC emissions increased by 25 times, and $\mathrm{CO}$ emissions increased by nearly 50 times. These trends are typical for rich operation. However, $\mathrm{NO}_{\mathrm{x}}$ emissions also increased, by nearly 3 times, during rich operation on RFG. Replicate tests confirmed this result, and all other observations indicated the vehicle was operating rich. Although high $\mathrm{NO}_{\mathrm{x}}$ exhaust emissions are not expected during rich operation, it was outside the scope of the program to investigate this phenomenon any further. Rich operation on all other fuels (M85, E85, CNG, and LPG) resulted in high HC and CO exhaust emissions, and low $\mathrm{NO}_{\mathrm{x}}$ emissions.

\section{Alcohols, Aldehydes and Ketones}

Samples were analyzed for alcohols, aldehydes, and ketones from the three liquid fuels. Table 16 summarizes the individual compounds for each fuel. Detailed data for individual tests are given in Appendix D. In general, these emissions followed expected trends. With rich operation, all alcohols, aldehydes, and ketones increased when compared to RFG at a stoichiometric fuel/air equivalence ratio. In addition, the alcohol and aldehydes with the corresponding carbon number to the alcohol in the fuel produced higher concentrations in the exhaust when compared to the tests with RFG under rich operation. These results were used to calculate OMNMHCE. 
Table 16. Composite Emission Results for Alcohols, Aldehydes, and Ketones

\begin{tabular}{||l|c|c|c|c||}
\hline \multirow{2}{*}{} & \multicolumn{4}{|c|}{ Composite Results, mg/mi } \\
\cline { 2 - 5 } & RFG-BASE & RFG-3 & M85 & E85 \\
\hline \hline Formaldehyde & 1.27 & 18.13 & 85.85 & 14.93 \\
\hline Acetaldehyde & 0.36 & 23.81 & 4.37 & 476.10 \\
\hline Acrolein & 0.00 & 1.25 & 0.02 & 0.36 \\
\hline Acetone & 0.00 & 13.03 & 1.73 & 8.19 \\
\hline Propionaldehyde & 0.00 & 4.18 & 0.76 & 1.65 \\
\hline Crotonaldehyde & 0.17 & 2.33 & 0.17 & 0.89 \\
\hline isobutyraldehyde and methyl ethyl ketone & 0.04 & 2.21 & 0.76 & 1.21 \\
\hline Benzaidehyde & 0.09 & 6.20 & 1.13 & 1.08 \\
\hline Hexanaldehyde & 0.94 & 0.40 & 0.00 & 0.00 \\
\hline Methanol & 1.99 & 66.41 & 2149 & 98.33 \\
\hline Ethanol & 0.22 & 1.31 & 0.00 & 1172 \\
\hline
\end{tabular}

The proposed Title 13, California Code of Regulations, Section 1960.1 includes a formaldehyde standard for 1992 and subsequent model-year light-duty vehicles. For TLEV and LEV, the standard is $9.3 \mathrm{mg} / \mathrm{km}$ (15 $\mathrm{mg} / \mathrm{mi}$ ) with an intermediate in-use compliance standard for $31,000 \mathrm{~km}(50,000 \mathrm{mi})$ of $14 \mathrm{mg} / \mathrm{km}(23 \mathrm{mg} / \mathrm{mi})$ and $9.3 \mathrm{mg} / \mathrm{km}(15 \mathrm{mg} / \mathrm{mi})$, respectively. ULEV must meet $5.0 \mathrm{mg} / \mathrm{km}(8 \mathrm{mg} / \mathrm{mi})$ with a $31,000 \mathrm{~km}(50,000 \mathrm{mi})$ in-use standard of $7.5 \mathrm{mg} / \mathrm{km}$ (12 mg/mi). With RFG at the baseline condition, the emission rate of formaldehyde was well below the proposed standard. When the vehicle was operated fuel-rich with RFG and M85, the formaldehyde emissions exceeded the proposed standard by about $20 \%$ and $470 \%$, respectively. The fuel rich test with E85 was just able to meet the $9.3 \mathrm{mg} / \mathrm{km}$ (15 $\mathrm{mg} / \mathrm{mi}$ ) formaldehyde standard.

\section{Total Particulate Mass and Particle Size Distribution}

Prior to performing emission tests on the vehicle, background samples were taken from the ambient air in the laboratory and from the CVS background (in the dilution tunnel) to determine the sizes of the particles that pass through the CVS filter box. Figure 15 shows the ambient and CVS background results from these measurements. The largest particles in the laboratory ambient air had equivalent aerodynamic diameters larger than 3 $\mu \mathrm{m}$. These particles accounted for more than $50 \%$ of the particle mass in the laboratory ambient air. After the air was passed through the CVS filter box, nearly all the large particles were removed, and the largest contribution to the CVS background became particles in the $0.16 \mu \mathrm{m}$ to $0.52 \mu \mathrm{m}$ equivalent aerodynamic diameter size range. The CVS background was estimated to contribute less than $0.03 \%$ of the total mass collected on all of the impactor stages during each test on the vehicle. In addition, the filter box was capable of removing about $84 \%$ of the laboratory ambient air particle mass and about $51 \%$ of the ambient particle mass in the equivalent aerodynamic diameter range produced by the vehicle. In summary, the background contributions to the total mass collected by the impactor were small; therefore, no background corrections were made for the measurements. 


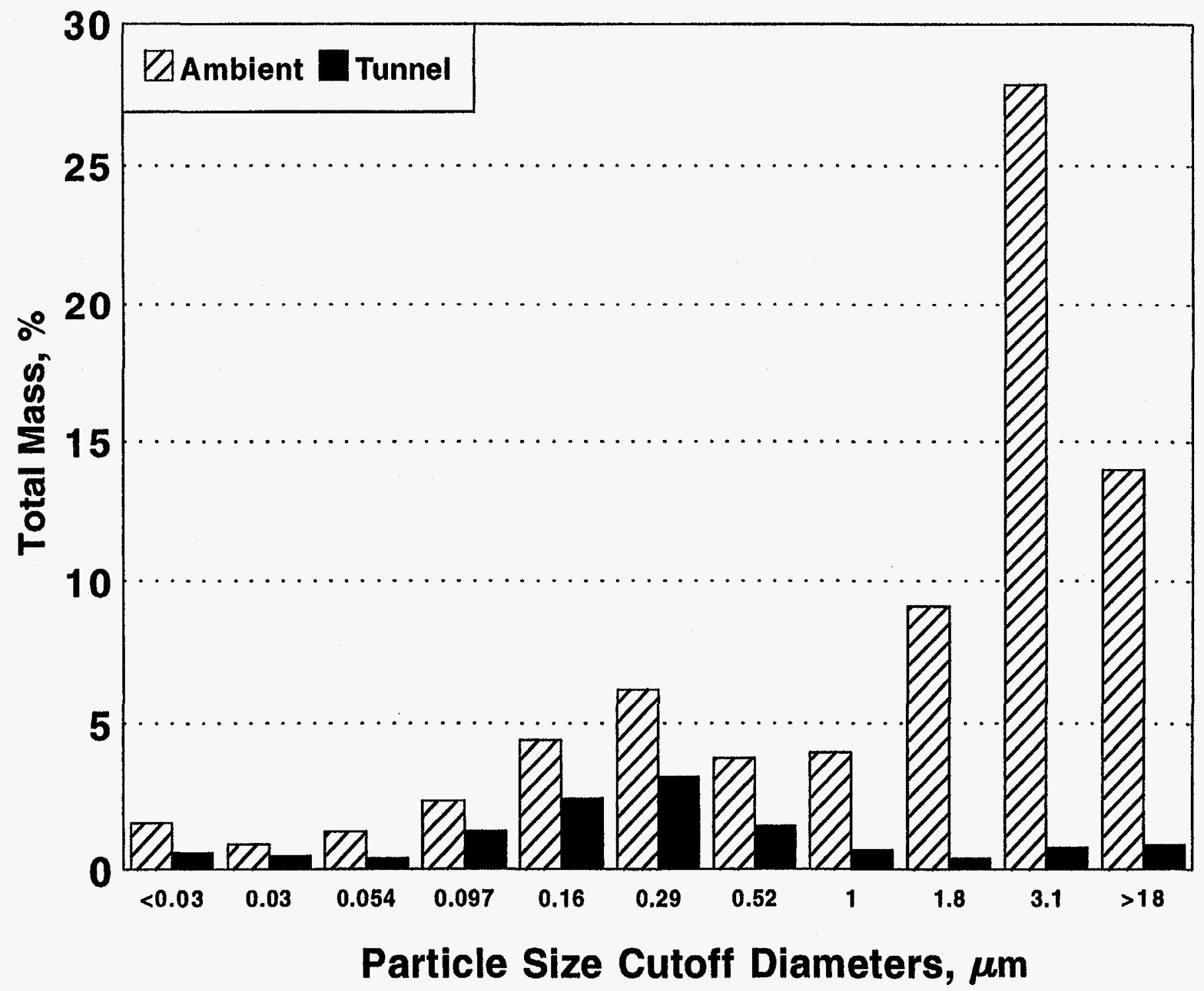

Fiqure 15. Particle Size Distribution of Laboratory Amblent and CVS Background Particulate 
The general trend in particle size distribution for particulate mass from this spark-ignited engine was significantly different from typical results for particulate mass from diesel (compression-ignition) engines. In the case of diesel engines, the majority of the particles are smaller than about $0.8 \mu \mathrm{m}$ equivalent aerodynamic diameter, and a significant percentage occurs in the range less than $0.2 \mu \mathrm{m} .{ }^{(18-19)}$ Very few particles are larger than about $1 \mu \mathrm{m}$.

In these experiments with a spark-ignited engine, the results at a stoichiometric fuel/air equivalence ratio indicated that the particle size range containing most of the mass was less than about $0.2 \mu \mathrm{m}$ (about $65 \%$ of the total particulate mass), and the next largest percentage of particle cutoff diameters was in the 2 to $3 \mu \mathrm{m}$ range (about 26\%). Figure 16 shows the equivalent aerodynamic diameter particulate emission rates compared by fuel, and Figure 17 illustrates the mass percentage of each particle size cutoff in relation to the equivalent aerodynamic diameter. Table 17 and Figure 18 present the particle size distribution data for each fuel.

When the vehicle was operated fuel rich with RFG, more total mass of particulate was collected, and a significant shift in the particle size distribution occurred when compared to the baseline condition with RFG. Total particulate mass increased by a factor of almost four, from $5.5 \mathrm{mg} / \mathrm{mi}$ to $19.9 \mathrm{mg} / \mathrm{mi}$. The percentage of particle mass was greatest in the less than $0.2 \mu \mathrm{m}$ size range. The second most abundant particle size was in the greater than $3 \mu \mathrm{m}$ range. Percentages of particle mass in each equivalent aerodynamic diameter range decreased from the range greater than $3 \mu \mathrm{m}$ to a minimum at the $0.2-0.4 \mu \mathrm{m}$ range.

In the case of M85, the total particulate rate was about twice that for the RFG baseline and about $35 \%$ less than that for RFG rich operation. The most abundant particle mass was in the size range less than $0.2 \mu \mathrm{m}$. Percentages of particle mass in the size ranges from $0.7 \mu \mathrm{m}$ to greater than $3 \mu \mathrm{m}$ were relatively constant, and the percentages for the 0.2 to $0.7 \mu \mathrm{m}$ ranges were less than half of the larger sizes.

For E85, the total particulate mass was also about twice the RFG baseline, and $47 \%$ less than RFG rich operation. With this fuel, the largest percentages of particle mass were in the 1 to $2 \mu \mathrm{m}$ range and in the below $0.2 \mu \mathrm{m}$ range. In addition, the percentage of particle mass increased from smaller to larger particle sizes with a maximum in the 1 to $2 \mu \mathrm{m}$ range, and then decreased toward the larger particle sizes.

With rich LPG operation, the total particulate rate was $24 \%$ less than the RFG baseline condition, and 79\% less than the RFG rich condition. Most of the particle mass was in the size range less than $0.2 \mu \mathrm{m}$ (about $60 \%$ of the total particle mass). The percentages of particle mass in the size range from $0.4 \mu \mathrm{m}$ to $0.2 \mu \mathrm{m}$ and greater than $3 \mu \mathrm{m}$ were the next highest. 


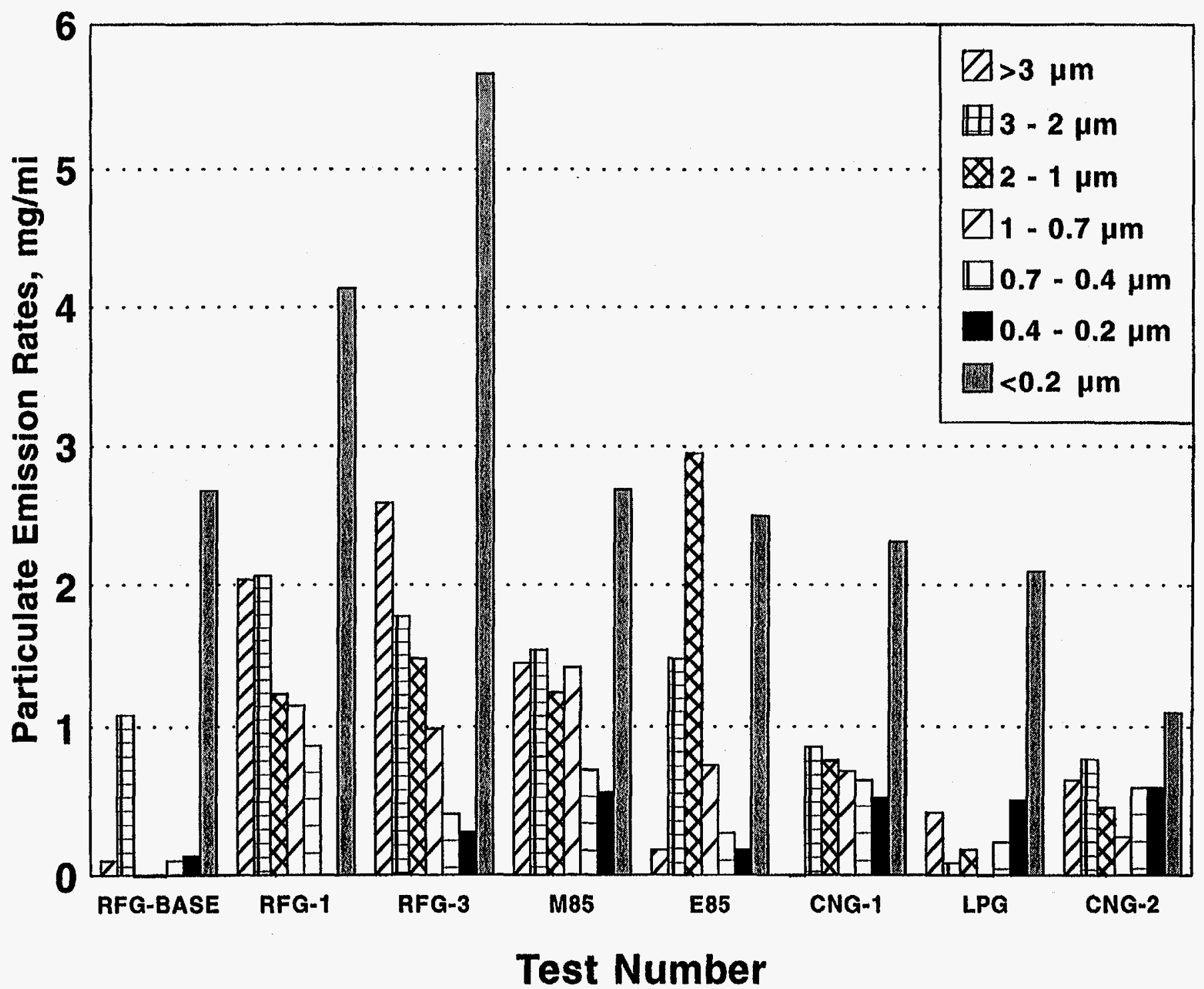

Flgure 16. Particle Size Distribution by Fuel 


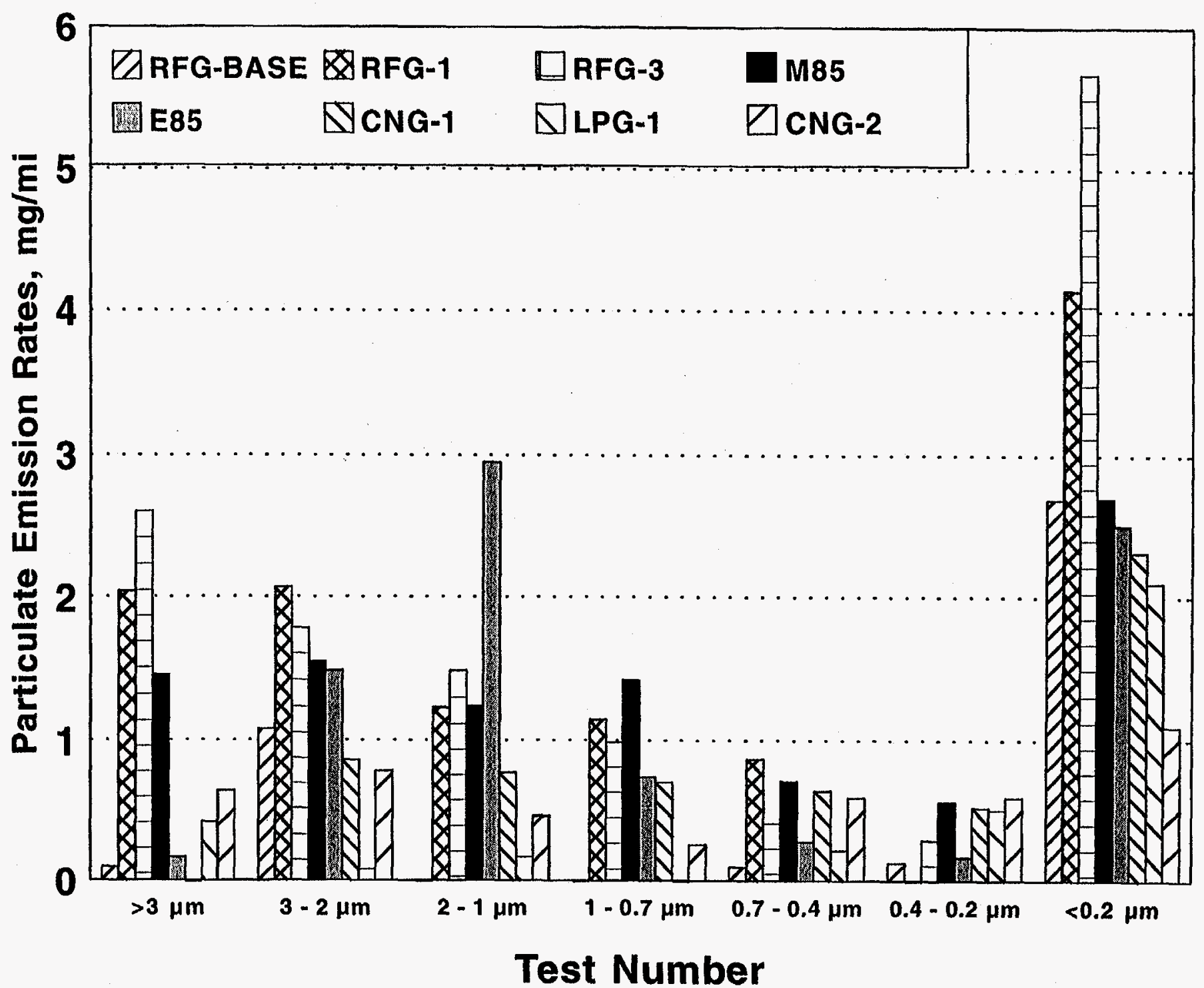

Figure 17. Equivalent Aerodynamic Dlameter Particle Size Distribution 
Table 17. Particle Size Distribution "

\begin{tabular}{|c|c|c|c|c|c|c|c|c|c|c|c|c|c|c|c|}
\hline \multirow{3}{*}{$\begin{array}{c}\text { Test } \\
\text { Number }\end{array}$} & \multicolumn{15}{|c|}{ Partlcle Size Cutoff Diameters } \\
\hline & \multicolumn{2}{|c|}{$>3 \mu \mathrm{m}$} & \multicolumn{2}{|c|}{$3-2 \mu \mathrm{m}$} & \multicolumn{2}{|c|}{$2-1 \mu \mathrm{m}$} & \multicolumn{2}{|c|}{$1-0.7 \mu \mathrm{m}$} & \multicolumn{2}{|c|}{$0.7 \cdot 0.4 \mu \mathrm{m}$} & \multicolumn{2}{|c|}{$0.4-0.2 \mu \mathrm{m}$} & \multicolumn{2}{|c|}{$<0.2 \mu \mathrm{m}$} & \multirow{2}{*}{$\begin{array}{c}\text { Total } \\
\text { Partlculate, } \\
\text { mg/ml }\end{array}$} \\
\hline & Percent & $\mathrm{mg} / \mathrm{ml}$ & Percent & $\mathrm{mg} / \mathrm{ml}$ & Porcent & $\mathrm{mg} / \mathrm{ml}$ & Percent & $\mathrm{mg} / \mathrm{mi}$ & Percent & $\mathrm{mg} / \mathrm{ml}$ & Percent & $\mathbf{m g} / \mathbf{m l}$ & Percent & $\mathbf{m g} / \mathbf{m l}$ & \\
\hline RFG-BASE & 2.5 & 0.10 & 26.4 & 1.08 & 0.0 & 0.00 & 0.0 & 0.00 & 2.5 & 0.10 & 3.1 & 0.13 & 65.4 & 2.68 & 4.1 \\
\hline RFG & 19.6 & 2.59 & 13.5 & 1.78 & 11.2 & 1.48 & 7.5 & 0.99 & 3.1 & 0.41 & 2.2 & 0.29 & 42.9 & 5.66 & 13.2 \\
\hline M85 & 15.1 & 1.45 & 16.0 & 1.54 & 12.9 & 1.24 & 14.8 & 1.42 & 7.4 & 0.71 & 5.8 & 0.56 & 28 & 2.69 & 9.6 \\
\hline E85 & 2.1 & 0.17 & 17.8 & 1.48 & 35.6 & 2.95 & 8.9 & 0.74 & 3.4 & 0.28 & 2.1 & 0.17 & 30.1 & 2.50 & 8.3 \\
\hline LPG & 12.0 & 0.42 & 2.4 & 0.08 & 4.8 & 0.17 & 0.0 & 0.00 & 6.4 & 0.22 & 14.4 & 0.50 & 60 & 2.10 & 3.5 \\
\hline $\mathrm{CNG}$ & 14.5 & 0.64 & 17.8 & 0.78 & 10.4 & 0.46 & 5.8 & 0.26 & 13.3 & 0.59 & 13.3 & 0.59 & 24.9 & 1.10 & 4.4 \\
\hline $\mathrm{CNG}^{\mathrm{b}}$ & 0.0 & 0.00 & 14.9 & 0.86 & 13.2 & 0.77 & 12.1 & $0.70^{\circ}$ & 11.0 & 0.64 & 8.9 & 0.52 & 39.9 & 2.31 & 5.8 \\
\hline
\end{tabular}




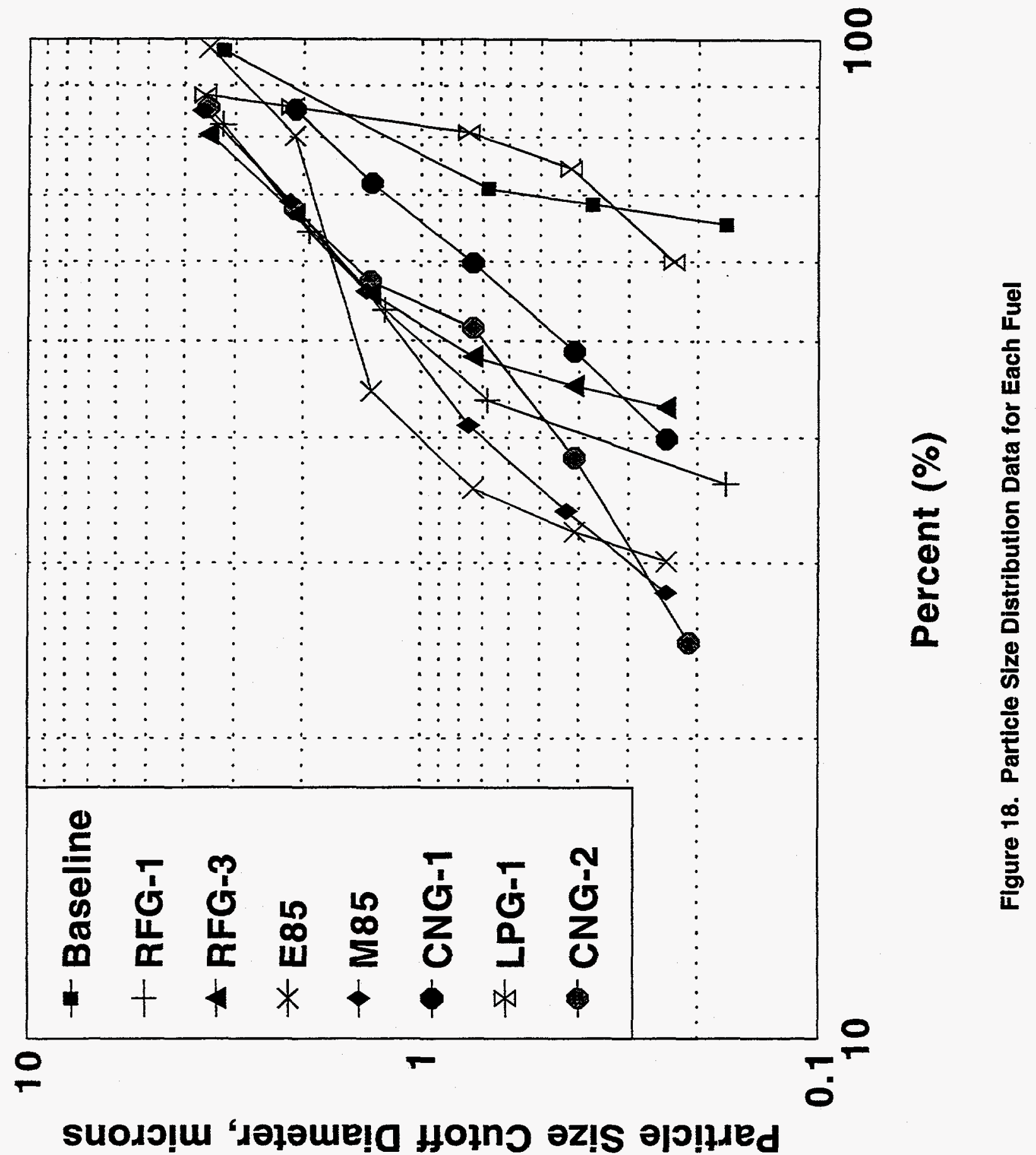


The tests on CNG were performed with both lean and rich equivalence ratios. When the vehicle was operated rich, the total particulate rate was about $24 \%$ more than that for the RFG baseline, and about $66 \%$ less than the RFG rich condition. As with the other fuels, the particle size range less than $0.2 \mu \mathrm{m}$ contained the majority of the particulate mass. The distribution of mass within the other particle sizes was relatively even through the size ranges, but the 0.7 to $1 \mu \mathrm{m}$ range produced the smallest percentage of mass, and the 2 to $3 \mu \mathrm{m}$ range resulted in the second highest percentage of mass. When CNG was run with a lean fuel/air equivalence ratio, the particle size ranges were relatively constant except for lower levels in the 0.7 to $0.2 \mu \mathrm{m}$ range and higher levels in the less than $0.2 \mu \mathrm{m}$ range

\section{H. Discussion}

\section{Regulated Emissions}

The regulated emissions followed typical trends for changes to the fuel/air equivalence ratio. When the vehicle operated fuel rich, THC and NMHC (gasoline, CNG, and LPG), OMHCE, and OMNMHCE (methanol and ethanol), and CO increased; and fuel economy decreased. For RFG at a rich fuel/air equivalence ratio, the $\mathrm{NO}_{\mathrm{x}}$ increased, and for the alcohol fuels and gaseous fuels, the $\mathrm{NO}_{\mathrm{x}}$ decreased when compared to the baseline condition with RFG. This increased $\mathrm{NO}_{x}$ emission rate during rich RFG operation was unexpected, and further investigation is needed to understand this observed phenomenon.

\section{Particle Size Distribution}

One possible mechanism may aid in explaining the particle size distribution test results: the agglomeration of smaller particles into larger ones. The mechanism for particle agglomeration suggests that very small particles (less than $0.2 \mu \mathrm{m}$ ) are produced by combustion of the fuel and any excess lubricating oil in the cylinder. As the temperature of the exhaust cools, the smaller particles begin to agglomerate as they pass through the exhaust system. In addition, some of the heavier gaseous $\mathrm{HC}$ also begin to condense into aerosols or adsorb onto the particles. These particles and aerosols further agglomerate until the agglomerated particles range from about 2 to $3 \mu \mathrm{m}$ in size.

The majority of the fuels had the largest percentage of particle mass in the equivalent aerodynamic diameter range smaller than about $0.2 \mu \mathrm{m}$. An exception to this observation was E85, which had 35\% of the particle mass in the 1 to $2 \mu \mathrm{m}$ range, and only about $30 \%$ of the total particle mass in the smaller than $0.2 \mu \mathrm{m}$ range. As the particles pass out of the cylinder and through the exhaust system, agglomeration begins, resulting in larger particles. This agglomeration mechanism appears to depend on the fuel as well as the total particulate mass in the exhaust stream.

When the engine was operated at a fuel/air equivalence ratio greater than stoichiometric (fuel rich operation), the total mass of particles increased, when compared to the RFG baseline condition, with four of the five fuels. The tests with LPG were the exception, yielding less total particulate mass than the RFG baseline condition. RFG under rich operation gave the highest total particulate mass rate; however, this rate was well below the $80 \mathrm{mg} / \mathrm{mi}$ particulate standard for 1996 model year light-duty vehicles. The trend toward 
particle agglomeration was also more pronounced with RFG under fuel rich operation, probably because of the increase in total particulate mass.

With M85 and E85 at a rich fuel/air equivalence ratio, the total particulate mass was about a factor of 2 greater than RFG at a stoichiometric fuel/air ratio. For M85, particle agglomeration was distributed evenly between about $0.7 \mu \mathrm{m}$ and the greater than $3 \mu \mathrm{m}$ equivalent aerodynamic diameter range. The particle size distribution indicated a lesser tendency toward particle agglomeration. More total particulate mass was measured for M85 than for E85, but the E85 measurements suggested a greater tendency toward particle agglomeration. With E85, particle agglomeration reached a maximum in the 1 to $2 \mu \mathrm{m}$ range, and the total mass of particulate in this size range was greater than the less than $0.2 \mu \mathrm{m}$ equivalent aerodynamic particle diameter range. These trends for the alcohol fuels may be explained in terms of their combustion characteristics at atmospheric conditions. The luminosity of a methanol flame in air is very low, because there is less tendency to form soot particles as it burns. Ethanol, on the other hand, has a comparatively luminous flame because of the formation of soot particles. ${ }^{(20-22)}$ If a similar mechanism is present with the in-cylinder combustion, the size distribution of particle mass in the exhaust can be at least partially explained in terms of the alcohol's tendency to form soot particles. With CNG and LPG under rich operation, the total mass of particulate was smaller than with the liquid fuels. These results indicated that particle agglomeration may not as predominant with the gaseous fuels as compared to the liquid fuels.

\section{Conclusions and Observations}

Some conclusions and observations that can be drawn from this study include:

- Baseline emissions for the test vehicle met the 1994 EPA Tier 1 standards.

- This vehicle operated satisfactorily on five different fuels, and fuel/air enrichment produced, for the most part, expected changes in gaseous emissions and fuel economy with all five fuels. For RFG at a rich fuel/air equivalence ratio, the $\mathrm{NO}_{\mathrm{x}}$ increased, and for the alcohol fuels and gaseous fuels the $\mathrm{NO}_{\mathrm{x}}$ decreased when compared to the baseline condition with RFG. This increased $\mathrm{NO}_{\mathrm{x}}$ emission with RFG was unexpected, and further investigation is needed to understand this observed phenomenon.

- Even while operating at rich fuel/air equivalence ratios, total particulate mass did not exceed the 1996 light-duty vehicle particulate standard for any of the fuels. In fact, particulate emissions while running rich on LPG were actually lower than those measured during the baseline run on gasoline.

- While operating fuel-rich, the trend for total particulate mass was LPG $\cong$ CNG $<\mathrm{E} 85 \cong \mathrm{M} 85<\mathrm{RFG}$. 
- Particle agglomeration appeared to be one of the mechanisms for larger particle formation; smaller particles agglomerated to form different equivalent aerodynamic diameter ranges depending on the fuel. Predominant particle agglomeration ranges by fuel were:

RFG - greater than $3 \mu \mathrm{m}$

M85 - broad range from 0.7 to greater than $3 \mu \mathrm{m}$

E85 - 1 to $2 \mu \mathrm{m}$

LPG - greater than $3 \mu \mathrm{m}$

CNG - 2 to $3 \mu \mathrm{m}$.

- For all fuels except LPG, the mass percentage of particles with sizes greater than $0.7 \mu \mathrm{m}$ (indicative of particle agglomeration), were quite similar: 52\%, $59 \%, 64 \%$, and $49 \%$ for RFG, M85, E85, and CNG, respectively.

- Gaseous fuels produced a lower percentage of larger particles than the liquid fuels.

\section{J. Recommendations}

In general, these tests indicated that the size distribution of spark-ignited engine particulate could be characterized, and that sufficient quantity of particulate mass could be collected to measure these trends. Although it is unknown whether the observed trends in particulate mass formation would be the same during stoichiometric operation, this study indicates the potential alternative fuels have to reduce particulate emissions from light-duty vehicles. However, additional work is needed to better characterize the particulate emissions from spark-ignited engines according to composition and to understand the production mechanisms and sources of the particulate during combustion. 


\section{References}

1. Code of Federal Regulations, Title 40, Part 86, Subpart B.

2. Series 220 Instruction Manual, Bulletin No. 12-78-220IM, Sierra Instruments, Inc.

3. Oil and Gas Journal, June 13, 1994.

4. State of California Air Resources Board, "Proposed Amendments to Low Emission Vehicle Regulations," May 1, 1995.

5. IMPCO Technical Information Brochure.

6. Knepper, J.C., Koehl, W.J., Benson, J.D., Burns, V.R., Forse, Jr., R.A., Hochhauser, A.M., Leppard, W.R., Rapp, L.A., and Reuter, R.M., "Fuel Effects in Auto/Oil High Emitting Vehicles," SAE Paper 930137, 1993.

7. Code of Federal Regulations, Title 40, Part 86, Subpart C.

8. California Code of Regulations, Title 13.

9. EPA Notice of Proposed Rulemaking, "Proposed Regulations for Revisions of the Federal Test Procedure for Emissions from Motor Vehicles."

10. Enns, P., German, J., and Markey, J., EPA's Survey of In-Use Patterns: "Implications for Mobile Source Emission Inventories," U.S. EPA Office of Mobile Sources, Certification Division, 2565 Plymouth Road, Ann Arbor, MI 48104.

11. Schatz, O., "Cold Start Improvements With a Heat Store," SAE 910305, SAE International Congress and Exposition, Detroit, Michigan, February 25 - March 1, 1991.

12. Ivanov, A.N., Vyboishikov, F.P., Misevich, B.S., and Tsvetkov, L.V., "Heat Accumulator for Electric Car Heating," Elektrotekhnika, Vol. 59, No. 11, 1988.

13. Federal Test Procedure Review Project: Preliminary Technical Report, U.S. Environmental Protection Agency, EPA 4200-R-93-007, May 1993.

14. Howe, F.D., "Smoky and Ml-Smelling Exhausts," Transactions of the Society of Automotive Engineers, 1910.

15. Code of Federal Regulations, Title 40, Part 86, Subpart A.

16. Marrack, D. "All PM-10 Are Not Biologically Equal," Particulate Matter: Health and Regulatory Issues, VIP-49, Proceedings of an International Specialty Conference, Air \& Waste Management Association, April 4-6, 1995. 
17. King, S., "Natural Gas as a Stationary Engine and Vehicular Fuel," SAE Paper 912364, 1991.

18. Marple, V.A., Rubow, K.L., and Olson, B.A., "Diesel Exhaust/Mine Dust Virtual Impactor Personal Aerosol Sampler: Design, Calibration and Field Evaluation," Aerosol Science and Technology, No. 22, pg. 140-150, 1995.

19. Marple, V.A., Rubow, K.., and Behm, S.M., "A Microorifice Uniform Deposit Impactor (MOUDI): Description, Calibration, and Use," Aerosol Science and Technology, No. 14, pg. 434-446, 1991.

20. Fanick, E.R., Smith, L.R., and Baines, T.M., "Safety Related Additives for Methanol Fuel," SAE Paper 841378, presented at the Fuels and Lubricants Meetings in Baltimore, Maryland, October 8-11, 1984.

21. Fanick, E.R., Smith, L.R., Russell, J.A., Likos, W.E., and Ahuja, M., "Laboratory Evaluation of Safety-Related Additives for Neat Methanol Fuel," SAE paper 902156, presented at the International Fuels and Lubricants Meeting and Exposition, Tulsa, Oklahoma, October 22-25, 1990.

22. Fanick, E.R., Smith, L.R., Ahuja, M.S., Albu, S., Santoro, S.J., Leonard, J.H., McCormack, M.C., and Wiens, F.J., "Laboratory Evaluation of Additives for Flame Luminosity Improvement in Neat Methanol Fuel," SAE paper 930379, presented at the 1993 SAE International Congress and Exposition, Detroit, MI, March 1-5, 1993. 


\section{Appendix A}

Detailed Computer Printouts of the FTP Regulated Emission Test Results from Task 1

\begin{tabular}{|c|c|c|c|}
\hline Fuel & Catalyst & Test No. & Page No. \\
\hline $\begin{array}{l}\text { CP2 } \\
\text { (pre-conversion) }\end{array}$ & Yes & $\begin{array}{c}\text { BL-CP2-01 } \\
\text { BL-CP2-02S }\end{array}$ & $\begin{array}{l}A-1 \\
A-2\end{array}$ \\
\hline $\begin{array}{l}\text { CP2 } \\
\text { (post conversion) }\end{array}$ & Yes & $\begin{array}{l}\text { BLB-CP2-01 } \\
\text { BLB-CP2-02S }\end{array}$ & $\begin{array}{l}A-3 \\
A-4\end{array}$ \\
\hline RFA & Yes & $\begin{array}{l}\text { BLB-RFA-01 } \\
\text { BLB-RFA-02S }\end{array}$ & $\begin{array}{l}A-5 \\
A-6\end{array}$ \\
\hline $100 \%$ n-Butane & Yes & $\begin{array}{c}100 \mathrm{NB}-01 \\
100 \mathrm{NB}-02 \mathrm{~S}\end{array}$ & $\begin{array}{l}A-7 \\
A-8\end{array}$ \\
\hline HD-5 LPG & Yes & $\begin{array}{l}\text { HD5-01 } \\
\text { HD5-02S }\end{array}$ & $\begin{array}{l}A-9 \\
A-10\end{array}$ \\
\hline $\begin{array}{l}90 \% \text { n-Butane } \\
10 \% \text { Isobutane }\end{array}$ & Yes & $\begin{array}{l}90 \mathrm{NB} / 10 \mathrm{IB}-01 \\
90 \mathrm{NB} / 10 \mathrm{BB}-02 S\end{array}$ & $\begin{array}{l}A-11 \\
A-12\end{array}$ \\
\hline $\begin{array}{l}90 \% \text { n-Butane } \\
10 \% \text { Mixed Butenes }\end{array}$ & Yes & $\begin{array}{c}90 \mathrm{NB} / 10 \mathrm{BE}-01 \\
90 \mathrm{NB} / 10 \mathrm{BE}-02 \mathrm{~S}\end{array}$ & $\begin{array}{l}A-13 \\
A-14\end{array}$ \\
\hline $\begin{array}{l}60 \% \text { n-Butane } \\
40 \% \text { Isobutane }\end{array}$ & Yes & $\begin{array}{c}60 \mathrm{NB} / 40 \mathrm{IB}-01 \\
60 \mathrm{NB} / 40 \mathrm{IB}-025\end{array}$ & $\begin{array}{l}A-15 \\
A-16\end{array}$ \\
\hline $\begin{array}{l}80 \% \text { n-Butane } \\
20 \% \text { Propane }\end{array}$ & Yes & $\begin{array}{c}\text { 80NB/20NP-01 } \\
80 \mathrm{NB} / 20 \mathrm{NP}-02 S\end{array}$ & $\begin{array}{l}A-17 \\
A-18\end{array}$ \\
\hline $\begin{array}{l}90 \% \text { n-Butane } \\
10 \% \text { n-Pentane }\end{array}$ & Yes & $\begin{array}{c}\text { 90NB/10PE-01 } \\
\text { 90NB/10PE-02S }\end{array}$ & $\begin{array}{l}\text { A-19 } \\
\text { A-20 }\end{array}$ \\
\hline $\begin{array}{l}50 \% \text { n-Butane } \\
50 \% \text { Propane }\end{array}$ & Yes & $\begin{array}{c}\text { 50NB/50NP-01 } \\
\text { 50NB/50NP-02S }\end{array}$ & $\begin{array}{l}\mathrm{A}-21 \\
\mathrm{~A}-22 \\
\end{array}$ \\
\hline $\begin{array}{l}20 \% \text { n-Butane } \\
80 \% \text { Propane }\end{array}$ & Yes & $\begin{array}{l}20 \mathrm{NB} / 80 \mathrm{P}-02 \mathrm{~S} \\
20 \mathrm{NB} / 80 \mathrm{P}-03\end{array}$ & $\begin{array}{l}\mathrm{A}-23 \\
\mathrm{~A}-24\end{array}$ \\
\hline $\begin{array}{l}100 \% \text { n-Butane } \\
\text { (repeat tests) }\end{array}$ & Yes & $\begin{array}{c}100 \mathrm{NB}-03 \\
100 \mathrm{NB}-04 \mathrm{~S} \\
\end{array}$ & $\begin{array}{l}A-25 \\
A-26 \\
\end{array}$ \\
\hline $\begin{array}{l}\text { CP2 } \\
\text { (repeat tests) }\end{array}$ & Yes & $\begin{array}{l}\text { BLB-CP2-03 } \\
\text { BLB-CP2-04S }\end{array}$ & $\begin{array}{l}\mathrm{A}-27 \\
\mathrm{~A}-28\end{array}$ \\
\hline $100 \%$ n-Butane & No & $\begin{array}{c}100 \mathrm{NB}(\mathrm{NC})-01 \\
100 \mathrm{NB}(\mathrm{NC})-025 \\
100 \mathrm{NB}(\mathrm{NC})-03\end{array}$ & $\begin{array}{l}A-29 \\
A-30 \\
A-31 \\
\end{array}$ \\
\hline $100 \%$ Propane & No & $\begin{array}{c}100 P(N C)-01 \\
100 P(N C)-02 S\end{array}$ & $\begin{array}{l}A-32 \\
A-33 \\
\end{array}$ \\
\hline $\begin{array}{l}50 \% \text { n-Butane } \\
50 \% \text { Propane }\end{array}$ & No & $\begin{array}{c}50 N B / 50 P(N C)-01 \\
50 N B / 50 P(N C)-02 S\end{array}$ & $\begin{array}{l}A-34 \\
A-35 \\
\end{array}$ \\
\hline
\end{tabular}




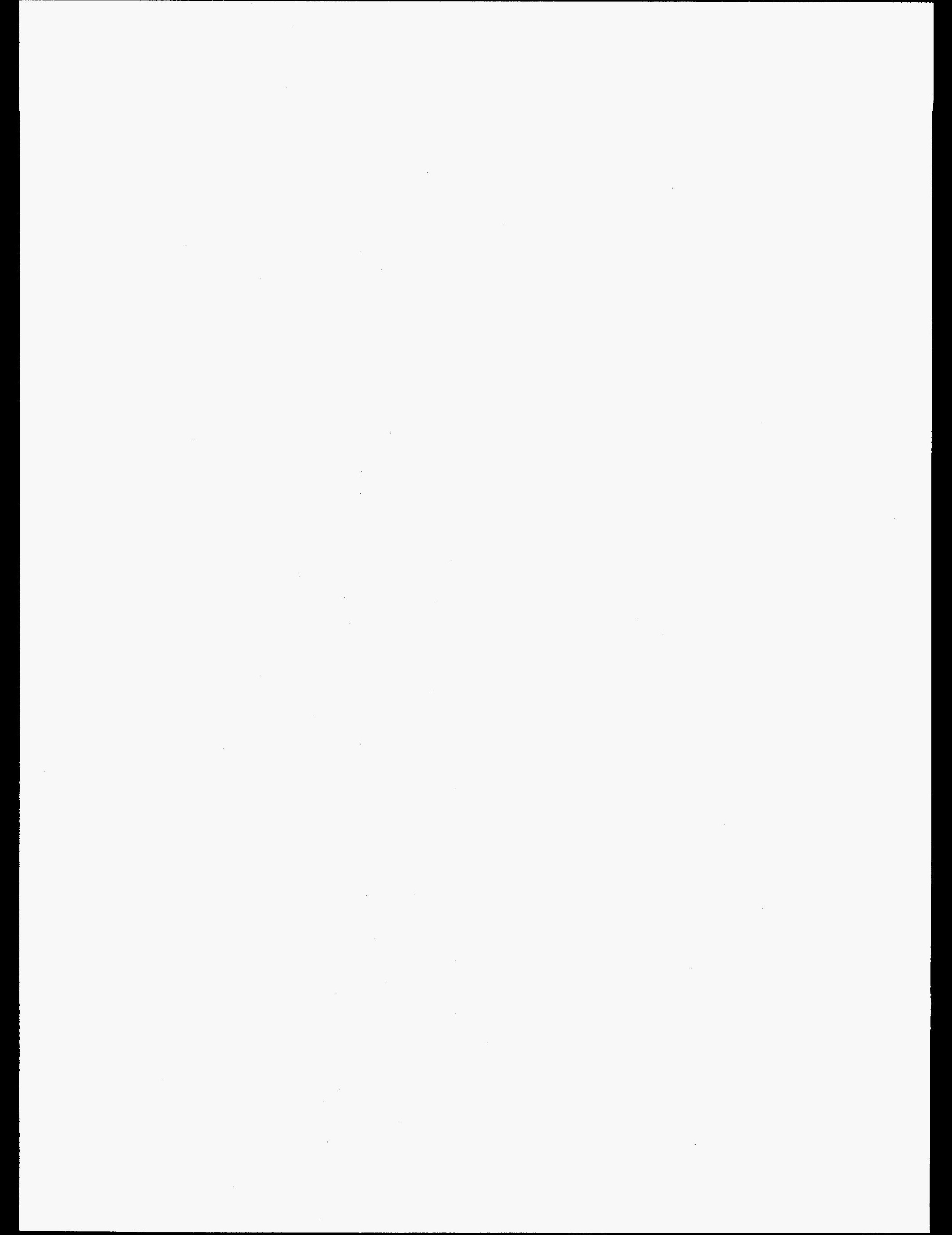


VEHICLE NOHBER GA2

VEHICLE HODEL 94 PONT GRANDAH

FIKGIIR $2.3 \mathrm{~L}(138 \mathrm{CID})-4$

TRAHSHISSION 24

ODONETER

1529 MILES ( $2460 \mathrm{KM})$

BAROMETER 29.20 II HG (741.7 NII HG) DRI BULB TEMPERAIURE $73.0^{\circ} \mathrm{F}\left(22.8^{\circ} \mathrm{C}\right)$ RELATIVE HOITDITY 65.4 PCT.

BAG KUIBER

BAG DESCRIPTIOH

ROW TINE SECORDS

DRY/WET CORRECTIOH FACTOR, SAYP/BACR

WRASURED DISTAHCE YILES (RK)

BLOWER FLOF RATE SCFH (SCH)

GAS IETER FLON RATE SCFH (SCH)

TOTAL FLON SCF (SCH)

EC SALPLE YETER/RAHGE/PPY (BAG)

BC BCRCRD HETER/RAHGR/PPI

CO SAIPLE METER/RAHGE/PPI

C BCRGRD HETER/RANGE/PPI

CO2 SAYPLE METER/RAHGE/PCT

CO2 BCKGRD METER/RAMGE/PCT

HOX SAYPIE METER/RAHGE/PPH (BAG) (D)

WOX BCRGRD VEFER/RAHGE/PPH

CH4 SAIPLE PPI (1.160)

CH4 BCKGPD PPH

$$
\begin{gathered}
1 \\
\text { COLD TRAHSIENT } \\
(0-505 \text { SEC. }) \\
505.2 \\
.971 / .981 \\
3.67(5.90) \\
320.5(9.08) \\
.00(.00) \\
2698 .(76.4)
\end{gathered}
$$

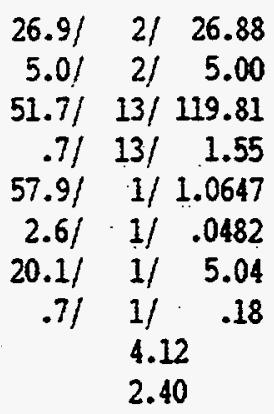

DILUTON FACTOR

BC COHCEHTRATION PPY

CO COHCEITRATION PFH

$\mathrm{CO} 2$ CONCENTRATTON PCT

NOX COHCEITRATION PPH

CH4 CONCENTRATION PPH

NHIC COHCAHTRATION PPI

FIDHC HASS GRAYS

CO MASS GRAYS

CO2 MASS GRAHS

HOX MASS GRAYS

CH4 MASS GRAMS

HUIAC HASS GRAYS (FID)

FOEL HASS KG

FUEL ECONOHY IPG (L/10ORM)

12.33

22.29

113.38

1.0204

4.88

1.92

20.07

1.009

10.086

1427.59

.736

.098

.884

.468

$21.88(10.75)$

3-BAG COIPOSITE RESULTS

FUEL ECONOHY IPG (L/100KY) $21.85(10.77)$

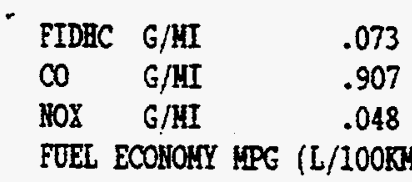

.073

7
TEST BL-CP2-01

DATE 8/22/95 ROI

DYHO 3 BAG CART 2

ACTOAL ROAD LOAD $6.60 \mathrm{EP}$ ( $4.92 \mathrm{KN}$ )

TEST WEIGHF 3375 LBS ( $1530 \mathrm{KG}$ )

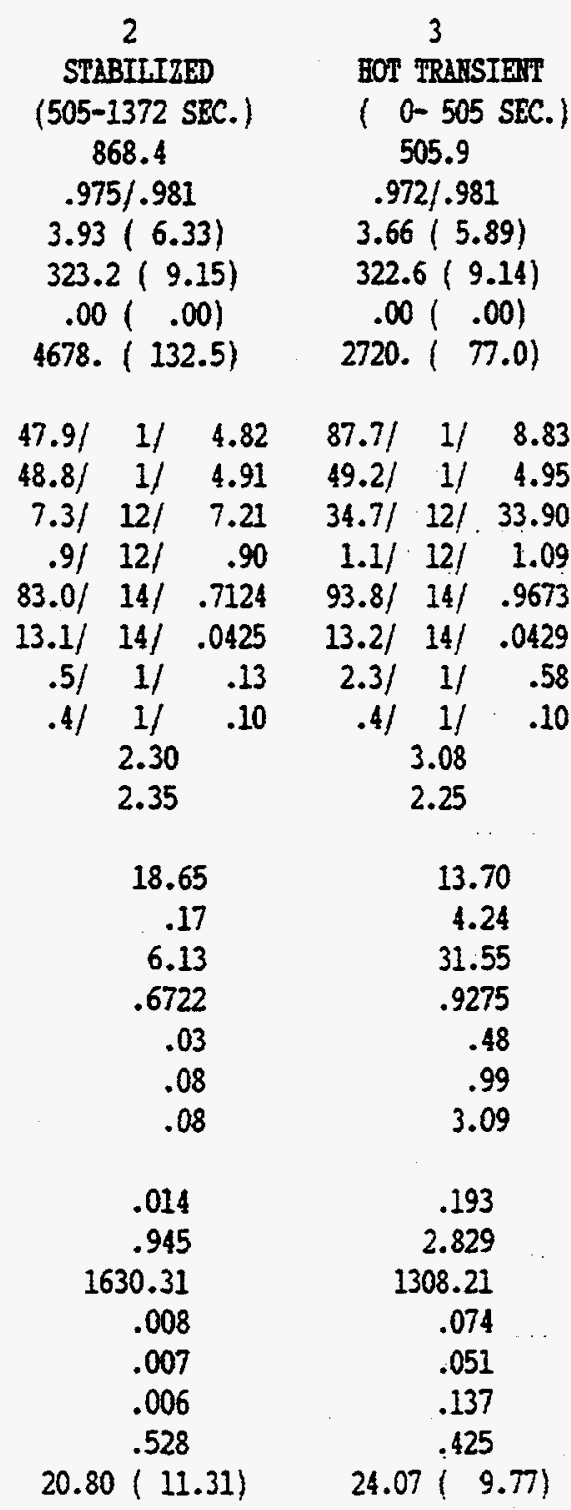

$20.80(11.31$

$24.07(9.77$
GASOLITE PHASE II BH-1968-P

FUEL DEISIIY $6.163 \mathrm{LB} / \mathrm{GAL}$

B $.137 \quad C .843 \quad 0.020 \quad \& .000$

HOX FUMIDIFY C.F. 1.032

$\begin{array}{lll}\text { CH4 } & \text { G/MI } & .010 \\ \text { WHHC } & \text { G/HI } & .061\end{array}$




\section{COIPOTER PROGRAY LDF 1.2-R 3-BAG CARB FTP VEHICLE RIISSIOI RESULTS \\ VEHICLE NOUBER GA2 \\ VERICLE HODEL 94 PONT GRANAK \\ BRGIFE 2.3 L (138 CID) -4 \\ TRAHSISSSTON 14 \\ ODONETER $\quad 1543$ MILES ( $2482 \mathrm{KM})$ \\ TBST BL-CP2-02S \\ DATE 8/23/95 ROH \\ DHNO 3 BAG CART 2 \\ ACTUAL ROAD LOAD $6.60 \mathrm{IPP}$ ( $4.92 \mathrm{KM}$ ) \\ TEST WEIGET 3375 IBS ( 1530 RG)}

BAROHETER 29.16 IN HG (740.7 MI HG)

RRLATIVE HOUIDITY 69.0 PCT.

BAG IUIBER

BAG DESCRIPITOA

ROI TINE SECOIDS

DRY/WET CORRECTIOI FACTOR, SAIP/BACR

VIFASURED DISTANCE MILRS (RM)

BLONER RLON RATE-SCFI (SCAN)

GAS HETER FLON RATR SCFH (SCAN)

TOTAL FLON SCP (SCH)

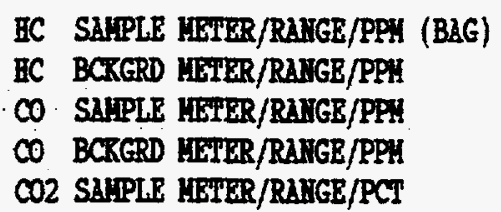

CO2 BCKERD BETER/RIHGE/PCT

FOX SAYPLE YETER/RAMGE/PPN (BAG) (D)

WOX BCKERD IIETER/RAHGE/PPH

CH4 SAIPLE PPY (1.160)

CH4 BCRERD PPI

DILUTION FACTOR

HC COHCEMTRRATIOH PPH

CO COHCETIRATIOH PPI

$\mathrm{CO} 2$ COHCERTRATIOH PCT

HOX COHCEMTRRATIOH PPH

CH4 COHCERTRATION PPH

IHEC COHCETTRATION PPH

THC MLSS GRAYS

$\infty$ HASS GRALS

CO2 WISS GRALS

MOX MASS GRALS

CH4 MASS GRIHS

IIHC WSS GRAYS (FID)

FUEL MLSS RG

FOEL ECOHOHY MPG (L/100RH)
DRY BULB TEYPERRTURE $72.0^{\circ} \mathrm{F}\left(22.2^{\circ} \mathrm{C}\right)$

$$
\begin{array}{cc}
1 & 2 \\
\text { COLD TRAHSIENT } & \text { STABILIZED } \\
(0-505 \text { SEC. }) & (505-1372 \text { SEC. }) \\
505.6 & 869.0 \\
.971 / .981 & .974 / .981 \\
3.61(5.81) & 3.87(6.23) \\
319.8(9.06) & 322.1(9.12) \\
.13(.00) & .14(.00) \\
2696 .(76.4) & 4668 .(132.2)
\end{array}
$$
$\begin{array}{llll}27.1 / \quad 2 / 27.08 & \end{array}$
$5.7 / 2 / \quad 5.70$
$52.5 / 13 / 121.81$
$.6 / \cdot 13 / 1.33$
$57.0 / 1 / 1.0478$
2.4/ 1/ .0445
$19.3 /$ I/ 4.84
$.4 / 1 / \quad .10$
4.02
2.38

12.53

21.84

115.39

1.0069

4.75

1.83

19.72

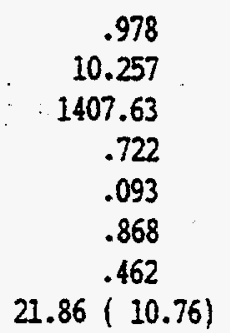

.978

.722

.093

.462

$21.86(10.76)$
$60.6 /$ 1/ 6.10

$59.21 \quad 1 / \quad 5.96$

$\begin{array}{llll}7.6 / & 12 / & 7.50\end{array}$

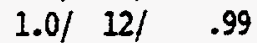

$82.5 / 14 / \quad .7026$

$12.5 / 14 / \quad .0402$

.4) $1 / \quad .10$

$.31 \quad 1 / \quad .08$

2.31

2.34

18.91

.46

6.31

.6644

.03

.09

.35

.046

.971

1608.12

.008

.008

.027

.521

$20.76(11.33)$
GASOLIIE PHASE II IA-1968-P PURL DEISITY $6.163 \mathrm{LB} / \mathrm{GAL}$ $\begin{array}{llllllllll}\text { H } .137 & \mathrm{C} .843 & 0.020 & \mathrm{X} .000\end{array}$

FOX HUIDITY C.F. 1.041

$$
\begin{aligned}
& 3 \\
& \text { HOT TRAMSIEMTT } \\
& (0-505 \text { SEC. }) \\
& 506.0 \\
& .972 / .981 \\
& 3.64(5.86) \\
& -321.8(9.11) \\
& .13(.00) \\
& 2715 .(76.9)
\end{aligned}
$$

$9.9 / \quad 2 / \quad 9.89$

$6.21,2 / \quad 6.20$

$31.2 / \quad 12 / \quad 30.48$

$.9 / 12 / \quad .90$

93.1/ $14 / \quad .9479$

$12.9 / 14 / \quad .0418$

$1.9 / 1 / \quad .48$

$.6) \quad 1 / .15$

3.04

2.34

13.98

4.14

28.42

.9091

.34

.87

3.13

.190

2.544

1279.76

.052

.045

.139

.416

$24.46(9.62)$

3-BAG CONPOSITE RESULTS

$\begin{array}{lll}\text { THC } & \text { G/AI } & .077 \\ \text { CO } & \text { G/MI } & .912 \\ \text { NOX } & \text { G/AI } & .046\end{array}$

FOEL ECOHONY HPG (L/100RM)
$\mathrm{CH} 4$

NIRC

G/MI

G/NI

CARBOHYL G/HI

NHOG G/MI
.010

.064

.003

$.067 \quad(\mathrm{RAF}=1.00)$ 
VETICLE HURBER GA2

VEHICLE HODEL 94 POIT GRAIDAY

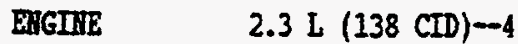

IRAHSIISSION 24

ODONETER $\quad 1855$ YIHLBS ( $2984 \mathrm{KI}$ )

BARONETER 29.18 II HG (741.2 IN EG)

RELAITVE HUIIDITY 73.5 PCI.

BAG MUIBER

BAG DESCRIPTIOH

ROI TIIE SECOMDS

DRY/WET CORRFCIIOI FACTOR, SAYP/BACR

IBRASURBD DISTAMCE BIIES (R:)

BLOMER FLON RATE SCFI (SCMIN)

GAS METER FLON RATB SCFH (SCAN)

TOTAL FLON SCP (SCI)

FC SAYPLE HITER/RAYGB/PPI (BIG)

BC BCRGRD MEIER/RANGE/PPH

CO SAYPLE / IETER/RAYGE/PPH

CO BCRERD YETER/RAYGR/PPH

CO2 SAYPLE IETER/RAYGE/PCT

CO2-BCRERD MELER/RAHGE/PCT

HOX SAIPLE ULTER/RAHGR/PPI (BגG) (D)

HOX BCRGRD HETBR/RAYGE/PPH

CH4 SAMPLE PPH (1.160)

CH4 BCRGRD PPI

DILOTIOA PACTOR

BC CORCAHTRTIOH PPH

CO COHCBTRATIOA PPH

$\mathrm{CO}_{2}$ CONCBNTATIOH PCT

HOX COHCRHIRATIOI PPX

CH4 CONCWITRTIOI PPI

IRHC CONCERTRATOA PFI

FIDEC MASS GRAYS

CO . MLSS GRUYS

$\mathrm{CO} 2$ : MASS GRAYS

HOX MASS GRAYS

CH4. MASS GRASS

MIIC MASS GRAYS (FID)

FUEL HASS RG

FUEय, ECOHOIY IPG (L/10ORH)
IBST BLB-CP2-01

DLTE $9 / 16 / 95$ RUI

DHO 3 BAG CART 2

ACIUAL ROAD LOAD 6.60 IP ( $4.92 \mathrm{KN}$ )

TEST VEIGHT 3375 IBS ( 1530 RG)
DRY BULB TEPPERATURR $73.0^{\circ} \mathrm{F}\left(22.8^{\circ} \mathrm{C}\right)$

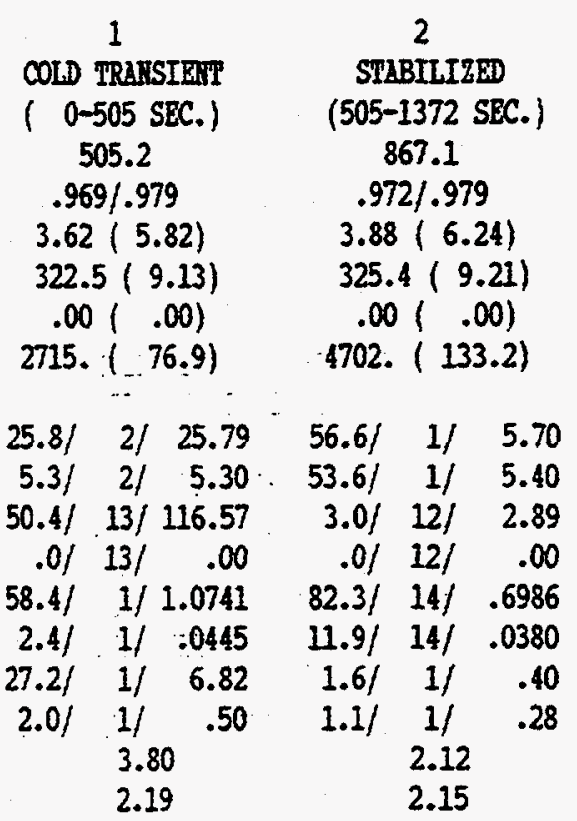

\subsection{3}

20.92

111.34

1.0332

6.36

1.79

18:85

.953

9.967

1454.57

1:017

.092

.836

.477

$21.21(11.09)$
19.02

.59

2.79

.6626

.14

.08

.49

.046

.432

1615.54

.039

.007

.038

.523

$20.71(11.36)$
GASOLIITE PHASE II EN-1968-P

FUEL DEISTTY 6.163 IB/GLI

$\begin{array}{llll}\text { H } .137 & \mathrm{C} .843 \quad 0.020 \quad \& .000\end{array}$

CALIFORYIA PHASR 2

BOX BUIDITY C.F. 1.087

3

EOT TRAISIFNT

( 0 - 505 SEC.)

505.0

$.970 / .979$

$3.62(5.83)$

$323.1(9.15)$

$.00(.00)$

2719. ( 77.0$)$

$85.5 /$ 1/ 8.61

$53.1 / \ldots 1 / \quad 5.35$

$15.9 / \cdot 12 / \cdot 15.38$

$.1 /: 12 \% \quad 10$

$93.5 / 14 / \cdots .9589$

$11.9 / 14 / \quad .0380$

2.1/ $1 / \quad .53$

$.5 / .1 \% \quad .13$

2.79

2.19

13.84

3.65

$14: 64$

.9237

.41

.76

2.77

$: 166$

1.312

1302.31

.066

.039

.123

.423

$23.95(9.82)$

3-BAG COIPOSITE RESULTS

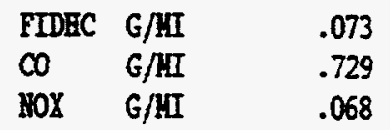

FUEL ECONOUY IPG (L/100R) $21.64(10.87)$

$\begin{array}{lll}\text { CHA } & \text { G/MI } & .009 \\ \text { HRHC } & \text { G/NI } & .062\end{array}$




\begin{tabular}{|c|c|}
\hline VERICLE HOLBER & Ga2 \\
\hline VBRICLE HODRL & 94 POII GRAIDAY \\
\hline MGIHE & $2.3 \mathrm{I}(138 \mathrm{CID})--4$ \\
\hline IRAHSUISSIC & 24 \\
\hline ODOMI & 1843 KIILES ( 2965 RI) \\
\hline
\end{tabular}

BAROUIEIER 29.24 III HG (742.7 IfH HG) RRTAFIVE HOYIDITY 61.9 PCT.

BAG IVIBER

BAG DESCRIPITOH

ROI TIE SECOIDS

DRY/WEI CORRECTIOH BACTOR, SAP/BACR

ITASURED DISTANCE HILES (KII)

BLONER FLON RAFE-SCFY (SCHI)

GLS HETER FLON RAIE SCFI (SCAY)

TOTAL FLON SCF (SCI)

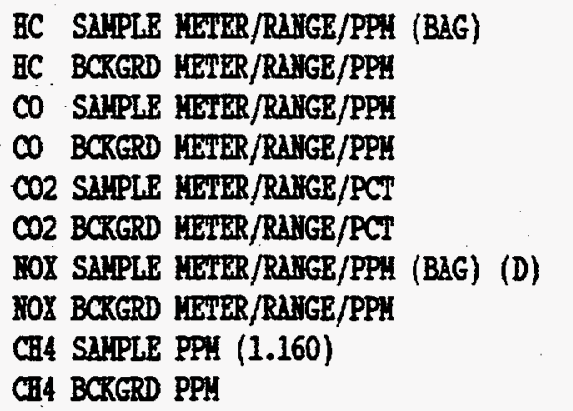

DILUTIOA FACTOR

EC COICENTRATTOR PPH

CO COMCOMIRATIOH PPI

$\mathrm{CO}_{2}$ COHCEIIRATIOH PCT

HOX COICAHTRATOH PPI

CH4 COMCEPIRATIOR PPH

MTC COHCRIRANTON PPH

$\begin{array}{ll}\text { THC } & \text { MASS GRAYS } \\ \text { CO } & \text { MASS GRAYS } \\ \text { CO2 } & \text { HASS GRAYS } \\ \text { HOX } & \text { HASS GRAYS } \\ \text { CH4 } & \text { MASS GRAYS } \\ \text { MIRC } & \text { HLSS GRAYS (FID) } \\ \text { FUEL } & \text { HASS KG }\end{array}$

FUEL ECOYOMY IPG (L/100RH)
TRST BLB-CP2-02S

DATE $9 / 15 / 95$ RUI

DYIO 3 BAG CART 2

ACIUAL ROAD LOAD $6.60 \mathrm{HP}$ ( $4.92 \mathrm{KN}$ )

TEST WETGHP 3375 LBS ( 1530 RG)
DRY BULB TEIPERATURE $74.0^{\circ} \mathrm{F}\left(23.3^{\circ} \mathrm{C}\right)$

$$
\begin{array}{cc}
1 & 2 \\
\text { COLD TRANSIEIT } & \text { STABILIZED } \\
(0-505 \text { SEC. }) & (505-1372 \text { SEC. }) \\
505.3 & 867.1 \\
.971 / .982 & .975 / .982 \\
3.62(5.82) & 3.87(6.22) \\
323.3(9.16) & 326.4(9.24) \\
.13(.00) & .14(.00) \\
2724 .(77.1) & 4719 .(133.6)
\end{array}
$$

$\begin{array}{rrr}23.9 / & 2 / & 23.89 \\ 5.5 / & 2 / & 5.50 \\ 90.3 / & 12 / & 90.40 \\ 1.3 / & 12 / & 1.25 \\ 58.7 / & 1 / & 1.0797 \\ 2.5 / & 1 / & .0463 \\ 25.8 / & 1 / & 6.47 \\ .0 / & 1 / & .00 \\ & 3.83 & \\ & 2.46\end{array}$

12.20

18.84

85.55

1.0372

6.47

1.57

17.02

.850

7.683

1464.92

.976

.081

.757

.479

$21.10(11.15)$

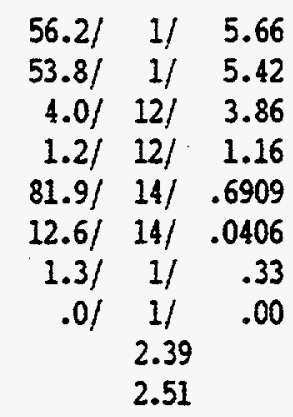

19.23

.52

2.65

.6524

.33

.01

.51

.040

.413

1596.23

.085

.001

.040

.517

$20.91(11.25)$
GASOLIIE PHASE II BI-1968-P FUEL DEHSITY $6.163 \mathrm{LB} / \mathrm{GML}$ H $.137 \quad C .843 \quad 0.020 \quad \mathrm{X} .000$

HOX HOLIDITY C.F. 1.023

3

HOT TRAISIERT

( 0- 505 SEC.)

505.5

$.973 / .982$

$3.61(5.81)$

$-324.6(9.19)$

$.13(.00)$

2736. ( 77.5$)$

$\begin{array}{rrr}83.0 / & 1 / & 8.36 \\ 55.2 / & 1 / & 5.56 \\ 18.0 / & 12 / & 17.42 \\ .9 / & 12 / & .87 \\ 92.4 / & 14 / & .9289 \\ 12.6 / & 14 / & .0406 \\ 2.5 / & 1 / & .63 \\ .0 / & 1 / & .00 \\ & 2.92 & \\ 2.48 & \end{array}$

14.29

3.19

15.97

.8912

.63

.61

2.48

.142

1.440

1264.21

.095

.032

.111

.410

$24.59(9.57)$

3-BAG COIPOSITR RESULTS

$\begin{array}{lll}\text { THC } & \text { G/MI } & .065 \\ C O & \text { G/MI } & .606 \\ \text { HOX } & \text { G/MI } & .075\end{array}$

FUEL ECONONY WPG (L/100KN)
$21.88(10.75)$
CH4 G/NI

CARBOHYL G/HT

NIOG G/MI
.007

.057

.001

$.058 \quad(R A F=1.00$ ) 


\begin{tabular}{|c|c|}
\hline VEHICLE NOYBER & GA2 \\
\hline VERICLE HODEL & 94 POWT GRAMDAY \\
\hline EHGIME & $2.3 \mathrm{~L}(138 \mathrm{CID})-4$ \\
\hline TRAHSYISSTOA & A4 \\
\hline ODOHETER & 1893 UILES ( 3045 RI) \\
\hline
\end{tabular}

BAROIETER 29.18 IN EG (741.2 $\mathrm{NH} \mathrm{HG)}$ RELATIVE HOKIDITY 69.0 PCT.

BAG NUYBBR

BAG DESCRIPIIOA

ROII TTHE SECOIDS

DRI/WET CORRECTIOA FACTOR, SAYP/BACK

YEASURED DISTAYICE MILES (RI)

BLOWER FLOW RATE SCFH (SCUN)

GAS IIETER FLON RATE SCFH (SCYH)

TOTAL FLON SCP (SCA)

BC SAMPLE HETER/RAMGE/PPI (BAG)

HC BCKGRD YETER/RAKGE/PPI

CO SAYPLE NEIER/RANGE/PPI

CO BCKGRD METER/RANGE/PPH

CO2 SAYPLE IETER/RANGE/PCT

CO2 BCRGRD HETER/RANGE/PCT

HOX SAYPLE LEHER/RANGE/PPH (BAG) (D)

HOX BCKGRD HETER/RAMGE/PPH

CH4 SALPLE PPH (1.160)

CH4 BCRGRD PPI

DILUTION FACTOR

BC COLCEMTRATIOA PPH

CO COHCRIPRAIOH PPN

$\mathrm{CO} 2$ CORCBIIRATIOR PCT

NOX CONCENIRATTON PPH

CH4 CORCEITRaTION PPA

RHPC CONCEITRATIOH PPH

FIDEC MASS GRAYS

CO MASS GRAYS

$\mathrm{CO}_{2}$ HaSS GRAVS

MOX : MASS GRAYS

CH4 MASS GRALS

ITHC MASS GRAYS (FID)

FUEL MASS RG

FUEL ECONOYY IPG (L/100RH)
TEST BIB-RFA-01

DAFE $9 / 17 / 95$ RON

DHO 3 BAG CART 2

ACTUAL ROAD LOAD $6.60 \mathrm{EP}$ ( $4.92 \mathrm{KW}$ )

TEST MEIGHT 3375 LBS ( 1530 RG)
GHSOLINE RFA BH-1700-F FOEL DERSITY $6.163 \mathrm{IB} / \mathrm{GAL}$ $\begin{array}{llllll}\text { H } .137 & \mathrm{C} .863 & 0.000 & \times .000\end{array}$ IIDUSTRY AVERAGE $\mathrm{RF}-\mathbf{A}$

HOX HUIIDITY C.F. 1.041

DRY BULB TERPERATURE $72.0^{\circ} \mathrm{F}\left(22.2^{\circ} \mathrm{C}\right)$

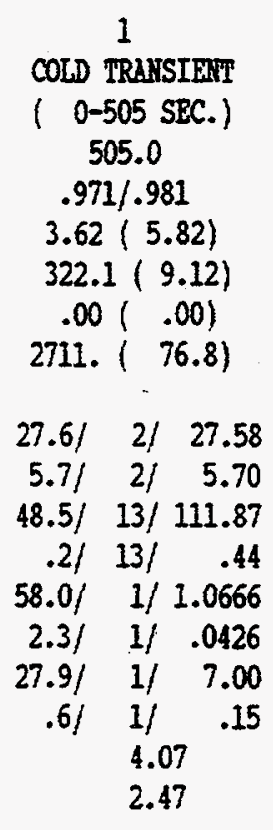

$$
\begin{gathered}
2 \\
\text { STABILIZED } \\
(505-1372 \text { SEC. }) \\
867.4 \\
.974 / .981 \\
3.84(6.19) \\
325.2(9.21) \\
.00(.00) \\
4701 .(133.1)
\end{gathered}
$$
$59.1 / 1 / 5.95$
$56.3 / 1 / 5.67$
4.6) $12 / 4.44$
$.0 / 12 / \quad .00$
$82.6 / 14 / .7045$
$12.0 / 14 / .0384$
$.8 / 1 / \quad .20$
$.6) \quad 1 / \quad .15$
2.46
2.50

\subsection{7}

22.35

106.66

1.0274

6.86

1.80

20.26

.992

9.533

1444.12

1.048

.092

.897

.463

$21.86(10.76)$

18.94

.58

4.28

.6682

.06

.09

.47

$$
.045
$$

.663

1628.72

.015

.008

.036

.516

$20.85(11.29)$

$87.5 / 1 / 8.81$

$53.9 / \quad 1 / \quad 5.43$

$22.8 / \quad 12 / 22.10$

$.01 \quad 12 / .00$

$93.8 / 14 / .9673$

$12.0 / 14 / .0384$

$2.2 / 1 / \quad .55$

$.4 / 1 /: .10$

2.88

2.34

$$
\begin{array}{r}
13.77 \\
3.78 \\
21.19 \\
.9317 \\
.46 \\
.71 \\
2.95 \\
\\
.169 \\
1.905 \\
316.71 \\
.070 \\
.036 \\
.131 \\
.418
\end{array}
$$

1316.71

$24.19(9.73)$

3-BAG COIPOSITE - RESULTS

$\begin{array}{lll}\text { FIDHC } & \text { G/NI } & .076 \\ \text { CO } & \text { G/MI } & .784 \\ \text { HOX } & \text { G/MI } & .068\end{array}$

FOEL ECOHOHY IPG (L/100RN) $21.90(10.74)$

$\begin{array}{lll}\text { CH4 } & \text { G/MI } & .009 \\ \text { IHHC } & \text { G/MI } & .067\end{array}$


VEHICLE NULBER GA2

VKHICLE MODEL 94 PONT GRAWDAM

BMGTNE $2.3 \mathrm{~L}(138 \mathrm{CID})-4$

TRAHSYISSION A4

ODOHETER 1904 MILES ( 3063 KM)

BAROHETER 29.18 II HG (741.2 HII EG) RELLTIVE HOHIDITY 61.0 PCT.

BAG ITUYBER

BAG DESCRIPITOH

ROT TINE SECONDS

DRY/WET CORRECTION FACTOR, SAIP/BRCR

IEASURED DISTAHCE UIILS (RI)

BLOWER FLOW RATE SCFI (SCMH)

GAS HETER FLON RATE SCFH (SCMH)

TOTAL FION SCF (SCH)

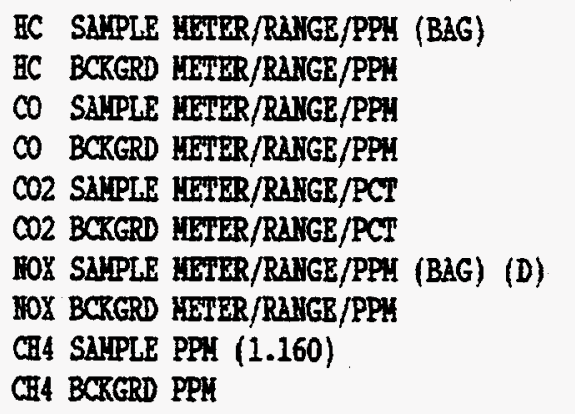

DILUTTON FACTOR

HC CONCEITRRATION POH

CO CONCEHTRATIOH PPH

CO2 CONCERTRATION PCT

NOX CONCEMTRATION PPH

CH4 CONCEATRATION PPH

MRAC CONCERTRATION PPY

THC HASS GRAMS

CO MRSS GRANS

CO2 HASS GRAHS

HOX MASS GRAHS

CH4 MASS GRAYS

MIEC MRSS GRAHS (FID)

FUEL MASS KG

FUEL ECOHONY IPG (L/10ORM)
TEST BLB-RFA-02S

DATE $9 / 18 / 95$ ROI

DWHO 3 BAG CART 2

ACTUAL ROAD LOAD $6.60 \mathrm{MP}$ ( $4.92 \mathrm{KN}$ )

TEST WEIGET 3375 LBS ( $1530 \mathrm{KG}$ )
GASOLINE RFA RH-1700-? FERL DERSITI $6.163 \mathrm{LB} / \mathrm{GAL}$ I $.137 \quad C .853 \quad 0.000 \quad \& .000$ IIDUSTRY AVERAGE $\mathrm{R}^{\mathrm{R}-\mathrm{A}}$

HOY HUIDITY C.F. .993
DRY BOLB TEMPERATURE $72.0^{\circ} \mathrm{P}\left(22.2^{\circ} \mathrm{C}\right)$

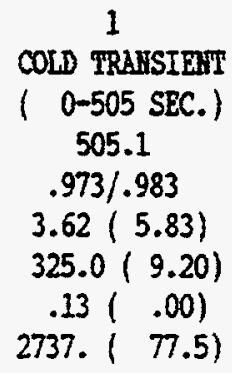
$24.3 / 2 / 24.29$
$5.01 \quad 2 / \quad 5.00$
$85.6 / 12 / 85.51$
$.6 / 12 / \quad .58$
$58.1 / 1 / 1.0685$
$2.6 / 1 / \quad .0482$
$31.6 / 1 / 7.92$
$.5 /$ 1) .13
3.67
2.33

12.38

19.69

81.52

1.0241

7.81

1.52

17.92

.895

7.356

1453.27

1.149

.079

.801

.464

$21.81(10.79)$

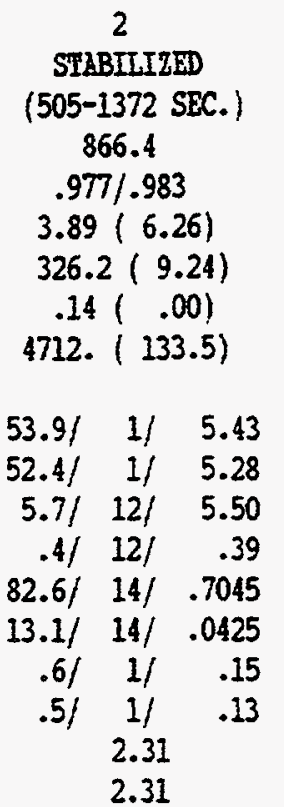

18.94

.43

4.96

.6642

.03

.12

.29

.036

.771

1622.95

.008

.011

.023

.514

21.16 ( 11.12)
3

HOT TRAHSTEITI

( 0 - 505 SEC.) 505.4

$.974 / .983$

$3.64(5.86)$

- $324.4(9.19)$

$.13(.00)$

2733. (77.4)

82.91 I/ 8.35

$53.2 / \quad 1 / \quad 5.36$

$21.6 / 12 / 20.93$

$.6 / 12 / \quad .58$

$93.2 / 14 / .9506$

$13.2 / 14 / \quad .0429$

$3.21 \quad 1 / \quad .80$

.7) $1 / \quad .18$

2.73

2.27

14.01

3.37

19.60

.9108

.64

.63

2.65

.153

1.767

1290.81

.094

.032

.118

.409

$24.85(9.47)$

3-BAG CONPOSITE . RESULTS

$\begin{array}{llr}\text { THC } & \text { G/HI } & .068 \\ C O & \text { G/HI } & .657 \\ \text { NOX } & \text { G/MI } & .074 \\ \text { FOEL ECONOHY HPG } & \text { (L/100RI) }\end{array}$

$22.22(10.59)$

$\begin{array}{ll}\text { CH4 } & \text { G/HI } \\ \text { MIHC } & \text { G/HI } \\ \text { CARBOWYL G/HI } \\ \text { MHOG G/MI }\end{array}$

.008

.058

.001

.059 (RAF $=1.00)$ 
SOUTHITEST RESEARCH IHSTITUTE - DEPARTHMII OP EISSIOUS RESEARCH

COLPOTER PROGRAY LDT 1.2-R 3-BAG CARB FIP VERICLE EISSIOM RESULTS

PROJECT HO. 08-6969-001

VERICLE MUIBER GA2

VEHICLE HODEL 94 PONT GRANAY

BIGIIE $\quad 2.3 \mathrm{~L}(138 \mathrm{CID})-4$

TRAHSHISSIOR 14

ODOKETER $\quad 1636$ IIILES ( 2632 RI)

BAROUETER 29.14 II HG (740.2 IH EG)

RPLATIVE HUITDITY 69.8 PCT.

BAG IOIBER

BAG DESCRIPIIOH

RUI TIHE SECOHDS

DRY/WET CORRECTIOR FACTOR, SAKP/BACK

WEASURED DISTAHCE MITES (RI)

BLOWRR FLOW RATE SCFH (SCHI)

GAS HETER FLON RATE SCFY (SCHY)

TOTLL FLON SCF (SCI)

HC SAYPLE IITTER/RAHCE/PPY (BAG)

BC BCRGRD HETER/RLRGE/PPI

CO SALPLE IETER/RANGE/PPH

CO BCKGRD WETER/PA1_ER/PPH

CO2 SAYPLE IELRR/RAUGE/PCT

CO2 BCKGRD NETER/RMLGE/PCI

HOY SAYPLE IIETER/RAIGE/PPY (BAG) (D)

BOX BCRGRD METER/RAMGE/PPH

CH4 SAYPLE PPH (1.160)

CH4 BCRGRD PPH

DILDTION PACTOR

BC COHCEHTRATION PPH

CO COICERTRATION PPH

$\mathrm{CO}_{2}$ CONCEATRATION PCT

HOX CONCRHTRATION PPH

CH4 CONCRITRATION PPA

NREC CONCERTRATION PPH

FIDHC MASS GRAYS

co MRSS GRAYS

$\mathrm{CO}_{2}$ HASS GRAYS

HOX MaSS GRAYS

CH4 MASS GRAYS

BHTC MASS GRAMS (FTD)

FURL MASS KG
FUEL ECOHOMY IPG (L/100RM)
TRST 1001B-01

DATE $9 / 7 / 95$ ROH

DWHO 3 BLG CART 2

ACTOAL ROAD LOAD 6.60 IPP ( $4.92 \mathrm{KN}$ )

TRST WEIGHT 3375 IRS ( $1530 \mathrm{KG}$ )
BUTARE BUTAME

FUEL DAISITI $4.830 \mathrm{IB} / \mathrm{GAL}$

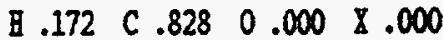

MOX HOIIDTTY C.F. 1.078

DRY BULB TERPERATURE $74.0^{\circ} \mathrm{F}\left(23.3^{\circ} \mathrm{C}\right)$

$$
\begin{gathered}
1 \\
\text { COLD TRAHSIEIT } \\
(0-505 \text { SEC. }) \\
505.5 \\
.967 / .979 \\
3.64(5.86) \\
319.9(9.06) \\
.00(.00) \\
2695 .(76.3)
\end{gathered}
$$

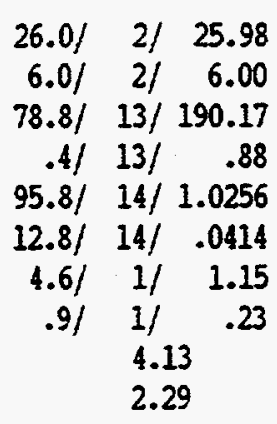

11.47

20.51

180.73

.9878

.95

2.04

18.15

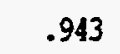

16.058

1380.36

.149

.104

.799

.465

$17.16(13.71)$

3-BAG COIPOSITE RESULTS

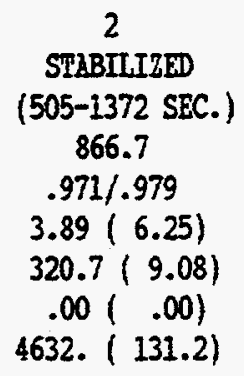

2

STABIIIZED

(505-1372 SEC.)

866.7

$.971 / .979$

$3.89(6.25)$

$320.7(9.08)$

$.00(.00)$

4632. (131.2)
$58.8 / 1 / 5.92$
$56.8 /$ i) 5.72
$19.0 / 12 / 18.61$
$.5 / 12 / \quad .50$
$81.7 / 14 / .6871$
$12.6 / 14 / .0406$
$.7 / 1 / \quad .18$
$.7 / 1 / \quad .18$
2.38
2.22

17.41

.53

17.45

.6488

.01

.28

.20

.042

2.664

1558.09

.003

.025

.015

.515

$16.53(14.23)$

$10.7 / 2 / 10.69$

$6.3 / 2 / \quad 6.30$

$68.3 / 12 / 67.60$

.7) $12 / \quad .70$

91.4/ $14 / .9027$

$12.8 / 14 / .0414$

$.8 / \quad 1 / \quad .20$

4.10

2.30

13.19

4.87

64.08

.8644

.04

1.98

2.58

.225

5.709

1211.12

.006

.101

.114

.403

$19.82(11.87)$
$.71 / \quad .18$ $\begin{array}{llr}\text { PIDHC } & \text { G/II } & .076 \\ \infty & \text { G/II } & 1.704 \\ \text { HOX } & \text { G/III } & .009\end{array}$

FURL ECOHOYY IPG (L/100RI)

$17.48(13.46)$ 
SOUTHEEST RESEARCH INSTITUTE - DEPARTIEAT OP EUISSTOIS RESEARCH

CONPUTER PROGRAN LDT 1.2-R

3-BAG CARB FTP VEHICLE EMISSION RESULTS

PROJECT NO. 08-6969-001

VAHICLE NUIBER GA2

VEHICLE HODEL 94 POHT GRALDAY

EHGIME $2.3 \mathrm{~L}(138 \mathrm{CDD})-4$

TRAMSHSSIOH 24

ODOHETER $\quad 1624$ KILES ( $2613 \mathrm{RI})$

BARONETER 29.23 II HG (742.4 IH HG) RRLATTVE HUIDITY 65.8 PCT.

BIG NU:BER

BAG DESCRIPITIOI

ROY TIR SECOHDS

DRY/WET CORRECTIOU RACTOR, SAYP/BACR

MEASURED DISTAHCR YITLS (KM)

BLOWER FLON RATE SCFH (SCHY)

GAS YETER FLON RATE SCFH (SCAM)

TORAL FLON SCP (SCH)

BC SAYPLE HETZR/RAKGE/PPI (BAG)

BC BCRGRD METER/RAHGE/PPI

CO SAIPLE ILFER/RAHEE/PPI

CO BCRGRD IERIER/RAHGE/PPI

CO2 SAHPLE HIGIBR/RAKGE/PCT

C2 BCRGRD IBTER/RAYGE/PCT

HOX SAYPLE IIGTER/RAKGE/PPI (BAG) (D)

HOX BCRGRD WETER/RAIGE/PPY

CB4 SAYPLE PPI (1.160)

CF4 BCRGRD PPH

DILUTION BACTOR

HC CORCEITRATION PPH

CO COHCDHRRTIOH PPH

$\mathrm{CO} 2$ COICETIRATTOH PCT

HOX COHCEHTRATIOH PPH

CH4 CONCEMTRATION PPI

MHEC CONCEITRATTOH PPH

\begin{tabular}{|c|c|}
\hline THC & HASS GRAHS \\
\hline$C O$ & MASS GRALS \\
\hline$\infty 02$ & MASS GRAYS \\
\hline NOX & HASS GRAYS \\
\hline CH4 & HASS GRAIS \\
\hline RHC & MASS GRAMS (FID) \\
\hline FUEL & MASS RG \\
\hline
\end{tabular}

TEST 100:1B-02S

DATE $9 / 6 / 95$ RUI

DHO 3 BAG CART 2

ACTUAL ROAD LOAD $6.60 \mathrm{IPP}$ ( $4.92 \mathrm{KN}$ )

TRST WETEHT 3375 IBS ( 1530 KG)
DRY BOLB TREPRERTURE $74.0^{\circ} \mathrm{F}\left(23.3^{\circ} \mathrm{C}\right)$

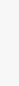

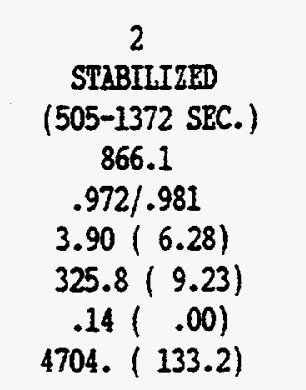

$29.3 / \quad 2 / 29.28$

$7.0 / 2 / 7.00$

$71.0 / 13 / 169.42$

$.3 / \quad 13 / \quad .66$

94.1/ 14/ .9758

$12.6 / 14 / \quad .0406$

$6.4 / 1 / 1.60$

$.6 / 1 / \quad .15$

4.21

2.45

12.06

22.87

161.52

.9385

1.47

1.96

20.59

1.035

14.618

1335.84

.229

.102

.923

.449

$17.70(13.29)$
$69.4 / 1 / 6.99$

$70.8 / 1 / 7.13$

$\begin{array}{llll}19.8 / & 12 / & 19.39\end{array}$

1.2/ $12 / 1.19$

$81.3 / 14 / .6794$

$12.8 / 14 / \quad .0414$

$.7 / 1 / \quad .18$

$.6 / 1 / .15$

2.47

2.32

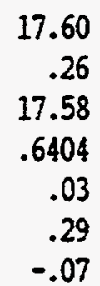

.030

2.727

1562.10

.009

.026

.000

.517

$16.55(14.21)$
BOTAHE BUTANE

FUEL DENSITY $4.830 \mathrm{LB} / \mathrm{GAL}$

B $.172 \quad C .828 \quad 0.000 \quad 8.000$

HOY HOUIDITY C.P. 1.049

3

HOT TRAHSIEMT

( 0 - 505 SEC.)

505.2

$.970 / .981$

$3.62(5.83)$

$-324.2(9.18)$

$.13(.00)$

2731. ( 77.3)

$13.3 / 2 / \quad 13.29$

$6.9 / 2 / 6.90$

$45.4 / 13 / 104.25$

$.7 / 13 / 1.55$

$90.4 / 14 / .8772$

$12.9 / 14 / \quad .0418$

$.7 / 1 / \quad .18$

$.5 / 1 / \quad .13$

4.60

2.29

13.51

6.91

98.59

.8386

.06

2.48

4.03

.309

8.877

1187.42

.009

.128

.180

.397

$20.00(11.76)$

3-BAG COAPOSITP RESULTS

$\begin{array}{llr}\text { THC } & \text { G/MI } & .086 \\ \infty & \text { G/MI } & 1.869 \\ \text { MOX } & \text { G/MI } & .015\end{array}$

CH4

G/HI

.019

MEHC

G/HI

.066

FOX G/HI ECOHOHY IPG (L/100XN)

CARBOHYL G/HI

.001

HHOG G/MI

$.068 \quad(R \lambda F=1.00)$ 
VERTCLE ROIBER GA2

VEHICLE HODEL 94 PONT GRAMDAH

BIGIIE $2.3 \mathrm{~L}(138 \mathrm{CID})-4$

TRAHSIISSION A4

ODOHETER

1943 MIIIES ( 3126 RI)
TEST HD5-01

DATE $9 / 19 / 95 \quad$ RON

DHO 3 BAG CART 2

ACTUAL ROAD LOAD $6.60 \mathrm{KP}$ ( $4.92 \mathrm{KN})$

TEST WEIGHI 3375 IBS ( 1530 RG)
BAROIETER 29.07 IN HG (738.4 MH BG) DRY BOLB TRPERATURE $74.0^{\circ} \mathrm{F}\left(23.3^{\circ} \mathrm{C}\right)$ REL.ATIVE BOHIDITY 69.8 PCT.

BAG HUMBER

BAG DESCRIPTION

RDI TIIT SECOIDS

DRY/WET CORRECTION PACTOR, SAIP/BACR

IEXSURED DISTAHCE YITES (RW)

BLONER FLON RATE SCFH (SCYN)

GAS IETER FLON RATE SCFI (SCII)

TOTAL FLOF SCF (SCH)

BC SAIPIE YETER/RAKGE/PFA (BAG)

BC BCRERD METER/RAHGE/PPI

CO SALPLE MEIER/RAKGE/PPI

CO BCRERD METER/RAHGE/PPH

CO2 SAIPLE IEIER/RAYGE/PCT

CO2 BCRGRD UEIER/RLIGR/PCT

HOX SAIPLE WELER/RAIGE/PPY (BAG) (D)

HOX BCKGRD YETER/RAHGE/PPN

CH4 SAYPLE PPI (1.160)

CH4 BCRGRD PPH

DILUTION FACTOR

HC CONCENIRATION PPH

CO COHCEMTRAIIOA PPH

$\mathrm{CO} 2$ CONCENTRATION PCT

HOX COHCEYRRATIOA PPY

CH4 CORCEHTRATIOH PPH

NHBC CONCEMTRATION PPH

BC MASS GRAYS

CO MLSS GRAHS

$\mathrm{CO} 2$ HASS GRAYS

MOX HASS GRAHS

CES HASS GRAIS

HHC HASS GRAYS (FID)

FUEL MASS KG

FUEL ECOHOHY UPG (L/10ORI)

$$
\begin{gathered}
1 \\
\text { COLD TRAHSIEHT } \\
(0-505 \text { SEC. }) \\
504.9 \\
.967 / .979 \\
3.63(5.83) \\
322.5(9.13) \\
.00(.00) \\
2714 .(76.9)
\end{gathered}
$$

$23.1 / 2 / 23.09$

$5.7 / 2 / \quad 5.70$

$35.7 / \quad 12 / 34.78$

$.5 / 12 / \quad .48$

$93.6 / 14 / \quad .9617$

$12.7 / \quad 14 / .0410$

$10.2 / \quad 1 / 2.56$

$.611 / \quad .15$

2.83

2.25

12.02

17.86

32.78

.9241

2.42

.77

16.98

.839

2.933

1300.27

.384

.039

.796

.437

$16.60(14.17)$
$61.2 / 1 / 6.16$

$59.4 / 1 / 5.98$

$4.9 / \quad 12 / \quad 4.73$

$.2 / 12 / \quad .19$

$80.0 / 14 / .6552$

$12.6 / 14 / .0406$

$.9 / 1 / \quad .23$

$.5 / 1 / \quad .13$

2.13

2.19

17.71

.52

4.37

.6169

.11

.06

.45

.042

.674

1496.16

.029

.006

.036

.500

$15.59(15.09)$
PROPAIE

FUEL DERSITY $4.410 \mathrm{LB} / \mathrm{GAL}$ $\begin{array}{llllll}\text { В } .183 & \mathrm{C} .817 & 0.000 & \times .000\end{array}$ $100 \%$ PROPANE

HOX HOHIDITY C.F. 1.080

$$
\begin{gathered}
3 \\
\text { HOT TRAMSIWTT } \\
(0-505 \text { SEC. }) \\
505.1 \\
.968 / .979 \\
3.64(5.85) \\
321.9(9.12) \\
.00(.00) \\
2710 .(76.7)
\end{gathered}
$$

3-BAG COIPOSITE RESULTS

$\begin{array}{lll}\text { HC } & \text { G/MI } & .060 \\ \text { CO } & \text { G/NI } & .323 \\ \text { HOX } & \text { G/MI } & .048\end{array}$

FUEI ECOHOHT IPG (L/100RI) $16.61(14.17)$
CH4

G/MI

WHEC

G/RI
$71.8 / 1 / 7.23$

$56.6 / 1 / 5.70$

$10.7 / 12 / 10.34$

$.1 / 12 / \quad .10$

$89.6 / 14 / \quad .8575$

$12.7 / 14 / \quad .0410$

7.9/ 1/ 1.98

$.7 / 1 / \quad .18$

2.13

13.53

1.95

9.81

.8196

1.82

.77

1.06

.091

.876

1151.41

.288

.040

.049

.385

$18.87(12.46)$ 
PROJECT 10. 08-6969-001

TEST HD5-02S

DATE $9 / 20 / 95$ RUN

DYWO 3 BAG CART 2

ACTUAL ROAD LOAD $6.60 \mathrm{HP}$ ( $4.92 \mathrm{kN}$ )

TEST WEIGHT 3375 LBS ( $1530 \mathrm{KG)}$
VEBICLE NUIBER GA2

VRHICLE MODEL 94 PONT GRANDAH

BHEIIT $2.3 \mathrm{~L}(138 \mathrm{CID})--4$

TRAISIISSION A4

ODOALTER 1957 MILES ( 3148 KM)

BaRONETER $29.20 \mathrm{IN} \mathrm{HG} \mathrm{(741.7} \mathrm{MH} \mathrm{HG)}$ RELLTIVE HOLIDITY 60.9 PCT.

BAG NOLIBER

BAG DESCRIPIIOI

ROH TTHE SECONDS

DRY/WET CORRECTION FACTOR, SAMP/BACK

MERSURED DISTAHCE MILES (RM)

BLOFIR FLOF RATE SCFH (SCHM)

GAS HETER FLOW RRTE SCFH (SCHN)

TOTAL FLOF SCP (SCH)

HC SAYPLE HETER/RANGE/PPH (BAG)

HC BCKERD UETER/RAMGE/PPW

$\infty$ SAMPLE UETER/RAMGE/PPH

CO BCRGRD UETRR/RANGE/PPI

CO2 SAIPLE URTER/RAMGE/PCT

CO2 DEFERD YETER/RANGE/PCT

HOX SAYPLE HETER/RANGE/PPH (BAG) (D)

HOX BCEERD HETER/RANGE/PPH

CH4 SAYPLE PPH (1.140)

CH4 BCRERD PPII

DILUTIOI FACTOR

HC CONCENTRATIOA PPH

CO COICENTRATIOI PPH

$\mathrm{CO2}$ ore

HOX COICERTPRAPTOH PPH

CH4 COHCGATRATION PPH

INHC CONCENTRATION PPH
DRY BULB TEAPERATORE $72.0^{\circ} \mathrm{F}\left(22.2^{\circ} \mathrm{C}\right)$

$$
\begin{gathered}
1 \\
\text { COLD TRANSIBITI } \\
(0-505 \text { SEC. }) \\
505.2 \\
.970 / .983 \\
3.65(5.88) \\
325.5(9.22) \\
.00(.00) \\
2740 .(77.6)
\end{gathered}
$$

$20.5 / 20.49$

$4.9 / 2 / \quad 4.90$

$34.3 / \quad 12 / \quad 33.40$

53.5/ $1 / \quad .9824$

$2.5 / \quad 1 / 2.0463$

$9.7 / \quad 1 / 2.43$

HC HASS GRAMS

CO HASS GRAHS

$\mathrm{CO}_{2}$ HASS GRAHS

MOX HASS GRAHS

CH4 MASS GRAHS

WHAC HASS GRAYS (FID)

FUEL HASS KG

FURL ECONOHI UPG (L/100RH)
$.4 / \quad 12 / \quad .39$

$.9 / \quad 1 / \quad .23$

2.62

2.14

\subsection{7}

16.03

31.63

.9400

2.23

.66

15.25

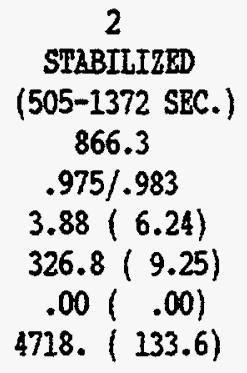

2

STABILIZED

(505-1372 SEC.)

866.3

$.975 / .983$

$3.88(6.24)$

$326.8(9.25)$

$.00(.00)$

4718. (133.6)

$52.8 / \quad 1 / \quad 5.32$

$52.2 / \quad 1 / \quad 5.26$

$4.6 / 12 / \quad 4.44$

$.4 / 12 / \quad .39$

$\begin{array}{llll}79.9 / & 14 / & .6534\end{array}$

$12.2 / 14 / .0391$

$\begin{array}{lll}1.8 / 1 / & .45\end{array}$

$.411 / \quad .10$

2.07

2.11

\subsection{6}

.36

3.93

.6165

.36

.08

.26

.029

.611

1508.12

.090

.003

.021

.504

$16.29(14.44)$

$15.39(15.28)$
PROPAIT 100\%

PUEL DETSITT $4.410 \mathrm{LB} / \mathrm{GAL}$

$\begin{array}{lllll}\text { H. } & .183 & C .817 & 0.000 \quad 8.000\end{array}$

HOX BOHIDITY C.F. .993

3

EOT TRANSIENT

( 0 - 505 SEC.)

505.2

$.972 / .983$

$3.61(5.81)$

$326.6(9.25)$

$.00(.00)$

2750. ( 77.9)

$68.5 / 1 / 6.90$

$52.9 / \quad 1 / 5.33$

$11.8 / 12 / 11.40$

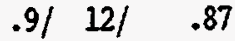

$88.9 / 14 / \quad .8407$

$12.3 / \quad 14 / \quad .0395$

$5.7 / 1 / 1.43$

$.3 / \quad 1 / \quad .08$

2.76

2.12

13.80

1.96

10.17

.8041

1.36

.79

1.05

.093

.922

1146.39

.201

.041

.050

.384

$18.82(12.50)$

3-BAG COHPOSITE RESULTS

$\begin{array}{lll}\text { HC } & \text { G/MI } & .054 \\ \infty & \text { G/MI } & .315 \\ \text { IOX } & \text { G/MI } & .046\end{array}$

FUEL ECONOHY UPG (L/10ORH) 16.42 (14.33) $\begin{array}{lll}\text { CH4 } & \text { G/MI } & .006 \\ \text { WIHC } & \text { G/MI } & .048\end{array}$ 
SOUTHWEST RESEARCH IISTITUTE - DEPARIMEMT OF EMISSTOHS RRSEARCH

COIPOTER PROGRAH LDT 1.2-R

VERICLE NOTBER GA2

VERICLE HODEL 94 PONI GRAKDAY

BMGINE $2.3 \mathrm{~L}$ (138 CID) -4

TRAISUISSTON A4

ODOMETER

1724 MILES ( 2773 XY)

BAROHETER 29.18 IN EG (741.2 HH EG)

RELLTIVE HDHIDITY 62.4 PCT.

BAG NUIBER

BAG DESCRIPIIOH

RUI TITE SECONDS

DRY/WET CORRECTIOI FACTOR, SALP/BACR

UERSURED DISTAMCE UIILSS (RI)

BLONER FLON RATE SCFH (SCHA)

GAS METER FLON RATE SCFH (SCAN)

TOTAL FLON SCF (SCH)

BC SAYPLE METER/RAIGE/PPN (BAG)

BC BCRERD HETER/RAHGE/PPH

$\infty$ SAIPLE HETER/RAIGR/PPH

$\infty$ BCRGRD HETER/RHHGE/PPH

CO2 SALPLE HETER/RAHGE/PCT

CO2 BCRERD METER/RAHGE/PCT

KOX SAIPLE YIETER/RANGE/PPH (BAG) (D)

HOZ BCRERD HETER/RAHGE/PPH

CB4 SAIPLE PPH (1.160)

CH4 BCRERD PPH

DILUTION FACTOR

HC COMCEXTRATIOH PPM

CO COHCEHTRATTOH PPH

CO2 COHCEMTRATIOH PCT

HOX CONCEHTRATIOH PPM

CH4 COHCWMTRATIOH PPH

MHAC COHCERTRATION PPH

FIDEC MASS GRAMS

$C O$ HASS GRAIS

CO2 MASS GRAYS

HOY MRSS GRAIS

CH4 HISS GRALS

MHEC MASS GRAKS (FID)

FOEL MASS RG

FOEL ECONONY HPG (L/10OKN)
3-BAG CARB FTP VEAICLE EIISSIOH RESULTS

TEST 90NB/10IB-01

DATE $9 / 9 / 95$ RUII

DHFO 3 BAG CART 2

ACTOAL ROAD LOAD $6.60 \mathrm{BP}$ ( $4.92 \mathrm{KN})$

TEST WETGHT 3375 LBS ( 1530 KG)

PROJECT 10. 08-6969-001

BDTARTE BUTARE

FUEL DEISITY $4.859 \mathrm{LB} / \mathrm{GAL}$

H $.170 \quad C \quad .830 \quad 0 \quad .000 \quad \& .000$

$90 \%$ BUTANE

10\% ISOBUTAHE

NOX HUIIDITY C.P. 1.041

DRY BULB TEIPERATURE $75.0^{\circ} \mathrm{F}\left(23.9^{\circ} \mathrm{C}\right)$

$$
\begin{gathered}
1 \\
\text { COLD TRAISIEETT } \\
(0-505 \text { SEC. }) \\
506.1 \\
.969 / .981 \\
3.63(5.84) \\
321.5(9.10) \\
.00(.00) \\
2712 .(76.8)
\end{gathered}
$$

$\begin{array}{rrr}28.2 / & 2 / & 28.18 \\ 6.2 / & 2 / & 6.20 \\ 81.8 / & 13 / & 198.23 \\ .5 / & 13 / & 1.10 \\ 54.0 / & 1 / & .9917 \\ 2.6 / & 1 / & .0482 \\ 3.7 / & 1 / & .93 \\ .7 / & 1 / & .18 \\ & 4.06 & \\ & 2.16 & \end{array}$

11.92

22.51

188.88

.9476

.77

2.08

20.10

1.040
16.887

1332.36

.117

.106

.890

.448

$17.86(13.17)$

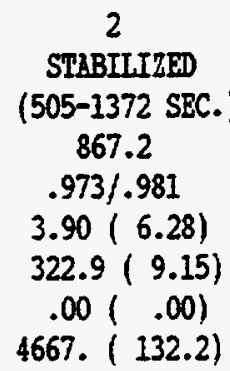

$63.9 / \quad 1 / 6.43$

$62.3 /$ if 6.27

$20.2 / \quad 12 / 19.78$

$1.1 / 12 / 1.09$

81.3/ 14/ .6794

$12.8 / 14 / \quad .0414$

$.6 / 1 / \quad .15$

$.6) 1 / .15$

2.43

2.33

17.72

.51

18.07

.6404

.01

.23

.24

.041

2.780

1549.71

.002

.021

.019

.511

$16.82(13.98)$
3

HOT TRAISIEXT

( 0- 505 SEC.)

505.6

$.970 / .981$

$3.63(5.84)$

321.7 ( 9.11$)$

$.00(.00)$

2711. ( 76.8)

$10.4 / 2 / \quad 10.39$

$6.1 / 2 / \quad 6.10$

$59.5 / 12 / 58.61$

$.9 / \quad 12 / \quad .90$

90.4/ 14/ .8772

$12.8 / 14 / \quad .0414$

$.6) \quad$ y .15

$.6 / 1 / \quad .15$

4.25

2.47

13.67

4.74

55.47

.8389

.01

1.97

2.46

.219

4.958

1179.17

.002

.101

.109

.391

$20.48(11.49)$

3-BAG COIPOSITE RESULTS

$$
\begin{array}{llr}
\text { FIDAC } & \text { G/NI } & .081 \\
\infty & \text { G/MI } & 1.709 \\
\text { HOX } & \text { G/HI } & .007
\end{array}
$$

FUEL ECONOHY UPG (L/100NH) 17.93 (13.12)

$\begin{array}{lll}\text { CH4 } & \text { G/MI } & .016 \\ \text { MIIC } & \text { G/MI } & .062\end{array}$


SOUTHWEST RESEARCH INSTITUTE - DEPARTHENT OF EHISSIONS RESEARCH

COIPUTER PROGRAH LDT $1.2-R$

VEHICLE BOHBER GA2

VEHICLE HODEL 94 PONT GRAKDAH

ETGINE

TRARSMISSION A4

ODOHETER

3-B3G CARB FTP VEHICLE EYISSION RESULTS

PROJECT NO. 08-6969-001

BAROHETER 29.14 IN HG (740.2 HM BG)

RELLATIVE HOUIDITY 66.7 PCT.

BAG NUYBER

BAG DESCRIPITION

ROI TINE SECOIDS

DRY/WET CORRECTION FACTOR, SAYP/BACK

UEASURED DISTAHCE YILEES (RI)

BLOWER FLOW RATE SCFN (SCMN)

GAS HETER FLON RATE SCFH (SCHY)

TOTAL FLON SCP (SCM)

HC SAMPLE METER/RANGE/PPY (BAG)

BC BCRGRD YIETER/RAHGE/PPH

CO SAHPLE HETER/RAMGE/PPN

CO BCRERD HETER/RANGE/PPH

CO2 SAYPLE HETER/RAHGE/PCT

C22 BCRGRD HETBR/RAMGE/PCT

NOX SAYPLE HETER/RAMGE/PPH (BAG) (D)

HOX BCRGRD HETER/RANGE/PPH

CH4 SAYPLE PPH (1.160)

CB4 BCRGRD PPI

DILUTIOH FACTOR

BC CONCERTRATION PPH

CO COLCEATRATIOH PPH

$\mathrm{CO}_{2}$ COHCENTRATION PCT

NOX CONCEMTRRATION PPH

CH4 CONCEHTRATION PPH

NIIHC COHCEYTRATION PPH

THC MASS GRALS

CO MASS GRAHS

CO2 MASS GRAHS

HOX MLSS GRAKS

CH4 HASS GRAHS

MTHC MASS GRANS (FID)

FUEL HASS RG

FOEL ECONONI MPE (L/100RN)

TEST 90RB/10IB-02S

DATE $9 / 8 / 95 \quad$ RON

DYHO 3 BAG CART 2

SCTOAL ROAD LOAD $6.60 \mathrm{AP}$ (4.92 KT)

TEST WETGHT 3375 LBS ( $1530 \mathrm{KG}$ )

DRY BULB PEIPERATURE $76.0^{\circ} \mathrm{F}\left(24.4^{\circ} \mathrm{C}\right)$

$$
\begin{array}{cc}
1 & 2 \\
\text { COLD TRAISIRTT } & \text { STABILIZDD } \\
(0-505 \text { SEC. }) & (505-1372 \text { SEC. }) \\
506.2 & 867.2 \\
.967 / .979 & .971 / .979 \\
3.66(5.89) & 3.89(6.27) \\
319.8(9.06) & 321.3(9.10) \\
.13(.00) & .14(.00) \\
2699 .(76.4) & 4646 .(131.6)
\end{array}
$$

$\begin{array}{rrr}25.6 / & 2 / & 25.59 \\ 7.4 / & 2 / & 7.40 \\ 80.4 / & 13 / & 194.47 \\ .6 / & 13 / & 1.33 \\ 55.0 / & 1 / & 1.0104 \\ 2.6 / & 1 / & .0482 \\ 3.9 / & 1 / & .98 \\ 1.1 / & 1 / & .28 \\ & 3.65 & \\ & 2.18 & \end{array}$

11.72

18.82

184.73

.9663

.73

1.66

16.89

.843

16.437

1352.21

.116

.085

.745

.454

$17.76(13.24)$
$68.1 /$ if 6.86

$70.8 /$ 1/ 7.13

$20.6 / \quad 12 / 20.16$

$1.0 / 12 / \quad .99$

82.1/ 14/ .6948

$13.5 / 14 / \quad .0441$

$.9 / \quad 1 / \quad .23$

$.9 / \quad 1 / \quad .23$

2.23

2.23

\subsection{3}

.14

18.50

.6532

.01

.14

$-.02$

.015

2.834

1573.66

.004

.012

.000

.519

$16.54(14.23)$
BOTARE BOTANE

FUEL DEISITY 4.859 IB/GAL $\begin{array}{llllll}\text { स } .170 & C .830 & 0.000 & \searrow .000\end{array}$ $90 \%$ BOTARE $10 \%$ ISOBUTARE

HOX HDKIDITY C.P. 1.089

3

HOT TRAHSIENT

( 0- 505 SEC.)

505.4

$.968 / .979$

$3.67(5.90)$

$319.9(9.06)$

$.13(.00)$

2696. ( 76.3)

86.4/ 1/ 8.70

$61.4 / 1 / \quad 6.18$

$\begin{array}{llll}48.7 / & 12 / & 47.73\end{array}$

$.7 / \quad 12 / \quad .70$

$\begin{array}{llllll}90.7 / & 14 / & .8848\end{array}$

$13.1 / 14 / \quad .0425$

2.4/ $1 / \quad .60$

$.9 / \quad 1 / \quad .23$

3.44

2.16

13.58

2.97

45.14

.8454

.39

1.44

1.30

.134

4.012

1181.71

.062

.073

.057

.391

20.67 ( 11.38$)$

3-BAG CONPOSITE RESULTS

$\begin{array}{llr}\text { THC } & \text { G/MI } & .060 \\ C D & \text { G/MI } & 1.613 \\ \text { HOX } & \text { G/MI } & .012\end{array}$

FURL ECOHONY UPG (L/100:M)
$17.79(13.22)$

$\begin{array}{crrr}\text { CH4 } & \text { GNI } & .012 \\ \text { NHHC } & \text { G/II } & .047 \\ \text { CARBOHIL G/MI } & .001 \\ \text { NHOG } & \text { G/MI } & .048 \quad(R A F=1.00)\end{array}$


SOUTHITEST RESEARCH INSTITUTE - DEPARTUEHTI OP EIISSTONS RESEARCH

COHPOTER PROGRAY LDT 1.2-R 3-BAG CARB FTP VEHICLE EYISSIOH RESULTS

PROJECT 10. 08-6969-001

VEHICLE ROLBER GA2

VERICLE HODEL 94 POHT GRAKDAY!

ERGIFE $2.3 \mathrm{~L}(138 \mathrm{CID})-4$

TRAISHISSION 14

ODONETER 1724 MILES ( $2773 \mathrm{KN}$ )

BAROHETER 29.25 IN HG (743.0 HN HG) RELLATIVE HUIIDITY 58.6 PCT.

BAG WTOBBER

BAG DESCRIPTIOI

RIN TIYE SECONDS

DRY/WET CORRECTIOH FACTOR, SAIP/BACR

MEASURED DISTAMCE UIIES (RII)

BLOFIRR FLON RATE SCEH (SCYII)

GAS HETER FLON RATE SCFH (SCMI)

TORAL FLON SCF (SCM)

BC SAMPLE METER/RAMGE/PPM (BAG)

BC BCKGRD HETER/RANGE/PPN

CO SAIPLE VETER/RAHER/PPW

$C O$ BCKERD HETER/RANGE/PPH

CO2 SAYPLE UETER/RANGE/PCT

CO2 BCKERD METER/RAHGE/PCT

HOX SAIPLE WETER/RAYGE/PPM (BLG) (D)

HOX BCRGRD HETER/RANGE/PPI

CR4 SAHPLE PPH (1.160)

CH4 BCRERD PPI

DILUTTON FACTOR

BC CONCEMTRATTON PPH

CO CONCEHTRATION PPH

$\mathrm{CO} 2$ COHCEHTRATION PCT

NOX CONCEITRATTON PPN

CH4 CONCENTRATIOI PPH

MIHC CONCEMTRATTON PPH

FIDEC MASS GRAYS

CO HASS GRAYS

$\mathrm{CO} 2$ MASS GRAYS

HOX HASS GRAHS

CF4 MASS GRAYS

MIEC MASS GRAYS (FID)

FUEL MASS RG

FOEL ECOHOHY UPG (L/100RI)
TEST 901B/10BE-01

DATE $9 / 10 / 95 \quad$ ROH

DWHO 3 BAG CART 2

ACTOAL ROAD LOAD $6.60 \mathrm{KP}$ ( $4.92 \mathrm{KN})$ TEST WEICAT 3375 LBS ( $1530 \mathrm{KG)}$

DRY BULB TEHPERATURE $75.0^{\circ} \mathrm{F}\left(23.9^{\circ} \mathrm{C}\right)$

$$
\begin{gathered}
2 \\
\text { STLBILILED } \\
(505-1372 \text { SEC. }) \\
867.1 \\
.974 / .982 \\
3.90(6.28) \\
323.3(9.16) \\
.00(.00) \\
4672 .(132.3)
\end{gathered}
$$

58.3/ 1/ 5.87

$57.0 / \quad 1 / 5.74$

$20.0 / 12 / 19.58$

$.3 / \quad 12 / \quad .30$

$81.2 / \quad 14 / \quad .6775$

12.7/ 14/ .0410

$.6 / 1 / \quad .15$

$.6) 1 / \quad .15$

2.21

2.11

17.77

.45

18.64

.6388

.01

.22

.20

.036

2.871

1547.66

.002

.019

.015

.511

$18.03(13.05)$

$17.05(13.80)$

BUTARE BOTAYE

FUEL DERSITY $4.916 \mathrm{LB} / \mathrm{GAL}$

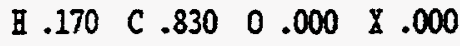

$90 \%$ BOTARE

$10 \%$ MIXED BUTEIE

HOX HUYIDITY C.F. 1.015

3

HOT TRAYSIEYT

$(0-505$ SEC. $)$

505.7

$.971 / .982$

$3.62(5.83)$

$322.5(9.13)$

$.00(.00)$

2718. ( 77.0)

$95.9 / \quad 1 / \quad 9.65$

$67.8 / \quad 1 / 6.83$

$56.9 / 12 / 55.97$

$.31 \quad 121 \quad .30$

$90.7 / 14 / \quad .8848$

$12.8 / 14 / \quad .0414$

$.5 / \quad 1 / \quad .13$

.4) $1 / \quad .10$

3.48

2.06

$$
\begin{array}{r}
13.56 \\
3.33 \\
53.54 \\
.8465 \\
.03 \\
1.57 \\
1.51 \\
\\
.154 \\
4.799 \\
1193.10 \\
.005 \\
.081 \\
.067 \\
.395
\end{array}
$$

$20.46(11.50)$

3-BAG COMPOSITE RRSULTS

$\begin{array}{llrl}\text { FIDHC } & \text { G/MI } & .067 & \\ \infty & \text { G/MI } & 1.510 & \\ \text { HOX } & \text { G/MI } & .011 & \\ \text { FOEL ECOHONY IPG } & (L / 100 \mathrm{NM}) & 18.09 & (13.00)\end{array}$

$\begin{array}{lll}\text { CH4 } & \text { G/MI } & .013 \\ \text { NIHC } & \text { G/MI } & .052\end{array}$ 
VEHICLE NUIBER GA2

VAIICLE YODEL 94 POIT GRAMLY

EMGIIE 2.3 L (138 CID) -4

TRAHSHISSTOR A4

ODOIETER

1724 YILLS ( 2773 (N)

BAROMETER 29.27 II HG (743.5 HH HG)

RELATIVE BUIIDIFY 61.4 PCT.

BAG NDIBBER

BAG DESCRIPTIOH

ROR TIRE SECOHDS

DRY/WET CORRECTION FACTOR, SAYP/BACK

HFASURED DISTAHCE IIIIES (IXI)

BLONER FLON RATE SCFI (SCWI)

GaS IETER FLON RATE SCFH (SON)

TOTAL FLON SCF (SCY)

BC SAYPLE HETER/RARGE/PPM (BAG)

HC BCRGRD HETER/RAYGE/PPW

CO SAYPLE HETER/RATER/PPH

CO BCRGRD YETER/RAFGR/PPH

CO2 SAIPLE HETER/RAKGE/PCT

CO2 BCRERD IETER/RAFGR/PCT

HOX SAYPLE METER/RMYGE/PPH (BAG) (D)

HOX BCRERD HETER/RAIGE/PPY

CH4 SAYPLE PPH (1.160)

CH4 BCRGRD PPN

DILUTION FACTOR

BC CONCERTRATIOH PPH

CO CORCBHTRATTOH PPH

$\mathrm{CO} 2$ CONCENTRAPTOH PCT

NOX COHCERTRATIOH PPH

CiH4 CONCENTRATION PPH

MHHC COHCENTRATIOH PPY

\begin{tabular}{|c|c|}
\hline $\mathrm{THC}$ & MASS GRAYS \\
\hline $\mathrm{CO}$ & MASS GRAHS \\
\hline $\mathrm{CO} 2$ & MASS GRAYS \\
\hline $\mathrm{HOX}$ & HASS GRAYS \\
\hline CII 4 & MASS GRAYS \\
\hline NHPC & MaSS GRAYS (FD) \\
\hline FUEL & MLSS RG \\
\hline
\end{tabular}

TEST 901B/10BE-02S

DATE $9 / 11 / 95$ RUI

DHIO 3 BAG CART 2

ACTOAL ROAD LOAD $6.60 \mathrm{HP}$ ( $4.92 \mathrm{KN}$ ) TEST WEIGHT 3375 LBS ( 1530 KG)

DRY BOLB TEYPERATURE $73.0^{\circ} \mathrm{F}\left(22.8^{\circ} \mathrm{C}\right)$

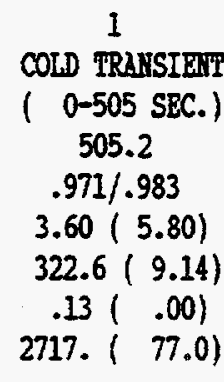

$23.9 / \quad 2 / \quad 23.89$

$6.7 / 2 / \quad 6.70$

$70.8 / 13 / 168.89$

$.5 / 13 / 1.10$

$94.7 / 14 / .9930$

$13.5 / 14 / .0441$

$5.5 / 1 / \quad 1.38$

$.7) \quad 1 / \quad .18$

3.85

2.48

11.95

17.75

160.82

.9527

1.22

1.58

15.91

.797

14.407

1342.12

.180

.081

.706

.450

$17.87(13.16)$

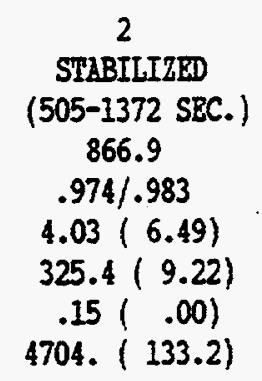

$84.8 / \quad 1 / 8.54$

$86.1 /$ I/ 8.67

$18.8 / 12 / 18.42$

$1.0 / 12 / \quad .99$

81.4/ 14/ .6813

$13.4 / 14 / .0437$

$.7 / 1 / \quad .18$

$.6) 1 / .15$

2.53

2.48

17.67

.36

16.85

.6401

.03

.19

.14

.028

2.614

1561.30

.009

.017

.010

.515

$17.46(13.48)$
BUTARE BUTARE

FUEL DEISITY 4.916 LB/GLL

$\begin{array}{lllllllllll}\text { H } .170 & C & .830 & 0 & 0.000 & \& & .000\end{array}$

908 BUTARE

10\% MIXED BDTERE

HOX HOIIDITY C.F. 1.007

3

HOT IRAISIERT

( 0- 505 SBC.)

505.2

$.972 / .983$

$3.62(5.83)$

$-323.1(9.15)$

$.13(.00)$

2721. ( 77.1$)$

$\begin{array}{lll}13.21 & 2 / \quad 13.19\end{array}$

$8.7 / 2 / \quad 8.69$

$68.6 / 12 / 67.90$

$\begin{array}{lll}.8 / & 12 / \quad .80\end{array}$

$90.9 / 14 / \quad .8898$

13.4/ 14/ .0437

.6) $1 / \quad .15$

$.6 / 1 / \quad .15$

4.05

2.34

13.46

5.14

64.49

.8494

.01

1.89

2.95

.230

5.787

1198.54

.002

.097

.131

.397

$20.32(11.57)$

3-BAG CONPOSITE RESULTS

$\begin{array}{llr}\text { TEC } & \text { G/III } & .066 \\ C D & \text { G/MI } & 1.584 \\ \text { WOX } & \text { G/III } & .011 \\ \text { FOEL ECOHONY IPG } & (\mathrm{L} / 100 \mathrm{~N})\end{array}$

.066

.584
.011

NHS

G/MI

.014

CARBOIYL G/MI

.051

FUEL ECONOIY IPG (L/100NI)

$18.25(12.89)$

IIOC

G/HII

$.052 \quad(R A F=1.00)$ 
SOUTHNRST RESEARCH IRSTITUTE - DEPARTIITIT OF EUISSIONS RESEARCH

COHPOTER PROCRAH LDT 1.2-R 3-BAG CARB FIP VERICLE BMISSIOI RESULTS

PROJECT HO. 08-6969-001

VEIICLE NUIBRR GA2

VEHICLE HODEL 94 PONT GRATAH

EIGTE $2.3 \mathrm{~L}$ (138 CID $)-4$

TRAHSHSSION $\mathrm{A4}$

ODONETER $\quad 1800$ MILES ( 2896 RN)

BLROWLTZR 29.28 II HG (743.7 M HG)

RELATIVE HUIIDITY 69.8 PCT.

BAG IIUIBER

BAG DESCRIPTTOY

ROI TIIE SECOHDS

DRY/WER CORRBCIIOA PACTOR, SAYP/BACR

IEASURED DISTAICE HILRS (IN)

BLONER FLON RATE SCEN (SCUY)

GAS HETER FON RATE SCFN (SCHA)

TOIAL FLON SCP (SCI)

BC SAYPLE HEIER/RATGE/PPY (BAG)

EC BCRERD IETER/RAHEE/PPH

CO SLIPLE IETER/RATEB/PPI

CO BCRERD YIFTER/RAKGE/PPH

CO2 SAIPLE IETER/RAHEE/PCT

CO2 BCRGRD LIETER/RMYGE/PCP

HOZ SAHPLE YETER/RAHGE/PPH (BAG) (D)

HOX BCKGRD KIETER/RAMGE/PPY

CR4 SAIPLE PPI (1.160)

CH4 BCRGRD PPI

DILUTTOA PACTOR

BC COACERTRATOH PPH

CO COMCETIRATIOH PPH

$\mathrm{CO} 2$ CONCEHTRATIOI PCT

BOX CONCERTRETIOA PPI

CB4 COACRHTRATION PPS

IRIPC COHCEIIIRATTOH PFH

FIDAC MASS GRAYS

CO MASS GRAIS

$\mathrm{CO} 2$ MASS GRAYS

HOX WASS GRAIS

CH4 MASS GRAYS

IIITC NASS GRALS (FD)

FUEL MASS RG

FUEL ECOHOYY IPG (L/100R)
TPST $60 \mathrm{LB} / 40 \mathrm{IB}-01$

DATR $9 / 14 / 95$ RUI

DHO 3 BAG CARI 2

ACIUAL ROAD LOAD 6.60 HP ( $4.92 \mathrm{KN}$ )

TEST WEIGHP 3375 IBS ( 1530 RG)

DRY BOLB TEIPERATURE $74.0^{\circ} \mathrm{F}\left(23.3^{\circ} \mathrm{C}\right)$
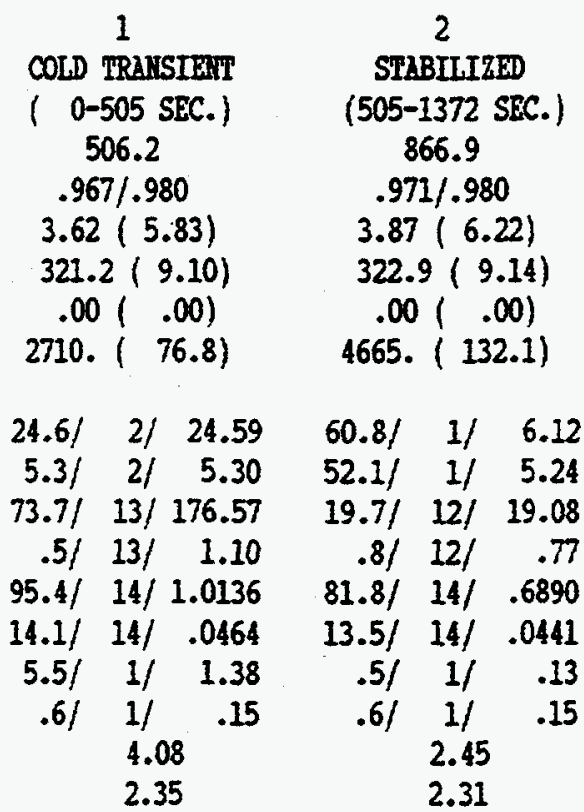

11.63

19.74

167.60

.9712

1.24

1.93

17.50

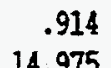

1364.75

.196

.099

.775

.459

$17.23(13.65)$

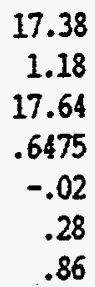

2.714

1566.15

.000

.024

.065

.518

$16.28(14.45)$
BUTALE BUTAIE

FOEL DBHSITY $4.806 \mathrm{IB/GLI}$ B. $.172 \quad C .828 \quad 0.000 \quad \& .000$ 60\% BUTARE $40 \%$ ISOBUTAIE

HOX HOITDITY C.F. 1.076

3

HOT TRANSIEHT

( 0 - 505 SBC. $)$

505.1

$.969 / .980$

$3.61(5.80)$

$323.4(9.16)$

$.00(.00)$

2723. ( 77.1)

$90.7 / 1 / 9.13$

$51.4 / 1 / 5.17$

$50.9 / 12 / 49.94$

$\begin{array}{llll}.6 / 12 / \quad .58 & 12 /\end{array}$

$91.0 / 14 / .8924$

$14.3 / \quad 14 / \quad .0472$

$.8 / 1 / \quad .20$

$.5 / \quad 1 / \quad .13$

3.73

2.30

13.38

4.34

47.30

.8487

.08

1.60

2.49

.202

4.246

1198.21

.013

.082

.111

.397

$19.79(11.89)$

3-BAG COIPOSITE RESULTS

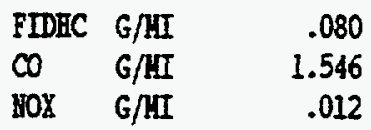

FUEL ECOFOIY IPG (L/100RI) 17.34 (13.57)

$\begin{array}{lll}\text { CE4 } & \text { G/III } & .015 \\ \text { WHBC } & \text { G/MI } & .062\end{array}$




\section{COUPUTER PROGRAY LDT 1.2-R 3-BAG CARB FTP VEHICLE EIISSTOY RRSULTS PROJECT HO. 08-6969-001 \\ RRSBRRCH}

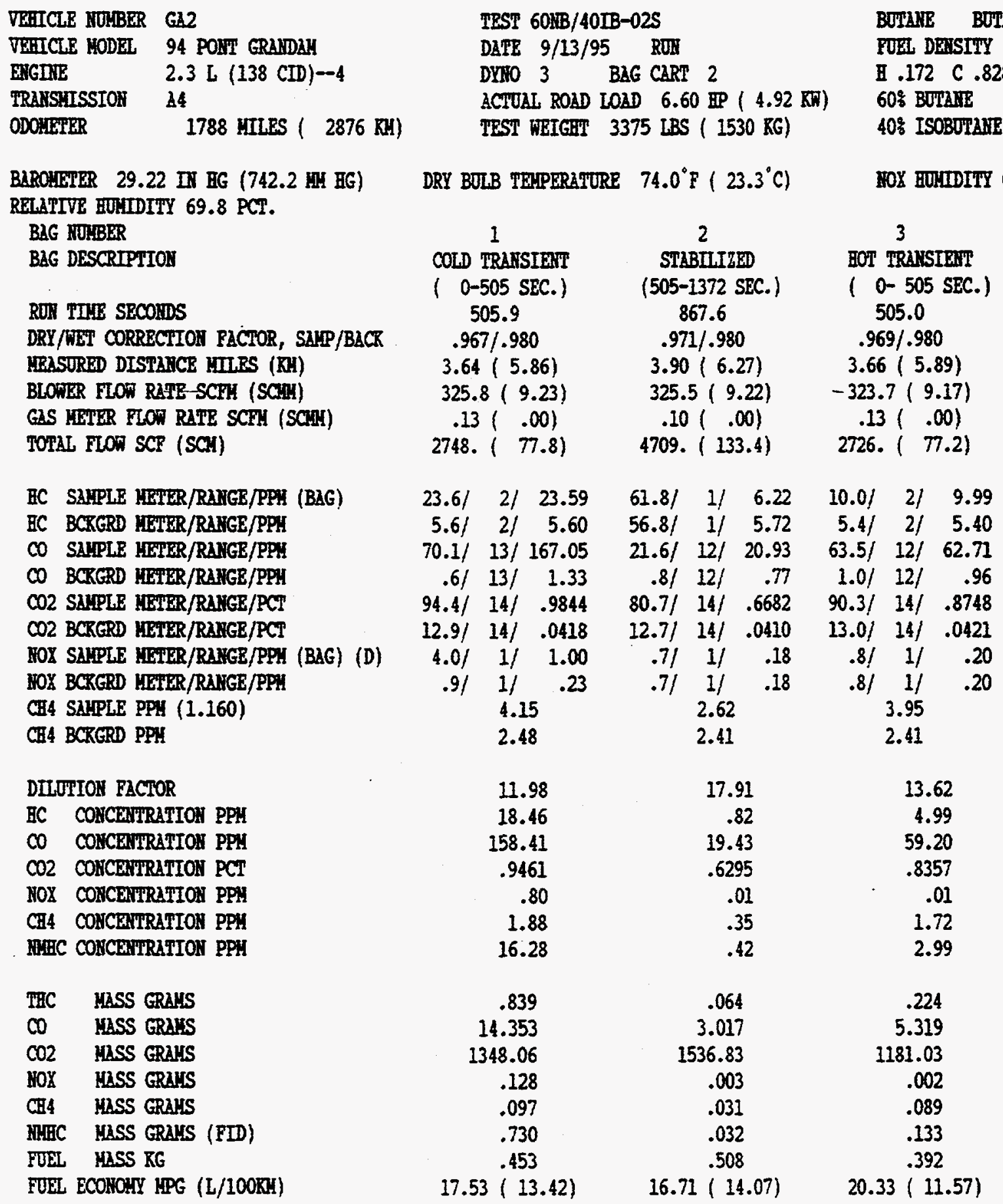

3-BAG COMPOSITE RESULTS

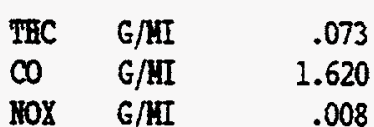

FUER ECOHOHY HPG (L/100RM)
TRST 6OIB/40IB-02S

DATE $9 / 13 / 95$ RUI

DYISO 3 BAG CART 2

ACTOAL ROAD LOAD $6.60 \mathrm{EP}$ ( $4.92 \mathrm{KN})$
BUTARE BUTAME

MOX HOHTDITY C.P. 1.077

3

0- 505 SEC.)

505.0

$3.66(5.89)$

$323.7(9.17)$

$.13(.00)$

$\begin{array}{lll}10.01 & 21 & 9.99\end{array}$

5.4) $2 / \quad 5.40$

$63.5 /$ 12/ 62.71

1.0/ $12 / \quad .96$

$90.3 / \quad 14 / \quad .8748$

3.01 14) .0421

$.8 / 1 / \quad .20$

3.95

13.62

4.99

59.20

1.72

2.99

.224

5.319

.002

.089

.133

20.33 (11.57) 
VEHICLE IUIBER GA2

VEHICLE HODEL 94 POIT GRAWDAN

BRGIIE $\quad 2.3 \mathrm{~L}(138 \mathrm{CID})-4$

TRAMSHISSIOH $\quad 44$

ODOLIEIER
TEST 80RB/20/P-01

DATE $9 / 21 / 95 \quad$ RDN

DHO 3 BAG CART 2

ACIVAL ROAD LOAD 6.60 IP ( $4.92 \mathrm{kN}$ )

TEST FEIEHP 3375 IBS ( 1530 RG)
BUTAYE PROPANE

FUR DBISITI $4.748 \mathrm{LB} / \mathrm{GAL}$

II $.175 \quad C .825 \quad 0.000 \quad \& .000$ 80\% BUT2IIE

20\% PROPAKE

BOX BUIIDITY C.F. 1.059

3

1
COLD TRARSIBTP
$(0-505$ SEC. $)$
506.3
$.968 / .980$
$3.64(5.86)$
$320.7(9.08)$
$.00(.00)$
$2706 .(76.6)$

21.6/ 2/ 21.59

$5.3 / 2 / \quad 5.30$

$67.2 / 13 / 259.44$

$.5 / 13 / 1.10$

$94.3 / 14 / .9815$

$13.7 / 14 / \quad .0448$

$4.7 / 1 / 1.18$

$.6 / 1 / \quad .15$

3.66

2.40

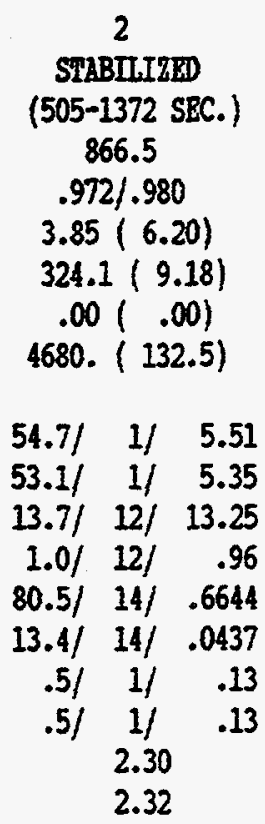

11.92

16.73

151.33

.9404

1.04

1.47

15.03

MHC COHCEITRRYTOY PPI

FIDEC MASS GRAYS

$C O$ HASS GRAYS

$\mathrm{CO}_{2}$ MASS GRARS

HOX HASS GRAYS

CH4 HASS GRAYS

WIRC HASS GRALS (FID)

FUII HASS RG

PUEL BCOHONY IPG (L/100KH)
.776

13.502

1319.51

.162

.075

.664

.444

$17.64(13.33)$

\subsection{7}

.46

11.86

.6232

.01

.11

.34

.037

1.830

1512.33

.002

.010

.026

.501

$16.55(14.22)$
FOT TRAKSIRIT

( 0 - 505 SEC.)

505.4

$.969 / .980$

$3.59(5.78)$

$322.3(9.13)$

$.00(.00)$

2715. (76.9)

$87.0 / 1 / 8.76$

$53.1 / 1 / 5.35$

$44.9 / \quad 12 / \quad 43.93$

$.7 / 12 / \quad .68$

$89.6 / 14 / .8575$

$13.3 / 14 / .0433$

1.1) $1 / .28$

$.5 / 1 / .13$

3.74

2.37

13.80

3.80

41.48

.8174

.16

1.54

2.01

.177

3.713

1150.68

.025

.079

.089

.383

$20.21(11.64)$

3-BAG CONPOSITE RESULTS

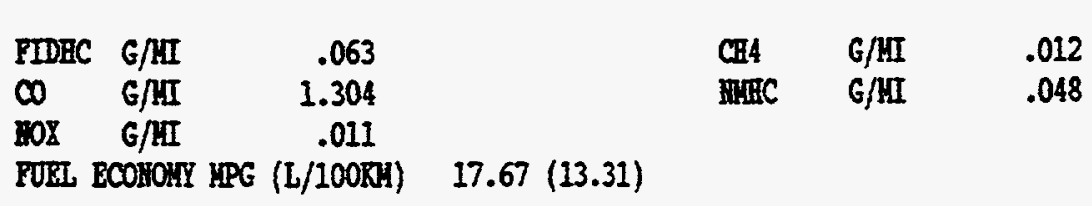


VEHICLE NOUBER GA2

\begin{tabular}{|c|c|}
\hline VRHICLE HODEL & 94 PONP GRANDAH \\
\hline EMGINE & 2.3 I (138 CID $)-4$ \\
\hline TRAISIISSION & 24 \\
\hline ODOAETER & 2008 KIILES । \\
\hline
\end{tabular}

BAROUETER 29.33 II HG (745.0 WA FG) RELATIVE HDHDITY 40.5 PCT.

BAG WOIBRR

BAG DESCRIPITOH

ROI TTYE SECONDS

DRY/WET CORRECTIOI FACTOR, SAMP/BACR

YEASURED DISTAYICR YILES (RI)

BLONER FLON RRTE SCFY (SCHI)

GAS WETER FLOF RATE SCFM (SCNI)

TOTAL FION SCR (SCH)

BC SAMPLE METER/RAHGE/PPH (BAG)

HC BCKGED HETER/RANGE/PPH

CO SAMPLE HETER/RAMGE/PPM

CO BCKGRD YETER/RATEE/PPH

CO2 SAMPLE HETER/RAIGE/PCT

CO2 BCEGRD HETER/RANGE/PCP

MOX SAMPLE HETER/RAIGE/PPH (BAG) (D)

HOX BCKCRD HETER/RAHEE/PPH

CH4 SAMPLE PPU (1.150)

CH4 BCKGRD PPH

DILOTION BACTOR

BC CONCENTRATTON PPH

CO COHCBNIRATION PPH

$\mathrm{CO} 2$ COHC HATHAITOH PCT

HOX COHCENIRAMTION PPHI

CH4 CONCENTRATION PPH

WHPC CONCENTRATTON PPH

FIDEC UASS GRALS

$\infty$ HASS GRAES

$\mathrm{CO} 2$ HASS GRAHS

HOX HASS GRAHS

CH4 MRSS GRAHS

WIIC WBSS GRAUS (PID)

FUEL MASS KG

PURL BCOHONY IPG (L/10ORH)
TRST 80RB/20NP-02S

DATE $9 / 22 / 95$ ROI

DHW 3 BAG CART 2

ACTUAL ROAD LOAD $6.60 \mathrm{IPP}$ ( $4.92 \mathrm{KIN}$ )

TEST WEIGET 3375 LBS ( 1530 RG)
BDTANE PROPANE

FOEL DEMSTIY $4.748 \mathrm{LB} / \mathrm{GAL}$

H $.175 \quad C .825 \quad 0.000 \quad \& .000$

80\% BUTANE

20\% PROPANE

FOY HUYIDITY C.F. $\quad .877$

3

$$
\begin{aligned}
& \text { COLD TR2HSIDVT } \\
& (0-505 \text { SEC. }) \\
& 505.5 \\
& .977 / .990 \\
& 3.64(5.85) \\
& 323.5(9.16) \\
& .00(.00) \\
& 2726 .(77.2)
\end{aligned}
$$

$\begin{array}{rcr}21.3 / & 2 / & 21.29 \\ 3.4 / & 2 / & 3.40 \\ 45.0 / & 13 / & 103.28 \\ .0 / & 13 / & .00 \\ 94.5 / & 14 / & .9872 \\ 12.1 / & 14 / & .0387 \\ 7.0 / & 1 / & 1.76 \\ .6 / & 1 / & .15 \\ & 3.11 & \\ & 1.99 & \end{array}$

11.91

18.17

99.62

.9517

1.62

1.28

16.70

.849

8.952

1345.08

.209

.066

.743

.451

$17.37(13.54)$

\section{2}

STABILIZED

(505-1372 SBC.)

866.4

$.981 / .990$

$3.89(6.25)$

$328.7(9.31)$

$.00(.00)$

4746. (134.4)

$\begin{array}{rrr}35.0 / & 1 / & 3.52 \\ 35.6 / & 1 / & 3.58 \\ 10.4 / & 12 / & 10.05 \\ .0 / & 12 / & .00 \\ 80.0 / & 14 / & .6552 \\ 12.1 / & 14 / & .0387 \\ .5 / & 1 / & .13 \\ .1 / & 1 / & .03 \\ & 1.95 & \\ & 1.99 & \end{array}$

18.13

.14

9.77

.6186

.10

.07

.06

.011

1.528

1522.43

.023

.006

.005

.505

$16.59(14.18)$
HOT TRAISIERT

( 0- 505 SEC.)

505.2

$.979 / .990$

$3.64(5.86)$

$328.3(9.30)$

$.00(.00)$

2765. ( 78.3)

$11.0 / 2 f \quad 10.99$

$3.5 / 2 / \quad 3.50$

$79.0 / \quad 12 / 78.65$

$.0 / \quad 12 / \quad .00$

$89.3 / \quad 14 / \quad .8503$

12.1/ $14 / \quad .0387$

$.6 / 1 / \quad .15$

$.5 / 1 / \quad .13$

3.94

2.03

13.86

7.75

76.11

.8143

.03

2.05

5.39

.367

6.937

1167.28

.004

.107

.243

.390

$20.10(11.71)$

3-BAG COMPOSITE RESULTS

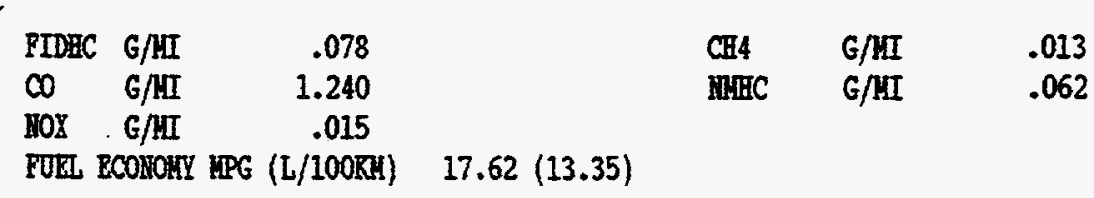


COHPUTER PROCRAH LDT 1.2-R

VEHICLE HUHBER G32

VEHICLE HODEL 94 PONT GRATDAH

EHGITE $2.3 \mathrm{~L}(138 \mathrm{CID})-4$

TRARSHISSIOH 14

ODOHETER 2081 GILES ( 3348 RH)

BAPCHETER $29.12 \mathrm{II} \mathrm{HG}$ (739.6 $\mathrm{HAG}$ BG)

RELATIVE HO:IDITT 64.5 PCT.

BAG KOERERR

BAG DESCRIPTION

RON TTHE SECONDS

DRY/WET CORRECTTOII FACTOR, SARP/BACR

HEASURED DISTAWCE HILES (RH)

BLOWER FLOF RRTE SCFH (SCHA)

GAS HETER FLOFI RATE SCFH (SCAN)

TOTAL PLOW SCP (SCH)

BC SARPLE HRTER/RANGE/PPHA (BAG)

BC BCRGRD HETER/RAHGE/PPH

CO SAHPLE HETER/RANGE/PPH

CO BCKGRD HETER/RAHGE/PPH

CO2 SAFPLE HETER/RAMGE/PCT

CO2 BCRGRD HEPER/RARGE/PCT

HOX SAFPLE HEPER/RANGE/PPH (BAG) (D)

HOY BCRERD HETER/RAHGE/PPH

CB4 SAFPLE PPH (1.150)

CF4 BCRERD PPH

DILOTIOH FACTOR

HC COHCBNTRATTON PPH

CD COHCXIHRATIOA PPH

$\mathrm{CO} 2$ CONCERTRATION PCT

HOY CONCEHTRATTOH PPH

CH4 COHCEHTRaTION PPH

MHEC CONCEMTRATIOA PPH

FIDHC MASS GRAHS

$\infty$ HRSS GRAHS

CO2 HRSS GRAHS

HOY HESS GRAHS

CH4 HASS GRAHS

HRHC HASS GRAHS (RTD)

FUEL HASS KG

FOEL ECONOHY HPG (L/100XH)
3-BAG CARB FIP VEHICLE ERISSIOI RESULTS

TEST 901B/10PE-01

DATE 9/27/95 RON

DHO 3 BAG CART 2

ACTUAL ROAD LOAD $6.60 \mathrm{BP}$ (4.92 KN)

TEST WEIGRT 3375 LBS ( 1530 KG)

PROJECT 10. 08-6969-001

BUTARE PERTIANE

FUEL DEUISITY $4.916 \mathrm{LB} / \mathrm{GLL}$

H $.172 \quad C \quad .828 \quad 0.000 \quad \& .000$

$90 \%$ BCTANE

10\% PERTAME

BOA BUHTDITY C.F. 1.002

DRY BULB TERPERATURE $71.0^{\circ} \mathrm{F}\left(21.7^{\circ} \mathrm{C}\right)$

$$
\begin{gathered}
1 \\
\text { COLD TRANSIRNT } \\
(0-505 \text { SRC. }) \\
506.2 \\
.971 / .983 \\
3.63(5.84) \\
325.1(9.21) \\
.00(.00) \\
2743 .(77.7)
\end{gathered}
$$

$28.7 / 2 / 28.68$

$5.01 \quad 2 / \quad 5.00$

$57.2 / 14 / 259.41$

.1/ $14 / \quad .40$

$94.3 / 14 / \quad .9815$

$12.6 / 14 / .0406$

$5.7 / 1 / 1.43$

$.5 / \quad 1 / \quad .13$

4.53

2.78

11.90

24.11

247.94

.9443

1.31

1.98

21.83

1.129

22.425

1343.08

.196

.103

.978

.456

$17.78(13.23)$

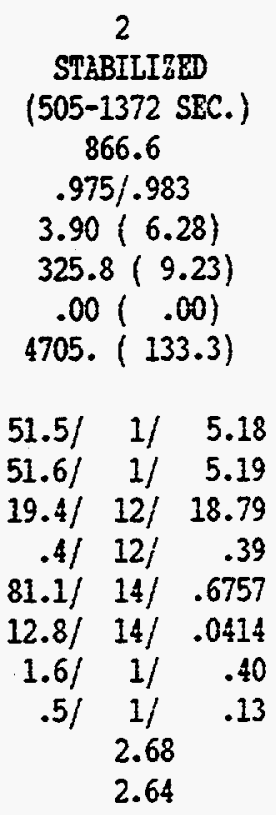

17.72

.28

17.75

.6366

.28

.19

.06

.023

2.754

1553.10

.072

.017

.005

.514

$16.95(13.88)$
3

HOT TRAHSIENT

( 0- 505 SEC.)

505.7

$.972 / .983$

$3.64(5.85)$

$322.9(9.14)$

$.00(.00)$

2721. ( 77.1)

$9.9 / \quad 2 / \quad 9.89$

$5.0 / 2 / \quad 5.00$

$82.6 / 12 / 82.39$

$.5 / 12 / \quad .48$

$90.4 / 14 / .8772$

$.7 / 1 / \quad .18$

.7/ $1 / \quad .18$

4.76

2.62

13.56

5.27

78.61

.8382

.01

2.33

2.59

.245

7.054

1182.78

.002

.120

.115

.394

$20.59(11.42)$
$13.0 / 14 / .0421$

3-BEG COAPOSITE RESULTS

$\begin{array}{llr}\text { PIDHC } & \text { G/HI } & .086 \\ C & \text { G/HI } & 2.178 \\ \text { WOY } & \text { G/HI } & .021\end{array}$

FUEL ECOAOHY HPG (E/100KH $) \quad 18.02(13.06)$

$\begin{array}{lll}\text { CH4 } & \text { G/HI } & .017 \\ \text { HHHC } & \text { G/HI } & .065\end{array}$


CONPUTER PROERAN IDT 1.2-R

VBHICLE NOUBER GA2

VEHICLE HODEL 94 PONT GRANDAH

BWGIIE $2.3 \mathrm{~L}(138 \mathrm{CID})-4$

IRRAYSHISSION $\mathbf{4}$

ODOWETER 2133 HILES ( $3431 \mathrm{KM}$ )

BAROIETER 29.14 II HG (740.2 $\mathrm{kH} \mathrm{HG)}$

REILITVE HUITDITY 62.0 PCT.

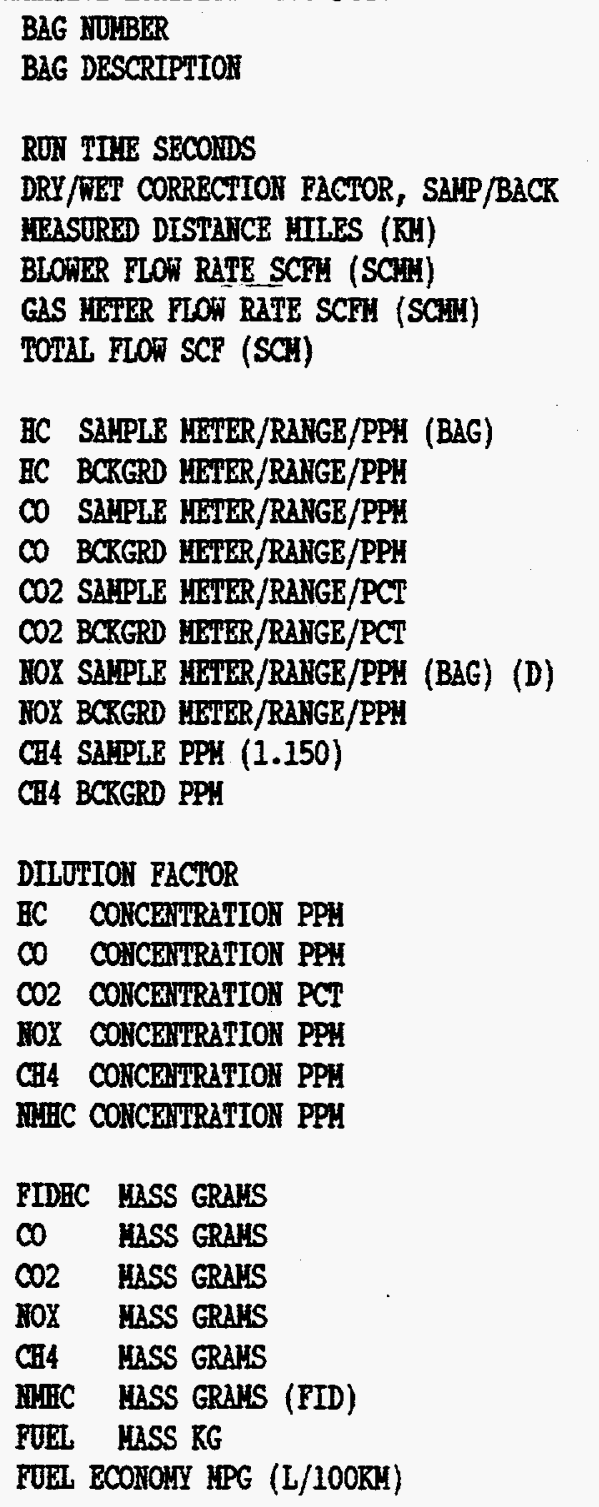

RESEARCH

PROJECI 10. 08-6969-001

DRY BULB TEIPERATURE $74.0^{\circ} \mathrm{F}\left(23.3^{\circ} \mathrm{C}\right)$
TEST 90RB/10PE-02S

DATE $9 / 28 / 95$ RUI

DHO 3 BAG CART 2

ACTUAL ROAD LOAD $6.60 \mathrm{EP}$ ( $4.92 \mathrm{KN}$ ) TEST WEIGHT 3375 LBS ( 1530 RG)
BOTZME PENTANE

FUEL DERSITY $4.916 \mathrm{LB} / \mathrm{GAL}$

H $.172 \quad C .828 \quad 0.000 \quad .000$ $90 \%$ BUTAKE

$10 \%$ PEMTIATE

HOX HOMIDITY C.F. 1.025

\begin{tabular}{|c|c|c|c|c|c|c|c|c|}
\hline & 1 & & & 2 & & & 3 & \\
\hline COLD & TRAMS & IEAP & SI & BILI2 & & HOT & TRAHS] & IBHT \\
\hline 10 & $505 S$ & BC.) & 1505 & 1372 & SBC.) & 10 & -505 & SEC.) \\
\hline & 305.5 & & & 66.9 & & & 05.4 & \\
\hline & $0 / .98$ & & & $3 / .98$ & & .97 & $1 / .982$ & \\
\hline 3.63 & 15. & 84) & 3.88 & 16. & 24) & 3.61 & 15.8 & \\
\hline 322. & 519 & $.13)$ & 324. & 719 & $.20)$ & 322. & 819. & .14) \\
\hline .00 & 1. & (0)) & .00 & 1. & 00) & .00 & 1.0 & D) \\
\hline 2717. & 17 & 6.9) & 4691. & 13 & 2.9) & 2719 & 17 & $7.0)$ \\
\hline $22.4 /$ & $2 /$ & 22.39 & 49.01 & 1) & 4.93 & $93.6 /$ & $1 /$ & 9.42 \\
\hline $4.9 /$ & $2 /$ & 4.90 & $47.8 /$ & 1) & 4.81 & $46.8 /$ & 1) & 4.71 \\
\hline $49.8 /$ & $14 /$ & 221.07 & $20.5 /$ & $12 /$ & 19.86 & 70.81 & 121 & 70.19 \\
\hline .41 & $14 /$ & 1.62 & .41 & $12 /$ & .39 & .41 & 121 & .39 \\
\hline 94.71 & $14)$ & .9930 & $81.0 /$ & $14 /$ & .6738 & $90.0 /$ & $14 /$ & .8673 \\
\hline 12.81 & $14 /$ & .0414 & $12.6 /$ & $14 /$ & .0406 & $12.9 /$ & $14 /$ & .0418 \\
\hline $4.3 /$ & 1) & 1.08 & $1.1 /$ & 1) & .28 & .91 & 1) & .23 \\
\hline .91 & $1 /$ & .23 & .51 & $1 /$ & .13 & .91 & 1) & .23 \\
\hline & 3.9 & & & 2.28 & & & 4.14 & \\
\hline & 2.2 & & & 2.27 & & & 2.21 & \\
\hline & 11. & & & 17. & & & 13.7 & \\
\hline & 17. & & & & 39 & & 5.0 & \\
\hline & 210 & & & 18. & & & 67.0 & \\
\hline & .95 & & & .63 & & & .828 & \\
\hline & & 87 & & & 16 & & .0 & 02 \\
\hline & 1. & & & & 14 & & 2.1 & \\
\hline & 15. & & & & 24 & & 2.6 & \\
\hline & .83 & & & .03 & & & .235 & \\
\hline & 18.83 & & & 2.90 & & & 6.013 & \\
\hline & 345.4 & & & 545.7 & & & 168.32 & \\
\hline & .13 & & & .04 & & & .002 & \\
\hline & .09 & & & .01 & & & .108 & \\
\hline & .70 & & & .01 & & & $.11 ?$ & \\
\hline & .45 & & & .51 & & & .385 & \\
\hline 17.8 & $1 / 1$ & 3.21) & 16.9 & 211 & $3.91)$ & 20.7 & 3 (1) & $1.35)$ \\
\hline
\end{tabular}

3-BAG COIPOSTTE RESULTS

$\begin{array}{llr}\text { FIDHC } & \text { G/MI } & .070 \\ \text { CO } & \text { G/MI } & 1.925 \\ \text { HOX } & \text { G/MI } & .013\end{array}$

FOEL ECOHOHY UPG (L/10ORM) $18.04(13.04)$
CH4 G/MI $\quad .015$

IHIFC G/MI $\quad .052$ 
COMPOTER PROGRAM LDT 1.2-R

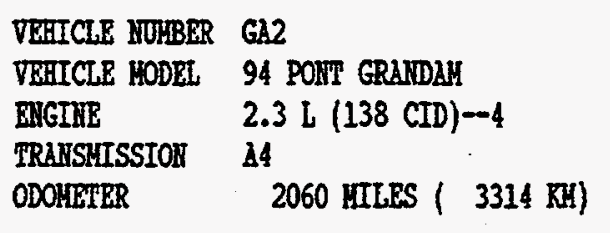

BAROAETER 29.14 IV HG (740.2 WI EG) RELLPTVE HOHIDITY 53.2 PCT.

BAG ITUBER

BAG DESCRIPTIOI

REI TIFE SECOHDS

DRY/WET CORRECTION FACTOR, SAMP/BICR

IIEASURED DISTANCE YILLES (IN)

BLONER FLOW RATE SCFY (SCHM)

GAS HETER FLOH RATE SCFH (SCHA)

TOTAL FLOW SCF (SCH)

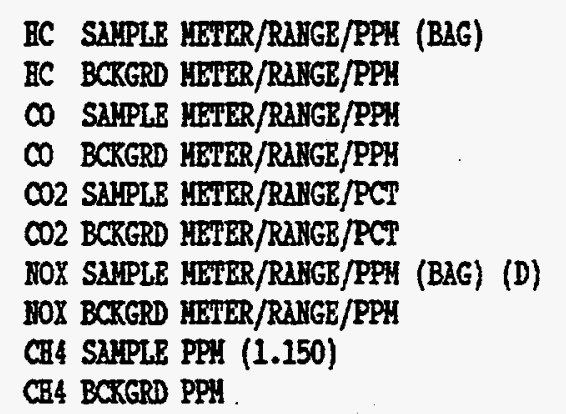

DILUTIOII FACTOR

BC CONCERTRAFTOY PPH

$\infty$ CONCERTRATTOR PPI

CO2 COICERTRATION PCT

HOX COICENTRATTOH PPH

C14 CONCEYTRATION PPH

HRIC CORCERTRATTOH PPY

FIDHC MASS GRAYS

$\infty$ MRS GRAYS

$\mathrm{CO}_{2}$ MASS GRAMS

HOX MASS GRAYS

CR4 MASS GRAMS

MTIC IRSS GRAMS (FID)

FOEL HASS KG

FUEL BCONONY IPG $(\mathrm{L} / 100 \mathrm{RH})$

PROJECT 10. 08-6969-001

TEST 50NB/50NP-01

DATE $9 / 25 / 95$ RON

DYNO 3 BAG CART 2

ICTOAL ROAD LOAD $6.60 \mathrm{HP}$ ( $4.92 \mathrm{KW}$ )

TEST WEIGHT 3375 LBS ( $1530 \mathrm{KG}$ )
BUTANE PROPANE

FUEL DEISITY $4.558 \mathrm{LB} /$ GLL

H. $.178 \quad C .822 \quad 0.000 \quad \searrow .000$

$50 \%$ BUTANE

50\% PROPARE

SOX BUUIDITY C.P. .951

DEY BULB TRTPERATURR $72.0^{\circ} \mathrm{F}\left(22.2^{\circ} \mathrm{C}\right)$

$$
\begin{gathered}
1 \\
\text { COLD TRARSIERTP } \\
(0-505 \text { SEC. }) \\
504.8 \\
.973 / .985 \\
3.63(5.84) \\
322.9(9.14) \\
.00(.00) \\
2717 .(76.9)
\end{gathered}
$$
$23.8 / 2 / 23.79$
$4.91 \quad 21 \quad 4.90$
$43.3 / 12 / \quad 42.33$
$1.2 / 12 / \quad 1.16$
$94.3 / 14 / .9815$
$13.7 / \quad 14 / \quad .0448$
$7.8 /$ if 1.96
$.9 / \quad 1 / \quad .23$
3.02
2.29

11.94

19.30

39.61

.9404

1.75

.92

18.24

.902

3.547

1324.63

.245

.047

.809

.443

$16.94(13.89)$

3

BOT TRAKSIENT

( 0 - 505 SEC.)

505.0

$.974 / .985$

$3.63(5.84)$

$324.0(9.18)$

$.00(.00)$

2727. (77.2)

$91.6 /$ I/ 9.22

49.7/ 1/ 5.00

$32.9 / 12 / 32.01$

$\begin{array}{llll}.8 / & 12 / \quad .77\end{array}$

$\begin{array}{llll}89.2 / & 14 / \quad .8479\end{array}$

$12.9 / 14 / \quad .0418$

$\begin{array}{lll}.7 / & 1 / & .18\end{array}$

$.3) 1 / \quad .08$

3.19

2.22

13.85

4.58

30.14

.8091

.11

1.13

3.29

.215

2.710

1144.22

.015

.058

.186

.382

19.67 ( 11.96$)$

3-BAG COMPOSITE RESULTS

$\begin{array}{lll}\text { PIDEC } & \text { G/MI } & .072 \\ \infty & \text { G/MI } & .608 \\ \text { HOX } & \text { G/MI } & .015\end{array}$

FUEL ECONOHY HPG (L/10ORH) 16.93 (13.90)

$\begin{array}{lll}\text { CB4 } & \text { G/MI } & .008 \\ \text { MHPC } & \text { G/MI } & .060\end{array}$




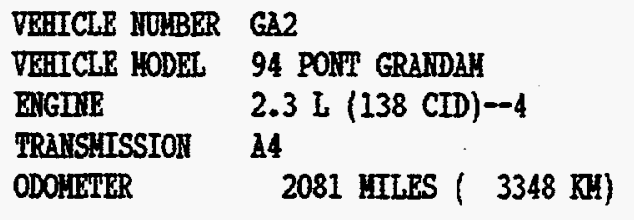

BARONETER 29.11 IN HG (739.4 WH HG) RELATTVE HUIIDITY 52.6 PCT.

BAG ITUVBR

BAG DESCRIPTION

ROI TITE SECONDS

DRY/WET CORRECTION RACTOR, SAYP/BACR

HERSORRD DISTANCE YIILES (RM)

BLONER FLOH RATE SCTH (SCHH)

GAS HETER FLOF RATTE SCFH (SCMH)

FOTAL FLOWI SCP (SCH)

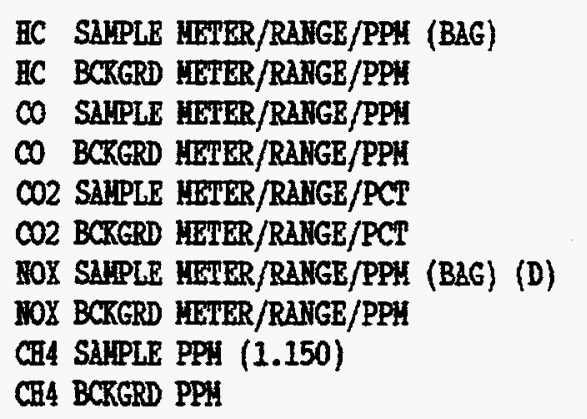

DILUTIOI PACTOR

HC COSCERTRATION PPH

CO CONCEHFRATION PPH

CO2 COHCENTRLTION PCT

HOX COICENTRATION PPH

CH4 COBCENTIRATTOH PPH

WHHC COMCERITRATIOI PPH

PIDHC MASS GRAHS

$C D$ HRSS GRAHS

$\mathrm{CO} 2$ HASS GRAHS

BOX HASS GRAHS

CH4 MASS GRAHS

HIIIC HBSS GRAHS (FID)

FURL HASS KG

FUEL BCOMOHY IPG (L/100RN)
TEST 50NB/50IP-02S

DATE $9 / 26 / 95$ ROH

DWHO 3 BAG CART 2

ACTUAL ROAD LOAD $6.60 \mathrm{MP}$ ( $4.92 \mathrm{KN}$ )

TEST WEIGHIT 3375 LBS (1530 RG)

DRY BOLB TERPERATORE $71.0^{\circ} \mathrm{F}\left(21.7^{\circ} \mathrm{C}\right)$
BUTARE PROPANE

FUEL DETSITY $4.558 \mathrm{LB} / \mathrm{GAL}$

$\begin{array}{llllll}\text { HI } .178 & \text { C } .822 & 0 & .000 & \text { X } & .000\end{array}$

$50 \%$ BOTARE

50\% PROPANE

WOX BOIIDITY C.P. .940

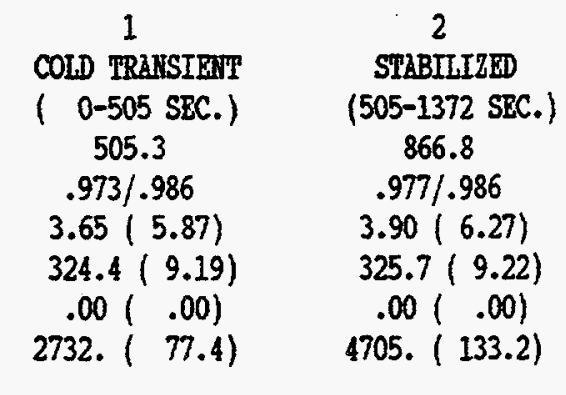

$20.6 / 21 \quad 20.59$

$\begin{array}{llll}5.2 / & 2 / & 5.20\end{array}$

$64.3 / 12 / 63.53$

$.4 / \quad 12 / \quad .39$

$94.3 / 14 / \quad .9815$

$13.3 / 14 / .0433$

$7.5 / 1 / 1.88$

$.5 / \quad 1 / \quad .13$

3.13

2.44

11.92

15.83

60.67

.9418

1.77

.90

14.80

.744

5.465

1334.10

.245

.046

.660

.447

$16.88(13.93)$

3

HOT TRARSIERT

( 0- 505 SBC. $)$

504.5

$.975 / .986$

$3.63(5.84)$

$323.8(9.17)$

$.00(.00)$

2722. ( 77.1$)$

3-BAG COIPOSITE RESULTS

$\begin{array}{rrrrrr}50.7 / & 1 / & 5.10 & 88.9 / & 1 / & 8.95 \\ 53.8 / & 1 / & 5.42 & 51.7 / & 1 / & 5.20 \\ 8.8 / & 12 / & 8.50 & 34.6 / & 12 / & 33.69 \\ .3 / & 12 / & .29 & .5 / & 12 / & .48 \\ 80.5 / & 14 / & .6644 & 89.5 / & 14 / & .8551 \\ 13.2 / & 14 / & .0429 & 13.6 / & 14 / & .0444 \\ .8 / & 1 / & .20 & .9 / & 1 / & .23 \\ .4 / & 1 / & .10 & .3 / & 1 / & .08 \\ 2.35 & & & 3.30 & \\ & 2.42 & & & 2.41 & \end{array}$

13.73

4.12

32.02

.8139

.16

1.07

2.90

.193

2.874

1148.80

.022

.055

.129

.383

$19.59(12.01)$

$15.91(14.78)$

.057

$\begin{array}{lll}\text { FIDHC } & \text { G/MI } & .057 \\ C^{\circ} & \text { G/MI } & .693 \\ \text { HOX } & \text { G/MI } & .019\end{array}$

CH4 G/HI $\quad .008$

IIIHC G/MI $\quad .047$ 
VREICLE NOHBER GA2

VRHICLE HODEL 94 PONT GRANDAM

ENGINE $2.3 \mathrm{~L}(138 \mathrm{CID})-4$

TRANSHISSION A4

ODOHETER

2306 UILES ( 3710 RM)

\section{TRST 20IB/80P-02S}

DATE $11 / 16 / 95$ RDI

DITO 3 BAG CART 2

ACTUAL ROAD LOAD $6.60 \mathrm{EP}(4.92 \mathrm{kN})$

TEST MEIGHF 3375 LBS ( 1530 RG)

BAROAETER $29.35 \mathrm{IN}$ HG (745.5 HI HG) DRY BULB TEMPERATURE $71.0^{\circ} \mathrm{P}\left(21.7^{\circ} \mathrm{C}\right)$ RELATIVE HIUIDITY 34.2 PCT.

BAG WUMBER

BAG DESCRIPTION

RUN TIIE SECOHDS

DRY/WET CORRECTIOH FACTOR, SAMP/BACR

UERASURED DISTAWCE HILBS (RM)

BLONER FLOF RRTE SCFH (SCHN)

GAS HETER FLON RATE SCFU (SCHN)

TOTAL FLON SCF (SCH)

HC SAYPLE HETER/RAYGE/PPI (BAG)

HC BCRERD IIETER/RAIGE/PPH

CO SAYPLE LETER/RAHGE/PPI

CO BCRERD UETER/RANGR/PPH

CO2 SAYPLE HETER/RAHGE/PCT

CO2 BCRGRD HETER/RANGE/PCT

NOX SAMPLE HETER/RANGE/PPH (BAG) (D)

BOX BCKGRD METER/RANGE/PPH

CH4 SAHPLE PPH (1.160)

CH4 BCRERD PPH

DILUTION FACTOR

HC CONCERTPRATTON PPA

CO COHCERTRATION PPH

CO2 CONCENTRARTION PCT

HOX COHCBRTRATION PPM

CH4 CONCENIRATIOI PPM

WNAC COHCENTRATION PPM

FIDHC MASS GRAKS

CO HaSS GRAUS

$\mathrm{CO} 2$ HASS GRAKS

HOX MASS GRAHS

CH4 HASS GRAMS

MHHC MASS GRAYS (FID)

FOEL MASS RG

FUEL ECONOHY MPG (L/10ORU)

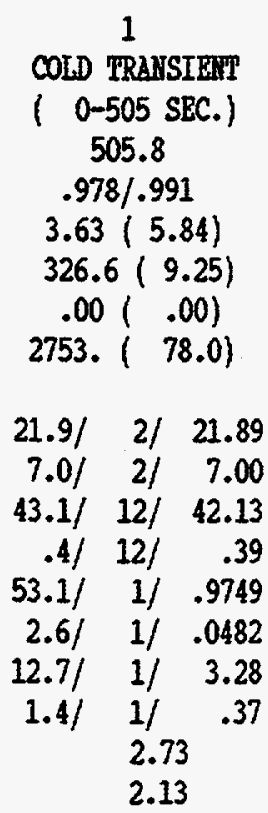

11.91

15.48

40.36

.9308

2.94

.78

14.57

.736

3.664

1328.73

.376

.041

.655

.446

$16.12(14.59)$

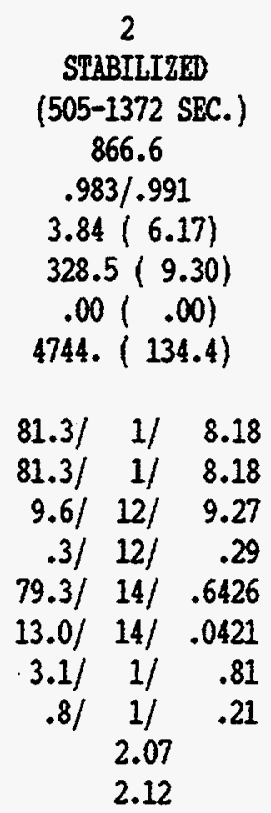

18.14

.45

8.76

.6028

.61

.07

.37

.037

1.371

1482.64

.134

.006

.029

.495

$15.34(15.33)$

PROJECT 10. 08-6990-001

20\%BDTANE 80\% PROP

FUEL DEISITY $4.366 \mathrm{IB} / \mathrm{GAL}$

स $.181 \quad C .819 \quad 0.000 \quad \& .000$

HOY HUMIDITY C.F. $\quad .856$

3

HOT TRANSIERT

( $0-505$ SEC. $)$

505.2

$.980 / .991$

$3.64(5.85)$

$327.2(9.27)$

$.00(.00)$

2755. ( 78.0)

$10.2 / 2 / \quad 10.19$

$7.9 / 2 / \quad 7.90$

$23.1 / 12 / 22.40$

$.0 / \quad 12 / \quad .00$

$88.9 / 14 / \quad .8407$

12.9/ 14/ .0418

$5.011 / 1.30$

$.6 / 1 / \quad .16$

3.19

2.14

13.85

2.87

21.71

.8019

1.15

1.21

1.46

.136

1.972

1145.68

.148

.063

.066

.383

$18.80(12.51)$

3-BAG COIPOSITE RESULTS

$$
\begin{array}{lll}
\text { FIDHC } & \text { G/MI } & .058 \\
\text { CO } & \text { G/MI } & .545 \\
\text { HOX } & \text { G/MI } & .051
\end{array}
$$

FUEL ECONOHY IPG (L/10ORW) $16.36(14.38)$

$\begin{array}{lll}\text { CH4 } & \text { G/MI } & .008 \\ \text { HWHC } & \text { G/HI } & .047\end{array}$


SOUTHTEST RESEARCH IHSTTTUTE - DEPARTHETIT OF RUISSIONS RESEARCH

COLPUTER PROGRAH LDT 1.3-R

3-BAC CLRB FTP VERTCLE EUTSSIOH RESULTS

PROJECT YO. 08-6990-001

VERICLE HOLBER GA2

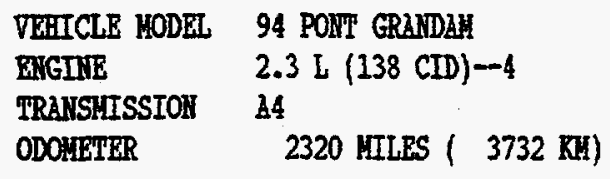

BARONETER 29.22 IN BG (742.2 WM BG)

RELLAIVE HUIIDITY 57.0 PCT.

BAG HUYBER

BAG DESCRIPIION

ROW TIME SECONDS

DRY/WET CORRECTION FLCTOR, SAYP/BACR

IEASURED DISTANCE YIILES (RH)

BLONER FLOW RATE SCFH (SCHN)

GAS METER FLOA RAIE SCTH (SCHA)

TOIAL FLOW SCF (SCH)

BC SAYPLE VETER/RAHGE/PPH (BAG)

EC BCRGRD HETER/RANGE/PPH

CO SAMPLE WETER/RANGE/PPH

CO BCRERD HETER/RAHGE/PPH

$\mathrm{CO} 2$ SAIPLE METER/RANGE/PCT

CO2 BCRGRD AETER/RAHGE/PCT

NOX SAIPLE YEFER/RANGE/PPH (BAG) (D)

NOX BCRGRD HETER/RANGE/PPI

CH4 SAYPLE PPH (1.160)

CH4 BCRGRD PPM

DILUTIOA FACIOR

HC COHCENTRATIOI PPA

CO CONCEIIRATION PPH

$\mathrm{CO} 2$ CORCENIRATIOR PCT

FOX CONCEHTRATION PPH

CH4 CORCRITRATIOH PPH

NHEC CONCEIITAMTOH PPH

FIDHC MASS GRAYS

$C O$ HASS GRAHS

$\mathrm{CO} 2$ HASS GRAYS

HOX HASS GRAHS

CH4 HASS GRAUS

MIIC MASS GRAYS (FID)

FURL HASS RG

FOEL ECONONY MPG (L/100KW)
TEST 201B/80P-03

DATE $11 / 17 / 95$ RDN

DYNO 3 BAG CART 2

ACTOAL ROAD LOAD $6.60 \mathrm{EP}$ ( $4.92 \mathrm{KW}$ )

TEST WEIGHI 3375 IBS ( 1530 KG)
20\%BUTANE 80\% PROP

FUEL DEISTIY $4.366 \mathrm{IB} / \mathrm{GAL}$

B .181 $\quad$ C $.819 \quad 0.000 \quad 8.000$

DRY BULB TEIPRRATURE $72.0^{\circ} \mathrm{F}\left(22.2^{\circ} \mathrm{C}\right)$

$$
\begin{gathered}
1 \\
\text { COLD TRRANSIENT } \\
(0-505 \text { SEC. }) \\
507.1 \\
.972 / .984 \\
3.62(5.82) \\
322.6(9.14) \\
.00(.00) \\
2726 .(77.2)
\end{gathered}
$$
$21.7 / 2 / 21.69$
$6.3 / 2 / \quad 6.30$
$66.4 / 12 / 65.67$
$.6 / 12 / \quad .58$
$93.8 / 14 / \quad .9673$
$12.8 / 14 / .0414$
$7.2 / \quad 1 / 1.87$
$.2111 \quad .05$
3.05
2.25
$63.0 / 1 / 6.34$
$64.4 /$ I/ 6.48
$10.5 / 12 / 10.14$
$.6 / 12 / \quad .58$
$80.0 / 14 / .6552$
$13.0 / 14 / .0421$
$.8 / 1 / \quad .21$
$.411 / \quad .10$
2.18
2.16

11.98

15.92

62.47

.9294

1.82

.99

14.77

.749

5.615

1313.78

.261

.051

.658

.442

$16.21(14.51)$

17.80

.22

9.27

.6155

.11

.14

.06

.018

1.429

1492.15

.027

.013

.004

.498

$15.30(15.38)$

$92.8 / 1 / \quad 9.34$

$66.9 / \quad 1 / 6.73$

$\begin{array}{llll}28.2 / & 12 / & 27.39\end{array}$

$.5 / \quad 12 / \quad .48$

$89.4 / 14 / .8527$

$12.9 / 14 / \quad .0418$

1.2/ $1 / .31$

$.6 / \quad 1 / \quad .16$

3.28

2.14

13.65

3.10

25.91

.8140

.17

1.29

1.60

.145

2.320

1146.26

.024

.066

.071

.383

$18.58(12.66)$

3-BAG COMPOSITE RRSULTS

$\begin{array}{lll}\text { FIDHC } & \text { G/MI } & .057 \\ \text { CO } & \text { G/MI } & .693 \\ \text { HOX } & \text { G/MI } & .021\end{array}$

CH4 G/HI $\quad .010$

NHEC G/MI $\quad .044$

FUEL ECOKOHY IPG (L/100RN) $16.30(14.43)$ - 
COAPUTER PROCRH LDI $1.2-R$

VERICLB HOEBER GA2

VRHICLE HODEL 94 PONT GRANDAH

BMIEIHE 2.3 L (138 CID $)-4$

TRANSHISSION 24

ODOHETER

2233 KILES ( 3592 RH)
3-BAG CARB FTP VEHICLE EHISSIOI RESULTS

TEST 100NB-03

DATE $10 / 3 / 95$ RTN

DYMO 3 BAG CART 2

ACTULE ROAD LOAD 6.60 IP ( $4.92 \mathrm{kN})$

TEST WEIGEP 3375 LBS ( $1530 \mathrm{KG}$ )

BaROHETER 29.03 IN HG (737.4 HAM BG) BRY BULB TRPPERATURE $73.0^{\circ} \mathrm{F}\left(22.8^{\circ} \mathrm{C}\right)$ RET.ATIVE HOHIDITY 53.9 PCT.

BAG NOEBER

BAG DESCRIPTION

RDW TIFE SECONDS

DRY/WET CORRECTTON FACTOR, SAYP/BACR

UEASORED DISTAHCE HILES (RA)

BLONER FLON RATE SCFH (SCAR)

GAS HETER FLOW RATE SCFM (SCHH)

TOTAL FLON SCT (SCH)

BC SAKPLE YIETER/RAHGR/PPA (BAG)

HC BCKGRD HETER/RAHCE/PPH

CO SALPLE HETER/RaHGE/PPH

CO BCRERD HETER/RAYGER/PPH

CO2 SAFPLE AETKR/RAPGE/PCT

CO2 BCRGRD HETER/RANGE/PCT

HOX SAYPLE HETER/RAHGE/PPH (BAG) (D)

HOX BCRGRD HETER/RAMGE/PPY

CH4 SAHPLE PPH (1.150)

CH4 BCRERD PPH

$$
\begin{gathered}
1 \\
\text { COLD TRANSIEMT } \\
(0-505 \text { SEC.) } \\
505.6 \\
.973 / .985 \\
3.61(5.81) \\
324.4(9.19) \\
.00(\quad .00) \\
2733 .(77.4)
\end{gathered}
$$

$25.9 / 2 / 25.88$

$4.3 / \quad 2 / \quad 4.30$

$92.3 / 13 / 226.62$

$.1 / \quad 13 / \quad .22$

$52.7 / 1 / \quad .9675$

$2.3 / \quad 1 / \quad .0426$

$4.81 \quad 1 / \quad 1.20$

$.2) \quad 1 / \quad .05$

3.77

2.07

12.10

21.94

217.56

.9284

1.16

1.87

19.80

1.024

19.607

1315.74

.166

.096

.884

.445

$17.76(13.24)$
2

STABILIZED

(505-1372 SEC.)

867.1

$.976 / .985$

$3.84(6.18)$

$325.1(9.21)$

$.00(.00)$

4698. (133.1)

41.3/ 1/ 4.16

$40.8 /$ 1/ 4.11

$17.7 / \quad 12 / 17.13$

$.3 / \quad 12 / \quad .29$

$80.3 / 14 / .6607$

12.1/ 14/ .0387

$.5 / \quad 1 / \quad .13$

$.011 / \quad .00$

2.13

2.09

18.11

.28

16.31

.6241

.13

.15

.10

.022

2.527

1520.46

.031

.014

.008

.503

$16.75(14.05)$
PROJECI 150. 08-6990-001

BUT2ME BUTARE

FUEL DEPSITY $4.830 \mathrm{IB} / \mathrm{GAL}$

H $.172 \quad C .828 \quad 0.000$ \& .000

HOL BOHIDITY C.F. .966

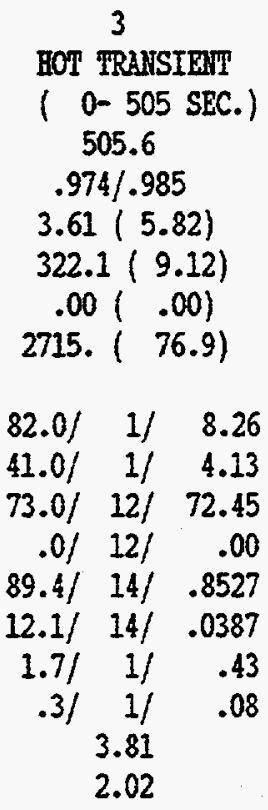

13.95

4.42

69.81

.8167

.36

1.93

2.21

.205

6.248

1149.51

.051

.099

.098

.383

$20.70(11.36)$

3-BAG COFPOSITE RESULTS

$\begin{array}{llr}\text { FIDHC } & \text { G/HI } & .078 \\ \text { Co } & \text { G/HI } & 1.948 \\ \text { HOX } & \text { G/HI } & .018\end{array}$

FOEL ECONOHI HPG (L/100RA) 17.93 (13.12) $\begin{array}{lll}\text { CB4 } & \text { G/HI } & .015 \\ \text { NHEC } & \text { G/HI } & .060\end{array}$ 
SOUTHILST RESEARCB INSTITUTE - DEPARTHEIIIT OR EHISSIOHS RESEARCH

COHPUTER PROCRAH LDT 1.2-R

3-BAG CARB FTP VEHTCLE BHISSTON RESULTS

PROJECI H0. 08-6990-001

VEHICLE HOSBER GA2

VEHICLE HODEL 94 POAT GRAKDAH

BMEIHE $2.3 \mathrm{~L}$ (138 CID) --4

TRAHSHSSION $\quad$ A4

ODDSETER 2247 HILES ( 3615 RH)

BAROHETER 28.90 IN HG (734.1 HA BG) RELLTTVE BDHDITY 46.7 PCT.

BAG ITOKBER

BAG DESCRIPTIOH

ROH TIFE SECOIDS

DRI/WET CORRECTIOA FACIOR, SARP/BACK

HEASURED DISTANCE GILES (RH)

BLONER FLOH RaTE SCFH (SCHA)

GAS HETIER FLOF RBTE SCFH (SCFH)

TOTAE PLOW SCP (SCA)

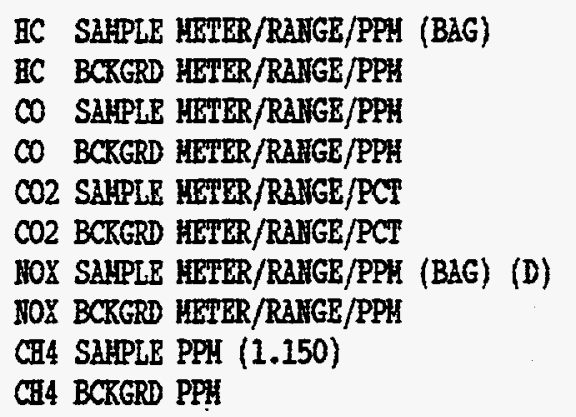

DILOTTON FACTOR

HC COHCEITRATION PPH

CO CONCEPTRATION PPH

$\mathrm{CO} 2$ COHCEHTRATTON PCT

HOX CONCEITRATTON PPH

CB4 CONCERTRATION PPH

HIIC COHCEHTRATION PPH

PIDHC HASS GRAHS

CO HaSS GRAHS

$\mathrm{CO} 2$ HLSS GRAHS

HOX HLSS GRAHS

CH4 HaSS GRAHS

HATC HASS GRAHS (FID)

POEL HASS RG

FUEL ECOROHI SPG (L/100RH)
TEST 100HB-04S

DATE $10 / 4 / 95$ REH

DHTO 3 BAE CART 2

ACTUAL ROAD LOAD $6.60 \mathrm{IP}$ ( $4.92 \mathrm{~km}$ )

TRST NEICHI' 3375 LBS ( $1530 \mathrm{KG}$ )
BOTANE BOTARE

FUEL DEHSITY $4.830 \mathrm{iB} / \mathrm{GAL}$

H $.172 \quad C .828 \quad 0.000 \quad 8.000$

WOX HUIDITY C.F. .928

DRY BULB TRKPERATURE $73.0^{\circ} \mathrm{F}\left(22.8^{\circ} \mathrm{C}\right)$

$$
\begin{gathered}
1 \\
\text { COLD TRAHSIEHT } \\
(0-505 \text { SEC. }) \\
504.8 \\
.974 / .987 \\
3.61(5.80) \\
320.8(9.09) \\
.00(.00) \\
2699 .(76.4)
\end{gathered}
$$

$24.01 \quad 21 \quad 23.99$

$6.2 / 2 / \quad 6.20$

$93.7 / 13 / 230.41$

$.5 / 13 / 1.10$

$94.7 / 14 / .9930$

$13.4 / 14 / \quad .0437$

$4.3 / \quad 1 / 1.08$

$.4) 1 / \quad .10$

3.89

2.21

11.80

18.31

220.81

.9531

.99

1.87

16.17

.844

19.651

1333.86

.134

.095

.713

.451

$17.52(13.43)$

$$
\begin{gathered}
2 \\
\text { STABILIZED } \\
(505-1372 \text { SEC. }) \\
867.1 \\
.978 / .987 \\
3.82(6.14) \\
321.4(9.10) \\
.00(.00) \\
4645 .(131.5)
\end{gathered}
$$

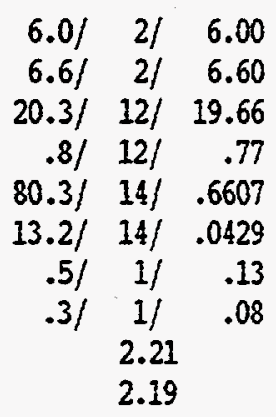

18.10

$-.24$

18.36

.6202

.05

.15

$-.40$

.000

2.811

1493.63

.013

.013

.000

.494

$16.93(13.90)$
3

HOT TRAHSIERT

( 0 - 505 SEC.)

504.3

$.976 / .987$

$3.57(5.74)$

$320.1(9.06)$

$-.00(.00)$

2690. (76.2)

$\begin{array}{lll}10.2 / 2 / & 10.19\end{array}$

$\begin{array}{lll}6.21 & 21 & 6.20\end{array}$

$77.3 / \quad 12 / 76.89$

$\begin{array}{llll}1.01 & 12 / \quad .96\end{array}$

$90.0 / 14 / \quad .8673$

$13.0 / 14 / .0421$

$2.011 / \quad .50$

$.4) 1 / \quad .10$

3.76

2.19

13.71

4.45

73.36

.8283

.41

1.73

2.46

.204

6.507

1155.32

.055

.088

.108

.385

$20.33(11.57)$

3-BEG COAPOSITE RESULIS

$\begin{array}{llr}\text { FIDBC } & \text { G/HI } & .065 \\ C & \text { G/HI } & 2.020 \\ \text { NOX } & \text { G/HI } & .014\end{array}$

PURL ECONOHY HPG (L/100KR)

$17.90(13.14)$ 
COHPUTER PROCRAH LDT 1.2-R S-BAG CARB FTP VEHICLE EIISSION RESULTS PROJECT HO. 08-6990-001

VEHICLE MOIBER GR2

VEHICLE HODEL 94 POHI GRANDAH

BRGIIE 2.3 L $(138 \mathrm{CID})-4$

TRANSIIISSIOI 24

ODOHETER 2174 IIILS ( $3497 \mathrm{KH})$

BAROHITIRR 29.08 III HG (738.6 WI BG)

RELATIVE HIJTDITY 45.8 PCT.

BAG NOIBER

BAG DESCRIPTION

ROX TTME SECONDS

DRY/WET CORRECTIOI FACTOR, SAIP/BACR

HRASURBD DISTARCE KILPS (RU)

BLONER FLOF RRTE SCFI (SCIII)

GAS IEWIER FLON RATE SCFI (SCHH)

TOTAL FLON SCP (SCA)

HC SAYPLE YETER/RAFGE/PPA (BAG)

HC BCKGRD IETER/RANGE/PPY

CO SAIPLE HETER/RAKGE/PPY

CD BCRGRD HETER/RZHGE/PPH

CO2 SAIPLE HETER/RAYGE/PCT

CO2 BCKGRD IETER/RAFGE/PCT

HOX BCKGRD IETER/RAIGE/PPI

CH4 SAYPLE PPI (1.150)

CB4 BCKGRD PPI

DILUTION FACTOR

BC CONCEIMTRATIOA PPY

$\infty$ CORCENIRATIOR PPH

$\mathrm{CO}_{2}$ COHCERIRATIOI PCT

HOX COHCERTRATION PPH

CH4 COHCEIIRATIOH PPH

IHAC COHCEHIRATIOH PPH

FIDEC MASS GRAMS

CO MASS GRANS

$\mathrm{CO} 2$ HASS GRAHS

HOX HASS GRAYS

CH4 MASS GRAHS

IIIEC MASS GRAYS (FID)

FUEL ECONOHY UPG (L/100RU)
HOX SAYPLE HEIER/RAYGE/PPY (BAG) (D)

FURL WaSS RG
TEST BLB-CP2-03

DATE $9 / 29 / 95$ ROH

DHO 3 BAG CART 2

ACIUAL ROAD LOLD $6.60 \mathrm{EP}$ ( $4.92 \mathrm{KN}$ )

TEST WEIGHT 3375 LBS ( 1530 KG)
GASOLIHE PHASE II BU-1968-F

FUEL DEISITY $6.163 \mathrm{LB} / \mathrm{GAL}$

H $.137 \quad C .843 \quad 0.020 \quad X .000$

HOX HUIIDITY C.F. .957

DRY BULB TERPERAYURE $77.0^{\circ} \mathrm{F}\left(25.0^{\circ} \mathrm{C}\right)$
1

COLD TRANSIEMT

( 0-505 SEC.)

505.3

$.975 / .985$

$3.63(5.84)$

$324.5(9.19)$

$.00(.00)$

2733. ( 77.4)

$22.9 / 2 / 22.89$

$4.7 / 2 / \quad 4.70$

$47.2 / 13 / 108.66$

$.4 / 13 / \quad .88$

$56.8 / 1 / 1.0441$

2.5/ if .0463

21.4/ 1/ 5.37

$1.0 / 1 / \quad .25$

4.06

2.62

12.59

18.56

104.02

1.0014

5.14

1.65

16.67

.851
9.374
1419.19
.727
.085
.744
.465
$21.82(10.78)$

3-BAG COMPOSITE RESULTS

$$
\begin{gathered}
2 \\
\text { STABILIZED } \\
(505-1372 \text { SEC. }) \\
867.2 \\
.979 / .985 \\
3.89(6.26) \\
325.1(9.21) \\
.00(.00) \\
4699 .(133.1)
\end{gathered}
$$

$47.0 /$ 1/ 4.73

46.91 1/ 4.72

$2.8 / 21 / 2.70$

$.6 / 12 / \quad .58$

$81.5 / 14 / .6832$

$12.8 / \quad 14 / \quad .0414$

1.3/ $1 / .33$

$\begin{array}{lll}.5 / 1 / & .13\end{array}$

2.54

2.61

19.46

.25

2.08

.6440

.21

.07

.18

.020

.323

1569.18

.050

.006

.014

.508

$21.39(11.00)$

$73.7 / 1 / 7.42$

$48.5 / \quad 1 / 4.88$

$20.7 / \quad 12 / 20.05$

$.5 / 12 / \quad .48$

92.2/ $14 / .9236$

$13.0 / 14 / .0421$

$2.6 / 1 / \quad .65$

$.4 / 11 \quad .10$

3.03

2.54

14.37

2.88

18.95

.8844

.56

.67

2.11

.131

1.699

1247.03

.079

.034

.094

.405

$24.99(9.41)$ $\begin{array}{lll}\text { FIDHC } & \text { G/MI } & .061 \\ \text { CO } & \text { G/MI } & .708 \\ \text { FOZ } & \text { G/III } & .054\end{array}$

FUEL ECOHOHY IPG (L/100RI)
$22.39(10.51)$ 
COHPUTER PROGRAH LDT 1.2-R

VEHICLE NOEBER GA2

TEHICLE HODEL 94 PONT GRADBH

BIGIIR $2.3 \mathrm{~L}$ (138 CID) -4

TRAHSHISSIOII A4

ODOHETER 2195 HILES ( 3531 R月)

BAROHETER $29.00 \mathrm{III}$ HG (736.6 HA HG) RRLATIVE HOHIDITY 57.7 PCT.

BAG WJIBBER

BaG DESCRIPITION

RUI TIHE SECONDS

DRY/WET CORRECTION FACTOR, SAYP/BACK

HEASURBD DISTANCE HILES (RH)

BLOWER FLOW RATE SCFH (SCXH)

GAS HETER FIOF RATE SCFH (SCHH)

TOTAL BLON SCP (SCH)

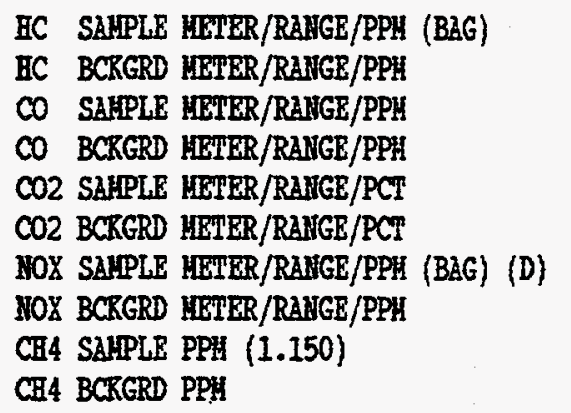

DILOTIOH FACTOR

HC COHCEHTRRTION PPH

CO COHCENTRATION PPH

CO2 CONCENTRATION PCT

HOX COHCENTRATTON PPH

CB4 CONCEVIRATTON PPH

MABC CONCWHTRATION PPH

PIDHC MASS GRAHS

CO HASS GRAHS

$\mathrm{CO} 2$ HASS GRAHS

NOX YRSS GRAHS

CH4 HASS GRAHS

MHPC HASS GRAHS (FID)

FUEL HRSS KG

FUEL BCOMOHI HPG (L/100RH)

SOUTHWEST RESEARCE IHSTTTOTE - DEPARTHETT OF BRISSIOHS RESEARCH

3-BAG CARB FTP VEHICLE BUISSION RESUIIS

PROJECT 10. 08-6990-001

TEST BLB-CP2-04S

DATE $10 / 2 / 95 \quad$ ROI

DINIO 3 BaE CART 2

ACTOAL ROAD LOAD $6.60 \mathrm{HP}$ ( $4.92 \mathrm{~kW})$

TEST WEIGHT 3375 LBS (1530 KG)

DRY BULB TERPRRATURE $73.0^{\circ} \mathrm{F}\left(22.8^{\circ} \mathrm{C}\right)$

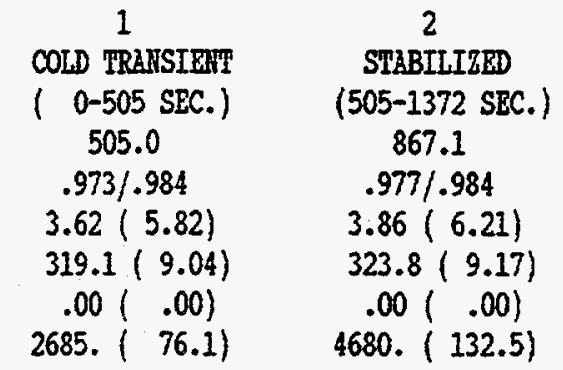

$23.7 / \quad 2 / \quad 23.69$

$4.612 / \quad 4.60$

$52.7 / 13 / 122.31$

$.5 / 13 / 1.10$

$58.3 / 1 / 1.0722$

$2.7 /$ 1/ .0500

$28.3 / 1 / 7.10$

$.4 / 1 / \quad .10$

4.14

2.43

12.25

19.46

116.45

1.0262

7.00

1.91

17.27

.877

10.311

1428.96

1.006

.097

.757

.469

$21.57(10.91)$ $\begin{array}{rrr}45.6 / & 1 / & 4.59 \\ 45.3 / & 1 / & 4.56 \\ 3.3 / & 12 / & 3.18 \\ 1.0 / & 12 / & .96 \\ 82.4 / & 14 / & .7006 \\ 13.1 / & 14 / & .0425 \\ 2.7 / & 1 / & .68 \\ .4 / & 1 / & .10 \\ & 2.24 & \\ & 2.70 & \end{array}$

18.97

.27

2.18

.6603

.58

$-.31$

.63

.021

.337

1602.37

.146

.000

.048

.519

$20.78(11.32)$
GASOLIHE PHBSE II BH-1968-F FURL DEISITY $6.163 \mathrm{LB} / \mathrm{GAL}$

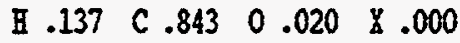
CALIFORIIL PHASE 2

NOX HUHIDITY C.F. .988

3

HOF TR2AISIEATT

( 0 - 505 SEC.)

505.3

$.974 / .984$

$3.60(5.79)$

$322.0(9.12)$

$.00(.00)$

2712. ( 76.8)

$71.6 / \quad 1 / 7.21$

$45.8 / \quad 1 / \quad 4.61$

$19.8 / 12 / 19.18$

$.8 / \quad 12 / \quad .77$

$92.7 / \quad 14 / \quad .9370$

$13.2 / \quad 14 / \quad .0429$

$3.6 / 1 / \quad .90$

$.4 / 1 / \quad .10$

2.74

2.20

14.16

2.92

17.76

.8971

.81

.69

2.13

.133

1.588

1261.34

.117

.035

.094

.409

$24.59(9.57)$

3-BAG COHPOSITE RESULTS

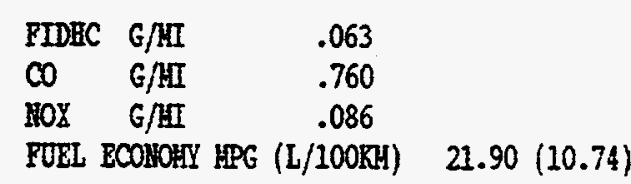

$\begin{array}{lll}\text { CH4 } & \text { G/MI } & .008 \\ \text { IHHC } & \text { G/MI } & .057\end{array}$ 
COMPOTER PROGRAY LDT 1.3-R

VEHICLE NOUBER GA2

VRHICLE HODEL 94 POST GRARDAM

BNGINE

TRANSHISSTON

ODOHETER

2.3 I $(138 \mathrm{CDD})-4$

14

2376 MILES ( $3822 \mathrm{KH})$
3-BAG CARB FIP VKHICLE EUISSIOI RESULTS

PEST 1001B(BC)-01

DATE 11/21/95 ROH

DHIO 3 BAG CART 2

ICTUAL ROAD LOAD $6.60 \mathrm{HP}$ ( $4.92 \mathrm{KN}$ )

TEST WEIGEP 3375 LBS ( 1530 KG)

BAROAETER 29.44 II HG (747.8 MM HG) DRY BULB TBMPERATURE $72.0^{\circ} \mathrm{P}\left(22.2^{\circ} \mathrm{C}\right)$ BRLLATIVE HUIIDITY 53.1 PCT.

BAG HOHBER

BAG DRSCRIPIIOI

RON TIME SECORDS

DRY/WEP CORRRCTTON PACTOR, SAMP/BACR

WEASURED DISTAHCE WIILES (RM)

BLONIR FLON RATE SCFM (SCHI)

GAS HETER FLON RATE SCHH (SCAH)

TOPAL FLON SCP (SCH)

HC SAMPLE HETER/RANGE/PPH (BAG)

BC BCKGRD IETIER/RAMGE/PPH

CO SAYPLE METIER/RAMGE/PPH

CO BCKGRD LETIER/RATGR/PPII

CO2 SAYPLE HETER/RANGE/PCT

CO2 BCRGRD METIER/RAFGR/PCT

MOZ SAYPLR UETER/RAVGE/PPM (BRG) (D)

MOX BCRGRD HRTER/RAHGR/PPH

CH4 SAIPLE PPN (1.150)

CB4 BCKGRD PPU

DILUTIOA FACTOR

BC COHCERTRATTON PPM

CO COHCERTPRATION PPH

CO2 CONCERTPRATION PCT

HOZ COMCTMTRATTOA PPH

CH4 CONCENTRATION PPM

MHAC COHCEMTRATTON PPH

PIDHC MASS GRAHS

CO HASS GRAHS

$\mathrm{CO} 2$ HASS GRAHS

HOX HASS GRAHS

CH4 MASS GRAMS

IIHC WASS GRAHS (FID)

FUEL MASS KG

1

COLD TRAHSIEAT

( 0-505 SBC.)

505.7

$.974 / .986$

$3.61(5.82)$

$329.4(9.33)$

$.00(.00)$

2776. ( 78.6)

$\begin{array}{rrr}11.3 / & 3 / & 112.75 \\ 1.4 / & 3 / & 13.97 \\ 71.5 / & 1 / 688.69 \\ .3 / & 1 / & 2.10 \\ 92.4 / & 14 / & .9289 \\ 13.6 / & 14 / & .0444 \\ 45.1 / & 3 / 112.98 \\ .0 / & 3 / & .00 \\ & 5.76 & \\ & 2.18\end{array}$

11.89

99.95

660.60

.8882

112.98

3.76

95.63

4.746

60.467

1278.61

16.100

.197

4.336

.458

$17.45(13.48)$
2

STABILIZED

(505-1372 SEC.)

867.1

$.978 / .986$

$3.85(6.19)$

$330.0(9.35)$

$.00(.00)$

4769. (135.1)

$63.3 / 2 / \quad 63.26$

$13.7 / 2 / 13.69$

$62.8 / 14 / 289.50$

$.3 / 14 / 1.21$

78.6/ 14/ .6301

$13.3 / 14 / .0433$

$56.5 / 2 / \quad 56.70$

$.0121 \quad .00$

4.11

2.14

18.00

50.33

279.31

.5892

56.70

2.09

47.93

4.105

43.914

1456.96

13.879

.188

3.732

.508

$16.75(14.05)$
PROJECT 10. 08-6990-001

BUTANE $100 \%$

FUEL DEISITY $4.878 \mathrm{LB} / \mathrm{GAL}$

H $.173 \quad C .827 \quad 0.000 \quad \& .000$

HOX HOUIDITY C.F. .948

3

HOT TRAHSIEIVT

( 0 - 505 SEC.)

505.2

$.975 / .986$

$3.62(5.82)$

$327.6(9.28)$

$.00(.00)$

2759. ( 78.1)

$84.5 / 2 / \quad 84.45$

$14.8 / 2 / 14.79$

$55.4 / 1 / 500.89$

$.3 / 1 / 2.10$

$87.8 / 14 / \quad .8150$

$13.2 / 14 / .0429$

$45.2 / 3 / 113.23$

$.2) \quad 3 / \quad .50$

5.19

2.14

13.72

70.74

481.20

.7752

112.76

3.21

67.04

3.337

43.766

1108.80

15.968

.167

3.020

.392

$20.41(11.52)$

3-BAG COLPOSITE RRSULTS

$\begin{array}{llr}\text { PIDHC } & \text { G/MI } & 1.078 \\ \infty & \text { G/NI } & 12.712 \\ \text { HOY } & \text { G/HI } & 4.007\end{array}$

FOEL ECONOHY SPG (L/100RU) 17.80 (13.22)

$\begin{array}{lll}\text { CH4 } & \text { G/MI } & .049 \\ \text { IINHC } & \text { G/MI } & .981\end{array}$


TEST 100IB(IC) -025

DATE $11 / 20 / 95$ RDN

DHIO 3 BAG CARP 2

BCTOAL ROAD LOAD $6.60 \mathrm{EP}$ ( $4.92 \mathrm{kN}$ )

TEST WEIGHT 3375 LBS ( 1530 KG)
COIPOTER PROGRAH LDT 1.3-R

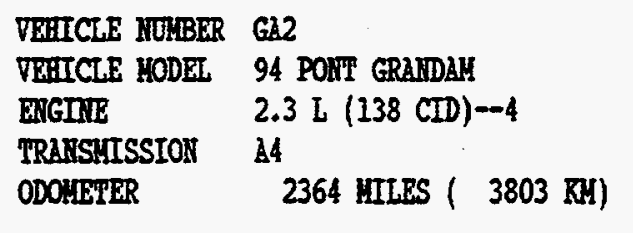

BAROUETER 29.30 II HG (744.2 MI HG) RRLLTIVE HUIIDITY 57.0 PCT.

BAG IUUZBER

BAG DESCRIPTIOII

ROI TTHE SECONDS

DRY/WET CORRECTTOI FACTOR, SAYP/BACK

HEASORED DISTAMCE YIILES (RU)

BLONER FLON RATE SCPH (SCNH)

GAS HETER FLON RATE SCFH (SCHH)

TOLAL FLOF SCF (SCH)

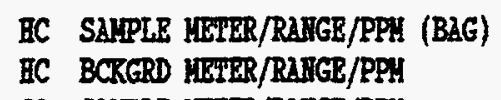

CO SAYPLE HETER/RANGE/PPH

CO BCKGERD UETER/RAHGE/PPH

CO2 SAYPLE HETER/RABGE/PCI

CO2 BCKGRD HETER/RANGR/PCT

HOX SAFPLE HETER/RUHGR/PPH (BAG) (D)

BOZ BCKGRD IETER/RAIGE/PPII

CH4 SARPLE PPI (1.150)

CFI BCKGRD PPY

DILUTTOH FACTOR

BC CORCERIRATTOI PPY

CO COMCEHTRATTOI PPH

$\mathrm{CO} 2$ COHCEHTRATTOI PCT

BOX COHCENTRRATION PPH

CH4 COICRMTRATION PPH

INIHC COICEHTRATION PPH

\begin{tabular}{|c|c|}
\hline FIDEC & IRSS GRAYS \\
\hline$\infty$ & HRSS GRMHS \\
\hline $\mathrm{CO2}$ & MRSS GRAHS \\
\hline HOX & MASS GRMS \\
\hline CH4 & HASS GRAHS \\
\hline MRTC & LASS GRAMS (FID) \\
\hline $\begin{array}{l}\text { FOEL } \\
\text { FURI }\end{array}$ & $\begin{array}{l}\text { MASS KG } \\
\text { COHOWY YPG (I/100 }\end{array}$ \\
\hline
\end{tabular}

DRY BULB TEYPERATURE $72.0^{\circ} \mathrm{P}\left(22.2^{\circ} \mathrm{C}\right)$
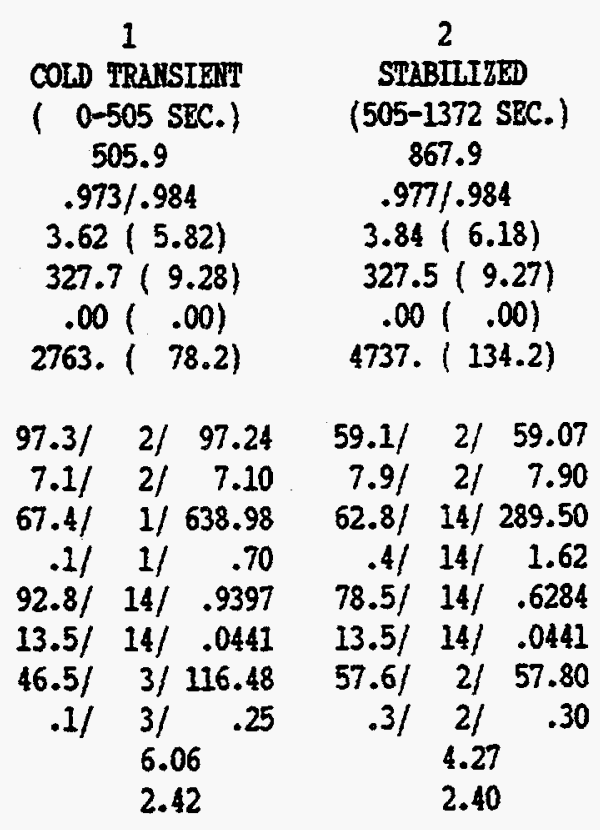

11.84

90.75

613.08

.8994

116.26

3.85

86.32

4.288
55.848
1288.44
16.868
.201
3.895
.459
$17.45(13.48)$

4.288

23.848

16.868

.201

(13.48)

3-BAG CONPOSITE RESULTS

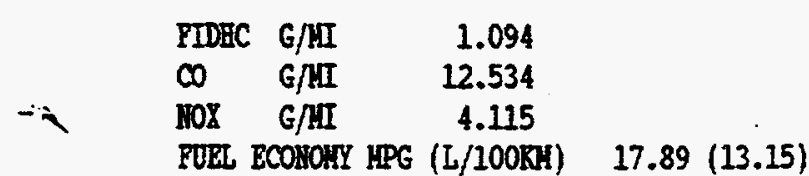

18.06
51.61
278.58
.5868
57.52
2.00
49.31
4.181
43.509
441.16
14.308
.179
3.814
.503
$(13.92)$

$16.90(13.92)$
BUT2ARE $100 \%$

FUEL DEISITY $4.878 \mathrm{LB} / \mathrm{GAL}$

$\begin{array}{lllll}\text { B } .173 & \mathrm{C} .827 & 0.000 \quad \& \quad .000\end{array}$

HOX HUIDITY C.P. .970

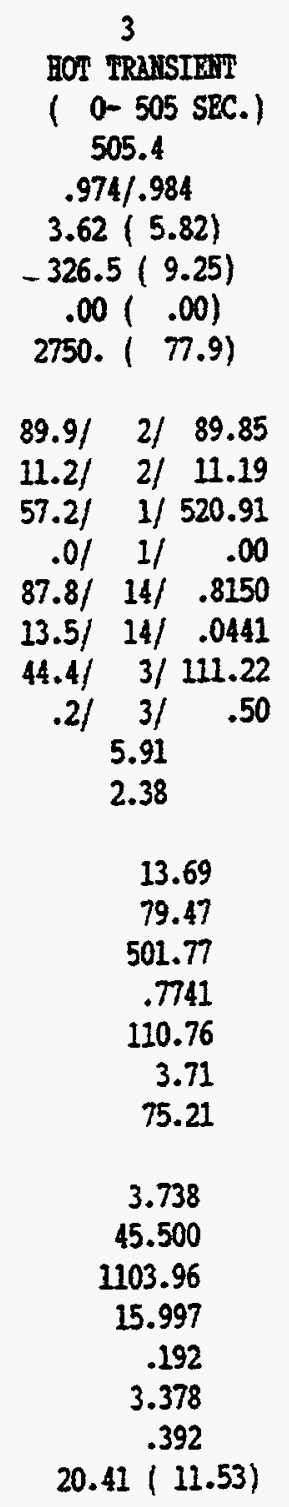

RESERRCH

PROTECT HO. 08-6990-001 


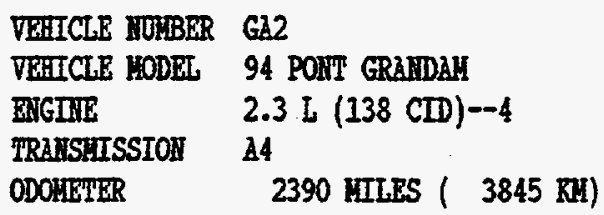

BaROUETER 29.23 II HG (742.4 WM HG) RELLATIVE HUIDDITY 47.2 PCT.

BAG MOUBER

BAG DESCRIPTIOI

ROH TITR SECOIDS

DRY/WET CORRECTION FACTOR, SAIP/BACK

UERSURED DISTAMCE YILLES (RI)

BLOFER FLOH RATE SCFH (SCMH)

GAS VETER FLON RATE SCRI (SCYM)

TOTAL FLOW SCF (SCY)

HC SAUPLE METER/RaITEE/PPH (BaG)

IC BCRERD HETLR/RAHEE/PPH

$C O$ SAYPLE HETER/RANGE/PPII

$\infty$ BCRERD HETER/RAHEE/PPH

CO2 SAYPLE METER/RRAGE/PCT

CO2 BCRERD HETER/RATGE/PCT

HOX SAIPLE NETER/RATGE/PPA (BAG) (D)

HOX BCRGRD HETER/RaHEE/PFH

CH4 SAYPLE PPH (1.150)

CH4 BCRGRD PPH

\section{DILDTOA FACTOR \\ HC CONCENTRRATTOH PPH \\ CO COHCERTRATTOH PPH \\ $\mathrm{CO2}$ COHCENTRATIOH PCT \\ WOX CONCEHTRATTON PPH \\ CH4 COMCENTRATION PPH \\ IIHC COHCENTRETTON PPH}

FIDHC MBSS GRAUS

$\infty$ HASS GRAISS

$\mathrm{CO2}$ HASS GRAHS

HOZ HASS GRAYSS

CH4 HRSS GRAHS

IIHFC HASS GRAHS (PID)

FUEL HASS RG

FUEL ECONONY UPG (L/100XI)
TEST 100IB(TC) -03

DATE 11/22/95 ROT

DEDO 3 BAG CART 2

ACTUAL ROAD LOAD $6.60 \mathrm{IPP}$ ( $4.92 \mathrm{KN}$ )

IEST AEIGHT 3375 LBS ( 1530 KG)
DRY BOLB TEYPERATURE $74.0^{\circ} \mathrm{P}\left(23.3^{\circ} \mathrm{C}\right)$

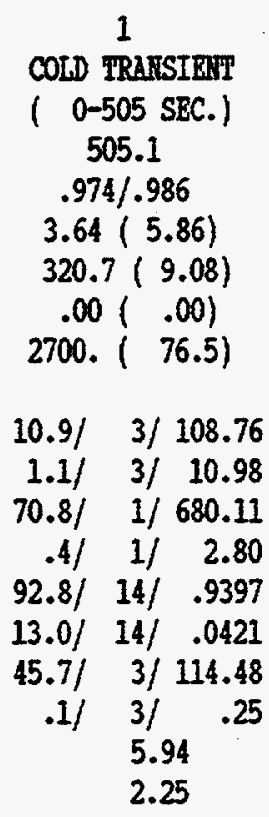

\subsection{7 \\ 98.71 \\ 652.85 \\ .9012 \\ 114.25 \\ 3.88 \\ 94.26}

4.558

58.107

1261.37

15.640

.198

4.155

.451

17.86 ( 13.17$)$

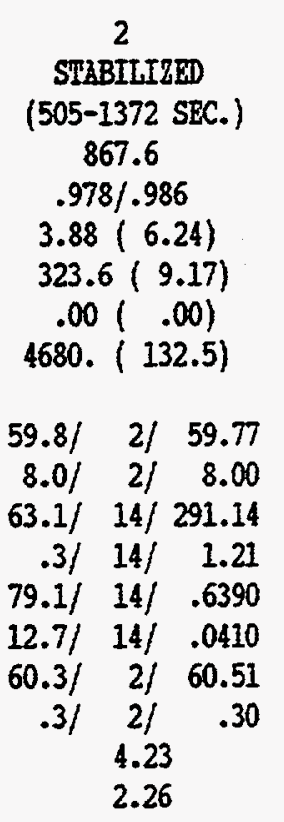

17.77

52.22

281.39

.6003

60.23

2.10

49.81

$$
4.179
$$

43.416

1456.58

14.293

.185

3.806

.508

$16.91(13.91)$
BUTANE $100 \%$

FORL DERSITY $4.878 \mathrm{LB} / \mathrm{GAL}$

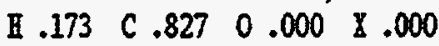

BOX HUEIDITY C.F. $\quad .936$

$$
3
$$

HOT TRANSIEWT

$(0-505$ SEC. $)$

505.3

$.976 / .986$

$3.63(5.84)$

$322.4(9.13)$

$.00(.00)$

2715. ( 76.9)

$77.0 / 2 / \quad 76.96$

$5.81 \quad 2 / \quad 5.80$

$57.5 j$ 1/ 524.28

.4) $1 / 2.80$

88.2/ $14 / \quad .8242$

12.7/ 14/ .0410

44.3/ 3/110.97

.2) $3 / \quad .50$

5.37

2.32

\subsection{6}

71.59

504.01

.7862

110.51

3.23

67.88

3.324

45.117

1106.83

15.215

.165

3.009

.392

$20.46(11.50)$

3-BAE CONPOSITE RESULIS

$$
\begin{array}{llr}
\text { FIDHC } & \text { G/MI } & 1.069 \\
\infty & \text { G/MI } & 12.524 \\
\text { HOX } & \text { G/MI } & 3.951
\end{array}
$$

FOEL ECONOMY IPG (L/100RM)

$17.98(13.08)$ 
COAPUTIR PROGRAH IDT 1.3-R

VEHICLE WUIBER GR2

TERICLE HODRL 94 PONT GRANDAH

EHGINE $2.3 \mathrm{~L}(138 \mathrm{CD})--4$

TRAISHISSIOH $\quad 84$

ODOHETER 2437 HILLES ( 3921 KM)

BAROHETER 29.32 III HE (744.7 III BG)

RRLATIVE HOIDITI 26.4 PCT.

BAG IUUBBER

BAG DESCRIPITOA

ROII TIII SECOMS

DRI/WET CORRECTIOI FACTOR, SAYP/BACK

YERASURED DISTAMCE YILES (NI)

BLONTRR FLON RRIE-SCFM (SCMA)

GAS HETER FLON RATE SCFH (SCHY)

TOTAL FLON SCF (SCI)

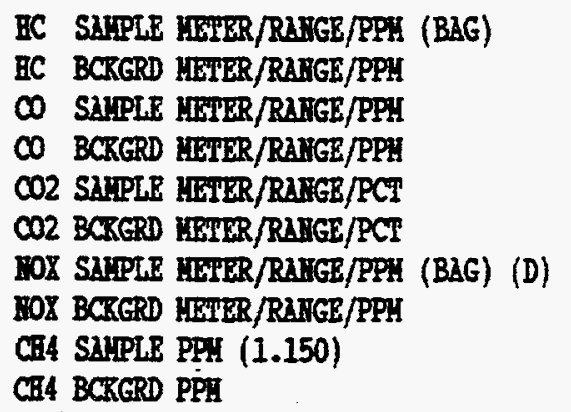

DILOTIOH FACTOR

BC CONCERIRRTION PPH

C COACEMTRRTIOA PPH

$\mathrm{CO} 2$ COHCENTRATION PCT

HOX COSCENTRATION PPH

CH4 COHCEMTRRATION PPM

IIHC COACRITRRTION PPY

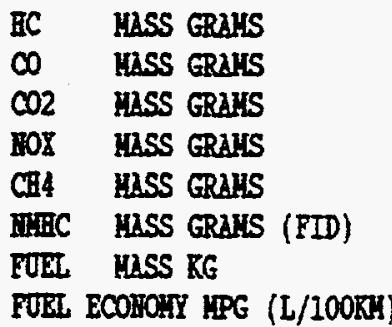

3-BAG CARB FTP VERTCLE BMISSION RESULTS

TEST 100P(ITC) -01

DATE 11/29/95 RUII

DIHO 3 BAG CART 2

ACTUAL ROAD LOAD $6.60 \mathrm{HP}$ (4.92 $\mathrm{NN}$ )

TEST WEIGHT 3375 LBS ( $1530 \mathrm{KG}$ )
PROJECT NO. 08-6990-001

PROPANE $100 \%$

FUEL DERSITY 4.410 LB/GAL

$\begin{array}{lllllllllll}\text { II } & .183 & \mathrm{C} & .817 & 0 & 0.000 & \mathrm{X} & .000\end{array}$

HOX HUIDIFY C.F. $\quad .823$

DRY BULB IXIPERRTURE $70.0^{\circ} \mathrm{F}\left(21.1^{\circ} \mathrm{C}\right)$

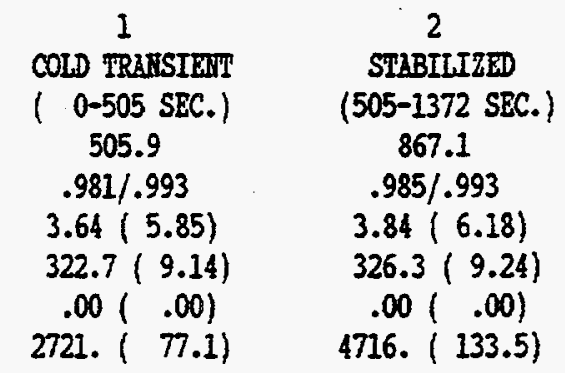

$85.8 / 2 / \cdot 85.75$

$\begin{array}{llll}48.9 / 2 / 28.87 & 21\end{array}$

$4.6) \quad 2 / \quad 4.60$

$4.7) \quad 2 / \quad 4.70$

$71.9 / 14 / 340.21$

48.6/ 14/ 215.01

$.4 / 14 / 1.62$

.4/ 14/ 1.62

51.1) 1/ .9377

2.6/ $1 / \quad .0482$

$\begin{array}{llll}78.3 / & 14 / & .6249\end{array}$

13.5/ 14/ .0441

$52.6 / 3 / 131.77$

$.21 \quad 3 / \quad .50$

4.73

2.27

$68.8 / 2 / \quad 69.04$

$.6) \quad 2 / \quad .60$

3.91

2.22

11.87
81.54
328.39
.8935
131.31
2.64
78.50

17.86

44.44

208.52

.5833

68.47

1.81

42.35

3.839

29.458

1260.51

15.926

.136

3.692

.441

$16.51(14.25)$
3.626

32.420

1426.14

14.395

.161

3.453

.497

$15.46(15.21)$
3

HOT TRANSIERTI

( 0- 505 SEC.)

504.9

$.982 / .993$

$3.63(5.84)$

$-325.0(9.21)$

$.00(.00)$

2735. ( 77.5)

66.4/ 2/ 66.36

$4.51 \quad 21 \quad 4.50$

$72.1 / 14 / 341.34$

$1.0 / 14 / 4.04$

$89.4 / \quad 14 / \quad .8527$

14.4/ 14/ .0476

49.8/ 3/ 124.75

$.21 \quad 3 / \quad .50$

4.57

2.22

13.02

62.21

327.94

.8088

124.29

2.52

59.31

2.944

29.573

1146.97

15.155

.130

2.805

.402

$18.06(13.02)$

3-BAG COMPOSITE RESULTS

$\begin{array}{llr}\text { HC } & \text { G/MI } & .930 \\ \text { CO } & \text { G/MI } & 8.287 \\ \text { HOX } & \text { G/MI } & 3.998\end{array}$

FUEL ECONONY HPG (L/100XN) $16.34(14.40)$
CA4

IIIIC
G/MI

G/II
.039

.888 
VEHICLE NUUBERR GA2

VRHICLE HODELI 94 PONT GRANDAH

HIGINE

TRAHSUISSTON 14

ODOMETER

PROJECT 10. 08-6969-001

TEST 100P(NC)-02S

DATE $11 / 30 / 95 \quad$ RUS

DWTO 3 BLE CART 2

ACTVAL ROAD IOAD $6.60 \mathrm{IPP}$ ( $4.92 \mathrm{KN}$ )

TEST WRIGHT 3375 IBS ( $1530 \mathrm{KG}$ )
BARONETER 29.32 WI HG (744.7 MM EG) DRY BULB TBRTPERaTURE $70.0^{\circ} \mathrm{F}\left(21.1^{\circ} \mathrm{C}\right)$ RELATIVE HDIIDITY 29.8 PCT.

BAG HOIBER

BAG DESCRTPTIOII

RON TTHE SECORD

DRY/WET CORRECTIOI EACTOR, SAIP/BACK

VRASURED DISTAMCE HILES (RII)

BLONER FLON RATE SCFH (SCNI)

GAS HETER FLOF RATE SCFH (SCAN)

TOTAL FLOW SCP (SCM)

EC SAHPLE HETER/RAMGE/PPM (BAG)

IC BCRGRD HETER/RAMGE/PPH

CO SAYPLE HETER/RAIEE/PPH

CO BCRERD HETER/RAWGE/PPH

CO2 SAYPLE HETER/RAIGE/PCT

$\mathrm{CO2}$ BCRERD UETER/RAYGE/PCT

MOZ SAYPLE UETER/RANGE/PPH (BAG) (D)

HOY BCRGRD UETER/RARGE/PPH

CH4 SAYPLE PPH (1.150)

CH4 BCRGRD PPH

DILUTTOH FACTOR

HC COHCERTRATION PPH

CO COHCEITRATTON PPM

$\mathrm{CO2}$ CONCEMTRATTON PCT

HOX COHCAMTRATION PPH

CH4 COHCEHIRATION PPH

WIFC COHCEATRATIOI PPI

HC MASS GRAHS

CO MRS GRAHS

$\mathrm{CO} 2$ MASS GRAUS

HOX HASS GRAHS

CH4 HASS GRAWS

WHIC MRSS GRAYS (FID)

FUEL MASS RG

FUEL ECOHOIY YPG (L/10ORU)

$$
\begin{gathered}
1 \\
\text { COLD TRANSIENT } \\
(0-505 \text { SEC. }) \\
505.6 \\
.980 / .992 \\
3.61(5.81) \\
323.5(9.16) \\
.00(.00) \\
2726 .(77.2)
\end{gathered}
$$

$79.1 / 2 / 79.05$

$4.7 / \quad 2 / \quad 4.70$

$69.4 / 14 / 326.08$

$.5 / 14 / 2.02$

$92.2 / 14 / .9236$

$13.8 / 14 / \quad .0452$

$50.5 / 3 / 126.50$

$.3 / \quad 3 / \quad .75$

4.86

2.45

12.07

74.75

314.07

.8821

125.82

2.61

71.74

3.526

28.227

1246.82

15.520

.134

3.381

.435

$16.60(14.17)$

$$
\begin{gathered}
2 \\
\text { STABILIZED } \\
(505-1372 \text { SEC. }) \\
866.4 \\
.984 / .992 \\
3.85(6.19) \\
328.8(9.31) \\
.00(.00) \\
4748 .(134.5)
\end{gathered}
$$

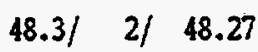

$4.9 / 2 / \quad 4.90$

41.7/ 14/ 181.01

$.5 / 14 / 2.02$

78.4/ 14/ .6266

13.9/ 14/ .0456

$25.8 / 3 / \quad 64.63$

$.31 \quad 3 / \quad .75$

4.19

2.52

17.91

43.65

174.73

.5836

63.92

1.81

41.57

3.586

27.351

1436.58

13.733

.162

3.412

.498

$15.45(15.23)$
PROPANE $100 \%$

FUEL DERISITY $4.410 \mathrm{IB} / \mathrm{GAL}$

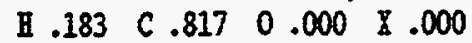

HOX BUHIDITY C.F. .836

3

HOT TRANSIETT

( 0 - 505 SEC. $)$

504.7

$.981 / .992$

$3.63(5.84)$

$326.9(9.26)$

$.00(.00)$

2750. ( 77.9$)$

$63.9 / 2 / 63.86$

$4.9 / 2 / \quad 4.90$

$69.6 / 14 / 327.20$

$.4 / 14 / 1.62$

$88.9 / 14 / \quad .8407$

$14.6 / 14 / \quad .0484$

$50.0 / 3 / 125.25$

.3) $3 / \quad .75$

4.76

2.53

$$
\begin{array}{r}
13.23 \\
59.34 \\
316.15 \\
.7960 \\
124.56 \\
2.43 \\
56.55
\end{array}
$$

2.824

28.666

1135.01

15.501

.126

2.688

.397

$18.28(12.87)$

3-BAG COAPOSIIE RESULTS

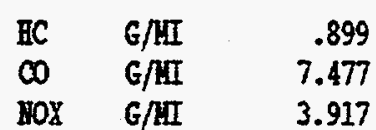

FUEL ECOHOHY IPG (L/100RI)
.899

.477

3.917

$16.39(14.35)$ $\begin{array}{lll}\text { CH4 } & \text { G/II } & .039 \\ \text { HHC } & \text { G/MI } & .857\end{array}$ 
SOOTHWTST RESEARCH INSTITUTE - DEPARTHEMT OF EHISSTONS RESEARCH

COKPUTER PROGRAH LDT 1.3-R 3-BAG CARB FIP VEHICLE EMISSTON RESULTS

PROJECT 10. 08-6969-001

$\begin{array}{ll}\text { VEHICLE HOHBER } & \text { GA2 } \\ \text { VEHTCLE HODEL } & 94 \text { PONT GRADDAM } \\ \text { BHGINE } & 2.3 \text { L (138 CID) }-4 \\ \text { TRAHSHISSTON } & \text { A4 } \\ \text { ODOULTER } & 2487 \text { MILES ( } 4001 \mathrm{KM})\end{array}$

BAROMETER 29.15 IN BG (740.4 WM BG) RELATIVE HDHIDITY 52.6 PCT.

BAG NOYBER

BBG DESCRIPTION

RUN TIME SECONDS

DRY/WET CORRECTION FACTOR, SAMP/BACR

HEASURED DISTANCE MILES (RH)

BLOFIER FLOW RATE SCFH (SCIM)

GAS METER FLOW RATE SCTH (SCWM)

TOTAL FLOW SCF (SCH)

EC SAMPLE METER/RANGE/PPH (BAG)

BC BCRGRD METER/RANGE/PPU

$C O$ SAIPLE HETER/RARGE/PPH

CO BCRGRD METER/RANGE/PPH

CO2 SAMPLE METER/RANGE/PCT

CO2 BCRGRD METER/RANGE/PCT

HOX SAMPLE METER/RAFGE/PPH (BAG) (D)

NOX BCRGRD METER/RANGE/PPH

CH4 SAMPLE PPH (1.150)

CH4 BCKGRD PPH

DILUTION FACTOR

HC CONCERTRATION PPH

CO CONCEYTRATION PPH

$\mathrm{CO} 2$ CONCENTRATION PCT

HOX CONCEYTRATION PPH

CH4 CONCENTRATION PPH

WMHC CONCENTRATION PPH

FIDHC MASS GRAHS

$C O$ MASS GRAHS

$\mathrm{CO} 2$ MASS GRAHS

HOY HASS GRAHS

CHA MASS GRAHS

MWHC HASS GRAMS (FID)

POEL MASS RG

FUEL ECONOWY MPG (L/10ORH)
TEST 50NB/50P(HC) -0 :

DATE $12 / 1 / 95$ RON

DinO 3 BAG CARP 2

ACTOAL ROAD LOAD $6.60 \mathrm{HP}$ ( $4.92 \mathrm{~kW}$ )

TEST WEIGHT 3375 LBS ( 1530 KG)

DRY BOLB TEMPERATURE $71.0^{\circ} \mathrm{F}\left(21.7^{\circ} \mathrm{C}\right)$

$$
\begin{array}{cc}
1 & 2 \\
\text { COLD TRANSIENT } & \text { STBBILIZED } \\
(0-505 \text { SEC. }) & (505-1372 \text { SEC. }) \\
505.5 & 866.4 \\
.975 / .986 & .978 / .986 \\
3.64(5.86) & 3.85(6.19) \\
323.4(9.16) & 324.6(9.19) \\
.00(.00) & .00(.00) \\
2724 .(77.2) & 4687 .(132.7)
\end{array}
$$

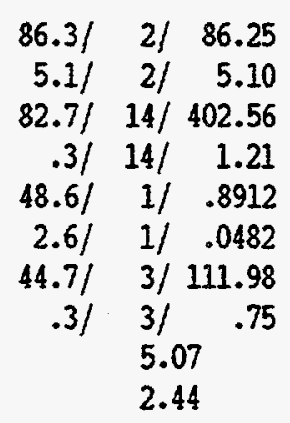

12.57

81.56

386.40

.8468

111.28

2.82

78.32

3.821

34.707

1196.18

15.419

.145

3.484

.419

$17.96(13.10)$
$50.0 / 2 f \quad 49.97$

$5.4 / 2 / \quad 5.40$

$50.4 / 14 / 224.11$

$.4 / 14 / 1.62$

76.7/ 14/ .5975

13.0/ 14/ .0421

$57.0 / 2 / 57.20$

$.6 / 2 / \quad .60$

4.05

2.41

18.90

44.86

215.73

.5576

56.63

1.77

42.83

3.616

33.336

1355.05

13.499

.157

3.278

.471

$16.88(13.94)$
50\% BOTAN 50\% PROP

FOEL DETSITY $4.558 \mathrm{IB} / \mathrm{GAL}$

$\begin{array}{lllll}\text { \# } .178 & \text { C } .822 & 0.000 & \times .000\end{array}$

HOX BUIDDTYY C.F. $\quad .939$

3

HOT TRANSIENT

( 0- 505 SEC.)

504.9

$.976 / .986$

$3.63(5.85)$

$-323.1(9.15)$

$.00(.00)$

2719. $(77.0)$

$\begin{array}{llll}68.8 / 2 / 2 / 28 & 68.76\end{array}$

$5.4 / 2 / \quad 5.40$

$77.3 / 14 / 371.16$

$.3 / 14 / 1.21$

$86.5 / \quad 14 / \quad .7857$

12.9/ 14/ .0418

$44.3 / 3 / 110.97$

.1) $3 / \quad .25$

4.86

2.38

14.24

63.74

357.07

.7469

110.74

2.64

60.70

2.980

32.004

1052.79

15.312

.136

2.695

.369

$20.34(11.57)$

3-BÄG CONPOSITE RESULTS

$\begin{array}{llr}\text { PIDHC } & \text { G/MI } & .930 \\ \text { CO } & \text { G/MI } & 8.887 \\ \text { HOI } & \text { G/MI } & 3.856\end{array}$

PUEL ECONOHY IPG (L/100KM) 17.96 (13.09)
CB4 G/HI $\quad .040$

MHEC G/MI $\quad .843$ 
COIPUTER PROGRAM LDT 1.3-R

VRHICLE HOHBER GA2

VKRTCLE HODEL 94 PONT GRANDAH

BIGTHE

IRAHSIISSION A4

ODOHETER 2509 IILLES ( 4036 RN)

BAROHETER 29.23 II HG (742.4 HM BG) RRLLATIVE HUIIDITY 40.6 PCT.

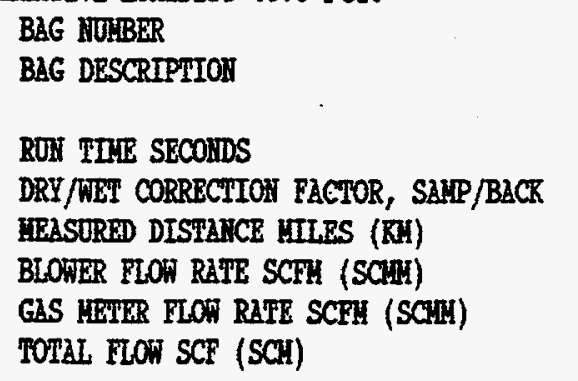
BC SAHPLE HETER/RAHGE/PPH (BAG)
BC BCKERD YETLR/RATEE/PPH
CO SAIPLE HEFER/RANGE/PPH
CO BCKGRD HETER/RAIGE/PPH
CO2 SAIPLE HETER/RAHGE/PCT
CO2 BCKGRD HETER/RAIFER/PCT
HOY SAMPLE HETER/RAYGE/PPI (BAG) (D)
NOX BCRERD HETER/R3HGE/PPH
CH4 SAHPLE PPH (1.150)
CH4 BCRGRD PPH

DILUTIOI BACTOR

HC COSCERTTRATION PPH

CO CORCEYTPATIOH PPY

$\mathrm{CO2}$ COHCEMTRATTON PCT

HOX CORCERTRRTIOH PPY

CH4 COHCERTRATION PPH

HWHC CONCERTRRATION PPY

FIDEC MASS GRAIS

CO MASS GRALS

$\mathrm{CO2}$ MLSS GRAMS

HOI MBSS GRAMS

CH4 HASS GRAHS

IWHC MBSS GRALS (FID)

FUEL HASS KG

FUEL ECOAOHY IPPG (L/100NH)

3-BAG CARB FTP VRHICLE EIISSIOH RESULTS

TEST $50 \mathrm{NB} / 5 \mathrm{OP}(\mathrm{NC})-02 \mathrm{~S}$

DATE $12 / 4 / 95$ ROD

DHIO 3 BAG CART 2

ACTUAI ROAD LOAD $6.60 \mathrm{IP}$ ( $4.92 \mathrm{KN}$ )

TEST WEIGHT 3375 LBS (1530 KG)

DRY BULB TENPERaTURE $70.0^{\circ} \mathrm{F}\left(21.1^{\circ} \mathrm{C}\right)$

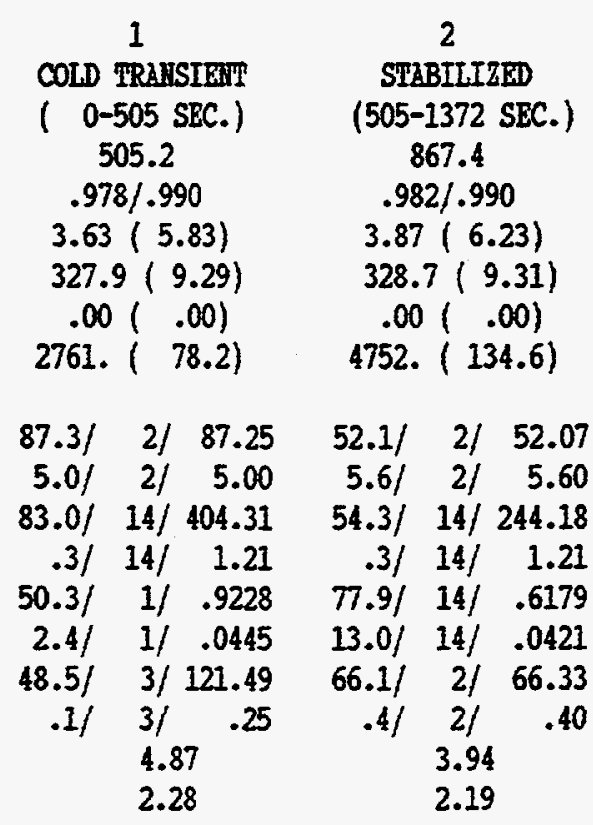

12.16

82.66

389.37

.8819

121.26

2.78

79.46

3.925
35.441

1262.46

15.913

.145

3.583

.442

$16.97(13.86)$

1.87

44.63

3.823

37.035

1424.30

14.896

.168

3.463

.496

$16.13(14.59)$

PROJECT 10. 08-6969-001

50\% BUTAII $50 \%$ PROP

FOEL DETSITY $4.558 \mathrm{LB} / \mathrm{GAL}$

$\begin{array}{lllllll}\text { H } .178 & \text { C. } .822 & 0 & .000 & \mathbb{8} & .000\end{array}$

HOY HUYDITY C.F. $\quad .878$

$$
\begin{aligned}
& 3 \\
& \text { HOT TRARSIBRT } \\
& (0-505 \text { SEC. }) \\
& 505.7 \\
& .979 / .990 \\
& 3.61(5.81) \\
& 327.4(9.27) \\
& .00(.00) \\
& 2759 .(78.1)
\end{aligned}
$$

$70.3 / 2 / \quad 70.26$

$5.6 / 2 / \quad 5.60$

$82.7 / 14 / 402.56$

$.3 / 14 / 1.21$

$87.0 / 14 / .7968$

13.1/ 14/ .0425

$46.6 / 3 / 116.74$

$.2 / \quad 3 / \quad .50$

4.91

2.20

14.00

65.06

388.82

.7573

116.27

2.87

61.77
3.087

35.372

1083.52

15.249

.149

2.783

.381

$19.57(12.02)$

3-BAG COIPOSITE RESULTS

$$
\begin{array}{llr}
\text { PIDHC } & \text { G/MI } & .971 \\
\text { CO } & \text { G/MI } & 9.675 \\
\text { HOL } & \text { G/MI } & 4.065
\end{array}
$$

FUEL ECORONY IPG (L/100RM)
CH4 G/HI

IILHC G/MI
.042

.880 

Appendix B

FTP Detailed Speciation Data from Task 1

\begin{tabular}{|c|c|c|c|}
\hline Fuel & Catalyst & Test No. & Page No. \\
\hline $\begin{array}{l}\text { CP2 } \\
\text { (pre-conversion) }\end{array}$ & Yes & BL-CP2-02S & B-1 \\
\hline $\begin{array}{l}\text { CP2 } \\
\text { (post conversion) }\end{array}$ & Yes & BLB-CP2-02S & B-6 \\
\hline RFA & Yes & BLB-RFA-02S & $B-11$ \\
\hline $100 \%$ n-Butane & Yes & 100NB-02S & B-16 \\
\hline HD-5 LPG & Yes & HD5-02S & B-21 \\
\hline $\begin{array}{l}90 \% \text { n-Butane } \\
10 \% \text { Isobutane }\end{array}$ & Yes & $90 \mathrm{NB} / 10 \mathrm{OB}-025$ & B-26 \\
\hline $\begin{array}{l}90 \% \text { n-Butane } \\
10 \% \text { Mixed Butenes }\end{array}$ & Yes & 90NB/10BE-02S & B-31 \\
\hline $\begin{array}{l}60 \% \text { n-Butane } \\
40 \% \text { Isobutane }\end{array}$ & Yes & $60 \mathrm{NB} / 40 \mathrm{OB}-02 \mathrm{~S}$ & B-36 \\
\hline $\begin{array}{l}80 \% \text { n-Butane } \\
20 \% \text { Propane }\end{array}$ & Yes & $80 \mathrm{NB} / 20 \mathrm{NP}-02 \mathrm{~S}$ & $B-41$ \\
\hline $\begin{array}{l}90 \% \text { n-Butane } \\
10 \% \text { n-Pentane }\end{array}$ & Yes & 90NB/10PE-02S & $B-46$ \\
\hline $\begin{array}{l}50 \% \text { n-Butane } \\
50 \% \text { Propane }\end{array}$ & Yes & 50NB/50NP-02S & B-51 \\
\hline $\begin{array}{l}20 \% \text { n-Butane } \\
80 \% \text { Propane }\end{array}$ & Yes & $20 \mathrm{NB} / 80 \mathrm{P}-02 \mathrm{~S}$ & B-56 \\
\hline $\begin{array}{l}100 \% \text { n-Butane } \\
\text { (repeat tests) }\end{array}$ & Yes & $100 N B-04 S$ & B-61 \\
\hline $\begin{array}{l}\text { CP2 } \\
\text { (repeat tests) }\end{array}$ & Yes & BLB-CP2-04S & B-66 \\
\hline $100 \%$ n-Butane & No & $100 \mathrm{NB}(\mathrm{NC})-02 \mathrm{~S}$ & $\mathrm{~B}-71$ \\
\hline $100 \%$ Propane & No & $100 \mathrm{P}(\mathrm{NC})-02 \mathrm{~S}$ & B-76 \\
\hline $\begin{array}{l}50 \% \text { n-Butane } \\
50 \% \text { Propane }\end{array}$ & No & $50 \mathrm{NB} / 50 \mathrm{P}(\mathrm{NC})-02 \mathrm{~S}$ & $B-81$ \\
\hline
\end{tabular}



TEST NO: BL-CP2-O2S

TEST CAR: 1994 PONTIAC GRAND AM

TEST DATE: $8 / 23 / 95$

TEST FUEL: EM-1968-F

\begin{tabular}{|c|c|c|c|c|c|c|c|c|c|c|c|c|}
\hline \multirow[b]{2}{*}{ COMPOUND } & \multicolumn{3}{|c|}{ BAG 1} & \multicolumn{3}{|c|}{ BAG 2} & \multicolumn{3}{|c|}{ BAG 3} & \multicolumn{3}{|c|}{ FTP } \\
\hline & MG/MI & $\begin{array}{l}\text { WEIGHT } \\
\text { NMOG \% } \\
\end{array}$ & $\begin{array}{l}\text { OZONE, } \\
\text { MGMI }\end{array}$ & MG/MI & $\begin{array}{l}\text { WEIGHT } \\
\text { NMOG \% } \\
\end{array}$ & $\begin{array}{l}\text { OZONE } \\
\text { MG/MI }\end{array}$ & MG/MI & $\begin{array}{l}\text { WEIGHT } \\
\text { NMOG } \% \\
\end{array}$ & $\begin{array}{l}\text { OZONE } \\
\text { MG/MI }\end{array}$ & MG/MI & $\begin{array}{l}\text { WEIGHT } \\
\text { NMOQ } \%\end{array}$ & $\begin{array}{l}\text { OZONE } \\
\text { MG/MI }\end{array}$ \\
\hline METHANE & 25.8 & & 0.4 & 2.1 & & 0.0 & 12.4 & & 0.2 & 9.8 & & 0.15 \\
\hline ETHANE & 5.3 & 2.35 & 1.3 & 0.0 & 0.03 & 0.0 & 1.6 & 4.24 & 0.4 & 1.6 & 2.53 & 0.39 \\
\hline ETHYLENE & 12.8 & 5.66 & 93.4 & 0.0 & 0.30 & 0.2 & 1.2 & 3.06 & 8.4 & 3.0 & 4.88 & 21.80 \\
\hline PROPANE & 0.1 & 0.05 & 0.1 & 0.0 & 0.00 & 0.0 & 0.1 & 0.24 & 0.0 & 0.0 & 0.08 & 0.02 \\
\hline PROPYLENE & 11.1 & 4.90 & 104.3 & 0.0 & 0.13 & 0.1 & 1.0 & 2.63 & 9.4 & 2.6 & 4.21 & 24.27 \\
\hline ACETYLENE & 6.5 & 2.87 & 3.3 & 0.0 & 0.00 & 0.0 & 0.0 & 0.05 & 0.0 & 1.4 & 2.21 & 0.68 \\
\hline PROPADIENE & 0.0 & 0.00 & 0.0 & 0.0 & 0.00 & 0.0 & 0.0 & 0.00 & 0.0 & 0.0 & 0.00 & 0.00 \\
\hline BUTANE & 3.0 & 1.32 & 3.1 & 0.0 & 0.00 & 0.0 & 0.4 & 0.99 & 0.4 & 0.7 & 1.18 & 0.74 \\
\hline TRANS-2-BUTENE & 0.9 & 0.39 & 8.9 & 0.0 & 0.00 & 0.0 & 0.1 & 0.30 & 1.1 & 0.2 & 0.36 & 2.15 \\
\hline 1-BUTENE & 1.2 & 0.63 & 10.7 & 0.0 & 0.00 & 0.0 & 0.1 & 0.30 & 1.0 & 0.3 & 0.46 & 2.51 \\
\hline 2-METHYLPROPENE (ISOBUTYLENE) & 9.6 & 4.25 & 51.1 & 0.1 & 1.20 & 0.5 & 1.4 & 3.72 & 7.5 & 2.4 & 3.97 & 12.91 \\
\hline 2,2-DIMETHYLPROPANE (NEOPENTANE) & 0.1 & 0.05 & 0.0 & 0.0 & 0.00 & 0.0 & 0.0 & 0.00 & 0.0 & 0.0 & 0.04 & $\overline{0.01}$ \\
\hline PROPYNE & 0.0 & 0.00 & 0.0 & 0.0 & 0.00 & 0.0 & 0.0 & 0.00 & 0.0 & 0.0 & 0.00 & 0.00 \\
\hline 1,3-BUTADIENE & 1.2 & 0.63 & 13.1 & 0.0 & 0.00 & 0.0 & 0.0 & 0.00 & 0.0 & 0.2 & 0.41 & $\overline{2.71}$ \\
\hline 2-METHYLPROPANE (ISOBUTANE) & 0.0 & 0.00 & 0.0 & 0.0 & 0.00 & 0.0 & 0.1 & 0.16 & 0.1 & 0.0 & 0.03 & 0.02 \\
\hline 1-BUTYNE & 0.0 & 0.00 & 0.0 & 0.0 & 0.00 & 0.0 & 0.0 & 0.00 & 0.0 & 0.0 & 0.00 & 0.00 \\
\hline METHANOL & 0.0 & 0.00 & 0.0 & 0.0 & 0.00 & 0.0 & 0.0 & 0.00 & 0.0 & 0.0 & 0.00 & 0.00 \\
\hline CIS-2-BUTENE & 0.7 & 0.29 & 6.5 & 0.0 & 0.00 & 0.0 & 0.1 & 0.19 & 0.7 & 0.2 & 0.26 & $\overline{1.66}$ \\
\hline 3-METHYL-1-BUTENE & 0.0 & 0.00 & 0.0 & 0.0 & 0,00 & 0.0 & 0.0 & 0.00 & 0.0 & 0.0 & 0.00 & 0.00 \\
\hline ETHANOL & 0.0 & 0.00 & 0.0 & 0.0 & 0.00 & 0.0 & 0.0 & 0.00 & 0.0 & 0.0 & 0.00 & 0.00 \\
\hline 2-METHYLBUTANE (ISOPENTANE) & 18.5 & 8.18 & 25.6 & 0.3 & 4.38 & 0.4 & 3.4 & 9.07 & 4.7 & 5.0 & 8.10 & 6.85 \\
\hline 2-BUTYNE & 0.0 & 0.00 & 0.0 & 0.0 & 0.00 & 0.0 & 0.0 & 0.00 & 0.0 & 0.0 & 0.00 & 0.00 \\
\hline 1-PENTENE & 0.0 & 0.00 & 0.0 & 0.0 & 0.00 & 0.0 & 0.0 & 0.00 & 0.0 & 0.0 & 0.00 & 0.00 \\
\hline 2-METHYL-1-BUTENE & 0.6 & 0.27 & 3.0 & 0.0 & 0.00 & 0.0 & 0.1 & 0.33 & 0.6 & 0.2 & 0.26 & 0.78 \\
\hline PENTANE & 2.0 & 0.90 & 2.1 & 0.2 & 2.86 & 0.2 & 0.4 & 0.96 & 0.4 & 0.6 & 1.03 & 0.66 \\
\hline UNIDENTIFIED C6 OLEFINS & 0.0 & 0.00 & 0.0 & 0.0 & 0.00 & 0.0 & 0.0 & 0.00 & 0.0 & 0.0 & 0.00 & 0.00 \\
\hline 2-METHYL-1,3-BUTADIENE & 0.8 & 0.34 & 7.0 & 0.0 & 0.00 & 0.0 & 0.2 & 0.43 & 1.5 & 0.2 & 0.34 & 1.86 \\
\hline TRANS-2-PENTENE & 0.5 & 0.22 & 4.5 & 0.0 & 0.00 & 0.0 & 0.1 & 0.25 & 0.8 & 0.1 & 0.21 & 1.16 \\
\hline 3,3-DIMETHYL-1-BUTENE & 0.0 & 0.00 & 0.0 & 0.0 & 0.00 & 0.0 & 0.0 & 0.00 & 0.0 & 0.0 & 0.00 & 0.00 \\
\hline CIS-2-PENTENE & 0.3 & 0.13 & 2.6 & 0.0 & 0.00 & 0.0 & 0.0 & 0.00 & 0.0 & 0.1 & 0.10 & 0.62 \\
\hline 2-METHYL-2-BUTENE & 1.4 & 0.62 & 9.0 & 0.1 & 0.94 & 0.4 & 0.1 & 0.31 & 0.8 & 0.4 & 0.59 & 2.32 \\
\hline CYCLOPENTADIENE & 0.4 & 0.17 & 2.9 & 0.0 & 0.00 & 0.0 & 0.0 & 0.00 & 0.0 & 0.1 & 0.13 & 0.60 \\
\hline 2,2-DIMETHYLBUTANE & 0.3 & 0.12 & 0.2 & 0.0 & 0.00 & 0.0 & 0.1 & 0.30 & 0.1 & 0.1 & 0.14 & 0.07 \\
\hline CYCLOPENTENE & 0.1 & 0.06 & 1.0 & 0.0 & 0.00 & 0.0 & 0.0 & 0.00 & 0.0 & 0.0 & 0.04 & 0.20 \\
\hline 4-METHYL-1-PENTENE & 0.2 & 0.07 & 0.7 & 0.0 & 0.00 & 0.0 & 0.0 & 0.00 & 0.0 & 0.0 & 0.05 & 0.14 \\
\hline 3-METHYL-1-PENTENE & 0.1 & 0.05 & 0.6 & 0.0 & 0.00 & 0.0 & 0.0 & 0.00 & 0.0 & 0.0 & 0.04 & 0.11 \\
\hline CYCLOPENTANE & 0.0 & 0.00 & 0.0 & 0.0 & 0.00 & 0.0 & 0.0 & 0.00 & 0.0 & 0.0 & 0.00 & 0.00 \\
\hline 2,3-DIMETHYLBUTANE & 1.5 & 0.67 & 1.6 & 0.1 & 0.78 & 0.1 & 0.4 & 1.11 & $\overline{0.4}$ & 0.6 & 0.75 & $\overline{0.49}$ \\
\hline MTBE & 11,6 & 5.10 & 7.2 & 0.0 & 0.00 & 0.0 & 0.0 & 0.00 & 0.0 & 2.4 & 3.91 & 1.49 \\
\hline
\end{tabular}




\begin{tabular}{|c|c|c|c|c|c|c|c|c|c|c|c|c|}
\hline \multirow[b]{2}{*}{ COMPOUND } & \multicolumn{3}{|c|}{ BAG 1} & \multicolumn{3}{|c|}{ BAG 2} & \multicolumn{3}{|c|}{ BAG 3} & \multicolumn{3}{|c|}{ FTP } \\
\hline & MGMI & \begin{tabular}{|l|} 
WEIGHT \\
NMOG \% \\
\end{tabular} & $\begin{array}{l}\text { OZONE, } \\
\text { MG/MI }\end{array}$ & MG/MI & $\begin{array}{l}\text { WEIGHT } \\
\text { NMOQ } \% \\
\end{array}$ & $\begin{array}{l}\text { OZONE } \\
\text { MG/MI }\end{array}$ & MG/MI & $\begin{array}{l}\text { WEIGHT } \\
\text { NMOQ \% }\end{array}$ & $\begin{array}{l}\text { OZONE } \\
\text { MGIMI }\end{array}$ & MG/MI & $\begin{array}{l}\text { WEIGHT } \\
\text { NMOG \% }\end{array}$ & $\begin{array}{l}\text { OZONE } \\
\text { MG/MI }\end{array}$ \\
\hline 2,3-DIMETHYL-1-BUTENE & 0.0 & 0.00 & 0.0 & 0.0 & 0.00 & 0.0 & 0.0 & 0.00 & 0.0 & 0.0 & 0.00 & 0.00 \\
\hline 4-METHYL-CIS-2-PENTENE & 0.0 & 0.00 & 0.0 & 0.0 & 0.00 & 0.0 & 0.0 & 0.00 & 0.0 & 0.0 & 0.00 & 0.00 \\
\hline 2-METHYLPENTANE & 1.6 & 0.71 & 2.4 & $\overline{0.3}$ & 3.69 & 0.4 & 0.8 & 2.22 & 1.3 & 0.7 & 1.15 & 1.08 \\
\hline 4-METHYL-TRANS-2-PENTENE & 0.0 & 0.00 & 0.0 & 0.0 & 0.00 & 0.0 & 0.0 & 0.00 & 0.0 & 0.0 & 0.00 & 0.00 \\
\hline 3-METHYLPENTANE & 1.3 & 0.55 & 1.9 & 0.1 & 1.69 & 0.2 & 0.7 & 1.72 & 1.0 & $\overline{0.6}$ & $\overline{0.82}$ & $\overline{0.77}$ \\
\hline 2-METHYL-1-PENTENE & 0.2 & 0.07 & 0.7 & 0.0 & 0.00 & 0.0 & 0.0 & 0.00 & 0.0 & 0.0 & 0.06 & $\overline{0.14}$ \\
\hline 1-HEXENE & 0.2 & 0.07 & 0.7 & 0.0 & 0.00 & 0.0 & 0.0 & 0.00 & 0.0 & 0.0 & 0.05 & 0.15 \\
\hline HEXANE & 0.9 & 0.40 & 0.9 & 0.2 & 2.77 & 0.2 & 0.4 & 1.19 & 0.4 & 0.4 & 0.68 & 0.41 \\
\hline UNIDENTIFIED C6 OLEFINS & 0.3 & 0.14 & 2.1 & 0.0 & 0.00 & 0.0 & 0.0 & 0.00 & 0.0 & 0.1 & 0.11 & 0.44 \\
\hline TRANS-3-HEXENE & 0.2 & 0.09 & 1.4 & 0.0 & 0.00 & 0.0 & 0.0 & 0.00 & 0.0 & 0.0 & 0.07 & 0.28 \\
\hline CIS-3-HEXENE & 0.3 & 0.13 & 2.0 & 0.0 & 0.15 & 0.1 & 0.1 & 0.26 & 0.7 & 0.1 & 0.16 & 0.64 \\
\hline TRANS-2-HEXENE & 0.0 & 0.00 & 0.0 & 0.0 & 0.00 & 0.0 & 0.0 & 0.00 & 0.0 & 0.0 & 0.00 & 0.00 \\
\hline 3-METHYL-TRANS-2-PENTENE & 0.4 & 0.17 & 2.5 & 0.0 & 0.00 & 0.0 & 0.0 & 0.00 & 0.0 & 0.1 & 0.13 & 0.52 \\
\hline 2-METHYL-2-PENTENE & 0.3 & 0.11 & 1.7 & 0.0 & 0.00 & 0.0 & 0.0 & 0.00 & 0.0 & 0.1 & 0.08 & 0.35 \\
\hline 3-METHYLCYCLOPENTENE & 0.0 & 0.00 & 0.0 & 0.0 & 0.00 & 0.0 & 0.0 & 0.00 & 0.0 & 0.0 & 0.00 & 0.00 \\
\hline CIS-2-HEXENE & 0.1 & 0.06 & 0.8 & 0.0 & 0.00 & 0.0 & 0.0 & 0.00 & 0.0 & 0.0 & 0.05 & 0.20 \\
\hline ETBE & 0.0 & 0.00 & 0.0 & 0.0 & 0.00 & 0.0 & 0.0 & 0.00 & 0.0 & 0.0 & 0.00 & 0.00 \\
\hline 3-METHYL-CIS-2-PENTENE & 0,3 & 0.14 & 2.2 & 0.0 & 0.00 & 0.0 & 0.1 & 0.23 & 0.6 & 0.1 & 0.15 & 0.61 \\
\hline 2,2-DIMETHYLPENTANE & 0.6 & 0.25 & 0.8 & 0.1 & 1.01 & 0.1 & 0.5 & 1.29 & 0.7 & 0.3 & 0.48 & 0.41 \\
\hline METHYLCYCLOPENTANE & 0.1 & 0.04 & 0.3 & 0.0 & 0.00 & 0.0 & 0.0 & 0.00 & 0.0 & 0.0 & 0.03 & 0.06 \\
\hline 2,4-DIMETHYLPENTANE & 4.3 & 1.90 & 7.7 & 0.1 & 0.90 & 0.1 & 1.2 & 3.06 & 2.1 & $\overline{1.2}$ & 2.03 & 2.22 \\
\hline 2,3,3-TRIMETHYL-1-BUTENE & 0.0 & 0.00 & 0.0 & 0.0 & 0.00 & 0.0 & 0.0 & 0.00 & 0.0 & 0.0 & 0.00 & 0.00 \\
\hline 2,2,3-TAIMETHYLBUTANE & 0.2 & 0.10 & 0.3 & 0.0 & 0.52 & 0.1 & 0.1 & 0.36 & 0.2 & 0.1 & 0.17 & 0.14 \\
\hline 3,4-DIMETHYL-1-PENTENE & 0.0 & 0.00 & 0.0 & 0.0 & 0.00 & 0.0 & 0.0 & 0.00 & 0.0 & 0.0 & 0.00 & 0.00 \\
\hline 1-METHYLCYCLOPENTENE & 0.3 & 0.14 & 1.9 & 0.2 & 2.38 & 1.0 & 0.3 & 0.68 & 1.6 & 0.2 & 0.38 & 1.30 \\
\hline BENZENE & 10.0 & 4.40 & 4.2 & 0.3 & 3.38 & 0.1 & 3.5 & 9.26 & 1.5 & 3.2 & 5.16 & 1.33 \\
\hline 3-METHYL-1-HEXENE & 0.0 & 0.00 & 0.0 & 0.0 & 0.00 & 0.0 & 0.0 & 0.00 & 0.0 & 0.0 & 0.00 & 0.00 \\
\hline 3,3-DIMETHYLPENTANE & 0.2 & 0.07 & 0.1 & 0.0 & 0.00 & 0.0 & 0.0 & 0.00 & 0.0 & 0.0 & 0.08 & 0.02 \\
\hline CYCLOHEXANE & 0.2 & 0.09 & 0.3 & 0.0 & 0.00 & 0.0 & 0.2 & 0.50 & 0.2 & 0.1 & 0.16 & 0.12 \\
\hline 2-METHYLHEXANE & 0.0 & 0.00 & 0.0 & 0.0 & 0.00 & 0.0 & 0.0 & 0.00 & 0.0 & 0.0 & 0.00 & 0.00 \\
\hline 2,3-DIMETHYLPENTANE & 8.5 & 3.74 & 12.8 & 0.1 & 1.81 & 0.2 & 2.3 & 6.97 & 3.4 & 2.5 & 4.00 & 3.70 \\
\hline 1,1-DIMETHYLCYCLOPENTANE & 0.0 & 0.00 & 0.0 & 0.0 & 0.00 & 0.0 & 0.0 & 10.00 & 0.0 & 0.0 & 0.00 & 0.00 \\
\hline CYCLOHEXENE & 0.0 & 0.00 & 0.0 & 0.0 & 0.00 & 0.0 & 0.0 & 0.00 & 0.0 & 0.0 & 0.00 & 0.00 \\
\hline 3-METHYLHEXANE & 0.0 & 0.00 & 0.0 & 0.1 & 1.19 & 0.1 & 0.6 & 1.54 & 0.8 & 0.2 & 0.34 & 0.29 \\
\hline CIS-1,3-DIMETHYLCYCLOPENTANE & 0.2 & 0.09 & 0.5 & 0.0 & 0.00 & 0.0 & 0.1 & 0.24 & 0.2 & 0.1 & 0.11 & 0.18 \\
\hline 3-ETHYLPENTANE & 0.0 & 0.00 & 0.0 & 0.0 & 0.00 & 0.0 & 0.0 & 0.00 & 0.0 & 0.0 & 0.00 & 0.00 \\
\hline TRANS-1,2-DIMETHYLCYCLOPENTANE & 0.0 & 0.00 & 0.0 & 0.0 & 0.00 & 0.0 & 0.0 & 0.00 & 0.0 & 0.0 & 0.00 & 0.00 \\
\hline TRANS-1,3-DIMETHYLCYCLOPENTANE & 0.0 & 0.00 & 0.0 & 0.0 & 0.00 & 0.0 & 0.0 & 0.00 & 0.0 & 0.0 & 0.00 & 0.00 \\
\hline 1-HEPTENE & 0.0 & 0.00 & 0.0 & 0.0 & 0.00 & 0.0 & 0.0 & 0.00 & 0.0 & 0.0 & 0.00 & 0.00 \\
\hline 2,2,4-TAIMETHYLPENTANE & 17.1 & 7.55 & 15.9 & 0.2 & 2.88 & 0.2 & 4.0 & 10.68 & 3.7 & 4.8 & 7.77 & 4.43 \\
\hline 2-METHYL-1-HEXENE & 0.0 & 0.00 & 0.0 & 0.0 & 0.00 & 0.0 & 0.0 & 0.00 & 0.0 & 0.0 & 0.00 & 0.00 \\
\hline TRANS-3-HEPTENE & 0.0 & 0.00 & 0.0 & 0.0 & 0.00 & 0.0 & 0.0 & 0.00 & 0.0 & 0.0 & 0.00 & 0.00 \\
\hline HEPTANE & 1.2 & 0.51 & 0.8 & 0.0 & 0.21 & 0.0 & 0.2 & 0.60 & 0.2 & 0.3 & 0.61 & 0.25 \\
\hline UNIDENTIFIED C7 & 1.1 & 0.48 & 4.8 & $\overline{0.0}$ & 0.37 & 0.1 & 0.3 & 0.87 & 1.5 & 0.3 & 0.64 & 1.60 \\
\hline
\end{tabular}




\begin{tabular}{|c|c|c|c|c|c|c|c|c|c|c|c|c|}
\hline \multirow[b]{2}{*}{ COMPOUND } & \multicolumn{3}{|c|}{ BAG 1} & \multicolumn{3}{|c|}{ BAG 2} & \multicolumn{3}{|c|}{ BAG 3 } & \multicolumn{3}{|c|}{ FTP } \\
\hline & MG/MI & $\begin{array}{l}\text { WEIGHT } \\
\text { NMOG \% }\end{array}$ & $\begin{array}{l}\text { OZONE, } \\
\text { MG/MI }\end{array}$ & MG/MI & $\begin{array}{l}\text { WEIGHT } \\
\text { NMOG } \%\end{array}$ & $\begin{array}{l}\text { OZONE } \\
\text { MG/MI }\end{array}$ & MG/MI & $\begin{array}{l}\text { WEIGHT } \\
\text { NMOQ } \%\end{array}$ & $\begin{array}{l}\text { OZONE } \\
\text { MG/MI }\end{array}$ & MG/MI & $\begin{array}{l}\text { WEIGHT } \\
\text { NMOG \% }\end{array}$ & $\begin{array}{l}\text { OZONE } \\
\text { MQ/MI }\end{array}$ \\
\hline 2-METHYL-2-HEXENE & 0.0 & 0.00 & 0.0 & 0.0 & 0.00 & 0.0 & 0.0 & 0.00 & 0.0 & 0.0 & 0.00 & 0.00 \\
\hline 3-METHYL-TRANS-3-HEXENE & 0.0 & 0.00 & 0.0 & 0.0 & 0.00 & 0.0 & 0.0 & 0.00 & 0.0 & 0.0 & 0.00 & 0.00 \\
\hline TRANS-2-HEPTENE & 0.1 & 0.05 & 0.7 & 0.0 & 0.00 & 0.0 & 0.0 & 0.00 & 0.0 & 0.0 & 0.04 & 0.16 \\
\hline 3-ETHYL-CIS-2-PENTENE & 0.0 & 0.00 & 0.0 & 0.0 & 0.00 & 0.0 & 0.0 & 0.00 & 0.0 & 0.0 & 0.00 & 0.00 \\
\hline 2,4,4-TRIMETHYL-1-PENTENE & 0.2 & 0.07 & 0.4 & 0.0 & 0.00 & 0.0 & 0.0 & 0.00 & 0.0 & 0.0 & 0.06 & 0.09 \\
\hline 2,2,4-TRIMETHYL-1-PENTENE & 0.0 & 0.00 & 0.0 & 0.0 & 0.00 & 0.0 & 0.0 & 0.00 & 0.0 & 0.0 & 0.00 & 0.00 \\
\hline 2,3-DIMETHYL-2-PENTENE & 0.0 & 0.00 & 0.0 & 0.0 & 0.00 & 0.0 & 0.0 & 0.00 & 0.0 & 0.0 & 0.00 & 0.00 \\
\hline CIS-2-HEPTENE & 0.1 & 0.05 & 0.6 & 0.0 & 0.00 & 0.0 & 0.0 & 0.00 & 0.0 & 0.0 & 0.04 & 0.13 \\
\hline METHYLCYCLOHEXANE & 0.2 & 0.09 & 0.4 & 0.0 & 0.00 & 0.0 & 0.0 & 0.00 & 0.0 & 0.0 & 0.07 & 0.07 \\
\hline CIS-1,2-DIMETHYLCYCLOPENTANE & 0.0 & 0.00 & 0.0 & 0.0 & 0.00 & 0.0 & $\overline{0.0}$ & 0.00 & 0.0 & $\overline{0.0}$ & 0.00 & 0.00 \\
\hline 2,2-DIMETHYLHEXANE & 1.7 & 0.77 & 2.1 & 0.0 & 0.00 & 0.0 & 0.4 & 1.09 & 0.5 & 0.5 & 0.78 & 0.67 \\
\hline 1,1,3-TRIMETHYLCYCLOPENTANE & 0.0 & 0.00 & 0.0 & 0.0 & 0.00 & 0.0 & 0.0 & 0.00 & 0.0 & 0.0 & 0.00 & 0.00 \\
\hline 2,4,4-TRIMETHYL-2-PENTENE & 0.0 & 0.00 & 0.0 & 0.0 & 0.00 & 0.0 & 0.0 & 0.00 & 0.0 & 0.0 & 0.00 & 0.00 \\
\hline 2,2,3-TAIMETHYLPENTANE & 0.0 & 0.00 & 0.0 & 0.0 & 0.00 & 0.0 & 0.0 & 0.00 & 0.0 & 0.0 & 0.00 & 0.00 \\
\hline 2,5-DIMETHYLHEXANE & 0.0 & 0.00 & 0.0 & 0.0 & 0.00 & 0.0 & 0.0 & 0.00 & 0.0 & 0.0 & 0.00 & 0.00 \\
\hline ETHYLCYCLOPENTANE & 0.0 & 0.00 & 0.0 & 0.0 & 0.00 & 0.0 & 0.0 & 0.00 & 0.0 & 0.0 & 0.00 & 0.00 \\
\hline 2,4-DIMETHYLHEXANE & 2.2 & 0.98 & 3.3 & 0.0 & 0.00 & 0.0 & 0.4 & 1.13 & 0.6 & 0.6 & 0.95 & 0.87 \\
\hline 1-TRANS-2-CIS-4-TRIMETHYLCYCLOPENTANE & 0.0 & 0.00 & 0.0 & 0.0 & 0.00 & 0.0 & 0.0 & 0.00 & 0.0 & 0.0 & 0.00 & 0.00 \\
\hline 3,3-DIMETHYLHEXANE & 0.1 & 0.04 & 0.1 & 0.0 & 0.00 & 0.0 & 0.0 & 0.00 & 0.0 & 0.0 & 0.03 & 0.02 \\
\hline 1-TRANS-2-CIS-3-TRIMETHYLCYCLOPENTANE & 0.0 & 0.00 & 0.0 & 0.0 & 0.00 & 0.0 & 0.0 & 0.00 & 0.0 & 0.0 & 0.00 & 0.00 \\
\hline 2,3,4-TRIMETHYLPENTANE & 0.0 & 0.00 & 0.0 & $\overline{0.0}$ & 0.00 & 0.0 & 0.0 & 0.00 & 0.0 & 0.0 & 0.00 & 0.00 \\
\hline 2,3,3-TRIMETHYLPENTANE & 3.9 & 1.72 & 4.7 & 0.1 & 0.99 & $\overline{0.1}$ & 0.9 & 2.32 & 1.1 & 1.1 & 1.78 & 1.31 \\
\hline TOLUENE & 18.6 & 8.22 & 50.8 & 0.6 & 7.52 & 1.5 & 2.6 & 6.90 & 7.1 & 4.9 & 7.95 & 13.30 \\
\hline 2,3-DIMETHYLHEXANE & 2.9 & 1.27 & 3.8 & 0.0 & 0.00 & 0.0 & 0.0 & 0.00 & 0.0 & 0.6 & 0.98 & 0.79 \\
\hline 1,1,2-TRIMETHYLCYCLOPENTANE & 0.0 & 0.00 & 0.0 & 0.0 & 0.00 & 0.0 & 0.0 & 0.00 & 0.0 & 0.0 & 0.00 & 0.00 \\
\hline 2-METHYLHEPTANE & 1.4 & 0.60 & 1.3 & 0.0 & 0.00 & 0.0 & 0.2 & 0.57 & 0.2 & 0.3 & 0.56 & 0.33 \\
\hline 3,4-DIMETHYLHEXANE & 0.0 & 0.00 & 0.0 & 0.0 & 0.00 & 0.0 & 0.0 & 0.00 & 0.0 & 0.0 & 0.00 & 0.00 \\
\hline 2,2,4,4-TETRAMETHYLPENTANE & 0.0 & 0.00 & 0.0 & 0.0 & 0.00 & 0.0 & 0.0 & 0.00 & 0.0 & 0.0 & 0.00 & 0.00 \\
\hline 4-METHYLHEPTANE & 0.4 & 0.19 & 0.5 & 0.0 & 0.00 & 0.0 & 0.0 & 0.00 & 0.0 & 0.1 & 0.14 & 0.10 \\
\hline 2-METHYL-3-ETHYLPENTANE & 0.0 & 0.00 & 0.0 & 0.0 & 0.00 & 0.0 & 0.0 & 0.00 & 0.0 & 0.0 & 0.00 & 0.00 \\
\hline 2,6-DIMETHYLHEPTANE & 0.0 & 0.00 & 0.0 & $\overline{0.0}$ & 0.00 & 0.0 & 0.0 & 0.00 & 0.0 & 0.0 & 0.00 & 0.00 \\
\hline 3-METHYLHEPTANE & 1.5 & 0.67 & 1.5 & 0.0 & 0.00 & 0.0 & 0.3 & 0.80 & 0.3 & 0.4 & 0.85 & 0.39 \\
\hline 1-CIS,2-TAANS,3-TRIMETHYLCYCLOPENTANE & 0.0 & 0.00 & 0.0 & 0.0 & 0.00 & 0.0 & 0.0 & 0.00 & 0.0 & 0.0 & 0.00 & 0.00 \\
\hline CIS-1,3-DIMETHYLCYCLOHEXANE & 0.0 & 0.00 & 0.0 & 0.0 & 0.00 & 0.0 & 0.0 & 0.00 & 0.0 & 0.0 & 0.00 & 0.00 \\
\hline TRANS-1,4-DIMETHYLCYCLOHEXANE & 1.7 & 0.74 & 3.3 & 0.0 & 0.00 & 0.0 & $\overline{0.4}$ & 1.07 & 0.8 & 0.5 & 0.75 & 0.89 \\
\hline 3-ETHYLHEXANE & 0.0 & 0.00 & 0.0 & 0.0 & 0.00 & 0.0 & 0.0 & 0.00 & 0.0 & 0.0 & 0.00 & 0.00 \\
\hline 2,2,5-TRIMETHYLHEXANE & 0.0 & 0.00 & 0.0 & 0.0 & 0.00 & 0.0 & 0.0 & 0.00 & 0.0 & 0.0 & 0.00 & 0.00 \\
\hline CIS-1-METHYL-3-ETHYLCYCLOPENTANE & 0.0 & 0.00 & 0.0 & 0.0 & 0.00 & 0.0 & 0.0 & 0.00 & 0.0 & 0.0 & 0.00 & 0.00 \\
\hline 1,1-DIMETHYLCYCLOHEXANE & 0.0 & 0.00 & 0.0 & 0.0 & 0.00 & 0.0 & 0.0 & 0.00 & 0.0 & 0.0 & 0.00 & 0.00 \\
\hline TRANS-1-METHYL-2-ETHYLCYCLOPENTANE & 0.0 & 0.00 & 0.0 & 0.0 & 0.00 & 0.0 & 0.0 & 0.00 & 0.0 & 0.0 & 0.00 & 0.00 \\
\hline 1-METHYL-1-ETHYL-CYCLOPENTANE & 0.0 & 0.00 & 0.0 & 0.0 & 0.00 & 0.0 & 0.0 & 0.00 & 0.0 & 0.0 & 0.00 & 0.00 \\
\hline 2,4,4-TRIMETHYLLEXANE & 0.0 & 0.00 & 0.0 & 0.0 & 0.00 & 0.0 & 0.0 & 0.00 & 0.0 & 0.0 & 0.00 & 0.00 \\
\hline 2,2,4-TRIMETHYLHEXANE & 0.0 & 0.00 & 0.0 & 0.0 & 0.00 & 0.0 & 0.0 & 0.00 & 0.0 & 0.0 & 0.00 & 0.00 \\
\hline TRANS-1,2-DIMETHYLCYCLOHEXANE & 0.0 & 0.00 & 0.0 & 0.0 & 0.00 & 0.0 & 0.0 & 0.00 & 0.0 & 0.0 & 0.00 & 0.00 \\
\hline
\end{tabular}




\begin{tabular}{|c|c|c|c|c|c|c|c|c|c|c|c|c|}
\hline \multirow[b]{2}{*}{ COMPOUND } & \multicolumn{3}{|c|}{ BAG 1} & \multicolumn{3}{|c|}{ BAG 2} & \multicolumn{3}{|c|}{ BAG 3} & \multicolumn{3}{|c|}{ FTP } \\
\hline & MG/MI & $\begin{array}{l}\text { WEIGHT } \\
\text { NMOG } \%\end{array}$ & $\begin{array}{l}\text { OZONE, } \\
\text { MG/MI }\end{array}$ & MG/MI & $\begin{array}{l}\text { WEIGHT } \\
\text { NMOG } \%\end{array}$ & $\begin{array}{l}\text { OZONE } \\
\text { MG/MI }\end{array}$ & MG/MI & $\begin{array}{l}\text { WEIGHT } \\
\text { NMOQ } \%\end{array}$ & $\begin{array}{l}\text { OZONE } \\
\text { MAMMI }\end{array}$ & MG/MI & $\begin{array}{l}\text { WEIGHT } \\
\text { NMOG } \%\end{array}$ & $\begin{array}{l}\text { OZONE } \\
\text { MG/MI }\end{array}$ \\
\hline 1-OCTENE & 0.1 & 0.04 & 0.2 & 0.0 & 0.00 & 0.0 & 0.0 & 0.00 & 0.0 & 0.0 & 0.03 & 0.05 \\
\hline TRANS-4-OCTENE & 0.0 & 0.00 & 0.0 & 0.0 & 0.00 & 0.0 & 0.0 & 0.00 & 0.0 & 0.0 & 0.00 & 0.00 \\
\hline OCTANE & 0.9 & 0.39 & 0.5 & 0.0 & 0.00 & 0.0 & 0.2 & 0.42 & 0.1 & 0.2 & 0.37 & 0.14 \\
\hline UNIDENTIFIED C8 & 1.4 & 0.62 & 4.8 & 0.1 & 1.15 & 0.3 & 0.1 & 0.30 & 0.4 & 0.4 & 0.60 & 1.25 \\
\hline TRANS-2-OCTENE & 0.1 & 0.05 & 0.6 & 0.0 & 0.00 & 0.0 & 0.0 & 0.00 & 0.0 & 0.0 & 0.04 & 0.12 \\
\hline TRANS-1,3-DIMETHYLCYCLOHEXANE & 0.1 & 0.06 & 0.2 & 0.0 & 0.00 & 0.0 & 0.0 & 0.00 & 0.0 & 0.0 & 0.04 & 0.05 \\
\hline CIS-1,4-DIMETHYLCYCLOHEXANE & $\overline{0.0}$ & 0.00 & 0.0 & 0.0 & 0.00 & 0.0 & $\overline{0.0}$ & 0.00 & 0.0 & 0.0 & 0.00 & 0.00 \\
\hline CIS-2-OCTENE & 0.0 & 0.00 & 0.0 & 0.0 & 0.00 & 0.0 & 0.0 & 0.00 & 0.0 & 0.0 & 0.00 & 0.00 \\
\hline 2,3,5-TRIMETHYLHEXANE & 0.2 & 0.11 & 0.3 & 0.0 & 0.00 & 0.0 & 0.0 & 0.00 & 0.0 & 0.0 & 0.08 & 0.06 \\
\hline CIS-1-METHYL-2-ETHYLCYCLOPENTANE & 0.0 & 0.00 & 0.0 & 0.0 & 0.00 & 0.0 & 0.0 & 0.00 & 0.0 & 0.0 & 0.00 & 0.00 \\
\hline 2-METHYL-2-ETHYLHEPTANE & 0.0 & 0.00 & 0.0 & 0.0 & 0.00 & 0.0 & 0.0 & 0.00 & 0.0 & 0.0 & 0.00 & 0.00 \\
\hline 2,4-DIMETHYLHEPTANE & 0.1 & 0.03 & 0.1 & 0.3 & 4.07 & 0.4 & 0.0 & 0.11 & 0.1 & 0.2 & 0.29 & 0.24 \\
\hline 4,4-DIMETHYLHEPTANE & 0.0 & 0.00 & 0.0 & 0.0 & 0.00 & 0.0 & 0.0 & 0.00 & 0.0 & 0.0 & 0.00 & 0.00 \\
\hline CIS-1,2-DIMETHYLCYCLOHEXANE & 0.2 & 0.09 & 0.4 & 0.0 & 0.00 & 0.0 & 0.0 & 0.00 & 0.0 & 0.0 & 0.07 & 0.09 \\
\hline ETHYLCYCLOHEXANE & 0.3 & 0.14 & 0.6 & 0.0 & 0.00 & 0.0 & 0.1 & 0.23 & 0.2 & 0.1 & 0.15 & 0.18 \\
\hline PROPYLCYCLOHEXANE & 0.0 & 0.00 & 0.0 & 0.0 & 0.00 & 0.0 & 0.0 & 0.00 & 0.0 & 0.0 & 0.00 & 0.00 \\
\hline 2-METHYL-4-ETHYLHEXANE & 0.0 & 0.00 & 0.0 & 0.0 & 0.00 & 0.0 & 0.0 & 0.00 & 0.0 & 0.0 & 0.00 & 0.00 \\
\hline 2,6-DIMETHYLHEPTANE & 0.0 & 0.00 & 0.0 & 0.0 & 0.00 & 0.0 & 0.0 & 0.00 & 0.0 & 0.0 & 0.00 & 0.00 \\
\hline 1,1,3-TRIMETHYLCYCLOHEXANE & 0.0 & 0.00 & 0.0 & 0.0 & 0.00 & 0.0 & 0.0 & 0.00 & 0.0 & 0.0 & 0.00 & 0.00 \\
\hline 2,5-DIMETHYLHEPTANE & 0.0 & 0.00 & 0.0 & 0.0 & 0.00 & 0.0 & 0.0 & 0.00 & 0.0 & 0.0 & 0.00 & 0.00 \\
\hline 3,3-DIMETHYLHEPTANE & 0.0 & 0.00 & 0.0 & 0.0 & 0.00 & 0.0 & 0.0 & 0.00 & 0.0 & 0.0 & 0.00 & 0.00 \\
\hline 3,6-DIMETHYLHEPTANE & 0.0 & 0.00 & 0.0 & 0.0 & 0.00 & 0.0 & 0.0 & 0.00 & 0.0 & 0.0 & 0.00 & 0.00 \\
\hline ETHYLBENZENE & $\overline{\theta .1}$ & 2.67 & 16.4 & 0.2 & 2.07 & 0.4 & 0.6 & 1.60 & 1.8 & 1.6 & 2.45 & 4.08 \\
\hline 2,3-DIMETHYLHEPTANE & 0.0 & 0.00 & 0.0 & 0.0 & 0.00 & 0.0 & 0.0 & 0.00 & 0.0 & 0.0 & 0.00 & 0.00 \\
\hline$m-\& p-X Y L E N E$ & 13.5 & 5.96 & 99.6 & 0.2 & 2.26 & 1.2 & 1.2 & 3.14 & 8.7 & 3.2 & 6.25 & 23.72 \\
\hline 4-METHYLOCTANE & 0.0 & 0.00 & 0.0 & 0.0 & 0.00 & 0.0 & 0.0 & 0.00 & 0.0 & 0.0 & 0.00 & 0.00 \\
\hline 2-METHYLOCTANE & 0.7 & 0.31 & 0.8 & 0.0 & 0.00 & 0.0 & 0.1 & 0.33 & 0.1 & 0.2 & 0.30 & 0.21 \\
\hline 3-METHYLOCTANE & 0.4 & 0.19 & 0.6 & 0.0 & 0.00 & 0.0 & 0.0 & 0.00 & 0.0 & 0.1 & 0.15 & 0.10 \\
\hline STYRENE & 0.0 & 0.00 & 0.0 & 0.0 & 0.00 & 0.0 & 0.0 & 0.00 & 0.0 & 0.0 & 0.00 & 0.00 \\
\hline o-XYLENE & 4.6 & 2.04 & 29.9 & 0.1 & 1.71 & 0.8 & 0.6 & 1.47 & 3.6 & 1.2 & 1.02 & 7.62 \\
\hline 2,4,6-TRIMETHYLHEXANE & 0.0 & 0.00 & 0.0 & 0.0 & 0.00 & 0.0 & 0.0 & 0.00 & 0.0 & 0.0 & 0.00 & 0.00 \\
\hline 1-NONENE & 0.4 & 0.18 & 0.8 & 0.0 & 0.00 & 0.0 & 0.0 & $\begin{array}{ll} & 0.00 \\
\end{array}$ & 0.0 & 0.1 & 0.14 & 0.19 \\
\hline NONANE & 0.3 & 0.14 & 0.2 & 0.0 & 0.00 & 0.0 & 0.1 & 0.23 & 0.0 & 0.1 & 0.15 & 0.06 \\
\hline ISOPAOPYLBENZENE (CUMENE) & 0.2 & 0.08 & 0.4 & 0.0 & 0.00 & 0.0 & 0.0 & 0.00 & 0.0 & 0.0 & 0.06 & 0.09 \\
\hline 2,2-DIMETHYLOCTANE & 0.0 & 0.00 & 0.0 & 0.0 & 0.00 & 0.0 & 0.0 & 0.00 & 0.0 & 0.0 & 0.00 & 0.00 \\
\hline 2,4-DIMETHYLOCTANE & 0.0 & 0.00 & 0.0 & 0.0 & 0.00 & 0.0 & 0.0 & 0.00 & 0.0 & 0.0 & 0.00 & 0.00 \\
\hline n-PAOPYLBENZENE & 0.7 & 0.31 & 1.5 & 0.0 & 0.00 & 0.0 & 0.1 & 0.24 & 0.2 & 0.2 & 0.28 & $\overline{0.36}$ \\
\hline 1-METHYL-3-ETHYLBENZENE & 2.9 & 1.28 & 20.9 & 0.0 & 0.00 & 0.0 & 0.3 & 0.73 & 2.0 & 0.7 & 1.11 & 4.88 \\
\hline 1-METHYL -4-ETHYLBENZENE & 1.2 & 0.55 & 8.0 & 0.0 & 0.00 & 0.0 & 0.2 & 0.44 & 1.2 & 0.3 & 0.50 & 2.19 \\
\hline 1,3,6-TRIMETHYLBENZENE & 1.2 & 0.52 & 12.0 & 0.0 & 0.00 & 0.0 & 0.1 & 0.31 & 1.2 & 0.3 & 0.45 & 2.81 \\
\hline 1-METHYL-2-ETHYLBENZENE & 0.8 & 0.39 & 6.4 & 0.0 & 0.00 & 0.0 & 0.2 & 0.49 & 1.3 & 0.2 & 0.38 & 1.70 \\
\hline 1,2,4-TRIMETHYLBENZENE & 3.4 & 1.52 & 30.4 & 0.0 & 0.00 & 0.0 & 0.2 & 0.50 & 1.7 & 0.8 & 1.25 & 6.77 \\
\hline DECANE & 0.0 & 0.00 & 0.0 & 0.0 & 0.17 & 0.0 & 0.0 & 0.00 & 0.0 & 0.0 & 0.01 & 0.00 \\
\hline ISOBUTYLBENZENE & 0.0 & 0.00 & 0.0 & 0.0 & 0.16 & 0.0 & 0.0 & 0.00 & 0.0 & 0.0 & 0.01 & 0.01 \\
\hline
\end{tabular}




\begin{tabular}{|c|c|c|c|c|c|c|c|c|c|c|c|c|}
\hline \multirow[b]{2}{*}{ COMPOUND } & \multicolumn{3}{|c|}{ BAG 1} & \multicolumn{3}{|c|}{ BAG 2} & \multicolumn{3}{|c|}{ BAG 3} & \multicolumn{3}{|c|}{ FTP } \\
\hline & MG/MI & $\begin{array}{l}\text { WEIGHT } \\
\text { NMOG \% }\end{array}$ & $\begin{array}{l}\text { OZONE, } \\
\text { MG/MI }\end{array}$ & Ma/MI & $\begin{array}{l}\text { WEIGHT } \\
\text { NMOG } \%\end{array}$ & $\begin{array}{l}\text { OZONE } \\
\text { MGIMI }\end{array}$ & Ma/MI & $\begin{array}{l}\text { WEIGHT } \\
\text { NMOG } \%\end{array}$ & $\begin{array}{l}\text { OZONE } \\
\text { MG/MI }\end{array}$ & MG/MI & $\begin{array}{l}\text { WEIGHT } \\
\text { NMOQ } \%\end{array}$ & $\begin{array}{l}\text { OZONE } \\
\text { MG/MI }\end{array}$ \\
\hline METHYLPROPYLBENZENE & 0.0 & 0.00 & 0.0 & 0.0 & 0.00 & 0.0 & 0.0 & 0.00 & 0.0 & 0.0 & 0.00 & 0.00 \\
\hline S-BUTYLBENZENE & 0.2 & 0.09 & 0.4 & 0.0 & 0.00 & 0.0 & 0.0 & 0.00 & 0.0 & 0.0 & 0.07 & 0.08 \\
\hline 1-METHYL-3-ISOPROPYLBENZENE & 0.6 & 0.28 & 3.4 & 0.0 & 0.00 & 0.0 & 0.0 & 0.00 & 0.0 & 0.1 & 0.20 & 0.71 \\
\hline 1,2,3-TRIMETHYLBENZENE & 0.0 & 0.00 & 0.0 & 0.0 & 0.00 & 0.0 & 0.0 & 0.00 & 0.0 & 0.0 & 0.00 & 0.00 \\
\hline 1-METHYL-4-ISOPROPYLBENZENE & 0.0 & 0.00 & 0.0 & 0.0 & 0.00 & 0.0 & 0.0 & 0.00 & $\overline{0.0}$ & 0.0 & 0.00 & 0.00 \\
\hline INDAN & 0.2 & 0.11 & 0.3 & 0.0 & 0.00 & 0.0 & 0.0 & 0.00 & 0.0 & 0.1 & 0.08 & 0.05 \\
\hline 1-METHYL-2-ISOPROPYLBENZENE & 0.2 & 0.10 & 1.4 & 0.0 & 0.00 & 0.0 & 0.0 & 0.00 & 0.0 & 0.0 & 0.08 & 0.29 \\
\hline 1,3-DIETHYLBENZENE & 0.0 & 0.00 & 0.0 & 0.0 & 0.00 & 0.0 & 0.0 & 0.00 & 0.0 & 0.0 & 0.00 & 0.00 \\
\hline 1,4-DIETHYLBENZENE & 0.3 & 0.13 & 1.8 & 0.0 & 0.00 & 0.0 & 0.0 & 0.00 & 0.0 & 0.1 & 0.10 & 0.38 \\
\hline 1-METHYL-3-N-PROPYLBENZENE & 0.1 & 0.06 & 0.8 & 0.0 & 0.00 & 0.0 & 0.0 & 0.00 & 0.0 & 0.0 & 0.04 & 0.17 \\
\hline 1-METHYL-4-N-PROPYLBENZENE & 0.6 & 0.23 & 3.3 & 0.0 & 0.00 & 0.0 & 0.0 & 0.00 & 0.0 & 0.1 & 0.17 & 0.69 \\
\hline 1,2 DIETHYLBENZENE & 0.0 & 0.00 & 0.0 & 0.0 & 0.00 & 0.0 & 0.0 & 0.00 & 0.0 & 0.0 & 0.00 & 0.00 \\
\hline 1-METHYL-2-N-PROPYLBENZENE & 0.0 & 0.00 & 0.0 & 0.0 & 0.00 & 0.0 & $\overline{0.0}$ & 0.00 & 0.0 & 0.0 & 0.00 & 0.00 \\
\hline 1,4-DIMETHYL-2-ETHYLBENZENE & 0.3 & 0.12 & 2.5 & 0.0 & 0.00 & 0.0 & 0.0 & 0.00 & 0.0 & 0.1 & 0.09 & 0.52 \\
\hline 1,3-DIMETHYL-4-ETHYLBENZENE & 0.0 & 0.00 & 0.0 & 0.0 & 0.00 & 0.0 & 0.0 & 0.00 & 0.0 & 0.0 & 0.00 & 0.00 \\
\hline 1,2-DIMETHYL-4-ETHYLBENZENE & 0.4 & 0.18 & 3.6 & 0.0 & 0.00 & 0.0 & 0.0 & 0.00 & 0.0 & 0.1 & 0.13 & 0.75 \\
\hline 1,3-DIMETHYL-2-ETHYLBENZENE & 0.0 & 0.00 & 0.0 & 0.0 & 0.00 & 0.0 & 0.0 & 0.00 & 0.0 & 0.0 & 0.00 & 0.00 \\
\hline UNDECANE & 0.0 & 0.00 & 0.0 & 0.0 & 0.00 & 0.0 & 0.0 & 0.00 & 0.0 & 0.0 & 0.00 & 0.00 \\
\hline 1,2-DIMETHYL-3-ETHYLBENZENE & 0.0 & 0.00 & 0.0 & 0.0 & 0.00 & 0.0 & 0.0 & 0.00 & 0.0 & 0.0 & 0.00 & 0.00 \\
\hline 1,2,4,5-TETRAMETHYLBENZENE & 0.1 & 0.06 & 1.2 & 0.0 & 0.00 & 0.0 & 0.0 & 0.00 & 0.0 & 0.0 & 0.04 & 0.25 \\
\hline 2-METHYLBUTYLBENZENE (sec AMYLBENZENE) & 0.0 & 0.00 & 0.0 & 0.0 & 0.00 & 0.0 & 0.0 & 0.00 & 0.0 & 0.0 & 0.00 & 0.00 \\
\hline 3,4 DIMETHYLCUMENE & 0.0 & 0.00 & 0.0 & 0.0 & 0.00 & 0.0 & 0.0 & 0.00 & 0.0 & 0.0 & 0.00 & 0.00 \\
\hline 1,2,3,5-TETRAMETHYLBENZENE & 0.0 & 0.00 & 0.0 & 0.0 & 0.00 & 0.0 & 0.0 & 0.00 & 0.0 & 0.0 & 0.00 & 0.00 \\
\hline TEAT-1-BUT-2-METHYLBENZENE & 0.0 & 0.00 & 0.0 & 0.0 & 0.00 & 0.0 & 0.0 & 0.00 & 0.0 & 0.0 & 0.00 & 0.00 \\
\hline 1,2,3,4-TETRAMETHYLBENZENE & 0.0 & 0.00 & 0.0 & 0.0 & 0.00 & 0.0 & 0.0 & 0.00 & 0.0 & 0.0 & 0.00 & 0.00 \\
\hline N-PENT-BENZENE & 0.0 & 0.00 & 0.0 & 0.0 & 0.00 & 0.0 & 0.0 & 0.00 & 0.0 & 0.0 & 0.00 & 0.00 \\
\hline TEAT-1-BUT-3,5-DIMETHYLBENZENE & 0.0 & 0.00 & 0.0 & 0.0 & 0.00 & 0.0 & 0.0 & 0.00 & 0.0 & 0.0 & 0.00 & 0.00 \\
\hline NAPHTHALENE & 0.0 & 0.00 & 0.0 & 0.0 & 0.00 & 0.0 & 0.0 & 0.00 & 0.0 & 0.0 & 0.00 & 0.00 \\
\hline DODECANE & 0.0 & 0.00 & 0.0 & 0.0 & 0.00 & 0.0 & 0.0 & 0.00 & 0.0 & 0.0 & 0.00 & 0.00 \\
\hline UNIDENTIFIED $\mathrm{Cg}-\mathrm{C} 12+$ & 3.2 & 1.43 & 12.3 & $0 . \overline{4}$ & 5.56 & 1.6 & 0.6 & 1.50 & 2.2 & 1.0 & 1.70 & 3.97 \\
\hline FORMALDEHYDE & 1.4 & 0.62 & 10.0 & 0.0 & 0.00 & 0.0 & 0.0 & 0.00 & 0.0 & 0.3 & 0.47 & 2.08 \\
\hline ACETALDEHYDE & 0.8 & 0.28 & 3.5 & 0.4 & 4.75 & 1.9 & 0.0 & 0.09 & 0.2 & 0.3 & 0.63 & 1.78 \\
\hline ACROLEIN & 0.1 & 0.05 & 0.8 & 0.0 & 0.00 & 0.0 & 0.0 & 0.00 & 0.0 & 0.0 & 0.04 & 0.17 \\
\hline ACETONE & 1.6 & 0.65 & 0.8 & 1.7 & 23.38 & 1.0 & 1.4 & 3.61 & 0.8 & 1.6 & 2.57 & 0.88 \\
\hline PROPIONALDEHYDE & 0.4 & 0.19 & 2.8 & 0.3 & 4.68 & 2.3 & 0.0 & 0.00 & 0.0 & 0.3 & 0.44 & 1.75 \\
\hline CROTONALDEHYDE & 0.0 & 0.00 & 0.0 & 0.0 & 0.00 & 0.0 & 0.0 & 0.00 & 0.0 & 0.0 & 0.00 & 0.00 \\
\hline ISOBUTYRALDEHYDE & 0.1 & 0.05 & 0.6 & 0.1 & 1.78 & 0.7 & 0.1 & 0.28 & 0.6 & 0.1 & 0.20 & 0.63 \\
\hline METHYLETHYL. KETONE & 0.1 & 0.05 & 0.1 & 0.1 & 1.78 & 0.2 & 0.1 & 0.28 & 0.1 & 0.1 & 0.20 & 0.14 \\
\hline BENZALDEHYDE & 0.2 & 0.10 & -0.1 & 0.3 & 4.31 & -0.2 & 0.2 & 0.62 & -0.1 & 0.3 & 0.45 & -0.16 \\
\hline HEXANALDEHYDE & 0.0 & 0.00 & 0.0 & 0.0 & 0.00 & 0.0 & 0.0 & 0.00 & 0.0 & 0.0 & 0.00 & 0.00 \\
\hline
\end{tabular}


TEST NO: BLB-CP2-O2S

TEST CAR: 1994 PONTIAC GRANDAM

TEST DATE: $9 / 15 / 95$

TEST FUEL: EM-1968-F

\begin{tabular}{|c|c|c|c|c|c|c|c|c|c|c|c|c|}
\hline \multirow[b]{2}{*}{ COMPOUND } & \multicolumn{3}{|c|}{$\overline{B A G 1}$} & \multicolumn{3}{|c|}{ BAG 2} & \multicolumn{3}{|c|}{$\overline{B A G ~} 3$} & \multicolumn{3}{|c|}{ FTP } \\
\hline & MG/MI & \begin{tabular}{|l|} 
WEIGHT \\
NMOG $\%$
\end{tabular} & $\begin{array}{l}\text { OZONE, } \\
\text { MG/MI }\end{array}$ & MGMI & \begin{tabular}{|l|} 
WEIGHT \\
NMOG $\%$
\end{tabular} & $\begin{array}{l}\text { OZONE } \\
\text { MG/MI }\end{array}$ & Ma/MI & $\begin{array}{l}\text { WEIGHT } \\
\text { NMOG \% }\end{array}$ & $\begin{array}{l}\text { OZONE } \\
\text { MG/MI }\end{array}$ & MG/MI & $\begin{array}{l}\text { WEIGHT } \\
\text { NMOG } \%\end{array}$ & $\begin{array}{l}\text { OZONE } \\
\text { MG/MI }\end{array}$ \\
\hline METHANE & 22.4 & & 0.3 & 0.3 & & 0.0 & 8.9 & & 0.1 & 7.2 & & $\overline{0.11}$ \\
\hline ETHANE & 5.4 & 2.79 & 1.4 & 0.0 & 0.06 & 0.0 & 1.0 & 4.08 & 0.3 & 1.4 & 2.72 & 0.35 \\
\hline ETHYLENE & 12.8 & 6.62 & 93.7 & 0.0 & 0.19 & 0.1 & 0.9 & 3.64 & 6.4 & 2.9 & 5.65 & 21.30 \\
\hline PROPANE & 0.1 & 0.08 & 0.1 & 0.0 & 0.00 & 0.0 & 0.0 & 0.00 & 0.0 & 0.0 & 0.04 & 0.01 \\
\hline PROPYLENE & 11.1 & 5.70 & 104.0 & 0.0 & 0.00 & 0.0 & 0.0 & 0.00 & 0.0 & 2.3 & $\overline{4.44}$ & 21.61 \\
\hline ACETYLENE & 6.7 & 3.46 & 3.4 & 0.0 & 0.02 & 0.0 & 0.0 & 0.00 & 0.0 & 1.4 & 2.70 & 0.70 \\
\hline PROPADIENE & 0.0 & 0.00 & 0.0 & 0.0 & 0.00 & 0.0 & 0.0 & 0.00 & 0.0 & 0.0 & 0.00 & 0.00 \\
\hline BUTANE & 1.8 & 0.95 & 1.8 & 0.4 & 4.64 & 0.4 & 0.3 & 1.16 & 0.3 & 0.7 & 1.30 & 0.69 \\
\hline TRANS-2-BUTENE & 0.9 & 0.46 & 8.9 & 0.0 & 0.00 & 0.0 & 0.1 & 0.39 & 1.0 & $\overline{0.2}$ & $\overline{0.41}$ & 2.12 \\
\hline 1-BUTENE & 1.2 & 0.64 & 11.1 & 0.0 & 0.00 & 0.0 & 0.1 & 0.38 & 0.9 & 0.3 & 0.65 & 2.54 \\
\hline 2-METHYLPROPENE (ISOBUTYLENE) & 7.0 & 3.63 & 37.4 & 0.0 & 0.00 & 0.0 & 1.1 & 4.51 & 6.0 & 1.8 & 3.43 & 9.42 \\
\hline 2,2-DIMETHYLPROPANE (NEOPENTANE) & 0.1 & 0.05 & 0.0 & 0.0 & 0.00 & 0.0 & 0.0 & 0.00 & 0.0 & 0.0 & 0.04 & 0.01 \\
\hline PROPYNE & 0.0 & 0.00 & 0.0 & 0.0 & 0.00 & 0.0 & 0.0 & 0.00 & 0.0 & 0.0 & 0.00 & 0.00 \\
\hline 1,3-BUTADIENE & 1.4 & 0.73 & 15.4 & 0.0 & 0.00 & 0.0 & 0.0 & 0.00 & 0.0 & 0.3 & 0.57 & 3.19 \\
\hline 2-METHYLPAOPANE (ISOBUTANE) & 0.3 & 0.13 & 0.3 & 0.1 & 1.03 & 0.1 & 0.0 & 0.04 & 0.0 & 0.1 & 0.20 & 0.12 \\
\hline 1-BUTYNE & 0.0 & 0.00 & 0.0 & 0.0 & 0.00 & 0.0 & 0.0 & 0.00 & 0.0 & 0.0 & 0.00 & 0.00 \\
\hline METHANOL & 0.0 & 0.00 & 0.0 & 0.0 & 0.00 & 0.0 & 0.0 & 0.00 & 0.0 & 0.0 & 0.00 & 0.00 \\
\hline CIS-2-BUTENE & 0.7 & 0.34 & 6.6 & 0.0 & 0.00 & 0.0 & 0.1 & 0.26 & 0.6 & 0.2 & $\overline{0.30}$ & 1.54 \\
\hline 3-METHYL-1-BUTENE & 0.0 & 0.00 & 0.0 & 0.0 & 0.00 & 0.0 & 0.0 & 0.00 & 0.0 & 0.0 & 0.00 & 0.00 \\
\hline ETHANOL & 0.0 & 0.00 & 0.0 & 0.0 & 0.00 & 0.0 & 0.0 & 0.00 & 0.0 & 0.0 & 0.00 & 0.00 \\
\hline 2-METHYLBUTANE (ISOPENTANE) & 12.9 & 6.67 & 17.8 & 0.6 & 6.49 & 0.8 & 2.2 & 8.97 & 3.1 & 3.6 & 6.96 & 4.97 \\
\hline 2-BUTYNE & 0.0 & 0.00 & 0.0 & 0.0 & 0.00 & 0.0 & 0.0 & 0.00 & 0.0 & $\overline{0.0}$ & 0.00 & 0.00 \\
\hline 1-PENTENE & 0.0 & 0.00 & 0.0 & 0.0 & 0.00 & 0.0 & 0.1 & 0.47 & 0.7 & 0.0 & 0.08 & 0.20 \\
\hline 2-METHYL-1-BUTENE & 0.6 & 0.31 & 3.0 & 0.0 & 0.00 & 0.0 & 0.1 & $\overline{0.45}$ & 0.6 & 0.2 & 0,30 & 0.77 \\
\hline PENTANE & 1.7 & 0.86 & 1.7 & 0.4 & 4.93 & 0.5 & 0.4 & 1.56 & 0.4 & 0.7 & 1.31 & 0.70 \\
\hline UNIDENTIFIED C6 OLEFINS & 0.0 & 0.00 & 0.0 & 0.0 & 0.00 & 0.0 & 0.0 & 0.00 & 0.0 & 0.0 & 0.00 & 0.00 \\
\hline 2-METHYL-1,3-BUTADIENE & 0.9 & 0.44 & 7.8 & 0.0 & 0.00 & 0.0 & 0.2 & 10.74 & 1.7 & 0.2 & 0.44 & 2.08 \\
\hline TRANS-2-PENTENE & 0.4 & 0.21 & 3.7 & 0.0 & 0.00 & 0.0 & 0.1 & 0.41 & 0.9 & 0.1 & 0.22 & 1.01 \\
\hline 3,3-DIMETHYL-1-BUTENE & 0.0 & 0.00 & 0.0 & 0.0 & 0.00 & 0.0 & 0.0 & 0.00 & 0.0 & 0.0 & 0.00 & 0.00 \\
\hline CIS-2-PENTENE & 0.2 & 0.10 & 1.8 & 0.0 & 0.00 & 0.0 & 0.0 & 0.00 & 0.0 & 0.0 & 0.08 & 0.37 \\
\hline 2-METHYL-2-BUTENE & 1.1 & 0.58 & 7.2 & 0.0 & 0.00 & 0.0 & 0.0 & 0.00 & 0.0 & 0.2 & 0.46 & 1.49 \\
\hline CYCLOPENTADIENE & $0 . \overline{0}$ & 0.23 & 3.6 & 0.0 & 0.00 & 0.0 & 0.0 & 0.00 & 0.0 & 0.1 & 0.18 & 0.72 \\
\hline 2,2-DIMETHYLBUTANE & 0.3 & 0.16 & 0.2 & 0.0 & 0.00 & 0.0 & 0.0 & 0.00 & 0.0 & 0.1 & 0.12 & 0.05 \\
\hline CYCLOPENTENE & 0.1 & 0.05 & 0.8 & 0.0 & 0.00 & 0.0 & 0.0 & 0.00 & 0.0 & 0.0 & 0.04 & 0.17 \\
\hline 4-METHYL-1-PENTENE & 0.1 & 0.06 & 0.6 & 0.0 & 0.00 & 0.0 & 0.0 & 0.00 & 0.0 & 0.0 & 0.05 & 0.10 \\
\hline 3-METHYL-1-PENTENE & 0.1 & 0.06 & 0.5 & 0.0 & 0.00 & 0.0 & 0.0 & 0.00 & 0.0 & 0.0 & 0.05 & 0.11 \\
\hline CYCLOPENTANE & 0.0 & 0.00 & 0.0 & 0.0 & 0.00 & 0.0 & 0.0 & 0.00 & 0.0 & 0.0 & 0.00 & 0.00 \\
\hline 2,3-DIMETHYLBUTANE & 1.2 & 0.60 & 1.3 & 0.3 & 3.01 & 0.3 & 0.3 & 1.24 & 0.3 & 0.5 & 0.90 & 0.60 \\
\hline MTBE & 8.3 & 4.29 & 5.2 & 0.0 & 0.00 & 0.0 & 0.0 & 0.00 & 0.0 & 1.7 & 3.34 & 1.07 \\
\hline
\end{tabular}




\begin{tabular}{|c|c|c|c|c|c|c|c|c|c|c|c|c|}
\hline \multirow[b]{2}{*}{ COMPOUND } & \multicolumn{3}{|c|}{ BAG 1} & \multicolumn{3}{|c|}{ BAG 2} & \multicolumn{3}{|c|}{ BAG 3} & \multicolumn{3}{|c|}{ FTP } \\
\hline & MG/MI & $\begin{array}{l}\text { WEIGHT } \\
\text { NMOG } \%\end{array}$ & $\begin{array}{l}\text { OZONE, } \\
\text { MG/MI }\end{array}$ & MG/MI & $\begin{array}{l}\text { WEIGHT } \\
\text { NMOG \% }\end{array}$ & $\begin{array}{l}\text { OZONE } \\
\text { MG/MI }\end{array}$ & MGMI & $\begin{array}{l}\text { WEIGHT } \\
\text { NMOQ \% }\end{array}$ & $\begin{array}{l}\text { OZONE } \\
\text { MGMIMI }\end{array}$ & Ma/MI & $\begin{array}{l}\text { WEIGHT } \\
\text { NMOG \% }\end{array}$ & $\begin{array}{l}\text { OZONE } \\
\text { MG/MI }\end{array}$ \\
\hline 2,3-DIMETHYL-1-8UTENE & 0.0 & 0.00 & 0.0 & 0.0 & 0.00 & 0.0 & 0.0 & 0.00 & 0.0 & 0.0 & 0.00 & 0.00 \\
\hline 4-METHYL-CIS-2-PENTENE & 0.0 & 0.00 & 0.0 & 0.0 & 0.00 & 0.0 & 0.0 & 0.00 & 0.0 & 0.0 & 0.00 & 0.00 \\
\hline 2-METHYLPENTANE & 1.7 & 0.88 & 2.6 & 1.0 & 10.99 & 1.5 & 0.7 & 2.82 & 1.1 & 1.1 & 2.03 & 1.61 \\
\hline 4-METHYL-TRANS-2-PENTENE & 0.0 & 0.00 & 0.0 & 0.0 & 0.00 & 0.0 & 0.0 & 0.00 & 0.0 & 0.0 & 0.00 & 0.00 \\
\hline 3-METHYLPENTANE & 1.2 & 0.60 & 1.8 & 0.6 & 5.79 & 0.8 & 0.4 & 1.63 & 0.6 & 0.6 & 1.10 & 0.94 \\
\hline 2-METHYL-1-PENTENE & 0.2 & 0.08 & 0.7 & 0.1 & 0.87 & 0.3 & 0.1 & 0.21 & 0.2 & 0.1 & 0.17 & 0.38 \\
\hline 1-HEXENE & 0.2 & 0.08 & 0.7 & 0.1 & 0.87 & 0.3 & 0.1 & 0.21 & 0.2 & 0.1 & 0.17 & 0.38 \\
\hline HEXANE & 0.8 & 0.46 & 0.9 & 0.6 & 6.50 & 0.6 & 0.4 & 1.41 & 0.3 & 0.6 & 1.12 & 0.57 \\
\hline UNIDENTIFIED C 6 OLEFINS & 0.2 & 0.13 & 1.7 & 0.0 & 0.00 & 0.0 & 0.0 & 0.00 & 0.0 & 0.1 & 0.10 & 0.34 \\
\hline TRANS-3-HEXENE & 0.2 & 0.10 & 1.3 & 0.0 & 0.00 & 0.0 & 0.1 & $\overline{0.43}$ & 0.7 & 0.1 & 0.14 & 0.48 \\
\hline CIS-3-HEXENE & 0.2 & 0.10 & 1.4 & 0.0 & 0.45 & 0.3 & 0.0 & 0.17 & 0.3 & 0.1 & 0.14 & 0.50 \\
\hline TRANS-2-HEXENE & 0.0 & 0.00 & 0.0 & 0.0 & 0.00 & $\overline{0.0}$ & 0.0 & 0.00 & 0.0 & 0.0 & 0.00 & 0.00 \\
\hline 3-METHYL-TRANS-2-PENTENE & 0.3 & 0.17 & 2.2 & 0.0 & 0.00 & 0.0 & 0.1 & 0.35 & 0.6 & 0.1 & 0.18 & 0.63 \\
\hline 2-METHYL-2-PENTENE & 0.2 & 0.13 & 1.7 & 0.0 & 0.00 & $\overline{0.0}$ & 0.1 & 0.35 & 0.6 & 0.1 & 0.15 & 0.51 \\
\hline 3-METHYLCYCLOPENTENE & 0.0 & 0.00 & 0.0 & 0.0 & 0.00 & $\overline{0.0}$ & 0.0 & 0.00 & 0.0 & 0.0 & 0.00 & 0.00 \\
\hline CIS-2-HEXENE & 0.0 & 0.00 & 0.0 & 0.0 & 0.00 & 0.0 & 0.0 & 0.00 & 0.0 & 0.0 & 0.00 & 0.00 \\
\hline ETBE & 0.0 & 0.00 & 0.0 & 0.0 & 0.00 & $\overline{0.0}$ & 0.0 & 0.00 & 0.0 & 0,0 & 0.00 & 0.00 \\
\hline 3-METHYL-CIS-2-PENTENE & 0.3 & 0.14 & 1.9 & 0.0 & 0.00 & 0.0 & 0.1 & 0.38 & 0.6 & 0.1 & 0.16 & 0.55 \\
\hline 2,2-DIMETHYLPENTANE & $\overline{0.6}$ & 0.31 & 0.9 & 0.2 & 2.27 & $\overline{0.3}$ & 0.2 & 0.64 & 0.2 & 0.3 & 0.53 & 0.38 \\
\hline METHYLCYCLOPENTANE & 0.1 & 0.05 & 0.3 & 0.0 & 0.00 & 0.0 & 0.0 & 0.00 & 0.0 & 0.0 & 0.04 & 0.05 \\
\hline 2,4-DIMETHYLPENTANE & 3.8 & 1.94 & 6.7 & 0.3 & 3.02 & 0.5 & 0,8 & 3.19 & 1.4 & 1.1 & 2.20 & 2.03 \\
\hline 2,3,3-TRIMETHYL-1-BUTENE & 0.0 & 0.00 & 0.0 & 0.0 & 0.00 & 0.0 & 0.0 & 0.00 & 0.0 & 0.0 & 0.00 & 0.00 \\
\hline 2,2,3-TRIMETHYLBUTANE & 0.3 & 0.14 & 0.4 & $\overline{0.1}$ & 1.03 & 0.1 & 0.0 & 0.13 & 0.0 & 0.1 & 0.22 & $\overline{0.15}$ \\
\hline 3,4-DIMETHYL-1-PENTENE & 0.0 & 0.00 & 0.0 & 0.0 & 0.00 & 0.0 & 0.0 & 0.00 & 0.0 & 0.0 & 0.00 & 0.00 \\
\hline 1-METHYLCYCLOPENTENE & 0.3 & 0.16 & 1.8 & 0.2 & 2.48 & 1.2 & 0.1 & 0.34 & 0.5 & 0.2 & 0.39 & 1.14 \\
\hline BENZENE & 7.8 & 4.04 & 3.3 & 0.2 & 2.10 & 0.1 & 2.5 & 10.04 & 1.1 & 2.4 & 4.66 & 1.01 \\
\hline 3-METHYL-1-HEXENE & 0.0 & 0.00 & 0.0 & 0.0 & 0.00 & 0.0 & 0.0 & 0.00 & 0.0 & 0.0 & 0.00 & 0.00 \\
\hline 3,3-DIMETHYLPENTANE & 0.2 & 0.13 & 0.2 & 0.1 & 1.64 & 0.1 & 0.1 & 0.48 & 0.1 & 0.2 & 0.31 & $\overline{0.11}$ \\
\hline CYCLOHEXANE & 0.2 & 0.12 & 0.3 & 0.0 & 0.00 & 0.0 & 0.2 & 0.82 & 0.3 & 0.1 & 0.20 & 0.13 \\
\hline 2-METHYLHEXANE & 0.0 & 0.00 & 0.0 & 0.0 & 0.00 & 0.0 & 0.0 & 0.00 & 0.0 & 0.0 & 0.00 & 0.00 \\
\hline 2,3-DIMETHYLPENTANE & 7.7 & 3.96 & 11.6 & 0.6 & 7.05 & 0.9 & 1.7 & 6.62 & 2.5 & 2.4 & 4.59 & 3.58 \\
\hline 1,1-DIMETHYLCYCLOPENTANE & 0.0 & 0.00 & 0.0 & 0.0 & 0.00 & 0.0 & 0.0 & 0.00 & 0.0 & 0.0 & 0.00 & 0.00 \\
\hline CYCLOHEXENE & 0.0 & 0.00 & 0.0 & 0.0 & 0.00 & 0.0 & 0.0 & 0.00 & 0.0 & 0.0 & 0.00 & 0.00 \\
\hline 3-METHYLHEXANE & 1.3 & 0.69 & 1.8 & 0.2 & 2.80 & 0.3 & 0.4 & 1.48 & 0.5 & 0.5 & 0.98 & 0.71 \\
\hline CIS-1,3-DIMETHYLCYCLOPENTANE & 0.2 & 0.08 & 0.4 & 0.1 & 0.72 & 0.2 & 0.0 & 0.12 & 0.1 & 0.1 & 0.14 & 0.18 \\
\hline 3-ETHYLPENTANE & 0.0 & 0.00 & 0.0 & 0.0 & 0.00 & 0.0 & 0.0 & 0.00 & 0.0 & 0.0 & 0.00 & 0.00 \\
\hline TRANS-1,2-DIMETHYLCYCLOPENTANE & 0.0 & 0.00 & 0.0 & 0.0 & 0.00 & 0.0 & 0.0 & 0.00 & 0.0 & 0.0 & 0.00 & 0.00 \\
\hline TRANS-1,3-DIMETHYLCYCLOPENTANE & 0.0 & 0.00 & 0.0 & 0.0 & 0.00 & 0.0 & 0.0 & 0.00 & 0.0 & 0.0 & 0.00 & 0.00 \\
\hline 1-HEPTENE & 0.0 & 0.00 & 0.0 & 0.0 & 0.00 & 0.0 & 0.0 & 0.00 & 0.0 & 0.0 & 0.00 & 0.00 \\
\hline 2,2,4-TAIMETHYLPENTANE & 13.4 & 6.89 & 12.4 & 0.5 & 5.98 & 0.6 & 3.3 & 13.26 & 3.1 & 4.0 & 7.68 & 3.68 \\
\hline 2-METHYL-1-HEXENE & 0.0 & 0.00 & 0.0 & 0.0 & 0.00 & 0.0 & 0.0 & 0.00 & 0.0 & 0.0 & 0.00 & 0.00 \\
\hline TRANS-3-HEPTENE & 0.0 & 0.00 & 0.0 & 0.0 & 0.00 & 0.0 & 0.0 & 0.00 & 0.0 & 0.0 & 0.00 & 0.00 \\
\hline HEPTANE & 0.8 & 0.44 & 0.7 & 0.0 & 0.09 & 0.0 & 0.1 & 0.42 & 0.1 & 0.2 & 0.40 & 0.17 \\
\hline UNIDENTIFIED C7 & 1.0 & 0.61 & 4.4 & 0.0 & 0.00 & 0.0 & 0.0 & 0.00 & 0.0 & 0.2 & 0.39 & 0.92 \\
\hline
\end{tabular}




\begin{tabular}{|c|c|c|c|c|c|c|c|c|c|c|c|c|}
\hline \multirow[b]{2}{*}{ COMPOUND } & \multicolumn{3}{|c|}{ BAG 1 } & \multicolumn{3}{|c|}{ BAG 2} & \multicolumn{3}{|c|}{ BAG 3} & \multicolumn{3}{|c|}{ FTP } \\
\hline & MGIMI & $\begin{array}{l}\text { WEIGHT } \\
\text { NMOG \% }\end{array}$ & $\begin{array}{l}\text { OZONE, } \\
\text { MG/MI }\end{array}$ & MG/MI & \begin{tabular}{|l|} 
WEIGHT \\
NMOQ \% \\
\end{tabular} & $\begin{array}{l}\text { OZONE } \\
\text { MGMMI }\end{array}$ & MG/MI & \begin{tabular}{|l|} 
WEIGHT \\
NMOG \% \\
\end{tabular} & $\begin{array}{l}\text { OZONE } \\
\text { MG/MI }\end{array}$ & MG/MI & $\begin{array}{l}\text { WEIGHT } \\
\text { NMOG } \%\end{array}$ & $\begin{array}{l}\text { OZONE } \\
\text { MG/MI }\end{array}$ \\
\hline 2-METHYL-2-HEXENE & 0.2 & 0.09 & 1.0 & 0.0 & 0.00 & 0.0 & 0.0 & 0.00 & 0.0 & 0.0 & 0.07 & 0.21 \\
\hline 3-METHYL-TRANS-3-HEXENE & 0.0 & 0.00 & 0.0 & 0.0 & 0.00 & 0.0 & 0.0 & 0.00 & 0.0 & 0.0 & 0.00 & 0.00 \\
\hline TRANS-2-HEPTENE & 0.1 & 0.05 & 0.6 & 0.0 & 0.00 & 0.0 & 0.0 & 0.00 & 0.0 & 0.0 & 0.04 & 0.13 \\
\hline 3-ETHYL-CIS-2-PENTENE & 0.0 & 0.00 & 0.0 & 0.0 & 0.00 & 0.0 & 0.0 & 0.00 & 0.0 & 0.0 & 0.00 & 0.00 \\
\hline 2,4,4-TRIMETHYL-1-PENTENE & 0.1 & 0.06 & 0.3 & 0.0 & 0.00 & 0.0 & 0.0 & 0.00 & 0.0 & 0.0 & 0.05 & 0.07 \\
\hline 2,2,4-TRIMETHYL-1-PENTENE & 0.0 & 0.00 & 0.0 & 0.0 & 0.00 & 0.0 & 0.0 & 0.00 & 0.0 & 0.0 & 0.00 & 0.00 \\
\hline 2,3-DIMETHYL-2-PENTENE & 0.0 & 0.00 & 0.0 & 0.0 & 0.00 & 0.0 & 0.0 & 0.00 & 0.0 & 0.0 & 0.00 & 0.00 \\
\hline CIS-2-HEPTENE & 0.0 & 0.00 & 0.0 & 0.0 & 0.00 & 0.0 & 0.0 & 0.00 & 0.0 & 0.0 & 0.00 & 0.00 \\
\hline METHYLCYCLOHEXANE & 0.1 & 0.07 & 0.3 & 0.0 & 0.00 & 0.0 & 0.0 & 0.00 & 0.0 & 0.0 & 0.06 & 0.08 \\
\hline CIS-1,2-DIMETHYLCYCLOPENTANE & 0.0 & 0.00 & 0.0 & 0.0 & 0.00 & 0.0 & 0.0 & 0.00 & 0.0 & 0.0 & 0.00 & 0.00 \\
\hline 2,2-DIMETHYLHEXANE & 1.2 & 0.62 & 1.5 & 0.2 & 2.33 & 0.2 & 0.3 & 1.06 & 0.3 & 0.4 & 0.83 & 0.62 \\
\hline 1,1,3-TAIMETHYLCYCLOPENTANE & 0.0 & 0.00 & 0.0 & 0.0 & 0.00 & 0.0 & 0.0 & 0.00 & 0.0 & 0.0 & 0.00 & 0.00 \\
\hline 2,4,4-TRIMETHYL-2-PENTENE & 0.0 & 0.00 & 0.0 & 0.0 & 0.00 & 0.0 & 0.0 & 0.00 & 0.0 & 0.0 & 0.00 & 0.00 \\
\hline 2,2,3-TRIMETHYLPENTANE & 0.0 & 0.00 & 0.0 & 0.0 & 0.00 & 0.0 & 0.0 & 0.00 & 0.0 & 0.0 & 0.00 & 0.00 \\
\hline 2,5-DIMETHYLHEXANE & 0.0 & 0.00 & 0.0 & 0.0 & 0.00 & 0.0 & 0.0 & 0.00 & 0.0 & 0.0 & 0.00 & 0.00 \\
\hline ETHYLOYCLOPENTANE & 0.0 & 0.00 & 0.0 & 0.0 & 0.00 & 0.0 & 0.0 & 0.00 & 0.0 & 0.0 & 0.00 & 0.00 \\
\hline 2,4-DIMETHYLHEXANE & 1.5 & 0.77 & 2.2 & 0.1 & 1.68 & 0.2 & 0.3 & 1.24 & 0.5 & 0.5 & 0.91 & 0.70 \\
\hline 1-TRANS-2-CIS-4-TRIMETHYLCYCLOPENTANE & 0.0 & 0.00 & 0.0 & 0.0 & 0.00 & 0.0 & 0.0 & 0.00 & 0.0 & 0.0 & 0.00 & 0.00 \\
\hline 3,3-DIMETHYLHEXANE & 0.0 & 0.00 & 0.0 & 0.0 & 0.00 & 0.0 & 0.0 & 0.00 & 0.0 & 0.0 & 0.00 & 0.00 \\
\hline 1-TRANS-2-CIS-3-TAIMETHYLCYCLOPENTANE & 0.0 & 0.00 & 0.0 & 0.0 & 0.00 & 0.0 & 0.0 & 0.00 & 0.0 & 0.0 & 0.00 & 0.00 \\
\hline 2,3,4-TAIMETHYLPENTANE & 0.0 & 0.00 & 0.0 & 0.0 & 0.00 & 0.0 & 0.0 & 0.00 & 0.0 & 0.0 & 0.00 & 0.00 \\
\hline 2,3,3-TRIMETHYLPENTANE & 2.7 & 1.41 & 3.3 & 0.0 & 0.13 & 0.0 & 0.7 & 2.76 & 0.8 & 0.8 & 1.48 & 0.92 \\
\hline TOLUENE & 17.4 & 8.98 & 47.5 & 0.9 & 10.50 & 2.5 & 2.2 & 8.92 & 6.1 & 4.7 & 9.10 & 12.86 \\
\hline 2,3-DIMETHYLHEXANE & 2.0 & 1.02 & 2.6 & 0.0 & 0.00 & 0.0 & 0.0 & 0.00 & 0.0 & 0.4 & 0.80 & 0.65 \\
\hline 1,1,2-TAIMETHYLCYCLOPENTANE & 0.0 & 0.00 & 0.0 & 0.0 & 0.00 & 0.0 & 0.0 & 0.00 & 0.0 & 0.0 & 0.00 & 0.00 \\
\hline 2-METHYLHEPTANE & 1.0 & 0.63 & 1.0 & 0.0 & 0.00 & 0.0 & 0.2 & 0.82 & 0.2 & 0.3 & 0.52 & 0.26 \\
\hline 3,4-DIMETHYLHEXANE & 0.0 & 0.00 & 0.0 & 0.0 & 0.00 & 0.0 & 0.0 & 0.00 & 0.0 & 0.0 & 0.00 & 0.00 \\
\hline 2,2,4,4-TETRAMETHYLPENTANE & 0.0 & 0.00 & 0.0 & 0.0 & 0.00 & 0.0 & 0.0 & 0.00 & 0.0 & 0.0 & 0.00 & 0.00 \\
\hline 4-METHYLHEPTANE & 0.3 & 0.17 & 0.4 & 0.0 & 0.00 & 0.0 & 0.2 & 0.83 & 0.2 & 0.1 & 0.24 & 0.15 \\
\hline 2-METHYL-3-ETHYLPENTANE & 0.0 & 0.00 & 0.0 & 0.0 & 0.00 & 0.0 & 0.0 & 0.00 & 0.0 & 0.0 & 0.00 & 0.00 \\
\hline 2,6-DIMETHYLHEPTANE & 0.0 & 0.00 & 0.0 & 0.0 & 0.00 & 0.0 & 0.0 & 0.00 & 0.0 & 0.0 & 0.00 & 0.00 \\
\hline 3-METHYLHEPTANE & 1.0 & 0.53 & 1.0 & 0.0 & 0.00 & 0.0 & 0.0 & 10.00 & 0.0 & 0.2 & 0.41 & 0.21 \\
\hline 1-CIS,2-TRANS,3-TRIMETHYLCYCLOPENTANE & 0.0 & 0.00 & 0.0 & 0.0 & 0.00 & 0.0 & 0.0 & 0.00 & 0.0 & 0.0 & 0.00 & 0.00 \\
\hline CIS-1,3-DIMETHYLCYCLOHEXANE & 0.0 & 0.00 & 0.0 & 0.0 & 0.00 & 0.0 & 0.0 & 0.00 & 0.0 & 0.0 & 0.00 & 0.00 \\
\hline TRANS-1,4-DIMETHYLCYCLOHEXANE & 1.2 & 0.62 & 2.3 & 0.0 & 0.00 & 0.0 & 0.3 & 1.34 & 0.6 & 0.3 & 0.68 & 0.66 \\
\hline 3-ETHYLHEXANE & 0.0 & 0.00 & 0.0 & 0.0 & 0.00 & 0.0 & 0.0 & 0.00 & 0.0 & 0.0 & 0.00 & 0.00 \\
\hline 2,2,5-TAIMETHYLHEXANE & 0.0 & 0.00 & 0.0 & 0.0 & 0.00 & 0.0 & 0.0 & 0.00 & 0.0 & 0.0 & 0.00 & 0.00 \\
\hline CIS-1-METHYL-3-ETHYLCYCLOPENTANE & 0.0 & 0.00 & 0.0 & 0.0 & 0.00 & 0.0 & 0.0 & 0.00 & 0.0 & 0.0 & 0.00 & 0.00 \\
\hline 1,1-DIMETHYLCYCLOHEXANE & 0.0 & 0.00 & 0.0 & 0.0 & 0.00 & 0.0 & 0.0 & 0.00 & 0.0 & 0.0 & 0.00 & 0.00 \\
\hline TRANS-1-METHYL-2-ETHYLCYCLOPENTANE & 0.0 & 0.00 & 0.0 & 0.0 & 0.00 & 0.0 & 0.0 & 0.00 & 0.0 & 0.0 & 0.00 & 0.00 \\
\hline 1-METHYL-1-ETHYL-CYCLOPENTANE & 0.0 & 0.00 & 0.0 & 0.0 & 0.00 & 0.0 & 0.0 & 0.00 & 0.0 & 0.0 & 0.00 & 0.00 \\
\hline 2,4,4-TAIMETHYLHEXANE & 0.0 & 0.00 & 0.0 & 0.0 & 0.00 & 0.0 & 0.0 & 0.00 & 0.0 & 0.0 & 0.00 & 0.00 \\
\hline 2,2,4-TAIMETHYLHEXANE & 0.0 & 0.00 & 0.0 & 0.0 & 0.00 & 0.0 & 0.0 & 0.00 & 0.0 & 0.0 & 0.00 & 0.00 \\
\hline TRANS-1,2-DIMETHYLCYCLOHEXANE & 0.0 & 0.00 & 0.0 & 0.0 & 0.00 & 0.0 & 0.0 & 0.00 & 0.0 & 0.0 & 0.00 & 0.00 \\
\hline
\end{tabular}




\begin{tabular}{|c|c|c|c|c|c|c|c|c|c|c|c|c|}
\hline \multirow[b]{2}{*}{ COMPOUND } & \multicolumn{3}{|c|}{ BAG 1} & \multicolumn{3}{|c|}{ BAG 2} & \multicolumn{3}{|c|}{ BAG 3} & \multicolumn{3}{|c|}{ FTP } \\
\hline & MG/MI & $\begin{array}{l}\text { WEIGHT } \\
\text { NMOG \% }\end{array}$ & $\begin{array}{l}\text { OZONE, } \\
\text { MGMI }\end{array}$ & MG/MI & $\begin{array}{l}\text { WEIGHT } \\
\text { NMOG \% }\end{array}$ & $\begin{array}{l}\text { OZONE } \\
\text { MG/MI }\end{array}$ & MG/MI & $\begin{array}{l}\text { WEIGHT } \\
\text { NMOG \% }\end{array}$ & $\begin{array}{l}\text { OZONE } \\
\text { MG/MI }\end{array}$ & MG/MI & $\begin{array}{l}\text { WEIGHT } \\
\text { NMOG \% }\end{array}$ & $\begin{array}{l}\text { OZONE } \\
\text { MG/MI }\end{array}$ \\
\hline 1-OCTENE & 0.1 & 0.08 & 0.4 & 0.0 & 0.00 & 0.0 & 0.0 & 0.00 & 0.0 & 0.0 & 0.06 & 0.08 \\
\hline TRANS-4-OCTENE & 0.0 & 0.00 & 0.0 & 0.0 & 0.00 & 0.0 & 0.0 & 0.00 & 0.0 & 0.0 & 0.00 & 0.00 \\
\hline OCTANE & 0.6 & 0.32 & 0.4 & 0.0 & 0.00 & 0.0 & 0.1 & 0.60 & 0.1 & 0.2 & 0.33 & 0.10 \\
\hline UNIDENTIFIED C8 & 0.9 & 0.46 & 3.0 & 0.1 & 1.46 & 0.4 & 0.1 & 0.60 & 0.6 & 0.3 & 0.57 & 1.00 \\
\hline TRANS-2-OCTENE & 0.1 & 0.05 & 0.5 & 0.0 & 0.00 & 0.0 & 0.0 & 0.00 & 0.0 & 0.0 & 0.04 & 0.11 \\
\hline TRANS-1,3-DIMETHYLCYCLOHEXANE & 0.0 & 0.00 & 0.0 & 0.0 & 0.00 & 0.0 & 0.0 & 0.00 & 0.0 & 0.0 & 0.00 & 0.00 \\
\hline CIS-1,4-DIMETHYLCYCLOHEXANE & 0.0 & 0.00 & 0.0 & 0.0 & 0.00 & 0.0 & 0.0 & 0.00 & 0.0 & 0.0 & 0.00 & 0.00 \\
\hline CIS-2-OCTENE & 0.0 & 0.00 & 0.0 & 0.0 & 0.00 & 0.0 & 0.0 & 0.00 & 0.0 & 0.0 & 0.00 & 0.00 \\
\hline 2,3,5-TAIMETHYLHEXANE & 0.2 & 0.00 & 0.2 & 0.0 & 0.00 & 0.0 & 0.0 & 0.00 & 0.0 & 0.0 & 0.07 & 0.04 \\
\hline C15-1-METHYL-2-ETHYLCYCLOPENTANE & 0.0 & 0.00 & 0.0 & 0.0 & 0.00 & 0.0 & 0.0 & 0.00 & 0.0 & 0.0 & 0.00 & 0.00 \\
\hline 2-METHYL-2-ETHYLHEPTANE & 0.0 & 0.00 & 0.0 & 0.0 & 0.00 & 0.0 & 0.0 & 0.00 & 0.0 & 0.0 & 0.00 & 0.00 \\
\hline 2,4-DIMETHYLHEPTANE & 0.1 & 0.07 & 0.2 & 0.2 & 2.31 & 0.3 & 0.5 & 2.10 & 0.7 & 0.3 & 0.53 & 0.37 \\
\hline 4.4-DIMETHYLHEPTANE & 0.0 & 0.00 & 0.0 & 0.0 & 0.00 & 0.0 & 0.0 & 0.00 & 0.0 & 0.0 & 0.00 & 0.00 \\
\hline CIS-1,2-DIMETHYLCYCLOHEXANE & 0.2 & 0.10 & 0.4 & 0.0 & 0.00 & 0.0 & 0.0 & 0.00 & 0.0 & 0.0 & 0.08 & 0.08 \\
\hline ETHYLCYCLOHEXANE & 0.3 & 0.14 & 0.5 & 0.0 & 0.00 & 0.0 & 0.0 & 0.00 & 0.0 & 0.1 & 0.11 & 0.11 \\
\hline PROPYLCYCLOHEXANE & 0.0 & 0.00 & 0.0 & 0.0 & 0.00 & 0.0 & 0.0 & 0.00 & 0.0 & 0.0 & 0.00 & 0.00 \\
\hline 2-METHYL-A-ETHYLHEXANE & 0.0 & 0.00 & 0.0 & 0.0 & 0.00 & 0.0 & 0.0 & 0.00 & 0.0 & 0.0 & 0.00 & 0.00 \\
\hline 2,6-DIMETHYLHEPTANE & 0.0 & 0.00 & 0.0 & 0.0 & 0.00 & 0.0 & 0.0 & 0.00 & 0.0 & 0.0 & 0.00 & 0.00 \\
\hline 1,1,3-TRIMETHYLCYCLOHEXANE & 0.0 & 0.00 & 0.0 & 0.0 & 0.00 & 0.0 & 0.0 & 0.00 & 0.0 & 0.0 & 0.00 & 0.00 \\
\hline 2,5-DIMETHYLHEPTANE & 0.0 & 0.00 & 0.0 & 0.0 & 0.00 & 0.0 & 0.0 & 0.00 & 0.0 & 0.0 & 0.00 & 0.00 \\
\hline 3,3-DIMETHYLHEPTANE & 0.0 & 0.00 & 0.0 & 0.0 & 0.00 & 0.0 & 0.0 & 0.00 & 0.0 & 0.0 & 0.00 & 0.00 \\
\hline 3,6-DIMETHYLHEPTANE & 0.0 & 0.00 & 0.0 & 0.0 & 0.00 & 0.0 & 0.0 & 0.00 & 0.0 & 0.0 & 0.00 & 0.00 \\
\hline ETHYLBENZENE & 5.3 & 2.73 & 14.3 & 0.1 & 0.63 & 0.1 & 0.9 & 3.72 & 2.5 & 1.4 & 2.68 & 3.74 \\
\hline 2,3-DIMETHYLHEPTANE & 0.0 & 0.00 & 0.0 & 0.0 & 0.00 & 0.0 & 0.0 & 0.00 & 0.0 & 0.0 & 0.00 & 0.00 \\
\hline m-\&p-XYLENE & 12.1 & 6.22 & 89.1 & 0.2 & 1.73 & 1.1 & 0.0 & 0.00 & 0.0 & 2.6 & 5.00 & 19.10 \\
\hline 4-METHYLOCTANE & 0.0 & 0.00 & 0.0 & 0.0 & 0.00 & 0.0 & 0.0 & 0.00 & 0.0 & 0.0 & 0.00 & 0.00 \\
\hline 2-METHYLOCTANE & 0.6 & 0.27 & 0.6 & 0.0 & 0.00 & 0.0 & 0.0 & 0.00 & 0.0 & 0.1 & 0.21 & 0.12 \\
\hline 3-METHYLOCTANE & 0.3 & 0.15 & 0.3 & 0.0 & 0.00 & 0.0 & 0.0 & 0.00 & 0.0 & 0.1 & 0.12 & 0.07 \\
\hline STYRENE & 0.0 & 0.00 & 0.0 & 0.0 & 0.00 & 0.0 & 0.0 & 0.00 & 0.0 & 0.0 & 0.00 & 0.00 \\
\hline O-XYLENE & 4.0 & 2.08 & 26.0 & 0.1 & 1.58 & 0.9 & 0.4 & 1.48 & 2.4 & 1.0 & 1.95 & 6.53 \\
\hline 2,4,6-TAIMETHYLHEXANE & 0.0 & 0.00 & 0.0 & 0.0 & 0.00 & 0.0 & 0.0 & 0.00 & 0.0 & 0.0 & 0.00 & 0.00 \\
\hline 1-NONENE & 0.3 & 0.13 & 0.6 & 0.0 & 0.00 & 0.0 & 0.0 & 0.00 & 0.0 & 0.1 & 0.10 & 0.12 \\
\hline NONANE & 0.2 & 0.11 & 0.1 & 0.0 & 0.00 & 0.0 & 0.0 & 0.00 & 0.0 & 0.0 & 0.09 & 0.02 \\
\hline ISOPROPYLBENZENE (CUMENE) & 0.2 & 0.08 & 0.3 & 0.0 & 0.00 & 0.0 & 0.0 & 0.00 & 0.0 & 0.0 & 0.06 & 0.07 \\
\hline 2,2-DIMETHYLOCTANE & 0.1 & 0.07 & 0.1 & 0.0 & 0.00 & 0.0 & 0.0 & 0.00 & 0.0 & 0.0 & 0.05 & 0.03 \\
\hline 2,4-DIMETHYLOCTANE & 0.0 & 0.00 & 0.0 & 0.0 & 0.00 & 0.0 & 0.0 & 0.00 & 0.0 & 0.0 & 0.00 & 0.00 \\
\hline n-PROPYLBENZENE & 0.5 & 0.28 & 1.2 & 0.0 & 0.00 & 0.0 & 0.0 & 0.00 & 0.0 & 0.1 & 0.22 & 0.24 \\
\hline 1-METHYL-3-ETHYLBENZENE & 2.6 & 1.34 & 18.8 & 0.0 & 0.00 & 0.0 & 0.1 & 0.41 & 0.7 & 0.6 & 1.10 & 4.10 \\
\hline 1-METHYL-4-ETHYLBENZENE & 1.1 & 0.54 & 7.6 & 0.0 & 0.00 & 0.0 & 0.0 & 0.00 & 0.0 & 0.2 & 0.42 & 1.68 \\
\hline 1,3,5-TRIMETHYLBENZENE & 1.0 & 0.53 & 10.5 & 0.0 & 0.00 & 0.0 & 0.0 & 0.00 & 0.0 & 0.2 & 0.42 & 2.17 \\
\hline 1-METHYL-2-ETHYLBENZENE & 0.8 & 0.44 & 6.2 & 0.0 & 0.00 & 0.0 & 0.0 & 0.00 & 0.0 & 0.2 & 0.35 & 1.29 \\
\hline 1,2,4-TRIMETHYLBENZENE & 2.8 & 1.52 & 26.0 & 0.0 & 0.00 & 0.0 & 0.1 & 0.33 & 0.7 & 0.6 & 1.23 & 6.61 \\
\hline DECANE & 0.1 & 0.06 & 0.1 & 0.1 & 0.95 & 0.0 & 0.0 & 0.00 & 0.0 & 0.1 & 0.13 & 0.03 \\
\hline ISOBUTYLBENZENE & 0.1 & 0.05 & 0.2 & 0.1 & 0.89 & 0.1 & 0.0 & 0.00 & 0.0 & 0.1 & 0.12 & 0.12 \\
\hline
\end{tabular}




\begin{tabular}{|c|c|c|c|c|c|c|c|c|c|c|c|c|}
\hline \multirow[b]{2}{*}{ COMPOUND } & \multicolumn{3}{|c|}{ BAG 1} & \multicolumn{3}{|c|}{ BAG 2} & \multicolumn{3}{|c|}{ BAG 3} & \multicolumn{3}{|c|}{ FTP } \\
\hline & MG/MI & $\begin{array}{l}\text { WEIGHT } \\
\text { NMOQ \% }\end{array}$ & $\begin{array}{l}\text { OZONE, } \\
\text { MG/MI }\end{array}$ & MG/MI & $\begin{array}{l}\text { WEIGHT } \\
\text { NMOQ } \%\end{array}$ & $\begin{array}{l}\text { OZONE } \\
\text { MG/MI }\end{array}$ & MG/MI & $\begin{array}{l}\text { WEIGHT } \\
\text { NMOG } \%\end{array}$ & $\begin{array}{l}\text { OZONE } \\
\text { MG/MI }\end{array}$ & Ma/MI & $\begin{array}{l}\text { WEIGHT } \\
\text { NMOG \% }\end{array}$ & $\begin{array}{l}\text { OZONE } \\
\text { MG/MI }\end{array}$ \\
\hline METHYLPROPYLBENZENE & 0.0 & 0.00 & 0.0 & 0.0 & 0.00 & 0.0 & 0.0 & 0.00 & 0.0 & 0.0 & 0.00 & 0.00 \\
\hline S-BUTYLBENZENE & 0.0 & 0.00 & 0.0 & 0.0 & 0.00 & 0.0 & 0.0 & 0.00 & 0.0 & 0.0 & 0.00 & 0.00 \\
\hline 1-METHYL-3-ISOPROPYLEENZENE & 0.5 & 0.24 & 2.8 & 0.0 & 0.00 & 0.0 & 0.0 & 0.00 & 0.0 & 0.1 & $\overline{0.19}$ & 0.57 \\
\hline 1,2,3-TRIMETHYLBENZENE & 0.0 & 0.00 & 0.0 & 0.0 & 0.00 & 0.0 & 0.0 & 0.00 & 0.0 & 0.0 & 0.00 & 0.00 \\
\hline 1-METHYL-4-ISOPROPYLBENZENE & 0.0 & 0.00 & 0.0 & 0.0 & 0.00 & 0.0 & 0.0 & 0.00 & 0.0 & 0.0 & 0.00 & 0.00 \\
\hline INDAN & 0.2 & 0.11 & 0.2 & 0.0 & 0.00 & 0.0 & 0.0 & 0.00 & 0.0 & 0.0 & 0.09 & 0.05 \\
\hline 1-METHYL-2-ISOPROPYLEENZENE & 0.2 & 0.12 & 1.4 & 0.0 & 0.00 & 0.0 & 0.0 & 0.00 & 0.0 & 0.1 & 0.10 & 0.29 \\
\hline 1.3-DIETHYLBENZENE & 0.0 & 0.00 & 0.0 & 0.0 & 0.00 & 0.0 & 0.0 & 0.00 & 0.0 & 0.0 & 0.00 & 0.00 \\
\hline 1.4-DIETHYLBENZENE & 0.2 & 0.11 & 1.4 & 0.0 & 0.00 & 0.0 & 0.0 & 0.00 & 0.0 & 0.0 & 0.08 & 0.28 \\
\hline 1-METHYL-3-N-PROPYLBENZENE & 0.4 & 0.22 & 2.8 & 0.0 & 0.00 & 0.0 & 0.0 & 0.00 & 0.0 & 0.1 & 0.17 & 0.58 \\
\hline 1-METHYL-4-N-PROPYLBENZENE & 0.0 & 0.00 & 0.0 & 0.0 & 0.00 & $\overline{0.0}$ & 0.0 & 0.00 & 0.0 & 0.0 & 0.00 & 0.00 \\
\hline 1,2 DIETHYLBENZENE & 0.0 & 0.00 & 0.0 & 0.0 & 0.00 & 0.0 & 0.0 & 0.00 & 0.0 & 0.0 & 0.00 & 0.00 \\
\hline 1-METHYL-2-N-PAOPYLBENZENE & 0.0 & 0.00 & 0.0 & 0.0 & 0.00 & 0.0 & 0.0 & 0.00 & 0.0 & 0.0 & 0.00 & 0.00 \\
\hline 1,4-DIMETHYL-2-ETHYLBENZENE & 0.2 & 0.12 & 2.1 & 0.0 & 0.00 & 0.0 & 0.0 & 0.00 & 0.0 & 0.0 & 0.09 & 0.43 \\
\hline 1,3-DIMETHYL-4-ETHYLBENZENE & 0.0 & 0.00 & 0.0 & 0.0 & 0.00 & 0.0 & 0.0 & 0.00 & 0.0 & 0.0 & 0.00 & 0.00 \\
\hline 1,2-DIMETHYL-4-ETHYLBENZENE & 0.3 & 0.18 & 3.2 & 0.0 & 0.00 & 0.0 & 0.0 & 0.00 & 0.0 & 0.1 & 0.14 & 0.68 \\
\hline 1,3-DIMETHYL-2-ETHYLBENZENE & 0.0 & 0.00 & 0.0 & 0.0 & 0.00 & 0.0 & 0.0 & 0.00 & 0.0 & 0.0 & 0.00 & 0.00 \\
\hline UNDECANE & 0.0 & 0.00 & 0.0 & 0.0 & 0.00 & 0.0 & 0.0 & 0.00 & 0.0 & 0.0 & 0.00 & 0.00 \\
\hline 1,2-DIMETHYL-3-ETHYLBENZENE & 0.0 & 0.00 & 0.0 & 0.0 & 0.00 & 0.0 & 0.0 & 0.00 & 0.0 & 0.0 & 0.00 & 0.00 \\
\hline 1,2,4,6-TETRAMETHYLBENZENE & 0.2 & 0.09 & 1.6 & 0.0 & 0.00 & 0.0 & 0.0 & 0.00 & 0.0 & 0.0 & 0.07 & 0.34 \\
\hline 2-METHYLBUTYLBENZENE (Bec AMYLBENZENE) & 0.0 & 0.00 & 0.0 & 0.0 & 0.00 & 0.0 & 0.0 & 0.00 & 0.0 & 0.0 & 0.00 & 0.00 \\
\hline 3,4 DIMETHYLCUMENE & 0.0 & 0.00 & 0.0 & 0.0 & 0.00 & 0.0 & 0.0 & 0.00 & 0.0 & 0.0 & 0.00 & 0.00 \\
\hline $1,2,3,5$-TETAAMETHYLBENZENE & 0.0 & 0.00 & 0.0 & 0.0 & 0.00 & 0.0 & 0.0 & 0.00 & 0.0 & 0.0 & 0.00 & 0.00 \\
\hline TEAT-1-BUT-2-METHYLBENZENE & 0.0 & 0.00 & 0.0 & 0.0 & 0.00 & 0.0 & 0.0 & 0.00 & 0.0 & 0.0 & 0.00 & 0.00 \\
\hline 1,2,3,4-TETRAMETHYLBENZENE & 0.0 & 0.00 & 0.0 & 0.0 & 0.00 & 0.0 & 0.0 & 0.00 & 0.0 & 0.0 & 0.00 & 0.00 \\
\hline N-PENT-BENZENE & 0.0 & 0.00 & 0.0 & 0.0 & 0.00 & 0.0 & 0.0 & 0.00 & 0.0 & 0.0 & 0.00 & 0.00 \\
\hline TEAT-1-BUT-3,6-DIMETHYLBENZENE & 0.0 & 0.00 & 0.0 & 0.0 & 0.00 & 0.0 & 0.0 & 0.00 & 0.0 & 0.0 & 0.00 & 0.00 \\
\hline NAPHTHALENE & 0.0 & 0.00 & 0.0 & 0.0 & 0.00 & 0.0 & 0.0 & 0.00 & 0.0 & 0.0 & 0.00 & 0.00 \\
\hline DODECANE & 0.0 & 0.00 & 0.0 & 0.0 & 0.00 & 0.0 & 0.0 & 0.00 & 0.0 & 0.0 & 0.00 & 0.00 \\
\hline UNIDENTIFIED $\mathrm{C} \theta-\mathrm{C} 12+$ & 3.3 & 1.71 & 12.8 & 0.1 & 0.83 & 0.3 & 0.0 & 0.09 & 0.1 & 0.7 & 1.42 & 2.79 \\
\hline FOAMALDEHYDE & 2.0 & 1.06 & 14.6 & 0.0 & 0.00 & 0.0 & 0.0 & 0.00 & 0.0 & 0.4 & 0.82 & 3.04 \\
\hline ACETALDEHYDE & 0.7 & 0.38 & 4.1 & 0.0 & 0.00 & 0.0 & 0.0 & 0.00 & 0.0 & 0.2 & 0.30 & 0.86 \\
\hline ACROLEIN & 0.2 & 0.12 & 1.6 & 0.0 & 0.00 & 0.0 & 0.0 & 0.00 & 0.0 & 0.0 & 0.09 & 0.32 \\
\hline ACETONE & 0.2 & 0.12 & 0.1 & 0.0 & 0.00 & 0.0 & 0.0 & 0.00 & 0.0 & 0.0 & 0.09 & 0.03 \\
\hline PROPIONALDEHYDE & 0.0 & 0.00 & 0.0 & 0.0 & 0.00 & 0.0 & 0.0 & 0.00 & 0.0 & 0.0 & 0.00 & 0.00 \\
\hline CROTONALDEHYDE & 0.0 & 0.00 & 0.0 & 0.0 & 0.00 & 0.0 & 0.0 & 0.00 & 0.0 & 0.0 & 0.00 & 0.00 \\
\hline ISOBUTYRALDEHYDE & 0.0 & 0.00 & 0.0 & 0.0 & 0.00 & 0.0 & 0.0 & 0.00 & 0.0 & 0.0 & 0.00 & 0.00 \\
\hline METHYL ETHYL KETONE & 0.0 & 0.00 & 0.0 & 0.0 & 0.00 & 0.0 & 0.0 & 0.00 & 0.0 & 0.0 & 0.00 & 0.00 \\
\hline BENZALDEHYDE & 0.0 & 0.02 & -0.0 & 0.0 & 0.00 & 0.0 & 0.0 & 0.00 & 0.0 & 0.0 & 0.02 & -0.00 \\
\hline HEXANALDEHYDE & 0.0 & 0.00 & 0.0 & 0.0 & 0.00 & 0.0 & 0.0 & 0.00 & 0.0 & 0.0 & 0.00 & 0.00 \\
\hline
\end{tabular}


TEST NO: BLB-AFA-02S

TEST CAR: 1994 PONTIAC GRANDAM

TEST DATE: $9 / 18 / 95$

TEST FUEL: EM-1700-F

\begin{tabular}{|c|c|c|c|c|c|c|c|c|c|c|c|c|}
\hline \multirow[b]{2}{*}{ COMPOUND } & \multicolumn{3}{|c|}{ BAG 1} & \multicolumn{3}{|c|}{ BAG 2} & \multicolumn{3}{|c|}{ BAG 3} & \multicolumn{3}{|c|}{ FTP } \\
\hline & MG/MI & $\begin{array}{l}\text { WEIGHT } \\
\text { NMOG \% }\end{array}$ & $\begin{array}{l}\text { OZONE, } \\
\text { MQ/MI }\end{array}$ & MG/MI & $\begin{array}{l}\text { WEIGHT } \\
\text { NMOG } \%\end{array}$ & $\begin{array}{l}\text { OZONE } \\
\text { MG/MI }\end{array}$ & MGMI & $\begin{array}{l}\text { WEIGHT } \\
\text { NMOG \% }\end{array}$ & $\begin{array}{l}\text { OZONE } \\
\text { MG/MI }\end{array}$ & MG/MI & $\begin{array}{c}\text { WEIGHT } \\
\text { NMOG } \%\end{array}$ & $\begin{array}{l}\text { OZONE } \\
\text { MG/MI }\end{array}$ \\
\hline METHANE & 21.8 & & 0.3 & 2.8 & & 0.0 & 8.8 & & 0.1 & 8.4 & & 0.12 \\
\hline ETHANE & 4.8 & 2.31 & 1.2 & 0.0 & 0.53 & 0.0 & 1.4 & 4.53 & $\overline{0.3}$ & 1.4 & 2.54 & 0.36 \\
\hline ETHYLENE & 13.5 & 6.57 & 98.7 & 0.0 & 0.75 & 0.4 & 1.5 & 4.89 & 10.8 & 3.2 & 5.98 & 23.68 \\
\hline PROPANE & 0.2 & 0.10 & 0.1 & 0.0 & 0.00 & 0.0 & 0.0 & 0.12 & 0.0 & 0.1 & 0.10 & 0.03 \\
\hline PROPYLENE & $\overline{\theta .7}$ & 4.70 & 90.9 & 0.1 & 1.60 & 1.0 & 1.0 & 3.20 & 9.2 & 2.3 & 4.27 & 21.89 \\
\hline ACETYLENE & 6.4 & 3.12 & 3.2 & 0.0 & 0.23 & 0.0 & 0.0 & 0.03 & 0.0 & 1.3 & 2.47 & 0.67 \\
\hline PROPADIENE & 0.0 & 0.00 & 0.0 & 0.0 & 0.00 & 0.0 & 0.0 & 0.00 & 0.0 & 0.0 & 0.00 & 0.00 \\
\hline BUTANE & 5.2 & 2.53 & 5.3 & 0.1 & 1.69 & 0.1 & 1.3 & 4.22 & 1.3 & 1.5 & 2.74 & 1.52 \\
\hline TRANS-2-BUTENE & 0.8 & 0.39 & 8.1 & 0.0 & 0.00 & 0.0 & 0.2 & 0.53 & 1.6 & 0.2 & 0.39 & 2.12 \\
\hline 1-BUTENE & 1.4 & 0.67 & 12.3 & 0.0 & 0.00 & 0.0 & 0.1 & 0.28 & 0.7 & 0.3 & 0.58 & 2.74 \\
\hline 2-METHYLPROPENE (ISOBUTYLENE) & 3.3 & 1.62 & 17.7 & 0.0 & 0.00 & 0.0 & 0.4 & 1.34 & 2.2 & 0.8 & 1.48 & 4.28 \\
\hline 2,2-DIMETHYLPROPANE (NEOPENTANE) & 0.1 & 0.06 & 0.0 & 0.0 & 0.00 & 0.0 & 0.1 & 0.26 & 0.0 & 0.0 & 0.09 & $\overline{0.02}$ \\
\hline PROPYNE & 0.0 & 0.00 & 0.0 & 0.0 & 0.00 & 0.0 & 0.0 & 0.00 & 0.0 & 0.0 & 0.00 & 0.00 \\
\hline 1,3-BUTADIENE & 1.6 & 0.78 & 17.6 & 0.0 & 0.00 & 0.0 & 0.1 & 0.45 & 1.5 & 0.4 & 0.68 & 4.05 \\
\hline 2-METHYLPROPANE (ISOBUTANE) & 0.2 & 0.08 & 0.2 & 0.0 & 0.62 & 0.0 & 0.1 & 0.38 & 0.1 & 0.1 & 0.16 & 0.11 \\
\hline 1-BUTYNE & 0.0 & 0.00 & 0.0 & 0.0 & 0.00 & 0.0 & 0.0 & 0.00 & 0.0 & 0.0 & 0.00 & 0.00 \\
\hline METHANOL & 0.0 & 0.00 & 0.0 & 0.0 & 0.00 & 0.0 & 0.0 & 0.00 & 0.0 & 0.0 & 0.00 & 0.00 \\
\hline CIS-2-BUTENE & 0.1 & 0.03 & 0.6 & 0.0 & 0.00 & 0.0 & 0.1 & 0.41 & 1.2 & 0.0 & 0.08 & 0.46 \\
\hline 3-METHYL-1-BUTENE & 0.0 & 0.00 & 0.0 & 0.0 & 0.00 & 0.0 & 0.0 & 0.00 & 0.0 & 0.0 & 0.00 & 0.00 \\
\hline ETHANOL & 0.0 & 0.00 & 0.0 & 0.0 & 0.00 & 0.0 & 0.0 & 0.00 & 0.0 & 0.0 & 0.00 & 0.00 \\
\hline 2-METHYLBUTANE (ISOPENTANE) & 17.2 & 8.35 & 23.7 & 0.4 & 6.19 & 0.6 & 4.3 & 14.14 & 6.0 & 5.0 & 9.12 & 6.85 \\
\hline 2-BUTYNE & 0.0 & 0.00 & 0.0 & 0.0 & 0.00 & 0.0 & 0.0 & 0.00 & 0.0 & 0.0 & 0.00 & 0.00 \\
\hline 1-PENTENE & 0.4 & 0.19 & 2.4 & 0.0 & 0.00 & 0.0 & 0.1 & 0.30 & 0.6 & 0.1 & 0.20 & 0.66 \\
\hline 2-METHYL-1-BUTENE & 0.8 & 0.30 & 3.0 & 0.0 & 0.00 & 0.0 & 0.0 & 0.15 & 0.2 & 0.1 & 0.26 & 0.69 \\
\hline PENTANE & 2.1 & 1.03 & 2.2 & 0.2 & 2.80 & 0.2 & 0.4 & 1.38 & 0.4 & 0.7 & 1.20 & 0.68 \\
\hline UNIDENTIFIED C6 OLEFINS & 0.0 & 0.00 & 0.0 & 0.0 & 0.00 & 0.0 & 0.0 & 0.00 & 0.0 & 0.0 & 0.00 & 0.00 \\
\hline 2-METHYL-1,3-BUTADIENE & 0.9 & 0.43 & 8.1 & 0.0 & 0.00 & 0.0 & 0.1 & 0.36 & 1.0 & 0.2 & 0.39 & 1.95 \\
\hline TRANS-2-PENTENE & 0.7 & 0.32 & 6.8 & 0.0 & 0.00 & 0.0 & 0.1 & 0.37 & 1.0 & 0.2 & 0.31 & 1.47 \\
\hline 3,3-DIMETHYL-1-BUTENE & 0.0 & 0.00 & 0.0 & 0.0 & 0.00 & 0.0 & 0.0 & 0.00 & 0.0 & 0.0 & 0.00 & 0.00 \\
\hline CIS-2-PENTENE & 0.3 & 0.16 & 3.0 & 0.0 & 0.00 & 0.0 & 0.0 & 0.00 & 0.0 & 0.1 & 0.13 & 0.61 \\
\hline 2-METHYL-2-BUTENE & 1.2 & 0.59 & 7.8 & 0.0 & 0.00 & 0.0 & 0.2 & 0.51 & 1.0 & 0.3 & 0.54 & 1.89 \\
\hline CYCLOPENTADIENE & 0.6 & 0.25 & 3.9 & 0.0 & 0.00 & 0.0 & 0.0 & 0.00 & 0.0 & 0.1 & 0.19 & 0.80 \\
\hline 2,2-DIMETHYLBUTANE & 0.8 & 0.44 & 0.7 & 0.0 & 0.08 & 0.0 & 0.2 & 0.59 & 0.1 & 0.2 & 0.44 & 0.20 \\
\hline CYCLOPENTENE & 0.0 & 0.00 & 0.0 & 0.0 & 0.00 & 0.0 & 0.0 & 0.00 & 0.0 & 0.0 & 0.00 & 0.00 \\
\hline 4-METHYL-1-PENTENE & 0.0 & 0.00 & 0.0 & 0.0 & 0.00 & 0.0 & 0.1 & 0.28 & 0.4 & 0.0 & 0.04 & 0.10 \\
\hline 3-METHYL-1-PENTENE & 0.0 & 0.00 & 0.0 & 0.0 & 0.00 & 0.0 & 0.0 & 0.00 & 0.0 & 0.0 & 0.00 & 0.00 \\
\hline CYCLOPENTANE & 0.0 & 0.00 & 0.0 & 0.0 & 0.00 & 0.0 & 0.0 & 0.00 & 0.0 & 0.0 & 0.00 & 0.00 \\
\hline 2,3-DIMETHYLBUTANE & 1.4 & 0.70 & 1.5 & 0.1 & 2.00 & 0.1 & 0.4 & 1.24 & 0.4 & 0.6 & 0.86 & 0.50 \\
\hline MTBE & 0.0 & 0.00 & 0.0 & 0.0 & 0.00 & 0.0 & 0.0 & 0.00 & 0.0 & 0.0 & 0.00 & 0.00 \\
\hline
\end{tabular}




\begin{tabular}{|c|c|c|c|c|c|c|c|c|c|c|c|c|}
\hline \multirow[b]{2}{*}{ COMPOUND } & \multicolumn{3}{|c|}{ BAG 1} & \multicolumn{3}{|c|}{ BAG 2} & \multicolumn{3}{|c|}{ BAG 3} & \multicolumn{3}{|c|}{ FTP } \\
\hline & MG/MI & $\begin{array}{l}\text { WEIGHT } \\
\text { NMOG } \%\end{array}$ & $\begin{array}{l}\text { OZONE, } \\
\text { MG/MI }\end{array}$ & MG/MI & $\begin{array}{l}\text { WEIGHT } \\
\text { NMOQ } \%\end{array}$ & $\begin{array}{l}\text { OZONE } \\
\text { MG/MI }\end{array}$ & MG/MI & $\begin{array}{l}\text { WEIGHT } \\
\text { NMOG } \%\end{array}$ & $\begin{array}{l}\text { OZONE } \\
\text { MG/MI }\end{array}$ & MG/MI & $\begin{array}{l}\text { WEIGHT } \\
\text { NMOG } \%\end{array}$ & $\begin{array}{l}\text { OZONE } \\
\text { MG/MI }\end{array}$ \\
\hline 2,3-DIMETHYL-1-BUTENE & 0.0 & 0.00 & 0.0 & 0.0 & 0.00 & 0.0 & 0.0 & 0.00 & 0.0 & 0.0 & 0.00 & 0.00 \\
\hline 4-METHYL-CIS-2-PENTENE & 0.0 & 0.00 & 0.0 & 0.0 & 0.00 & 0.0 & 0.0 & 0.00 & 0.0 & 0.0 & 0.00 & 0.00 \\
\hline 2-METHYLPENTANE & 4.0 & 1.97 & $\overline{6.2}$ & 0.4 & 5.90 & 0.6 & 1.0 & 3.36 & 1.6 & 1.3 & 2.42 & 2.02 \\
\hline 4-METHYL-TRANS-2-PENTENE & 0.0 & 0.00 & 0.0 & 0.0 & 0.00 & 0.0 & 0.0 & 0.00 & 0.0 & 0.0 & 0.00 & 0.00 \\
\hline 3-METHYLPENTANE & 2.3 & 1.09 & 3.4 & 0.4 & 6.89 & 0.6 & 0.6 & 1.91 & 0.9 & 0.8 & 1.51 & 1.25 \\
\hline 2-METHYL-1-PENTENE & 0.2 & 0.09 & 0.8 & 0.0 & 0.00 & 0.0 & 0.0 & 0.16 & 0.2 & 0.1 & 0.09 & 0.23 \\
\hline 1-HEXENE & 0.3 & 0.12 & 1.1 & 0.0 & 0.00 & 0.0 & 0.0 & 0.16 & 0.2 & 0.1 & 0.12 & 0.29 \\
\hline HEXANE & 1.2 & 0.57 & 1.2 & 0.3 & 5.09 & 0.3. & 0.4 & 1.35 & 0.4 & 0.5 & 0.97 & 0.52 \\
\hline UNIDENTIFIED C6 OLEFINS & 0.6 & 0.28 & 3.9 & 0.0 & 0.00 & 0.0 & 0.0 & 0.00 & 0.0 & 0.1 & 0.22 & $\overline{0.81}$ \\
\hline TRANS-3-HEXENE & 0.3 & 0.14 & 1.9 & 0.1 & 2.25 & 1.0 & 0.1 & 0.33 & 0.7 & 0,2 & 0.30 & 1.08 \\
\hline CIS-3-HEXENE & 0.3 & 0.16 & 2.3 & 0.1 & 0.90 & 0.4 & 0.1 & 0.24 & 0.5 & $\overline{0.1}$ & 0.22 & 0.80 \\
\hline TRANS-2-HEXENE & 0.0 & 0.00 & 0.0 & 0.0 & 0.00 & 0.0 & 0.0 & 0.00 & 0.0 & 0.0 & 0.00 & 0.00 \\
\hline 3-METHYL-TRANS-2-PENTENE & 0.5 & 0.23 & 3.2 & 0.0 & 0.00 & 0.0 & 0.1 & 0.29 & 0.6 & 0.1 & 0.22 & $\overline{0.82}$ \\
\hline 2-METHYL-2-PENTENE & 0.3 & 0.15 & 2.1 & 0.0 & 0.00 & 0.0 & 0.1 & 0.31 & 0.8 & 0.1 & 0.17 & 0.62 \\
\hline 3-METHYLCYCLOPENTENE & 0.0 & 0.00 & 0.0 & 0.0 & 0.00 & 0.0 & 0.0 & 0.00 & 0.0 & 0.0 & 0.00 & 0.00 \\
\hline CIS-2-HEXENE & 0.2 & 0.11 & 1.5 & 0.0 & 0.00 & 0.0 & 0.0 & 0.00 & 0.0 & 0.0 & 0.09 & 0.32 \\
\hline ETBE & 0.0 & 0.00 & 0.0 & 0.0 & 0.00 & 0.0 & 0.0 & 0.00 & 0.0 & 0.0 & 0.00 & 0.00 \\
\hline 3-METHYL-CIS-2-PENTENE & 0.4 & 0.18 & 2.5 & 0.1 & 2.23 & 1.0 & $\overline{0.1}$ & 0.31 & 0.6 & 0.2 & 0.33 & 1.19 \\
\hline 2,2-DIMETHYLPENTANE & 0.9 & 0.43 & 1.2 & 0.2 & 3.05 & 0.3 & 0.2 & 0.65 & 0.3 & 0.3 & 0.63 & $\overline{0.48}$ \\
\hline METHYLCYCLOPENTANE & 0.0 & 0.00 & 0.0 & 0.0 & 0.00 & 0.0 & 0.0 & 0.00 & 0.0 & 0.0 & 0.00 & 0.00 \\
\hline 2,4-DIMETHYLPENTANE & 1.3 & 0.64 & 2.3 & 0.2 & 2.34 & 0.3 & 0.3 & 1.13 & 0.6 & 0.4 & 0.82 & 0.79 \\
\hline 2,3,3-TRIMETHYL-1-BUTENE & 0.0 & 0.00 & 0.0 & 0.0 & 0.00 & 0.0 & 0.0 & 0.00 & 0.0 & 0.0 & 0.00 & 0.00 \\
\hline 2,2,3-TAIMETHYLBUTANE & 0.2 & 0.08 & 0.2 & 0.1 & 1.25 & 0.1 & 0.0 & 0.12 & 0.0 & 0.1 & 0.16 & 0.11 \\
\hline 3,4-DIMETHYL-1-PENTENE & 0.0 & 0.00 & 0.0 & 0.0 & 0.00 & 0.0 & 0.0 & 0.00 & 0.0 & 0.0 & 0.00 & 0.00 \\
\hline 1-METHYLCYCLOPENTENE & 0.3 & 0.15 & 1.8 & 0.2 & 2.63 & 1.0 & 0.1 & 0.16 & $\overline{0.3}$ & 0.2 & 0.31 & 0.94 \\
\hline BENZENE & 13.4 & 6.53 & 5.6 & 0.0 & 0.11 & 0.0 & 3.3 & 10.77 & $\overline{1.4}$ & 3.7 & 6.79 & 1.55 \\
\hline 3-METHYL-1-HEXENE & 0.0 & 0.00 & 0.0 & 0.0 & 0.00 & 0.0 & 0.0 & 0.00 & 0.0 & 0.0 & 0.00 & 0.00 \\
\hline 3,3-DIMETHYLPENTANE & 0.4 & 0.21 & 0.3 & 0.0 & 0.00 & 0.0 & 0.2 & 0.64 & $\overline{0.1}$ & 0.1 & 0.27 & 0.10 \\
\hline CYCLOHEXANE & 0.3 & 0.14 & 0.4 & 0.0 & 0.00 & 0.0 & 0.0 & 0.00 & $\overline{0.0}$ & 0.1 & 0.11 & 0.08 \\
\hline 2-METHYLHEXANE & 0.0 & 0.00 & 0.0 & 0.0 & 0.00 & 0.0 & 0.0 & 0.00 & 0.0 & 0.0 & 0.00 & 0.00 \\
\hline 2,3-DIMETHYLPENTANE & 5.1 & 2.60 & 7.8 & 0.3 & 3.80 & 0.4 & 1.3 & 4.16 & 1.9 & 1.5 & 2.84 & 2.34 \\
\hline 1,1-DIMETHYLCYCLOPENTANE & 0.0 & 0.00 & 0.0 & 0.0 & 0.00 & 0.0 & 0.0 & 0.00 & 0.0 & 0.0 & 0.00 & 0.00 \\
\hline CYCLOHEXENE & 0.1 & 0.05 & 0.6 & 0.0 & 0.00 & 0.0 & 0.0 & 0.00 & 0.0 & 0.0 & 0.04 & 0.12 \\
\hline 3-METHYLHEXANE & 3.3 & 1.62 & 4.7 & 0.2 & 2.34 & 0.2 & 0.7 & 2.42 & 1.0 & 1.0 & 1.78 & 1.36 \\
\hline CIS-1,3-DIMETHYLCYCLOPENTANE & 0.3 & 0.13 & 0.7 & 0.0 & 0.36 & 0.1 & 0.1 & 0.19 & 0.1 & 0.1 & 0.15 & 0.21 \\
\hline 3-ETHYLPENTANE & 0.0 & 0.00 & 0.0 & 0.0 & 0.00 & 0.0 & 0.0 & 0.00 & 0.0 & 0.0 & 0.00 & 0.00 \\
\hline TRANS-1,2-DIMETHYLCYCLOPENTANE & 0.0 & 0.00 & 0.0 & 0.0 & 0.00 & 0.0 & 0.0 & 0.00 & 0.0 & 0.0 & 0.00 & 0.00 \\
\hline TRANS-1,3-DIMETHYLCYCLOPENTANE & 0.0 & 0.00 & 0.0 & 0.0 & 0.00 & 0.0 & 0.0 & 0.00 & 0.0 & 0.0 & 0.00 & 0.00 \\
\hline 1-HEPTENE & 0.0 & 0.00 & 0.0 & 0.0 & 0.00 & 0.0 & 0.0 & 0.00 & 0.0 & 0.0 & 0.00 & 0.00 \\
\hline 2,2,4-TRIMETHYLPENTANE & $3 . \overline{6}$ & 1.77 & 3.4 & 0.3 & 5.23 & 0.3 & 1.0 & 3.28 & 0.8 & 1.2 & 2.21 & $\overline{1.12}$ \\
\hline 2-METHYL-1-HEXENE & 0.0 & 0.00 & 0.0 & 0.0 & 0.00 & 0.0 & 0.0 & 0.00 & 0.0 & 0.0 & 0.00 & 0.00 \\
\hline TRANS-3-HEPTENE & 0.0 & 0.00 & 0.0 & 0.0 & 0.00 & 0.0 & 0.0 & 0.00 & 0.0 & 0.0 & 0.00 & 0.00 \\
\hline HEPTANE & 2.4 & 1.14 & 1.9 & 0.1 & 1.61 & 0.1 & 0.4 & 1.16 & 0.3 & 0.6 & 1.17 & 0.52 \\
\hline UNIDENTIFIED C7 & 1.2 & 0.61 & 5.6 & 0.1 & 1.71 & 0.5 & 0.2 & 0.73 & 1.0 & 0.4 & 0.69 & 1.70 \\
\hline
\end{tabular}




\begin{tabular}{|c|c|c|c|c|c|c|c|c|c|c|c|c|}
\hline \multirow[b]{2}{*}{ COMPOUND } & \multicolumn{3}{|c|}{ BAG 1} & \multicolumn{3}{|c|}{ BAG 2} & \multicolumn{3}{|c|}{ BAG 3} & \multicolumn{3}{|c|}{ FTP } \\
\hline & MG/MI & $\begin{array}{l}\text { WEIGHT } \\
\text { NMOQ \% }\end{array}$ & $\begin{array}{l}\text { OZONE, } \\
\text { MaMI }\end{array}$ & MG/MI & $\begin{array}{l}\text { WEIGHT } \\
\text { NMOG } \%\end{array}$ & $\begin{array}{l}\text { OZONE } \\
\text { MG/MI }\end{array}$ & MG/MI & $\begin{array}{l}\text { WEIGHT } \\
\text { NMOG } \%\end{array}$ & $\begin{array}{l}\text { OZONE } \\
\text { MG/MI }\end{array}$ & MG/MI & $\begin{array}{l}\text { WEIGHT } \\
\text { NMOG \% }\end{array}$ & $\begin{array}{l}\text { OZONE } \\
\text { MG/MI }\end{array}$ \\
\hline 2-METHYL-2-HEXENE & 0.0 & 0.00 & 0.0 & 0.0 & 0.00 & 0.0 & 0.0 & 0.00 & 0.0 & 0.0 & 0.00 & 0.00 \\
\hline 3-METHYL-TAANS-3-HEXENE & 0.0 & 0.00 & 0.0 & 0.0 & 0.00 & 0.0 & 0.0 & 0.00 & 0.0 & 0.0 & 0.00 & 0.00 \\
\hline TRANS-2-HEPTENE & 0.0 & 0.00 & 0.0 & 0.0 & 0.00 & 0.0 & 0.0 & 0.00 & 0.0 & 0.0 & 0.00 & 0.00 \\
\hline 3-ETHYL-CIS-2-PENTENE & 0.0 & 0.00 & 0.0 & 0.0 & 0.00 & 0.0 & 0.0 & 0.00 & 0.0 & 0.0 & 0.00 & 0.00 \\
\hline 2,4,4-TRIMETHYL-1-PENTENE & 0.2 & 0.10 & 0.6 & 0.0 & 0.00 & 0.0 & 0.0 & 0.00 & 0.0 & 0.0 & 0.08 & $\overline{0.12}$ \\
\hline 2,2,4-TRIMETHYL-1-PENTENE & 0.0 & 0.00 & 0.0 & 0.0 & 0.00 & 0.0 & 0.0 & 0.00 & 0.0 & 0.0 & 0.00 & 0.00 \\
\hline 2,3-DIMETHYL-2-PENTENE & 0.0 & 0.00 & 0.0 & 0.0 & 0.00 & 0.0 & 0.0 & 0.00 & 0.0 & 0.0 & 0.00 & 0.00 \\
\hline CIS-2-HEPTENE & 0.2 & 0.07 & 0.8 & 0.0 & 0.00 & 0.0 & 0.0 & 0.00 & 0.0 & 0.0 & 0.06 & 0.18 \\
\hline METHYLCYCLOHEXANE & 0.0 & 0.00 & 0.0 & 0.0 & 0.00 & 0.0 & 0.1 & 0.28 & 0.2 & 0.0 & 0.04 & $\overline{0.04}$ \\
\hline CIS-1,2-DIMETHYLCYCLOPENTANE & 0.0 & 0.00 & 0.0 & 0.0 & 0.00 & 0.0 & 0.0 & 0.00 & 0.0 & 0.0 & 0.00 & 0.00 \\
\hline 2,2-DIMETHYLHEXANE & 0.6 & 0.29 & 0.7 & 0.0 & 0.00 & 0.0 & 0.2 & 0.72 & 0.3 & 0.2 & 0.34 & 0.22 \\
\hline 1,1,3-TRIMETHYLCYCLOPENTANE & 0.0 & 0.00 & 0.0 & 0.0 & 0.00 & 0.0 & 0.0 & 0.00 & 0.0 & 0.0 & 0.00 & 0.00 \\
\hline 2,4,4-TRIMETHYL-2-PENTENE & 0.0 & 0.00 & 0.0 & 0.0 & 0.00 & 0.0 & 0.1 & 0.30 & 0.5 & 0.0 & 0.05 & 0.13 \\
\hline 2,2,3-TRIMETHYLPENTANE & 0.0 & 0.00 & 0.0 & 0.0 & 0.00 & 0.0 & 0.0 & 0.00 & 0.0 & 0.0 & 0.00 & 0.00 \\
\hline 2,5-DIMETHYLHEXANE & 0.0 & 0.00 & 0.0 & 0.0 & 0.00 & 0.0 & 0.0 & 0.00 & 0.0 & 0.0 & 0.00 & 0.00 \\
\hline ETHYLCYCLOPENTANE & 0.0 & 0.00 & 0.0 & 0.0 & 0.00 & 0.0 & 0.0 & 0.00 & 0.0 & 0.0 & 0.00 & 0.00 \\
\hline 2,4-DIMETHYLHEXANE & 0.8 & 0.40 & 1.2 & 0.0 & 0.00 & 0.0 & 0.2 & 0.72 & 0.3 & 0.2 & 0.43 & 0.35 \\
\hline 1-TRANS-2-CIS-4-TRIMETHYLCYCLOPENTANE & 0.0 & 0.00 & 0.0 & 0.0 & 0.00 & 0.0 & 0.0 & 0.00 & 0.0 & 0.0 & 0.00 & 0.00 \\
\hline 3,3-DIMETHYLHEXANE & 0.2 & 0.10 & 0.2 & 0.0 & 0.00 & 0.0 & 0.0 & 0.00 & 0.0 & 0.0 & 0.08 & 0.05 \\
\hline 1-TAANS-2-CIS-3-TRIMETHYLCYCLOPENTANE & 0.0 & 0.00 & 0.0 & 0.0 & 0.00 & 0.0 & 0.0 & 0.00 & 0.0 & 0.0 & 0.00 & 0.00 \\
\hline 2,3,4-TAIMETHYLPENTANE & 0.0 & 0.00 & 0.0 & 0.0 & 0,00 & 0.0 & 0.0 & 0.00 & 0.0 & 0.0 & 0.00 & 0.00 \\
\hline 2,3,3-TRIMETHYLPENTANE & 0.9 & 0.42 & 1.0 & 0.0 & 0.49 & 0.0 & 0.2 & 0.81 & 0.3 & $\overline{0.3}$ & 0.48 & 0.32 \\
\hline TOLUENE & 22.7 & 11.04 & 62.1 & 0.5 & 8.46 & 1.5 & 2.5 & 8.30 & 6.8 & 5.7 & $\overline{10.45}$ & 15.55 \\
\hline 2,3-DIMETHYLHEXANE & 0.7 & 0.35 & 1.0 & 0.0 & 0.00 & 0.0 & 0.0 & 0.00 & 0.0 & 0.2 & 0.28 & 0.20 \\
\hline 1,1,2-TAIMETHYLCYCLOPENTANE & 0.0 & 0.00 & 0.0 & 0.0 & 0.00 & 0.0 & 0.0 & 0.00 & 0.0 & 0.0 & 0.00 & 0.00 \\
\hline 2-METHYLHEPTANE & 1.2 & 0.67 & 1.1 & 0.0 & 0.00 & 0.0 & 0.2 & 0.67 & 0.2 & 0.3 & 0.55 & 0.29 \\
\hline 3,4-DIMETHYLHEXANE & 0.0 & 0.00 & 0.0 & 0.0 & 0.00 & 0.0 & 0.0 & 0.00 & 0.0 & 0.0 & 0.00 & 0.00 \\
\hline 2,2,4,4-TETRAMETHYLPENTANE & 0.0 & 0.00 & 0.0 & 0.0 & 0.00 & 0.0 & 0.0 & 0.00 & 0.0 & 0.0 & 0.00 & 0.00 \\
\hline 4-METHYLHEPTANE & 0.4 & 0.19 & 0.5 & 0.0 & 0.00 & 0.0 & 0.0 & 0.00 & 0.0 & 0.1 & 0.15 & 0.10 \\
\hline 2-METHYL-3-ETHYLPENTANE & 0.0 & 0.00 & 0.0 & 0.0 & 0.00 & 0.0 & 0.0 & 0.00 & 0.0 & 0.0 & 0.00 & 0.00 \\
\hline 2,6-DIMETHYLHEPTANE & 0.0 & 0.00 & 0.0 & 0.0 & 0.00 & 0.0 & 0.0 & 0.00 & 0.0 & 0.0 & 0.00 & 0.00 \\
\hline 3-METHYLHEPTANE & 1.3 & 0.63 & 1.3 & 0.0 & 0.00 & 0.0 & 0.2 & 0.74 & 0.2 & 0.3 & 0.61 & 0.33 \\
\hline 1-CIS,2-TAANS,3-TRIMETHYLCYCLOPENTANE & 0.0 & 0.00 & 0.0 & 0.0 & 0.00 & 0.0 & 0.0 & 0.00 & 0.0 & 0.0 & 0.00 & 0.00 \\
\hline CIS-1,3-DIMETHYLCYCLOHEXANE & 0.0 & 0.00 & 0.0 & 0.0 & 0.00 & 0.0 & 0.0 & 0.00 & 0.0 & 0.0 & 0.00 & 0.00 \\
\hline TRANS-1,4-DIMETHYLCYCLOHEXANE & 0.6 & 0.27 & 1.1 & 0.0 & 0.00 & 0.0 & 0.2 & 0.57 & 0.3 & 0.2 & 0.30 & 0.32 \\
\hline 3-ETHYLHEXANE & 0.0 & 0.00 & 0.0 & 0.0 & 0.00 & 0.0 & 0.0 & 0.00 & 0.0 & $\overline{0.0}$ & 0.00 & 0.00 \\
\hline 2,2,6-TRIMETHYLHEXANE & 0.1 & 0.07 & 0.1 & 0.0 & 0.00 & 0.0 & 0.0 & 0.00 & 0.0 & 0.0 & 0.05 & 0.03 \\
\hline CIS-1-METHYL-3-ETHYLCYCLOPENTANE & 0.0 & 0.00 & 0.0 & 0.0 & 0.00 & 0.0 & 0.0 & 0.00 & 0.0 & 0.0 & 0.00 & 0.00 \\
\hline 1,1-DIMETHYLCYCLOHEXANE & 0.0 & 0.00 & 0.0 & 0.0 & 0.00 & 0.0 & 0.0 & 0.00 & 0.0 & 0.0 & 0.00 & 0.00 \\
\hline TAANS-1-METHYL-2-ETHYLCYCLOPENTANE & 0.0 & 0.00 & 0.0 & 0.0 & 0.00 & 0.0 & $\overline{0.0}$ & 0.00 & 0.0 & 0.0 & 0.00 & 0.00 \\
\hline 1-METHYL-1-ETHYL-CYCLOPENTANE & 0.0 & 0.00 & 0.0 & 0.0 & 0.00 & 0.0 & $\overline{0.0}$ & 0.00 & 0.0 & 0.0 & 0.00 & 0.00 \\
\hline 2,4,4-TRIMETHYLHEXANE & 0.0 & 0.00 & 0.0 & 0.0 & 0.00 & 0.0 & 0.0 & 0.00 & 0.0 & 0.0 & 0.00 & 0.00 \\
\hline 2,2,4-TRIMETHYLHEXANE & 0.0 & 0.00 & 0.0 & 0.0 & 0.00 & 0.0 & 0.0 & 0.00 & 0.0 & 0.0 & 0.00 & 0.00 \\
\hline TRANS-1,2-DIMETHYLCYCLOHEXANE & 0.0 & 0.00 & 0.0 & 0.0 & 0.00 & $\overline{0.0}$ & $\overline{0.0}$ & 0.00 & 0.0 & 0.0 & 0.00 & 0.00 \\
\hline
\end{tabular}




\begin{tabular}{|c|c|c|c|c|c|c|c|c|c|c|c|c|}
\hline \multirow[b]{2}{*}{ COMPOUND } & \multicolumn{3}{|c|}{ BAG 1} & \multicolumn{3}{|c|}{ BAG 2} & \multicolumn{3}{|c|}{ BAG 3} & \multicolumn{3}{|c|}{ FTP } \\
\hline & MGMI & $\begin{array}{l}\text { WEIGHT } \\
\text { NMOG } \% \\
\end{array}$ & $\begin{array}{l}\text { OZONE, } \\
\text { MG/MI }\end{array}$ & MG/MI & $\begin{array}{l}\text { WEIGHT } \\
\text { NMOQ \% } \\
\end{array}$ & $\begin{array}{l}\text { OZONE } \\
\text { MG/MI }\end{array}$ & MG/MI & \begin{tabular}{|l|} 
WEIGHT \\
NMOQ \% \\
\end{tabular} & $\begin{array}{l}\text { OZONE } \\
\text { MGMI }\end{array}$ & Ma/MI & \begin{tabular}{|l|} 
WEIGHT \\
NMOG \% \\
\end{tabular} & $\begin{array}{l}\text { OZONE } \\
\text { MGMI }\end{array}$ \\
\hline 1-OCTENE & 0.1 & 0.06 & 0.4 & 0.0 & 0.00 & 0.0 & 0.0 & 0.00 & 0.0 & 0.0 & 0.05 & 0.07 \\
\hline TAANS-4-OCTENE & 0.0 & 0.00 & 0.0 & 0.0 & 0.00 & 0.0 & 0.0 & 0.00 & 0.0 & 0.0 & 0.00 & 0.00 \\
\hline OCTANE & 0.6 & 0.30 & 0.4 & 0.0 & 0.00 & 0.0 & 0.1 & 0.42 & 0.1 & 0.2 & 0.30 & 0.10 \\
\hline UNIDENTIFIED C8 & 1.3 & 0.62 & 4.3 & 0.1 & 1.14 & 0.2 & 0.2 & 0.62 & 0.6 & 0.4 & 0.65 & 1.21 \\
\hline TRANS-2-OCTENE & 0.2 & 0.10 & 1.0 & 0.0 & 0.00 & 0.0 & 0.0 & 0.00 & 0.0 & 0.0 & 0.07 & 0.22 \\
\hline TRANS-1,3-DIMETHYLCYCLOHEXANE & 0.1 & 0.05 & 0.2 & 0.0 & 0.00 & 0.0 & 0.0 & 0.00 & 0.0 & 0.0 & 0.04 & 0.04 \\
\hline CIS-1,4-DIMETHYLCYCLOHEXANE & 0.0 & 0.00 & 0.0 & 0.0 & 0.00 & 0.0 & 0.0 & 0.00 & 0.0 & $\overline{0.0}$ & 0.00 & 0.00 \\
\hline CIS-2-OCTENE & 0.0 & 0.00 & 0.0 & 0.0 & 0.00 & 0.0 & 0.0 & 0.00 & 0.0 & 0.0 & 0.00 & 0.00 \\
\hline 2,3,5-TAIMETHYLHEXANE & 0.0 & 0.00 & 0.0 & 0.0 & 0.00 & 0.0 & 0.0 & 0.00 & 0.0 & 0.0 & 0.00 & 0.00 \\
\hline CIS-1-METHYL-2-ETHYLCYCLOPENTANE & 0.0 & 0.00 & 0.0 & 0.0 & 0.00 & 0.0 & 0.0 & 0.00 & 0.0 & 0.0 & 0.00 & 0.00 \\
\hline 2-METHYL-2-ETHYLHEPTANE & 0.0 & 0.00 & 0.0 & 0.0 & 0.00 & 0.0 & 0.0 & 0.00 & 0.0 & 0.0 & 0.00 & 0.00 \\
\hline 2,4-DIMETHYLHEPTANE & 0.0 & 0.01 & 0.0 & 0.2 & 3.82 & 0.3 & 0.1 & 0.32 & 0.1 & 0.2 & 0.29 & 0.21 \\
\hline 4,4-DIMETHYLHEPTANE & 0.0 & 0.00 & 0.0 & 0.0 & 0.00 & 0.0 & 0.0 & 0.00 & 0.0 & 0.0 & 0.00 & 0.00 \\
\hline CIS-1,2-DIMETHYLCYCLOHEXANE & 0.2 & 0.11 & 0.4 & 0.0 & 0.00 & 0.0 & 0.0 & 0.00 & 0.0 & 0.0 & 0.09 & 0.09 \\
\hline ETHYLCYCLOHEXANE & 0.2 & 0.12 & 0.5 & 0.0 & 0.00 & 0.0 & 0.0 & 0.00 & 0.0 & 0.1 & 0.09 & 0.10 \\
\hline PROPYLCYCLOHEXANE & 0.0 & 0.00 & 0.0 & 0.0 & 0.00 & 0.0 & 0.0 & 0.00 & 0.0 & 0.0 & 0.00 & 0.00 \\
\hline 2-METHYL-4-ETHYLHEXANE & 0.0 & 0.00 & 0.0 & 0.0 & 0.00 & 0.0 & 0.0 & 0.00 & 0.0 & 0.0 & 0.00 & 0.00 \\
\hline 2,6-DIMETHYLHEPTANE & 0.0 & 0.00 & 0.0 & 0.0 & 0.00 & 0.0 & 0.0 & 0.00 & 0.0 & 0.0 & 0.00 & 0.00 \\
\hline 1,1,3-TRIMETHYLCYCLOHEXANE & 0.0 & 0.00 & 0.0 & 0.0 & 0.00 & 0.0 & 0.0 & 0.00 & 0.0 & 0.0 & 0.00 & 0.00 \\
\hline 2,5-DIMETHYLHEPTANE & 0.0 & 0.00 & 0.0 & 0.0 & 0.00 & 0.0 & 0.0 & 0.00 & 0.0 & 0.0 & 0.00 & 0.00 \\
\hline 3,3-DIMETHYLHEPTANE & 0.0 & 0.00 & 0.0 & 0.0 & 0.00 & 0,0 & 0.0 & 0.00 & 0.0 & 0.0 & 0.00 & 0.00 \\
\hline 3,5-DIMETHYLHEPTANE & 0.0 & 0.00 & 0.0 & 0.0 & 0.00 & 0.0 & 0.0 & 0.00 & 0.0 & 0.0 & 0.00 & 0.00 \\
\hline ETHYLBENZENE & 6.4 & 3.09 & 17.2 & 0.2 & 2.83 & 0.5 & 0.5 & 1.68 & 1.4 & 1.6 & 2.86 & 4.21 \\
\hline 2,3-DIMETHYLHEPTANE & 0.0 & 0.00 & 0.0 & 0.0 & 0.00 & 0.0 & 0.0 & 0.00 & 0.0 & 0.0 & 0.00 & 0.00 \\
\hline$m-\& p-X Y L E N E$ & 13.7 & 6.63 & 100.8 & 0.1 & 1.15 & 0.5 & 1.0 & 3.33 & 7.5 & 3.2 & 6.78 & 23.25 \\
\hline 4-METHYLOCTANE & 0.0 & 0.00 & 0.0 & 0.0 & 0.00 & 0.0 & 0.0 & 0.00 & 0.0 & 0.0 & 0.00 & 0.00 \\
\hline 2-METHYLOCTANE & 0.6 & 0.28 & 0.6 & 0.0 & 0.00 & 0.0 & 0.2 & 0.49 & 0.2 & 0.2 & 0.29 & 0.18 \\
\hline 3-METHYLOCTANE & 0.3 & 0.15 & 0.4 & 0.0 & 0.00 & 0.0 & 0.0 & 0.00 & 0.0 & 0.1 & 0.12 & 0.07 \\
\hline STYRENE & 0.0 & 0.00 & 0.0 & 0.0 & 0.00 & 0.0 & 0.0 & 0.00 & 0.0 & 0.0 & 0.00 & 0.00 \\
\hline O-XYLENE & 4.9 & 2.38 & 31.7 & 0.1 & 2.31 & 1.0 & 0.5 & 1.71 & 3.4 & $1: 2$ & 2.28 & 8.01 \\
\hline 2,4,6-TRIMETHYLHEXANE & 0.0 & 0.00 & 0.0 & 0.0 & 0.00 & 0.0 & 0.0 & 0.00 & 0.0 & 0.0 & 0.00 & 0.00 \\
\hline 1-NONENE & 0.2 & 0.09 & 0.4 & 0.0 & 0.00 & 0.0 & 0.0 & 10.00 & 0.0 & 0.0 & 0.07 & 0.09 \\
\hline NONANE & 0.3 & 0.16 & 0.2 & 0.0 & 0.00 & 0.0 & 0.0 & 0.00 & 0.0 & 0.1 & 0.12 & 0.04 \\
\hline ISOPROPYLBENZENE (CUMENE) & 0.2 & 0.10 & 0.6 & 0.0 & 0.00 & 0.0 & 0.0 & 0.00 & 0.0 & 0.0 & 0.08 & 0.10 \\
\hline 2,2-DIMETHYLOCTANE & 0.3 & 0.14 & 0.3 & 0.0 & 0.00 & 0.0 & 0.0 & 0.00 & 0.0 & 0.1 & 0.11 & 0.08 \\
\hline 2,4-DIMETHYLOCTANE & 0.0 & 0.00 & 0.0 & 0.0 & 0.00 & 0.0 & 0.0 & 0.00 & 0.0 & 0.0 & 0.00 & 0.00 \\
\hline n-PROPYLBENZENE & 0.9 & 0.43 & 1.8 & 0.0 & 0.00 & 0.0 & 0.0 & 0.00 & 0.0 & 0.2 & 0.33 & 0.38 \\
\hline 1-METHYL-3-ETHYLBENZENE & 3.8 & 1.83 & 27.1 & 0.0 & 0.00 & 0.0 & 0.2 & 0.74 & 1.6 & 0.8 & 1.65 & 6.08 \\
\hline 1-METHYL-4-ETHYLBENZENE & 1.5 & 0.71 & 10.5 & 0.0 & 0.00 & 0.0 & 0.1 & 0.38 & 0.8 & 0.3 & 0.61 & 2.40 \\
\hline 1,3,5-TRIMETHYLBENZENE & $\overline{1.6}$ & 0.75 & 15.6 & 0.0 & 0.00 & 0.0 & 0.2 & 0.67 & 1.8 & 0.4 & 0.67 & 3.71 \\
\hline 1-METHYL-2-ETHYLBENZENE & 1.4 & 0.70 & 10.4 & 0.0 & 0.00 & 0.0 & 0.1 & 0.27 & 0.6 & 0.3 & 0.69 & 2.32 \\
\hline 1,2,4-TRIMETHYLBENZENE & 4.4 & 2.11 & 38.4 & 0.0 & 0.00 & 0.0 & 0.1 & 0.39 & 1.0 & 0.9 & 1.71 & 8.25 \\
\hline DECANE & 0.2 & 0.08 & 0.1 & 0.1 & 1.58 & 0.0 & 0.0 & 0.00 & 0.0 & 0.1 & 0.17 & 0.04 \\
\hline ISOBUTYLBENZENE & 0.1 & 0.05 & 0.2 & 0.1 & 1.49 & 0.2 & 0.0 & 0.00 & 0.0 & 0.1 & 0.13 & 0.14 \\
\hline
\end{tabular}




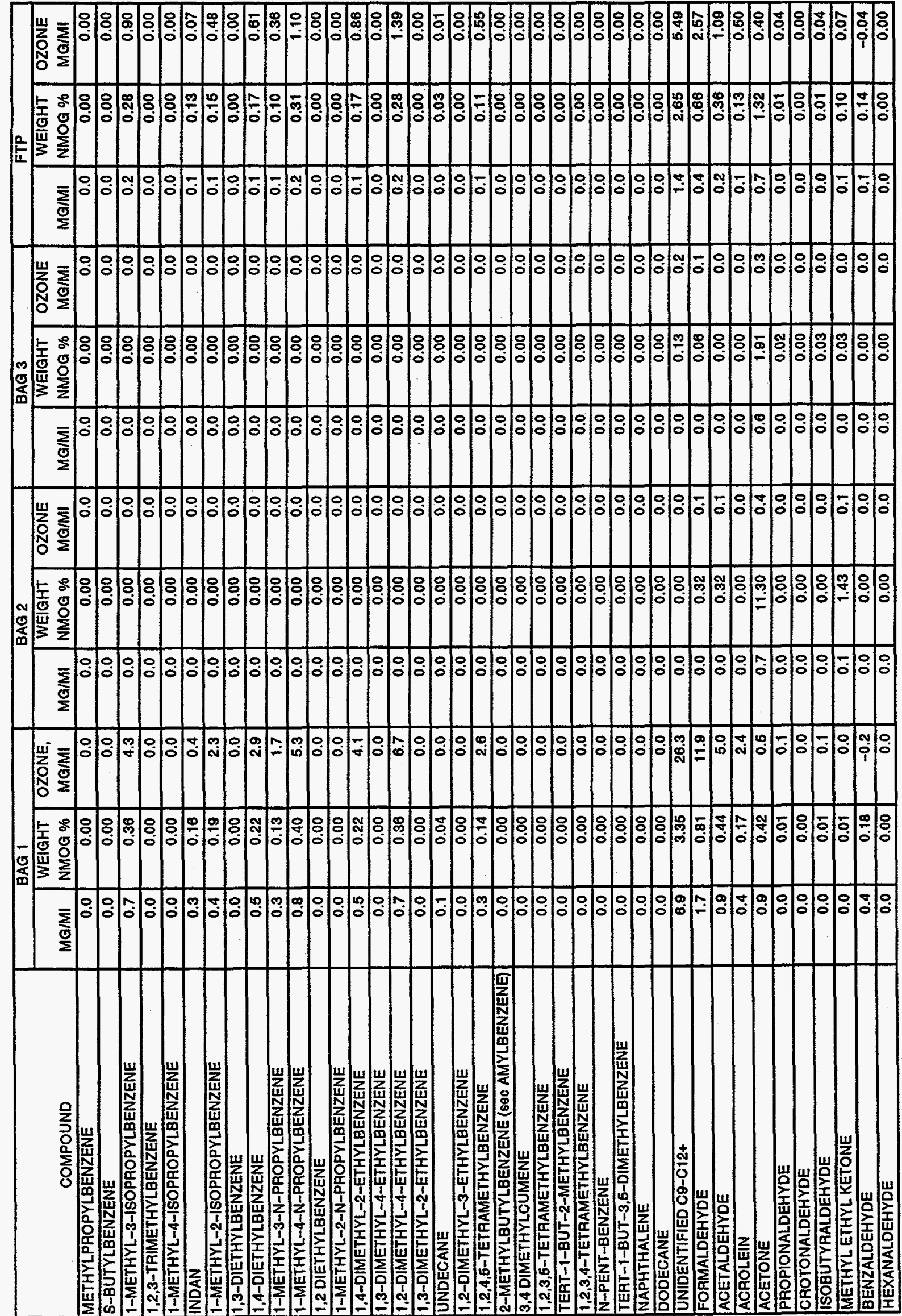


TEST NO: 100NB-02S

TEST CAR: 1994 PONTIAC GRANDAM

TEST DATE: $9 / 6 / 95$

TEST FUEL: BUTANE

\begin{tabular}{|c|c|c|c|c|c|c|c|c|c|c|c|c|}
\hline \multirow[b]{2}{*}{ COMPOUND } & \multicolumn{3}{|c|}{ BAG 1} & \multicolumn{3}{|c|}{ BAG 2} & \multicolumn{3}{|c|}{ BAG 3} & \multicolumn{3}{|c|}{ FTP } \\
\hline & MGMI & $\begin{array}{l}\text { WEIGHT } \\
\text { NMOG } \%\end{array}$ & $\begin{array}{l}\text { OZONE, } \\
\text { MGMI }\end{array}$ & MG/MI & $\begin{array}{l}\text { WEIGHT } \\
\text { NMOG \% }\end{array}$ & $\begin{array}{l}\text { OZONE } \\
\text { MG/MI }\end{array}$ & MG/MI & $\begin{array}{l}\text { WEIGHT } \\
\text { NMOG } \%\end{array}$ & $\begin{array}{l}\text { OZONE } \\
\text { MG/MI }\end{array}$ & MG/MI & $\begin{array}{l}\text { WEIQHT } \\
\text { NMOQ } \%\end{array}$ & $\begin{array}{l}\text { OZONE } \\
\text { MQMI }\end{array}$ \\
\hline METHANE & 28.1 & & 0.4 & 6.7 & & 0.1 & 35.4 & & 0.5 & 19.0 & & 0.28 \\
\hline ETHANE & 3.2 & 1.29 & 0.8 & 0.0 & 1.15 & 0.0 & 3.5 & 8.08 & 0.9 & 1.7 & 2.55 & 0.41 \\
\hline ETHYLENE & 19.0 & 7.60 & 138.8 & 0.0 & 1.60 & 0.2 & 4.3 & 9.88 & 31.7 & 5.2 & 7.93 & 37.59 \\
\hline PROPANE & 0.4 & 0.15 & 0.2 & 0.0 & 0.00 & 0.0 & 0.2 & 0.37 & 0.1 & 0.1 & 0.19 & 0.06 \\
\hline PROPYLENE & 8.1 & 3.22 & 76.8 & 0.0 & 0.73 & 0.1 & 1.1 & 2.61 & 10.8 & 2.0 & 3.07 & 18.76 \\
\hline ACETYLENE & 5.4 & 2.16 & 2.7 & 0.1 & 6.06 & 0.0 & 0.0 & 0.09 & 0.0 & 1.2 & 1.82 & 0.59 \\
\hline PROPADIENE & 0.0 & 0.00 & 0.0 & 0.0 & 0.00 & 0.0 & 0.0 & 0.00 & 0.0 & 0.0 & 0.00 & 0.00 \\
\hline BUTANE & 204.7 & 81.67 & 208.8 & 0.7 & 38.50 & 0.7 & 29.4 & 66.73 & 30.0 & 50.9 & 78.24 & 51.88 \\
\hline TRANS-2-BUTENE & 0.3 & 0.14 & 3.5 & 0.0 & 0.00 & 0.0 & 0.3 & 0.63 & 2.7 & 0.1 & 0.23 & 1.48 \\
\hline 1-BUTENE & 0.7 & 0.28 & 6.1 & 0.0 & 0.00 & 0.0 & 0.2 & 0.44 & 1.7 & 0.2 & 0.30 & 1.75 \\
\hline 2-METHYLPROPENE (ISOBUTYLENE) & 0.1 & 0.03 & 0.4 & 0.0 & 0.00 & 0.0 & 0.4 & 0.82 & 1.9 & 0.1 & 0.18 & 0.61 \\
\hline 2,2-DIMETHYLPROPANE (NEOPENTANE) & 0.0 & 0.00 & 0.0 & 0.0 & 0.00 & 0.0 & 0.0 & 0.01 & 0.0 & 0.0 & 0.00 & 0.00 \\
\hline PROPYNE & 0.0 & 0.00 & 0.0 & 0.0 & 0.00 & 0.0 & 0.0 & 0.00 & 0.0 & 0.0 & 0.00 & 0.00 \\
\hline 1,3-BUTADIENE & 0.2 & 0.06 & 1.7 & 0.0 & 0.00 & 0.0 & 0.0 & 0.00 & 0.0 & 0.0 & 0.05 & 0.36 \\
\hline 2-METHYLPROPANE (ISOBUTANE) & 0.0 & 0.00 & 0.0 & 0.0 & 0.00 & 0,0 & 0.0 & 0.00 & 0.0 & 0.0 & 0.00 & 0.00 \\
\hline 1-BUTYNE & 0.0 & 0.00 & 0.0 & 0.0 & 0.00 & 0.0 & 0.0 & 0.00 & 0.0 & 0.0 & 0.00 & 0.00 \\
\hline METHANOL & 0.0 & 0.00 & 0.0 & 0.0 & 0.00 & 0.0 & 0.0 & 0.00 & 0.0 & 0.0 & 0.00 & 0.00 \\
\hline CIS-2-BUTENE & 0.4 & 0.15 & 3.7 & 0.0 & 0.00 & 0.0 & 0.2 & 0.48 & 2.1 & 0.1 & 0.21 & 1.35 \\
\hline 3-METHYL-1-BUTENE & 0.0 & 0.00 & 0.0 & 0.0 & 0.00 & 0.0 & 0.0 & 0.00 & 0.0 & 0.0 & 0.00 & 0.00 \\
\hline ETHANOL & 0.0 & 0.00 & 0.0 & 0.0 & 0.00 & 0.0 & 0.0 & 0.00 & 0.0 & 0.0 & 0.00 & 0.00 \\
\hline 2-METHYLBUTANE (ISOPENTANE) & 1.6 & 0.65 & 2.2 & 0.0 & 0.00 & 0.0 & 1.7 & 3.78 & 2.3 & 0.8 & 1.22 & 1.09 \\
\hline 2-BUTYNE & 0.0 & 0.00 & 0.0 & 0.0 & 0.00 & 0.0 & 0.0 & 0.00 & 0.0 & 0.0 & 0.00 & 0.00 \\
\hline 1-PENTENE & 0.0 & 0.00 & 0.0 & 0.0 & 0.00 & 0.0 & 0.0 & 0.00 & 0.0 & 0.0 & 0.00 & 0.00 \\
\hline 2-METHYL-1-BUTENE & 0.0 & 0.00 & 0.0 & 0.0 & 0.00 & 0.0 & 0.0 & 0.00 & 0.0 & 0.0 & 0.00 & 0.00 \\
\hline PENTANE & 0.2 & 0.08 & 0.2 & 0.0 & 0.00 & 0.0 & 0.2 & 0.46 & 0.2 & 0.1 & 0.14 & 0.08 \\
\hline UNIDENTIFIED C5 OLEFINS & 0.0 & 0.00 & 0.0 & 0.0 & 0.00 & 0.0 & 0.0 & 0.00 & 0.0 & 0.0 & 0.00 & 0.00 \\
\hline 2-METHYL-1,3-BUTADIENE & 0.0 & 0.00 & 0.0 & 0.0 & 0.00 & 0.0 & 0.0 & 10.00 & 0.0 & 0.0 & 0.00 & 0.00 \\
\hline TRANS-2-PENTENE & 0.1 & 0.04 & 0.8 & 0.0 & 0.00 & 0.0 & 0.0 & 0.00 & 0.0 & 0.0 & 0.03 & 0.17 \\
\hline 3,3-DIMETHYL-1-BUTENE & 0.0 & 0.00 & 0.0 & 0.0 & 0.00 & 0.0 & 0.0 & 0.00 & 0.0 & 0.0 & 0.00 & 0.00 \\
\hline CIS-2-PENTENE & 0.0 & 0.00 & 0.0 & 0.0 & 0.00 & 0.0 & 0.0 & 0.00 & 0.0 & 0.0 & 0.00 & 0.00 \\
\hline 2-METHYL-2-BUTENE & 0.2 & 0.08 & 1.0 & 0.1 & 3.64 & 0.4 & 0.0 & 0.06 & 0.2 & 0.1 & 0.11 & 0.48 \\
\hline CYCLOPENTADIENE & 0.0 & 0.00 & 0.0 & 0.0 & 0.00 & 0.0 & 0.0 & 0.00 & 0.0 & 0.0 & 0.00 & 0.00 \\
\hline 2,2-DIMETHYLBUTANE & 0.0 & 0.01 & 0.0 & 0.0 & 1.05 & 0.0 & 0.0 & 0.00 & 0.0 & 0.0 & 0.02 & 0.01 \\
\hline CYCLOPENTENE & 0.0 & 0.00 & 0.0 & 0.0 & 0.00 & 0.0 & 0.0 & 0.00 & 0.0 & 0.0 & 0.00 & 0.00 \\
\hline 4-METHYL-1-PENTENE & 0.0 & 0.00 & 0.0 & 0.0 & 0.00 & 0.0 & 0.0 & 0.00 & 0.0 & 0.0 & 0.00 & 0.00 \\
\hline 3-METHYL-1-PENTENE & 0.0 & 0.00 & 0.0 & 0.0 & 0.09 & 0.0 & 0.0 & 0.06 & 0.1 & 0.0 & 0.01 & 0.04 \\
\hline CYCLOPENTANE & 0.0 & 0.00 & 0.0 & 0.0 & 0.00 & 0.0 & 0.0 & 0.00 & 0.0 & 0.0 & 0.00 & 0.00 \\
\hline 2,3-DIMETHYLBUTANE & 0.1 & 0.05 & 0.1 & 0.1 & 3.67 & 0.1 & 0.1 & 0.28 & 0.1 & 0.1 & 0.14 & 0.10 \\
\hline MTBE & 0.0 & 0.00 & 0.0 & 0.0 & 0.00 & 0.0 & 0.0 & 0.00 & 0.0 & 0.0 & 0.00 & 0.00 \\
\hline
\end{tabular}




\begin{tabular}{|c|c|c|c|c|c|c|c|c|c|c|c|c|}
\hline \multirow[b]{2}{*}{ COMPOUND } & \multicolumn{3}{|c|}{ BAG 1} & \multicolumn{3}{|c|}{$\overline{B A G 2}$} & \multicolumn{3}{|c|}{ BAG 3} & \multicolumn{3}{|c|}{ FTP } \\
\hline & MG/MI & $\begin{array}{l}\text { WEIGHT } \\
\text { NMOG \% } \\
\end{array}$ & $\begin{array}{l}\text { OZONE, } \\
\text { MG/MI }\end{array}$ & $\mathrm{MG} / \mathrm{MI}$ & \begin{tabular}{|l|} 
WEIGHT \\
NMOQ \% \\
\end{tabular} & $\begin{array}{l}\text { OZONE } \\
\text { MG/MI }\end{array}$ & MG/MI & $\begin{array}{l}\text { WEIGHT } \\
\text { NMOG } \%\end{array}$ & $\begin{array}{l}\text { OZONE } \\
\text { MG/MI }\end{array}$ & MG/MI & \begin{tabular}{|l|} 
WEIGHT \\
NMOG $\%$ \\
\end{tabular} & $\begin{array}{l}\text { OZONE } \\
\text { MG/MI }\end{array}$ \\
\hline 2,3-DIMETHYL-1-BUTENE & 0.0 & 0.00 & 0.0 & 0.0 & 0.00 & 0.0 & 0.0 & 0.00 & 0.0 & 0.0 & 0.00 & 0.00 \\
\hline 4-METHYL-CIS-2-PENTENE & 0.0 & 0.00 & 0.0 & 0.0 & 0.00 & 0.0 & 0.0 & 0.00 & 0.0 & 0.0 & 0.00 & 0.00 \\
\hline 2-METHYLPENTANE & 0.3 & 0.11 & 0.4 & 0.0 & 0.00 & 0.0 & 0.2 & 0.46 & 0.3 & 0.1 & 0.17 & 0.17 \\
\hline 4-METHYL-TRANS-2-PENTENE & 0.0 & 0.00 & 0.0 & 0.0 & 0.00 & 0.0 & 0.0 & 0.00 & 0.0 & 0.0 & 0.00 & 0.00 \\
\hline 3-METHYLPENTANE & 0.1 & 0.06 & 0.2 & 0.0 & 0.00 & 0.0 & 0.1 & 0.31 & 0.2 & 0.1 & 0.10 & 0.10 \\
\hline 2-METHYL-1-PENTENE & 0.0 & 0.00 & 0.0 & 0.0 & 0.00 & 0.0 & 0.0 & 0.00 & 0.0 & 0.0 & 0.00 & 0.00 \\
\hline 1-HEXENE & 0.0 & 0.00 & 0.0 & 0.0 & 0.00 & 0.0 & 0.0 & 0.00 & 0.0 & 0.0 & 0.00 & 0.00 \\
\hline HEXANE & 0.2 & 0.07 & 0.2 & 0.0 & 0.00 & 0.0 & 0.0 & 0.06 & 0.0 & 0.0 & 0.06 & 0.04 \\
\hline UNIDENTIFIED C 6 OLEFINS & 0.0 & 0.00 & 0.0 & 0.0 & 0.00 & 0.0 & 0.0 & 0.00 & 0.0 & 0.0 & 0.00 & 0.00 \\
\hline TRANS-3-HEXENE & 0.0 & 0.00 & 0.0 & 0.0 & 0.00 & 0.0 & 0.0 & 0.00 & 0.0 & 0.0 & 0.00 & 0.00 \\
\hline CIS-3-HEXENE & 0.0 & 0.00 & 0.0 & 0.0 & 0.00 & 0.0 & 0.0 & 0.01 & 0.0 & 0.0 & 0.00 & 0.01 \\
\hline TRANS-2-HEXENE & 0.0 & 0.00 & 0.0 & 0.0 & 0.00 & 0.0 & 0.0 & 0.00 & 0.0 & 0.0 & 0.00 & 0.00 \\
\hline 3-METHYL-TRANS-2-PENTENE & 0.0 & 0.00 & 0.0 & 0.0 & 0.00 & 0.0 & 0.0 & 0.00 & 0.0 & 0.0 & 0.00 & 0.00 \\
\hline 2-METHYL-2-PENTENE & 0.0 & 0.00 & 0.0 & 0.0 & 0.00 & 0.0 & 0.0 & 0.00 & 0.0 & 0.0 & 0.00 & 0.00 \\
\hline 3-METHYLCYCLOPENTENE & 0.0 & 0.00 & 0.0 & 0.0 & 0.00 & 0.0 & 0.0 & 0.00 & 0.0 & 0.0 & 0.00 & 0.00 \\
\hline CIS-2-HEXENE & 0.0 & 0.00 & 0.0 & 0.0 & 0.00 & 0.0 & 0.0 & 0.00 & 0.0 & 0.0 & 0.00 & 0.00 \\
\hline ETBE & 0.0 & 0.00 & 0.0 & 0.0 & 0.00 & 0.0 & 0.0 & 0.00 & 0.0 & 0.0 & 0.00 & 0.00 \\
\hline 3-METHYL-CIS-2-PENTENE & 0.0 & 0.00 & 0.0 & 0.0 & 0.00 & 0.0 & 0.0 & 0.00 & 0.0 & 0.0 & 0.00 & 0.00 \\
\hline 2,2-DIMETHYLPENTANE & 0.1 & 0.03 & 0.1 & 0.0 & 0.00 & 0.0 & 0.1 & 0.14 & 0.1 & 0.0 & 0.05 & 0.04 \\
\hline METHYLCYCLOPENTANE & 0.0 & 0.00 & 0.0 & 0.0 & 0.00 & 0.0 & 0.0 & 0.00 & 0.0 & 0.0 & 0.00 & 0.00 \\
\hline 2,4-DIMETHYLPENTANE & 0.2 & 0.06 & 0.3 & 0.1 & 5.35 & 0.2 & 0.1 & 0.25 & 0.2 & 0.1 & 0.18 & 0.21 \\
\hline 2,3,3-TRIMETHYL-1-BUTENE & 0.0 & 0.00 & 0.0 & 0.0 & 0.00 & 0.0 & 0.0 & 0.00 & 0.0 & 0.0 & 0.00 & 0.00 \\
\hline 2,2,3-TRIMETHYLBUTANE & 0.0 & 0.01 & 0.0 & 0.1 & 5.05 & 0.1 & 0.0 & 0.08 & 0.0 & 0.1 & 0.10 & 0.09 \\
\hline 3,4-DIMETHYL-1-PENTENE & 0.0 & 0.00 & 0.0 & 0.0 & 0.00 & 0.0 & 0.0 & 0.00 & 0.0 & 0.0 & 0.00 & 0.00 \\
\hline 1-METHYLCYCLOPENTENE & 0.1 & 0.02 & 0.3 & 0.0 & 0.00 & 0.0 & 0.0 & 0.00 & 0.0 & 0.0 & 0.02 & 0.06 \\
\hline BENZENE & 0.5 & 0.18 & 0.2 & 0.0 & 0.00 & 0.0 & 0.2 & 0.34 & 0.1 & 0.1 & 0.21 & 0.06 \\
\hline 3-METHYL-1-HEXENE & 0.0 & 0.00 & 0.0 & 0.0 & 0.00 & 0.0 & 0.0 & 0.00 & 0.0 & 0.0 & 0.00 & 0.00 \\
\hline 3,3-DIMETHYLPENTANE & 0.0 & 0.01 & 0.0 & 0.0 & 0.00 & 0.0 & 0.0 & 0.00 & 0.0 & 0.0 & 0.01 & 0.00 \\
\hline CYCLOHEXANE & 0.0 & 0.00 & 0.0 & 0.3 & 16.84 & 0.4 & 0.0 & 0.00 & 0.0 & 0.2 & 0.26 & 0.21 \\
\hline 2-METHYLHEXANE & 0.0 & 0.00 & 0.0 & 0.0 & 0.00 & 0.0 & 0.0 & 0.00 & 0.0 & 0.0 & 0.00 & 0.00 \\
\hline 2,3-DIMETHYLPENTANE & 0.3 & 0.11 & 0.4 & 0.0 & 0.00 & 0.0 & 0.2 & 0.44 & 0.3 & 0.1 & 0.17 & 0.17 \\
\hline 1,1-DIMETHYLCYCLOPENTANE & 0.0 & 0.00 & 0.0 & 0.0 & 0.00 & 0.0 & 0.0 & 0.00 & 0.0 & 0.0 & 0.00 & 0.00 \\
\hline CYCLOHEXENE & 0.0 & 0.00 & 0.0 & 0.0 & 0.00 & 0.0 & 0.0 & 0.00 & 0.0 & 0.0 & 0.00 & 0.00 \\
\hline 3-METHYLHEXANE & 0.0 & 0.00 & 0.0 & 0.0 & 0.00 & 0.0 & 0.0 & 0.00 & 0.0 & 0.0 & 0.00 & 0.00 \\
\hline CIS-1,3-DIMETHYLCYCLOPENTANE & 0.0 & 0.00 & 0.0 & 0.0 & 0.00 & 0.0 & 0.1 & 0.20 & 0.2 & 0.0 & 0.04 & 0.08 \\
\hline 3-ETHYLPENTANE & 0.0 & 0.00 & 0.0 & 0.0 & 0.00 & 0.0 & 0.0 & 0.00 & 0.0 & 0.0 & 0.00 & 0.00 \\
\hline TRANS-1,2-DIMETHYLCYCLOPENTANE & 0.0 & 0.00 & 0.0 & 0.0 & 0.00 & 0.0 & 0.0 & 0.00 & 0.0 & 0.0 & 0.00 & 0.00 \\
\hline TRANS-1,3-DIMETHYLCYCLOPENTANE & 0.0 & 0.00 & 0.0 & 0.0 & 0.00 & 0.0 & 0.0 & 0.00 & 0.0 & 0.0 & 0.00 & 0.00 \\
\hline 1-HEPTENE & 0.0 & 0.00 & 0.0 & 0.0 & 0.00 & 0.0 & 0.0 & 0.00 & 0.0 & 0.0 & 0.00 & 0.00 \\
\hline 2,2,4-TRIMETHYLPENTANE & 0.4 & 0.14 & 0.3 & 0.0 & 1.38 & 0.0 & 0.2 & 0.55 & 0.2 & 0.2 & 0.24 & 0.14 \\
\hline 2-METHYL-1-HEXENE & 0.0 & 0.00 & 0.0 & 0.0 & 0.00 & 0.0 & 0.0 & 0.00 & 0.0 & 0.0 & 0.00 & 0.00 \\
\hline TRANS-3-HEPTENE & 0.0 & 0.00 & 0.0 & 0.0 & 0.00 & 0.0 & 0.0 & 0.00 & 0.0 & 0.0 & 0.00 & 0.00 \\
\hline HEPTANE & 0.0 & 0.00 & 0.0 & 0.1 & 3.38 & 0.1 & 0.0 & 0.00 & 0.0 & 0.0 & 0.05 & 0.08 \\
\hline UNIDENTIFIED C7 & 0.0 & 0.00 & 0.0 & 0.0 & 0.87 & 0.1 & 0.0 & 0.07 & 0.1 & 0.0 & 0.03 & 0.08 \\
\hline
\end{tabular}




\begin{tabular}{|c|c|c|c|c|c|c|c|c|c|c|c|c|}
\hline \multirow[b]{2}{*}{ COMPOUND } & \multicolumn{3}{|c|}{ BAG 1} & \multicolumn{3}{|c|}{ BAG 2} & \multicolumn{3}{|c|}{ BAG 3} & \multicolumn{3}{|c|}{$\overline{\text { FTP }}$} \\
\hline & MGMI & $\begin{array}{l}\text { WEIGHT } \\
\text { NMOG \% }\end{array}$ & $\begin{array}{l}\text { OZONE, } \\
\text { MG/MI }\end{array}$ & MG/MI & $\begin{array}{l}\text { WEIGHT } \\
\text { NMOG \% }\end{array}$ & $\begin{array}{l}\text { OZONE } \\
\text { MG/MI }\end{array}$ & MG/MI & $\begin{array}{l}\text { WEIGHT } \\
\text { NMOG \% }\end{array}$ & $\begin{array}{l}\text { OZONE } \\
\text { MG/MI }\end{array}$ & MGMI & $\begin{array}{l}\text { WEIGHT } \\
\text { NMOG \% }\end{array}$ & $\begin{array}{l}\text { OZONE } \\
\text { MG/MI }\end{array}$ \\
\hline 2-METHYL-2-HEXENE & 0.0 & 0.00 & 0.0 & 0.0 & 0.00 & 0.0 & 0.0 & 0.00 & 0.0 & 0.0 & 0.00 & 0.00 \\
\hline 3-METHYL-TRANS-3-HEXENE & $\overline{0.0}$ & 0.00 & 0.0 & 0.0 & 0.00 & 0.0 & 0.0 & 0.00 & 0.0 & 0.0 & 0.00 & 0.00 \\
\hline TAANS-2-HEPTENE & 0.0 & 0.00 & 0.0 & 0.0 & 0.00 & 0.0 & 0.0 & 0.00 & 0.0 & 0.0 & 0.00 & 0.00 \\
\hline 3-ETHYL-CIS-2-PENTENE & 0.0 & 0.00 & 0.0 & 0.0 & 0.00 & 0.0 & 0.0 & 0.00 & 0.0 & 0.0 & 0.00 & 0.00 \\
\hline 2,4,4-TRIMETHYL-1-PENTENE & 0.0 & 0.00 & 0.0 & 0.0 & 0.00 & 0.0 & 0.0 & 0.00 & 0.0 & 0.0 & 0.00 & 0.00 \\
\hline 2,2,4-TRIMETHYL-1-PENTENE & 0.0 & 0.00 & 0.0 & 0.0 & 0.00 & 0.0 & 0.0 & 0.00 & 0.0 & 0.0 & 0.00 & 0.00 \\
\hline 2,3-DIMETHYL-2-PENTENE & 0.0 & 0.00 & 0.0 & 0.0 & 0.00 & 0.0 & 0.0 & 0.00 & 0.0 & 0.0 & 0.00 & 0.00 \\
\hline CIS-2-HEPTENE & 0.0 & 0.00 & 0.0 & 0.0 & 0.00 & 0.0 & 0.0 & 0.00 & 0.0 & 0.0 & 0.00 & 0.00 \\
\hline METHYLCYCLOHEXANE & 0.0 & 0.00 & 0.0 & 0.0 & 0.00 & 0.0 & 0.0 & 0.00 & 0.0 & 0.0 & 0.00 & 0.00 \\
\hline CIS-1,2-DIMETHYLCYCLOPENTANE & $\overline{0.0}$ & 0.00 & 0.0 & 0.0 & 0.00 & 0.0 & $\overline{0.0}$ & 0.00 & 0.0 & 0.0 & 0.00 & 0.00 \\
\hline 2,2-DIMETHYLHEXANE & 0.1 & 0.05 & 0.1 & 0.0 & 0.00 & 0.0 & 0.0 & 0.00 & 0.0 & 0.0 & 0.04 & 0.03 \\
\hline 1,1,3-TAIMETHYLCYCLOPENTANE & 0.0 & 0.00 & 0.0 & 0.0 & 0.00 & 0.0 & 0.0 & 0.00 & 0.0 & 0.0 & 0.00 & 0.00 \\
\hline 2,4,4-TRIMETHYL-2-PENTENE & 0.0 & 0.00 & 0.0 & 0.0 & 0.00 & 0.0 & 0.0 & 0.00 & 0.0 & 0.0 & 0.00 & 0.00 \\
\hline 2,2,3-TRIMETHYLPENTANE & $\overline{0.0}$ & 0.00 & 0.0 & 0.0 & 0.00 & 0.0 & 0.0 & 0.00 & 0.0 & 0.0 & 0.00 & 0.00 \\
\hline 2,5-DIMETHYLHEXANE & 0.0 & 0.00 & 0.0 & 0.0 & 0.00 & 0.0 & 0.0 & 0.00 & 0.0 & 0.0 & 0.00 & 0.00 \\
\hline ETHYLCYCLOPENTANE & 0.0 & 0.00 & 0.0 & 0.0 & 0.00 & 0.0 & 0.0 & 0.00 & 0.0 & 0.0 & 0.00 & 0.00 \\
\hline 2,4-DIMETHYLHEXANE & 0.0 & 0.01 & 0.0 & 0.0 & 0.00 & 0.0 & 0.0 & 0.02 & 0.0 & 0.0 & 0.01 & 0.01 \\
\hline 1-TRANS-2-CIS-4-TRIMETHYLCYCLOPENTANE & 0.0 & 0.00 & 0.0 & 0.0 & 0.00 & 0.0 & 0.0 & 0.00 & 0.0 & 0.0 & 0.00 & 0.00 \\
\hline 3,3-DIMETHYLHEXANE & 0.0 & 0.00 & 0.0 & 0.0 & 0.00 & 0.0 & 0.0 & 0.00 & 0.0 & 0.0 & 0.00 & 0.00 \\
\hline 1-TRANS-2-CIS-3-TRIMETHYLCYCLOPENTANE & 0.0 & 0.00 & 0.0 & 0.0 & 0.00 & 0.0 & 0.0 & 0.00 & 0.0 & 0.0 & 0.00 & 0.00 \\
\hline 2,3,4-TRIMETHYLPENTANE & 0.0 & 0.00 & 0.0 & 0.0 & 0.00 & 0.0 & 0.0 & 0.00 & 0.0 & 0.0 & 0.00 & 0.00 \\
\hline 2,3,3-TRIMETHYLPENTANE & 0.1 & 0.04 & 0.1 & 0.0 & 0.00 & 0.0 & 0.1 & 0.15 & 0.1 & 0.0 & 0.06 & 0.05 \\
\hline TOLUENE & 0.0 & 0.00 & 0.0 & 0.0 & 0.00 & 0.0 & 0.2 & 0.34 & 0.4 & 0.0 & 0.06 & 0.11 \\
\hline 2,3-DIMETHYLHEXANE & 0.0 & 0.00 & 0.0 & 0.0 & 0.00 & 0.0 & $\overline{0.0}$ & 0.00 & 0.0 & 0.0 & 0.00 & 0.00 \\
\hline 1,1,2-TRIMETHYLCYCLOPENTANE & 0.0 & 0.00 & 0.0 & 0.0 & 0.00 & 0.0 & 0.0 & 0.00 & 0.0 & 0.0 & 0.00 & 0.00 \\
\hline 2-METHYLHEPTANE & 0.0 & 0.00 & 0.0 & 0.0 & 0.00 & 0.0 & 0.0 & 0.00 & 0.0 & 0.0 & 0.00 & 0.00 \\
\hline 3,4-DIMETHYLHEXANE & 0.0 & 0.00 & 0.0 & 0.0 & 0.00 & 0.0 & 0.0 & 0.00 & 0.0 & 0.0 & 0.00 & 0.00 \\
\hline $2,2,4,4-T E T R A M E T H Y L P E N T A N E$ & 0.0 & 0.00 & 0.0 & 0.0 & 0.00 & 0.0 & 0.0 & 0.00 & 0.0 & 0.0 & 0.00 & 0.00 \\
\hline 4-METHYLHEPTANE & 0.0 & 0.00 & 0.0 & 0.0 & 0.00 & 0.0 & 0.0 & 0.00 & 0.0 & 0.0 & 0.00 & 0.00 \\
\hline 2-METHYL-3-ETHYLPENTANE & 0.0 & 0.00 & 0.0 & 0.0 & 0.00 & 0.0 & 0.0 & 0.00 & 0.0 & 0.0 & 0.00 & 0.00 \\
\hline 2,6-DIMETHYLHEPTANE & 0.0 & 0.00 & 0.0 & 0.0 & 0.00 & 0.0 & 0.0 & 0.00 & 0.0 & 0.0 & 0.00 & 0.00 \\
\hline 3-METHYLHEPTANE & 0.1 & 0.05 & 0.1 & 0.0 & 0.00 & 0.0 & 0.1 & 0.25 & 0.1 & 0.1 & 0.09 & 0.08 \\
\hline 1-GIS,2-TAANS,3-TAIMETHYLCYCLOPENTANE & 0.0 & 0.00 & 0.0 & 0.0 & 0.00 & 0.0 & 0.0 & 0.00 & 0.0 & 0.0 & 0.00 & 0.00 \\
\hline CIS-1,3-DIMETHYLCYCLOHEXANE & 0.0 & 0.00 & 0.0 & 0.0 & 0.00 & 0.0 & 0.0 & 0.00 & 0.0 & 0.0 & 0.00 & 0.00 \\
\hline TAANS-1,4-DIMETHYLCYCLOHEXANE & 0.0 & 0.00 & 0.0 & 0.0 & 0.00 & 0.0 & 0.0 & 0.00 & 0.0 & 0.0 & 0.00 & 0.00 \\
\hline 3-ETHYLHEXANE & 0.0 & 0.00 & 0.0 & 0.0 & 0.00 & 0.0 & 0.0 & 0.00 & 0.0 & 0.0 & 0.00 & 0.00 \\
\hline 2,2,6-TRIMETHYLHEXANE & 0.0 & 0.00 & 0.0 & 0.0 & 0.00 & 0.0 & 0.0 & 0.00 & 0.0 & 0.0 & 0.00 & 0.00 \\
\hline CIS-1-METHYL-3-ETHYLCYCLOPENTANE & 0.0 & 0.00 & 0.0 & 0.0 & 0.00 & 0.0 & 0.0 & 0.00 & 0.0 & 0.0 & 0.00 & 0.00 \\
\hline 1,1-DIMETHYLCYCLOHEXANE & 0.0 & 0.00 & 0.0 & 0.0 & 0.00 & 0.0 & $\overline{0.0}$ & 0.00 & 0.0 & 0.0 & 0.00 & 0.00 \\
\hline TRANS-1-METHYL-2-ETHYLCYCLOPENTANE & 0.0 & 0,00 & 0.0 & 0.0 & 0.00 & 0.0 & 0.0 & 0.00 & 0.0 & 0.0 & 0.00 & 0.00 \\
\hline 1-METHYL-1-ETHYL-CYCLOPENTANE & 0.0 & 0.00 & 0.0 & 0.0 & 0.00 & 0.0 & 0.0 & 0.00 & 0.0 & 0.0 & 0.00 & 0.00 \\
\hline 2,4,4-TAIMETHYLHEXANE & 0.0 & 0.00 & 0.0 & 0.0 & 0.00 & 0.0 & 0.0 & 0.00 & 0.0 & 0.0 & 0.00 & 0.00 \\
\hline 2,2,4-TRIMETHYLHEXANE & 0.0 & 0.00 & 0.0 & 0.0 & 0.00 & 0.0 & 0.0 & 0.00 & 0.0 & 0.0 & 0.00 & 0.00 \\
\hline TRANS-1,2-DIMETHYLCYCLOH & 0.0 & 0.00 & 0.0 & 0.0 & 0.00 & 0.0 & 0.0 & 0.00 & 0.0 & 0.0 & 0.00 & 0.00 \\
\hline
\end{tabular}




\begin{tabular}{|c|c|c|c|c|c|c|c|c|c|c|c|c|}
\hline \multirow[b]{2}{*}{ COMPOUND } & \multicolumn{3}{|c|}{ BAG 1} & \multicolumn{3}{|c|}{ BAG 2} & \multicolumn{3}{|c|}{ BAG 3} & \multicolumn{3}{|c|}{ FTP } \\
\hline & MG/MI & $\begin{array}{l}\text { WEIGHT } \\
\text { NMOG \% } \\
\end{array}$ & $\begin{array}{l}\text { OZONE, } \\
\text { MG/MI }\end{array}$ & MGMI & $\begin{array}{l}\text { WEIGHT } \\
\text { NMOG \% } \\
\end{array}$ & $\begin{array}{l}\text { OZONE } \\
\text { MG/MI }\end{array}$ & MG/MI & $\begin{array}{l}\text { WEIGHT } \\
\text { NMOG } \%\end{array}$ & $\begin{array}{l}\text { OZONE } \\
\text { MG/MI }\end{array}$ & MG/MI & \begin{tabular}{|l|} 
WEIGHT \\
NMOQ $\%$ \\
\end{tabular} & $\begin{array}{l}\text { OZONE } \\
\text { MG/MI }\end{array}$ \\
\hline 1-OCTENE & 0.0 & 0.00 & 0.0 & 0.0 & 0.00 & 0.0 & 0.0 & 0.00 & 0.0 & 0.0 & 0.00 & 0.00 \\
\hline TRANS-4-OCTENE & 0.0 & 0.00 & 0.0 & 0.0 & 0.00 & 0.0 & 0.0 & 0.00 & 0.0 & 0.0 & 0.00 & 0.00 \\
\hline OCTANE & 0.0 & 0.00 & 0.0 & 0.0 & 0.00 & 0.0 & 0.0 & 0.00 & 0.0 & 0.0 & 0.00 & 0.00 \\
\hline UNIDENTIFIED C8 & 0.0 & 0.01 & 0.1 & 0.0 & 0.00 & 0.0 & 0.0 & 0.02 & 0.0 & 0.0 & 0.01 & 0.03 \\
\hline TRANS-2-OCTENE & 0.0 & 0.00 & 0.0 & 0.0 & 0.00 & 0.0 & 0.0 & 0.00 & 0.0 & 0.0 & 0.00 & 0.00 \\
\hline TRANS-1,3-DIMETHYLCYCLOHEXANE & 0.0 & 0.00 & 0.0 & 0.0 & 0.00 & 0.0 & 0.0 & 0.00 & 0.0 & 0.0 & 0.00 & 0.00 \\
\hline CIS-1,4-DIMETHYLCYCLOHEXANE & 0.0 & 0.00 & 0.0 & 0.0 & 0.00 & 0.0 & 0.0 & 0.00 & 0.0 & 0.0 & 0.00 & 0.00 \\
\hline CIS-2-OCTENE & 0.0 & 0.00 & 0.0 & 0.0 & 0.00 & 0.0 & 0.0 & 0.00 & 0.0 & 0.0 & 0.00 & 0.00 \\
\hline 2,3,6-TRIMETHYLHEXANE & 0.0 & 0.00 & 0.0 & 0.0 & 0.00 & 0.0 & 0.0 & 0.00 & 0.0 & 0.0 & 0.00 & 0.00 \\
\hline CIS-1-METHYL-2-ETHYLCYCLOPENTANE & 0.0 & 0.00 & 0.0 & 0.0 & 0.00 & 0.0 & 0.0 & 0.00 & 0.0 & 0.0 & 0.00 & 0.00 \\
\hline 2-METHYL-2-ETHYLHEPTANE & 0.0 & 0.00 & 0.0 & 0.0 & 0.00 & 0.0 & 0.0 & 0.00 & 0.0 & 0.0 & 0.00 & 0.00 \\
\hline 2,4-DIMETHYLHEPTANE & 0.0 & 0.00 & 0.0 & 0.0 & 2.31 & 0.1 & 0.0 & 0.00 & 0.0 & 0.0 & 0.04 & 0.03 \\
\hline 4,4-DIMETHYLHEPTANE & 0.0 & 0.00 & 0.0 & 0.0 & 0.00 & 0.0 & 0.0 & 0.00 & 0.0 & 0.0 & 0.00 & 0.00 \\
\hline CIS-1,2-DIMETHYLCYCLOHEXANE & 0.0 & 0.00 & 0.0 & 0.0 & 0.00 & 0.0 & 0.0 & 0.00 & 0.0 & 0.0 & 0.00 & 0.00 \\
\hline ETHYLCYCLOHEXANE & 0.0 & 0.00 & 0.0 & 0.0 & 0.00 & 0.0 & 0.0 & 0.00 & 0.0 & 0.0 & 0.00 & 0.00 \\
\hline PROPYLCYCLOHEXANE & 0.0 & 0.00 & 0.0 & 0.0 & 0.00 & 0.0 & 0.0 & 0.00 & 0.0 & 0.0 & 0.00 & 0.00 \\
\hline 2-METHYL-4-ETHYLHEXANE & 0.0 & 0.00 & 0.0 & 0.0 & 0.00 & 0.0 & 0.0 & 0.00 & 0.0 & 0.0 & 0.00 & 0.00 \\
\hline 2,6-DIMETHYLHEPTANE & 0.0 & 0.00 & 0.0 & 0.0 & 0.00 & 0.0 & 0.0 & 0.00 & 0.0 & 0.0 & 0.00 & 0.00 \\
\hline 1,1,3-TAIMETHYLCYCLOHEXANE & 0.0 & 0.00 & 0.0 & 0.0 & 0.00 & 0.0 & 0.0 & 0.00 & 0.0 & 0.0 & 0.00 & 0.00 \\
\hline 2,6-DIMETHYLHEPTANE & 0.0 & 0.00 & 0.0 & 0.0 & 0.00 & 0.0 & 0.0 & 0.00 & 0.0 & 0.0 & 0.00 & 0.00 \\
\hline 3,3-DIMETHYLHEPTANE & 0.0 & 0.00 & 0.0 & 0.0 & 0.00 & 0.0 & 0.0 & 0.00 & 0.0 & 0.0 & 0.00 & 0.00 \\
\hline 3,6-DIMETHYLHEPTANE & 0.0 & 0.00 & 0.0 & 0.0 & 0.00 & 0.0 & 0.0 & 0.00 & 0.0 & 0.0 & 0.00 & 0.00 \\
\hline ETHYLBENZENE & 0.0 & 0.02 & 0.1 & 0.0 & 0.00 & 0.0 & 0.0 & 0.00 & 0.0 & 0.0 & 0.01 & 0.02 \\
\hline 2,3-DIMETHYLHEPTANE & 0.0 & 0.00 & 0.0 & 0.0 & 0.00 & 0.0 & 0.0 & 0.00 & 0.0 & 0.0 & 0.00 & 0.00 \\
\hline m-\& p-XYLENE & 0.1 & 0.05 & 1.0 & 0.0 & 0.00 & 0.0 & 0.0 & 0.00 & 0.0 & 0.0 & 0.04 & 0.20 \\
\hline 4-METHYLOCTANE & 0.0 & 0.00 & 0.0 & 0.0 & 0.00 & 0.0 & 0.0 & 0.00 & 0.0 & 0.0 & 0.00 & 0.00 \\
\hline 2-METHYLOCTANE & 0.0 & 0.00 & 0.0 & 0.0 & 0.00 & 0.0 & 0.0 & 0.00 & 0.0 & 0.0 & 0.00 & 0.00 \\
\hline 3-METHYLOCTANE & 0.0 & 0.00 & 0.0 & 0.0 & 0.00 & 0.0 & 0.0 & 0.00 & 0.0 & 0.0 & 0.00 & 0.00 \\
\hline STYAENE & 0.0 & 0.00 & 0.0 & 0.0 & 0.00 & 0.0 & 0.0 & 0.00 & 0.0 & 0.0 & 0.00 & 0.00 \\
\hline O-XYLENE & 0.0 & 0.02 & 0.2 & 0.0 & 0.00 & 0.0 & 0.1 & 0.22 & 0.6 & 0.0 & 0.05 & 0.22 \\
\hline 2,4,6-TAIMETHYLHEXANE & 0.0 & 0.00 & 0.0 & 0.0 & 0.00 & 0.0 & 0.0 & 0.00 & 0.0 & 0.0 & 0.00 & 0.00 \\
\hline 1-NONENE & 0.0 & 0.00 & 0.0 & 0.0 & 0.00 & 0.0 & 0.0 & 0.00 & $0 . \overline{0}$ & 0.0 & 0.00 & 0.00 \\
\hline NONANE & 0.0 & 0.00 & 0.0 & 0.0 & 0.00 & 0.0 & 0.0 & 0.00 & 0.0 & 0.0 & 0.00 & 0.00 \\
\hline ISOPAOPYLBENZENE (CUMENE) & 0.0 & 0.00 & 0.0 & 0.0 & 0.00 & 0.0 & 0.0 & 0.00 & 0.0 & 0.0 & 0.00 & 0.00 \\
\hline 2,2-DIMETHYLOCTANE & 0.0 & 0.00 & 0.0 & 0.0 & 0.00 & 0.0 & 0.0 & 0.00 & 0.0 & 0.0 & 0.00 & 0.00 \\
\hline 2,4-DIMETHYLOCTANE & 0.0 & 0.00 & 0.0 & 0.0 & 0.00 & 0.0 & 0.0 & 0.00 & 0.0 & 0.0 & 0.00 & 0.00 \\
\hline n-PROPYLBENZENE & 0.0 & 0.00 & 0.0 & 0.0 & 0.00 & 0.0 & 0.0 & 0.00 & 0.0 & 0.0 & 0.00 & 0.00 \\
\hline 1-METHYL-3-ETHYLBENZENE & 0.0 & 0.02 & 0.3 & 0.0 & 0.00 & 0.0 & 0.1 & 0.24 & 0.8 & 0.0 & 0.06 & 0.28 \\
\hline 1-METHYL-4-ETHYLBENZENE & 0.1 & 0.03 & 0.6 & 0.0 & 0.00 & 0.0 & 0.0 & 0.00 & 0.0 & 0.0 & 0.03 & 0.12 \\
\hline 1,3,5-TRIMETHYLBENZENE & 0.1 & 0.03 & 0.8 & 0.0 & 0.00 & 0.0 & 0.0 & 0.00 & 0.0 & 0.0 & 0.03 & 0.17 \\
\hline 1-METHYL-2-ETHYLBENZENE & 0.0 & 0.00 & 0.0 & 0.0 & 0.00 & 0.0 & 0.0 & 0.00 & 0.0 & 0.0 & 0.00 & 0.00 \\
\hline 1,2,4-TRIMETHYLBENZENE & 0.1 & 0.02 & 0.5 & 0.0 & 0.00 & 0.0 & 0.1 & 0.19 & 0.7 & 0.0 & 0.05 & 0.30 \\
\hline DECANE & 0.0 & 0.02 & 0.0 & 0.0 & 0.00 & 0.0 & 0.0 & 0.00 & 0.0 & 0.0 & 0.01 & 0.00 \\
\hline ISOBUTYLBENZENE & 0.0 & 0.01 & 0.1 & 0.0 & 0.00 & 0.0 & 0.0 & 0.00 & 0.0 & 0.0 & 0.01 & 0.01 \\
\hline
\end{tabular}




\begin{tabular}{|c|c|c|c|c|c|c|c|c|c|c|c|c|}
\hline \multirow[b]{2}{*}{ COMPOUND } & \multicolumn{3}{|c|}{$\overline{B A G 1}$} & \multicolumn{3}{|c|}{$\overline{B A G 2}$} & \multicolumn{3}{|c|}{ BAG 3} & \multicolumn{3}{|c|}{ FTP } \\
\hline & MG/MI & $\begin{array}{l}\text { WEIGHT } \\
\text { NMOG \% }\end{array}$ & $\begin{array}{l}\text { OZONE, } \\
\text { MGMI }\end{array}$ & MG/MI & $\begin{array}{l}\text { WEIGHT } \\
\text { NMOG } \%\end{array}$ & $\begin{array}{l}\text { OZONE } \\
\text { MG/MI }\end{array}$ & MG/MI & $\begin{array}{l}\text { WEIGHT } \\
\text { NMOG } \%\end{array}$ & $\begin{array}{l}\text { OZONE } \\
\text { MGMII }\end{array}$ & MG/MI & $\begin{array}{l}\text { WEIGHT } \\
\text { NMOG } \%\end{array}$ & $\begin{array}{l}\text { OZONE } \\
\text { MG/MI }\end{array}$ \\
\hline METHYLPROPYLBENZENE & 0.0 & 0.00 & 0.0 & 0.0 & 0.00 & 0.0 & 0.0 & 0.00 & 0.0 & 0.0 & 0.00 & 0.00 \\
\hline S-BUTYLBENZENE & 0.0 & 0.00 & 0.0 & 0.0 & 0.00 & 0.0 & 0.0 & 0.00 & 0.0 & 0.0 & 0.00 & 0.00 \\
\hline 1-METHYL-3-1SOPROPYLBENZENE & 0.0 & 0.00 & 0.0 & 0.0 & 0.00 & 0.0 & 0.0 & 0.00 & 0.0 & 0.0 & 0.00 & 0.00 \\
\hline 1,2,3-TRIMETHYLBENZENE & 0.0 & 0.00 & 0.0 & 0.0 & 0.00 & 0.0 & 0.0 & 0.00 & 0.0 & 0.0 & 0.00 & 0.00 \\
\hline 1-METHYL-4-1SOPROPYLBENZENE & 0.0 & 0.00 & 0.0 & 0.0 & 0.00 & 0.0 & 0.0 & 0.00 & 0.0 & 0.0 & 0.00 & 0.00 \\
\hline INDAN & 0.0 & 0.00 & 0.0 & 0.0 & 0.00 & 0.0 & 0.0 & 0.00 & 0.0 & 0.0 & 0.00 & 0.00 \\
\hline 1-METHYL-2-ISOPROPYLBENZENE & 0.0 & 0.00 & 0.0 & 0.0 & 0.00 & 0.0 & 0.0 & 0.00 & 0.0 & 0.0 & 0.00 & 0.00 \\
\hline 1,3-DIETHYLBENZENE & 0.0 & 0.00 & 0.0 & 0.0 & 0.00 & 0.0 & 0.0 & 0.00 & 0.0 & 0.0 & 0.00 & 0.00 \\
\hline 1,4-DIETHYLBENZENE & 0.0 & 0.00 & 0.0 & 0.0 & 0.00 & 0.0 & 0.0 & 0.00 & 0.0 & 0.0 & 0.00 & 0.00 \\
\hline 1-METHYL-3-N-PROPYLBENZENE & 0.0 & 0.00 & 0.0 & 0.0 & 0.00 & 0.0 & 0.0 & 0.00 & 0.0 & 0.0 & 0.00 & 0.00 \\
\hline 1-METHYL-4-N-PROPYLBENZENE & 0.0 & 0.00 & 0.0 & 0.0 & 0.00 & 0.0 & 0.0 & 0.00 & 0.0 & 0.0 & 0.00 & 0.00 \\
\hline 1,2 DIETHYLBENZENE & 0.0 & 0.00 & 0.0 & 0.0 & 0.00 & 0.0 & $0: 0$ & 0.00 & 0.0 & 0.0 & 0.00 & 0.00 \\
\hline 1-METHYL-2-N-PROPYLBENZENE & 0.0 & 0.00 & 0.0 & 0.0 & 0.00 & 0.0 & 0.0 & 0.00 & 0.0 & 0.0 & 0.00 & 0.00 \\
\hline 1,4-DIMETHYL-2-ETHYLBENZENE & 0.0 & 0.00 & 0.0 & 0.0 & 0.00 & 0.0 & 0.0 & 0.00 & 0.0 & $\overline{0.0}$ & 0.00 & 0.00 \\
\hline 1,3-DIMETHYL-4-ETHYLBENZENE & 0.0 & 0.00 & 0.0 & 0.0 & 0.00 & 0.0 & 0.0 & 0.00 & 0.0 & 0.0 & 0.00 & 0.00 \\
\hline 1,2-DIMETHYL-4-ETHYLBENZENE & 0.0 & 0.00 & 0.0 & 0.0 & 0.00 & 0.0 & 0.0 & 0.00 & 0.0 & 0.0 & 0.00 & 0.00 \\
\hline 1,3-DIMETHYL-2-ETHYLBENZENE & 0.0 & 0.00 & 0.0 & 0.0 & 0.00 & 0.0 & 0.0 & 0.00 & 0.0 & 0.0 & 0.00 & 0.00 \\
\hline UNNDECANE & 0.0 & 0.00 & 0.0 & 0.0 & 0.00 & 0.0 & 0.0 & 0.00 & 0.0 & 0.0 & 0.00 & 0.00 \\
\hline 1,2-DIMETHYL-3-ETHYLBENZENE & 0.0 & 0.00 & 0.0 & 0.0 & 0.00 & 0.0 & 0.0 & 0.00 & 0.0 & 0.0 & 0.00 & 0.00 \\
\hline 1,2,4,5-TETAAMETHYLBENZENE & 0.3 & 0.12 & 2.8 & 0.0 & 0.00 & 0.0 & 0.0 & 0.00 & 0.0 & 0.1 & 0.10 & 0.67 \\
\hline 2-METHYLBUTYLBENZENE (8EC AMYLBENZENE) & 0.0 & 0.00 & 0.0 & 0.0 & 0.00 & 0.0 & 0.0 & 0.00 & 0.0 & 0.0 & 0.00 & 0.00 \\
\hline 3,4 DIMETHYLCUMENE & 0.0 & 0.00 & 0.0 & 0.0 & 0.00 & 0.0 & 0.0 & 0.00 & 0.0 & 0.0 & 0.00 & 0.00 \\
\hline $1,2,3,5$-TETRAMETHYLBENZENE & 0.0 & 0.00 & 0.0 & 0.0 & 0.00 & 0.0 & 0.0 & 0.00 & 0.0 & $\overline{0.0}$ & 0.00 & 0.00 \\
\hline TERT-1-BUT-2-METHYLBENZENE & 0.0 & 0.00 & 0.0 & 0.0 & 0.00 & 0.0 & 0.0 & 0.00 & 0.0 & 0.0 & 0.00 & 0.00 \\
\hline $1,2,3,4$-TETAAMETHYLBENZENE & 0.0 & 0.00 & 0.0 & 0.0 & 0.00 & 0.0 & 0.0 & 0.00 & 0.0 & 0.0 & 0.00 & 0.00 \\
\hline N-PENT-BENZENE & 0.0 & 0.00 & 0.0 & 0.0 & 0.00 & 0.0 & 0.0 & 0.00 & 0.0 & $\overline{0.0}$ & 0.00 & 0.00 \\
\hline TERT-1-BUT-3,5-DIMETHYLBENZENE & 0.0 & 0.00 & 0.0 & 0.0 & 0.00 & 0.0 & 0.0 & 0.00 & 0.0 & 0.0 & 0.00 & 0.00 \\
\hline NAPHTHALENE & 0.0 & 0.00 & 0.0 & 0.0 & 0.00 & 0.0 & 0.0 & 0.00 & 0.0 & 0.0 & 0.00 & 0.00 \\
\hline DODECANE & 0.0 & 0.00 & 0.0 & 0.0 & 0.00 & 0.0 & 0.0 & 0.00 & 0.0 & 0.0 & 0.00 & 0.00 \\
\hline UNIDENTIFIED C $9-\mathrm{C} 12+$ & 0.1 & 0.02 & 0.2 & 0.0 & 0.00 & 0.0 & 0.0 & 0.00 & 0.0 & 0.0 & 0.02 & 0.05 \\
\hline FORMALDEHYDE & 2.1 & 0.82 & 14.7 & 0.1 & 6.33 & 0.9 & 0.4 & 0.88 & 2.8 & 0.6 & 0.82 & 4.26 \\
\hline ACETALDEHYDE & 0.6 & 0.25 & 3.4 & 0.0 & 0.00 & 0.0 & 0.0 & 10.00 & 0.0 & 0.1 & 0.20 & 0.71 \\
\hline ACROLEIN & 0.0 & 0.00 & 0.0 & 0.0 & 0.00 & 0.0 & 0.0 & 0.00 & 0.0 & 0.0 & 0.00 & 0.00 \\
\hline ACETONE & 0.0 & 0.00 & 0.0 & 0.0 & 0.00 & 0.0 & 0.0 & 0.00 & 0.0 & 0.0 & 0.00 & 0.00 \\
\hline PAOPIONALDEHYDE & 0.0 & 0.01 & 0.2 & 0.0 & 0.00 & 0.0 & 0.0 & 0.00 & 0.0 & 0.0 & 0.01 & 0.04 \\
\hline CROTONALDEHYDE & 0.0 & 0.00 & 0.0 & 0.1 & 2.96 & 0.3 & 0.0 & 0.02 & 0.0 & 0.0 & 0.05 & 0.17 \\
\hline ISOBUTYRALDEHYDE & 0.0 & 0.00 & 0.0 & 0.0 & 0.00 & 0.0 & 0.0 & 0.00 & 0.0 & 0.0 & 0.00 & 0.00 \\
\hline METHYLETHYL KETONE & 0.0 & 0.00 & 0.0 & 0.0 & 0.00 & 0.0 & 0.0 & 0.00 & 0.0 & 0.0 & 0.00 & 0.00 \\
\hline BENZALDEHYDE & 0.0 & 0.00 & 0.0 & 0.0 & 0.00 & 0.0 & 0.0 & 0.00 & 0.0 & $\overline{0.0}$ & 0.00 & 0.00 \\
\hline HEXANALDEHYDE & 0.0 & 0.00 & 0.0 & 0.0 & 0.00 & 0.0 & 0.0 & 0.00 & 0.0 & 0.0 & 0.00 & 0.00 \\
\hline
\end{tabular}


TEST NO: HD5-02S

TEST CAR: 1994 PONTIAC GRANDAM

TEST DATE: 9/20/95

TEST FUEL: PROPANE

\begin{tabular}{|c|c|c|c|c|c|c|c|c|c|c|c|c|}
\hline \multirow[b]{2}{*}{ COMPOUND } & \multicolumn{3}{|c|}{ BAG 1} & \multicolumn{3}{|c|}{$\overline{B A G 2}$} & \multicolumn{3}{|c|}{ BAG 3} & \multicolumn{3}{|c|}{ FTP } \\
\hline & MG/MI & $\begin{array}{l}\text { WEIGHT } \\
\text { NMOG \% } \\
\end{array}$ & $\begin{array}{l}\text { OZONE, } \\
\text { MG/MI }\end{array}$ & MG/MI & \begin{tabular}{|l|} 
WEIGHT \\
NMOG $\%$ \\
\end{tabular} & $\begin{array}{l}\text { OZONE } \\
\text { MG/MI }\end{array}$ & MG/MI & \begin{tabular}{|l|} 
WEIGHT \\
NMOG \% \\
\end{tabular} & $\begin{array}{l}\text { OZONE } \\
\text { MG/MI }\end{array}$ & MG/MI & $\begin{array}{l}\text { WEIGHT } \\
\text { NMOG \% } \\
\end{array}$ & $\begin{array}{l}\text { OZONE } \\
\text { MG/MI }\end{array}$ \\
\hline METHANE & 9.3 & & 0.1 & 1.8 & & 0.0 & 11.4 & & 0.2 & 6.0 & & 0.08 \\
\hline ETHANE & 1.9 & 1.07 & 0.5 & 0.0 & 0.00 & 0.0 & 0.8 & 6.20 & 0.2 & 0.6 & 1.43 & 0.15 \\
\hline ETHYLENE & 9.7 & 5.58 & 70.6 & 0.0 & 0.34 & 0.1 & 0.1 & 0.96 & 0.8 & 2.1 & 4.95 & 15.00 \\
\hline PROPANE & 138.0 & 80.06 & 66.7 & 0.2 & 5.01 & 0.1 & 8.7 & 71.84 & 4.2 & 31.5 & 75.67 & 15.10 \\
\hline PROPYLENE & 5.0 & 2.85 & 46.5 & 0.0 & 0.00 & 0.0 & 0.0 & 0.39 & 0.4 & 1.0 & 2.61 & 9.83 \\
\hline ACETYLENE & 1.4 & 0.81 & 0.7 & 0.0 & 0.00 & 0.0 & 0.0 & 0.03 & 0.0 & 0.3 & 0.71 & 0.16 \\
\hline PROPADIENE & 0.0 & 0.00 & 0.0 & 0.0 & 0.00 & 0.0 & 0.0 & 0.00 & 0.0 & 0.0 & 0.00 & 0.00 \\
\hline BUTANE & 3.4 & 1.95 & 3.5 & 0.4 & 8.83 & 0.4 & 0.3 & 2.33 & 0.3 & 1.0 & 2.33 & 0.89 \\
\hline TAANS-2-BUTENE & 0.1 & 0.03 & 0.6 & 0.0 & 0.00 & 0.0 & 0.0 & 0.00 & 0.0 & 0.0 & 0.03 & 0.12 \\
\hline 1-BUTENE & 0.2 & 0.11 & 1.7 & 0.0 & 0.00 & 0.0 & 0.0 & 0.00 & 0.0 & 0.0 & 0.09 & 0.35 \\
\hline 2-METHYLPROPENE (ISOBUTYLENE) & 0.1 & 0.03 & 0.3 & 0.0 & 0.00 & 0.0 & 0.0 & 0.00 & 0.0 & 0.0 & 0.03 & 0.06 \\
\hline 2,2-DIMETHYLPROPANE(NEOPENTANE) & 0.0 & 0.00 & 0.0 & 0.0 & 0.00 & 0.0 & 0.0 & 0.00 & 0.0 & 0.0 & 0.00 & 0.00 \\
\hline PROPYNE & 0.0 & 0.00 & 0.0 & 0.0 & 0.00 & 0.0 & 0.0 & 0.00 & 0.0 & 0.0 & 0.00 & 0.00 \\
\hline 1,3-BUTADIENE & 0.0 & 0.02 & 0.5 & 0.0 & 0.00 & 0.0 & 0.0 & 0.00 & 0.0 & 0.0 & 0.02 & 0.10 \\
\hline 2-METHYLPROPANE (ISOBUTANE) & 0.7 & 0.40 & 0.8 & 0.0 & 0.44 & 0.0 & 0.0 & 0.27 & 0.0 & 0.2 & 0.39 & 0.20 \\
\hline 1-BUTYNE & 0.0 & 0.00 & 0.0 & 0.0 & 0.00 & 0.0 & 0.0 & 0.00 & 0.0 & 0.0 & 0.00 & 0.00 \\
\hline METHANOL & 0.0 & 0.00 & 0.0 & 0.0 & 0.00 & 0.0 & 0.0 & 0.00 & 0.0 & 0.0 & 0.00 & 0.00 \\
\hline CIS-2-8UTENE & 0.0 & 0.00 & 0.0 & 0.0 & 0.00 & 0.0 & 0.0 & 0.00 & 0.0 & 0.0 & 0.00 & 0.00 \\
\hline 3-METHYL-1-BUTENE & 0.0 & 0.00 & 0.0 & 0.0 & 0.00 & 0.0 & 0.0 & 0.00 & 0.0 & 0.0 & 0.00 & 0.00 \\
\hline ETHANOL & 0.0 & 0.00 & 0.0 & 0.0 & 0.00 & 0.0 & 0.0 & 0.00 & 0.0 & 0.0 & 0.00 & 0.00 \\
\hline 2-METHYLBUTANE (ISOPENTANE) & 2.9 & 1.70 & 4.1 & 0.3 & 8.33 & 0.5 & 0.3 & 2.58 & 0.4 & 0.9 & 2.10 & 1.20 \\
\hline 2-BUTYNE & 0.0 & 0.00 & 0.0 & 0.0 & 0.00 & 0.0 & 0.0 & 0.00 & 0.0 & 0.0 & 0.00 & 0.00 \\
\hline 1-PENTENE & 0.0 & 0.00 & 0.0 & 0.0 & 0.00 & 0.0 & 0.0 & 0.00 & 0.0 & 0.0 & 0.00 & 0.00 \\
\hline 2-METHYL-1-BUTENE & 0.0 & 0.00 & 0.0 & 0.0 & 0.00 & 0.0 & 0.0 & 0.00 & 0.0 & 0.0 & 0.00 & 0.00 \\
\hline PENTANE & 0.4 & 0.20 & 0.4 & 0.2 & 5.95 & 0.2 & 0.1 & 1.03 & 0.1 & 0.2 & 0.56 & 0.24 \\
\hline UNIDENTIFIED C5 OLEFINS & 0.0 & 0.00 & 0.0 & 0.0 & 0.00 & 0.0 & 0.0 & 0.00 & 0.0 & 0.0 & 0.00 & 0.00 \\
\hline 2-METHYL-1,3-BUTADIENE & 0.0 & 0.00 & 0.0 & 0.0 & 0.00 & 0.0 & 0.0 & 0.00 & 0.0 & 0.0 & 0.00 & 0.00 \\
\hline TRANS-2-PENTENE & 0.1 & 0.06 & 0.8 & 0.0 & 0.00 & 0.0 & 0.0 & 0.00 & 0.0 & 0.0 & 0.05 & 0.20 \\
\hline 3,3-DIMETHYL-1-BUTENE & 0.0 & 0.00 & 0.0 & 0.0 & 0.00 & 0.0 & 0.0 & 0.00 & 0.0 & 0.0 & 0.00 & 0.00 \\
\hline CIS-2-PENTENE & 0.0 & 0.00 & 0.0 & 0.0 & 0.00 & 0.0 & 0.0 & 0.00 & 0.0 & 0.0 & 0.00 & 0.00 \\
\hline 2-METHYL-2-BUTENE & 0.2 & 0.09 & 1.0 & 0.1 & 2.20 & 0.8 & 0.0 & 0.00 & 0.0 & 0.1 & 0.19 & 0.51 \\
\hline CYCLOPENTADIENE & 0.0 & 0.00 & 0.0 & 0.0 & 0.00 & 0.0 & 0.0 & 0.00 & 0.0 & 0.0 & 0.00 & 0.00 \\
\hline 2,2-DIMETHYLBUTANE & 0.1 & 0.07 & 0.1 & 0.0 & 0.00 & 0.0 & 0.0 & 0.00 & 0.0 & 0.0 & 0.08 & 0.02 \\
\hline CYCLOPENTENE & 0.0 & 0.00 & 0.0 & 0.0 & 0.00 & 0.0 & 0.0 & 0.00 & 0.0 & 0.0 & 0.00 & 0.00 \\
\hline 4-METHYL-1-PENTENE & $0 . \overline{0}$ & 0.00 & 0.0 & 0.0 & 0.00 & 0.0 & 0.0 & 0.00 & 0.0 & 0.0 & 0.00 & 0.00 \\
\hline 3-METHYL-1-PENTENE & 0.0 & 0.00 & 0.0 & 0.0 & 0.00 & 0.0 & 0.0 & 0.00 & 0.0 & 0.0 & 0.00 & 0.00 \\
\hline CYCLOPENTANE & 0.0 & 0.00 & 0.0 & 0.0 & 0.00 & 0.0 & 0.0 & 0.00 & 0.0 & 0.0 & 0.00 & 0.00 \\
\hline 2,3-DIMETHYLBUTANE & 0.2 & 0.12 & 0.2 & 0.1 & 2.23 & 0.1 & 0.1 & 0.43 & 0.1 & 0.1 & 0.25 & 0.11 \\
\hline MTBE & 0.0 & 0.00 & 0.0 & 0.0 & 0.00 & 0.0 & 0.0 & 0.00 & 0.0 & 0.0 & 0.00 & 0.00 \\
\hline
\end{tabular}




\begin{tabular}{|c|c|c|c|c|c|c|c|c|c|c|c|c|}
\hline \multirow[b]{2}{*}{ COMPOUND } & \multicolumn{3}{|c|}{$\mathrm{BAQ1}$} & \multicolumn{3}{|c|}{ BAG 2} & \multicolumn{3}{|c|}{ BAG 3} & \multicolumn{2}{|r|}{ FTP } & \multirow[b]{2}{*}{$\begin{array}{l}\text { OZONE } \\
\text { MGMI }\end{array}$} \\
\hline & MG/MI & $\begin{array}{l}\text { WEIGHT } \\
\text { NMOG } \%\end{array}$ & $\begin{array}{l}\text { OZONE, } \\
\text { MG/MI }\end{array}$ & MG/MI & \begin{tabular}{|l|} 
WEIGHT \\
NMOG \% \\
\end{tabular} & $\begin{array}{l}\text { OZONE } \\
\text { MG/MI }\end{array}$ & $M G / M I$ & \begin{tabular}{|l|} 
WEIGHT \\
NMOG $\%$ \\
\end{tabular} & $\begin{array}{l}\text { OZONE } \\
\text { MG/MI }\end{array}$ & MG/MI & $\begin{array}{l}\text { WEIGHT } \\
\text { NMOQ \% }\end{array}$ & \\
\hline 2,3-DIMETHYL-1-BUTENE & 0.0 & 0.00 & 0.0 & 0.0 & 0.00 & 0.0 & 0.0 & 0.00 & 0.0 & 0.0 & 0.00 & 0.00 \\
\hline 4-METHYL-CIS-2-PENTENE & 0.0 & 0.00 & 0.0 & 0.0 & 0.00 & 0.0 & 0.0 & 0.00 & 0.0 & 0.0 & 0.00 & 0.00 \\
\hline 2-METHYLPENTANE & 0.6 & 0.37 & 1.0 & 0.2 & 4.80 & 0.3 & 0.1 & 1.01 & 0.2 & 0.3 & 0.64 & 0.41 \\
\hline 4-METHYL-TRANS-2-PENTENE & 0.0 & 0.00 & 0.0 & 0.0 & 0.00 & 0.0 & 0.0 & 0.00 & 0.0 & 0.0 & 0.00 & 0.00 \\
\hline 3-METHYLPENTANE & 0.3 & 0.16 & 0.4 & 0.2 & 3.77 & 0.2 & 0.1 & 0.63 & 0.1 & 0.2 & 0.37 & 0.24 \\
\hline 2-METHYL-1-PENTENE & 0.0 & 0.00 & 0.0 & 0.0 & 0.00 & 0.0 & 0.0 & 0.00 & 0.0 & 0.0 & 0.00 & 0.00 \\
\hline 1-HEXENE & 0.0 & 0.00 & 0.0 & 0.0 & 0.00 & 0.0 & 0.0 & 0.00 & 0.0 & 0.0 & 0.00 & 0.00 \\
\hline HEXANE & 0.2 & 0.11 & 0.2 & 0.2 & 4.95 & 0.2 & 0.1 & 0.59 & 0.1 & 0.2 & 0.39 & 0.16 \\
\hline UNIDENTIFIED C 6 OLEFINS & 0.0 & 0.00 & 0.0 & 0.0 & 0.00 & 0.0 & 0.0 & 0.00 & 0.0 & 0.0 & 0.00 & 0.00 \\
\hline TRANS-3-HEXENE & 0.0 & 0.00 & 0.0 & 0.0 & 0.00 & 0.0 & 0.0 & 0.00 & 0.0 & 0.0 & 0.00 & 0.00 \\
\hline CIS-3-HEXENE & 0.1 & 0.05 & 0.6 & 0.0 & 0.00 & 0.0 & 0.0 & 0.00 & 0.0 & 0.0 & 0.04 & 0.12 \\
\hline TAANS-2-HEXENE & 0.0 & 0.00 & 0.0 & 0.0 & 0.00 & 0.0 & 0.0 & 0.00 & 0.0 & 0.0 & 0.00 & 0.00 \\
\hline 3-METHYL-TRANS-2-PENTENE & 0.0 & 0.00 & 0.0 & 0.0 & 0.00 & 0.0 & 0.0 & 0.00 & 0.0 & 0.0 & 0.00 & 0.00 \\
\hline 2-METHYL-2-PENTENE & 0.0 & 0.00 & 0.0 & 0.0 & 0.00 & 0.0 & 0.0 & 0.00 & 0.0 & 0.0 & 0.00 & 0.00 \\
\hline 3-METHYLCYCLOPENTENE & 0.0 & 0.00 & 0.0 & 0.0 & 0.00 & 0.0 & 0.0 & 0.00 & 0.0 & 0.0 & 0.00 & 0.00 \\
\hline CIS-2-HEXENE & 0.0 & 0.00 & 0.0 & 0.0 & 0.00 & 0.0 & 0.0 & 0.00 & 0.0 & 0.0 & 0.00 & 0.00 \\
\hline ETBE & $\overline{0.0}$ & 0.00 & 0.0 & 0.0 & 0.00 & 0.0 & 0.0 & 0.00 & 0.0 & 0.0 & 0.00 & 0.00 \\
\hline 3-METHYL-CIS-2-PENTENE & 0.0 & 0.00 & 0.0 & 0.0 & 0.00 & 0.0 & 0.2 & 2.02 & 1.6 & 0.1 & 0.16 & 0.45 \\
\hline 2,2-DIMETHYLPENTANE & 0.1 & 0.07 & 0.2 & 0.1 & 1.85 & 0.1 & 0.0 & 0.17 & 0.0 & 0.1 & 0.16 & 0.10 \\
\hline METHYLCYCLOPENTANE & 0.0 & 0.00 & 0.0 & 0.0 & 0.00 & 0.0 & 0.0 & 0.00 & 0.0 & 0.0 & 0.00 & 0.00 \\
\hline 2,4-DIMETHYLPENTANE & 0.1 & 0.07 & 0.2 & 0.1 & 1.85 & 0.1 & 0.0 & 0.00 & 0.0 & 0.1 & 0.16 & 0.12 \\
\hline 2,3,3-TRIMETHYL-1-BUTENE & 0.0 & 0.00 & 0.0 & 0.0 & 0.00 & 0.0 & 0.0 & 0.00 & 0.0 & 0.0 & 0.00 & 0.00 \\
\hline 2,2,3-TAIMETHYLBUTANE & 0.1 & 0.06 & 0.1 & 0.0 & 0.00 & 0.0 & 0.0 & 0.00 & 0.0 & 0.0 & 0.05 & 0.03 \\
\hline 3,4-DIMETHYL-1-PENTENE & 0.0 & 0.00 & 0.0 & 0.0 & 0.00 & 0.0 & 0.0 & 0.00 & 0.0 & 0.0 & 0.00 & 0.00 \\
\hline 1-METHYLCYCLOPENTENE & 0.1 & 0.04 & 0.4 & 0.3 & 6.69 & 1.5 & 0.0 & 0.00 & 0.0 & 0.2 & 0.37 & 0.87 \\
\hline BENZENE & 0.3 & 0.20 & 0.1 & 0.2 & 5.34 & 0.1 & 0.2 & 1.79 & 0.1 & 0.2 & 0.58 & 0.10 \\
\hline 3-METHYL-1-HEXENE & 0.0 & 0.00 & 0.0 & 0.0 & 0.00 & 0.0 & 0.0 & 0.00 & 0.0 & 0.0 & 0.00 & 0.00 \\
\hline 3,3-DIMETHYLPENTANE & 0.0 & 0.00 & 0.0 & 0.0 & 0.00 & 0.0 & 0.0 & 0.00 & 0.0 & 0.0 & 0.00 & 0.00 \\
\hline CYCLOHEXANE & 0.0 & 0.00 & 0.0 & 0.0 & 0.00 & 0.0 & 0.0 & 0.00 & 0.0 & 0.0 & 0.00 & 0.00 \\
\hline 2-METHYLHEXANE & 0.0 & 0.00 & 0.0 & 0.0 & 0.00 & 0.0 & 0.0 & 0.00 & 0.0 & 0.0 & 0.00 & 0.00 \\
\hline 2,3-DIMETHYLPENTANE & 0.4 & 0.23 & 0.6 & 0.0 & 1.16 & 0.1 & 0.1 & 0.55 & 0.1 & 0.1 & 0.30 & 0.19 \\
\hline 1,1-DIMETHYLCYCLOPENTANE & 0.0 & 0.00 & 0.0 & 0.0 & 0.00 & 0.0 & 0.0 & 10.00 & 0.0 & 0.0 & 0.00 & 0.00 \\
\hline CYCLOHEXENE & 0.0 & 0.00 & 0.0 & 0.0 & 0.00 & 0.0 & 0.0 & 0.00 & 0.0 & 0.0 & 0.00 & 0.00 \\
\hline 3-METHYLHEXANE & 0.2 & 0.12 & 0.3 & 0.0 & 0.88 & 0.0 & 0.1 & 0.44 & 0.1 & 0.1 & 0.19 & 0.11 \\
\hline CIS-1,3-DIMETHYLCYCLOPENTANE & 0.0 & 0.00 & 0.0 & 0.0 & 0.00 & 0.0 & 0.1 & 0.75 & 0.2 & 0.0 & 0.06 & 0.06 \\
\hline 3-ETHYLPENTANE & 0.0 & 0.00 & 0.0 & 0.0 & 0.00 & 0.0 & 0.0 & 0.00 & 0.0 & 0.0 & 0.00 & 0.00 \\
\hline TRANS-1,2-DIMETHYLCYCLOPENTANE & 0.0 & 0.00 & 0.0 & 0.0 & 0.00 & 0.0 & 0.0 & 0.00 & 0.0 & 0.0 & 0.00 & 0.00 \\
\hline TRANS-1,3-DIMETHYLCYCLOPENTANE & 0.0 & 0.00 & 0.0 & 0.0 & 0.00 & 0.0 & 0.0 & 0.00 & 0.0 & 0.0 & 0.00 & 0.00 \\
\hline 1-HEPTENE & 0.0 & 0.00 & 0.0 & 0.0 & 0.00 & 0.0 & 0.0 & 0.00 & 0.0 & 0.0 & 0.00 & 0.00 \\
\hline 2,2,4-TRIMETHYLPENTANE & 0.2 & 0.14 & 0.2 & 0.3 & 6.64 & 0.2 & 0.1 & 0.65 & 0.1 & 0.2 & 0.50 & 0.19 \\
\hline 2-METHYL-1-HEXENE & 0.0 & 0.00 & 0.0 & 0.0 & 0.00 & 0.0 & 0.0 & 0.00 & 0.0 & 0.0 & 0.00 & 0.00 \\
\hline TRANS-3-HEPTENE & 0.0 & 0.00 & 0.0 & 0.0 & 0.00 & 0.0 & 0.0 & 0.00 & 0.0 & 0.0 & 0.00 & 0.00 \\
\hline HEPTANE & 0.1 & 0.08 & 0.1 & 0.1 & 1.67 & 0.1 & 0.0 & 0.00 & 0.0 & 0.1 & 0.13 & 0.04 \\
\hline UNIDENTIFIED C7 & 0.3 & 0.17 & 1.3 & 0.3 & 7.36 & 1.3 & 0.0 & 0.06 & 0.0 & 0.2 & 0.62 & 0.97 \\
\hline
\end{tabular}




\begin{tabular}{|c|c|c|c|c|c|c|c|c|c|c|c|c|}
\hline \multirow[b]{2}{*}{ COMPOUND } & \multicolumn{3}{|c|}{ BAG 1} & \multicolumn{3}{|c|}{$\overline{B A G 2}$} & \multicolumn{3}{|c|}{ EAG 3} & \multicolumn{3}{|c|}{ FTP } \\
\hline & MG/MI & $\begin{array}{l}\text { WEIGHT } \\
\text { NMOQ \% } \\
\end{array}$ & $\begin{array}{l}\text { OZONE, } \\
\text { MGMII }\end{array}$ & MG/MI & $\begin{array}{l}\text { WEIGHT } \\
\text { NMOG \% } \\
\end{array}$ & $\begin{array}{l}\text { OZONE } \\
\text { MGMM }\end{array}$ & MGMI & $\begin{array}{l}\text { WEGHT } \\
\text { NMOG \% }\end{array}$ & $\begin{array}{l}\text { Orome } \\
\text { menal }\end{array}$ & menM & $\begin{array}{l}\text { WETGHT } \\
\text { NMOG \% } \\
\end{array}$ & $\begin{array}{l}\text { OZONE } \\
\text { MG/MI }\end{array}$ \\
\hline 2-METHYL-2-HEXENE & 0.0 & 0.00 & 0.0 & 0.0 & 0.00 & 0.0 & a.0 & $\mathbf{0 . 0 0}$ & $\mathbf{0 . 0}$ & 0.0 & 0.00 & 0.00 \\
\hline 3-METHYL-TRANS-3-HEXENE & 0.0 & 0.00 & $0: 0$ & 0.0 & 0.00 & 0.0 & aco & ano & 0.0 & $\mathbf{a . 0}$ & 0.00 & 0.00 \\
\hline TRANS-2-HEPTENE & 0.0 & 0.00 & 0.0 & 0.0 & 0.00 & a. & E.0. & 9.00 & $\mathbf{0 . 0}$ & $\overline{\mathbf{a . 0}}$ & 0.00 & 0.00 \\
\hline 3-ETHYL-CIS-2-PENTENE & 0.0 & 0.00 & 0.0 & 0.0 & 0.00 & 0.0 & 0.0 & a.0. & $\mathbf{E . 0}$ & 0.0 & 0.00 & 0.00 \\
\hline 2,4,4-TRIMETHYL-1-PENTENE & 0.0 & 0.00 & 0.0 & 0.0 & 0.00 & 0.0 & a.a & 0.00 & 0.0 & 0.0 & 0.00 & 0.00 \\
\hline 2,2,4-TRIMETHYL-1-PENTENE & 0.0 & 0.00 & 0.0 & 0.0 & 0.00 & 0.0 & $\overline{0.0}$ & E.00 & a.0 & 0.0 & 0.00 & 0.00 \\
\hline 2,3-DIMETHYL-2-PENTENE & 0.0 & 0.00 & 0.0 & 0.0 & 0.00 & 0.0 & 0.0 & acos & 0.0 & 0.0 & 0.00 & 0.00 \\
\hline CIS-2-HEPTENE & 0.0 & 0.00 & 0.0 & 0.0 & 0.00 & 0.0 & 0.0 & a.ma & 0.0 & 6.0 & 0.00 & 0.00 \\
\hline METHYLCYCLOHEXANE & 0.0 & 0.00 & 0.0 & 0.0 & 0.00 & 0.0 & 0.0 & 0.00 & a.0 & 0.0 & 0.00 & 0.00 \\
\hline CIS-1,2-DIMETHYLCYCLOPENTANE & 0.0 & 0.00 & 0.0 & 0.0 & 0.00 & 0.0 & 0.0 & 0.00 & 0.0 & 0.0 & 0.00 & 0.00 \\
\hline 2,2-DIMETHYLHEXANE & 0.1 & 0.08 & 0.1 & 0.0 & 0.00 & 0.0 & a.a & 0.04 & 0.0 & 0.0 & 0.05 & 0.02 \\
\hline 1,1,3-TRIMETHYLCYCLOPENTANE & 0.0 & 0.00 & 0.0 & 0.0 & 0.00 & 0.0 & 0.0. & 0.0 .0 & a.o & 0.0 & 0.00 & 0.00 \\
\hline 2,4,4-TRIMETHYL-2-PENTENE & 0.0 & 0.00 & 0.0 & 0.0 & 0.00 & a.a & E.a & a.c. & $\mathbf{c o s}$ & 0.0 & 0.00 & 0.00 \\
\hline 2,2,3-TRIMETHYLPENTANE & 0.0 & 0.00 & 0.0 & 0.0 & 0.00 & 0.0 & 0.0 & acou & an & $\mathbf{0 . 0}$ & 0.00 & 0.00 \\
\hline 2,5-DIMETHYLHEXANE & 0.0 & 0.00 & 0.0 & 0.0 & 0.00 & en & 0.0 & Q.00 & $a .0$ & 0.0 & 0.00 & 0.00 \\
\hline ETHYLCYCLOPENTANE & 0.0 & 0.00 & $\mathbf{0 . 0}$ & 0.0 & 0.00 & $\mathbf{a . 0}$ & 0.9 & Q.00 & 0.0 & 0.0 & 0.00 & 0.00 \\
\hline 2,4-DIMETHYLHEXANE & 0.0 & 0.00 & 0.0 & 0.0 & 0.00 & 0.0 & 0.0 & a.00 & 0.6 & 0.0 & 0.00 & 0.00 \\
\hline 1-TRANS-2-CIS-4-TRIMETHYLCYCLOPENTANE & 0.0 & 0.00 & 0.0 & 0.0 & 0.00 & 0.0 & 0.0 & 0.00 & 0.0 & 0.0 & 0.00 & 0.00 \\
\hline 3,3-DIMETHYLHEXANE & 0.0 & 0.00 & 0.0 & 0.0 & 0.00 & 0.0 & 0.0 & 0.00 & a.0 & 0.0 & 0.00 & 0.00 \\
\hline 1-TRANS-2-CIS-3-TRIMETHYLCYCLOPENTANE & 0.0 & 0.00 & 0.0 & 0.0 & 0.00 & a.o & 0.0 & 0.00 & 0.0 & 0.0 & 0.00 & 0.00 \\
\hline 2,3,4-TAIMETHYLPENTANE & 0.0 & 0.00 & 0.0 & 0.0 & 0.00 & 0.0 & 0.0 & a.00 & Q.0 & 0.0 & 0.00 & 0.00 \\
\hline 2,3,3-TAIMETHYLPENTANE & 0.1 & 0.05 & 0.1 & 0.1 & 1.28 & a.1 & a.d & Q40 & $\mathbf{a . t}$ & 0.1 & 0.14 & 0.07 \\
\hline TOLUENE & 0.8 & 0.43 & 2.1 & 0.1 & 2.26 & 0.2 & Q.3. & 278 & a.g & 6.3 & 0.71 & 0.81 \\
\hline 2,3-DIMETHYLLEXANE & 0.0 & 0.00 & 0.0 & 0.0 & 0.00 & 0.0 & a.d) & 0.00 & a.0 & 0,0 & 0.00 & 0.00 \\
\hline 1,1,2-TRIMETHYLCYCLOPENTANE & 0.0 & 0.00 & 0.0 & 0.0 & 0.00 & 0.0 & $\mathbf{0 . 0}$ & 0.00 & 0.0 & 0.0 & 0.00 & 0.00 \\
\hline 2-METHYLHEPTANE & 0.0 & 0.00 & 0.0 & 0.0 & 0.00 & 0.0 & Q.0 & 0.00 & 0,0 & 0.0 & 0.00 & 0.00 \\
\hline 3,4-DIMETHYLHEXANE & 0.0 & 0.00 & 0.0 & 0.0 & 0.00 & 0.0 & 0.0 & 0.00 & a.0. & 0.0 & 0.00 & 0.00 \\
\hline 2,2,4,4-TETRAMETHYLPENTANE & 0.0 & 0.00 & 0.0 & 0.0 & 0.00 & 0.0 & 0.0 & $0: 00$ & a.a & a.a & 0.00 & 0.00 \\
\hline 4-METHYLHEPTANE & 0.0 & 0.00 & 0.0 & 0.0 & 0.00 & $\overline{0.0}$ & 0.0 & a.a & 0.0 & 0.0 & 0.00 & 0.00 \\
\hline 2-METHYL-3-ETHYLPENTANE & 0.0 & 0.00 & 0.0 & 0.0 & 0.00 & 0.0 & 0.0 & Q00 & 0.0 & 6.0 & 0.00 & 0.00 \\
\hline 2,6-DIMETHYLHEPTANE & 0.0 & 0.00 & 0.0 & 0.0 & 0.00 & 0.0 & 0.0 & $\mathbf{0 , 0 0}$ & 0.0 & 0.0 & 0.00 & 0.00 \\
\hline 3-METHYLHEPTANE & 0.1 & 0.05 & 0.1 & 0.0 & 0.00 & 0.0 & 0.0 & 0.00 & $\overline{0.0}$ & 0.0 & 0.05 & 0.02 \\
\hline 1-CIS,2-TRANS,3-TRIMETHYLCYCLOPENTANE & 0.0 & 0.00 & 0.0 & 0.0 & 0.00 & 0.0 & 0.0 & 0.00 & a.a & 0.0 & 0.00 & 0.00 \\
\hline CIS-1,3-DIMETHYLCYCLOHEXANE & 0.0 & 0.00 & 0.0 & 0.0 & 0.00 & 0.0 & 0.0 & 0.00 & a.a & 0.0 & 0.00 & 0.00 \\
\hline TRANS-1.4-DIMETHYLCYCLOHEXANE & 0.0 & 0.00 & 0.0 & 0.0 & 0.00 & 0.0 & 0.0 & 0.00 & 0.0 & 0.0 & 0.00 & 0.00 \\
\hline 3-ETHYLHEXANE & 0.0 & 0.00 & 0.0 & 0.0 & 0.00 & 0.0 & 0.0 & 0.00 & 0.0 & 0.0 & 0.00 & 0.00 \\
\hline 2,2,6-TRIMETHYLHEXANE & 0.0 & 0.00 & 0.0 & 0.0 & 0.00 & 0.0 & 0.0 & 0.00 & 0.0 & 0.0 & 0.00 & 0.00 \\
\hline CIS-1-METHYL-3-ETHYLCYCLOPENTANE & 0.0 & 0.00 & 0.0 & 0.0 & 0.00 & 0.0 & 0.0 & 0.00 & 0.0 & 0.0 & 0.00 & 0.00 \\
\hline 1,1-DIMETHYLCYCLOHEXANE & 0.0 & 0.00 & 0.0 & 0.0 & 0.00 & 0.0 & 0.0 & 0.00 & 0.0 & $\mathbf{0 . 0}$ & 0.00 & 0.00 \\
\hline TRANS-1-METHYL-2-ETHYLCYCLOPENTANE & 0.0 & 0.00 & 0.0 & 0.0 & 0.00 & 0.0 & 0.0 & a.0a & 0.0 & 0.0 & 0.00 & 0.00 \\
\hline 1-METHYL-1-ETHYL-CYCLOPENTANE & 0.0 & 0.00 & 0.0 & 0.0 & 0.00 & 0.0 & $\mathbf{0 . 0}$ & 0.00 & a, & 0.0 & 0.00 & 0.00 \\
\hline 2,4,4-TRIMETHYLHEXANE & 0.0 & 0.00 & 0.0 & 0.0 & 0.00 & 0.0 & $\mathbf{0 . 0}$ & 0.00 & 0.0 & Q.0 & 0.00 & 0.00 \\
\hline 2,2,4-TAIMETHYLHEXANE & 0.0 & 0.00 & 0.0 & 0.0 & 0.00 & 0.0 & $\mathbf{0 . 0}$ & 0.00 & 0.0 & 0.0 & 0.00 & 0.00 \\
\hline TRANS-1,2-DIMETHYLCYCLOHEXANE & 0.0 & 0.00 & 0.0 & 0.0 & 0.00 & 0.0 & 0.0 & 0.00 & 0.0 & 0.0 & 0.00 & 0.00 \\
\hline
\end{tabular}


ш

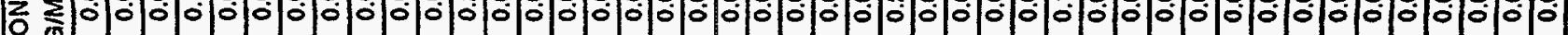

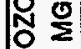

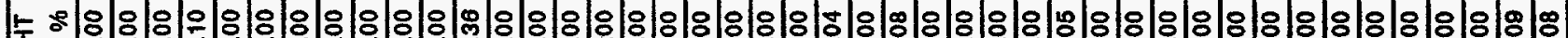
童 畏崖

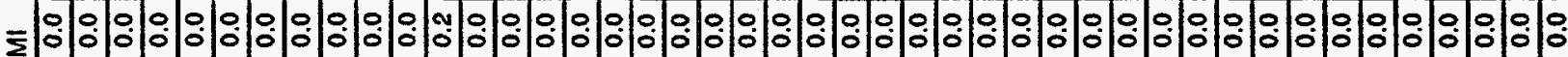
울

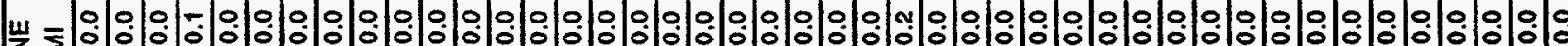

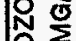

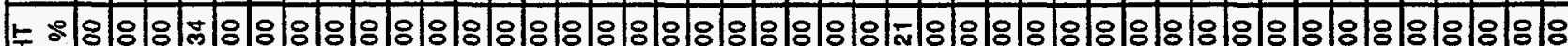
e 㐊

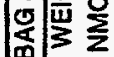

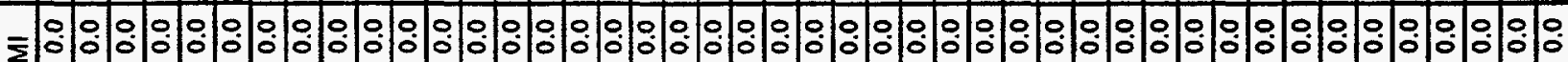
일

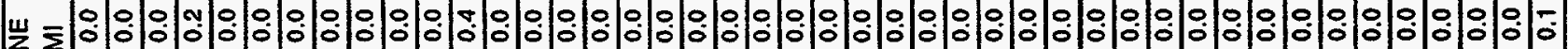
负 응

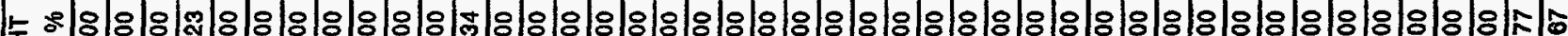
先 0 这空

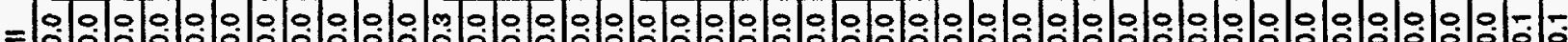
$\sum_{0}$

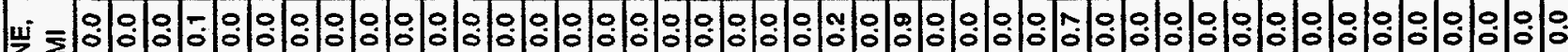
蛋

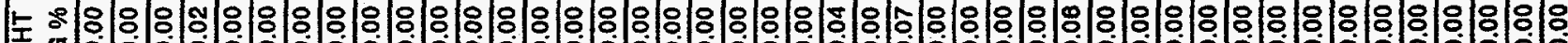
可崖

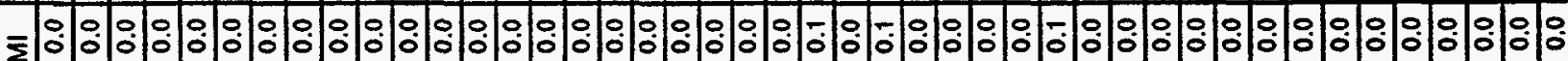
एँ

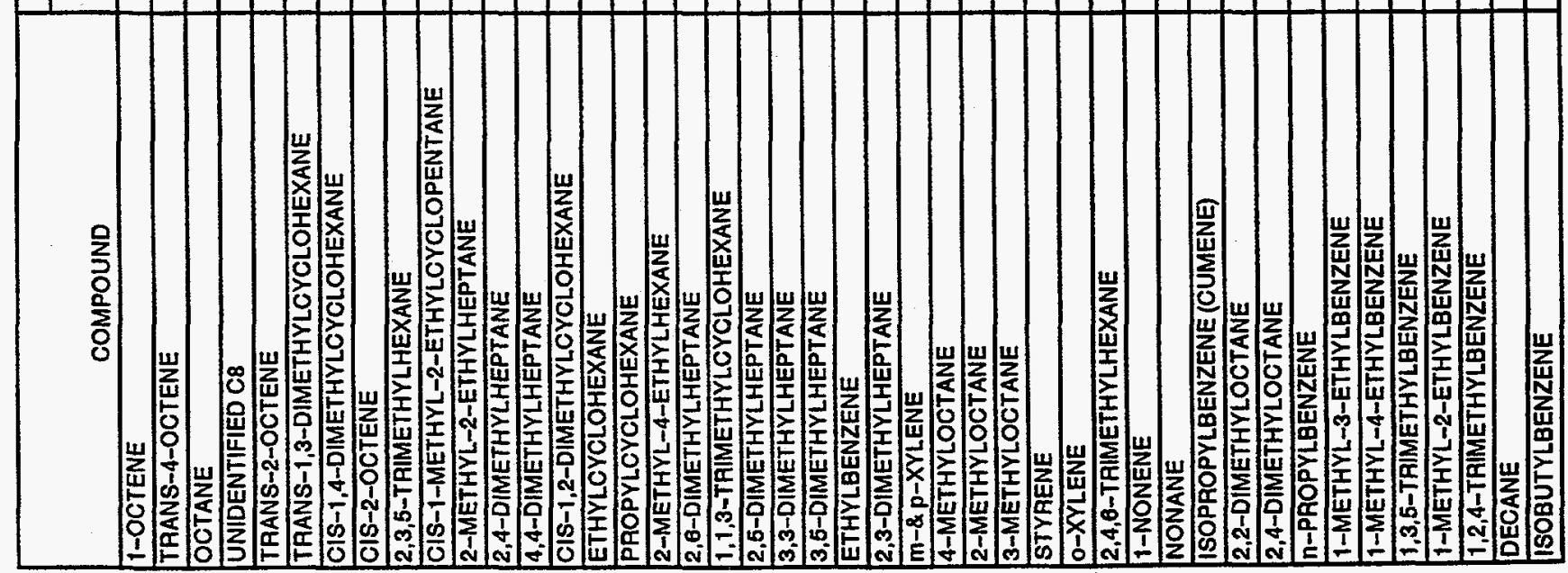




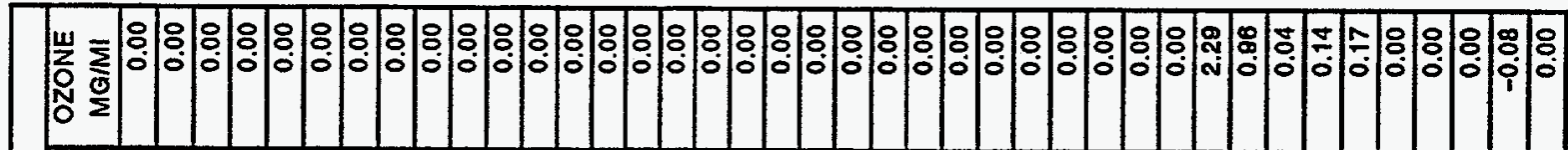

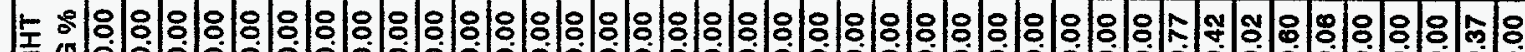
位离

$3 \leq$

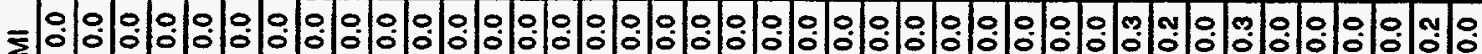
닌

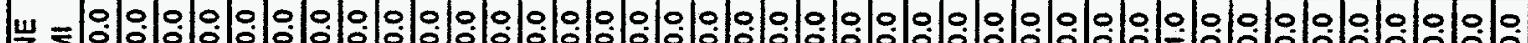
尽

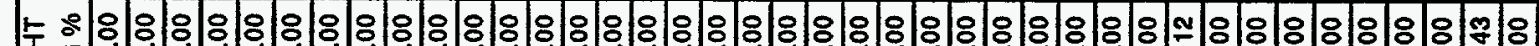
要这

\begin{tabular}{l} 
产 \\
\hline
\end{tabular}

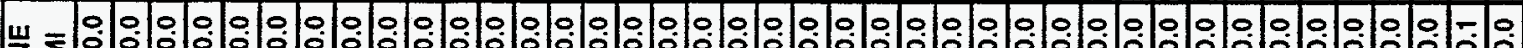

崖 N

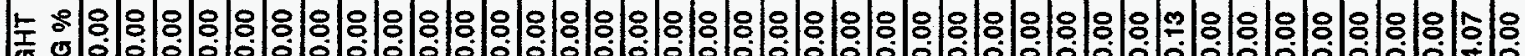

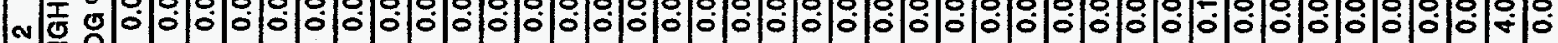
蒠 $\sum_{2}$

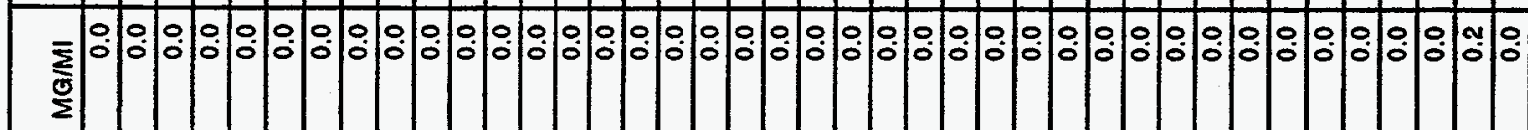

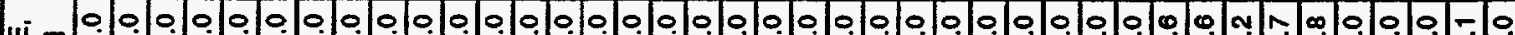

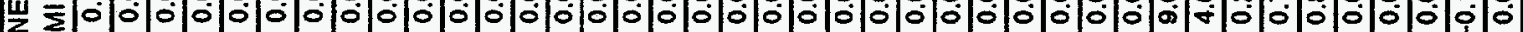
负 ํㅗㄹ

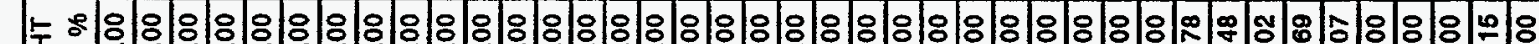
- 壬 察崖

$$
\text { 三 }
$$

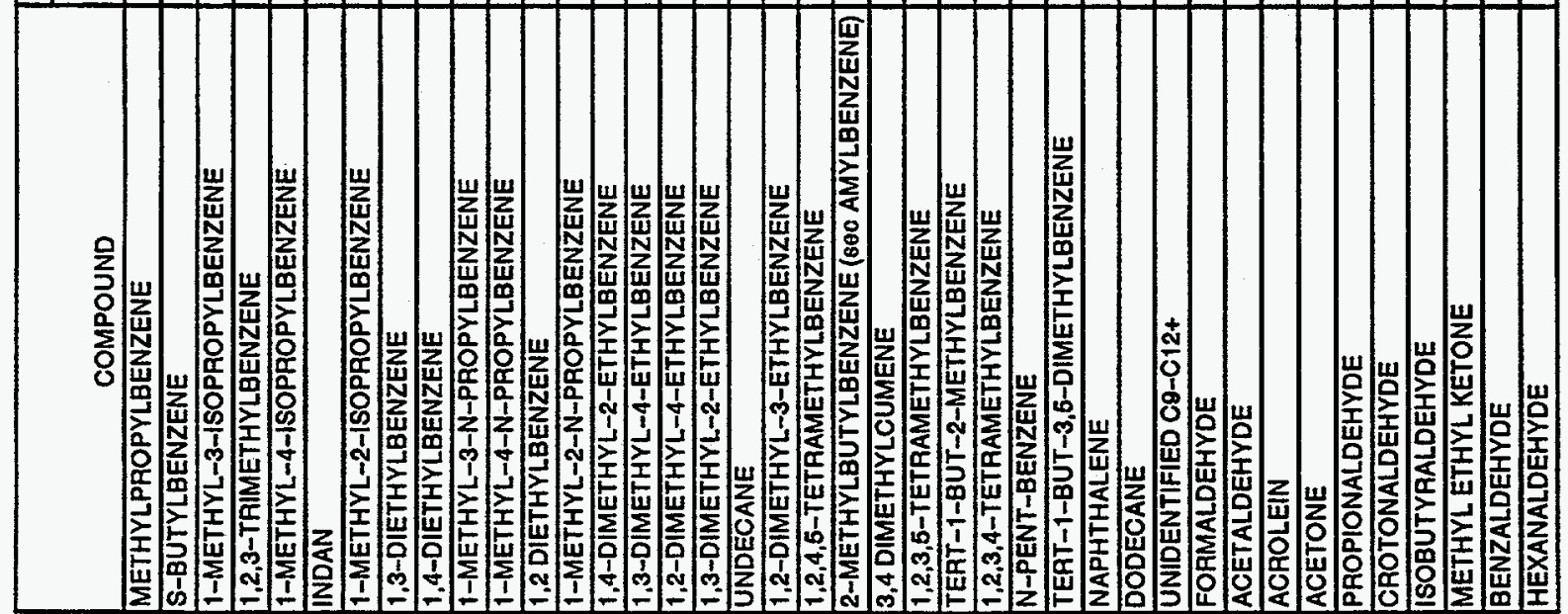


TEST NUMBER: 90NB/10/B-02S

TEST CAR: 1994 PONTIAC GRANDAM

TEST DATE: $9 / 8 / 95$

TEST FUEL: BUTANE

\begin{tabular}{|c|c|c|c|c|c|c|c|c|c|c|c|c|}
\hline \multirow[b]{2}{*}{ COMPOUND } & \multicolumn{3}{|c|}{$B A G 1$} & \multicolumn{3}{|c|}{ BAG 2} & \multicolumn{3}{|c|}{ BAG 3} & \multicolumn{3}{|c|}{ FTP } \\
\hline & MG/MI & $\begin{array}{l}\text { WEIGHT } \\
\text { NMOG } \%\end{array}$ & $\begin{array}{l}\text { OZONE, } \\
\text { MG/MI }\end{array}$ & MG/MI & $\begin{array}{l}\text { WEIGHT } \\
\text { NMOG } \%\end{array}$ & $\begin{array}{l}\text { OZONE } \\
\text { MG/MI }\end{array}$ & MG/MI & $\begin{array}{l}\text { WEIGHT } \\
\text { NMOG } \%\end{array}$ & $\begin{array}{l}\text { OZONE } \\
\text { MG/MI }\end{array}$ & MGMI & $\begin{array}{l}\text { WEIGHT } \\
\text { NMOG \% }\end{array}$ & $\begin{array}{l}\text { OZONE } \\
\text { MG/MI }\end{array}$ \\
\hline METHANE & 23.2 & & 0.3 & 3.1 & & 0.0 & 19.9 & & 0.3 & 11.9 & & 0.18 \\
\hline ETHANE & 2.5 & 1.40 & 0.6 & 0.0 & 0.57 & 0.0 & 2.2 & 12.11 & 0.8 & 1.1 & 2.62 & 0.29 \\
\hline ETHYLENE & 16.7 & 9.24 & 121.9 & 0.0 & 0.00 & 0.0 & $\overline{1.4}$ & 7.55 & 10.1 & 3.9 & 8.83 & 28.19 \\
\hline PROPANE & 0.3 & 0.14 & 0.1 & 0.0 & 0.00 & 0.0 & 0.1 & 0.77 & 0.1 & 0.1 & 0.21 & 0.04 \\
\hline PROPYLENE & 7.5 & 4.12 & 70.1 & 0.0 & 0.00 & 0.0 & 0.4 & 2.01 & 3.5 & 1.7 & 3.78 & 15.57 \\
\hline ACETYLENE & 9.8 & 5.43 & 4.9 & 0.0 & 0.00 & 0.0 & 1.4 & 7.74 & 0.7 & 2.4 & 5.57 & 1.22 \\
\hline PROPADIENE & 0.0 & 0.00 & 0.0 & 0.0 & 0.00 & 0.0 & 0.0 & 0.00 & 0.0 & 0.0 & 0.00 & 0.00 \\
\hline BUTANE & 122.0 & 67.42 & 124.4 & 0.0 & 0.00 & 0.0 & 10.0 & 54.53 & 10.2 & 28.2 & 64.37 & 28.76 \\
\hline TRANS-2-BUTENE & 0.2 & 0.13 & 2.3 & 0.0 & 0.00 & 0.0 & 0.0 & 0.00 & 0.0 & 0.0 & 0.11 & 0.48 \\
\hline 1-BUTENE & 0.5 & 0.29 & 4.7 & 0.0 & 0.00 & 0.0 & 0.1 & 0.41 & 0.7 & 0.1 & 0.30 & 1.17 \\
\hline 2-METHYLPROPENE (ISOBUTYLENE) & 0,7 & 0.39 & 3.7 & 0.0 & 0.00 & 0.0 & 0.1 & 0.59 & 0.8 & 0.2 & 0.40 & 0.93 \\
\hline 2,2-DIMETHYLPROPANE (NEOPENTANE) & 0.0 & 0.00 & 0.0 & 0.0 & 0.00 & 0.0 & 0.0 & 0.03 & 0.0 & 0.0 & 0.00 & 0.00 \\
\hline PROPYNE & 0.0 & 0.00 & 0.0 & 0.0 & 0.00 & 0.0 & 0.0 & 0.00 & 0.0 & 0.0 & 0.00 & 0.00 \\
\hline 1,3-BUTADIENE & 0.1 & 0.00 & 1.3 & 0.0 & 0.00 & 0.0 & 0.0 & 0.00 & 0.0 & 0.0 & 0.06 & 0.27 \\
\hline 2-METHYLPROPANE (ISOBUTANE) & 12.2 & 6.73 & 14.7 & 0.0 & 0.00 & 0.0 & 0.0 & 0.20 & 0.0 & 2.5 & 6.82 & 3.08 \\
\hline 1-BUTYNE & 0.0 & 0.00 & 0.0 & 0.0 & 0.00 & 0.0 & 0.0 & 0.00 & 0.0 & 0.0 & 0.00 & 0.00 \\
\hline METHANOL & 0.0 & 0.00 & 0.0 & 0.0 & 0.00 & 0.0 & 0.0 & 0.00 & 0.0 & 0.0 & 0.00 & 0.00 \\
\hline CIS-2-BUTENE & 0.0 & 0.00 & 0.0 & $\overline{0.0}$ & 0.00 & 0.0 & 0.0 & 0.00 & 0.0 & 0.0 & 0.00 & 0.00 \\
\hline 3-METHYL-1-BUTENE & 0.0 & 0.00 & 0.0 & 0.0 & 0.00 & 0.0 & 0.0 & 0.00 & 0.0 & 0.0 & 0.00 & 0.00 \\
\hline ETHANOL & 0.0 & 0.00 & 0.0 & 0.0 & 0.00 & 0.0 & 0.0 & 0.00 & 0.0 & 0.0 & 0.00 & 0.00 \\
\hline 2-METHYLBUTANE (ISOPENTANE) & 1.5 & 0.82 & 2.1 & 0.0 & 0.00 & 0.0 & 0.4 & 1.97 & 0.5 & 0.4 & 0.94 & 0.57 \\
\hline 2-BUTYNE & 0.0 & 0.00 & 0.0 & 0.0 & 0.00 & 0.0 & 0.0 & 0.00 & 0.0 & 0.0 & 0.00 & 0.00 \\
\hline I-PENTENE & 0.0 & 0.00 & 0.0 & 0.0 & 0.00 & 0.0 & 0.0 & 0.00 & 0.0 & 0.0 & 0.00 & 0.00 \\
\hline 2-METHYL-1-BUTENE & 0.1 & 0.05 & 0.5 & 0.1 & 7.27 & 0.7 & 0.0 & 0.00 & 0.0 & 0.1 & 0.21 & 0.46 \\
\hline PENTANE & 0.2 & 0.11 & 0.2 & 0.1 & 2.68 & 0.1 & 0.1 & 0.45 & 0.1 & 0.1 & 0.21 & 0.10 \\
\hline UNIDENTIFIED CS OLEFINS & 0.0 & 0.00 & 0.0 & 0.0 & 0.00 & 0.0 & 0.0 & 0.00 & 0.0 & 0.0 & 0.00 & 0.00 \\
\hline 2-METHYL-1,3-BUTADIENE & 0.0 & 0.00 & 0.0 & 0.0 & 0.00 & 0.0 & 0.0 & 10.00 & 0.0 & 0.0 & 0.00 & 0.00 \\
\hline TRANS-2-PENTENE & 0.0 & 0.02 & 0.3 & 0.0 & 0.00 & 0.0 & 0.0 & 0.00 & 0.0 & 0.0 & 0.02 & 0.07 \\
\hline 3,3-DIMETHYL-1-BUTENE & 0.0 & 0.00 & 0.0 & 0.0 & 0.00 & 0.0 & 0.0 & 0.00 & 0.0 & 0.0 & 0.00 & 0.00 \\
\hline CIS-2-PENTENE & 0.0 & 0.00 & 0.0 & 0.1 & 7.07 & 1.2 & 0.0 & 0.00 & 0.0 & 0.1 & 0.16 & 0.63 \\
\hline 2-METHYL-2-BUTENE & 0.2 & 0.09 & 1.0 & 0.0 & 0.00 & 0.0 & 0.0 & 0.22 & 0.3 & 0.0 & 0.10 & 0.29 \\
\hline CYCLOPENTADIENE & 0.0 & 0.00 & 0.0 & 0.0 & 0.00 & 0.0 & 0.0 & 0.00 & 0.0 & 0.0 & 0.00 & 0.00 \\
\hline 2,2-DIMETHYLBUTANE & 0.0 & 0.00 & 0.0 & 0.0 & 0.00 & 0.0 & 0.0 & 0.00 & 0.0 & 0.0 & 0.00 & 0.00 \\
\hline CYCLOPENTENE & 0.0 & 0.00 & 0.0 & 0.0 & 0.00 & 0.0 & 0.0 & 0.00 & 0.0 & 0.0 & 0.00 & 0.00 \\
\hline 4-METHYL-1-PENTENE & 0.0 & 0.00 & 0.0 & 0.0 & 0.00 & 0.0 & 0.0 & 0.00 & 0.0 & 0.0 & 0.00 & 0.00 \\
\hline 3-METHYL-1-PENTENE & 0.0 & 0.02 & 0.1 & 0.0 & 1.73 & 0.2 & 0.0 & 0.00 & 0.0 & 0.0 & 0.05 & 0.10 \\
\hline CYCLOPENTANE & 0.0 & 0.00 & 0.0 & 0.0 & 0.00 & 0.0 & 0.0 & 0.00 & 0.0 & 0.0 & 0.00 & 0.00 \\
\hline 2,3-DIMETHYLBUTANE & 0.1 & 0.06 & 0.1 & 0.0 & 2.38 & 0.1 & 0.0 & 0.26 & 0.1 & 0.1 & 0.14 & 0.07 \\
\hline MTBE & 0.0 & 0.00 & 0.0 & 0.0 & 0.00 & 0.0 & 0.0 & 0.00 & 0.0 & 0.0 & 0.00 & 0.00 \\
\hline
\end{tabular}




\begin{tabular}{|c|c|c|c|c|c|c|c|c|c|c|c|c|}
\hline \multirow[b]{2}{*}{ COMPOUND } & \multicolumn{3}{|c|}{ BAG 1} & \multicolumn{3}{|c|}{ BAG 2} & \multicolumn{3}{|c|}{ BAG 3} & \multicolumn{3}{|c|}{ FTP } \\
\hline & MG/MI & $\begin{array}{l}\text { WEIGHT } \\
\text { NMOG } \% \\
\end{array}$ & $\begin{array}{l}\text { OZONE, } \\
\text { MG/MI }\end{array}$ & $M G / M I$ & \begin{tabular}{|l|} 
WEIGHT \\
NMOG $\%$ \\
\end{tabular} & $\begin{array}{l}\text { OZONE } \\
\text { MG/MI }\end{array}$ & MG/MI & $\begin{array}{l}\text { WEIGHT } \\
\text { NMOG \% }\end{array}$ & $\begin{array}{l}\text { OZONE } \\
\text { MG/MI }\end{array}$ & MG/MI & $\begin{array}{l}\text { WEIGHT } \\
\text { NMOG \% }\end{array}$ & $\begin{array}{l}\text { OZONE } \\
\text { MG/MI }\end{array}$ \\
\hline 2,3-DIMETHYL-1-8UTENE & 0.0 & 0.00 & 0.0 & 0.0 & 0.00 & 0.0 & 0.0 & 0.00 & 0.0 & 0.0 & 0.00 & 0.00 \\
\hline 4-METHYL-CIS-2-PENTENE & 0.0 & 0.00 & 0.0 & 0.0 & 0.00 & 0.0 & 0.0 & 0.00 & 0.0 & 0.0 & 0.00 & 0.00 \\
\hline 2-METHYLPENTANE & 0.2 & 0.10 & 0.3 & 0.1 & 5.51 & 0.2 & 0.2 & 0.94 & 0.3 & 0.1 & 0.32 & 0.22 \\
\hline 4-METHYL-TRANS-2-PENTENE & 0.0 & 0.00 & 0.0 & 0.0 & 0.00 & 0.0 & 0.0 & 0.00 & 0.0 & 0.0 & 0.00 & 0.00 \\
\hline 3-METHYLPENTANE & 0.1 & 0.07 & 0.2 & 0.0 & 2.12 & 0.1 & 0.1 & 0.37 & 0.1 & 0.1 & 0.15 & 0.10 \\
\hline 2-METHYL-1-PENTENE & 0.0 & 0.00 & 0.0 & 0.0 & 0.00 & 0.0 & $\overline{0.0}$ & 0.00 & 0.0 & 0.0 & 0.00 & 0.00 \\
\hline 1-HEXENE & 0.0 & 0.00 & 0.0 & 0.0 & 0.00 & 0.0 & 0.0 & 0.00 & 0.0 & 0.0 & 0.00 & 0.00 \\
\hline HEXANE & 0.1 & 0.07 & 0.1 & 0.0 & 0.00 & 0.0 & 0.1 & 0.34 & 0.1 & 0.0 & 0.10 & 0.04 \\
\hline UNIDENTIFIED C6 OLEFINS & 0.0 & 0.00 & 0.0 & 0.0 & 0.00 & 0.0 & 0.0 & 0.00 & 0.0 & 0.0 & 0.00 & 0.00 \\
\hline TRANS-3-HEXENE & 0.1 & 0.05 & 0.6 & 0.0 & 0.00 & 0.0 & 0.0 & 0.00 & 0.0 & 0.0 & 0.04 & 0.12 \\
\hline CIS-3-HEXENE & 0.0 & 0.02 & 0.2 & 0.0 & 1.48 & 0.2 & 0.1 & 0.46 & 0.6 & 0.0 & 0.10 & 0.30 \\
\hline TRANS-2-HEXENE & 0.0 & 0.00 & 0.0 & 0.0 & 0.00 & 0.0 & 0.0 & 0.00 & 0.0 & 0.0 & 0.00 & 0.00 \\
\hline 3-METHYL-TRANS-2-PENTENE & 0.0 & 0.00 & 0.0 & 0.0 & 0.00 & 0.0 & 0.0 & 0.00 & 0.0 & 0.0 & 0.00 & 0.00 \\
\hline 2-METHYL-2-PENTENE & 0.0 & 0.00 & 0.0 & 0.0 & 0.00 & 0.0 & 0.0 & 0.00 & 0.0 & 0.0 & 0.00 & 0.00 \\
\hline 3-METHYLCYCLOPENTENE & 0.0 & 0.00 & 0.0 & 0.0 & 0.00 & 0.0 & 0.0 & 0.00 & 0.0 & 0.0 & 0.00 & 0.00 \\
\hline CIS-2-HEXENE & 0.0 & 0.00 & 0.0 & 0.0 & 0.00 & 0.0 & 0.0 & 0.00 & 0.0 & 0.0 & 0.00 & $\overline{0.00}$ \\
\hline ETBE & 0.0 & 0.00 & 0.0 & 0.0 & 0.00 & 0.0 & 0.0 & 0.00 & 0.0 & 0.0 & 0.00 & 0.00 \\
\hline 3-METHYL-CIS-2-PENTENE & 0.0 & 0.00 & 0.0 & 0.0 & 0.00 & 0.0 & 0.0 & 0.00 & 0.0 & 0.0 & 0.00 & 0.00 \\
\hline 2,2-DIMETHYLPENTANE & 0.0 & 0.02 & 0.0 & 0.0 & 0.64 & 0.0 & 0.0 & 0.22 & 0.1 & 0.0 & 0.06 & 0.03 \\
\hline METHYLCYCLOPENTANE & 0.0 & 0.00 & 0.0 & 0.0 & 0.00 & 0.0 & 0.0 & 0.00 & 0.0 & 0.0 & 0.00 & 0.00 \\
\hline 2,4-DIMETHYLPENTANE & 0.2 & 0.09 & 0.3 & 0.1 & 2.94 & 0.1 & 0.0 & 0.15 & 0.0 & 0.1 & 0.17 & 0.13 \\
\hline 2,3,3-TAIMETHYL-1-BUTENE & 0.0 & 0.00 & 0.0 & 0.0 & 0.00 & 0.0 & 0.0 & 0.00 & 0.0 & 0.0 & 0.00 & 0.00 \\
\hline 2,2,3-TRIMETHYLBUTANE & 0.0 & 0.00 & 0.0 & 0.0 & 2.32 & 0.1 & 0.0 & 0.00 & 0.0 & 0.0 & 0.08 & 0.03 \\
\hline 3,4-DIMETHYL-1-PENTENE & 0.0 & 0.00 & 0.0 & 0.0 & 0.00 & 0.0 & 0.0 & 0.00 & 0.0 & 0.0 & 0.00 & 0.00 \\
\hline 1-METHYLCYCLOPENTENE & 0.0 & 0.01 & 0.1 & 0.1 & 2.86 & 0.3 & 0.0 & 0.12 & 0.1 & 0.0 & 0.09 & $\overline{0.22}$ \\
\hline BENZENE & 0.2 & 0.09 & 0.1 & 0.1 & 4.47 & 0.0 & 0.1 & 0.37 & 0.0 & 0.1 & 0.22 & 0.04 \\
\hline 3-METHYL-1-HEXENE & 0.0 & 0.00 & 0.0 & 0.0 & 0.00 & 0.0 & 0.0 & 0.00 & 0.0 & 0.0 & 0.00 & 0.00 \\
\hline 3,3-DIMETHYLPENTANE & 0.0 & 0.00 & 0.0 & 0.0 & 0.00 & 0.0 & 0.0 & 0.15 & 0.0 & 0.0 & 0.02 & 0.01 \\
\hline CYCLOHEXANE & 0.0 & 0.00 & 0.0 & 0.0 & 0.00 & 0.0 & 0.0 & 0.00 & 0.0 & 0.0 & 0.00 & 0.00 \\
\hline 2-METHYLHEXANE & 0.0 & 0.00 & 0.0 & 0.0 & 0.00 & 0.0 & 0.0 & 0.00 & 0.0 & 0.0 & 0.00 & 0.00 \\
\hline 2,3-DIMETHYLPENTANE & 0.1 & 0.08 & 0.2 & 0.0 & 0.00 & 0.0 & 0.1 & 0.40 & 0.1 & 0.1 & 0.12 & 0.08 \\
\hline 1,1-DIMETHYLCYCLOPENTANE & 0.0 & 0.00 & 0.0 & 0.0 & 0.00 & 0.0 & 0.0 & 0.00 & 0.0 & 0.0 & 0.00 & 0.00 \\
\hline CYCLOHEXENE & 0.0 & 0.00 & 0.0 & 0.0 & 0.00 & 0.0 & 0.0 & 0.00 & 0.0 & 0.0 & 0.00 & 0.00 \\
\hline 3-METHYLHEXANE & 0.0 & 0.00 & 0.0 & 0.0 & 0.00 & 0.0 & 0.0 & 0.00 & 0.0 & 0.0 & 0.00 & 0.00 \\
\hline CIS-1,3-DIMETHYLCYCLOPENTANE & 0.0 & 0.00 & 0.0 & 0.0 & 0.00 & 0.0 & 0.0 & 0.00 & 0.0 & 0.0 & 0.00 & 0.00 \\
\hline 3-ETHYLPENTANE & 0.0 & 0.00 & 0.0 & 0.0 & 0.00 & 0.0 & 0.0 & 0.00 & 0.0 & 0.0 & 0.00 & 0.00 \\
\hline TRANS-1,2-DIMETHYLCYCLOPENTANE & 0.0 & 0.00 & 0.0 & 0.0 & 0.00 & 0.0 & 0.0 & 0.00 & 0.0 & 0.0 & 0.00 & 0.00 \\
\hline TRANS-1,3-DIMETHYLCYCLOPENTANE & 0.0 & 0.00 & 0.0 & 0.0 & 0.00 & 0.0 & 0.0 & 0.00 & 0.0 & 0.0 & 0.00 & 0.00 \\
\hline 1-HEPTENE & 0.0 & 0.00 & 0.0 & 0.0 & 0.00 & 0.0 & 0.0 & 0.00 & 0.0 & 0.0 & 0.00 & 0.00 \\
\hline 2,2,4-TRIMETHYLPENTANE & 0.4 & 0.21 & 0.4 & 0.1 & 6.18 & 0.1 & 0.1 & 0.52 & 0.1 & 0.2 & 0.38 & 0.16 \\
\hline 2-METHYL-1-HEXENE & 0.0 & 0.00 & 0.0 & 0.0 & 0.00 & 0.0 & 0.0 & 0.00 & 0.0 & 0.0 & 0.00 & 0.00 \\
\hline TRANS-3-HEPTENE & 0.0 & 0.00 & 0.0 & 0.0 & 0.00 & 0.0 & 0.0 & 0.00 & 0.0 & 0.0 & 0.00 & 0.00 \\
\hline HEPTANE & 0.0 & 0.00 & 0.0 & 0.0 & 0.00 & 0.0 & 0.0 & 0.00 & 0.0 & 0.0 & 0.00 & 0.00 \\
\hline UNIDENTIFIED C7 & 0.0 & 0.02 & 0.2 & 0.0 & 0.00 & 0.0 & 0.0 & 0.18 & 0.2 & 0.0 & 0.04 & 0.08 \\
\hline
\end{tabular}


专 造

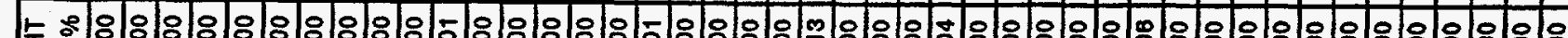
志

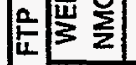

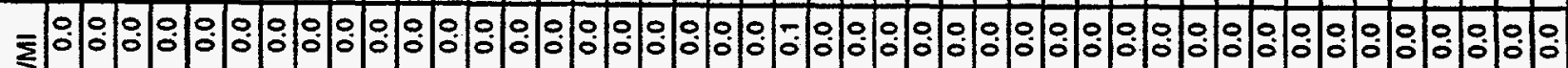

을

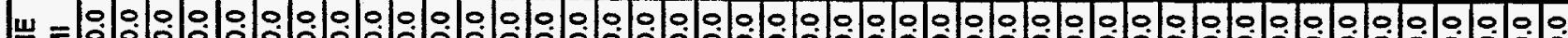

岁

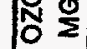

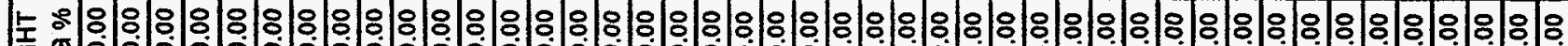
m 志

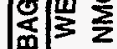

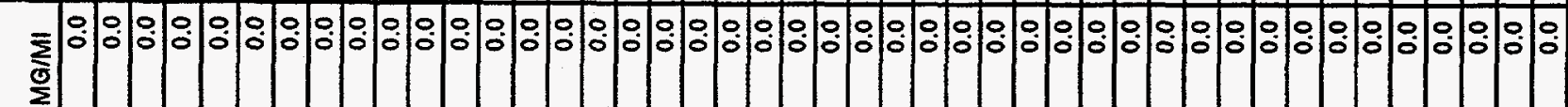

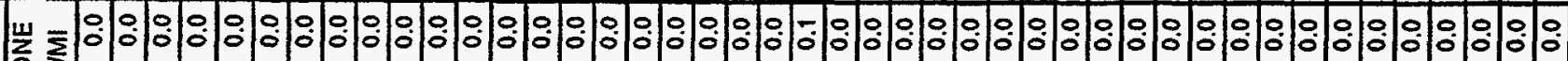
要

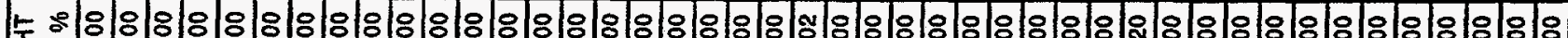
、 空离

- $000000000000000000-000000000000000000000$

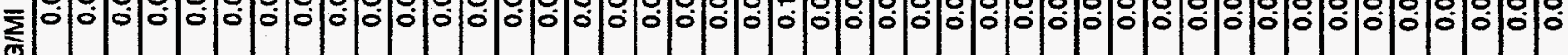
$\stackrel{5}{2}$

岁

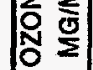

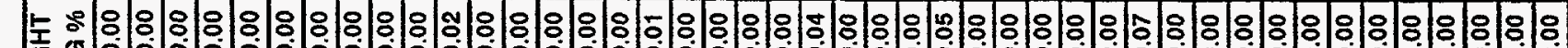
通要

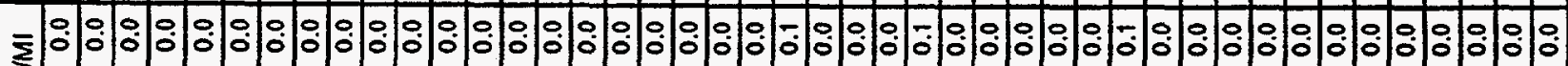
$\stackrel{\Xi}{2}$

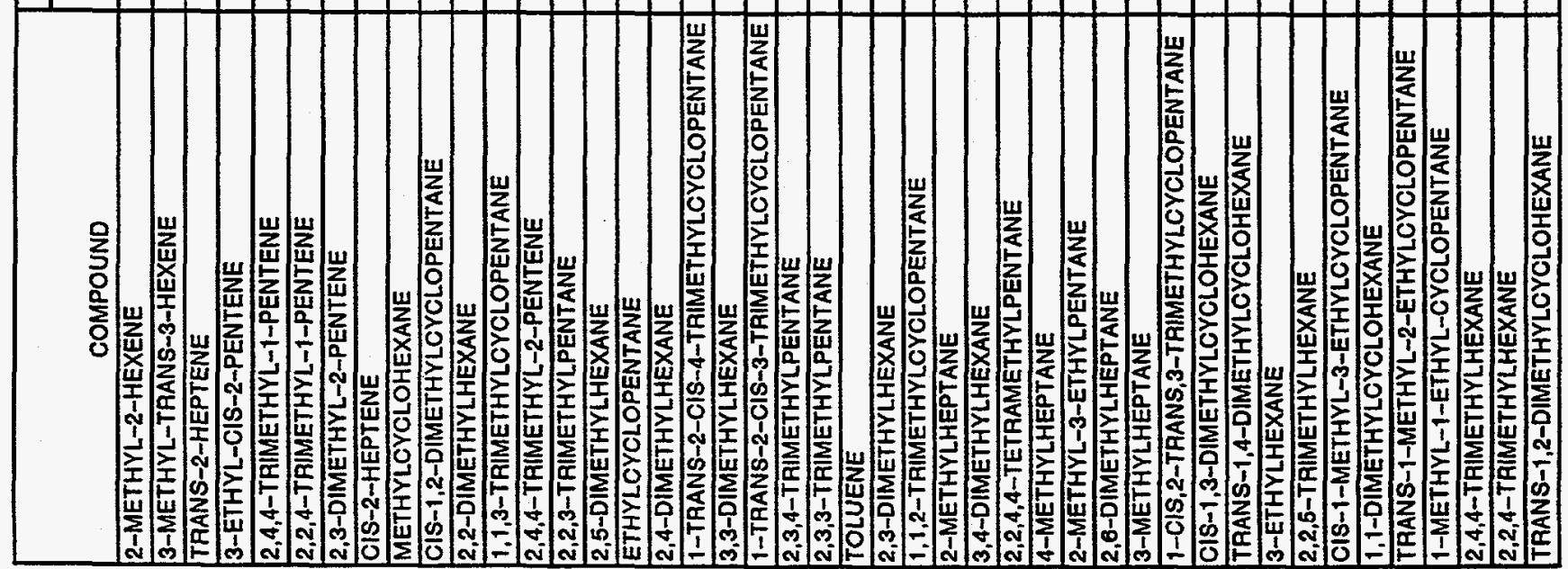




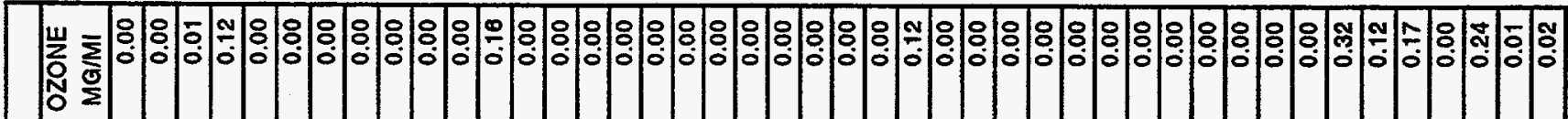

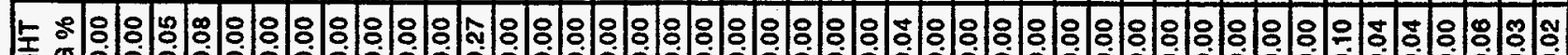

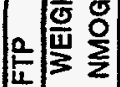

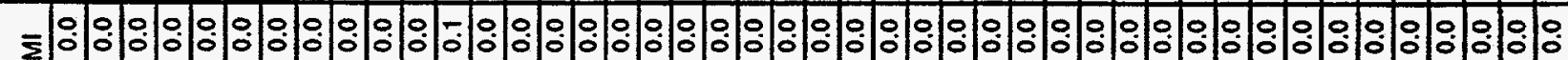

$\frac{\mathrm{o}}{2}$

岁

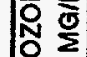

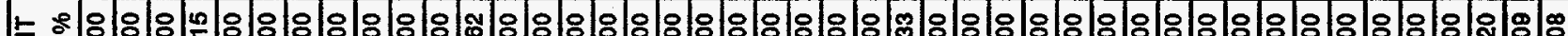
m 高高紊

«

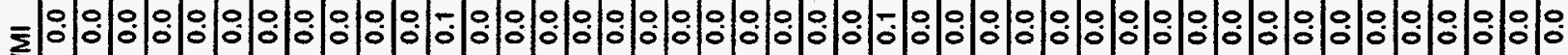

$\frac{\mathrm{O}}{2}$

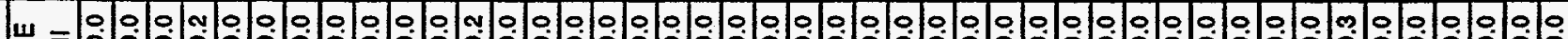

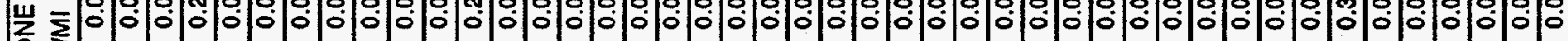
资

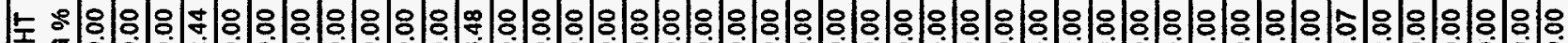
N 志 高要产

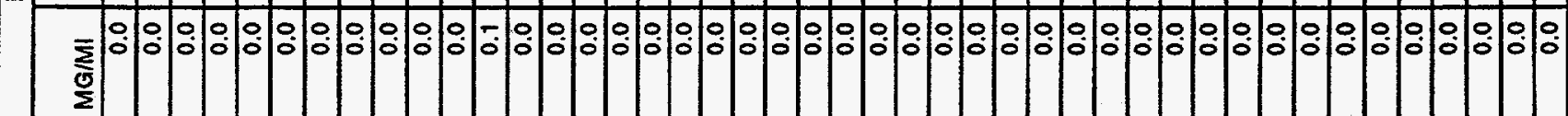

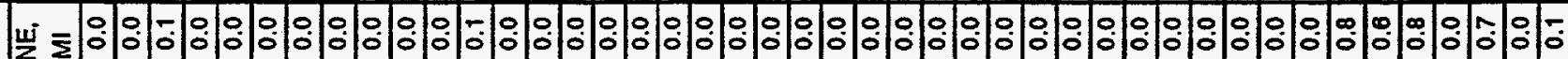
空

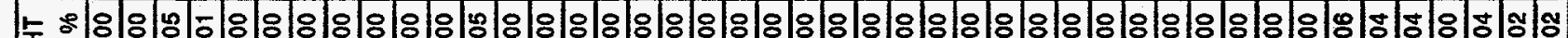
- 质 这高

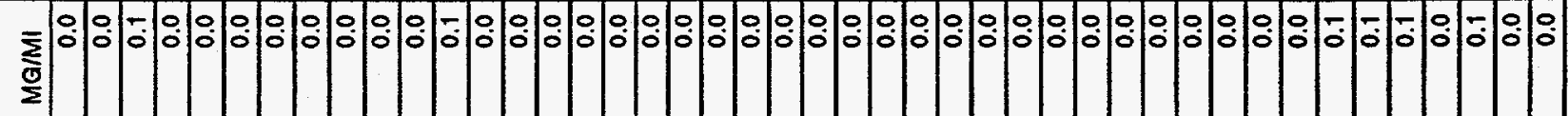

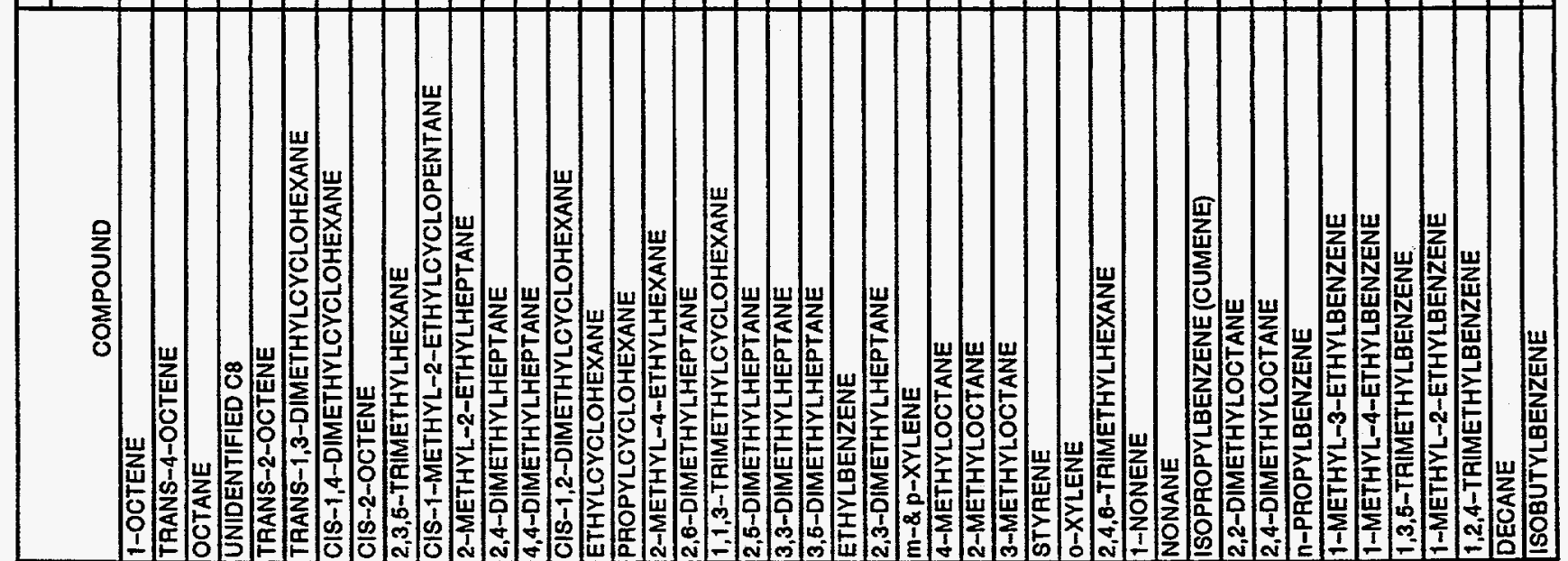




\begin{tabular}{|c|c|c|c|c|c|c|c|c|c|c|c|c|}
\hline \multirow[b]{2}{*}{ COMPOUND } & \multicolumn{3}{|c|}{$\overline{B A G ~} 1$} & \multicolumn{3}{|c|}{ BAG 2} & \multicolumn{3}{|c|}{ BAG 3} & \multicolumn{3}{|c|}{ FTP } \\
\hline & MG/MI & $\begin{array}{l}\text { WEIGHT } \\
\text { NMOG \% }\end{array}$ & $\begin{array}{l}\text { OZONE, } \\
\text { MG/MI }\end{array}$ & MG/MI & $\begin{array}{l}\text { WEIGHT } \\
\text { NMOQ } \% \\
\end{array}$ & $\begin{array}{l}\text { OZONE } \\
\text { MG/MI }\end{array}$ & MG/MI & $\begin{array}{l}\text { WEIGHT } \\
\text { NMOG } \% \\
\end{array}$ & $\begin{array}{l}\text { OZONE } \\
\text { MG/MI }\end{array}$ & MG/MI & $\begin{array}{l}\text { WEIGHT } \\
\text { NMOG \% }\end{array}$ & $\begin{array}{l}\text { OZONE } \\
\text { MG/MI }\end{array}$ \\
\hline METHYLPROPYLBENZENE & 0.0 & 0.00 & 0.0 & 0.0 & 0.00 & 0.0 & 0.0 & 0.00 & 0.0 & 0.0 & 0.00 & 0.00 \\
\hline S-BUTYLBENZENE & $\overline{0.0}$ & 0.00 & 0.0 & 0.0 & 0.00 & 0.0 & 0.0 & 0.00 & 0.0 & 0.0 & 0.00 & 0.00 \\
\hline 1-METHYL-3-ISOPROPYLBENZENE & 0.0 & 0.00 & 0.0 & 0.0 & 0.00 & 0.0 & 0.0 & 0.00 & 0.0 & 0.0 & 0.00 & 0.00 \\
\hline 1,2,3-TRIMETHYLBENZENE & 0.0 & 0.00 & 0.0 & 0.0 & 0.00 & 0.0 & 0.0 & 0.00 & 0.0 & $\overline{0.0}$ & 0.00 & 0.00 \\
\hline 1-METHYL-4-ISOPROPYLBENZENE & 0.0 & 0.00 & 0.0 & 0.0 & 0.00 & 0.0 & 0.0 & 0.00 & 0.0 & 0.0 & 0.00 & 0.00 \\
\hline INDAN & 0.0 & 0.00 & 0.0 & 0.0 & 0.00 & 0.0 & 0.0 & 0.00 & 0.0 & 0.0 & 0.00 & 0.00 \\
\hline 1-METHYL-2-1SOPROPYLBENZENE & 0.0 & 0.00 & 0.0 & 0.0 & 0.00 & 0.0 & 0.0 & 0.00 & 0.0 & 0.0 & 0.00 & 0.00 \\
\hline 1,3-DIETHYLBENZENE & 0.0 & 0.00 & 0.0 & 0.0 & 0.00 & 0.0 & 0.0 & 0.00 & 0.0 & 0.0 & 0.00 & 0.00 \\
\hline 1.4-DIETHYLBENZENE & 0.0 & 0.00 & 0.0 & 0.0 & 0.00 & 0.0 & 0.0 & 0.00 & 0.0 & 0.0 & 0.00 & 0.00 \\
\hline 1-METHYL-3-N-PROPYLBENZENE & 0.0 & 0.00 & 0.0 & 0.0 & 0.00 & 0.0 & 0.0 & 0.00 & 0.0 & 0.0 & 0.00 & 0.00 \\
\hline 1-METHYL-4-N-PROPYLBENZENE & 0.0 & 0.00 & 0.0 & 0.0 & 0.00 & 0.0 & 0.0 & 0.00 & 0.0 & 0.0 & 0.00 & 0.00 \\
\hline 1,2 DIETHYLBENZENE & 0.0 & 0.00 & 0.0 & 0.0 & 0.00 & 0.0 & 0.0 & 0.00 & 0.0 & 0.0 & 0.00 & 0.00 \\
\hline 1-METHYL-2-N-PROPYLBENZENE & 0.0 & 0.00 & 0.0 & 0.0 & 0.00 & 0.0 & 0.0 & 0.00 & 0.0 & 0.0 & 0.00 & 0.00 \\
\hline 1,4-DIMETHYL-2-ETHYLBENZENE & 0.0 & 0.00 & 0.0 & 0.0 & 0.00 & 0.0 & 0.0 & 0.00 & 0.0 & 0.0 & 0.00 & 0.00 \\
\hline 1,3-DIMETHYL-4-ETHYLBENZENE & 0.0 & 0.00 & 0.0 & 0.0 & 0.00 & 0.0 & 0.0 & 0.00 & 0.0 & 0.0 & 0.00 & 0.00 \\
\hline 1.2-DIMETHYL-4-ETHYLBENZENE & 0.0 & 0.00 & 0.0 & 0.0 & 0.00 & 0.0 & 0.0 & 0.00 & 0.0 & 0.0 & 0.00 & 0.00 \\
\hline 1,3-DIMETHYL-2-ETHYLBENZENE & 0.0 & 0.00 & 0.0 & 0.0 & 0.00 & 0.0 & 0.0 & 0.00 & 0.0 & 0.0 & 0.00 & 0.00 \\
\hline UNDECANE & 0.0 & 0.00 & 0.0 & 0.0 & 0.00 & 0.0 & 0.0 & 0.00 & 0.0 & 0.0 & 0.00 & 0.00 \\
\hline 1,2-DIMETHYL-3-ETHYLBENZENE & 0.0 & 0.00 & 0.0 & 0.0 & 0.00 & 0.0 & 0.0 & 0.00 & 0.0 & 0.0 & 0.00 & 0.00 \\
\hline 1,2,4,6-TETRAMETHYLBENZENE & 0.0 & 0.00 & 0.0 & 0.0 & 0.00 & 0.0 & 0.0 & 0.00 & 0.0 & 0.0 & 0.00 & 0.00 \\
\hline 2-METHYLBUTYLBENZENE (Bec AMYLBENZENE) & 0.0 & 0.00 & 0.0 & 0.0 & 0.00 & 0.0 & 0.0 & 0.00 & 0.0 & 0.0 & 0.00 & 0.00 \\
\hline 3,4 DIMETHYLCUMENE & 0.0 & 0.00 & 0.0 & 0.0 & 0.00 & 0.0 & 0.0 & 0.00 & 0.0 & 0.0 & 0.00 & 0.00 \\
\hline 1,2,3,6-TETRAMETHYLBENZENE & 0.0 & 0.00 & 0.0 & 0.0 & 0.00 & 0.0 & 0.0 & 0.00 & 0.0 & 0.0 & 0.00 & 0.00 \\
\hline TERT-1-BUT-2-METHYLBENZENE & 0.0 & 0.00 & 0.0 & 0.0 & 0.00 & 0.0 & 0.0 & 0.00 & 0.0 & 0.0 & 0.00 & 0.00 \\
\hline 1,2,3,4-TETRAMETHYLBENZENE & 0.0 & 0.00 & 0.0 & 0.0 & 0.00 & 0.0 & 0.0 & 0.00 & 0.0 & $\overline{0.0}$ & 0.00 & 0.00 \\
\hline N-PENT-BENZENE & 0.0 & 0.00 & 0.0 & 0.0 & 0.00 & 0.0 & 0.0 & 0.00 & 0.0 & 0.0 & 0.00 & 0.00 \\
\hline TEAT-1-BUT-3,5-DIMETHYLBENZENE & 0.0 & 0.00 & 0.0 & 0.0 & 0.00 & 0.0 & 0.0 & 0.00 & 0.0 & 0.0 & 0.00 & 0.00 \\
\hline NAPHTHALENE & 0.0 & 0.00 & 0.0 & 0.0 & 0.00 & 0.0 & 0.0 & 0.00 & 0.0 & 0.0 & 0.00 & 0.00 \\
\hline DODECANE & 0.0 & 0.00 & 0.0 & 0.0 & 0.00 & 0.0 & 0.0 & 0.00 & 0.0 & 0.0 & 0.00 & 0.00 \\
\hline UNIDENTIFIED C9-C12+ & 0.0 & 0.00 & 0.0 & 0.0 & 0.00 & 0.0 & 0.0 & 0.03 & 0.0 & 0.0 & 0.00 & 0.01 \\
\hline FORMALDEHYDE & 1.5 & 0.85 & 11.0 & 0.0 & 0.00 & 0.0 & 0.1 & 0.39 & 0.5 & 0.3 & 0.77 & 2.42 \\
\hline ACETALDEHYDE & 0.3 & 0.16 & 1.6 & 0.0 & 0.00 & 0.0 & 0.0 & 10.00 & 0.0 & 0.1 & 0.14 & 0.34 \\
\hline ACROLEIN & 0.2 & 0.11 & 1.4 & 0.0 & 0.00 & 0.0 & 0.0 & 0.00 & 0.0 & 0.0 & 0.09 & 0.28 \\
\hline ACETONE & 0.0 & 0.00 & 0.0 & 0.0 & 0.00 & 0.0 & 0.8 & 4.56 & 0.5 & 0.2 & 0.53 & 0.13 \\
\hline PAOPIONALDEHYDE & 0.0 & 0.00 & 0.0 & 0.0 & 0.00 & 0.0 & 0.0 & 0.00 & 0.0 & 0.0 & 0.00 & 0.00 \\
\hline CAOTONALDEHYDE & 0.0 & 0.00 & 0.0 & 0.0 & 0.00 & 0.0 & 0.0 & 0.00 & 0.0 & 0.0 & 0.00 & 0.00 \\
\hline ISOBUTYRALDEHYDE & 0.0 & 0.02 & 0.2 & 0.0 & 0.00 & 0.0 & 0.0 & 0.24 & 0.2 & 0.0 & 0.04 & 0.10 \\
\hline METHYLETHYL KETONE & 0.0 & 0.02 & 0.0 & 0.0 & 0.00 & 0.0 & 0.0 & 0.24 & 0.1 & 0.0 & 0.04 & 0.02 \\
\hline BENZALDEHYDE & 1.8 & 0.97 & -1.0 & 0.4 & 18.82 & -0.2 & 0.0 & 0.00 & 0.0 & 0.6 & 1.30 & -0.31 \\
\hline HEXANALDEHYDE & 0.0 & 0.00 & 0.0 & 0.3 & 14.86 & 1.1 & 0.0 & 0.00 & 0.0 & 0.2 & 0.34 & 0.57 \\
\hline
\end{tabular}


TEST NO: 90NB/10BE-02S

TEST CAR: 1994 PONTIAC GRANDAM

TEST DATE: $9 / 11 / 95$

TEST FUEL: BUTANE

\begin{tabular}{|c|c|c|c|c|c|c|c|c|c|c|c|c|}
\hline \multirow[b]{2}{*}{ COMPOUND } & \multicolumn{3}{|c|}{$B A G 1$} & \multicolumn{3}{|c|}{ BAG 2} & \multicolumn{3}{|c|}{ BAG 3} & \multicolumn{3}{|c|}{ FTP } \\
\hline & MGIMI & \begin{tabular}{|l|} 
WEIGHT \\
NMOG $\%$
\end{tabular} & $\begin{array}{l}\text { OZONE, } \\
\text { MG/MI }\end{array}$ & MG/MI & $\begin{array}{l}\text { WEIGHT } \\
\text { NMOG \% }\end{array}$ & $\begin{array}{l}\text { OZONE } \\
\text { MGMII }\end{array}$ & MG/MI & $\begin{array}{l}\text { WEIGHT } \\
\text { NMOQ \% }\end{array}$ & $\begin{array}{l}\text { OZONE } \\
\text { MG/MI }\end{array}$ & MG/MI & $\begin{array}{l}\text { WEIGHT } \\
\text { NMOG \% }\end{array}$ & $\begin{array}{l}\text { OZONE } \\
\text { MG/MI }\end{array}$ \\
\hline METHANE & 22.5 & & 0.3 & 4.2 & & 0.1 & 26.8 & & 0.4 & 14.0 & & 0.21 \\
\hline ETHANE & 2.6 & 1.47 & 0.6 & 0.0 & 0.95 & 0.0 & 2.5 & 8.19 & 0.6 & 1.2 & 2.70 & 0.30 \\
\hline ETHYLENE & 15.5 & 8.91 & 112.8 & 0.0 & 0.52 & 0.1 & 2.2 & 6.96 & 15.7 & 3.7 & 8.28 & 27.17 \\
\hline PROPANE & 0.2 & 0.14 & 0.1 & 0.0 & 0.00 & 0.0 & 0.1 & 0.36 & 0.1 & 0.1 & 0.18 & 0.04 \\
\hline PROPYLENE & 7.1 & 4.07 & 66.5 & 0.0 & 0.00 & 0.0 & 0.7 & 2.22 & 6.4 & 1.6 & 3.60 & 15.23 \\
\hline ACETYLENE & 5.5 & 3.19 & 2.8 & 0.0 & 0.54 & 0.0 & 0.0 & 0.08 & 0.0 & 1.1 & 2.53 & 0.57 \\
\hline PROPADIENE & 0.0 & 0.00 & 0.0 & 0.0 & 0.00 & 0.0 & 0.0 & 0.00 & 0.0 & 0.0 & 0.00 & 0.00 \\
\hline BUTANE & 122.6 & 70.59 & 125.1 & 0.1 & 3.36 & 0.1 & 20.7 & 66.96 & 21.1 & 30.5 & 67.76 & 31.11 \\
\hline TRANS-2-BUTENE & 2.9 & 1.65 & 28.5 & 0.0 & 0.00 & 0.0 & 0.1 & 0.22 & 0.7 & 0.6 & 1.33 & 5.98 \\
\hline 1-BUTENE & 3.0 & 1.72 & 26.6 & 0.0 & 0.00 & 0.0 & 0.0 & 0.00 & 0.0 & 0.6 & 1.34 & 5.39 \\
\hline 2-METHYLPAOPENE (ISOBUTYLENE) & 2.8 & 1.62 & 14.9 & 0.0 & 0.23 & 0.0 & 0.1 & 0.22 & 0.4 & 0.6 & 1.32 & 3.14 \\
\hline 2,2-DIMETHYLPROPANE (NEOPENTANE) & 0.1 & 0.03 & 0.0 & 0.0 & 0.00 & 0.0 & 0.0 & 0.00 & 0.0 & 0.0 & 0.03 & 0.00 \\
\hline PROPYNE & 0.0 & 0.00 & 0.0 & 0.0 & 0.00 & 0.0 & 0.0 & 0.00 & 0.0 & 0.0 & 0.00 & 0.00 \\
\hline 1,3-BUTADIENE & 0.4 & 0.20 & 3.8 & 0.0 & 0.00 & 0.0 & 0.0 & 0.00 & 0.0 & 0.1 & 0.16 & 0.78 \\
\hline 2-METHYLPROPANE (ISOBUTANE) & 0.0 & 0.00 & 0.0 & 0.0 & 0.32 & 0.0 & 0.0 & 0.00 & 0.0 & 0.0 & 0.01 & 0.01 \\
\hline 1-BUTYNE & 0.0 & 0.00 & 0.0 & 0.0 & 0.00 & 0.0 & 0.0 & 0.00 & 0.0 & 0.0 & 0.00 & 0.00 \\
\hline METHANOL & 0.0 & 0.00 & 0.0 & 0.0 & 0.00 & 0.0 & 0.0 & 0.00 & 0.0 & 0.0 & 0.00 & 0.00 \\
\hline CIS-2-BUTENE & 2.9 & 1.60 & 28.6 & 0.0 & 0.00 & 0.0 & 0.8 & 2.93 & 9.0 & 0.8 & 1.84 & 8.23 \\
\hline 3-METHYL-1-BUTENE & 0.0 & 0.00 & 0.0 & 0.0 & 0.00 & 0.0 & 0.0 & 0.00 & 0.0 & 0.0 & 0.00 & 0.00 \\
\hline ETHANOL & 0.0 & 0.00 & 0.0 & 0.0 & 0.00 & 0.0 & 0.0 & 0.00 & 0.0 & 0.0 & 0.00 & 0.00 \\
\hline 2-METHYLBUTANE (ISOPENTANE) & 2.0 & 1.18 & 2.8 & 0.5 & 17.79 & 0.7 & 1.2 & 3.98 & 1.7 & 1.0 & 2.22 & 1.38 \\
\hline 2-BUTYNE & 0.0 & 0.00 & 0.0 & 0.0 & 0.00 & 0.0 & 0.0 & 0.00 & 0.0 & 0.0 & 0.00 & 0.00 \\
\hline 1-PENTENE & 0.0 & 0.00 & 0.0 & 0.0 & 0.00 & 0.0 & 0.1 & 0.28 & 0.6 & 0.0 & 0.05 & 0.15 \\
\hline 2-METHYL-1-BUTENE & 0.0 & 0.00 & 0.0 & 0.0 & 0.00 & 0.0 & 0.1 & 0.46 & 0.7 & 0.0 & 0.08 & 0.19 \\
\hline PENTANE & 0.2 & 0.12 & 0.2 & 0.2 & 6.52 & 0.2 & 0.1 . & 0.44 & 0.1 & 0.2 & 0.38 & 0.18 \\
\hline UNIDENTIFIED C5 OLEFINS & 0.0 & 0.00 & 0.0 & 0.0 & 0.00 & 0.0 & 0.0 & 0.00 & 0.0 & 0.0 & 0.00 & 0.00 \\
\hline 2-METHYL-1,3-BUTADIENE & 0.0 & 0.00 & 0.0 & 0.0 & 0.00 & 0.0 & 0.0 & 0.00 & 0.0 & 0.0 & 0.00 & 0.00 \\
\hline TAANS-2-PENTENE & 0.1 & 0.06 & 0.9 & 0.0 & 0.00 & 0.0 & 0.0 & 0.00 & 0.0 & 0.0 & 0.05 & 0.19 \\
\hline 3,3-DIMETHYL-1-BUTENE & 0.0 & 0.00 & 0.0 & 0.0 & 0.00 & 0.0 & 0.0 & 0.00 & 0.0 & 0.0 & 0.00 & 0.00 \\
\hline CIS-2-PENTENE & 0.0 & 0.00 & 0.0 & 0.0 & 0.00 & 0.0 & 0.0 & 0.00 & 0.0 & 0.0 & 0.00 & 0.00 \\
\hline 2-METHYL-2-BUTENE & 0.1 & 0.05 & 0.6 & 0.0 & 0.00 & 0.0 & 0.0 & 0.06 & 0.1 & 0.0 & 0.05 & 0.16 \\
\hline CYCLOPENTADIENE & 0.0 & 0.00 & 0.0 & 0.0 & 0.00 & 0.0 & 0.0 & 0.00 & 0.0 & 0.0 & 0.00 & 0.00 \\
\hline 2,2-DIMETHYLBUTANE & 0.0 & 0.00 & 0.0 & 0.0 & 0.00 & 0.0 & 0.0 & 0.00 & 0.0 & 0.0 & 0.00 & 0.00 \\
\hline CYCLOPENTENE & 0.0 & 0.00 & 0.0 & 0.0 & 0.00 & 0.0 & 0.0 & 0.00 & 0.0 & 0.0 & 0.00 & 0.00 \\
\hline 4-METHYL-1-PENTENE & 0.0 & 0.00 & 0.0 & 0.0 & 0.00 & 0.0 & 0.0 & 0.00 & 0.0 & 0.0 & 0.00 & 0.00 \\
\hline 3-METHYL-1-PENTENE & 0.0 & 0.00 & 0.0 & 0.0 & 0.00 & 0.0 & 0.0 & 0.00 & 0.0 & 0.0 & 0.00 & 0.00 \\
\hline CYCLOPENTANE & 0.0 & 0.00 & 0.0 & 0.0 & 0.00 & 0.0 & 0.0 & 0.00 & 0.0 & 0.0 & 0.00 & 0.00 \\
\hline 2,3-DIMETHYLBUTANE & 0.1 & 0.06 & 0.1 & 0.1 & 2.62 & 0.1 & 0.1 & 0.21 & 0.1 & 0.1 & 0.17 & 0.08 \\
\hline MTBE & 0.0 & 0.00 & 0.0 & 0.0 & 0.00 & 0.0 & 0.0 & 0.00 & 0.0 & 0.0 & 0.00 & 0.00 \\
\hline
\end{tabular}




\begin{tabular}{|c|c|c|c|c|c|c|c|c|c|c|c|c|}
\hline \multirow[b]{2}{*}{ COMPOUND } & \multicolumn{3}{|c|}{ BAG 1} & \multicolumn{3}{|c|}{ BAG 2 } & \multicolumn{3}{|c|}{ BAG 3} & \multicolumn{3}{|c|}{ FTP } \\
\hline & MG/MI & $\begin{array}{l}\text { WEIGHT } \\
\text { NMOG \% }\end{array}$ & $\begin{array}{l}\text { OZONE, } \\
\text { MG/MI }\end{array}$ & MG/MI & $\begin{array}{l}\text { WEIGHT } \\
\text { NMOG \% }\end{array}$ & $\begin{array}{l}\text { OZONE } \\
\text { MGIMI }\end{array}$ & MG/MI & $\begin{array}{l}\text { WEIGHT } \\
\text { NMOQ \% } \\
\end{array}$ & $\begin{array}{l}\text { OZONE } \\
\text { MG/MI }\end{array}$ & MG/MI & $\begin{array}{l}\text { WEIGHT } \\
\text { NMOG } \%\end{array}$ & $\begin{array}{l}\text { OZONE } \\
\text { MG/MI }\end{array}$ \\
\hline 2,3-DIMETHYL-1-BUTENE & 0.0 & 0.00 & 0.0 & 0.0 & 0.00 & 0.0 & 0.0 & 0.00 & 0.0 & 0.0 & 0.00 & 0.00 \\
\hline 4-METHYL-CIS-2-PENTENE & 0.0 & 0.00 & 0.0 & 0.0 & 0.00 & 0.0 & 0.0 & 0.00 & 0.0 & 0.0 & 0.00 & 0.00 \\
\hline 2-METHYLPENTANE & 0.2 & 0.14 & $\overline{0.4}$ & 0.2 & 6.05 & 0.3 & 0.2 & 0.61 & 0.3 & 0.2 & 0.42 & 0.29 \\
\hline 4-METHYL-TRANS-2-PENTENE & 0.0 & 0.00 & 0.0 & 0.0 & 0.00 & 0.0 & 0.0 & 0.00 & 0.0 & 0.0 & 0.00 & 0.00 \\
\hline 3-METHYLPENTANE & 0.1 & 0.07 & 0.2 & 0.1 & 4.32 & $\overline{0.2}$ & 0.1 & 0.35 & 0.2 & $\overline{0.1}$ & 0.26 & 0.18 \\
\hline 2-METHYL-1-PENTENE & 0.0 & 0.00 & 0.0 & 0.0 & 0.00 & 0.0 & 0.0 & 0.00 & 0.0 & 0.0 & 0.00 & 0.00 \\
\hline 1-HEXENE & 0.0 & 0.00 & 0.0 & 0.0 & 0.00 & 0.0 & 0.0 & 0.00 & 0.0 & 0.0 & 0.00 & 0.00 \\
\hline HEXANE & 0.2 & 0.10 & 0.2 & 0.0 & 1.81 & 0.0 & 0.1 & 0.26 & 0.1 & 0.1 & 0.19 & 0.08 \\
\hline UNIDENTIFIED CB OLEFINS & 0.0 & 0.00 & 0.0 & 0.0 & 0.00 & 0.0 & 0.0 & 0.00 & 0.0 & 0.0 & 0.00 & 0.00 \\
\hline TRANS-3-HEXENE & 0.0 & 0.00 & 0.0 & 0.0 & 0.00 & 0.0 & 0.0 & 0.00 & 0.0 & 0.0 & 0.00 & 0.00 \\
\hline CIS-3-HEXENE & 0.0 & 0.00 & 0.0 & 0.0 & 0.00 & 0.0 & 0.0 & 0.00 & 0.0 & 0.0 & 0.00 & 0.00 \\
\hline TAANS-2-HEXENE & 0.0 & 0.00 & 0.0 & 0.0 & 0.00 & 0.0 & 0.0 & 0.00 & 0.0 & 0.0 & 0.00 & 0.00 \\
\hline 3-METHYL-TRANS-2-PENTENE & 0.0 & 0.00 & 0.0 & 0.0 & 0.00 & 0.0 & 0.0 & 0.00 & 0.0 & 0.0 & 0.00 & 0.00 \\
\hline 2-METHYL-2-PENTENE & 0.0 & 0.00 & 0.0 & 0.0 & 0.00 & 0.0 & 0.0 & 0.00 & 0.0 & 0.0 & 0.00 & 0.00 \\
\hline 3-METHYLCYCLOPENTENE & 0.0 & 0.00 & 0.0 & 0.0 & 0.00 & 0.0 & 0.0 & 0.00 & 0.0 & 0.0 & 0.00 & 0.00 \\
\hline CIS-2-HEXENE & 0.0 & 0.00 & 0.0 & 0.0 & 0.00 & 0.0 & 0.0 & 0.00 & 0.0 & 0.0 & 0.00 & 0.00 \\
\hline ETBE & 0.0 & 0.00 & 0.0 & 0.0 & 0.00 & 0.0 & 0.0 & 0.00 & 0.0 & 0.0 & 0.00 & 0.00 \\
\hline 3-METHYL-CIS-2-PENTENE & 0.0 & 0.00 & 0.0 & 0.0 & 0.00 & 0.0 & 0.0 & 0.00 & 0.0 & 0.0 & 0.00 & 0.00 \\
\hline 2,2-DIMETHYLPENTANE & 0.1 & 0.05 & 0.1 & 0.1 & 3.48 & 0.1 & 0.1 & 0.21 & 0.1 & 0.1 & 0.19 & 0.12 \\
\hline METHYLCYCLOPENTANE & 0.0 & 0.00 & 0.0 & 0.0 & 0.00 & 0.0 & 0.0 & 0.00 & 0.0 & 0.0 & 0.00 & 0.00 \\
\hline 2,4-DIMETHYLPENTANE & $\overline{0.2}$ & 0.11 & 0.3 & 0.1 & 2.34 & 0.1 & 0.0 & 0.13 & 0.1 & 0.1 & 0.19 & 0.15 \\
\hline 2,3,3-TRIMETHYL-1-BUTENE & 0.0 & 0.00 & 0.0 & 0.0 & 0.00 & 0.0 & 0.0 & 0.00 & 0.0 & 0.0 & 0.00 & 0.00 \\
\hline 2,2,3-TRIMETHYLBUTANE & 0.1 & 0.06 & 0.1 & 0.1 & 5.45 & 0.2 & 0.1 & 0.37 & 0.2 & 0.1 & $0.2 \theta$ & 0.17 \\
\hline 3,4-DIMETHYL-1-PENTENE & 0.0 & 0.00 & 0.0 & 0.0 & 0.00 & 0.0 & 0.0 & 0.00 & 0.0 & 0.0 & 0.00 & 0.00 \\
\hline 1-METHYLCYCLOOPENTENE & 0.1 & 0.07 & 0.7 & 0.1 & 4.95 & 0.8 & 0.0 & 0.00 & 0.0 & 0.1 & 0.21 & 0.54 \\
\hline BENZENE & 0.3 & 0.17 & 0.1 & 0.3 & 10.93 & 0.1 & 0.4 & 1.14 & 0.1 & 0.3 & 0.69 & 0.13 \\
\hline 3-METHYL-1-HEXENE & 0.0 & 0.00 & 0.0 & 0.0 & 0.00 & 0.0 & 0.0 & 0.00 & 0.0 & 0.0 & 0.00 & 0.00 \\
\hline 3,3-DIMETHYLPENTANE & 0.0 & 0.00 & 0.0 & 0.0 & 0.00 & 0.0 & 0.0 & 0.00 & 0.0 & 0.0 & 0.00 & 0.00 \\
\hline CYCLOHEXANE & 0.0 & 0.00 & 0.0 & 0.0 & 0.00 & 0.0 & 0.0 & 0.00 & 0.0 & 0.0 & 0.00 & 0.00 \\
\hline 2-METHYLHEXANE & 0.0 & 0.00 & 0.0 & 0.0 & 0.00 & 0.0 & 0.0 & 0.00 & 0.0 & 0.0 & 0.00 & 0.00 \\
\hline 2,3-DIMETHYLPENTANE & 0.3 & 0.17 & 0.5 & 0.1 & 4.56 & 0.2 & 0.1 & 0.22 & 0.1 & 0.1 & 0.32 & 0.22 \\
\hline 1,1-DIMETHYLCYCLOPENTANE & 0.0 & 0.00 & 0.0 & 0.0 & 0.00 & 0.0 & 0.0 & 0.00 & 0.0 & 0.0 & 0.00 & 0.00 \\
\hline CYCLOHEXENE & 0.0 & 0.00 & 0.0 & 0.0 & 0.00 & 0.0 & 0.0 & 0.00 & 0.0 & 0.0 & 0.00 & 0.00 \\
\hline 3-METHYLHEXANE & 0.1 & 0.04 & 0.1 & 0.0 & 1.02 & 0.0 & 0.0 & 0.14 & 0.1 & 0.0 & 0.09 & 0.05 \\
\hline CIS-1,3-DIMETHYLCYCLOPENTANE & 0.0 & 0.00 & 0.0 & 0.0 & 0.00 & 0.0 & 0.0 & 0.00 & 0.0 & 0.0 & 0.00 & 0.00 \\
\hline 3-ETHYLPENTANE & 0.0 & 0.00 & 0.0 & 0.0 & 0.00 & 0.0 & 0.0 & 0.00 & 0.0 & 0.0 & 0.00 & 0.00 \\
\hline TRANS-1,2-DIMETHYLCYCLOPENTANE & 0.0 & 0.00 & 0.0 & 0.0 & 0.00 & 0.0 & 0.0 & 0.00 & 0.0 & 0.0 & 0.00 & 0.00 \\
\hline TRANS-1,3-DIMETHYLCYCLOPENTANE & 0.0 & 0.00 & 0.0 & 0.0 & 0.00 & 0.0 & 0.0 & 0.00 & 0.0 & 0.0 & 0.00 & 0.00 \\
\hline 1-HEPTENE & 0.0 & 0.00 & 0.0 & 0.0 & 0.00 & 0.0 & 0.0 & 0.00 & 0.0 & 0.0 & 0.00 & 0.00 \\
\hline 2,2,4-TRIMETHYLPENTANE & 0.2 & 0.14 & 0.2 & 0.0 & 1.66 & 0.0 & 0.0 & 0.08 & 0.0 & 0.1 & 0.17 & 0.07 \\
\hline 2-METHYL-1-HEXENE & 0.0 & 0.00 & 0.0 & 0.0 & 0.00 & 0.0 & 0.0 & 0.00 & 0.0 & 0.0 & 0.00 & 0.00 \\
\hline TRANS-3-HEPTENE & 0.0 & 0.00 & 0.0 & 0.0 & 0.00 & 0.0 & 0.0 & 0.00 & 0.0 & 0.0 & 0.00 & 0.00 \\
\hline HEPTANE & 0.1 & 0.03 & 0.0 & 0.0 & 0.51 & 0.0 & 0.0 & 0.07 & 0.0 & 0.0 & 0.00 & 0.02 \\
\hline UNIDENTIFIED C7 & 0.0 & 0.00 & 0.0 & 0.0 & 0.00 & 0.0 & 0.0 & 0.00 & 0.0 & 0.0 & 0.00 & 0.00 \\
\hline
\end{tabular}




\begin{tabular}{|c|c|c|c|c|c|c|c|c|c|c|c|c|}
\hline \multirow[b]{2}{*}{ COMPOUND } & \multicolumn{3}{|c|}{ BAG 1} & \multicolumn{3}{|c|}{ BAG 2 } & \multicolumn{3}{|c|}{ BAG 3} & \multicolumn{3}{|c|}{ FTP } \\
\hline & MG/MI & $\begin{array}{l}\text { WEIGHT } \\
\text { NMOQ } \%\end{array}$ & $\begin{array}{l}\text { OZONE, } \\
\mathrm{MG} / \mathrm{MI}\end{array}$ & MG/MI & $\begin{array}{l}\text { WEIGHT } \\
\text { NMOQ } \%\end{array}$ & $\begin{array}{l}\text { OZONE } \\
\text { MG/MI }\end{array}$ & MG/MI & $\begin{array}{l}\text { WEIGHT } \\
\text { NMOG \% }\end{array}$ & $\begin{array}{l}\text { OZONE } \\
\text { MG/MI }\end{array}$ & MG/MI & $\begin{array}{l}\text { WEIGHT } \\
\text { NMOG \% }\end{array}$ & $\begin{array}{l}\text { OZONE } \\
\text { MGMI }\end{array}$ \\
\hline 2-METHYL-2-HEXENE & 0.0 & 0.00 & 0.0 & 0.0 & 0.00 & 0.0 & 0.0 & 0.00 & 0.0 & 0.0 & 0.00 & 0.00 \\
\hline 3-METHYL-TRANS-3-HEXENE & 0.0 & 0.00 & 0.0 & 0.0 & 0.00 & 0.0 & 0.0 & 0.00 & 0.0 & 0.0 & 0.00 & 0.00 \\
\hline TRANS-2-HEPTENE & 0.0 & 0.00 & 0.0 & 0.0 & 0.00 & 0.0 & 0.0 & 0.00 & 0.0 & 0.0 & 0.00 & 0.00 \\
\hline 3-ETHYL-CIS-2-PENTENE & 0.0 & 0.00 & 0.0 & 0.0 & 0.00 & 0.0 & 0.0 & 0.00 & 0.0 & 0.0 & 0.00 & 0.00 \\
\hline 2,4,4-TRIMETHYL-1-PENTENE & 0.0 & 0.00 & 0.0 & 0.0 & 0.00 & 0.0 & 0.0 & 0.00 & 0.0 & 0.0 & 0.00 & 0.00 \\
\hline 2,2,4-TRIMETHYL-1-PENTENE & 0.0 & 0.00 & 0.0 & 0.0 & 0.00 & 0.0 & 0.0 & 0.00 & 0.0 & 0.0 & 0.00 & 0.00 \\
\hline 2,3-DIMETHYL-2-PENTENE & 0.0 & 0.00 & 0.0 & 0.0 & 0.00 & 0.0 & 0.0 & 0.00 & 0.0 & 0.0 & 0.00 & 0.00 \\
\hline CIS-2-HEPTENE & 0.0 & 0.00 & 0.0 & 0.0 & 0.00 & 0.0 & 0.0 & 0.00 & 0.0 & 0.0 & 0.00 & 0.00 \\
\hline METHYLCYCLOHEXANE & 0.0 & 0.00 & 0.0 & 0.0 & 0.00 & 0.0 & 0.0 & 0.00 & 0.0 & 0.0 & 0.00 & 0.00 \\
\hline CIS-1,2-DIMETHYLCYCLOPENTANE & 0.0 & 0.00 & 0.0 & 0.0 & 0.00 & 0.0 & 0.0 & 0.00 & 0.0 & 0.0 & 0.00 & 0.00 \\
\hline 2,2-DIMETHYLHEXANE & 0.1 & 0.05 & 0.1 & 0.0 & 0.00 & 0.0 & 0.0 & 0.00 & 0.0 & 0.0 & 0.04 & 0.02 \\
\hline 1,1,3-TRIMETHYLCYCLOPENTANE & 0.0 & 0.00 & 0.0 & 0.0 & 0.00 & 0.0 & 0.0 & 0.00 & 0.0 & 0.0 & 0.00 & 0.00 \\
\hline 2,4,4-TRIMETHYL-2-PENTENE & 0.0 & 0.00 & 0.0 & 0.0 & 0.00 & 0.0 & 0.0 & 0.00 & 0.0 & 0.0 & 0.00 & 0.00 \\
\hline 2,2,3-TRIMETHYLPENTANE & 0.0 & 0.00 & 0.0 & 0.0 & 0.00 & 0.0 & 0.0 & 0.00 & 0.0 & 0.0 & 0.00 & 0.00 \\
\hline 2,5-DIMETHYLHEXANE & 0.0 & 0.00 & 0.0 & 0.0 & 0.00 & 0.0 & 0.0 & 0.00 & 0.0 & 0.0 & 0.00 & 0.00 \\
\hline ETHYLCYCLOPENTANE & 0.0 & 0.00 & 0.0 & 0.0 & 0.00 & 0.0 & 0.0 & 0.00 & 0.0 & 0.0 & 0.00 & 0.00 \\
\hline 2,4-DIMETHYLHEXANE & 0.0 & 0.00 & 0.0 & 0.0 & 0.00 & 0.0 & 0.0 & 0.00 & 0.0 & 0.0 & 0.00 & 0.00 \\
\hline 1-TRANS-2-CIS-4-TRIMETHYLCYCLOPENTANE & 0.0 & 0.00 & 0.0 & 0.0 & 0.00 & 0.0 & 0.0 & 0.00 & 0.0 & 0.0 & 0.00 & 0.00 \\
\hline 3,3-DIMETHYLHEXANE & 0.0 & 0.00 & 0.0 & 0.0 & 0.00 & 0.0 & 0.0 & 0.00 & 0.0 & $\overline{0.0}$ & 0.00 & 0.00 \\
\hline 1-TRANS-2-CIS-3-TRIMETHYLCYCLOPENTANE & 0.0 & 0.00 & 0.0 & 0.0 & 0.00 & 0.0 & 0.0 & 0.00 & 0.0 & 0.0 & 0.00 & 0.00 \\
\hline 2,3,4-TRIMETHYLPENTANE & 0.0 & 0.00 & 0.0 & 0.0 & 0.00 & 0.0 & 0.0 & 0.00 & 0.0 & 0.0 & 0.00 & 0.00 \\
\hline 2,3,3-TRIMETHYLPENTANE & 0.1 & 0.08 & 0.2 & 0.0 & 0.00 & 0.0 & 0.0 & 0.00 & 0.0 & 0.0 & 0.07 & 0.04 \\
\hline TOLUENE & 0.5 & 0.29 & 1.4 & 0.2 & 8.41 & 0.6 & 0.2 & 0.79 & 0.7 & $\overline{0.3}$ & 0.65 & 0.79 \\
\hline 2,3-DIMETHYLHEXANE & 0.0 & 0.00 & 0.0 & 0.0 & 0.00 & 0.0 & 0.0 & 0.00 & 0.0 & 0.0 & 0.00 & 0.00 \\
\hline 1,1,2-TRIMETHYLCYCLOPENTANE & 0.0 & 0.00 & 0.0 & 0.0 & 0.00 & 0.0 & 0.0 & 0.00 & 0.0 & 0.0 & 0.00 & 0.00 \\
\hline 2-METHYLHEPTANE & 0.0 & 0.00 & 0.0 & 0.0 & 0.00 & 0.0 & 0.0 & 0.00 & 0.0 & 0.0 & 0.00 & 0.00 \\
\hline 3,4-DIMETHYLHEXANE & 0.0 & 0.00 & 0.0 & 0.0 & 0.00 & 0.0 & 0.0 & 0.00 & 0.0 & 0.0 & 0.00 & 0.00 \\
\hline $2,2,4,4-T E T R A M E T H Y L P E N T A N E$ & 0.0 & 0.00 & 0.0 & 0.0 & 0.00 & 0.0 & 0.0 & 0.00 & 0.0 & 0.0 & 0.00 & 0.00 \\
\hline 4-METHYLHEPTANE & 0.0 & 0.00 & 0.0 & 0.0 & 0.00 & 0.0 & 0.0 & 0.00 & 0.0 & 0.0 & 0.00 & 0.00 \\
\hline 2-METHYL-3-ETHYLPENTANE & 0.0 & 0.00 & 0.0 & 0.0 & 0.00 & 0.0 & 0.0 & 0.00 & 0.0 & 0.0 & 0.00 & 0.00 \\
\hline 2,6-DIMETHYLHEPTANE & 0.0 & 0.00 & 0.0 & 0.0 & 0.00 & 0.0 & 0.0 & 0.00 & 0.0 & 0.0 & 0.00 & 0.00 \\
\hline 3-METHYLHEPTANE & 0.0 & 0.00 & 0.0 & 0.0 & 0.00 & 0.0 & 0.0 & 0.00 & 0.0 & 0.0 & 0.00 & 0.00 \\
\hline 1-CIS,2-TAANS,3-TRIMETHYLCYCLOPENTANE & 0.0 & 0.00 & 0.0 & 0.0 & 0.00 & 0.0 & 0.0 & 0.00 & 0.0 & 0.0 & 0.00 & 0.00 \\
\hline CIS-1,3-DIMETHYLCYCLOHEXANE & 0.0 & 0.00 & 0.0 & 0.0 & 0.00 & 0.0 & 0.0 & 0.00 & 0.0 & 0.0 & 0.00 & 0.00 \\
\hline TRANS-1,4-DIMETHYLCYCLOHEXANE & 0.0 & 0.00 & 0.0 & 0.0 & 0.00 & 0.0 & 0.0 & 0.00 & 0.0 & 0.0 & 0.00 & 0.00 \\
\hline 3-ETHYLHEXANE & 0.0 & 0.00 & 0.0 & 0.0 & 0.00 & 0.0 & 0.0 & 0.00 & 0.0 & 0.0 & 0.00 & 0.00 \\
\hline 2,2,5-TRIMETHYLHEXANE & 0.0 & 0.00 & 0.0 & 0.0 & 0.00 & 0.0 & 0.0 & 0.00 & 0.0 & 0.0 & 0.00 & 0.00 \\
\hline CIS-1-METHYL-3-ETHYLCYCLOPENTANE & 0.0 & 0.00 & 0.0 & 0.0 & 0.00 & 0.0 & 0.0 & 0.00 & 0.0 & 0.0 & 0.00 & 0.00 \\
\hline 1,1-DIMETHYLCYCLOHEXANE & 0.0 & 0.00 & 0.0 & 0.0 & 0.00 & 0.0 & 0.0 & 0.00 & 0.0 & 0.0 & 0.00 & 0.00 \\
\hline TRANS-1-METHYL-2-ETHYLCYCLOPENTANE & 0.0 & 0.00 & 0.0 & 0.0 & 0.00 & 0.0 & 0.0 & 0.00 & 0.0 & 0.0 & 0.00 & 0.00 \\
\hline 1-METHYL-1-ETHYL-CYCLOPENTANE & 0.0 & 0.00 & 0.0 & 0.0 & 0.00 & 0.0 & 0.0 & 0.00 & 0.0 & 0.0 & 0.00 & 0.00 \\
\hline 2,4,4-TRIMETHYLHEXANE & 0.0 & 0.00 & 0.0 & 0.0 & 0.00 & 0.0 & 0.0 & 0.00 & 0.0 & 0.0 & 0.00 & 0.00 \\
\hline 2,2,4-TRIMETHYLHEXANE & 0.0 & 0.00 & 0.0 & 0.0 & 0.00 & 0.0 & 0.0 & 0.00 & 0.0 & 0.0 & 0.00 & 0.00 \\
\hline TAANS-1,2-DIMETHYLCYCLOHEXANE & 0.0 & 0.00 & 0.0 & 0.0 & 0.00 & 0.0 & 0.0 & 0.00 & 0.0 & 0.0 & 0.00 & 0.00 \\
\hline
\end{tabular}


य ₹

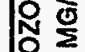

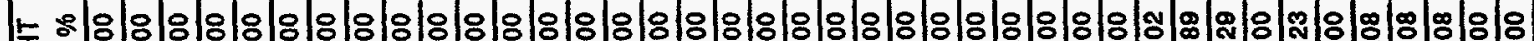
志 位崖部

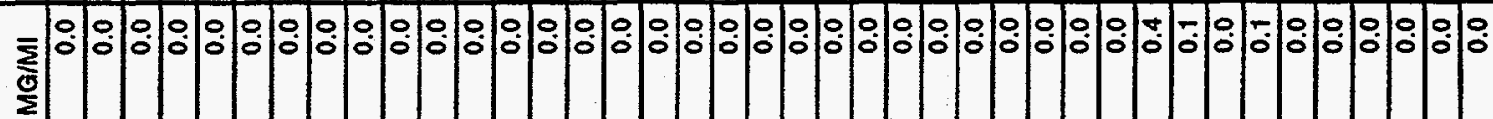

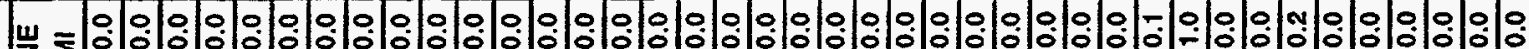
娄 领

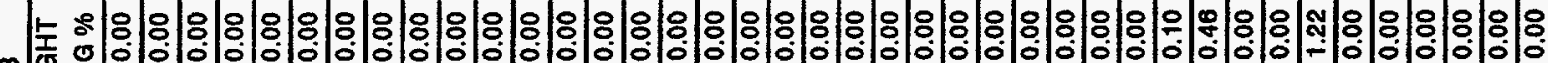
彭崖

$$
\text { 产 }
$$

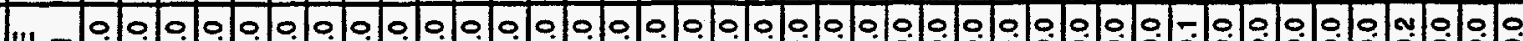

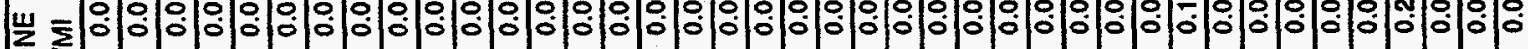
范 $\frac{0}{2}$

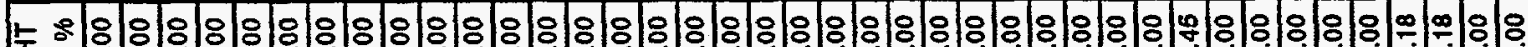
ง 这迹

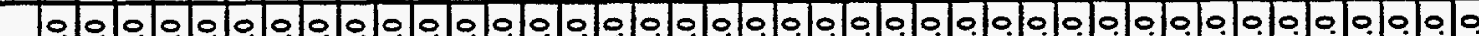

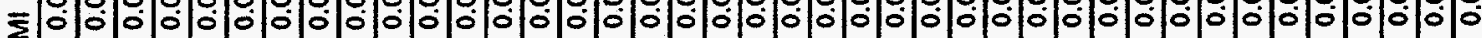
$\sum$

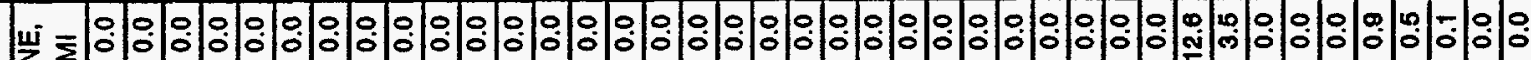

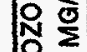

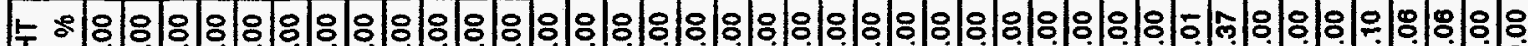
$-$

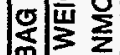

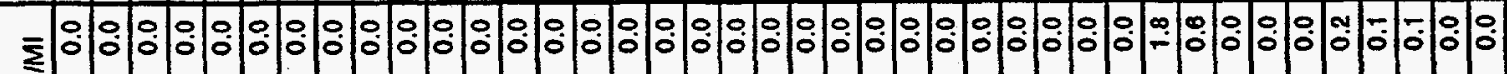

$\frac{\mathrm{O}}{2}$

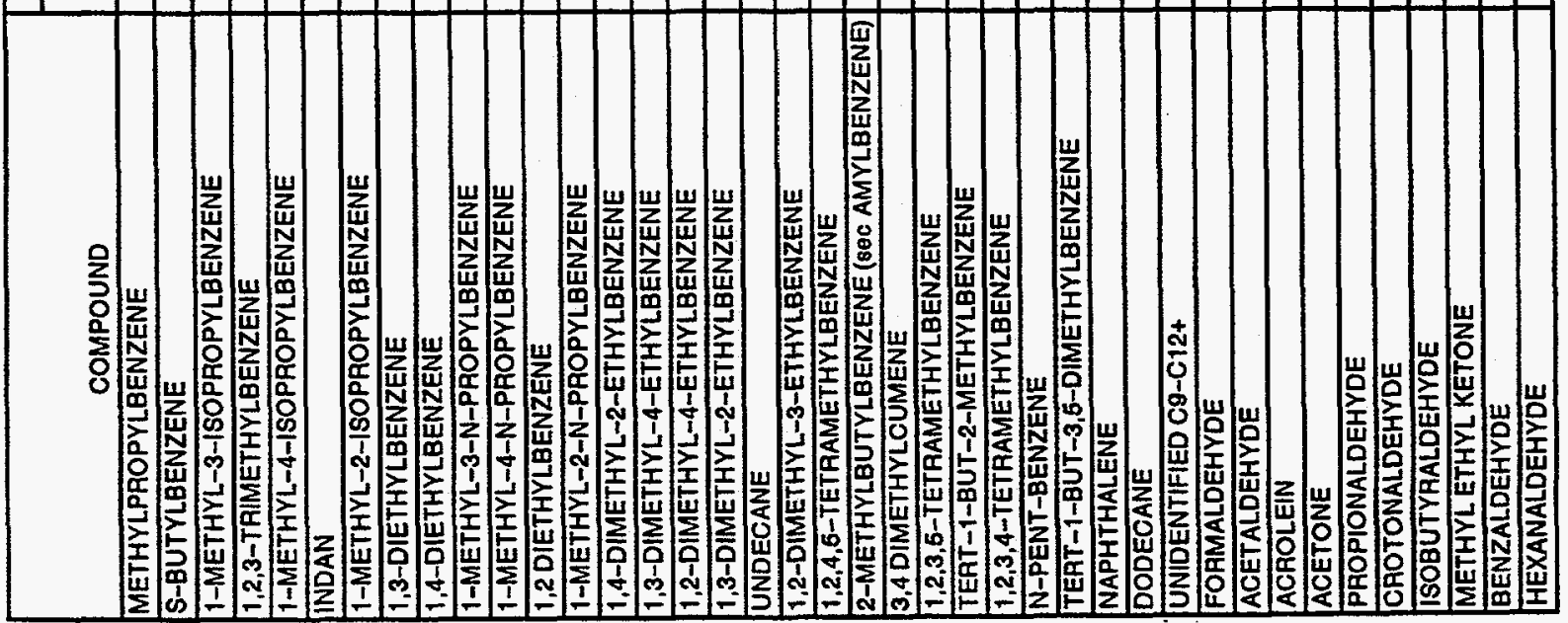




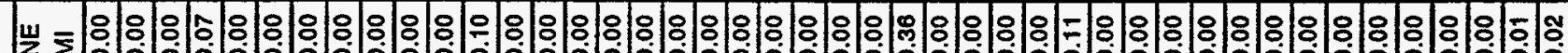
乏 ₹ 造 $\frac{0}{2}$

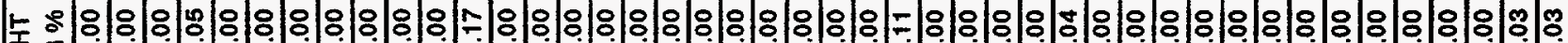

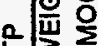

点 3

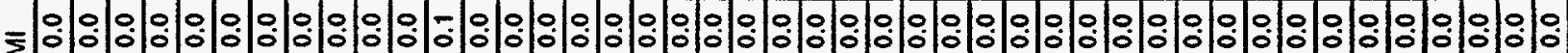

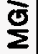

u

山 ₹ No

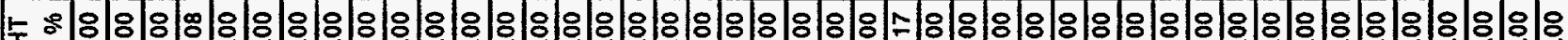
๓ I

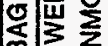

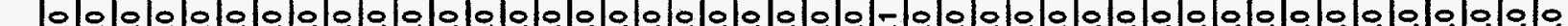
三

$\stackrel{\oplus}{\Sigma}$

山 = T్̂

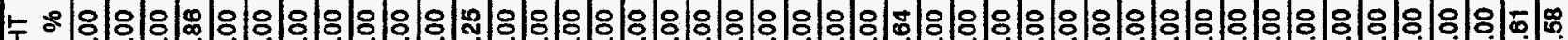
$\checkmark$ 志 एव:

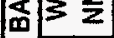

হ

$\stackrel{0}{\Sigma}$

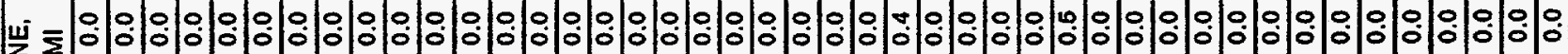
愘 N̦

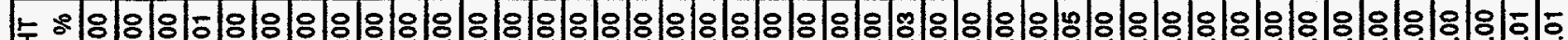

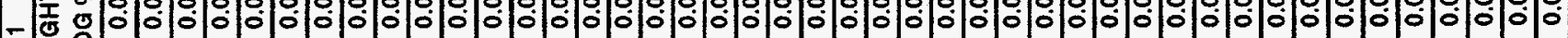
蓄鰝

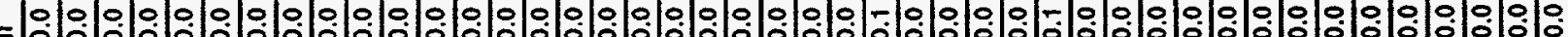

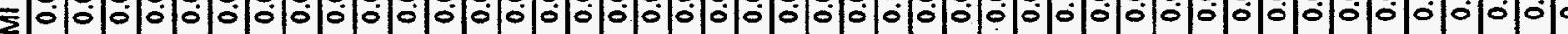
$\stackrel{\mathrm{O}}{\Sigma}$

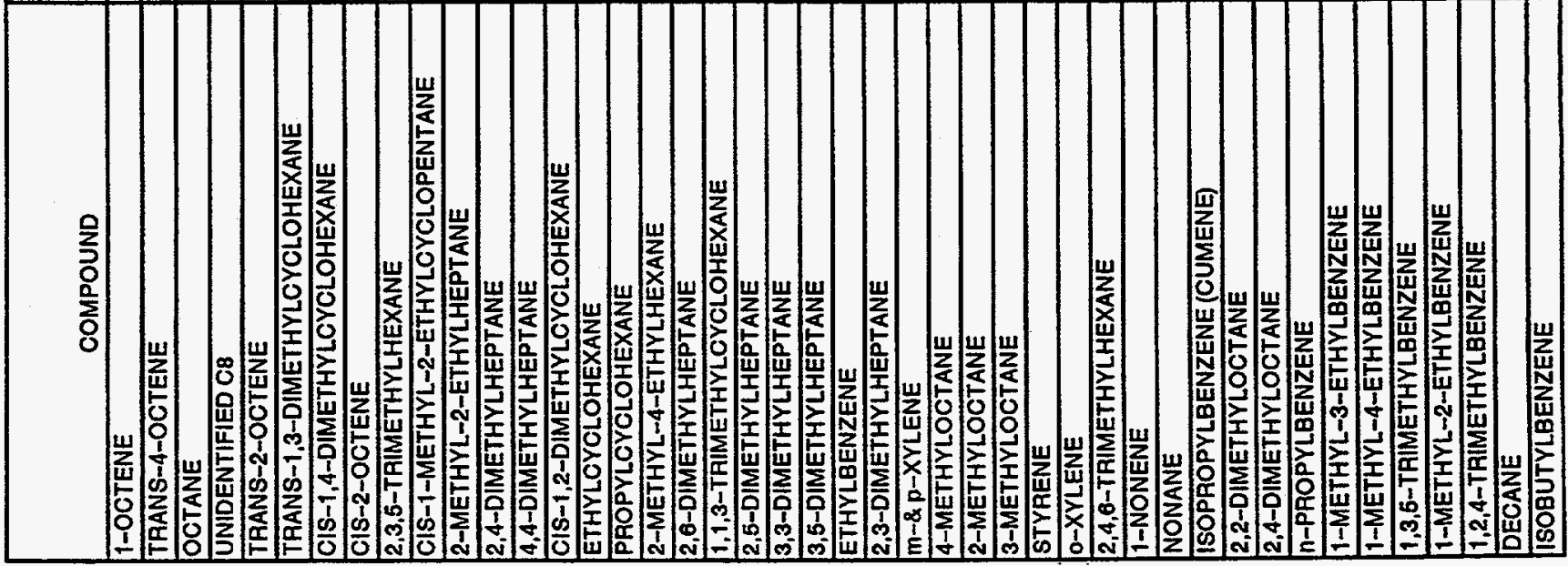


TEST NO: $60 \mathrm{NB} / 401 \mathrm{~B}-025$

TEST CAR: 1994 PONTIAC GRANDAM

TEST DATE: $9 / 13 / 95$

TEST FUEL: BUTANE

\begin{tabular}{|c|c|c|c|c|c|c|c|c|c|c|c|c|}
\hline \multirow[b]{2}{*}{ COMPOUND } & \multicolumn{3}{|c|}{$\overline{B A G 1}$} & \multicolumn{3}{|c|}{$\overline{B A G 2}$} & \multicolumn{3}{|c|}{$\overline{B A G 3}$} & \multicolumn{3}{|c|}{ FTP } \\
\hline & MG/MI & $\begin{array}{l}\text { WEIGHT } \\
\text { NMOG } \%\end{array}$ & $\begin{array}{l}\text { OZONE, } \\
\text { MG/MI }\end{array}$ & MG/MI & $\begin{array}{l}\text { WEIGHT } \\
\text { NMOG \% }\end{array}$ & $\begin{array}{l}\text { OZONE } \\
\text { MG/MI }\end{array}$ & MG/MI & $\begin{array}{l}\text { WEIGHT } \\
\text { NMOG \% }\end{array}$ & $\begin{array}{l}\text { OZONE } \\
\text { MG/MI }\end{array}$ & MG/MI & $\begin{array}{l}\text { WEIGHT } \\
\text { NMOQ } \%\end{array}$ & $\begin{array}{l}\text { OZONE } \\
\text { MG/MI }\end{array}$ \\
\hline METHANE & 26.6 & & 0.4 & 7.9 & & 0.1 & 24.3 & & 0.4 & 16.3 & & 0.24 \\
\hline ETHANE & 3.2 & 1.61 & 0.8 & 0.0 & 0.00 & 0.0 & 2.0 & 6.01 & 0.5 & 1.2 & 2.26 & 0.30 \\
\hline ETHYLENE & 14.2 & 7.18 & 103.8 & 0.0 & 0.00 & 0.0 & 1.7 & 5.01 & 12.2 & 3.4 & 6.34 & 24.91 \\
\hline PROPANE & 0.3 & 0.16 & 0.1 & 0.0 & 0.00 & 0.0 & 0.1 & 0.24 & 0.0 & 0.1 & 0.16 & 0.04 \\
\hline PROPYLENE & 8.5 & 4.30 & 80.2 & 0.0 & 0.00 & 0.0 & 0.5 & 1.64 & 5.1 & 1.9 & 3.57 & 18.08 \\
\hline ACETYLENE & 5.9 & 2.99 & 3.0 & 0.0 & 0.00 & 0.0 & 0.0 & 0.00 & 0.0 & 1.2 & 2.29 & 0.62 \\
\hline PROPADIENE & 0.0 & 0.00 & 0.0 & 0.0 & 0.00 & 0.0 & 0.0 & 0.00 & 0.0 & 0.0 & 0.00 & 0.00 \\
\hline BUTANE & 90.1 & 45.47 & 91.9 & 0.7 & 10.42 & 0.7 & 14.2 & 42.52 & 14.5 & 23.0 & 42,68 & 23.45 \\
\hline TRANS-2-BUTENE & 0.3 & 0.15 & 3.0 & 0.0 & 0.00 & 0.0 & 0.1 & 0.31 & 1.0 & 0.1 & 0.17 & 0.81 \\
\hline 1-BUTENE & 0.6 & 0.30 & 5.4 & 0.0 & 0.00 & 0.0 & 0.1 & 0.26 & 0.8 & 0.1 & 0.28 & 1.33 \\
\hline 2-METHYLPROPENE (ISOBUTYLENE) & 2.5 & 1.28 & 13.5 & 0.0 & 0.00 & 0.0 & 0.2 & 0.70 & 1.2 & 0.6 & 1.10 & 3.14 \\
\hline 2,2-DIMETHYLPROPANE (NEOPENTANE) & 0.0 & 0.00 & 0.0 & 0.0 & 0.00 & 0.0 & 0.0 & 0.00 & 0.0 & 0.0 & 0.00 & 0.00 \\
\hline PAOPYNE & 0.0 & 0.00 & 0.0 & 0.0 & 0.00 & 0.0 & 0.0 & 0.00 & 0.0 & 0.0 & 0.00 & 0.00 \\
\hline 1,3-BUTADIENE & 0.0 & 0.00 & 0.0 & 0.0 & 0.00 & 0.0 & 0.0 & 0.00 & 0.0 & 0.0 & 0.00 & 0.00 \\
\hline 2-METHYLPROPANE (ISOBUTANE) & 63.3 & 31.01 & 76.5 & 0.5 & 7.97 & 0.7 & 11.2 & 33.57 & 13.6 & 16.5 & 30.64 & 19.97 \\
\hline 1-BUTYNE & 0.0 & 0.00 & 0.0 & 0.0 & 0.00 & 0.0 & 0.0 & 0.00 & 0.0 & 0.0 & 0.00 & 0.00 \\
\hline METHANOL & 0.0 & 0.00 & 0.0 & 0.0 & 0.00 & 0.0 & 0.0 & 0.00 & 0.0 & 0.0 & 0.00 & 0.00 \\
\hline CIS-2-BUTENE & 0.1 & 0.05 & 1.0 & 0.0 & 0.00 & 0.0 & 0.0 & 0.00 & 0.0 & 0.0 & 0.04 & 0.21 \\
\hline 3-METHYL-1-BUTENE & 0.0 & 0.00 & 0.0 & 0.0 & 0.00 & 0.0 & 0.0 & 0.00 & 0.0 & 0.0 & 0.00 & 0.00 \\
\hline ETHANOL & 0.0 & 0.00 & 0.0 & 0.0 & 0.00 & 0.0 & 0.0 & 0.00 & 0.0 & 0.0 & 0.00 & 0.00 \\
\hline 2-METHYLBUTANE (ISOPENTANE) & 2.1 & 1.08 & 3.0 & 0.0 & 0.00 & 0.0 & 0.2 & 0.52 & 0.2 & 0.5 & 0.92 & 0.68 \\
\hline 2-BUTYNE & 0.0 & 0.00 & 0.0 & 0.0 & 0.00 & 0.0 & 0.0 & 0.00 & 0.0 & 0.0 & 0.00 & 0.00 \\
\hline 1-PENTENE & 0.0 & 0.00 & 0.0 & 0.0 & 0.25 & 0.1 & 0.0 & 0.00 & 0.0 & 0.0 & 0.02 & 0.06 \\
\hline 2-METHYL-1-BUTENE & 0.1 & 0.05 & 0.5 & 0.0 & 0.00 & 0.0 & 0.1 & 0.43 & 0.7 & 0.1 & 0.11 & 0.29 \\
\hline PENTANE & 0.2 & 0.11 & 0.2 & 0.3 & 4.03 & 0.3 & 0.2 & 0.53 & 0.2 & 0.2 & 0.44 & 0.25 \\
\hline UNIDENTIFIED C5 OLEFINS & 0.0 & 0.00 & 0.0 & 0.0 & 0.00 & 0.0 & 0.0 & 0.00 & 0.0 & 0.0 & 0.00 & 0.00 \\
\hline 2-METHYL-1,3-BUTADIENE & 0.0 & 0.00 & 0.0 & 0.0 & 0.00 & 0.0 & 0.0 & $1 \quad 0.00$ & 0.0 & 0.0 & 0.00 & 0.00 \\
\hline TRANS-2-PENTENE & 0.1 & 0.05 & 0.8 & 0.0 & 0.49 & 0.3 & 0.0 & 0.00 & 0.0 & 0.0 & 0.07 & 0.34 \\
\hline 3,3-DIMETHYL-1-BUTENE & 0.0 & 0.00 & 0.0 & 0.0 & 0.00 & 0.0 & 0.0 & 0.00 & 0.0 & 0.0 & 0.00 & 0.00 \\
\hline CIS-2-PENTENE & 0.0 & 0.00 & 0.0 & 0.0 & 0.00 & 0.0 & 0.0 & 0.00 & 0.0 & 0.0 & 0.00 & 0.00 \\
\hline 2-METHYL-2-BUTENE & 0.1 & 0.05 & 0.6 & 0.0 & 0.53 & 0.2 & 0.0 & 0.11 & 0.2 & 0.0 & 0.09 & 0.32 \\
\hline CYCLOPENTADIENE & 0.0 & 0.00 & 0.0 & 0.0 & 0.00 & 0.0 & 0.0 & 0.00 & 0.0 & 0.0 & 0.00 & 0.00 \\
\hline 2,2-DIMETHYLBUTANE & 0.0 & 0.00 & 0.0 & 0.1 & 2.05 & 0.1 & 0.0 & 0.00 & 0.0 & 0.1 & 0.13 & 0.06 \\
\hline CYCLOPENTENE & 0.0 & 0.00 & 0.0 & 0.0 & 0.00 & 0.0 & 0.0 & 0.00 & 0.0 & 0.0 & 0.00 & 0.00 \\
\hline 4-METHYL-1-PENTENE & 0.1 & 0.05 & $\overline{0.4}$ & 0.0 & 0.00 & 0.0 & 0.0 & 0.00 & 0.0 & 0.0 & 0.04 & 0.09 \\
\hline 3-METHYL-1-PENTENE & 0.1 & 0.05 & 0.6 & 0.0 & 0.00 & 0.0 & 0.0 & 0.00 & 0.0 & 0.0 & 0.04 & 0.10 \\
\hline CYCLOPENTANE & 0.0 & 0.00 & 0.0 & 0.0 & 0.00 & 0.0 & 0.0 & 0.00 & 0.0 & 0.0 & 0.00 & 0.00 \\
\hline 2,3-DIMETHYLBUTANE & 0.1 & 0.08 & $\overline{0.1}$ & 0.2 & 2.79 & 0.2 & 0.1 & 0.21 & 0.1 & 0.1 & 0.28 & 0.15 \\
\hline MTBE & 0.0 & 0.00 & 0.0 & 0.0 & 0.00 & 0.0 & 0.0 & 0.00 & 0.0 & 0.0 & 0.00 & 0.00 \\
\hline
\end{tabular}




\begin{tabular}{|c|c|c|c|c|c|c|c|c|c|c|c|c|}
\hline \multirow[b]{2}{*}{ COMPOUND } & \multicolumn{3}{|c|}{$\overline{\mathrm{BAG} 1}$} & \multicolumn{3}{|c|}{$\overline{B A G 2}$} & \multicolumn{3}{|c|}{ BAG 3} & \multicolumn{3}{|c|}{ FTP } \\
\hline & MG/MI & $\begin{array}{l}\text { WEIGHT } \\
\text { NMOG \% }\end{array}$ & $\begin{array}{l}\text { OZONE, } \\
\text { MG/MI }\end{array}$ & MG/MI & $\begin{array}{l}\text { WEIGHT } \\
\text { NMOG \% }\end{array}$ & $\begin{array}{l}\text { OZONE } \\
\text { MG/MI }\end{array}$ & MG/MI & $\begin{array}{l}\text { WEIGHT } \\
\text { NMOG } \%\end{array}$ & $\begin{array}{l}\text { OZONE } \\
\text { MG/MI }\end{array}$ & MG/MI & $\begin{array}{l}\text { WEIGHT } \\
\text { NMOG } \%\end{array}$ & $\begin{array}{l}\text { OZONE } \\
\text { MG/MI }\end{array}$ \\
\hline 2,3-DIMETHYL-1-BUTENE & 0.0 & 0.00 & 0.0 & $\overline{0.0}$ & 0.00 & 0.0 & 0.0 & 0.00 & 0.0 & 0.0 & 0.00 & 0.00 \\
\hline 4-METHYL-CIS-2-PENTENE & 0.0 & 0.00 & 0.0 & 0.0 & 0.00 & 0.0 & 0.0 & 0.00 & 0.0 & 0.0 & 0.00 & 0.00 \\
\hline 2-METHYLPENTANE & 0.3 & 0.13 & 0.4 & 0.6 & 9.33 & 1.0 & 0.3 & 0.87 & $\overline{0.4}$ & 0.5 & 0.85 & 0.70 \\
\hline 4-METHYL-TRANS-2-PENTENE & 0.0 & 0.00 & 0.0 & 0.0 & 0.00 & 0.0 & 0.0 & 0.00 & 0.0 & 0.0 & 0.00 & 0.00 \\
\hline 3-METHYLPENTANE & 0.2 & 0.08 & 0.2 & 0.4 & 6.12 & 0.6 & 0.2 & 0.46 & 0.2 & $\overline{0.3}$ & 0.54 & 0.44 \\
\hline 2-METHYL-1-PENTENE & 0.0 & 0.00 & 0.0 & 0.0 & 0.00 & 0.0 & 0.0 & 0.00 & 0.0 & 0.0 & 0.00 & 0.00 \\
\hline 1-HEXENE & 0.0 & 0.00 & 0.0 & 0.0 & 0.00 & 0.0 & 0.0 & 0.00 & 0.0 & 0.0 & 0.00 & 0.00 \\
\hline HEXANE & 0.2 & 0.10 & 0.2 & $\overline{0.4}$ & $\overline{5.46}$ & 0.4 & 0.1 & 0.38 & 0.1 & 0.3 & 0.50 & 0.26 \\
\hline UNIDENTIFIED C6 OLEFINS & 0.0 & 0.00 & 0.0 & 0.0 & 0.00 & 0.0 & 0.0 & 0.00 & 0.0 & 0.0 & 0.00 & 0.00 \\
\hline TRANS-3-HEXENE & 0.1 & 0.00 & 0.8 & 0.0 & 0.00 & 0.0 & 0.0 & 0.00 & 0.0 & 0.0 & 0.05 & 0.18 \\
\hline CIS-3-HEXENE & 0.1 & 0.03 & 0.4 & $\overline{0.2}$ & 2.33 & 1.1 & 0.0 & 0.00 & 0.0 & 0.1 & 0.17 & 0.63 \\
\hline TRANS-2-HEXENE & 0.0 & 0.00 & 0.0 & 0.0 & 0.00 & 0.0 & 0.0 & 0.00 & 0.0 & 0.0 & 0.00 & 0.00 \\
\hline 3-METHYL-TRANS-2-PENTENE & 0.0 & 0.00 & 0.0 & 0.0 & 0.00 & 0.0 & 0.0 & 0.00 & 0.0 & 0.0 & 0.00 & 0.00 \\
\hline 2-METHYL-2-PENTENE & 0.1 & 0.05 & 0.7 & 0.0 & 0.00 & 0.0 & 0.0 & 0.00 & 0.0 & $\overline{0.0}$ & 0.04 & $\overline{0.14}$ \\
\hline 3-METHYLCYCLOPENTENE & 0.0 & 0.00 & 0.0 & 0.0 & 0.00 & 0.0 & 0.0 & 0.00 & 0.0 & 0.0 & 0.00 & 0.00 \\
\hline CIS-2-HEXENE & 0.0 & 0.00 & 0.0 & 0.0 & 0.00 & 0.0 & 0.0 & 0.00 & 0.0 & 0.0 & 0.00 & 0.00 \\
\hline ETBE & 0.0 & 0.00 & 0.0 & 0.0 & 0.00 & 0.0 & 0.0 & 0.00 & 0.0 & 0.0 & 0.00 & 0.00 \\
\hline 3-METHYL-CIS-2-PENTENE & 0.0 & 0.00 & 0.0 & 0.0 & 0.00 & 0.0 & 0.0 & 0.00 & 0.0 & 0.0 & 0.00 & 0.00 \\
\hline 2,2-DIMETHYLPENTANE & 0.1 & 0.05 & 0.1 & 0.3 & 3.90 & 0.4 & 0.1 & 0.35 & 0.2 & 0.2 & 0.36 & 0.27 \\
\hline METHYLCYCLOPENTANE & 0.0 & 0.00 & 0.0 & 0.0 & 0.00 & 0.0 & 0.0 & 0.00 & 0.0 & 0.0 & 0.00 & 0.00 \\
\hline 2,4-DIMETHYLPENTANE & 0.1 & 0.07 & 0.3 & 0.2 & 2.59 & 0.3 & 0.1 & 0.25 & 0.1 & 0.1 & 0.27 & 0.26 \\
\hline 2,3,3-TRIMETHYL-1-BUTENE & 0.0 & 0.00 & 0.0 & 0.0 & 0.00 & 0.0 & 0.0 & 0.00 & 0.0 & 0.0 & 0.00 & 0.00 \\
\hline 2,2,3-TRIMETHYLBUTANE & 0.0 & 0.01 & 0.0 & 0.1 & 1.18 & 0.1 & 0.0 & 0.12 & 0.1 & 0.1 & 0.11 & 0.08 \\
\hline 3,4-DIMETHYL-1-PENTENE & 0.0 & 0.00 & 0.0 & 0.0 & 0.00 & 0.0 & 0.0 & 0.00 & 0.0 & 0.0 & 0.00 & 0.00 \\
\hline 1-METHYLCYCLOPENTENE & 0.1 & 0.05 & 0.6 & 0.0 & 0.00 & 0.0 & 0.0 & 0.04 & 0.1 & 0.0 & 0.05 & 0.14 \\
\hline BENZENE & 0.2 & 0.10 & 0.1 & 0.3 & 4.55 & 0.1 & 0.3 & 0.92 & $\overline{0.1}$ & 0.3 & 0.53 & $\overline{0.12}$ \\
\hline 3-METHYL-1-HEXENE & 0.0 & 0.00 & 0.0 & $\overline{0.0}$ & 0.00 & 0.0 & 0.0 & 0.00 & 0.0 & 0.0 & 0.00 & 0.00 \\
\hline 3,3-DIMETHYLPENTANE & 0.0 & 0.00 & 0.0 & 0.2 & 2.33 & 0.1 & 0.0 & 0.00 & 0.0 & $\overline{0.1}$ & 0.16 & 0.08 \\
\hline CYCLOHEXANE & 0.0 & 0.00 & 0.0 & 0.0 & 0.00 & 0.0 & 0.0 & 0.00 & 0.0 & 0.0 & 0.00 & 0.00 \\
\hline 2-METHYLHEXANE & 0.0 & 0.00 & 0.0 & 0.0 & 0.00 & 0.0 & 0.0 & 0.00 & 0.0 & 0.0 & 0.00 & 0.00 \\
\hline 2,3-DIMETHYLPENTANE & 0.3 & 0.13 & 0.4 & 0.5 & 6.65 & 0.7 & 0.1 & 0.34 & 0.2 & 0.3 & 0.59 & 0.48 \\
\hline 1,1-DIMETHYLCYCLOPENTANE & 0.0 & 0.00 & 0.0 & 0.0 & 0.00 & 0.0 & 0.0 & 0.00 & 0.0 & 0.0 & 0.00 & 0.00 \\
\hline CYCLOHEXENE & 0.0 & 0.00 & 0.0 & 0.0 & 0.00 & 0.0 & 0.0 & 0.00 & 0.0 & 0.0 & 0.00 & 0.00 \\
\hline 3-METHYLHEXANE & 0.1 & 0.04 & 0.1 & 0.1 & 1.54 & 0.1 & 0.0 & 0.03 & 0.0 & 0.1 & 0.14 & 0.10 \\
\hline CIS-1,3-DIMETHYLCYCLOPENTANE & 0.0 & 0.01 & 0.0 & 0.0 & 0.00 & 0.0 & 0.0 & 0.05 & 0.0 & 0.0 & 0.01 & 0.02 \\
\hline 3-ETHYLPENTANE & 0.0 & 0.00 & $\overline{0.0}$ & 0.0 & 0.00 & 0.0 & 0.0 & 0.00 & $\overline{0.0}$ & 0.0 & 0.00 & 0.00 \\
\hline TRANS-1,2-DIMETHYLCYCLOPENTANE & 0.0 & 0.00 & 0.0 & 0.0 & 0.00 & 0.0 & 0.0 & 0.00 & 0.0 & 0.0 & 0.00 & 0.00 \\
\hline TRANS-1,3-DIMETHYLCYCLOPENTANE & 0.0 & 0.00 & 0.0 & 0.0 & 0.00 & 0.0 & 0.0 & 0.00 & 0.0 & 0.0 & 0.00 & 0.00 \\
\hline 1-HEPTENE & 0.0 & 0.00 & 0.0 & 0.0 & 0.00 & 0.0 & 0.0 & 0.00 & 0.0 & 0.0 & 0.00 & 0.00 \\
\hline 2,2,4-TRIMETHYLPENTANE & 0.2 & 0.10 & 0.2 & 0.3 & 3.82 & 0.2 & 0.1 & 0.36 & 0.1 & 0.2 & 0.39 & 0.20 \\
\hline 2-METHYL-1-HEXENE & 0.0 & 0.00 & 0.0 & 0.0 & 0.00 & 0.0 & 0.0 & 0.00 & 0.0 & 0.0 & 0.00 & 0.00 \\
\hline TRANS-3-HEPTENE & 0.0 & 0.00 & 0.0 & 0.0 & 0.00 & 0.0 & 0.0 & 0.00 & 0.0 & 0.0 & 0.00 & 0.00 \\
\hline HEPTANE & 0.0 & 0.00 & 0.0 & 0.1 & 1.43 & 0.1 & 0.1 & 0.16 & 0.0 & 0.1 & 0.12 & 0.05 \\
\hline UNIDENTIFIED C7 & 0.1 & 0.06 & 0.5 & 0.1 & 1.49 & 0.6 & 0.1 & 0.27 & 0.4 & 0.1 & 0.19 & 0.46 \\
\hline
\end{tabular}




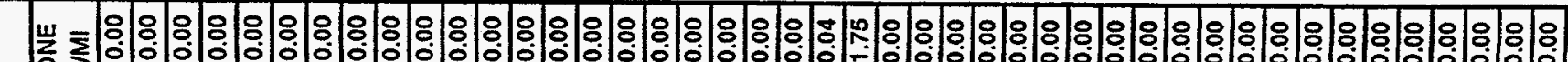
兑

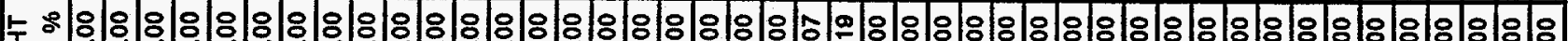
a

吾要要

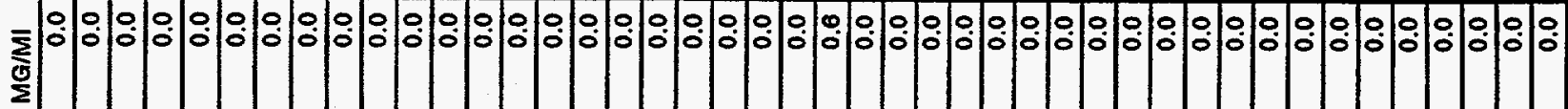

य ₹ ON

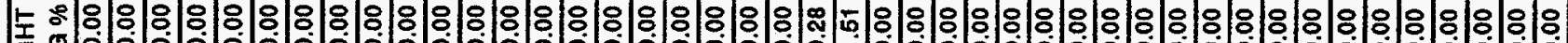

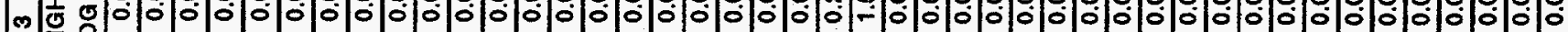
客岗

乏 $\stackrel{\mathcal{O}}{\Sigma}$

L 号

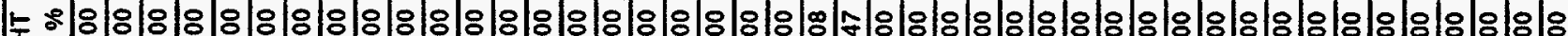

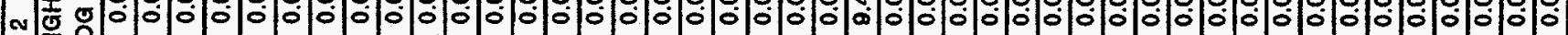
安

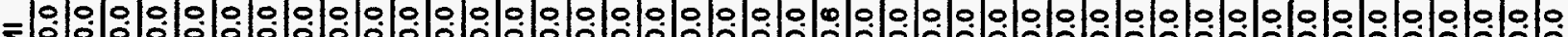
三 $\stackrel{\mathcal{S}}{\Sigma}$

山 엉

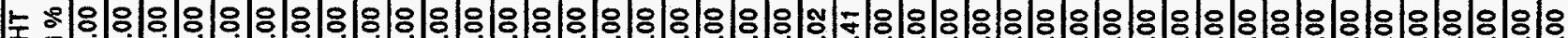
$-0$ 容岕

इ 을

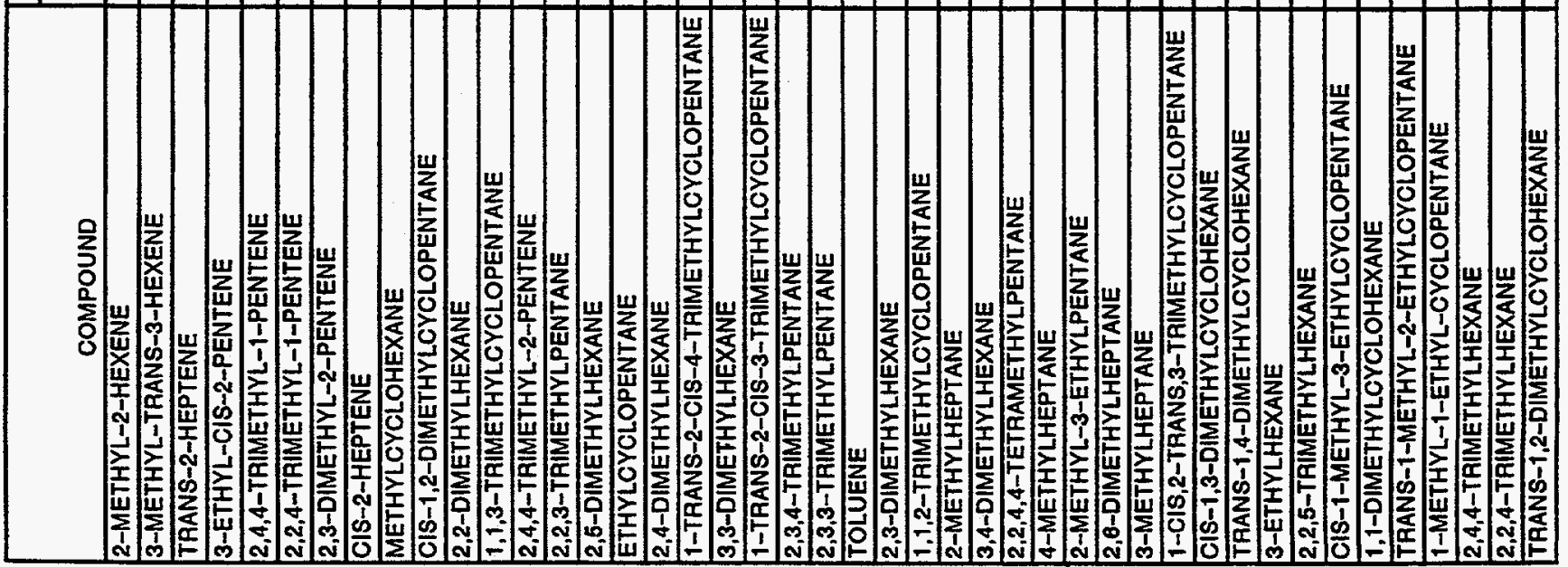




\begin{tabular}{|c|c|c|c|c|c|c|c|c|c|c|c|c|}
\hline \multirow[b]{2}{*}{ COMPOUND } & \multicolumn{3}{|c|}{ BAG 1} & \multicolumn{3}{|c|}{$\overline{B A G 2}$} & \multicolumn{3}{|c|}{ BAG 3} & \multicolumn{3}{|c|}{ FTP } \\
\hline & MG/MI & $\begin{array}{l}\text { WEIGHT } \\
\text { NMOG \% }\end{array}$ & $\begin{array}{l}\text { OZONE, } \\
\text { MG/MI }\end{array}$ & MG/MI & $\begin{array}{l}\text { WEIGHT } \\
\text { NMOG } \%\end{array}$ & $\begin{array}{l}\text { OZONE } \\
\text { MG/MI }\end{array}$ & MG/MI & $\begin{array}{l}\text { WEIGHT } \\
\text { NMOG } \%\end{array}$ & $\begin{array}{l}\text { OZONE } \\
\text { MG/MI }\end{array}$ & MG/MI & $\begin{array}{l}\text { WEIGHT } \\
\text { NMOG } \%\end{array}$ & $\begin{array}{l}\text { OZONE } \\
\text { MG/MI }\end{array}$ \\
\hline 1-OCTENE & 0.0 & 0.00 & 0.0 & 0.0 & 0.00 & 0.0 & 0.0 & 0.00 & 0.0 & 0.0 & 0.00 & 0.00 \\
\hline TRANS-4-OCTENE & 0.0 & 0.00 & 0.0 & 0.0 & 0.00 & 0.0 & 0.0 & 0.00 & 0.0 & 0.0 & 0.00 & 0.00 \\
\hline OCTANE & 0.0 & 0.00 & 0.0 & 0.0 & 0.00 & 0.0 & 0.0 & 0.00 & 0.0 & 0.0 & 0.00 & 0.00 \\
\hline UNIDENTIFIED CB & 0.0 & 0.00 & 0.0 & 0.1 & 1.27 & 0.3 & 0.0 & 0.06 & 0.1 & 0.0 & 0.09 & 0.17 \\
\hline TRANS-2-OCTENE & 0.0 & 0.00 & 0.0 & 0.0 & 0.00 & 0.0 & 0.0 & 0.00 & 0.0 & 0.0 & 0.00 & 0.00 \\
\hline TRANS-1,3-DIMETHYLCYCLOHEXANE & 0.0 & 0.00 & 0.0 & 0.0 & 0.00 & 0.0 & 0.0 & 0.00 & 0.0 & 0.0 & 0.00 & 0.00 \\
\hline CIS-1,4-DIMETHYLCYCLOHEXANE & 0.0 & 0.00 & 0.0 & 0.0 & 0.00 & 0.0 & 0.0 & 0.00 & 0.0 & 0.0 & 0.00 & 0.00 \\
\hline CIS-2-OCTENE & 0.0 & 0.00 & 0.0 & 0.0 & 0.00 & 0.0 & 0.0 & 0.00 & 0.0 & 0.0 & 0.00 & 0.00 \\
\hline 2,3,5-TAIMETHYLHEXANE & 0.0 & 0.00 & 0.0 & 0.0 & 0.00 & 0.0 & 0.0 & 0.00 & 0.0 & 0.0 & 0.00 & 0.00 \\
\hline CIS-1-METHYL-2-ETHYLCYCLOPENTANE & 0.0 & 0.00 & 0.0 & 0.0 & 0.00 & 0.0 & 0.0 & 0.00 & 0.0 & 0.0 & 0.00 & 0.00 \\
\hline 2-METHYL-2-ETHYLHEPTANE & 0.0 & 0.00 & 0.0 & 0.0 & 0.00 & 0.0 & 0.0 & 0.00 & 0.0 & 0.0 & 0.00 & 0.00 \\
\hline 2,4-DIMETHYLHEPTANE & 0.0 & 0.00 & 0.0 & 0.2 & 2.57 & 0.2 & 0.0 & 0.00 & 0.0 & 0.1 & 0.17 & 0.12 \\
\hline 4,4-DIMETHYLHEPTANE & 0.0 & 0.00 & 0.0 & 0.0 & 0.00 & 0.0 & 0.0 & 0.00 & 0.0 & 0.0 & 0.00 & 0.00 \\
\hline CIS-1,2-DIMETHYLCYCLOHEXANE & 0.0 & 0.00 & 0.0 & 0.0 & 0.00 & 0.0 & 0.0 & 0.00 & 0.0 & 0.0 & 0.00 & 0.00 \\
\hline ETHYLCYCLOHEXANE & 0.0 & 0.00 & 0.0 & 0.0 & 0.00 & $\overline{0.0}$ & 0.0 & 0.00 & 0.0 & 0.0 & 0.00 & 0.00 \\
\hline PROPYLCYCLOHEXANE & 0.0 & 0.00 & 0.0 & 0.0 & 0.00 & 0.0 & 0.0 & 0.00 & 0.0 & 0.0 & 0.00 & 0.00 \\
\hline 2-METHYL-4-ETHYLHEXANE & 0.0 & 0.00 & 0.0 & 0.0 & 0.00 & 0.0 & 0.0 & 0.00 & 0.0 & 0.0 & 0.00 & 0.00 \\
\hline 2,6-DIMETHYLHEPTANE & 0.0 & 0.00 & 0.0 & 0.0 & 0.00 & 0.0 & 0.0 & 0.00 & 0.0 & 0.0 & 0.00 & 0.00 \\
\hline 1,1,3-TRIMETHYLCYCLOHEXANE & 0.0 & 0.00 & 0.0 & 0.0 & 0.00 & 0.0 & 0.0 & 0.00 & 0.0 & 0.0 & 0.00 & 0.00 \\
\hline 2,5-DIMETHYLHEPTANE & 0.0 & 0.00 & 0.0 & 0.0 & 0.00 & 0.0 & 0.0 & 0.00 & 0.0 & 0.0 & 0.00 & 0.00 \\
\hline 3,3-DIMETHYLHEPTANE & 0.0 & 0.00 & 0.0 & 0.0 & 0.00 & 0.0 & 0.0 & 0.00 & 0.0 & 0.0 & 0.00 & 0.00 \\
\hline 3.5-DIMETHYLHEPTANE & 0.0 & 0.00 & 0.0 & 0.0 & 0.00 & 0.0 & 0.0 & 0.00 & 0.0 & 0.0 & 0.00 & 0.00 \\
\hline ETHYLBENZENE & 0.0 & 0.00 & 0.0 & 0.0 & 0.00 & 0.0 & 0.0 & 0.00 & 0.0 & 0.0 & 0.00 & 0.00 \\
\hline 2,3-DIMETHYLHEPTANE & 0.0 & 0.00 & 0.0 & 0.0 & 0.00 & 0.0 & 0.0 & 0.00 & 0.0 & 0.0 & 0.00 & 0.00 \\
\hline$m-\& p-X Y L E N E$ & 0.1 & 0.04 & 0.6 & 0.0 & 0.64 & 0.3 & 0.0 & 0.00 & 0.0 & 0.0 & 0.07 & 0.20 \\
\hline 4-METHYLOCTANE & 0.0 & 0.00 & 0.0 & 0.0 & 0.00 & 0.0 & 0.0 & 0.00 & 0.0 & 0.0 & 0.00 & 0.00 \\
\hline 2-METHYLOCTANE & 0.0 & 0.00 & 0.0 & 0.0 & 0.00 & 0.0 & 0.0 & 0.00 & 0.0 & 0.0 & 0.00 & 0.00 \\
\hline 3-METHYLOCTANE & 0.0 & 0.00 & 0.0 & 0.0 & 0.00 & 0.0 & 0.0 & 0.00 & 0.0 & 0.0 & 0.00 & 0.00 \\
\hline STYRENE & 0.0 & 0.00 & 0.0 & 0.0 & 0.00 & 0.0 & 0.0 & 0.00 & 0.0 & 0.0 & 0.00 & 0.00 \\
\hline O-XYLENE & 0.0 & 0.00 & 0.0 & 0.0 & 0.00 & 0.0 & 0.0 & 0.03 & 0.1 & 0.0 & 0.00 & 0.02 \\
\hline 2,4,6-TRIMETHYLHEXANE & 0.0 & 0.00 & 0.0 & 0.0 & 0.00 & 0.0 & 0.0 & 0.00 & 0.0 & 0.0 & 0.00 & 0.00 \\
\hline 1-NONENE & 0.0 & 0.00 & 0.0 & 0.0 & 0.00 & 0.0 & 0.0 & 0.00 & 0.0 & 0.0 & 0.00 & 0.00 \\
\hline NONANE & 0.0 & 0.00 & 0.0 & 0.0 & 0.00 & 0.0 & 0.0 & 0.00 & 0.0 & 0.0 & 0.00 & 0.00 \\
\hline ISOPROPYLBENZENE(CUMENE) & 0.0 & 0.00 & 0.0 & 0.0 & 0.00 & 0.0 & 0.0 & 0.00 & 0.0 & 0.0 & 0.00 & 0.00 \\
\hline 2,2-DIMETHYLOCTANE & 0.0 & 0.00 & 0.0 & 0.0 & 0.00 & 0.0 & 0.0 & 0.00 & 0.0 & 0.0 & 0.00 & 0.00 \\
\hline 2,4-DIMETHYLOCTANE & 0.0 & 0.00 & 0.0 & 0.0 & 0.00 & 0.0 & 0.0 & 0.00 & 0.0 & $\overline{0.0}$ & 0.00 & 0.00 \\
\hline n-PROPYLBENZENE & 0.0 & 0.00 & 0.0 & 0.0 & 0.00 & 0.0 & 0.0 & 0.00 & 0.0 & 0.0 & 0.00 & 0.00 \\
\hline 1-METHYL-3-ETHYLBENZENE & 0.0 & 0.00 & 0.0 & 0.0 & 0.00 & 0.0 & 0.0 & 0.00 & 0.0 & $\overline{0.0}$ & 0.00 & 0.00 \\
\hline 1-METHYL-4-ETHYLBENZENE & 0.0 & 0.00 & 0.0 & 0.0 & 0.00 & 0.0 & 0.0 & 0.00 & 0.0 & 0.0 & 0.00 & 0.00 \\
\hline 1,3,5-TRIMETHYLBENZENE & 0.0 & 0.00 & 0.0 & 0.0 & 0.00 & 0.0 & 0.0 & 0.00 & 0.0 & 0.0 & 0.00 & 0.00 \\
\hline 1-METHYL-2-ETHYLBENZENE & 0.0 & 0.00 & 0.0 & 0.0 & 0.00 & 0.0 & 0.0 & 0.00 & 0.0 & 0.0 & 0.00 & 0.00 \\
\hline 1,2,4-TRIMETHYLBENZENE & 0.0 & 0.00 & 0.0 & 0.0 & 0.00 & 0.0 & 0.0 & 0.00 & 0.0 & 0.0 & 0.00 & 0.00 \\
\hline DECANE & 0.0 & 0.00 & 0.0 & 0.1 & 0.79 & 0.0 & 0.0 & 0.00 & 0.0 & 0.0 & 0.05 & 0.01 \\
\hline ISOBUTYLBENZENE & 0.0 & 0.00 & 0.0 & 0.1 & 0.75 & 0.1 & 0.0 & 0.00 & 0.0 & 0.0 & 0.05 & 0.05 \\
\hline
\end{tabular}




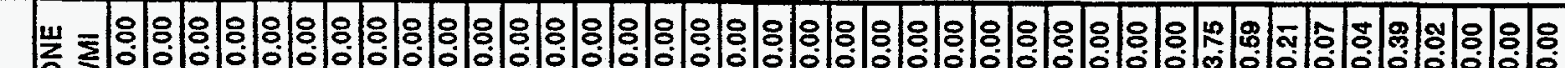
芯

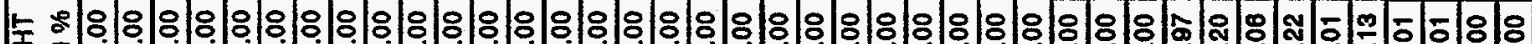
a

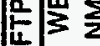

₹

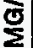

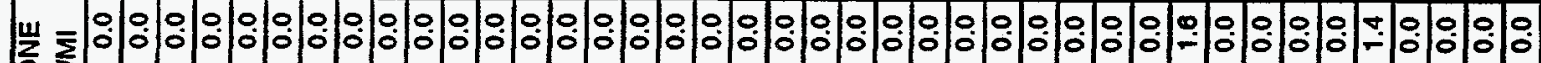
依

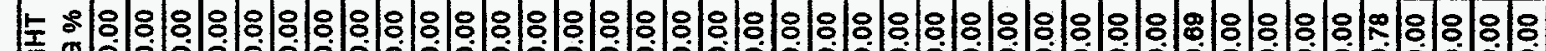
o) 安要 $\sum_{z}$

इ 을

щ ₹ 员

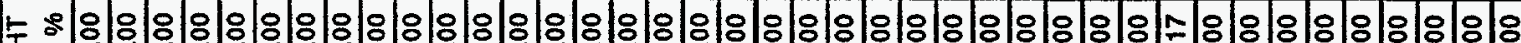
、志 安䎡

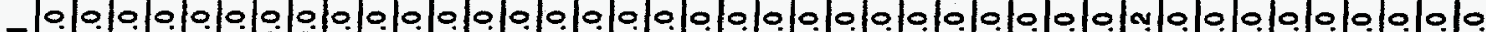
三 $\stackrel{0}{2}$

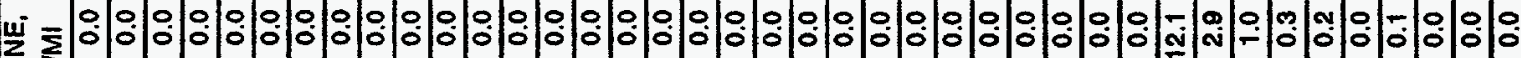
No $\frac{0}{\Sigma}$

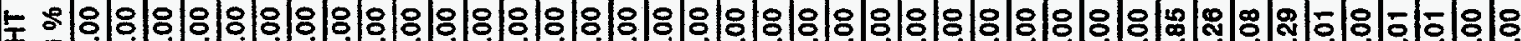
$-\underline{0}$

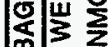

= $\stackrel{0}{\Sigma}$
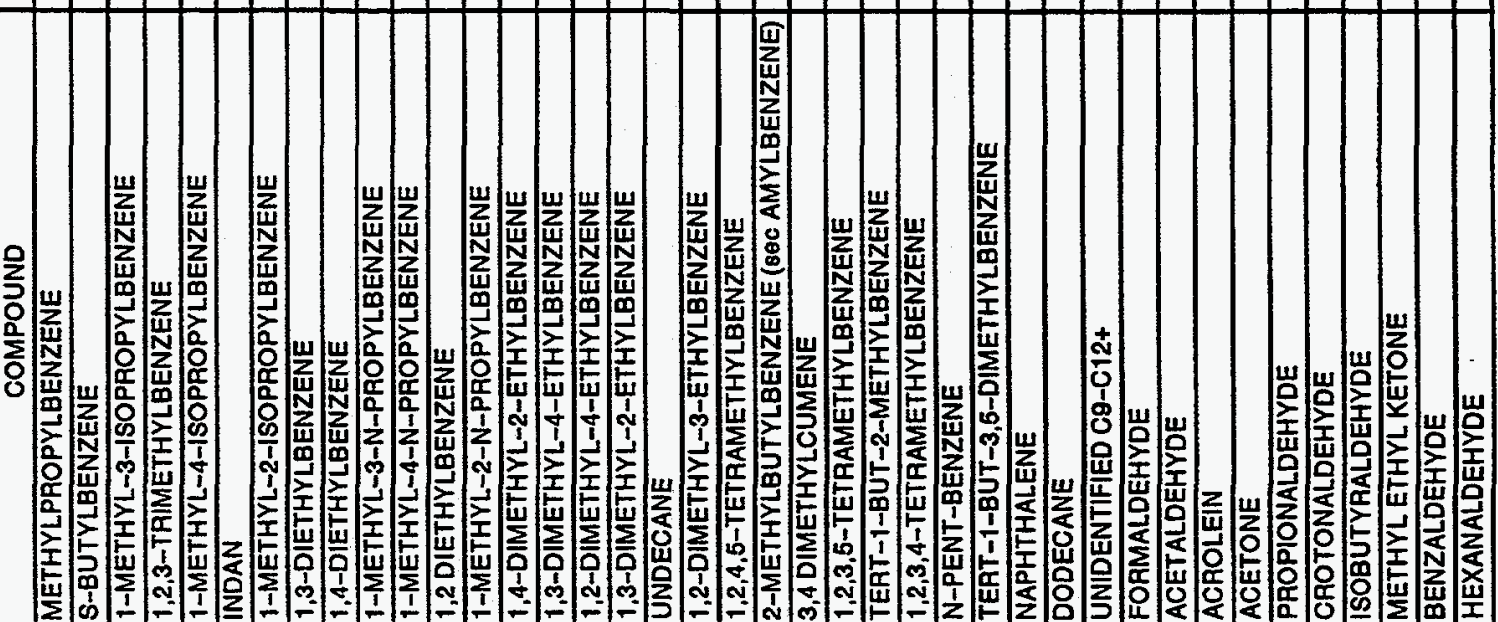
TEST NO: 80NB/20NP-02S

TEST CAR: 1994 PONTIAC GRANDAM

TEST DATE: 9/22/95

TEST FUEL: PROPANE

\begin{tabular}{|c|c|c|c|c|c|c|c|c|c|c|c|c|}
\hline \multirow[b]{2}{*}{ COMPOUND } & \multicolumn{3}{|c|}{$\overline{B A Q 1}$} & \multicolumn{3}{|c|}{$\overline{B A G 2}$} & \multicolumn{3}{|c|}{$\overline{B A G 3}$} & \multicolumn{3}{|c|}{ FTP } \\
\hline & Ma/MI & $\begin{array}{l}\text { WEIGHT } \\
\text { NMOG } \%\end{array}$ & $\begin{array}{l}\text { OZONE, } \\
\text { MG/MI }\end{array}$ & MG/MI & $\begin{array}{l}\text { WEIGHT } \\
\text { NMOG } \%\end{array}$ & $\begin{array}{l}\text { OZONE } \\
\text { MG/MI }\end{array}$ & Ma/MI & $\begin{array}{l}\text { WEIGHT } \\
\text { NMOG \% }\end{array}$ & $\begin{array}{l}\text { OZONE } \\
\text { MGMMI }\end{array}$ & MGIMI & $\begin{array}{l}\text { WEIGHT } \\
\text { NMOQ } \%\end{array}$ & $\begin{array}{l}\text { OZONE } \\
\text { MG/MI }\end{array}$ \\
\hline METHANE & $\overline{18.1}$ & & 0.3 & 1.5 & & 0.0 & 42.3 & & 0.6 & 16.2 & & $\overline{0.24}$ \\
\hline ETHANE & 2.5 & 1.21 & 0.6 & 0.0 & 1.91 & 0.0 & 10.7 & 20.72 & 2.7 & 3.5 & 5.96 & 0.87 \\
\hline ETHYLENE & $\overline{14.3}$ & 6.87 & 103.9 & 0.1 & 6.57 & 1.1 & 4.8 & 9.49 & 35.8 & 4.4 & 7.50 & 31.97 \\
\hline PROPANE & 68.9 & 33.24 & 33.1 & 0.1 & 2.47 & 0.0 & 11.9 & 23.05 & $\overline{5.7}$ & 17.6 & 30.16 & 8.46 \\
\hline PROPYLENE & $\overline{6.8}$ & 3.28 & 63.9 & 0.0 & 0.00 & 0.0 & 1.3 & 2.47 & 11.9 & 1.8 & 3.02 & 16.57 \\
\hline ACETYLENE & 3.5 & 1.68 & 1.7 & 0.0 & 0.00 & 0.0 & 0.0 & 0.00 & 0.0 & 0.7 & 1.24 & 0.36 \\
\hline PROPADIENE & 0.0 & 0.00 & 0.0 & 0.0 & 0.00 & 0.0 & 0.0 & 0.00 & 0.0 & 0.0 & 0.00 & 0.00 \\
\hline BUTANE & 101.0 & 48.68 & 103.0 & 0.2 & 8.49 & 0,2 & 18.3 & 35.60 & 18.7 & 26.1 & 44.72 & 28.68 \\
\hline TRANS-2-BUTENE & 0.2 & 0.11 & 2.3 & 0.0 & 0.00 & 0.0 & 0.0 & 0.00 & 0.0 & 0.0 & 0.08 & 0.48 \\
\hline 1-BUTENE & 0.6 & 0.22 & 4.1 & 0.0 & 0.00 & 0.0 & 0.2 & 0.43 & 2.0 & 0.2 & 0.27 & 1.40 \\
\hline 2-METHYLPROPENE (ISOBUTYLENE) & $\overline{0.1}$ & 0.03 & 0.3 & 0.0 & 0.00 & 0.0 & 0.2 & 0.37 & 1.0 & 0.1 & 0.11 & 0.34 \\
\hline 2,2-DIMETHYLPROPANE (NEOPENTANE) & 0.0 & 0.00 & 0.0 & 0.0 & 0.00 & 0.0 & 0.0 & 0.00 & 0.0 & 0.0 & 0.00 & 0.00 \\
\hline PROPYNE & 0.0 & 0.00 & 0.0 & 0.0 & 0.00 & 0.0 & 0.0 & 0.00 & 0.0 & 0.0 & 0.00 & 0.00 \\
\hline 1,3-BUTADIENE & 0.1 & 0.05 & 1.1 & 0.0 & 0.00 & 0.0 & 0.0 & 0.00 & 0.0 & 0.0 & 0.03 & 0.22 \\
\hline 2-METHYLPROPANE (ISOBUTANE) & 0.3 & 0.15 & 0.4 & 0.0 & 0.18 & 0.0 & 0.1 & 0.14 & 0.1 & 0.1 & 0.15 & 0.10 \\
\hline 1-BUTYNE & 0.0 & 0.00 & 0.0 & 0.0 & 0.00 & 0.0 & 0.0 & 0.00 & 0.0 & 0.0 & 0.00 & 0.00 \\
\hline METHANOL & 0.0 & 0.00 & 0.0 & 0.0 & 0.00 & 0.0 & 0.0 & 0.00 & 0.0 & 0.0 & 0.00 & 0.00 \\
\hline CIS-2-BUTENE & 0.0 & 0.00 & 0.0 & 0.0 & 0.00 & 0.0 & 0.0 & 0.00 & 0.0 & 0.0 & 0.00 & 0.00 \\
\hline 3-METHYL-1-BUTENE & 0.0 & 0.00 & 0.0 & 0.0 & 0.00 & 0.0 & 0.0 & 0.00 & 0.0 & 0.0 & 0.00 & 0.00 \\
\hline ETHANOL & 0.0 & 0.00 & 0.0 & 0.0 & 0.00 & 0.0 & 0.0 & 0.00 & 0.0 & 0.0 & 0.00 & 0.00 \\
\hline 2-METHYLBUTANE (ISOPENTANE) & 2.9 & 1.39 & 4.0 & 0.3 & 14.21 & 0.4 & 1.6 & 3.10 & 2.2 & 1.2 & 2.05 & 1.65 \\
\hline 2-BUTYNE & 0.0 & 0.00 & 0.0 & 0.0 & 0.00 & 0.0 & 0.0 & 0.00 & 0.0 & 0.0 & 0.00 & 0.00 \\
\hline 1-PENTENE & 0.0 & 0.00 & 0.0 & 0.0 & 0.00 & 0.0 & 0.0 & 0.00 & 0.0 & 0.0 & 0.00 & 0.00 \\
\hline 2-METHYL-1-BUTENE & 0.0 & 0.00 & 0.0 & 0.0 & 0.00 & 0.0 & 0.0 & 0.00 & 0.0 & 0.0 & 0.00 & 0.00 \\
\hline PENTANE & $\overline{0.3}$ & 0.14 & 0.3 & 0.1 & 2.98 & $\overline{0.1}$ & 0.2 & 0.32 & 0.2 & 0.1 & 0.24 & 0.14 \\
\hline UNIDENTIFIED C5 OLEFINS & 0.0 & 0.00 & 0.0 & 0.0 & 0.00 & 0.0 & 0.0 & 0.00 & 0.0 & 0.0 & 0.00 & 0.00 \\
\hline 2-METHYL-1,3-8UTADIENE & $\overline{0.0}$ & 0.00 & 0.0 & 0.0 & 0.00 & 0.0 & 0.0 & 0.00 & 0.0 & 0.0 & 0.00 & 0.00 \\
\hline TRANS-2-PENTENE & 0.0 & 0.00 & 0.0 & 0.0 & 0.00 & 0.0 & 0.0 & 0.00 & 0.0 & 0.0 & 0.00 & 0.00 \\
\hline 3,3-DIMETHYL-1-BUTENE & 0.0 & 0.00 & 0.0 & 0.0 & 0.00 & 0.0 & 0.0 & 0.00 & 0.0 & 0.0 & 0.00 & 0.00 \\
\hline CIS-2-PENTENE & 0.0 & 0.00 & 0.0 & 0.0 & 0.00 & 0.0 & 0.0 & 0.00 & 0.0 & 0.0 & 0.00 & 0.00 \\
\hline 2-METHYL-2-BUTENE & 0.1 & 0.04 & 0.6 & 0.0 & 1.57 & 0.2 & 0.0 & 0.00 & 0.0 & 0.0 & 0.06 & 0.23 \\
\hline CYCLOPENTADIENE & 0.0 & 0.00 & 0.0 & 0.0 & 0.00 & 0.0 & 0.0 & 0.00 & 0.0 & 0.0 & 0.00 & $\overline{0.00}$ \\
\hline 2,2-DIMETHYLBUTANE & 0.1 & 0.05 & 0.1 & 0.0 & 0.00 & 0.0 & 0.0 & 0.00 & 0.0 & 0.0 & 0.04 & 0.02 \\
\hline CYCLOPENTENE & $\overline{0.0}$ & 0.00 & 0.0 & 0.0 & 0.00 & 0.0 & 0.0 & 0.00 & 0.0 & 0.0 & 0.00 & 0.00 \\
\hline 4-METHYL-1-PENTENE & 0.0 & 0.00 & 0.0 & 0.0 & 0.00 & 0.0 & 0.0 & 0.00 & 0.0 & 0.0 & 0.00 & 0.00 \\
\hline 3-METHYL-1-PENTENE & 0.0 & 0.00 & 0.0 & 0.0 & 0.00 & 0.0 & 0.0 & 0.00 & 0.0 & 0.0 & 0.00 & 0.00 \\
\hline CYCLOPENTANE & 0.0 & 0.00 & 0.0 & 0.0 & 0.00 & 0.0 & 0.0 & 0.00 & 0.0 & 0.0 & 0.00 & 0.00 \\
\hline 2,3-DIMETHYLBUTANE & 0.1 & 0.07 & 0.2 & 0.1 & 3.07 & 0.1 & 0.1 & 0.24 & 0.1 & 0.1 & 0.17 & $\overline{0.11}$ \\
\hline MTBE & 0.0 & 0.00 & 0.0 & 0.0 & 0.00 & 0.0 & 0.0 & 0.00 & 0.0 & 0.0 & 0.00 & 0.00 \\
\hline
\end{tabular}




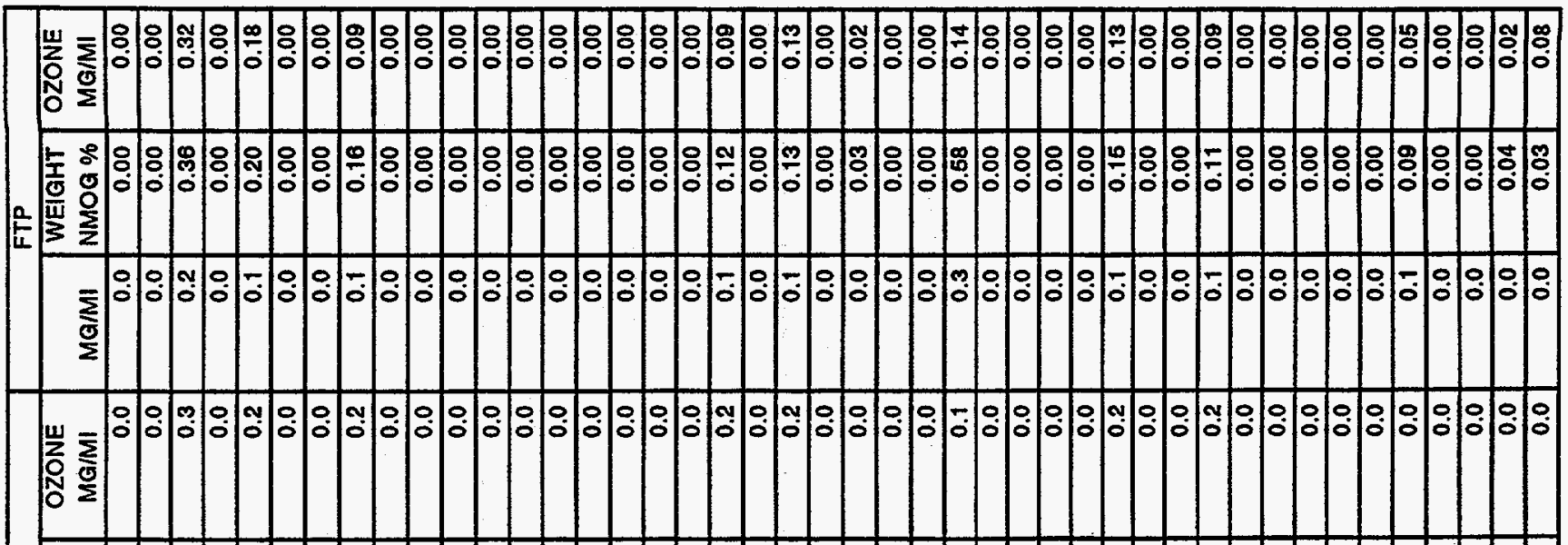

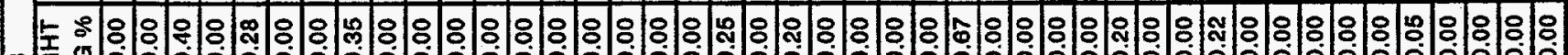
$\infty$ 응

这岕

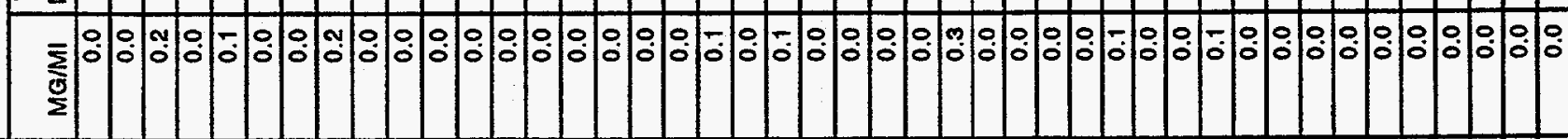

崖 옹

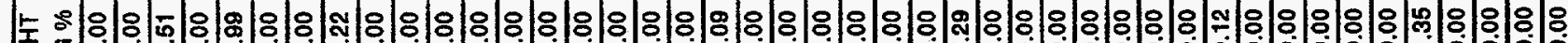

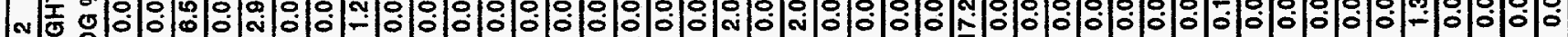
它

囟了

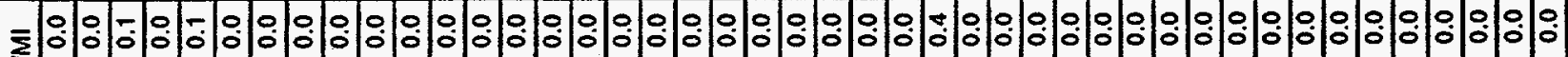

$$
\begin{aligned}
& \stackrel{\oplus}{\Sigma}
\end{aligned}
$$

山і ₹ 怘 $\frac{0}{\Sigma}$

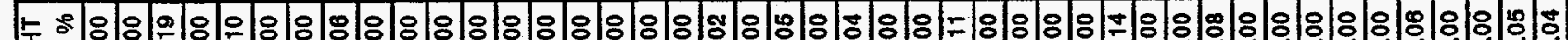

- II 它岕

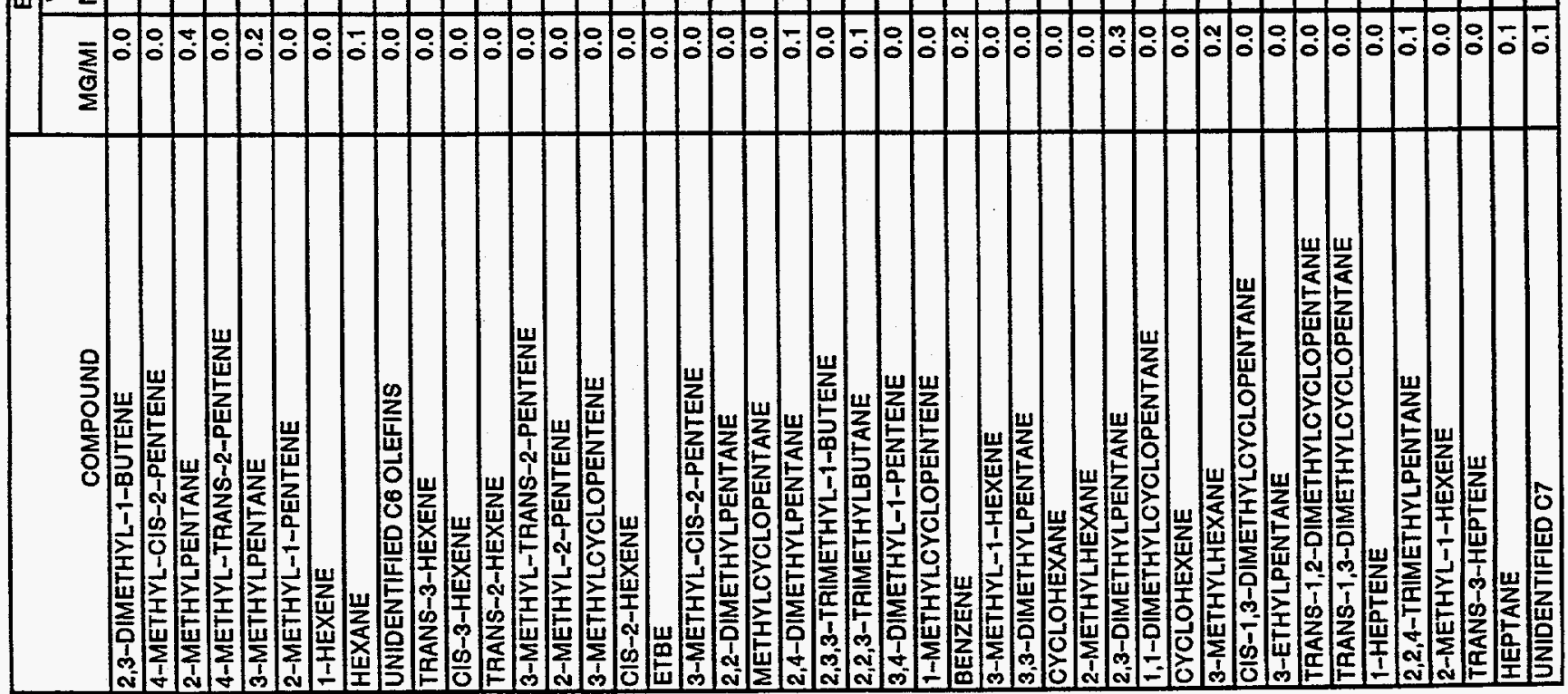




\begin{tabular}{|c|c|c|c|c|c|c|c|c|c|c|c|c|}
\hline \multirow[b]{2}{*}{ COMPOUND } & \multicolumn{3}{|c|}{$\overline{B A G 1}$} & \multicolumn{3}{|c|}{$B A Q_{2}$} & \multicolumn{3}{|c|}{ BAG 3} & \multicolumn{3}{|c|}{ FTP } \\
\hline & MG/MI & \begin{tabular}{|l|} 
WEIGHT \\
NMOG $\%$
\end{tabular} & $\begin{array}{l}\text { OZONE, } \\
\text { MG/MI }\end{array}$ & MG/MI & $\begin{array}{l}\text { WEIGHT } \\
\text { NMOG } \%\end{array}$ & $\begin{array}{l}\text { OZONE } \\
M G / M I\end{array}$ & MGMI & $\begin{array}{l}\text { WEIGHT } \\
\text { NMOG } \%\end{array}$ & $\begin{array}{l}\text { OZONE } \\
\text { MG/MI }\end{array}$ & MG/MI & $\begin{array}{l}\text { WEIGHT } \\
\text { NMOG } \%\end{array}$ & $\begin{array}{l}\text { OZONE } \\
\text { MG/MI }\end{array}$ \\
\hline 2-METHYL-2-HEXENE & 0.0 & 0.00 & 0.0 & 0.0 & 0.00 & 0.0 & 0.0 & 0.00 & 0.0 & 0.0 & 0.00 & 0.00 \\
\hline 3-METHYL-TRANS-3-HEXENE & 0.0 & 0.00 & 0.0 & 0.0 & 0.00 & $\overline{0.0}$ & 0.0 & 0.00 & $\overline{0.0}$ & 0.0 & 0.00 & 0.00 \\
\hline TRANS-2-HEPTENE & 0.0 & 0.00 & 0.0 & 0.0 & 0.00 & 0.0 & 0.0 & 0.00 & 0.0 & 0.0 & 0.00 & 0.00 \\
\hline 3-ETHYL-CIS-2-PENTENE & 0.0 & 0.00 & 0.0 & 0.0 & 0.00 & 0.0 & 0.0 & 0.00 & 0.0 & 0.0 & 0.00 & 0.00 \\
\hline 2,4,4-TRIMETHYL-1-PENTENE & 0.0 & 0.00 & 0.0 & 0.0 & 0.00 & 0.0 & 0.0 & 0.00 & 0.0 & $\overline{0.0}$ & 0.00 & 0.00 \\
\hline 2,2,4-TRIMETHYL-1-PENTENE & 0.0 & 0.00 & 0.0 & 0.0 & 0.00 & 0.0 & 0.0 & 0.00 & 0.0 & 0.0 & 0.00 & 0.00 \\
\hline 2,3-DIMETHYL-2-PENTENE & 0.0 & 0.00 & 0.0 & 0.0 & 0.00 & 0.0 & 0.0 & 0.00 & 0.0 & 0.0 & 0.00 & 0.00 \\
\hline CIS-2-HEPTENE & 0.0 & 0.00 & 0.0 & 0.0 & 0.00 & 0.0 & 0.0 & 0.00 & $\overline{0.0}$ & 0.0 & 0.00 & 0.00 \\
\hline METHYLCYCLOHEXANE & 0.0 & 0.00 & 0.0 & 0.0 & 0.00 & 0.0 & 0.0 & 0.00 & 0.0 & 0.0 & 0.00 & 0.00 \\
\hline CIS-1,2-DIMETHYLCYCLOPENTANE & 0.0 & 0.00 & 0.0 & 0.0 & 0.00 & $\overline{0.0}$ & 0.0 & 0.00 & $\overline{0.0}$ & 0.0 & 0,00 & 0.00 \\
\hline 2,2-DIMETHYLHEXANE & 0.0 & 0.00 & 0.0 & 0.0 & 0.00 & 0.0 & 0.0 & 0.00 & 0.0 & 0.0 & 0.00 & 0.00 \\
\hline 1,1,3-TRIMETHYLCYCLOPENTANE & 0.0 & 0.00 & 0.0 & 0.0 & 0.00 & 0.0 & 0.0 & 0.00 & 0.0 & 0.0 & 0.00 & 0.00 \\
\hline 2,4,4-TRIMETHYL-2-PENTENE & 0.0 & 0.00 & 0.0 & 0.0 & 0.00 & 0.0 & 0.0 & 0.00 & 0.0 & 0.0 & 0.00 & 0.00 \\
\hline 2,2,3-TRIMETHYLPENTANE & 0.0 & 0.00 & 0.0 & 0.0 & 0.00 & 0.0 & 0.0 & 0.00 & 0.0 & 0.0 & 0.00 & 0.00 \\
\hline 2,5-DIMETHYLHEXANE & 0.0 & 0.00 & 0.0 & 0.0 & 0.00 & 0.0 & 0.0 & 0.00 & 0.0 & 0.0 & 0.00 & 0.00 \\
\hline ETHYLCYCLOPENTANE & 0.0 & 0.00 & 0.0 & 0.0 & 0.00 & 0.0 & 0.0 & 0.00 & 0.0 & 0.0 & 0.00 & 0.00 \\
\hline 2,4-DIMETHYLHEXANE & 0.0 & 0.00 & $\overline{0.0}$ & 0.0 & 0.00 & 0,0 & 0.0 & 0.00 & 0.0 & 0.0 & 0.00 & 0.00 \\
\hline 1-TRANS-2-CIS-4-TRIMETHYLCYCLOPENTANE & 0.0 & 0.00 & 0.0 & 0.0 & 0.00 & 0.0 & 0.0 & 0.00 & 0.0 & 0.0 & 0.00 & 0.00 \\
\hline 3,3-DIMETHYLHEXANE & 0.0 & 0.00 & 0.0 & 0.0 & 0.00 & 0.0 & 0.0 & 0.00 & 0.0 & 0.0 & 0.00 & 0.00 \\
\hline 1-TRANS-2-CIS-3-TRIMETHYLCYCLOPENTANE & 0.0 & 0.00 & 0.0 & 0.0 & 0.00 & 0.0 & 0.0 & 0.00 & 0.0 & 0.0 & 0.00 & 0,00 \\
\hline 2,3,4-TRIMETHYLPENTANE & 0.0 & 0.00 & 0.0 & 0.0 & 0.00 & 0.0 & 0.0 & 0.00 & 0.0 & 0.0 & 0.00 & 0.00 \\
\hline 2,3,3-TRIMETHYLPENTANE & 0.0 & 0.00 & 0.0 & 0.0 & 0.00 & 0.0 & 0.0 & 0.00 & 0.0 & 0.0 & 0.00 & 0.00 \\
\hline TOLUENE & 0.4 & 0.20 & 1.1 & 0.2 & 7.69 & 0.5 & 0.2 & 0.39 & 0.5 & 0.2 & $\overline{0.39}$ & 0.62 \\
\hline 2,3-DIMETHYLHEXANE & 0.0 & 0.00 & 0.0 & 0.0 & 0.00 & 0.0 & 0.0 & 0.00 & 0.0 & 0.0 & 0.00 & 0.00 \\
\hline 1,1,2-TRIMETHYLCYCLOPENTANE & 0.0 & 0.00 & 0.0 & 0.0 & 0.00 & 0.0 & 0.0 & 0.00 & 0.0 & 0.0 & 0.00 & 0.00 \\
\hline 2-METHYLHEPTANE & 0.0 & 0.00 & 0.0 & 0.0 & 0.00 & 0.0 & 0.0 & 0.00 & 0.0 & 0.0 & 0.00 & 0.00 \\
\hline 3,4-DIMETHYLHEXANE & 0.0 & 0.00 & 0.0 & 0.0 & 0.00 & 0.0 & 0.0 & 0.00 & 0.0 & 0.0 & 0.00 & 0.00 \\
\hline 2,2,4,4-TETAAMETHYLPENTANE & 0.0 & 0.00 & 0.0 & 0.0 & 0.00 & 0.0 & 0.0 & 0.00 & 0.0 & 0.0 & 0.00 & 0.00 \\
\hline 4-METHYLHEPTANE & 0.0 & 0.00 & 0.0 & 0.0 & 0.00 & 0.0 & 0.0 & 0.00 & 0.0 & 0.0 & 0.00 & 0.00 \\
\hline 2-METHYL-3-ETHYLPENTANE & 0.0 & 0.00 & 0.0 & 0.0 & 0.00 & 0.0 & 0.0 & 0.00 & 0.0 & 0.0 & 0.00 & 0.00 \\
\hline 2,6-DIMETHYLHEPTANE & 0.0 & 0.00 & 0.0 & 0.0 & 0.00 & 0.0 & 0.0 & 0.00 & 0.0 & 0.0 & 0.00 & 0.00 \\
\hline 3-METHYLHEPTANE & 0.0 & 0.00 & 0.0 & 0.0 & 0.00 & 0.0 & 0.0 & 0.00 & 0.0 & 0.0 & 0.00 & 0.00 \\
\hline 1-CIS,2-TRANS,3-TRIMETHYLCYCLOPENTANE & 0.0 & 0.00 & 0.0 & 0.0 & 0.00 & 0.0 & 0.0 & 0.00 & 0.0 & 0.0 & 0.00 & 0.00 \\
\hline CIS-1,3-DIMETHYLCYCLOHEXANE & 0.0 & 0.00 & 0.0 & 0.0 & 0.00 & 0.0 & 0.0 & 0.00 & 0.0 & 0.0 & 0.00 & 0.00 \\
\hline TRANS-1,4-DIMETHYLCYCLOHEXANE & 0.0 & 0.00 & 0.0 & 0.0 & 0.00 & 0.0 & 0.0 & 0.00 & 0.0 & 0.0 & 0.00 & 0.00 \\
\hline 3-ETHYLHEXANE & 0.0 & 0.00 & 0.0 & 0.0 & 0.00 & 0.0 & 0.0 & 0.00 & 0.0 & 0.0 & 0.00 & 0.00 \\
\hline 2,2,5-TRIMETHYLHEXANE & 0.0 & 0.00 & 0.0 & 0.0 & 0.00 & 0.0 & 0.0 & 0.00 & 0.0 & 0.0 & 0.00 & 0.00 \\
\hline CIS-1-METHYL-3-ETHYLCYCLOPENTANE & 0.0 & 0.00 & 0.0 & 0.0 & 0.00 & 0.0 & 0.0 & 0.00 & 0.0 & 0.0 & 0.00 & 0.00 \\
\hline 1,1-DIMETHYLCYCLOHEXANE & 0.0 & 0.00 & 0.0 & 0.0 & 0.00 & 0.0 & 0.0 & 0.00 & 0.0 & 0.0 & 0.00 & 0.00 \\
\hline TRANS-1-METHYL-2-ETHYLCYCLOPENTANE & 0.0 & 0.00 & 0.0 & 0.0 & 0.00 & 0.0 & 0.0 & 0.00 & 0.0 & $\overline{0.0}$ & 0.00 & 0.00 \\
\hline 1-METHYL-1-ETHYL-CYCLOPENTANE & 0.0 & 0.00 & 0.0 & 0.0 & 0.00 & 0.0 & 0.0 & 0.00 & 0.0 & 0.0 & 0.00 & 0.00 \\
\hline 2,4,4-TRIMETHYLHEXANE & 0.0 & 0.00 & 0.0 & 0.0 & 0.00 & 0.0 & 0.0 & 0.00 & 0.0 & 0.0 & 0.00 & 0.00 \\
\hline 2,2,4-TAIMETHYLHEXANE & 0.0 & 0.00 & 0.0 & 0.0 & 0.00 & 0.0 & 0.0 & 0.00 & 0.0 & 0.0 & 0.00 & 0.00 \\
\hline TRANS-1,2-DIMETHYLCYCLOHEXANE & 0.0 & 0.00 & 0.0 & 0.0 & 0.00 & 0.0 & 0.0 & 0.00 & 0.0 & 0.0 & 0.00 & 0.00 \\
\hline
\end{tabular}




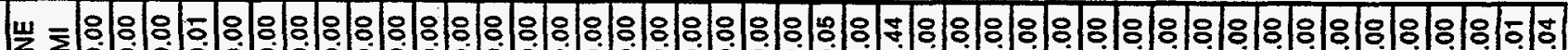

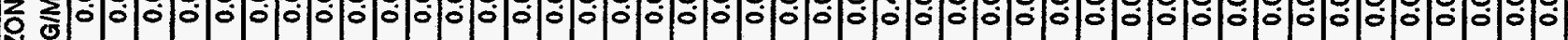
N

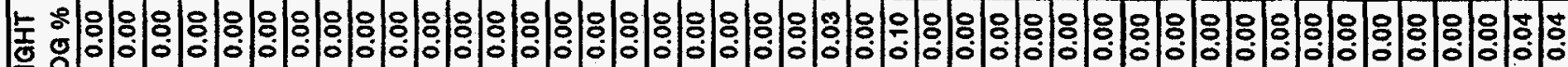
产 ํㅗㄴ

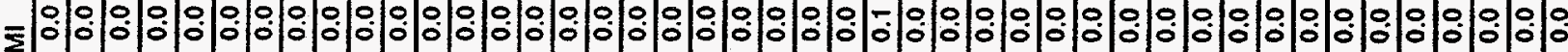

일

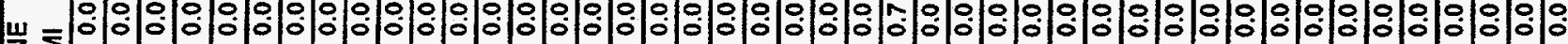
芯

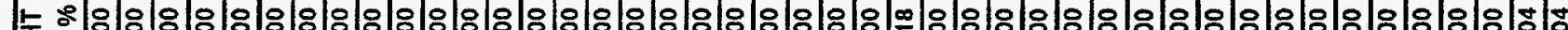
- 苼

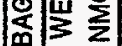

$100000000000000000000000-00000000000000000$

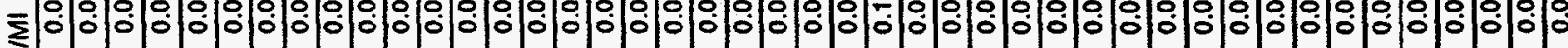
$\frac{\mathrm{O}}{2}$

崖

- 000000000000000000000000000000 응 న్ำ

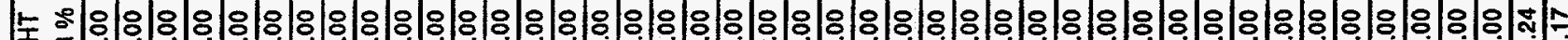

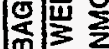

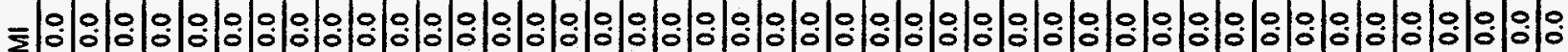
일

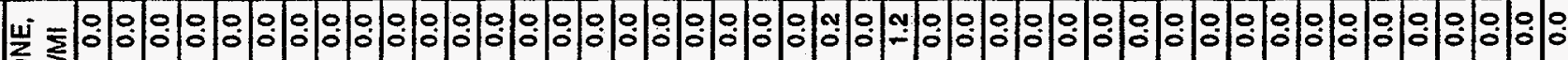
No

농ㅇㅇㅇㅇㅇㅇㅇㅇㅇㅇㅇㅇㅇㅇㅇㅇㅇㅇㅇㅇㅇㅇㅇㅇㅇㅇㅇㅇㅇㅇㅇㅇㅇㅇㅇㅇㅇㅇ으으. 通 高要

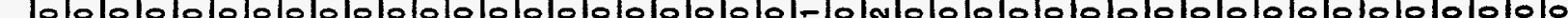

三 $\stackrel{\Xi}{\Sigma}$
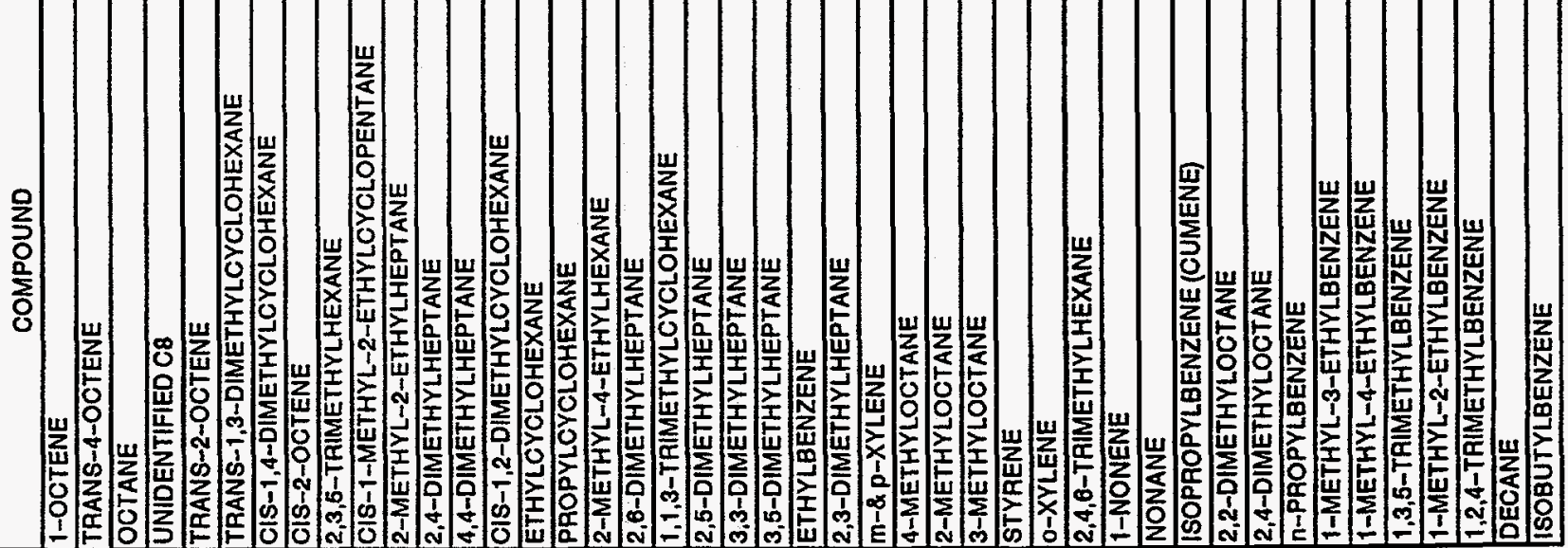


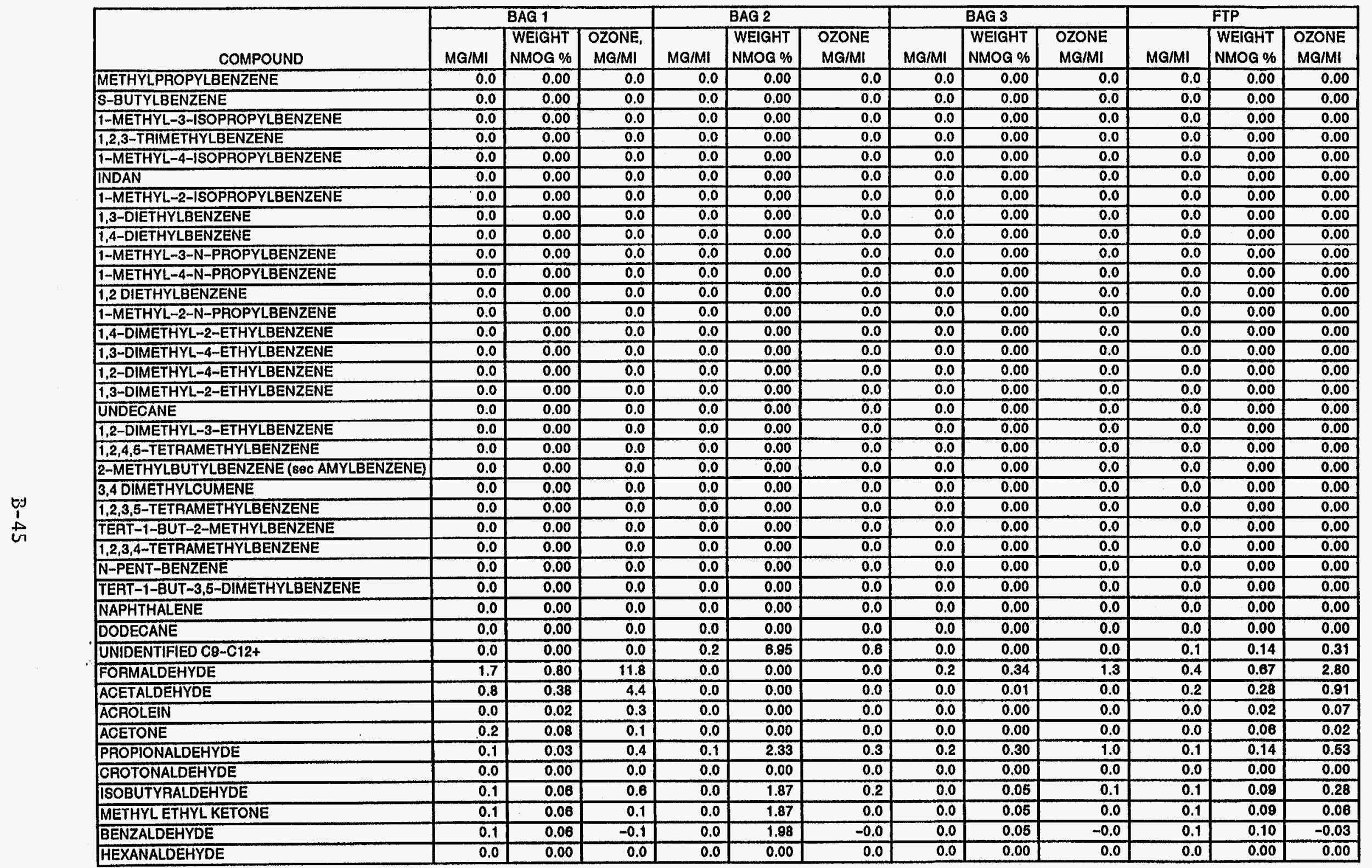


TEST NO: 90NB/1OPE-02S

TEST CAR: 1994 PONTIAC GRANDAM

TEST DATE: $9 / 28 / 85$

TEST FUEL: $90 \%$ BUTANE/10\% PENTANE

\begin{tabular}{|c|c|c|c|c|c|c|c|c|c|c|c|c|}
\hline \multirow[b]{2}{*}{ COMPOUND } & \multicolumn{3}{|c|}{$B A Q_{1}$} & \multicolumn{3}{|c|}{ BAG 2} & \multicolumn{3}{|c|}{ BAG 3} & \multicolumn{3}{|c|}{ FTP } \\
\hline & MG/MI & $\begin{array}{l}\text { WEIGHT } \\
\text { NMOG } \%\end{array}$ & $\begin{array}{l}\text { OZONE, } \\
\text { MG/MI }\end{array}$ & $\mathrm{MG} / \mathrm{MI}$ & $\begin{array}{l}\text { WEIGHT } \\
\text { NMOG } \%\end{array}$ & $\begin{array}{l}\text { OZONE } \\
\text { MG/MI }\end{array}$ & MG/MI & $\begin{array}{l}\text { WEIGHT } \\
\text { NHOG \% }\end{array}$ & $\begin{array}{l}\text { OZONE } \\
\text { MG/MI }\end{array}$ & MG/MI & $\begin{array}{l}\text { WEIGHT } \\
\text { NMOG } \%\end{array}$ & $\begin{array}{l}\text { OZONE } \\
\text { MG/MI }\end{array}$ \\
\hline METHANE & 25.6 & & 0.4 & 3.1 & & 0.0 & 29.9 & & 0.4 & 16.1 & & 0.22 \\
\hline ETHANE & 2.8 & 1.45 & 0.7 & 0.0 & 0.68 & 0.0 & 4.3 & 14.27 & 1.1 & $\overline{1.8}$ & 3.46 & 0.44 \\
\hline ETHYLENE & 19.1 & 9.83 & 139.1 & 0.0 & 0.76 & 0.3 & 1.8 & 5.93 & 13.0 & 4.5 & 8.73 & 32.62 \\
\hline PROPANE & 1.0 & 0.50 & 0.5 & 0.0 & 0.00 & 0.0 & 0.2 & 0.68 & 0.1 & 0.3 & 0.50 & 0.12 \\
\hline PROPYLENE & 7.7 & 3.98 & 72.7 & 0.0 & 0.00 & 0.0 & 0.5 & 1.79 & 5.0 & 1.8 & 3.42 & 16.49 \\
\hline ACETYLENE & 7.6 & 3.85 & 3.7 & 0.0 & 0.00 & 0.0 & 0.0 & 0.00 & 0.0 & 1.6 & 3.03 & 0.78 \\
\hline PROPADIENE & 0.0 & 0.00 & 0.0 & 0.0 & 0.00 & 0.0 & 0.0 & 0.00 & 0.0 & 0.0 & 0.00 & 0.00 \\
\hline BUTANE & 127.8 & 65.84 & 130.4 & 0.4 & 8.61 & 0.5 & 17.5 & 58.54 & 17.9 & 31.6 & 61.66 & 32.25 \\
\hline TRANS-2-BUTENE & 0.3 & 0.16 & 3.1 & 0.0 & 0.00 & 0.0 & 0.1 & 0.36 & 1.1 & 0.1 & 0.18 & 0.93 \\
\hline 1-BUTENE & 0.7 & 0.37 & 6.4 & 0.0 & 0.00 & 0.0 & 0.1 & 0.19 & 0.5 & 0.2 & 0.32 & 1.47 \\
\hline 2-METHYLPAOPENE (ISOBUTYLENE) & 0.1 & 0.04 & 0.4 & 0.0 & 0.00 & 0.0 & 0.1 & 0.20 & 0.3 & 0.0 & 0.08 & 0.16 \\
\hline 2,2-DIMETHYLPAOPANE (NEOPENTANE) & 0.1 & 0.04 & 0.0 & 0.0 & 0.00 & 0.0 & 0.0 & 0.00 & 0.0 & 0.0 & 0.03 & 0.01 \\
\hline PROPYNE & 0.0 & 0.00 & 0.0 & 0.0 & 0.00 & 0.0 & 0.0 & 0.00 & 0.0 & 0.0 & 0.00 & 0.00 \\
\hline 1,3-BUTADIENE & 0.1 & 0.07 & 1.5 & 0.0 & 0.00 & 0.0 & 0.0 & 0.00 & 0.0 & 0.0 & 0.06 & 0.31 \\
\hline 2-METHYLPROPANE (ISOBUTANE) & 0.2 & 0.08 & 0.2 & 0.0 & 0.86 & 0.1 & 0.0 & 0.11 & 0.0 & 0.1 & 0.12 & 0.08 \\
\hline 1-BUTYNE & 0.0 & 0.00 & 0.0 & 0.0 & 0.00 & 0.0 & 0.0 & 0.00 & 0.0 & 0.0 & 0.00 & 0.00 \\
\hline METHANOL & 0.0 & 0.00 & 0.0 & 0.0 & 0.00 & 0.0 & 0.0 & 0.00 & 0.0 & 0.0 & 0.00 & 0.00 \\
\hline CIS-2-BUTENE & 0.1 & 0.04 & 0.7 & 0.0 & 0.00 & 0.0 & 0.0 & 0.00 & 0.0 & 0.0 & 0.03 & 0.14 \\
\hline 3-METHYL-1-BUTENE & 0.0 & 0.00 & 0.0 & 0.0 & 0.00 & 0.0 & 0.0 & 0.00 & 0.0 & 0.0 & 0.00 & 0.00 \\
\hline ETHANOL & 0.0 & 0.00 & 0.0 & 0.0 & 0.00 & 0.0 & 0.0 & 0.00 & 0.0 & 0.0 & 0.00 & 0.00 \\
\hline 2-METHYLBUTANE (ISOPENTANE) & 2.8 & 1.47 & 3.8 & 0.6 & 11.94 & 0.9 & 0.3 & 0,91 & 0.4 & 1.0 & 1.93 & 1.37 \\
\hline 2-BUTYNE & 0.0 & 0.00 & 0.0 & 0.0 & 0.00 & 0.0 & 0.0 & 0.00 & 0.0 & 0.0 & 0.00 & 0.00 \\
\hline 1-PENTENE & 0.0 & 0.00 & 0.0 & 0.0 & 0.00 & 0.0 & 0.0 & 0.00 & 0.0 & 0.0 & 0.00 & 0.00 \\
\hline 2-METHYL-1-BUTENE & 0.1 & 0.08 & 0.6 & 0.0 & 0.00 & 0.0 & 0.0 & 0.00 & 0.0 & 0.0 & 0.05 & 0.12 \\
\hline PENTANE & 14.8 & 7.60 & 15.3 & 0.2 & 4.40 & 0.2 & 2.1 & 7.06 & 2.2 & $\overline{3.8}$ & 7.35 & 3.92 \\
\hline UNIDENTIFIED C6 OLEFINS & 0.0 & 0.00 & 0.0 & 0.0 & 0.00 & 0.0 & 0.0 & 0.00 & 0.0 & $\overline{0.0}$ & 0.00 & 0.00 \\
\hline 2-METHYL-1,3-BUTADIENE & 0.0 & 0.00 & 0.0 & 0.0 & 0.00 & 0.0 & 0.0 & 0.00 & 0.0 & 0.0 & 0.00 & 0.00 \\
\hline TAANS-2-PENTENE & 0.1 & 0.07 & 1.1 & 0.0 & 0.00 & 0.0 & 0.0 & 0.00 & 0.0 & $\overline{0.0}$ & 0.05 & 0.23 \\
\hline 3,3-DIMETHYL-1-BUTENE & 0.0 & 0.00 & 0.0 & 0.0 & 0.00 & 0.0 & 0.0 & 0.00 & 0.0 & $\overline{0.0}$ & 0.00 & 0.00 \\
\hline CIS-2-PENTENE & 0.1 & 0.05 & 0.8 & 0.0 & 0.00 & 0.0 & 0.0 & 0.00 & 0.0 & $\overline{0.0}$ & 0.04 & 0.16 \\
\hline 2-METHYL-2-BUTENE & 0.1 & 0.04 & 0.5 & 0.0 & 0.29 & 0.1 & 0.0 & 0.08 & 0.1 & 0.0 & 0.06 & 0.20 \\
\hline CYCLOPENTADIENE & 0.0 & 0.00 & 0.0 & 0.0 & 0.00 & 0.0 & 0.0 & 0.00 & 0.0 & 0.0 & 0.00 & 0.00 \\
\hline 2,2-DIMETHYLBUTANE & 0.1 & 0.06 & 0.1 & 0.0 & 0.00 & 0.0 & 0.0 & 0.00 & 0.0 & 0.0 & 0.05 & 0.02 \\
\hline CYCLOPENTENE & 0.0 & 0.00 & 0.0 & 0.0 & 0.00 & 0.0 & 0.0 & 0.00 & 0.0 & 0.0 & 0.00 & 0.00 \\
\hline 4-METHYL-1-PENTENE & 0.0 & 0.00 & 0.0 & 0.0 & 0.00 & 0.0 & 0.0 & 0.00 & 0.0 & 0.0 & 0.00 & 0.00 \\
\hline 3-METHYL-1-PENTENE & 0.1 & 0.05 & 0.4 & 0.0 & 0.00 & 0.0 & 0.0 & 0.00 & 0.0 & 0.0 & 0.04 & $0 . \overline{08}$ \\
\hline CYCLOPENTANE & 0.0 & 0.00 & 0.0 & 0.0 & 0.00 & 0.0 & 0.0 & 0.00 & 0.0 & 0.0 & 0.00 & 0.00 \\
\hline 2,3-DIMETHYLBUTANE & 0.2 & 0.09 & 0.2 & 0.1 & 1.08 & 0.1 & 0.1 & 0.25 & 0.1 & 0.1 & 0.17 & 0.09 \\
\hline MTBE & 0.0 & 0.00 & 0.0 & 0.0 & 0.00 & 0.0 & 0.0 & 0.00 & 0.0 & 0.0 & 0.00 & 0.00 \\
\hline
\end{tabular}




\begin{tabular}{|c|c|c|c|c|c|c|c|c|c|c|c|c|}
\hline \multirow[b]{2}{*}{ COMPOUND } & \multicolumn{3}{|c|}{ BAG 1} & \multicolumn{3}{|c|}{ BAG 2} & \multicolumn{3}{|c|}{ BAG 3} & \multicolumn{3}{|c|}{ FTP } \\
\hline & MG/MI & $\begin{array}{l}\text { WEIGHT } \\
\text { NMOG } \%\end{array}$ & $\begin{array}{l}\text { OZONE, } \\
\text { MG/MI }\end{array}$ & MG/MI & $\begin{array}{l}\text { WEIGHT } \\
\text { NMOG \% }\end{array}$ & $\begin{array}{l}\text { OZONE } \\
\text { MG/MI }\end{array}$ & $\mathrm{MG} / \mathrm{MI}$ & $\begin{array}{l}\text { WEIGHT } \\
\text { NMOQ \% }\end{array}$ & $\begin{array}{l}\text { OZONE } \\
\text { MG/MI }\end{array}$ & MG/MI & $\begin{array}{l}\text { WEIGHT } \\
\text { NMOQ } \%\end{array}$ & $\begin{array}{l}\text { OZONE } \\
\text { MG/MI }\end{array}$ \\
\hline 2,3-DIMETHYL-1-BUTENE & 0.0 & 0.00 & 0.0 & 0.0 & 0.00 & 0.0 & 0.0 & 0.00 & 0.0 & 0.0 & 0.00 & 0.00 \\
\hline 4-METHYL-CIS-2-PENTENE & 0.0 & 0.00 & 0.0 & 0.0 & 0.00 & 0.0 & 0.0 & 0.00 & 0.0 & 0.0 & 0.00 & 0.00 \\
\hline 2-METHYLPENTANE & 0.5 & 0.28 & 0.8 & 0.3 & 4.83 & 0.4 & 0.2 & 0.64 & 0.3 & 0.3 & 0.58 & 0.45 \\
\hline 4-METHYL-TRANS-2-PENTENE & 0.0 & 0.00 & 0.0 & 0.0 & 0.00 & 0.0 & 0.0 & 0.00 & 0.0 & 0.0 & 0.00 & 0.00 \\
\hline 3-METHYLPENTANE & 0.3 & 0.15 & 0.4 & 0.2 & 2.99 & 0.2 & 0.1 & 0.47 & 0.2 & 0.2 & 0.35 & 0.27 \\
\hline 2-METHYL-1-PENTENE & 0.0 & 0.00 & 0.0 & 0.0 & 0.00 & 0.0 & 0.0 & 0.00 & 0.0 & 0.0 & 0.00 & 0.00 \\
\hline 1-HEXENE & 0.0 & 0.00 & 0.0 & 0.0 & 0.00 & 0.0 & 0.0 & 0.00 & 0.0 & 0.0 & 0.00 & 0.00 \\
\hline HEXANE & 0.2 & 0.13 & 0.2 & 0.2 & 3.04 & 0.2 & 0.1 & 0.47 & 0.1 & 0.2 & 0.33 & $\overline{0.17}$ \\
\hline UNIDENTIFIED C6 OLEFINS & 0.0 & 0.00 & 0.0 & 0.0 & 0.00 & 0.0 & 0.1 & 0.33 & 0.7 & 0.0 & 0.05 & 0.18 \\
\hline TRANS-3-HEXENE & 0.0 & 0.00 & 0.0 & 0.0 & 0.00 & 0.0 & 0.0 & 0.00 & 0.0 & 0.0 & 0.00 & 0.00 \\
\hline CIS-3-HEXENE & 0.1 & 0.05 & 0.6 & 0.0 & 0.00 & 0.0 & 0.0 & 0.00 & 0.0 & 0.0 & 0.04 & 0.13 \\
\hline TRANS-2-HEXENE & 0.0 & 0.00 & 0.0 & 0.0 & 0.00 & 0.0 & 0.0 & 0.00 & 0.0 & 0.0 & 0.00 & 0.00 \\
\hline 3-METHYL-TRANS-2-PENTENE & 0.0 & 0.00 & 0.0 & 0.0 & 0.00 & 0.0 & 0.0 & 0.00 & 0.0 & 0.0 & 0.00 & 0.00 \\
\hline 2-METHYL-2-PENTENE & 0.0 & 0.00 & 0.0 & 0.0 & 0.00 & 0.0 & 0.0 & 0.00 & 0.0 & 0.0 & 0.00 & 0.00 \\
\hline 3-METHYLCYCLOPENTENE & 0.0 & 0.00 & 0.0 & 0.0 & 0.00 & 0.0 & 0.0 & 0.00 & 0.0 & 0.0 & 0.00 & 0.00 \\
\hline CIS-2-HEXENE & 0.0 & 0.00 & 0.0 & 0.0 & 0.00 & 0.0 & 0.0 & 0.00 & 0.0 & 0.0 & 0.00 & 0.00 \\
\hline ETBE & 0.0 & 0.00 & 0.0 & 0.0 & 0.00 & 0.0 & 0.0 & 0.00 & 0.0 & 0.0 & 0.00 & 0.00 \\
\hline 3-METHYL-CIS-2-PENTENE & 0.0 & 0.00 & 0.0 & 0.0 & 0.00 & 0.0 & 0.0 & 0.00 & 0.0 & 0.0 & 0.00 & 0.00 \\
\hline 2,2-DIMETHYLPENTANE & 0.1 & 0.05 & 0.1 & 0.1 & 1.39 & 0.1 & 0.1 & 0.28 & 0.1 & 0.1 & 0.16 & 0.11 \\
\hline METHYLCYCLOPENTANE & 0.0 & 0.00 & 0.0 & 0.0 & 0.00 & 0.0 & 0.0 & 0.00 & 0.0 & 0.0 & 0.00 & 0.00 \\
\hline 2,4-DIMETHYLPENTANE & 0.1 & 0.06 & 0.2 & 0.0 & 0.94 & 0.1 & 0.1 & 0.22 & 0.1 & 0.1 & 0.13 & $\overline{0.12}$ \\
\hline 2,3,3-TRIMETHYL-1-BUTENE & 0.0 & 0.00 & 0.0 & 0.0 & 0.00 & 0.0 & 0.0 & 0.00 & 0.0 & 0.0 & 0.00 & 0.00 \\
\hline 2,2,3-TRIMETHYLBUTANE & 0.1 & 0.08 & 0.2 & 0.1 & 1.11 & 0.1 & 0.1 & 0.28 & 0.1 & 0.1 & 0.15 & 0.10 \\
\hline 3,4-DIMETHYL-1-PENTENE & 0.0 & 0.00 & 0.0 & 0.0 & 0.00 & 0.0 & 0.0 & 0.00 & 0.0 & 0.0 & 0.00 & 0.00 \\
\hline 1-METHYLCYCLOPENTENE & 0.0 & 0.00 & 0.0 & 0.0 & 0.00 & 0.0 & 0.0 & 0.06 & 0.1 & 0.0 & 0.01 & 0.03 \\
\hline BENZENE & 0.6 & 0.28 & 0.2 & 0.2 & 3.58 & 0.1 & 0.3 & 0.92 & 0.1 & 0.3 & 0.66 & 0.12 \\
\hline 3-METHYL-1-HEXENE & 0.0 & 0.00 & 0.0 & 0.0 & 0.00 & 0.0 & 0.0 & 0.00 & 0.0 & 0.0 & 0.00 & 0.00 \\
\hline 3,3-DIMETHYLPENTANE & 0.0 & 0.00 & 0.0 & 0.0 & 0.00 & 0.0 & 0.0 & 0.00 & 0.0 & 0.0 & 0.00 & 0.00 \\
\hline CYCLOHEXANE & 0.0 & 0.00 & 0.0 & 0.0 & 0.00 & 0.0 & 0.0 & 0.00 & 0.0 & 0.0 & 0.00 & 0.00 \\
\hline 2-METHYLHEXANE & 0.0 & 0.00 & 0.0 & 0.0 & 0.00 & 0.0 & 0.0 & 0.00 & 0.0 & 0.0 & 0.00 & 0.00 \\
\hline 2,3-DIMETHYLPENTANE & 0.3 & 0.16 & 0.5 & 0.2 & 3.11 & 0.2 & 0.2 & 0.52 & 0.2 & 0.2 & 0.37 & 0.29 \\
\hline 1,1-DIMETHYLCYCLOPENTANE & 0.0 & 0.00 & 0.0 & 0.0 & 0.00 & 0.0 & 0.0 & 0.00 & 0.0 & 0.0 & 0.00 & 0.00 \\
\hline CYCLOHEXENE & 0.0 & 0.00 & 0.0 & 0.0 & 0.00 & 0.0 & 0.0 & 0.00 & 0.0 & 0.0 & 0.00 & 0.00 \\
\hline 3-METHYLHEXANE & 0.2 & 0.08 & 0.2 & 0.1 & 1.08 & 0.1 & 0.1 & 0.20 & 0.1 & $\overline{0.1}$ & 0.16 & 0.11 \\
\hline CIS-1,3-DIMETHYLCYCLOPENTANE & 0.1 & 0.05 & 0.2 & 0.1 & 2.38 & 0.3 & 0.0 & 0.00 & 0.0 & 0.1 & 0.16 & 0.21 \\
\hline 3-ETHYLPENTANE & 0.0 & 0.00 & 0.0 & 0.0 & 0.00 & 0.0 & 0.0 & 0.00 & 0.0 & 0.0 & 0.00 & 0.00 \\
\hline TAANS-1,2-DIMETHYLCYCLOPENTANE & $\overline{0.0}$ & 0.00 & 0.0 & 0.0 & 0.00 & 0.0 & 0.0 & 0.00 & 0.0 & 0.0 & 0.00 & 0.00 \\
\hline TAANS-1,3-DIMETHYLCYCLOPENTANE & 0.0 & 0.00 & 0.0 & 0.0 & 0.00 & 0.0 & 0.0 & 0.00 & 0.0 & 0.0 & 0.00 & 0.00 \\
\hline 1-HEPTENE & 0.0 & 0.00 & 0.0 & 0.0 & 0.00 & 0.0 & 0.0 & 0.00 & 0.0 & 0.0 & 0.00 & 0.00 \\
\hline 2,2,4-TRIMETHYLPENTANE & $\overline{0.2}$ & 0.12 & 0.2 & 0.2 & 4.10 & 0.2 & 0.2 & 0.51 & 0.1 & 0.2 & 0.39 & 0.19 \\
\hline 2-METHYL-1-HEXENE & 0.0 & 0.00 & 0.0 & 0.0 & 0.00 & 0.0 & 0.0 & 0.00 & 0.0 & 0.0 & 0.00 & 0.00 \\
\hline TRANS-3-HEPTENE & 0.0 & 0.00 & 0.0 & 0.0 & 0.00 & 0.0 & 0.0 & 0.00 & 0.0 & 0.0 & 0.00 & 0.00 \\
\hline HEPTANE & 0.0 & 0.01 & 0.0 & 0.0 & 0.74 & 0.0 & 0.0 & 0.00 & 0.0 & 0.0 & 0.05 & 0.02 \\
\hline UNIDENTIFIED C7 & 0.2 & 0.10 & 0.0 & 0.1 & 2.71 & 0.6 & 0.1 & 0.28 & 0.4 & $\overline{0.1}$ & 0.27 & 0.62 \\
\hline
\end{tabular}




\begin{tabular}{|c|c|c|c|c|c|c|c|c|c|c|c|c|}
\hline \multirow[b]{2}{*}{ COMPOUND } & \multicolumn{3}{|c|}{ BAG 1} & \multicolumn{3}{|c|}{ BAG 2} & \multicolumn{3}{|c|}{ BAC 3} & \multicolumn{3}{|c|}{ FTP } \\
\hline & MG/MI & $\begin{array}{l}\text { WEIGHT } \\
\text { NMOG \% }\end{array}$ & $\begin{array}{l}\text { OZONE, } \\
\text { MG/MI }\end{array}$ & MG/MI & \begin{tabular}{|l|} 
WEIGHT \\
NMOG $\%$
\end{tabular} & $\begin{array}{l}\text { OZONE } \\
\text { MG/MI }\end{array}$ & MG/MI & $\begin{array}{l}\text { WEIGHT } \\
\text { NMOG } \%\end{array}$ & $\begin{array}{l}\text { OZONE } \\
\text { MGMI }\end{array}$ & MG/MI & $\begin{array}{l}\text { WEIGHT } \\
\text { NMOG \% }\end{array}$ & $\begin{array}{l}\text { OZONE } \\
\text { MG/MI }\end{array}$ \\
\hline 2-METHYL-2-HEXENE & 0.0 & 0.00 & 0.0 & 0.0 & 0.00 & 0.0 & 0.0 & 0.00 & 0.0 & 0.0 & 0.00 & 0.00 \\
\hline 3-METHYL-TRANS-3-HEXENE & 0.0 & 0.00 & 0.0 & 0.0 & 0.00 & 0.0 & 0.0 & 0.00 & 0.0 & 0.0 & 0.00 & 0.00 \\
\hline TRANS-2-HEPTENE & 0.0 & 0.00 & 0.0 & 0.0 & 0.00 & 0.0 & 0.0 & 0.00 & 0.0 & 0.0 & 0.00 & 0.00 \\
\hline 3-ETHYL-CIS-2-PENTENE & 0.0 & 0.00 & 0.0 & 0.0 & 0.00 & 0.0 & 0.0 & 0.00 & 0.0 & 0.0 & 0.00 & 0.00 \\
\hline 2,4,4-TRIMETHYL-1-PENTENE & 0.0 & 0.00 & 0.0 & 0.0 & 0.00 & 0.0 & 0.0 & 0.00 & 0.0 & 0.0 & 0.00 & 0.00 \\
\hline 2,2,4-TRIMETHYL-1-PENTENE & 0.0 & 0.00 & 0.0 & 0.0 & 0.00 & 0.0 & 0.0 & 0.00 & 0.0 & 0.0 & 0.00 & 0.00 \\
\hline 2,3-DIMETHYL-2-PENTENE & 0.0 & 0.00 & 0.0 & 0.0 & 0.00 & 0.0 & 0.0 & 0.00 & 0.0 & 0.0 & 0.00 & 0.00 \\
\hline CIS-2-HEPTENE & 0.0 & 0.00 & 0.0 & 0.0 & 0.00 & 0.0 & 0.0 & 0.00 & 0.0 & 0.0 & 0.00 & 0.00 \\
\hline METHYLCYCLOHEXANE & 0.0 & 0.00 & 0.0 & 0.0 & 0.00 & 0.0 & 0.0 & 0.00 & 0.0 & 0.0 & 0.00 & 0.00 \\
\hline CIS-1,2-DIMETHYLCYCLOPENTANE & 0.0 & 0.00 & 0.0 & 0.0 & 0.00 & 0.0 & 0.0 & 0.00 & 0.0 & 0.0 & 0.00 & 0.00 \\
\hline 2,2-DIMETHYLHEXANE & 0.0 & 0.00 & 0.0 & 0.0 & 0.00 & 0.0 & 0.0 & 0.00 & 0.0 & 0.0 & 0.00 & 0.00 \\
\hline 1,1,3-TRIMETHYLCYCLOPENTANE & 0.0 & 0.00 & 0.0 & 0.0 & 0.00 & 0.0 & 0.0 & 0.00 & 0.0 & 0.0 & 0.00 & 0.00 \\
\hline 2,4,4-TRIMETHYL-2-PENTENE & 0.0 & 0.00 & 0.0 & 0.0 & 0.00 & 0.0 & 0.0 & 0.00 & 0.0 & 0.0 & 0.00 & 0.00 \\
\hline 2,2,3-TRIMETHYLPENTANE & 0.0 & 0.00 & 0.0 & 0.0 & 0.00 & 0.0 & 0.0 & 0.00 & 0.0 & 0.0 & 0.00 & 0.00 \\
\hline 2,5-DIMETHYLHEXANE & 0.0 & 0.00 & 0.0 & 0.0 & 0.00 & 0.0 & 0.0 & 0.00 & 0.0 & 0.0 & 0.00 & 0.00 \\
\hline ETHYLCYCLOPENTANE & 0.0 & 0.00 & 0.0 & 0.0 & 0.00 & 0.0 & 0.0 & 0.00 & 0.0 & 0.0 & 0.00 & 0.00 \\
\hline 2,4-DIMETHYLHEXANE & 0.0 & 0.00 & 0.0 & 0.0 & 0.00 & 0.0 & 0.0 & 0.00 & 0.0 & 0.0 & 0.00 & 0.00 \\
\hline 1-TRANS-2-CIS-4-TRIMETHYLCYCLOPENTANE & 0.0 & 0.00 & 0.0 & 0.0 & 0.00 & 0.0 & 0.0 & 0.00 & 0.0 & 0.0 & 0.00 & 0.00 \\
\hline 3,3-DIMETHYLHEXANE & 0.0 & 0.00 & 0.0 & 0.0 & 0.00 & 0.0 & 0.0 & 0.00 & 0.0 & 0.0 & 0.00 & 0.00 \\
\hline 1-TRANS-2-CIS-3-TRIMETHYLCYCLOPENTANE & 0.0 & 0.00 & 0.0 & 0.0 & 0.00 & 0.0 & 0.0 & 0.00 & 0.0 & 0.0 & 0.00 & 0.00 \\
\hline 2,3,4-TRIMETHYLPENTANE & 0.0 & 0.00 & 0.0 & 0.0 & 0.00 & 0.0 & 0.0 & 0.00 & 0.0 & 0.0 & 0.00 & 0.00 \\
\hline 2,3,3-TRIMETHYLPENTANE & 0.1 & 0.07 & 0.2 & 0.2 & 3.02 & 0.2 & 0.0 & 0.00 & 0.0 & 0.1 & 0.22 & 0.13 \\
\hline TOLUENE & 1.1 & 0.55 & 2.9 & 0.5 & 9.90 & 1.4 & 0.2 & 0.67 & 0.5 & 0.5 & 1.06 & 1.49 \\
\hline 2,3-DIMETHYLHEXANE & 0.0 & 0.00 & 0.0 & 0.0 & 0.00 & 0.0 & 0.0 & 0.00 & 0.0 & 0.0 & 0.00 & 0.00 \\
\hline 1,1,2-TRIMETHYLCYCLOPENTANE & 0.0 & 0.00 & 0.0 & 0.0 & 0.00 & 0.0 & 0.0 & 0.00 & 0.0 & 0.0 & 0.00 & 0.00 \\
\hline 2-METHYLHEPTANE & 0.0 & 0.00 & 0.0 & 0.0 & 0.00 & 0.0 & 0.0 & 0.00 & 0.0 & 0.0 & 0.00 & 0.00 \\
\hline 3,4-DIMETHYLHEXANE & 0.0 & 0.00 & 0.0 & 0.0 & 0.00 & 0.0 & 0.0 & 0.00 & 0.0 & 0.0 & 0.00 & 0.00 \\
\hline 2,2,4,4-TETRAMETHYLPENTANE & 0.0 & 0.00 & 0.0 & 0.0 & 0.00 & 0.0 & 0.0 & 0.00 & 0.0 & 0.0 & 0.00 & 0.00 \\
\hline 4-METHYLHEPTANE & 0.0 & 0.00 & 0.0 & 0.0 & 0.00 & 0.0 & 0.0 & 0.00 & 0.0 & 0.0 & 0.00 & 0.00 \\
\hline 2-METHYL-3-ETHYLPENTANE & 0.0 & 0.00 & 0.0 & 0.0 & 0.00 & 0.0 & 0.0 & 0.00 & 0.0 & 0.0 & 0.00 & 0.00 \\
\hline 2,6-DIMETHYLHEPTANE & 0.0 & 0.00 & 0.0 & 0.0 & 0.00 & 0.0 & 0.0 & 0.00 & 0.0 & 0.0 & 0.00 & 0.00 \\
\hline 3-METHYLHEPTANE & 0.0 & 0.00 & 0.0 & 0.0 & 0.00 & 0.0 & 0.0 & 0.00 & 0.0 & 0.0 & 0.00 & 0.00 \\
\hline 1-CIS,2-TRANS,3-TRIMETHYLCYCLOPENTANE & 0.0 & 0.00 & 0.0 & 0.0 & 0.00 & 0.0 & 0.0 & 0.00 & 0.0 & 0.0 & 0.00 & 0.00 \\
\hline CIS-1,3-DIMETHYLCYCLOHEXANE & 0.0 & 0.00 & 0.0 & 0.0 & 0.00 & 0.0 & 0.0 & 0.00 & 0.0 & 0.0 & 0.00 & 0.00 \\
\hline TRANS-1,4-DIMETHYLCYCLOHEXANE & 0.0 & 0.00 & 0.0 & 0.0 & 0.00 & 0.0 & 0.0 & 0.00 & 0.0 & 0.0 & 0.00 & 0.00 \\
\hline 3-ETHYLHEXANE & 0.0 & 0.00 & 0.0 & 0.0 & 0.00 & 0.0 & 0.0 & 0.00 & 0.0 & 0.0 & 0.00 & 0.00 \\
\hline 2,2,5-TRIMETHYLHEXANE & 0.0 & 0.00 & 0.0 & 0.0 & 0.00 & 0.0 & 0.0 & 0.00 & 0.0 & 0.0 & 0.00 & 0.00 \\
\hline CIS-1-METHYL-3-ETHYLCYCLOPENTANE & 0.0 & 0.00 & 0.0 & 0.0 & 0.00 & 0.0 & 0.0 & 0.00 & 0.0 & 0.0 & 0.00 & 0.00 \\
\hline 1.1-DIMETHYLCYCLOHEXANE & 0.0 & 0.00 & 0.0 & 0.0 & 0.00 & 0.0 & 0.0 & 0.00 & 0.0 & 0.0 & 0.00 & 0.00 \\
\hline TRANS-1-METHYL-2-ETHYLCYCLOPENTANE & 0.0 & 0.00 & 0.0 & 0.0 & 0.00 & 0.0 & 0.0 & 0.00 & 0.0 & 0.0 & 0.00 & 0.00 \\
\hline 1-METHYL-1-ETHYL-CYCLOPENTANE & 0.0 & 0.00 & 0.0 & 0.0 & 0.00 & 0.0 & 0.0 & 0.00 & 0.0 & 0.0 & 0.00 & 0.00 \\
\hline 2,4,4-TRIMETHYLHEXANE & 0.0 & 0.00 & 0.0 & 0.0 & 0.00 & 0.0 & 0.0 & 0.00 & 0.0 & 0.0 & 0.00 & 0.00 \\
\hline 2,2,4-TRIMETHYLHEXANE & 0.0 & 0.00 & 0.0 & 0.0 & 0.00 & 0.0 & 0.0 & 0.00 & 0.0 & 0.0 & 0.00 & 0.00 \\
\hline TRANS-1,2-DIMETHYLCYCLOHEXANE & 0.0 & 0.00 & 0.0 & 0.0 & 0.00 & 0.0 & 0.0 & 0.00 & 0.0 & 0.0 & 0.00 & 0.00 \\
\hline
\end{tabular}




\begin{tabular}{|c|c|c|c|c|c|c|c|c|c|c|c|c|}
\hline \multirow[b]{2}{*}{ COMPOUND } & \multicolumn{3}{|c|}{ BAG 1} & \multicolumn{3}{|c|}{ BAG 2} & \multicolumn{3}{|c|}{ BAG 3} & \multicolumn{3}{|c|}{ FTP } \\
\hline & MG/MI & $\begin{array}{l}\text { WEIGHT } \\
\text { NMOG } \%\end{array}$ & $\begin{array}{l}\text { OZONE, } \\
\text { MG/MI }\end{array}$ & MG/MI & $\begin{array}{l}\text { WEIGHT } \\
\text { NMOQ } \%\end{array}$ & $\begin{array}{l}\text { OZONE } \\
\text { MG/MI }\end{array}$ & MG/MI & $\begin{array}{l}\text { WEIGHT } \\
\text { NMOG } \%\end{array}$ & $\begin{array}{l}\text { OZONE } \\
\text { MG/MI }\end{array}$ & MG/MI & $\begin{array}{l}\text { WEIGHT } \\
\text { NMOG } \%\end{array}$ & $\begin{array}{l}\text { OZONE } \\
\text { MG/MI }\end{array}$ \\
\hline 1-OCTENE & 0.0 & 0.00 & 0.0 & 0.0 & 0.00 & 0.0 & 0.0 & 0.00 & 0.0 & 0.0 & 0.00 & 0.00 \\
\hline TRANS-4-OCTENE & 0.0 & 0.00 & 0.0 & 0.0 & 0.00 & 0.0 & 0.0 & 0.00 & 0.0 & 0.0 & 0.00 & 0.00 \\
\hline OCTANE & 0.0 & 0.00 & 0.0 & 0.0 & 0.00 & 0.0 & 0.0 & 0.00 & 0.0 & 0.0 & 0.00 & 0.00 \\
\hline UNIDENTIFIED C8 & 0.0 & 0.02 & 0.1 & 0.1 & 1.00 & 0.2 & 0.0 & 0.16 & 0.2 & 0.0 & 0.09 & 0.17 \\
\hline TRANS-2-OCTENE & 0.0 & 0.00 & 0.0 & 0.0 & 0.00 & 0.0 & 0.0 & 0.00 & 0.0 & 0.0 & 0.00 & 0.00 \\
\hline TRANS-1,3-DIMETHYLCYCLOHEXANE & 0.0 & 0.00 & 0.0 & 0.0 & 0.00 & 0.0 & 0.0 & 0.00 & 0.0 & 0.0 & 0.00 & 0.00 \\
\hline CIS-1,4-DIMETHYLCYCLOHEXANE & 0.0 & 0.00 & 0.0 & 0.0 & 0.00 & 0.0 & 0.0 & 0.00 & 0.0 & 0.0 & 0.00 & 0.00 \\
\hline CIS-2-OCTENE & 0.0 & 0.00 & 0.0 & 0.0 & 0.00 & 0.0 & 0.0 & 0.00 & 0.0 & 0.0 & 0.00 & 0.00 \\
\hline 2,3,5-TAIMETHYLHEXANE & 0.0 & 0.00 & 0.0 & 0.0 & 0.00 & 0.0 & 0.0 & 0.00 & 0.0 & 0.0 & 0.00 & 0.00 \\
\hline CIS-1-METHYL-2-ETHYLCYCLOPENTANE & 0.0 & 0.00 & 0.0 & 0.0 & 0.00 & 0.0 & 0.0 & 0.00 & 0.0 & 0.0 & 0.00 & 0.00 \\
\hline 2-METHYL-2-ETHYLHEPTANE & 0.0 & 0.00 & 0.0 & 0.0 & 0.00 & 0.0 & 0.0 & 0.00 & 0.0 & 0.0 & 0.00 & 0.00 \\
\hline 2,4-DIMETHYLHEPTANE & 0.1 & 0.06 & 0.1 & 0.2 & 3.60 & 0.3 & 0.1 & 0.33 & 0.1 & 0.1 & 0.28 & 0.19 \\
\hline 4,4-DIMETHYLHEPTANE & 0.0 & 0.00 & 0.0 & 0.0 & 0.00 & 0.0 & 0.0 & 0.00 & 0.0 & 0.0 & 0.00 & 0.00 \\
\hline CIS-1,2-DIMETHYLCYCLOHEXANE & 0.0 & 0.00 & 0.0 & 0.0 & 0.00 & 0.0 & 0.0 & 0.00 & 0.0 & 0.0 & 0.00 & 0.00 \\
\hline ETHYLCYCLOHEXANE & 0.0 & 0.00 & 0.0 & 0.0 & 0.00 & 0.0 & 0.0 & 0.00 & 0.0 & 0.0 & 0.00 & 0.00 \\
\hline PROPYLCYCLOHEXANE & 0.0 & 0.00 & 0.0 & 0.0 & 0.00 & 0.0 & 0.0 & 0.00 & 0.0 & 0.0 & 0.00 & 0.00 \\
\hline 2-METHYL-4-ETHYLHEXANE & 0.0 & 0.00 & 0.0 & 0.0 & 0.00 & 0.0 & 0.0 & 0.00 & 0.0 & 0.0 & 0.00 & 0.00 \\
\hline 2,6-DIMETHYLHEPTANE & 0.0 & 0.00 & 0.0 & 0.0 & 0.00 & 0.0 & 0.0 & 0.00 & 0.0 & 0.0 & 0.00 & 0.00 \\
\hline 1,1,3-TRIMETHYLCYCLOHEXANE & 0.0 & 0.00 & 0.0 & 0.0 & 0.00 & 0.0 & 0.0 & 0.00 & 0.0 & 0.0 & 0.00 & 0.00 \\
\hline 2,5-DIMETHYLHEPTANE & 0.0 & 0.00 & 0.0 & 0.0 & 0.00 & 0.0 & 0.0 & 0.00 & 0.0 & 0.0 & 0.00 & 0.00 \\
\hline 3,3-DIMETHYLHEPTANE & 0.0 & 0.00 & 0.0 & 0.0 & 0.00 & 0.0 & 0.0 & 0.00 & 0.0 & 0.0 & 0.00 & 0.00 \\
\hline 3,5-DIMETHYLHEPTANE & 0.0 & 0.00 & 0.0 & 0.0 & 0.00 & 0.0 & 0.0 & 0.00 & 0.0 & 0.0 & 0.00 & 0.00 \\
\hline ETHYLBENZENE & 0.1 & 0.06 & 0.2 & 0.0 & 0.00 & 0.0 & 0.0 & 0.08 & 0.1 & 0.0 & 0.05 & 0.07 \\
\hline 2,3-DIMETHYLHEPTANE & 0.0 & 0.00 & 0.0 & 0.0 & 0.00 & 0.0 & 0.0 & 0.00 & 0.0 & 0.0 & 0.00 & 0.00 \\
\hline$m-\& p-X Y L E N E$ & 0.2 & 0.08 & 1.2 & 0.0 & 0.96 & 0.4 & 0.0 & 0.16 & 0.4 & 0.1 & 0.14 & 0.63 \\
\hline 4-METHYLOCTANE & 0.0 & 0.00 & 0.0 & 0.0 & 0.00 & 0.0 & 0.0 & 0.00 & 0.0 & 0.0 & 0.00 & 0.00 \\
\hline 2-METHYLOCTANE & 0.0 & 0.00 & 0.0 & 0.0 & 0.00 & 0.0 & 0.0 & 0.00 & 0.0 & 0.0 & 0.00 & 0.00 \\
\hline 3-METHYLOCTANE & 0.0 & 0.00 & 0.0 & 0.0 & 0.00 & 0.0 & 0.0 & 0.00 & 0.0 & 0.0 & 0.00 & 0.00 \\
\hline STYAENE & 0.0 & 0.00 & 0.0 & 0.0 & 0.00 & 0.0 & 0.0 & 0.00 & 0.0 & 0.0 & 0.00 & 0.00 \\
\hline O-XYLENE & 0.1 & 0.06 & 0.8 & 0.0 & 0.00 & 0.0 & 0.0 & 0.00 & 0.0 & 0.0 & 0.05 & 0.16 \\
\hline 2,4,6-TRIMETHYLHEXANE & 0.0 & 0.00 & 0.0 & 0.0 & 0.00 & 0.0 & 0.0 & 0.00 & 0.0 & 0.0 & 0.00 & 0.00 \\
\hline 1-NONENE & 0.0 & 0.00 & 0.0 & 0.0 & 0.00 & 0.0 & 0.0 & 0.00 & 0.0 & 0.0 & 0.00 & 0.00 \\
\hline NONANE & 0.0 & 0.00 & 0.0 & 0.0 & 0.00 & 0.0 & 0.0 & 0.00 & 0.0 & 0.0 & 0.00 & 0.00 \\
\hline ISOPROPYLBENZENE (CUMENE) & 0.0 & 0.00 & 0.0 & 0.0 & 0.00 & 0.0 & 0.0 & 0.00 & 0.0 & 0.0 & 0.00 & 0.00 \\
\hline 2,2-DIMETHYLOCTANE & 0.0 & 0.00 & 0.0 & 0.0 & 0.00 & 0.0 & 0.0 & 0.00 & 0.0 & 0.0 & 0.00 & 0.00 \\
\hline 2,4-DIMETHYLOCTANE & 0.0 & 0.00 & 0.0 & 0.0 & 0.00 & 0.0 & 0.0 & 0.00 & 0.0 & 0.0 & 0.00 & 0.00 \\
\hline n-PROPYLBENZENE & 0.0 & 0.00 & 0.0 & 0.0 & 0.00 & 0.0 & 0.0 & 0.00 & 0.0 & 0.0 & 0.00 & 0.00 \\
\hline 1-METHYL-3-ETHYLBENZENE & 0.0 & 0.00 & 0.0 & 0.0 & 0.00 & 0.0 & 0.0 & 0.00 & 0.0 & 0.0 & 0.00 & 0.00 \\
\hline 1-METHYL-4-ETHYLBENZENE & 0.0 & 0.00 & 0.0 & 0.0 & 0.00 & 0.0 & 0.0 & 0.00 & 0.0 & 0.0 & 0.00 & 0.00 \\
\hline 1,3,5-TRIMETHYLBENZENE & 0.0 & 0.00 & 0.0 & 0.0 & 0.00 & 0.0 & 0.0 & 0.00 & 0.0 & 0.0 & 0.00 & 0.00 \\
\hline 1-METHYL-2-ETHYLBENZENE & 0.0 & 0.00 & 0.0 & 0.2 & 3.16 & 1.2 & 0.0 & 0.00 & 0.0 & 0.1 & 0.17 & 0.61 \\
\hline 1,2,4-TAIMETHYLBENZENE & 0.0 & 0.00 & 0.0 & 0.0 & 0.00 & 0.0 & 0.0 & 0.00 & 0.0 & 0.0 & 0.00 & 0.00 \\
\hline DECANE & 0.1 & 0.04 & 0.0 & 0.0 & 0.00 & 0.0 & 0.1 & 0.17 & 0.0 & 0.0 & 0.06 & 0.01 \\
\hline ISOBUTYLBENZENE & 0.1 & 0.03 & 0.1 & 0.0 & 0.00 & 0.0 & 0.0 & 0.16 & 0.1 & 0.0 & 0.05 & 0.05 \\
\hline
\end{tabular}




\begin{tabular}{|c|c|c|c|c|c|c|c|c|c|c|c|c|}
\hline \multirow[b]{2}{*}{ COMPOUND } & \multicolumn{3}{|c|}{ BAG 1} & \multicolumn{3}{|c|}{ BAG 2} & \multicolumn{3}{|c|}{ BAG 3} & \multicolumn{3}{|c|}{ FTP } \\
\hline & MG/MI & \begin{tabular}{|l|} 
WEIGHT \\
NMOQ \% \\
\end{tabular} & $\begin{array}{l}\text { OZONE, } \\
\text { MG/MI }\end{array}$ & MG/MI & \begin{tabular}{|l|} 
WEIGHT \\
NMOG $\%$ \\
\end{tabular} & $\begin{array}{l}\text { OZONE } \\
\text { MG/MI }\end{array}$ & MG/MI & \begin{tabular}{|l|} 
WEIGHT \\
NMOG $\%$ \\
\end{tabular} & $\begin{array}{l}\text { OZONE } \\
\text { MG/MI }\end{array}$ & MG/MI & \begin{tabular}{|l|} 
WEIGHT \\
NMOG $\%$ \\
\end{tabular} & $\begin{array}{l}\text { OZONE } \\
M G / M I\end{array}$ \\
\hline METHYLPROPYLBENZENE & 0.0 & 0.00 & 0.0 & 0.0 & 0.00 & 0.0 & 0.0 & 0.00 & 0.0 & 0.0 & 0.00 & 0.00 \\
\hline S-BUTYLBENZENE & 0.0 & 0.00 & 0.0 & 0.0 & 0.00 & 0.0 & 0.0 & 0.00 & 0.0 & 0.0 & 0.00 & 0.00 \\
\hline 1-METHYL-3-ISOPROPYLBENZENE & 0.0 & 0.00 & 0.0 & 0.0 & 0.00 & 0.0 & 0.0 & 0.00 & 0.0 & 0.0 & 0.00 & 0.00 \\
\hline 1,2,3-TRIMETHYLBENZENE & 0.0 & 0.00 & 0.0 & 0.0 & 0.00 & 0.0 & 0.0 & 0.00 & 0.0 & 0.0 & 0.00 & 0.00 \\
\hline 1-METHYL-4-ISOPROPYLBENZENE & 0.0 & 0.00 & 0.0 & 0.0 & 0.00 & 0.0 & 0.0 & 0.00 & 0.0 & 0.0 & 0.00 & 0.00 \\
\hline INDAN & 0.0 & 0.00 & 0.0 & 0.0 & 0.00 & 0.0 & 0.0 & 0.00 & 0.0 & 0.0 & 0.00 & 0.00 \\
\hline 1-METHYL-2-ISOPROPYLBENZENE & 0.0 & 0.00 & 0.0 & 0.0 & 0.00 & 0.0 & 0.0 & 0.00 & 0.0 & 0.0 & 0.00 & 0.00 \\
\hline 1,3-DIETHYLBENZENE & 0.0 & 0.00 & 0.0 & 0.0 & 0.00 & 0.0 & 0.0 & 0.00 & 0.0 & 0.0 & 0.00 & 0.00 \\
\hline 1,4-DIETHYLBENZENE & 0.0 & 0.00 & 0.0 & 0.0 & 0.00 & 0.0 & 0.0 & 0.00 & 0.0 & 0.0 & 0.00 & 0.00 \\
\hline 1-METHYL-3-N-PROPYLBENZENE & 0.0 & 0.00 & 0.0 & 0.0 & 0.00 & 0.0 & 0.0 & 0.00 & 0.0 & 0.0 & 0.00 & 0.00 \\
\hline 1-METHYL-4-N-PROPYLBENZENE & 0.0 & 0.00 & 0.0 & 0.0 & 0.00 & 0.0 & 0.0 & 0.00 & 0.0 & 0.0 & 0.00 & 0.00 \\
\hline 1,2 DIETHYLBENZENE & 0.0 & 0.00 & 0.0 & 0.0 & 0.00 & 0.0 & 0.0 & 0.00 & 0.0 & 0.0 & 0.00 & 0.00 \\
\hline 1-METHYL-2-N-PROPYLBENZENE & 0.0 & 0.00 & 0.0 & 0.0 & 0.00 & 0.0 & 0.0 & 0.00 & 0.0 & 0.0 & 0.00 & 0.00 \\
\hline 1.4-DIMETHYL-2-ETHYLBENZENE & 0.0 & 0.00 & 0.0 & 0.0 & 0.00 & 0.0 & 0.0 & 0.00 & 0.0 & 0.0 & 0.00 & 0.00 \\
\hline 1,3-DIMETHYL-4-ETHYLBENZENE & 0.0 & 0.00 & 0.0 & 0.0 & 0.00 & 0.0 & 0.0 & 0.00 & 0.0 & 0.0 & 0.00 & 0.00 \\
\hline 1,2-DIMETHYL-4-ETHYLBENZENE & 0.0 & 0.00 & 0.0 & 0.0 & 0.00 & 0.0 & 0.0 & 0.00 & 0.0 & 0.0 & 0.00 & 0.00 \\
\hline 1,3-DIMETHYL-2-ETHYLBENZENE & 0.0 & 0.00 & 0.0 & 0.0 & 0.00 & 0.0 & 0.0 & 0.00 & 0.0 & 0.0 & 0.00 & 0.00 \\
\hline UNDECANE & 0.0 & 0.00 & 0.0 & 0.0 & 0.00 & 0.0 & 0.0 & 0.00 & 0.0 & 0.0 & 0.00 & 0.00 \\
\hline 1,2-DIMETHYL-3-ETHYLBENZENE & 0.0 & 0.00 & 0.0 & 0.0 & 0.00 & 0.0 & 0.0 & 0.00 & 0.0 & 0.0 & 0.00 & 0.00 \\
\hline 1,2,4,5-TETRAMETHYLBENZENE & 0.0 & 0.00 & 0.0 & 0.0 & 0.00 & 0.0 & 0.0 & 0.00 & 0.0 & 0.0 & 0.00 & 0.00 \\
\hline 2-METHYLBUTYLBENZENE (BOC AMYLBENZENE) & 0.0 & 0.00 & 0.0 & 0.0 & 0.00 & 0.0 & 0.0 & 0.00 & 0.0 & 0.0 & 0.00 & 0.00 \\
\hline 3,4 DIMETHYLCUMENE & 0.0 & 0.00 & 0.0 & 0.0 & 0.00 & 0.0 & 0.0 & 0.00 & 0.0 & 0.0 & 0.00 & 0.00 \\
\hline 1,2,3,6-TETRAMETHYLBENZENE & 0.0 & 0.00 & 0.0 & 0.0 & 0.00 & 0.0 & 0.0 & 0.00 & 0.0 & 0.0 & 0.00 & 0.00 \\
\hline TERT-1-BUT-2-METHYLBENZENE & 0.0 & 0.00 & 0.0 & 0.0 & 0.00 & 0.0 & 0.0 & 0.00 & 0.0 & 0.0 & 0.00 & 0.00 \\
\hline 1,2,3,4-TETRAMETHYLBENZENE & $\overline{0.0}$ & 0.00 & 0.0 & 0.0 & 0.00 & 0.0 & 0.0 & 0.00 & 0.0 & 0.0 & 0.00 & 0.00 \\
\hline N-PENT-BENZENE & 0.0 & 0.00 & 0.0 & 0.0 & 0.00 & 0.0 & 0.0 & 0.00 & 0.0 & 0.0 & 0.00 & 0.00 \\
\hline TERT-1-BUT-3,6-DIMETHYLBENZENE & 0.0 & 0.00 & 0.0 & 0.0 & 0.00 & 0.0 & 0.0 & 0.00 & 0.0 & 0.0 & 0.00 & 0.00 \\
\hline NAPHTHALENE & 0.0 & 0.00 & 0.0 & 0.0 & 0.00 & 0.0 & 0.0 & 0.00 & 0.0 & 0.0 & 0.00 & 0.00 \\
\hline DODECANE & 0.0 & 0.00 & 0.0 & 0.0 & 0.00 & 0.0 & 0.0 & 0.00 & 0.0 & 0.0 & 0.00 & 0.00 \\
\hline UNIDENTIFIED C9-C12+ & 0.2 & 0.09 & 0.6 & 0.0 & 0.14 & 0.0 & 0.0 & 0.00 & 0.0 & 0.0 & 0.08 & 0.15 \\
\hline FOAMALDEHYDE & 1.6 & 0.83 & 11.6 & 0.3 & 4.89 & 1.8 & 0.4 & 1.26 & 2.7 & 0.6 & 1.11 & 4.07 \\
\hline ACETALDEHYDE & 0.7 & 0.34 & 3.7 & 0.0 & 0.16 & 0.0 & 0.0 & 10.00 & 0.0 & 0.1 & 0.28 & 0.79 \\
\hline ACROLEIN & 0.1 & 0.06 & 0.7 & 0.0 & 0.00 & 0.0 & 0.0 & 0.00 & 0.0 & 0.0 & 0.04 & 0.16 \\
\hline ACETONE & 0.4 & 0.23 & 0.2 & 0.0 & 0.84 & 0.0 & 0.1 & 0.19 & 0.0 & 0.1 & 0.25 & 0.07 \\
\hline PROPIONALDEHYDE & 0.3 & 0.17 & 2.1 & 0.2 & 4.15 & 1.4 & 0.2 & 0.79 & 1.6 & 0.2 & 0.48 & 1,60 \\
\hline CROTONALDEHYDE & 0.0 & 0.00 & 0.0 & 0.4 & 6.81 & 2.0 & 0.0 & 0.00 & 0.0 & 0.2 & 0.36 & 1.01 \\
\hline ISOBUTYRALDEHYDE & 0.0 & 0.02 & 0.2 & 0.0 & 0.00 & 0.0 & 0.0 & 0.00 & 0.0 & 0.0 & 0.02 & 0.05 \\
\hline METHYLETHYL KETONE & 0.0 & 0.02 & 0.0 & 0.0 & 0.00 & 0.0 & 0.0 & 0.00 & 0.0 & 0.0 & 0.02 & 0.01 \\
\hline BENZALDEHYDE & 0.0 & 0.00 & -0.0 & 0.0 & 0.79 & -0.0 & 0.2 & 0.51 & -0.1 & 0.1 & 0.12 & -0.04 \\
\hline HEXANALDEHYOE & 0.0 & 0.00 & 0.0 & 0.0 & 0.00 & 0.0 & 0.0 & 0.00 & 0.0 & 0.0 & 0.00 & 0.00 \\
\hline
\end{tabular}


TEST NO: 50NB/60NP-02S

TEST CAR: 1994 PONTIAC GRANDAM

TEST DATE: $9 / 26 / 95$

TEST FUEL: $60 \%$ BUTANE/50\% PROPANE

\begin{tabular}{|c|c|c|c|c|c|c|c|c|c|c|c|c|}
\hline \multirow[b]{2}{*}{ COMPOUND } & \multicolumn{3}{|c|}{$\overline{B A G 1}$} & \multicolumn{3}{|c|}{$\overline{B A G 2}$} & \multicolumn{3}{|c|}{ BAG 3} & \multicolumn{3}{|c|}{$\overline{F T P}$} \\
\hline & MG/MI & $\begin{array}{l}\text { WEIGHT } \\
\text { NMOG \% }\end{array}$ & $\begin{array}{l}\text { OZONE, } \\
\text { MGMI }\end{array}$ & MG/MI & $\begin{array}{l}\text { WEIGHT } \\
\text { NMOQ } \%\end{array}$ & $\begin{array}{l}\text { OZONE } \\
\text { MG/MI }\end{array}$ & MGMI & $\begin{array}{l}\text { WEIGHT } \\
\text { NMOG } \%\end{array}$ & $\begin{array}{l}\text { OZONE } \\
\text { MG/MI }\end{array}$ & MG/MI & $\begin{array}{l}\text { WEIGHT } \\
\text { NMOG \% }\end{array}$ & $\begin{array}{l}\text { OZONE } \\
\text { MG/MI }\end{array}$ \\
\hline METHANE & 12.6 & & 0.2 & 1.3 & & 0.0 & 16.2 & & 0.2 & 7.4 & & 0.11 \\
\hline ETHANE & 2.1 & 1.15 & 0.5 & 0.0 & 0.14 & 0.0 & 2.0 & 6.49 & 0.5 & 1.0 & 1.98 & 0.25 \\
\hline ETHYLENE & 11.3 & 6.19 & 82.3 & 0.0 & 0.38 & 0.1 & 1.3 & 3.67 & 9.7 & 2.7 & 5.45 & 19.85 \\
\hline PROPANE & 104.0 & 56.89 & 49.9 & 0.0 & 0.00 & 0.0 & 22.1 & 60.73 & 10.6 & 27.7 & 55.37 & 13.29 \\
\hline PROPYLENE & 5.7 & 3.14 & 53.8 & 0.0 & 0.00 & 0.0 & 0.4 & 1.09 & 3.7 & 1.3 & 2.60 & 12.20 \\
\hline ACETYLENE & 2.3 & 1.24 & 1.1 & 0.0 & 0.18 & 0.0 & 0.0 & 0.02 & 0.0 & 0.5 & 0.95 & 0.24 \\
\hline PROPADIENE & 0.0 & 0.00 & 0.0 & 0.0 & 0.00 & 0.0 & 0.0 & 0.00 & 0.0 & 0.0 & 0.00 & 0.00 \\
\hline BUTANE & 46.2 & 25.32 & 47.1 & 0.0 & 0.00 & 0.0 & 8.4 & 23.20 & 8.6 & 11.9 & 23.85 & 12.16 \\
\hline TRANS-2-BUTENE & 0.1 & 0.07 & 1.2 & 0.0 & 0.00 & 0.0 & 0.0 & 0.00 & 0.0 & 0.0 & 0.05 & 0.25 \\
\hline 1-BUTENE & 0.3 & 0.16 & 2.6 & 0.0 & 0.00 & 0.0 & 0.0 & 0.00 & 0.0 & 0.1 & 0.12 & 0.53 \\
\hline 2-METHYLPROPENE (ISOBUTYLENE) & 0.0 & 0.00 & 0.0 & 0.0 & 0.00 & 0.0 & 0.0 & 0.00 & 0.0 & 0.0 & 0.00 & 0.00 \\
\hline 2,2-DIMETHYLPROPANE (NEOPENTANE) & 0.0 & 0.00 & 0.0 & 0.0 & 0.00 & 0.0 & 0.0 & 0.00 & 0.0 & 0.0 & 0.00 & 0.00 \\
\hline PROPYNE & 0.0 & 0.00 & 0.0 & 0.0 & 0.00 & 0.0 & 0.0 & 0.00 & 0.0 & 0.0 & 0.00 & 0.00 \\
\hline 1,3-BUTADIENE & 0.1 & 0.04 & 0.7 & 0.0 & 0.00 & 0.0 & 0.0 & 0.00 & 0.0 & 0.0 & 0.03 & 0.16 \\
\hline 2-METHYLPROPANE (ISOBUTANE) & 0.2 & 0.10 & 0.2 & 0.0 & 0.00 & 0.0 & 0.0 & 0.08 & 0.0 & 0.0 & 0.00 & 0.05 \\
\hline 1-BUTYNE & 0.0 & 0.00 & 0.0 & 0.0 & 0.00 & 0.0 & 0.0 & 0.00 & 0.0 & 0.0 & 0.00 & 0.00 \\
\hline METHANOL & 0.0 & 0.00 & 0.0 & 0.0 & 0.00 & 0.0 & 0.0 & 0.00 & 0.0 & 0.0 & 0.00 & 0.00 \\
\hline CIS-2-BUTENE & 0.0 & 0.00 & 0.0 & 0.0 & 0.00 & 0.0 & 0.0 & 0.00 & 0.0 & 0.0 & 0.00 & 0.00 \\
\hline 3-METHYL-1-BUTENE & 0.0 & 0.00 & 0.0 & 0.0 & 0.00 & 0.0 & 0.0 & 0.00 & 0.0 & 0.0 & 0.00 & 0.00 \\
\hline ETHANOL & 0.0 & 0.00 & 0.0 & 0.0 & 0.00 & 0.0 & 0.0 & 0.00 & 0.0 & 0.0 & 0.00 & 0.00 \\
\hline 2-METHYLBUTANE (ISOPENTANE) & 2.5 & 1.37 & 3.5 & 0.0 & 0.00 & 0.0 & 0.0 & 0.00 & 0.0 & 0.5 & 1.04 & 0.72 \\
\hline 2-BUTYNE & 0.0 & 0.00 & 0.0 & 0.0 & 0.00 & 0.0 & 0.0 & 0.00 & 0.0 & 0.0 & 0.00 & 0.00 \\
\hline 1-PENTENE & 0.0 & 0.00 & 0.0 & 0.0 & 0.00 & 0.0 & 0.0 & 0.00 & 0.0 & 0.0 & 0.00 & 0.00 \\
\hline 2-METHYL-1-BUTENE & 0.0 & 0.00 & 0.0 & 0.0 & 0.00 & 0.0 & 0.0 & 0.00 & 0.0 & 0.0 & 0.00 & 0.00 \\
\hline PENTANE & 0.2 & 0.09 & 0.2 & 0.0 & 0.00 & 0.0 & 0.0 & 0.00 & 0.0 & 0.0 & 0.07 & 0.04 \\
\hline UNIDENTIFIED C5 OLEFINS & 0.0 & 0.00 & 0.0 & 0.0 & 0.00 & 0.0 & 0.0 & 0.00 & 0.0 & 0.0 & 0.00 & 0.00 \\
\hline 2-METHYL-1,3-BUTADIENE & 0.0 & 0.00 & 0.0 & 0.0 & 0.00 & 0.0 & 0.0 & 0.00 & 0.0 & 0.0 & 0.00 & 0.00 \\
\hline TRANS-2-PENTENE & 0.0 & 0.00 & 0.0 & 0.0 & 0.00 & 0.0 & 0.0 & 0.00 & 0.0 & 0.0 & 0.00 & 0.00 \\
\hline 3,3-DIMETHYL-1-BUTENE & 0.0 & 0.00 & 0.0 & 0.0 & 0.00 & 0.0 & 0.0 & 0.00 & 0.0 & 0.0 & 0.00 & 0.00 \\
\hline CIS-2-PENTENE & 0.0 & 0.00 & 0.0 & 0.0 & 0.00 & 0.0 & 0.0 & 0.00 & 0.0 & 0.0 & 0.00 & 0.00 \\
\hline 2-METHYL-2-BUTENE & 0.1 & 0.04 & 0.5 & 0.0 & 0.27 & 0.1 & 0.0 & 0.00 & 0.0 & 0.0 & 0.04 & 0.14 \\
\hline CYCLOPENTADIENE & 0.0 & 0.00 & 0.0 & 0.0 & 0.00 & 0.0 & 0.0 & 0.00 & 0.0 & 0.0 & 0.00 & 0.00 \\
\hline 2,2-DIMETHYLBUTANE & 0.1 & 0.05 & 0.1 & 0.0 & 0.00 & 0.0 & 0.0 & 0.00 & 0.0 & 0.0 & 0.04 & 0.02 \\
\hline CYCLOPENTENE & 0.0 & 0.00 & 0.0 & 0.0 & 0.00 & 0.0 & 0.0 & 0.00 & 0.0 & 0.0 & 0.00 & 0.00 \\
\hline 4-METHYL-1-PENTENE & 0.0 & 0.00 & 0.0 & 0.0 & 0.00 & 0.0 & 0.0 & 0.00 & 0.0 & 0.0 & 0.00 & 0.00 \\
\hline 3-METHYL-1-PENTENE & 0.0 & 0.00 & 0.0 & 0.0 & 0.00 & 0.0 & 0.0 & 0.00 & 0.0 & 0.0 & 0.00 & 0.00 \\
\hline CYCLOPENTANE & 0.0 & 0.00 & 0.0 & 0.0 & 0.00 & 0.0 & 0.0 & 0.00 & 0.0 & 0.0 & 0.00 & 0.00 \\
\hline 2,3-DIMETHYLBUTANE & 0.1 & 0.07 & 0.1 & 0.0 & 0.35 & 0.0 & 0.0 & 0.00 & 0.0 & 0.0 & 0.07 & 0.04 \\
\hline MTBE & 0.0 & 0.00 & 0.0 & 0.0 & 0.00 & 0.0 & 0.0 & 0.00 & 0.0 & 0.0 & 0.00 & 0.00 \\
\hline
\end{tabular}




\begin{tabular}{|c|c|c|c|c|c|c|c|c|c|c|c|c|}
\hline \multirow[b]{2}{*}{ COMPOUND } & \multicolumn{3}{|c|}{ BAG 1} & \multicolumn{3}{|c|}{ BAG 2 } & \multicolumn{3}{|c|}{ BAQ 3} & \multicolumn{3}{|c|}{ FTP } \\
\hline & MG/MI & $\begin{array}{l}\text { WEIGHT } \\
\text { NMOG } \%\end{array}$ & $\begin{array}{l}\text { OZONE, } \\
\text { MG/MI }\end{array}$ & MG/MI & $\begin{array}{l}\text { WEIGHT } \\
\text { NMOG } \%\end{array}$ & $\begin{array}{l}\text { OZONE } \\
\text { MG/MI }\end{array}$ & MG/MI & \begin{tabular}{|l|} 
WEIGHT \\
NMOG $\%$
\end{tabular} & $\begin{array}{l}\text { OZONE } \\
\text { MG/MI }\end{array}$ & MG/MI & $\begin{array}{l}\text { WEIGHT } \\
\text { NMOG \% }\end{array}$ & $\begin{array}{l}\text { OZONE } \\
\text { MG/MI }\end{array}$ \\
\hline 2,3-DIMETHYL-1-BUTENE & 0.0 & 0.00 & 0.0 & 0.0 & 0.00 & 0.0 & 0.0 & 0.00 & 0.0 & 0.0 & 0.00 & 0.00 \\
\hline 4-METHYL-CIS-2-PENTENE & 0.0 & 0.00 & 0.0 & 0.0 & 0.00 & 0.0 & 0.0 & 0.00 & 0.0 & 0.0 & 0.00 & 0.00 \\
\hline 2-METHYLPENTANE & 0.4 & 0.20 & 0.6 & 0.0 & 1.22 & 0.1 & 0.0 & 0.00 & 0.0 & 0.1 & 0.20 & 0.15 \\
\hline 4-METHYL-TRANS-2-PENTENE & 0.0 & 0.00 & 0.0 & 0.0 & 0.00 & 0.0 & 0.0 & $1 \quad 0.00$ & 0.0 & 0.0 & 0.00 & 0.00 \\
\hline 3-METHYLPENTANE & 0.2 & 0.10 & 0.3 & 0.0 & 0.10 & 0.0 & 0.0 & 0.12 & 0.1 & 0.1 & 0.10 & 0.08 \\
\hline 2-METHYL-1-PENTENE & 0.0 & 0.00 & 0.0 & 0.0 & 0.00 & 0.0 & 0.0 & 0.00 & 0.0 & 0.0 & 0.00 & 0.00 \\
\hline 1-HEXENE & 0.0 & 0.00 & 0.0 & 0.0 & 0.00 & 0.0 & 0.0 & 0.00 & 0.0 & 0.0 & 0.00 & 0.00 \\
\hline HEXANE & 0.1 & 0.06 & 0.1 & 0.1 & 2.69 & 0.1 & 0.0 & 0.00 & 0.0 & 0.1 & 0.15 & 0.08 \\
\hline UNIDENTIFIED C6 OLEFINS & 0.0 & 0.00 & 0.0 & 0.0 & 0.00 & 0.0 & 0.0 & 0.00 & 0.0 & 0.0 & 0.00 & 0.00 \\
\hline TRANS-3-HEXENE & 0.0 & 0.00 & 0.0 & 0.0 & 0.00 & 0.0 & 0.0 & 0.00 & 0.0 & 0.0 & 0.00 & 0.00 \\
\hline GIS-3-HEXENE & 0.1 & 0.05 & 0.6 & 0.0 & 0.00 & 0.0 & 0.0 & 0.00 & 0.0 & 0.0 & 0.04 & 0.12 \\
\hline TRANS-2-HEXENE & 0.0 & 0.00 & 0.0 & 0.0 & 0.00 & 0.0 & 0.0 & 0.00 & 0.0 & 0.0 & 0.00 & 0.00 \\
\hline 3-METHYL-TRANS-2-PENTENE & 0.1 & 0.05 & 0.6 & 0.0 & 0.00 & 0.0 & 0.0 & 0.00 & 0.0 & 0.0 & 0.04 & 0.13 \\
\hline 2-METHYL-2-PENTENE & 0.0 & 0.00 & 0.0 & 0.0 & 0.00 & 0.0 & 0.0 & 0.00 & 0.0 & 0.0 & 0.00 & 0.00 \\
\hline 3-METHYLCYCLOPENTENE & 0.0 & 0.00 & 0.0 & 0.0 & 0.00 & 0.0 & 0.0 & 0.00 & 0.0 & 0.0 & 0.00 & 0.00 \\
\hline CIS-2-HEXENE & 0.0 & 0.00 & 0.0 & 0.0 & 0.00 & 0.0 & 0.0 & 0.00 & 0.0 & 0.0 & 0.00 & 0.00 \\
\hline ETBE & 0.0 & 0.00 & 0.0 & 0.0 & 0.00 & 0.0 & 0.0 & 0.00 & 0.0 & 0.0 & 0.00 & 0.00 \\
\hline 3-METHYL-CIS-2-PENTENE & 0.0 & 0.00 & 0.0 & 0.0 & 0.00 & 0.0 & 0.0 & 0.00 & 0.0 & 0.0 & 0.00 & 0.00 \\
\hline 2,2-DIMETHYLPENTANE & 0.1 & 0.04 & 0.1 & 0.0 & 0.00 & 0.0 & 0.0 & 0.00 & 0.0 & 0.0 & 0.03 & 0.02 \\
\hline METHYLCYCLOPENTANE & 0.0 & 0.00 & 0.0 & 0.0 & 0.00 & 0.0 & 0.0 & 0.00 & 0.0 & 0.0 & 0.00 & 0.00 \\
\hline 2,4-DIMETHYLPENTANE & 0.0 & 0.03 & 0.1 & 0.0 & 0.01 & 0.0 & 0.0 & 0.02 & 0.0 & 0.0 & $\overline{0.02}$ & 0.02 \\
\hline 2,3,3-TRIMETHYL-1-BUTENE & 0.0 & 0.00 & 0.0 & 0.0 & 0.00 & 0.0 & 0.0 & 0.00 & 0.0 & 0.0 & 0.00 & 0.00 \\
\hline 2,2,3-TAIMETHYLBUTANE & 0.0 & 0.00 & 0.0 & 0.0 & 0.00 & 0.0 & 0.0 & 0.00 & 0.0 & 0.0 & 0.00 & 0.00 \\
\hline 3,4-DIMETHYL-1-PENTENE & 0.0 & 0.00 & 0.0 & 0.0 & 0.00 & 0.0 & 0.0 & 0.00 & 0.0 & 0.0 & 0.00 & 0.00 \\
\hline 1-METHYLCYCLOPENTENE & 0.0 & 0.00 & 0.0 & 0.0 & 0.00 & 0.0 & 0.0 & 0.00 & 0.0 & 0.0 & 0.00 & 0.00 \\
\hline BENZENE & 0.3 & 0.18 & 0.1 & 0.3 & 6.62 & 0.1 & 0.1 & 0.26 & 0.0 & 0.2 & 0.46 & 0.10 \\
\hline 3-METHYL-1-HEXENE & 0.0 & 0.00 & 0.0 & 0.0 & 0.00 & 0.0 & 0.0 & 0.00 & 0.0 & 0.0 & 0.00 & 0.00 \\
\hline 3,3-DIMETHYLPENTANE & 0.0 & 0.00 & 0.0 & 0.0 & 0.00 & 0.0 & 0.0 & 0.00 & 0.0 & 0.0 & 0.00 & 0.00 \\
\hline CYCLOHEXANE & 0.0 & 0.00 & 0.0 & 0.0 & 0.00 & 0.0 & 0.0 & 0.00 & 0.0 & 0.0 & 0.00 & 0.00 \\
\hline 2-METHYLHEXANE & 0.0 & 0.00 & 0.0 & 0.0 & 0.00 & 0.0 & 0.0 & 0.00 & 0.0 & 0.0 & 0.00 & 0.00 \\
\hline 2,3-DIMETHYLPENTANE & 0.2 & 0.12 & 0.3 & 0.0 & 0.00 & 0.0 & 0.0 & 0.00 & 0.0 & 0.0 & 0.09 & 0.07 \\
\hline 1,1-DIMETHYLCYCLOPENTANE & 0.0 & 0.00 & 0.0 & 0.0 & 0.00 & 0.0 & 0.0 & 10.00 & 0.0 & 0.0 & 0.00 & 0.00 \\
\hline CYCLOHEXENE & 0.0 & 0.00 & 0.0 & 0.0 & 0.00 & 0.0 & 0.0 & 0.00 & 0.0 & 0.0 & 0.00 & 0.00 \\
\hline 3-METHYLHEXANE & 0.1 & 0.08 & 0.1 & 0.0 & 0.00 & 0.0 & 0.0 & 0.02 & 0.0 & 0.0 & 0.05 & 0.03 \\
\hline CIS-1,3-DIMETHYLCYCLOPENTANE & 0.0 & 0.00 & 0.0 & 0.0 & 0.00 & 0.0 & 0.0 & 0.00 & 0.0 & 0.0 & 0.00 & 0.00 \\
\hline 3-ETHYLPENTANE & 0.0 & 0.00 & 0.0 & 0.0 & 0.00 & 0.0 & 0.0 & 0.00 & 0.0 & 0.0 & 0.00 & 0.00 \\
\hline TRANS-1,2-DIMETHYLCYCLOPENTANE & 0.0 & 0.00 & 0.0 & 0.0 & 0.00 & 0.0 & 0.0 & 0.00 & 0.0 & 0.0 & 0.00 & 0.00 \\
\hline TRANS-1,3-DIMETHYLCYCLOPENTANE & 0.0 & 0.00 & 0.0 & 0.0 & 0.00 & 0.0 & 0.0 & 0.00 & 0.0 & 0.0 & 0.00 & 0.00 \\
\hline 1-HEPTENE & 0.0 & $\overline{0.00}$ & 0.0 & 0.0 & 0.00 & 0.0 & 0.0 & 0.00 & 0.0 & 0.0 & 0.00 & 0.00 \\
\hline 2,2,4-TRIMETHYLPENTANE & 0.0 & 0.02 & 0.0 & 0.0 & 0.00 & 0.0 & 0.0 & 0.04 & 0.0 & 0.0 & 0.03 & 0.01 \\
\hline 2-METHYL-1-HEXENE & 0.0 & 0.00 & 0.0 & 0.0 & 0.00 & 0.0 & 0.0 & 0.00 & 0.0 & 0.0 & 0.00 & 0.00 \\
\hline TRANS-3-HEPTENE & 0.0 & 0.00 & 0.0 & 0.0 & 0.00 & 0.0 & 0.0 & 0.00 & 0.0 & 0.0 & 0.00 & 0.00 \\
\hline HEPTANE & 0.1 & 0.08 & 0.1 & 0.1 & 3.74 & 0.1 & 0.0 & 0.00 & 0.0 & 0.1 & 0,20 & 0.08 \\
\hline UNIDENTIFIED C7 & 0.0 & 0.00 & 0.0 & 0.0 & 0.00 & 0.0 & 0.0 & 0.00 & 0.0 & 0.0 & 0.00 & 0.00 \\
\hline
\end{tabular}




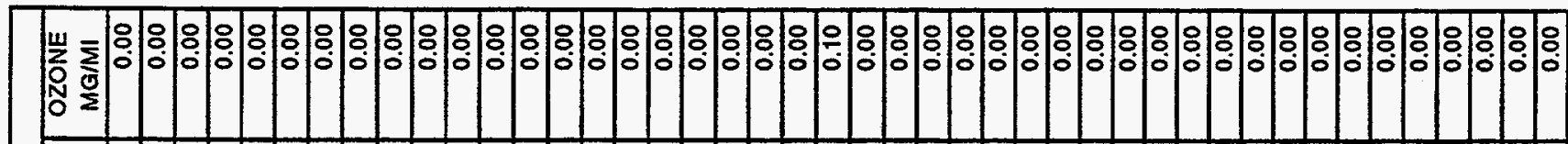

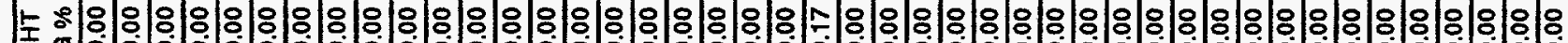

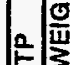

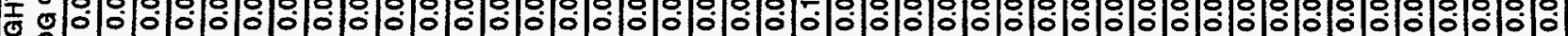
敦要 $\sum_{2}$

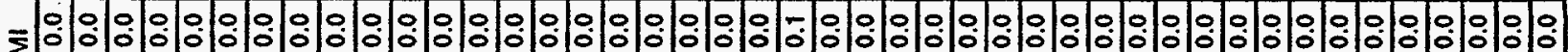
$\frac{\mathrm{O}}{2}$

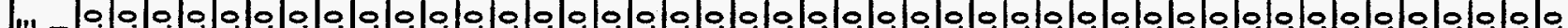
崖 N

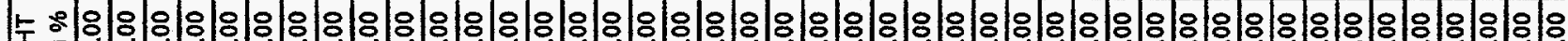
n

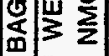

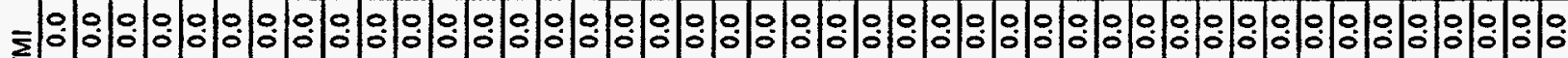
$\stackrel{0}{2}$

崖 帘

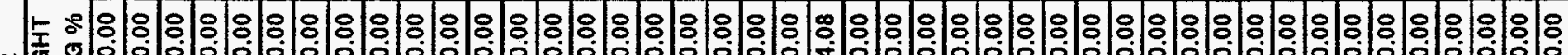

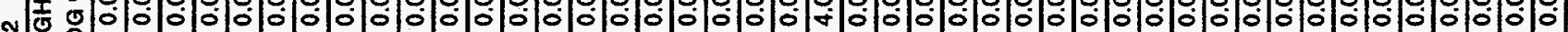
它岗

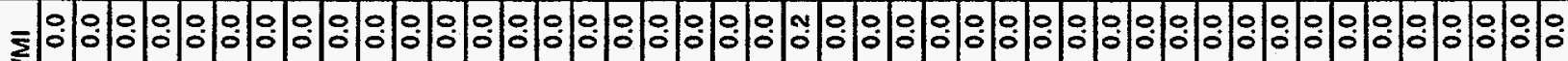
$\frac{0}{2}$

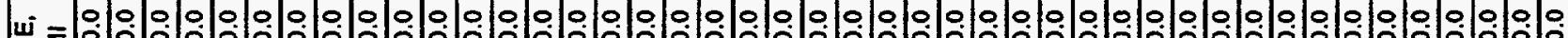

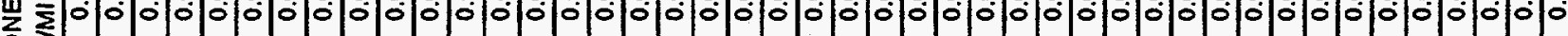
经

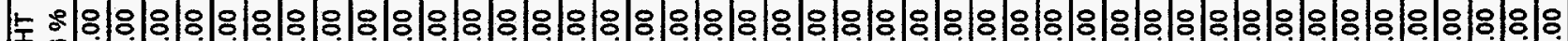
- I

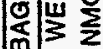

纹

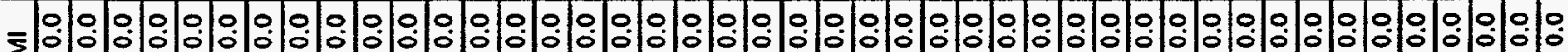
$\frac{0}{2}$

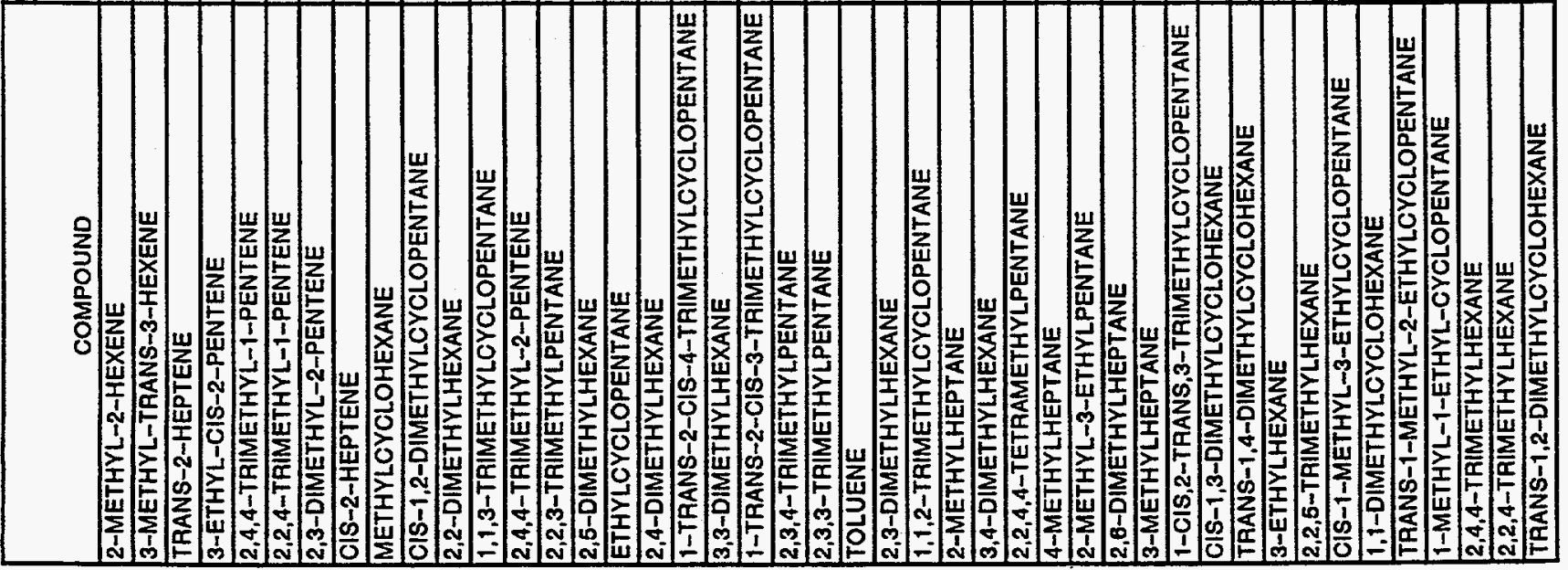




\begin{tabular}{|c|c|c|c|c|c|c|c|c|c|c|c|c|}
\hline \multirow[b]{2}{*}{ COMPOUND } & \multicolumn{3}{|c|}{ BAG 1} & \multicolumn{3}{|c|}{ BAG 2} & \multicolumn{3}{|c|}{ BAG 3} & \multicolumn{3}{|c|}{ FTP } \\
\hline & MG/MI & $\begin{array}{l}\text { WEIGHT } \\
\text { NMOQ \% }\end{array}$ & $\begin{array}{l}\text { OZONE, } \\
\text { MG/MI }\end{array}$ & MG/MI & $\begin{array}{l}\text { WEIGHT } \\
\text { NMOG } \%\end{array}$ & $\begin{array}{l}\text { OZONE } \\
\text { MG/MI }\end{array}$ & MG/MI & $\begin{array}{l}\text { WEIGHT } \\
\text { NMOG } \%\end{array}$ & $\begin{array}{l}\text { OZONE } \\
\text { MG/MI }\end{array}$ & MGMI & $\begin{array}{l}\text { WEIGHT } \\
\text { NMOG } \%\end{array}$ & $\begin{array}{l}\text { OZONE } \\
\text { MG/MI }\end{array}$ \\
\hline 1-OCTENE & 0.0 & 0.00 & 0.0 & 0.0 & 0.00 & 0.0 & 0.0 & 0.00 & 0.0 & 0.0 & 0.00 & 0.00 \\
\hline TRANS-4-OCTENE & 0.0 & 0.00 & 0.0 & 0.0 & 0.00 & 0.0 & 0.0 & 0.00 & 0.0 & 0.0 & 0.00 & 0.00 \\
\hline OCTANE & 0.0 & 0.00 & 0.0 & 0.0 & 0.00 & 0.0 & 0.0 & 0.00 & 0.0 & 0.0 & 0.00 & 0.00 \\
\hline UNIDENTIFIED C8 & 0.1 & 0.05 & 0.3 & 0.0 & 0.00 & 0.0 & 0.0 & 0.00 & 0.0 & 0.0 & 0.04 & 0.07 \\
\hline TRANS-2-OCTENE & 0.0 & 0.00 & 0.0 & 0.0 & 0.00 & 0.0 & 0.0 & 0.00 & 0.0 & 0.0 & 0.00 & 0.00 \\
\hline TRANS-1,3-DIMETHYLCYCLOHEXANE & 0.0 & 0.00 & 0.0 & 0.0 & 0.00 & 0.0 & 0.0 & 0.00 & 0.0 & 0.0 & 0.00 & 0.00 \\
\hline CIS-1,4-DIMETHYLCYCLOHEXANE & 0.0 & 0.00 & 0.0 & 0.0 & 0.00 & 0.0 & 0.0 & 0.00 & 0.0 & 0.0 & 0.00 & 0.00 \\
\hline CIS-2-OCTENE & 0.0 & 0.00 & 0.0 & 0.0 & 0.00 & 0.0 & 0.0 & 0.00 & 0.0 & 0.0 & 0.00 & 0.00 \\
\hline 2,3,6-TRIMETHYLHEXANE & 0.0 & 0.00 & 0.0 & 0.0 & 0.00 & 0.0 & 0.0 & 0.00 & 0.0 & 0.0 & 0.00 & 0.00 \\
\hline CIS-1-METHYL-2-ETHYLCYCLOPENTANE & 0.0 & 0.00 & 0.0 & 0.0 & 0.00 & 0.0 & 0.0 & 0.00 & 0.0 & 0.0 & 0.00 & 0.00 \\
\hline 2-METHYL-2-ETHYLHEPTANE & 0.0 & 0.00 & 0.0 & 0.0 & 0.00 & 0.0 & 0.0 & 0.00 & 0.0 & 0.0 & 0.00 & 0.00 \\
\hline 2,4-DIMETHYLHEPTANE & 0.0 & 0.00 & 0.0 & 0.2 & 4.16 & 0.2 & 0.0 & 0.00 & 0.0 & 0.1 & 0.17 & 0.12 \\
\hline 4,4-DIMETHYLHEPTANE & 0.0 & 0.00 & 0.0 & 0.0 & 0.00 & 0.0 & 0.0 & 0.00 & 0.0 & 0.0 & 0.00 & 0.00 \\
\hline CIS-1,2-DIMETHYLCYCLOHEXANE & 0.0 & 0.00 & 0.0 & 0.0 & 0.00 & 0.0 & 0.0 & 0.00 & 0.0 & 0.0 & 0.00 & 0.00 \\
\hline ETHYLCYCLOHEXANE & 0.0 & 0.00 & 0.0 & 0.0 & 0.00 & 0.0 & 0.0 & 0.00 & 0.0 & 0.0 & 0.00 & 0.00 \\
\hline PROPYLCYCLOHEXANE & 0.0 & 0.00 & 0.0 & 0.0 & 0.00 & 0.0 & 0.0 & 0.00 & 0.0 & 0.0 & 0.00 & 0.00 \\
\hline 2-METHYL-4-ETHYLHEXANE & 0.0 & 0.00 & 0.0 & 0.0 & 0.00 & 0.0 & 0.0 & 0.00 & 0.0 & 0.0 & 0.00 & 0.00 \\
\hline 2,6-DIMETHYLHEPTANE & 0.0 & 0.00 & 0.0 & 0.0 & 0.00 & 0.0 & 0.0 & 0.00 & 0.0 & 0.0 & 0.00 & 0.00 \\
\hline 1,1,3-TAIMETHYLCYCLOHEXANE & 0.0 & 0.00 & 0.0 & 0.0 & 0.00 & 0.0 & 0.0 & 0.00 & 0.0 & 0.0 & 0.00 & 0.00 \\
\hline 2,5-DIMETHYLHEPTANE & 0.0 & 0.00 & 0.0 & 0.0 & 0.00 & 0.0 & 0.0 & 0.00 & 0.0 & 0.0 & 0.00 & 0.00 \\
\hline 3,3-DIMETHYLLEPTANE & 0.0 & 0.00 & 0.0 & 0.0 & 0.00 & 0.0 & 0.0 & 0.00 & 0.0 & 0.0 & 0.00 & 0.00 \\
\hline 3,5-DIMETHYLHEPTANE & 0.0 & 0.00 & 0.0 & 0.0 & 0.00 & 0.0 & 0.0 & 0.00 & 0.0 & 0.0 & 0.00 & 0.00 \\
\hline ETHYLBENZENE & 0.0 & 0.01 & 0.0 & 0.0 & 0.00 & 0.0 & 0.0 & 0.00 & 0.0 & 0.0 & 0.01 & 0.01 \\
\hline 2,3-DIMETHYLHEPTANE & 0.0 & 0.00 & 0.0 & 0.0 & 0.00 & 0.0 & 0.0 & 0.00 & 0.0 & 0.0 & 0.00 & 0.00 \\
\hline$m-\& p-X Y L E N E$ & 0.0 & 0.00 & 0.0 & 0.0 & 0.00 & 0.0 & 0.0 & 0.00 & 0.0 & 0.0 & 0.00 & 0.00 \\
\hline 4-METHYLOCTANE & 0.0 & 0.00 & 0.0 & 0.0 & 0.00 & 0.0 & 0.0 & 0.00 & 0.0 & 0.0 & 0.00 & 0.00 \\
\hline 2-METHYLOCTANE & 0.0 & 0.00 & 0.0 & 0.0 & 0.00 & 0.0 & 0.0 & 0.00 & 0.0 & 0.0 & 0.00 & 0.00 \\
\hline 3-METHYLOCTANE & 0.0 & 0.00 & 0.0 & 0.0 & 0.00 & 0.0 & 0.0 & 0.00 & 0.0 & 0.0 & 0.00 & 0.00 \\
\hline STYRENE & 0.0 & 0.00 & 0.0 & 0.0 & 0.00 & 0.0 & 0.0 & 0.00 & 0.0 & 0.0 & 0.00 & 0.00 \\
\hline O-XYLENE & 0.0 & 0.00 & 0.0 & 0.0 & 0.00 & 0.0 & 0.1 & 0.24 & 0.6 & 0.0 & 0.05 & 0.15 \\
\hline 2,4,6-TRIMETHYLHEXANE & 0.0 & 0.00 & 0.0 & 0.0 & 0.00 & 0.0 & 0.0 & 0.00 & 0.0 & 0.0 & 0.00 & 0.00 \\
\hline 1-NONENE & 0.0 & 0.00 & 0.0 & 0.0 & 0.00 & 0.0 & 0.0 & 0.00 & 0.0 & 0.0 & 0.00 & 0.00 \\
\hline NONANE & 0.0 & 0.00 & 0.0 & 0.0 & 0.00 & 0.0 & 0.0 & 0.00 & 0.0 & 0.0 & 0.00 & 0.00 \\
\hline ISOPROPYLBENZENE (CUMENE) & 0.0 & 0.00 & 0.0 & 0.0 & 0.00 & 0.0 & 0.0 & 0.00 & 0.0 & 0.0 & 0.00 & 0.00 \\
\hline 2,2-DIMETHYLOCTANE & 0.0 & 0.00 & 0.0 & 0.0 & 0.00 & 0.0 & 0.0 & 0.00 & 0.0 & 0.0 & 0.00 & 0.00 \\
\hline 2,4-DIMETHYLOCTANE & 0.0 & 0.00 & 0.0 & 0.0 & 0.00 & 0.0 & 0.0 & 0.00 & 0.0 & 0.0 & 0.00 & 0.00 \\
\hline n-PROPYLBENZENE & 0.0 & 0.00 & 0.0 & 0.0 & 0.00 & 0.0 & 0.0 & 0.00 & 0.0 & 0.0 & 0.00 & 0.00 \\
\hline 1-METHYL-3-ETHYLBENZENE & 0.0 & 0.00 & 0.0 & 0.0 & 0.00 & 0.0 & 0.0 & 0.05 & 0.1 & 0.0 & 0.01 & 0.04 \\
\hline 1-METHYL-4-ETHYLBENZENE & 0.0 & 0.00 & 0.0 & 0.0 & 0.00 & 0.0 & 0.0 & 0.00 & 0.0 & 0.0 & 0.00 & 0.00 \\
\hline 1,3,5-TRIMETHYLBENZENE & 0.0 & 0.00 & 0.0 & 0.0 & 0.00 & 0.0 & 0.0 & 0.00 & 0.0 & 0.0 & 0.00 & 0.00 \\
\hline 1-METHYL-2-ETHYLBENZENE & 0.0 & 0.00 & 0.0 & 0.0 & 0.00 & 0.0 & 0.0 & 0.00 & 0.0 & 0.0 & 0.00 & 0.00 \\
\hline 1,2,4-TRIMETHYLBENZENE & 0.0 & 0.00 & 0.0 & 0.0 & 0.00 & 0.0 & 0.0 & 0.07 & 0.2 & 0.0 & 0.01 & 0.06 \\
\hline DECANE & 0.0 & 0.03 & 0.0 & 0.0 & 0.42 & 0.0 & 0.0 & 0.00 & 0.0 & 0.0 & 0.04 & 0.01 \\
\hline ISOBUTYLBENZENE & 0.0 & 0.02 & 0.1 & 0.0 & 0.40 & 0.0 & 0.0 & 0.00 & 0.0 & 0.0 & 0.04 & 0.03 \\
\hline
\end{tabular}




\begin{tabular}{|c|c|c|c|c|c|c|c|c|c|c|c|c|}
\hline \multirow[b]{2}{*}{ COMPOUND } & \multicolumn{3}{|c|}{$\overline{B A G 1}$} & \multicolumn{3}{|c|}{ BAG 2} & \multicolumn{3}{|c|}{ BAG 3} & \multicolumn{3}{|c|}{ FTP } \\
\hline & MG/MI & $\begin{array}{l}\text { WEIGHT } \\
\text { NMOG \% }\end{array}$ & $\begin{array}{l}\text { OZONE, } \\
\text { MG/MI }\end{array}$ & MG/MI & $\begin{array}{l}\text { WEIGHT } \\
\text { NMOG \% }\end{array}$ & $\begin{array}{l}\text { OZONE } \\
\text { MGMII }\end{array}$ & MG/MI & $\begin{array}{l}\text { WEIGHT } \\
\text { NMOG \% }\end{array}$ & $\begin{array}{l}\text { OZONE } \\
\text { MG/MI }\end{array}$ & MG/MI & $\begin{array}{l}\text { WEIGHT } \\
\text { NMOG } \%\end{array}$ & $\begin{array}{l}\text { OZONE } \\
\text { MG/MI }\end{array}$ \\
\hline METHYLPROPYLBENZENE & 0.0 & 0.00 & 0.0 & 0.0 & 0.00 & 0.0 & 0.0 & 0.00 & 0.0 & 0.0 & 0.00 & 0.00 \\
\hline S-BUTYLBENZENE & 0.0 & 0.00 & 0.0 & 0.0 & 0.00 & 0.0 & 0.0 & 0.00 & 0.0 & 0.0 & 0.00 & 0.00 \\
\hline 1-METHYL-3-1SOPROPYLBENZENE & 0.0 & 0.00 & 0.0 & 0.0 & 0.00 & 0.0 & 0.0 & 0.00 & 0.0 & 0.0 & 0.00 & 0.00 \\
\hline 1,2,3-TRIMETHYLBENZENE & 0.0 & 0.00 & 0.0 & 0.0 & 0.00 & 0.0 & 0.0 & 0.00 & 0.0 & 0.0 & 0.00 & 0.00 \\
\hline 1-METHYL-4-ISOPROPYLBENZENE & 0.0 & 0.00 & 0.0 & 0.0 & 0.00 & 0.0 & 0.0 & 0.00 & 0.0 & 0.0 & 0.00 & 0.00 \\
\hline INDAN & 0.0 & 0.00 & 0.0 & 0.0 & 0.00 & 0.0 & 0.0 & 0.00 & 0.0 & 0.0 & 0.00 & 0.00 \\
\hline 1-METHYL-2-ISOPROPYLBENZENE & 0.0 & 0.00 & 0.0 & 0.0 & 0.00 & 0.0 & 0.0 & 0.00 & 0.0 & 0.0 & 0.00 & 0.00 \\
\hline 1,3-DIETHYLBENZENE & 0.0 & 0.00 & 0.0 & 0.0 & 0.00 & 0.0 & 0.0 & 0.00 & 0.0 & 0.0 & 0.00 & 0.00 \\
\hline 1.4-DIETHYLBENZENE & 0.0 & 0.00 & 0.0 & 0.0 & 0.00 & 0.0 & 0.0 & 0.00 & 0.0 & 0.0 & 0.00 & 0.00 \\
\hline 1-METHYL-3-N-PROPYLBENZENE & 0.0 & 0.00 & 0.0 & 0.0 & 0.00 & 0.0 & 0.0 & 0.00 & 0.0 & 0.0 & 0.00 & 0.00 \\
\hline 1-METHYL-4-N-PROPYLBENZENE & 0.0 & 0.00 & 0.0 & 0.0 & 0.00 & 0.0 & 0.0 & 0.00 & 0.0 & 0.0 & 0.00 & 0.00 \\
\hline 1,2 DIETHYLBENZENE & 0.0 & 0.00 & 0.0 & 0.0 & 0.00 & 0.0 & 0.0 & 0.00 & 0.0 & 0.0 & 0.00 & 0.00 \\
\hline 1-METHYL-2-N-PROPYLBENZENE & 0.0 & 0.00 & 0.0 & 0.0 & 0.00 & 0.0 & 0.0 & 0.00 & 0.0 & 0.0 & 0.00 & 0.00 \\
\hline 1,4-DIMETHYL-2-ETHYLBENZENE & 0.0 & 0.00 & 0.0 & 0.0 & 0.00 & 0.0 & 0.0 & 0.00 & 0.0 & 0.0 & 0.00 & 0.00 \\
\hline 1,3-DIMETHYL-4-ETHYLBENZENE & 0.0 & 0.00 & 0.0 & 0.0 & 0.00 & 0.0 & 0.0 & 0.00 & 0.0 & 0.0 & 0.00 & 0.00 \\
\hline 1,2-DIMETHYL-4-ETHYLBENZENE & 0.0 & 0.00 & 0.0 & 0.0 & 0.00 & 0.0 & 0.0 & 0.00 & 0.0 & 0.0 & 0.00 & 0.00 \\
\hline 1,3-DIMETHYL-2-ETHYLBENZENE & 0.0 & 0.00 & 0.0 & 0.0 & 0.00 & 0.0 & 0.0 & 0.00 & 0.0 & 0.0 & 0.00 & 0.00 \\
\hline UNDECANE & 0.0 & 0.00 & 0.0 & 0.0 & 0.00 & 0.0 & 0.0 & 0.00 & 0.0 & 0.0 & 0.00 & 0.00 \\
\hline 1,2-DIMETHYL-3-ETHYLBENZENE & 0.0 & 0.00 & 0.0 & 0.0 & 0.00 & 0.0 & 0.0 & 0.00 & 0.0 & 0.0 & 0.00 & 0.00 \\
\hline $1,2,4,5$-TETRAMETHYLBENZENE & 0.0 & 0.00 & 0.0 & 0.0 & 0.00 & 0.0 & 0.0 & 0.00 & 0.0 & 0.0 & 0.00 & 0.00 \\
\hline 2-METHYLBUTYLBENZENE (seC AMYLBENZENE) & 0.0 & 0.00 & 0.0 & 0.0 & 0.00 & 0.0 & 0.0 & 0.00 & 0.0 & 0.0 & 0.00 & 0.00 \\
\hline 3,4 DIMETHYLCUMENE & 0.0 & 0.00 & 0.0 & 0.0 & 0.00 & 0.0 & 0.0 & 0.00 & 0.0 & 0.0 & 0.00 & 0.00 \\
\hline $1,2,3,6$-TETRAMETHYLBENZENE & 0.0 & 0.00 & 0.0 & 0.0 & 0.00 & 0.0 & 0.0 & 0.00 & 0.0 & $\overline{0.0}$ & 0.00 & 0.00 \\
\hline TERT-1-BUT-2-METHYLBENZENE & 0.0 & 0.00 & 0.0 & 0.0 & 0.00 & 0.0 & 0.0 & 0.00 & 0.0 & 0.0 & 0.00 & 0.00 \\
\hline 1,2,3,4-TETRAMETHYLBENZENE & 0.0 & 0.00 & 0.0 & 0.0 & 0.00 & 0.0 & 0.0 & 0.00 & 0.0 & 0.0 & 0.00 & 0.00 \\
\hline N-PENT-BENZENE & 0.0 & 0.00 & 0.0 & 0.0 & 0.00 & 0.0 & 0.0 & 0.00 & 0.0 & 0.0 & 0.00 & 0.00 \\
\hline TERT-1-BUT-3,6-DIMETHYLBENZENE & 0.0 & 0.00 & 0.0 & 0.0 & 0.00 & 0.0 & 0.0 & 0.00 & 0.0 & 0.0 & 0.00 & 0.00 \\
\hline NAPHTHALENE & 0.0 & 0.00 & 0.0 & 0.0 & 0.00 & 0.0 & 0.0 & 0.00 & 0.0 & 0.0 & 0.00 & 0.00 \\
\hline DODECANE & 0.0 & 0.00 & 0.0 & 0.0 & 0.00 & 0.0 & 0.0 & 0.00 & 0.0 & 0.0 & 0.00 & 0.00 \\
\hline UNIDENTIFIED C9-C12+ & 0.0 & 0.00 & 0.0 & 0.0 & 0.00 & 0.0 & 0.0 & 0.00 & 0.0 & 0.0 & 0.00 & 0.00 \\
\hline FOAMALDEHYDE & 1.6 & 0.89 & 11.6 & 0.2 & 5.76 & 1.7 & 0.4 & 1.07 & 2.8 & 0.6 & 1.13 & 4.02 \\
\hline ACETALDEHYDE & 1.1 & 0.61 & 6.2 & 0.7 & 16.63 & 3.7 & 0.0 & 0.00 & 0.0 & 0.8 & 1.16 & 3.19 \\
\hline ACROLEIN & 0.1 & 0.03 & 0.4 & 0.0 & 0.00 & 0.0 & 0.0 & 0.00 & 0.0 & 0.0 & 0.02 & 0.08 \\
\hline ACETONE & 0.0 & 0.00 & 0.0 & 0.0 & 0.00 & 0.0 & 0.0 & 0.00 & 0.0 & 0.0 & 0.00 & 0.00 \\
\hline PROPIONALDEHYDE & 0.3 & 0.19 & 2.2 & 0.6 & 13.62 & 3.6 & 0.0 & 0.00 & 0.0 & 0.4 & 0.71 & 2.31 \\
\hline CROTONALDEHYDE & 0.0 & 0.00 & 0.0 & 0.0 & 0.00 & 0.0 & 0.0 & 0.00 & 0.0 & 0.0 & 0.00 & 0.00 \\
\hline ISOBUTYRALDEHYDE & 0.6 & 0.31 & 2.9 & 0.5 & 12.73 & 2.7 & 0.3 & 0.80 & 1.5 & 0.5 & 0.92 & 2.42 \\
\hline METHYL ETHYL KETONE & 0.6 & 0.31 & 0.7 & 0.5 & 12.73 & 0.6 & 0.3 & 0.80 & 0.3 & 0.5 & 0.92 & 0.54 \\
\hline BENZALDEHYDE & 1.0 & 0.67 & -0.6 & 0.6 & 13.88 & -0.3 & 0.8 & 2.27 & -0.5 & 0.7 & 1.46 & -0.40 \\
\hline HEXANALDEHYDE & 0.0 & 0.00 & 0.0 & 0.0 & 0.00 & 0.0 & 0.0 & 0.00 & 0.0 & 0.0 & 0.00 & 0.00 \\
\hline
\end{tabular}


TEST NUMBER: 20NB/80P-02S

TEST CAR: 1994 PONTIAC GRANDAM

TEST DATE: 11/16/95

TEST FUEL: 20\% BUTANE/BO\% PROPANE

\begin{tabular}{|c|c|c|c|c|c|c|c|c|c|c|c|c|}
\hline \multirow[b]{2}{*}{ COMPOUND } & \multicolumn{3}{|c|}{$\overline{B A G 1}$} & \multicolumn{3}{|c|}{$\overline{B A G 2}$} & \multicolumn{3}{|c|}{ BAG 3} & \multicolumn{3}{|c|}{ FTP } \\
\hline & MG/MI & $\begin{array}{l}\text { WEIGHT } \\
\text { NMOG \% }\end{array}$ & $\begin{array}{l}\text { OZONE, } \\
\text { MG/MI }\end{array}$ & MGMI & $\begin{array}{l}\text { WEIGHT } \\
\text { NMOG } \%\end{array}$ & $\begin{array}{l}\text { OZONE } \\
\text { MQ/MI }\end{array}$ & MG/MI & $\begin{array}{l}\text { WEIGHT } \\
\text { NMQG \% }\end{array}$ & $\begin{array}{l}\text { OZONE } \\
\text { MG/MI }\end{array}$ & MG/MI & \begin{tabular}{|l|} 
WEIGHT \\
NMOG \%
\end{tabular} & $\begin{array}{l}\text { OZONE } \\
\text { MG/MI }\end{array}$ \\
\hline METHANE & 11.3 & & 0.2 & 1.6 & & 0.0 & 17.3 & & 0.3 & 8.0 & & 0.12 \\
\hline ETHANE & 2.3 & 1.18 & 0.6 & 0.0 & 0.00 & 0.0 & 0.0 & 0.00 & 0.0 & 0.5 & 1.00 & 0.12 \\
\hline ETHYLENE & 11.6 & 6.90 & 84.8 & 0.1 & 1.10 & 0.4 & 0.2 & 1.38 & 1.8 & 2.6 & 6.20 & 18.43 \\
\hline PROPANE & 134.2 & 67.98 & 64.4 & 3.5 & 72.37 & 1.7 & 13.5 & 75.60 & 6.5 & 33.5 & 68.98 & 16.10 \\
\hline PROPYLENE & 6.1 & 3.11 & 57.6 & 0.0 & 0.42 & 0.2 & 0.1 & 0.83 & 1.4 & 1.3 & 2.74 & 12.52 \\
\hline ACETYLENE & 1.9 & 0.98 & 0.9 & 0.0 & 0.14 & 0.0 & 0.0 & 0.02 & 0.0 & 0.4 & 0.82 & 0.20 \\
\hline PROPADIENE & 0.0 & 0.00 & $\overline{0.0}$ & 0.0 & 0.00 & 0.0 & 0.0 & 0.00 & 0.0 & 0.0 & 0.00 & 0.00 \\
\hline BUTANE & 35.0 & 17.75 & 35.7 & 0.4 & 7.72 & 0.4 & $\overline{2.4}$ & 13.27 & 2.4 & 8.2 & 16.79 & 8.33 \\
\hline TAANS-2-BUTENE & 0.1 & $\overline{0.04}$ & 0.7 & 0.0 & 0.00 & 0.0 & 0.0 & 0.00 & 0.0 & 0.0 & 0.03 & 0.15 \\
\hline 1-BUTENE & 0.3 & 0.13 & 2.3 & 0.0 & 0.00 & 0.0 & 0.0 & 0.00 & 0.0 & 0.1 & 0.11 & 0.49 \\
\hline 2-METHYLPROPENE (ISOBUTYLENE) & 0.1 & 0.05 & 0.5 & 0.0 & 0.00 & 0.0 & 0.0 & 0.00 & 0.0 & 0.0 & 0.04 & $\overline{0.11}$ \\
\hline 2,2-DIMETHYLPROPANE (NEOPENTANE) & 0.0 & 0.00 & 0.0 & 0.0 & 0.00 & 0.0 & 0.0 & 0.00 & 0.0 & 0.0 & 0.00 & 0.00 \\
\hline PROPYNE & 0.0 & 0.00 & 0.0 & 0.0 & 0.00 & 0.0 & 0.0 & 0.00 & 0.0 & 0.0 & 0.00 & 0.00 \\
\hline 1,3-BUTADIENE & 0.1 & 0.04 & 0.8 & 0.0 & 0.00 & 0.0 & 0.0 & 0.00 & 0.0 & 0.0 & 0.03 & 0.17 \\
\hline 2-METHYLPROPANE (ISOBUTANE) & 0.1 & 0.05 & 0.1 & 0.0 & 0.02 & 0.0 & 0.0 & 0.04 & 0.0 & 0.0 & 0.05 & 0.03 \\
\hline 1-BUTYNE & 0.0 & 0.00 & 0.0 & 0.0 & 0.00 & 0.0 & 0.0 & 0.00 & 0.0 & 0.0 & 0.00 & 0.00 \\
\hline METHANOL & 0.0 & 0.00 & 0.0 & 0.0 & 0.00 & 0.0 & 0.0 & 0.00 & 0.0 & 0.0 & 0.00 & 0.00 \\
\hline CIS-2-BUTENE & 0.0 & 0.00 & 0.0 & 0.0 & 0.00 & 0.0 & 0.0 & 0.00 & 0.0 & 0.0 & 0.00 & 0.00 \\
\hline 3-METHYL-1-BUTENE & 0.0 & 0.00 & 0.0 & 0.0 & 0.00 & 0.0 & 0.0 & 0.00 & 0.0 & 0.0 & 0.00 & 0.00 \\
\hline ETHANOL & 0.0 & 0.00 & 0.0 & 0.0 & 0.00 & 0.0 & 0.0 & 0.00 & 0.0 & 0.0 & 0.00 & 0.00 \\
\hline 2-METHYLBUTANE (ISOPENTANE) & 2.0 & 1.03 & 2.8 & 0.1 & 2.96 & $\overline{0.2}$ & 0.0 & 0.24 & 0.1 & 0.5 & 1.04 & 0.70 \\
\hline 2-BUTYNE & 0.0 & 0.00 & 0.0 & 0.0 & 0.00 & 0.0 & 0.0 & 0.00 & 0.0 & 0.0 & 0.00 & 0.00 \\
\hline 1-PENTENE & 0.0 & 0.00 & 0.0 & 0.0 & 0.00 & 0.0 & 0.0 & 0.00 & 0.0 & 0.0 & 0.00 & 0.00 \\
\hline 2-METHYL-1-BUTENE & 0.0 & 0.00 & $\overline{0.0}$ & 0.0 & 0.00 & 0.0 & 0.0 & 0.00 & 0.0 & 0.0 & 0.00 & 0.00 \\
\hline PENTANE & 0.1 & 0.07 & 0.1 & 0.0 & 0.00 & 0.0 & 0.0 & 0.19 & 0.0 & 0.0 & 0.08 & 0.04 \\
\hline UNIDENTIFIED C6 OLEFINS & 0.0 & 0.00 & 0.0 & 0.0 & 0.00 & 0.0 & 0.0 & 0.00 & 0.0 & 0.0 & 0.00 & 0.00 \\
\hline 2-METHYL-1,3-BUTADIENE & 0.0 & 0.00 & 0.0 & 0.0 & 0.00 & 0.0 & 0.0 & 10.00 & 0.0 & 0.0 & 0.00 & 0.00 \\
\hline TRANS-2-PENTENE & 0.0 & 0.00 & 0.0 & 0.0 & 0.00 & 0.0 & 0.0 & 0.00 & 0.0 & 0.0 & 0.00 & 0.00 \\
\hline 3,3-DIMETHYL-1-BUTENE & 0.0 & 0.00 & 0.0 & 0.0 & 0.00 & 0.0 & 0.0 & 0.00 & 0.0 & 0.0 & 0.00 & 0.00 \\
\hline CIS-2-PENTENE & 0.0 & 0.00 & 0.0 & 0.0 & 0.00 & 0.0 & 0.0 & 0.00 & 0.0 & 0.0 & 0.00 & 0.00 \\
\hline 2-METHYL-2-BUTENE & 0.1 & 0.04 & 0.5 & 0.0 & 0.00 & 0.0 & 0.0 & 0.00 & 0.0 & 0.0 & 0.03 & 0.11 \\
\hline CYCLOPENTADIENE & 0.0 & 0.00 & 0.0 & 0.0 & 0.00 & 0.0 & 0.0 & 0.00 & 0.0 & 0.0 & 0.00 & 0.00 \\
\hline 2,2-DIMETHYLBUTANE & 0.0 & 0.00 & 0.0 & 0.0 & 0.00 & 0.0 & 0.0 & 0.00 & 0.0 & 0.0 & 0.00 & 0.00 \\
\hline CYCLOPENTENE & 0.0 & 0.00 & 0.0 & 0.0 & 0.00 & 0.0 & 0.0 & 0.00 & 0.0 & 0.0 & 0.00 & 0.00 \\
\hline 4-METHYL-1-PENTENE & 0.0 & 0.00 & 0.0 & 0.0 & 0.00 & 0.0 & 0.0 & 0.00 & 0.0 & 0.0 & 0.00 & 0.00 \\
\hline 3-METHYL-1-PENTENE & 0.0 & 0.00 & 0.0 & 0.0 & 0.00 & 0.0 & 0.0 & 0.00 & 0.0 & 0.0 & 0.00 & 0.00 \\
\hline CYCLOPENTANE & 0.0 & 0.00 & 0.0 & 0.0 & 0.00 & 0.0 & 0.0 & 0.00 & 0.0 & 0.0 & 0.00 & 0.00 \\
\hline 2,3-DIMETHYLBUTANE & 0.0 & 0.00 & 0.0 & 0.0 & 0.00 & 0.0 & 0.1 & 0.63 & 0.1 & 0.0 & 0.08 & 0.03 \\
\hline MTBE & 0.0 & 0.00 & 0.0 & 0.0 & 0.00 & 0.0 & 0.0 & 0.00 & 0.0 & 0.0 & 0.00 & 0.00 \\
\hline
\end{tabular}


出 居

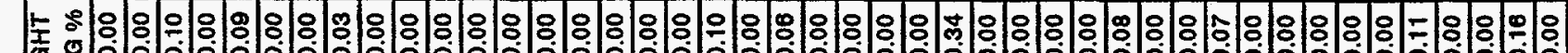

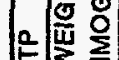

4. 3

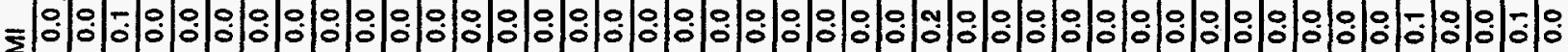
₹

o o n o v o o o o o o o o o o o o vo

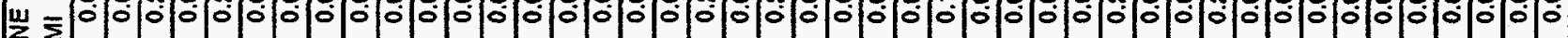
잉 일

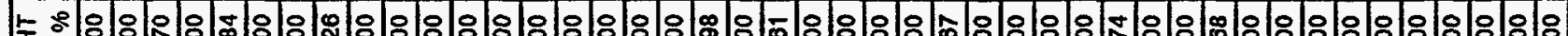

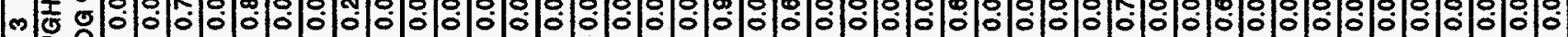
这崖

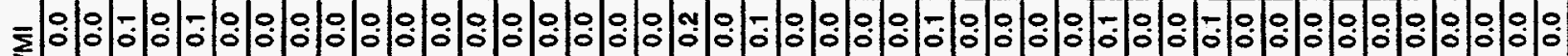

ङ

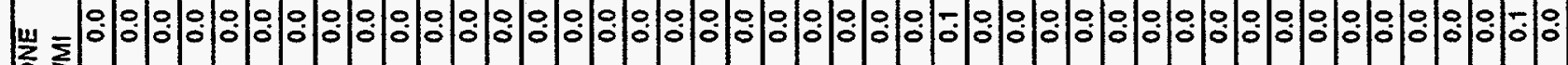

命

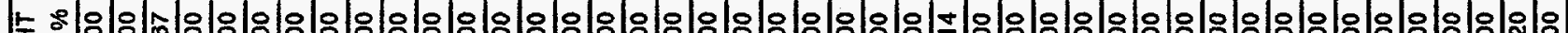
N 率要

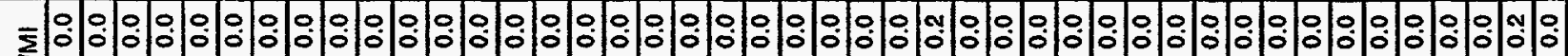
$\sum$

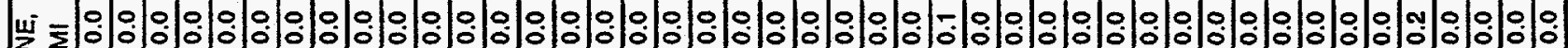
㣽

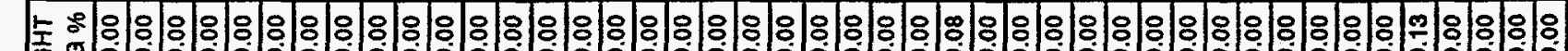
空

离高

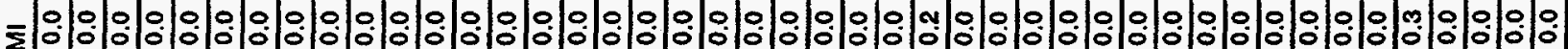
$\stackrel{\S}{\Sigma}$

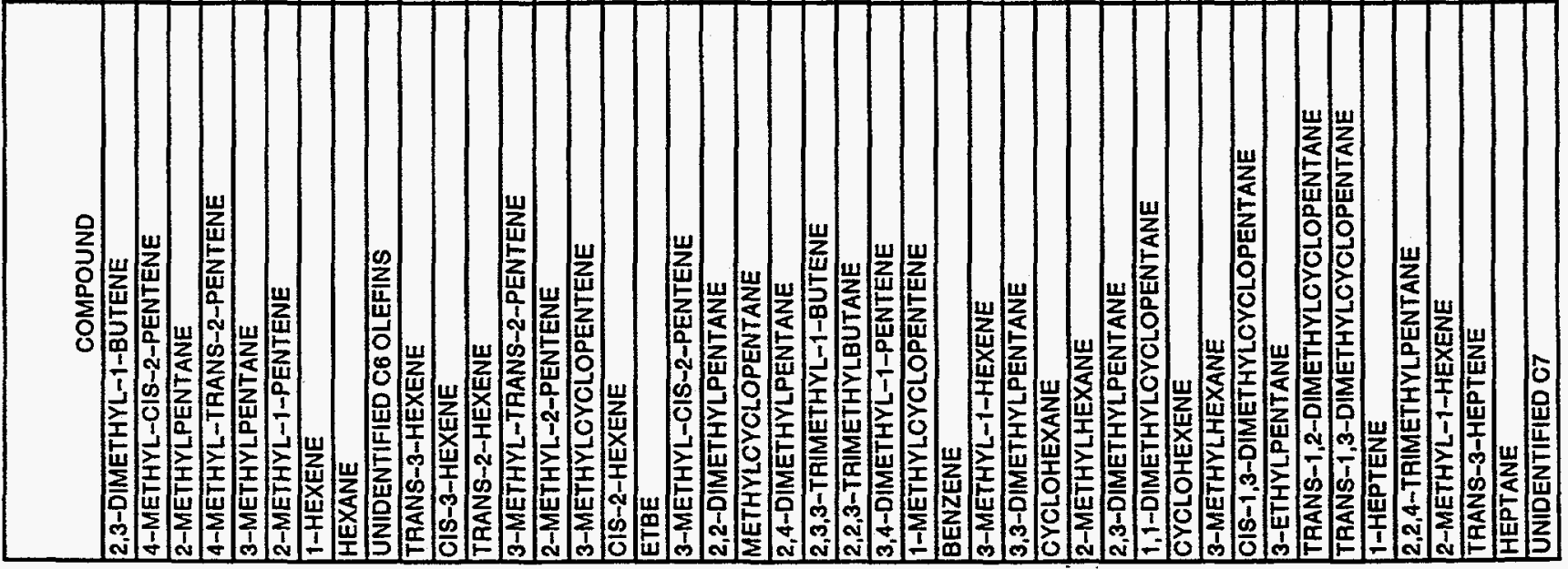




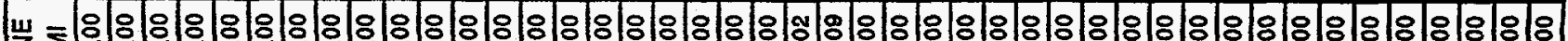
看 合 $\frac{1}{2}$

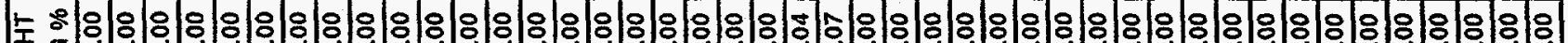

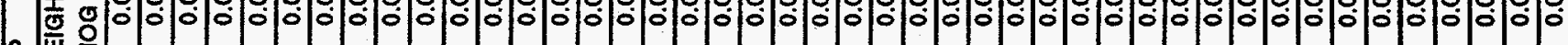

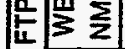

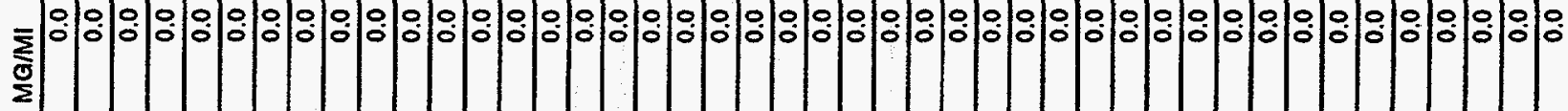

宸

10000000000 잉 这

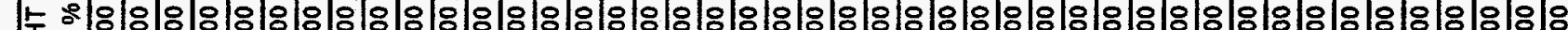

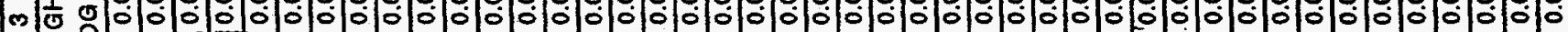
高要部

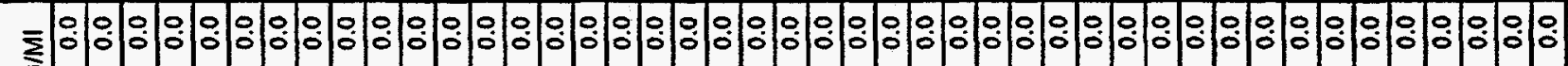
$\stackrel{5}{\Sigma}$

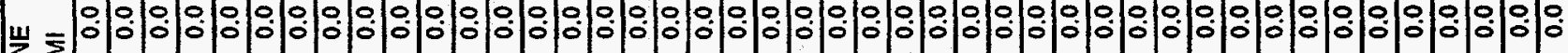
㣽

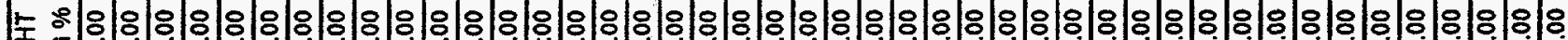
๙ 壬 要部

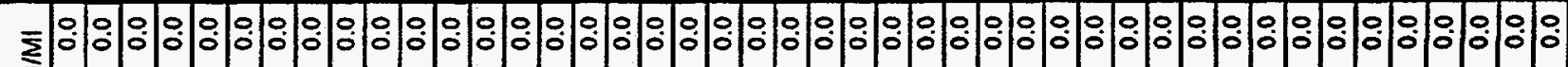
을

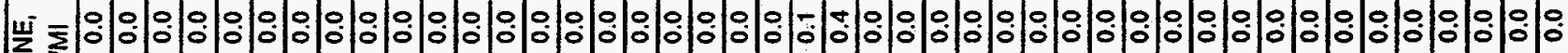
空

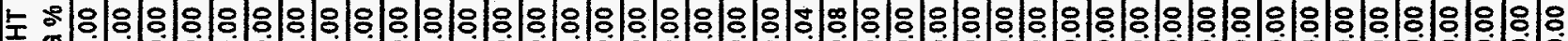
- 志 这要

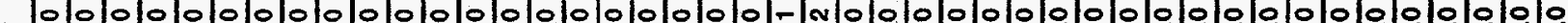

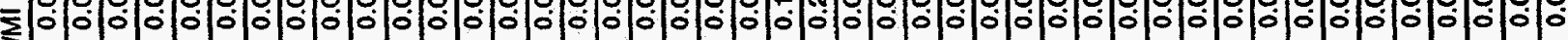
울

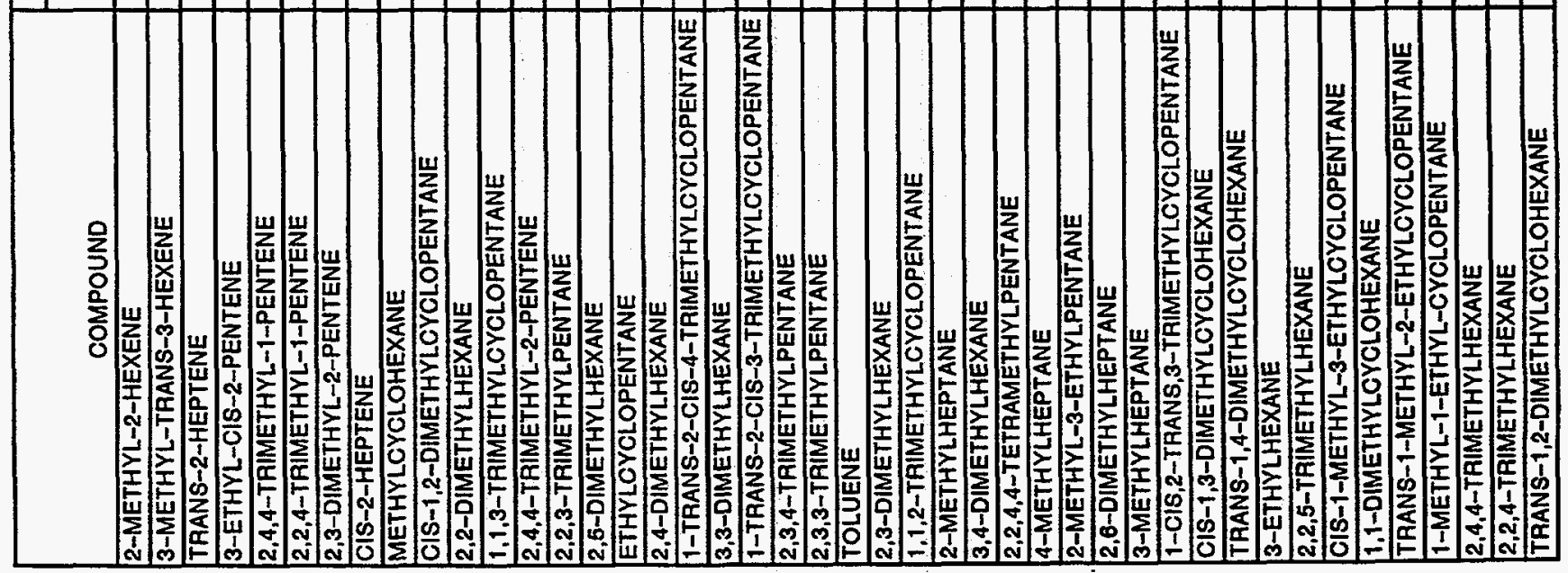


岁

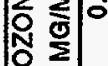

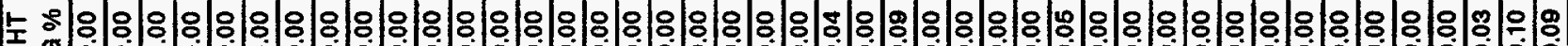
a

造运

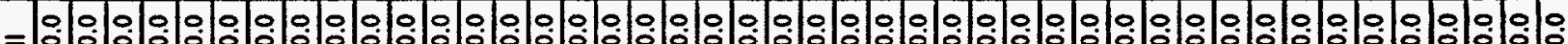
三 $\stackrel{\mathcal{S}}{\Sigma}$

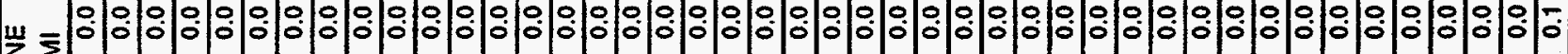
용

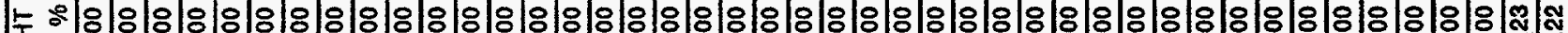
m I 这岗

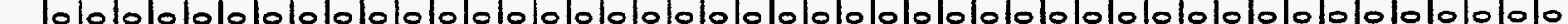
इ 旁

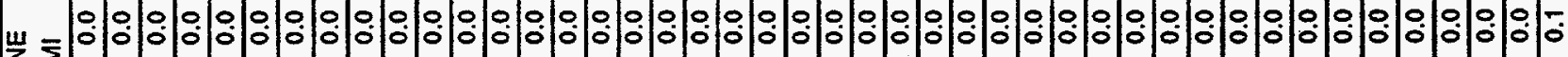

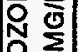

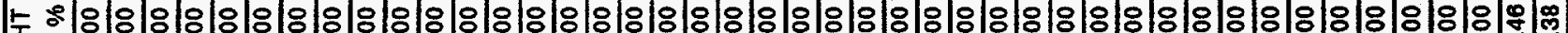
w 声 窟崖

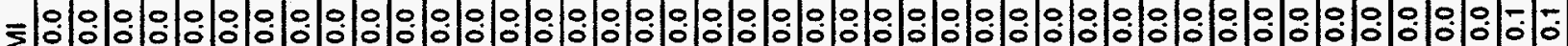

$\sum$

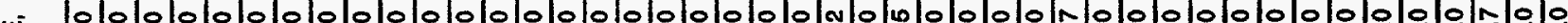
य 三 员

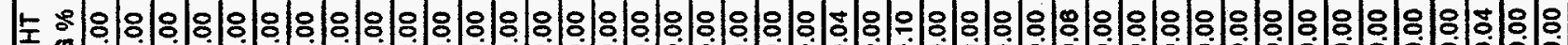
- 告 状

三 $\stackrel{0}{\Sigma}$

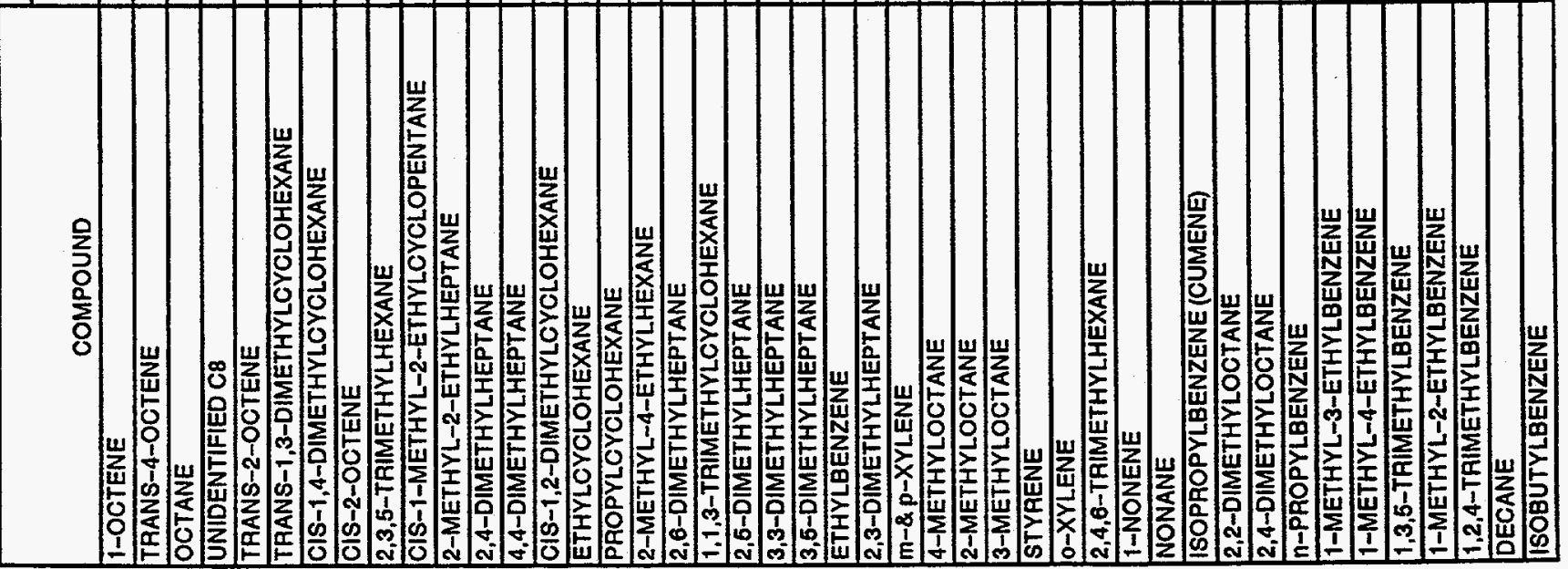




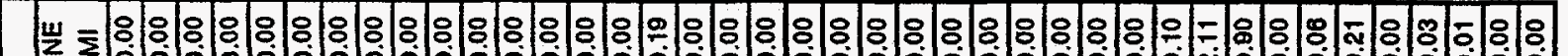
O 定

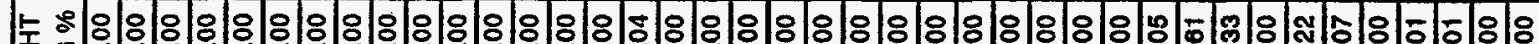
a

采

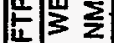

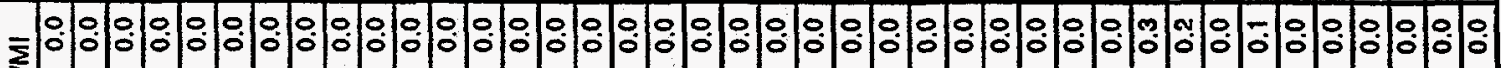

$\stackrel{2}{\frac{1}{3}}$

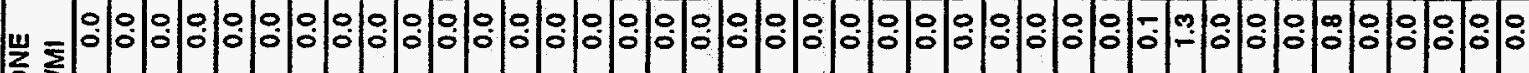
N

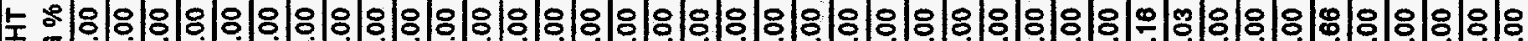

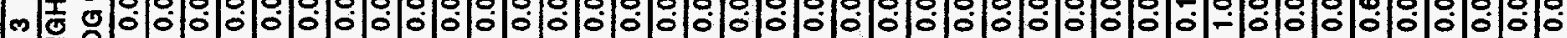

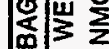

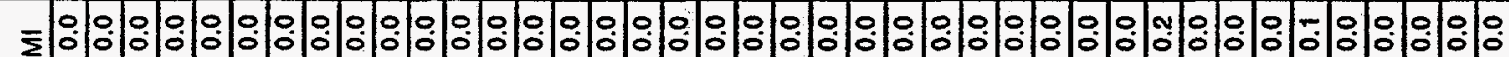
ङ

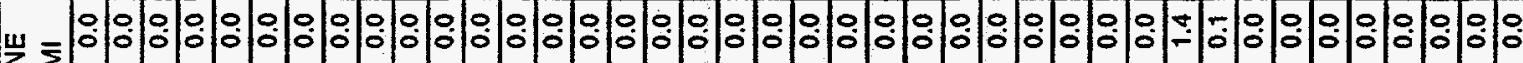
¿ N

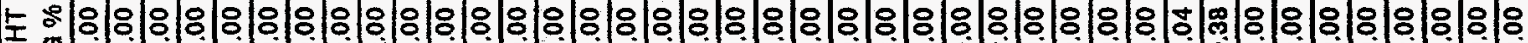
誌 要这

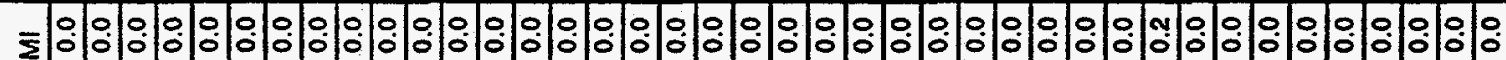
$\stackrel{5}{\Sigma}$

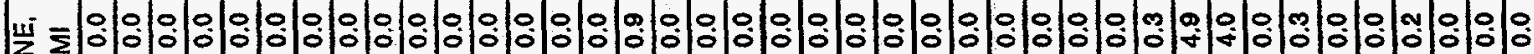

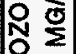

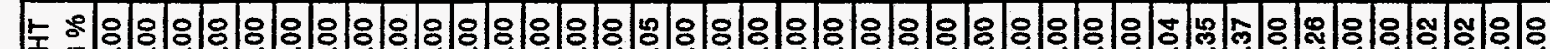

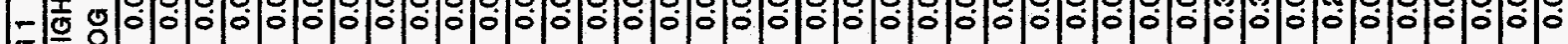

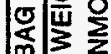

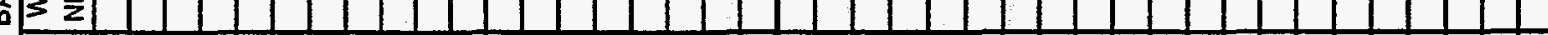

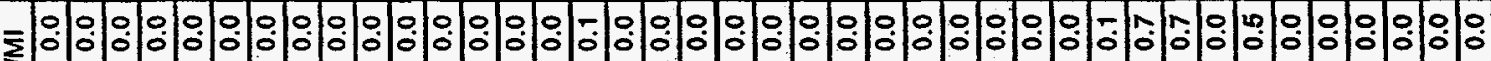
$\stackrel{5}{\Sigma}$

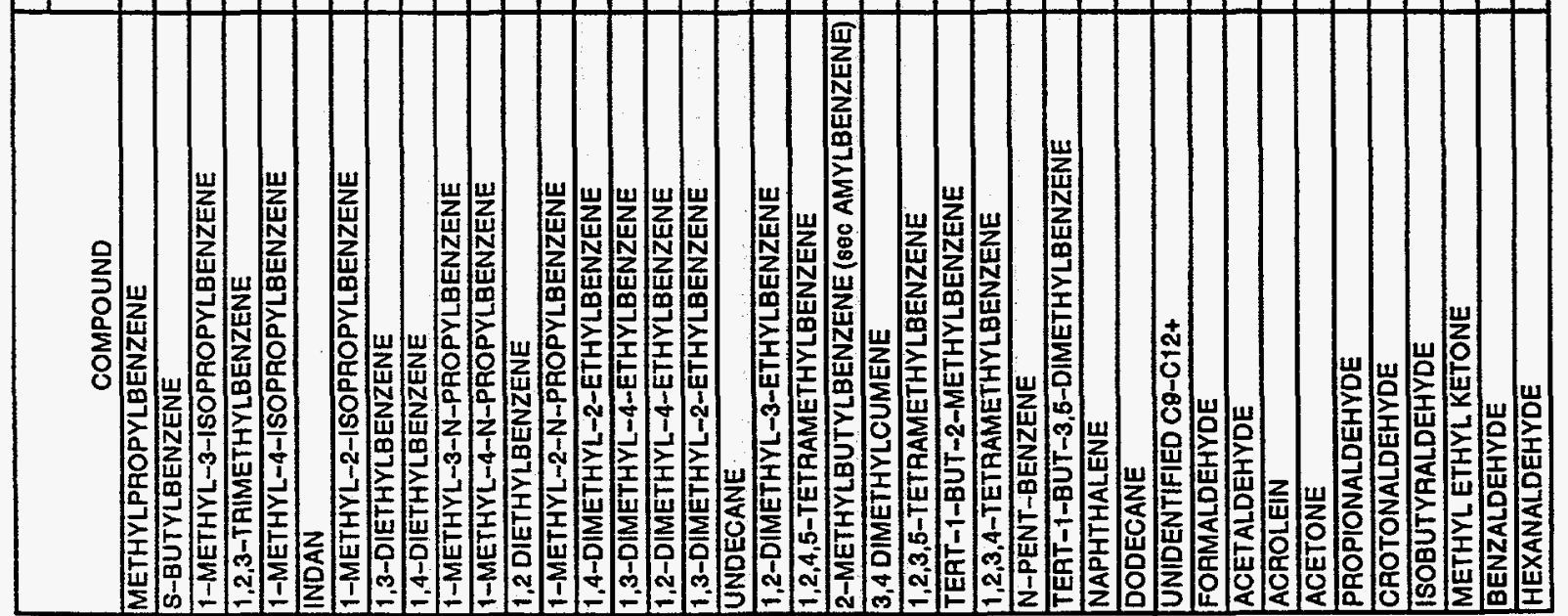


TEST NO: 100B-04S

TEST CAR: 1994 PONTIAC GRANDAM

TEST DATE: $10 / 4 / 95$

TEST FUEL: BUTANE

\begin{tabular}{|c|c|c|c|c|c|c|c|c|c|c|c|c|}
\hline \multirow[b]{2}{*}{ COMPOUND } & \multicolumn{3}{|c|}{$\overline{B A Q} 1$} & \multicolumn{3}{|c|}{ BAG 2} & \multicolumn{3}{|c|}{ BAG 3} & \multicolumn{3}{|c|}{ FTP } \\
\hline & MG/MI & $\begin{array}{l}\text { WEIGHT } \\
\text { NMOG } \%\end{array}$ & $\begin{array}{l}\text { OZONE, } \\
\text { MG/MI }\end{array}$ & MG/MI & $\begin{array}{l}\text { WEIGHT } \\
\text { NMOG } \%\end{array}$ & $\begin{array}{l}\text { OZONE } \\
\text { MG/MI }\end{array}$ & MG/MI & \begin{tabular}{|l|} 
WEIGHT \\
NMOG $\%$
\end{tabular} & $\begin{array}{l}\text { OZONE } \\
\text { MG/MI }\end{array}$ & MG/MI & $\begin{array}{l}\text { WEIGHT } \\
\text { NMOG } \%\end{array}$ & $\begin{array}{l}\text { OZONE } \\
\text { MG/MI }\end{array}$ \\
\hline METHANE & 26.3 & & 0.4 & 3.4 & & 0.1 & 24.6 & & 0.4 & 14.0 & & 0.21 \\
\hline ETHANE & 2.7 & 1.47 & 0.7 & 0.0 & 0.00 & 0.0 & 4.5 & 16.07 & 1.1 & 1.8 & 3.83 & 0.45 \\
\hline ETHYLENE & 17.8 & 9.72 & 130.0 & 0.0 & 0.00 & 0.0 & 2.9 & 9.71 & 21.0 & 4.5 & 9.64 & 32.95 \\
\hline PROPANE & 0.3 & 0.17 & 0.1 & 0.0 & 0.00 & 0.0 & 0.1 & 0.38 & 0.1 & 0.1 & 0.20 & 0.04 \\
\hline PROPYLENE & 7.5 & 4.06 & 70.0 & 0.0 & 0.00 & 0.0 & 0.8 & 2.60 & 7.3 & 1.8 & 3.77 & 18.63 \\
\hline ACETYLENE & 6.6 & 3.58 & 3.3 & 0.0 & 0.00 & 0.0 & 0.0 & 0.00 & 0.0 & 1.4 & 2.93 & 0.69 \\
\hline PROPADIENE & 0.0 & 0.00 & 0.0 & 0.0 & 0.00 & 0.0 & 0.0 & 0.00 & 0.0 & 0.0 & 0.00 & 0.00 \\
\hline BUTANE & 137.3 & 74.81 & 140.0 & 0.0 & 0.00 & 0.0 & 20.6 & 69.21 & 21.0 & 34.3 & 73.24 & 35.03 \\
\hline TRANS-2-BUTENE & $\overline{0.3}$ & 0.15 & 2,8 & 0.0 & 0.00 & 0.0 & 0.1 & 0.49 & 1.4 & 0.1 & 0.21 & 0.97 \\
\hline 1-BUTENE & 0.6 & 0.33 & 5.3 & 0.0 & 0.00 & 0.0 & 0.1 & 0.32 & 0.8 & 0.2 & 0.32 & $\overline{1.34}$ \\
\hline 2-METHYLPROPENE (ISOBUTYLENE) & $\overline{0.1}$ & 0.05 & 0.4 & 0.0 & 0.00 & 0.0 & 0.1 & 0.25 & 0.4 & 0.0 & 0.08 & 0.20 \\
\hline 2,2-DIMETHYLPROPANE (NEOPENTANE) & 0.0 & 0.00 & 0.0 & 0.0 & 0.00 & 0.0 & 0.0 & 0.00 & 0.0 & 0.0 & 0.00 & 0.00 \\
\hline PROPYNE & 0.0 & 0.00 & 0.0 & 0.0 & 0.00 & 0.0 & 0.0 & 0.00 & 0.0 & 0.0 & 0.00 & 0.00 \\
\hline 1,3-BUTADIENE & 0.1 & 0.07 & 1.3 & 0.0 & 0.00 & 0.0 & 0.0 & 0.00 & 0.0 & 0.0 & 0.05 & 0.27 \\
\hline 2-METHYLPROPANE (ISOBUTANE) & 0.1 & 0.07 & 0.2 & 0.0 & 0.00 & 0.0 & 0.0 & 0.00 & 0.0 & 0.0 & 0.06 & 0.03 \\
\hline 1-BUTYNE & 0.0 & 0.00 & 0.0 & 0.0 & 0.00 & 0.0 & 0.0 & 0.00 & 0.0 & 0.0 & 0.00 & 0.00 \\
\hline METHANOL & 0.0 & 0.00 & 0.0 & 0.0 & 0.00 & 0.0 & 0.0 & 0.00 & 0.0 & 0.0 & 0.00 & 0.00 \\
\hline CIS-2-BUTENE & 0.3 & 0.10 & 3.6 & 0.0 & 0.00 & 0.0 & 0.0 & 0.00 & 0.0 & 0.1 & 0.16 & 0.73 \\
\hline 3-METHYL-1-BUTENE & 0.0 & 0.00 & 0.0 & 0.0 & 0.00 & 0.0 & 0.0 & 0.00 & 0.0 & 0.0 & 0.00 & 0.00 \\
\hline ETHANOL & 0.0 & 0.00 & 0.0 & 0.0 & 0.00 & 0.0 & 0.0 & 0.00 & 0.0 & 0.0 & 0.00 & 0.00 \\
\hline 2-METHYLBUTANE (ISOPENTANE) & 2.6 & 1.42 & 3.6 & 0.0 & 0.00 & 0.0 & 0.0 & 0.00 & 0.0 & 0.6 & 1.16 & 0.75 \\
\hline 2-BUTYNE & 0.0 & 0.00 & 0.0 & 0.0 & 0.00 & 0.0 & 0.0 & 0.00 & 0.0 & 0.0 & 0.00 & 0.00 \\
\hline 1-PENTENE & 0.0 & 0.00 & 0.0 & 0.0 & 0.00 & 0.0 & 0.0 & 0.00 & 0.0 & 0.0 & 0.00 & 0.00 \\
\hline 2-METHYL-1-BUTENE & 0.0 & 0.00 & 0.0 & 0.0 & 0.00 & 0.0 & 0.1 & 0.32 & 0.5 & 0.0 & 0.06 & 0.13 \\
\hline PENTANE & 0.3 & 0.19 & 0.4 & 0.0 & 0.00 & 0.0 & 0.0 & 0.00 & 0.0 & 0.1 & 0.16 & 0.07 \\
\hline UNIDENTIFIED C5 OLEFINS & 0.0 & 0.00 & 0.0 & 0.0 & 0.00 & 0.0 & 0.0 & 0.00 & 0.0 & 0.0 & 0.00 & 0.00 \\
\hline 2-METHYL-1,3-BUTADIENE & 0.0 & 0.00 & 0.0 & 0.0 & 0.00 & 0.0 & 0.0 & 0.00 & 0.0 & 0.0 & 0.00 & 0.00 \\
\hline TRANS-2-PENTENE & 0.1 & 0.06 & 1.0 & 0.0 & 0.00 & 0.0 & 0.0 & 0.00 & 0.0 & 0.0 & 0.05 & 0.21 \\
\hline 3,3-DIMETHYL-1-BUTENE & 0.0 & 0.00 & 0.0 & 0.0 & 0.00 & 0.0 & 0.0 & 0.00 & 0.0 & 0.0 & 0.00 & 0.00 \\
\hline CIS-2-PENTENE & 0.0 & 0.00 & 0.0 & 0.0 & 0.00 & 0.0 & 0.0 & 0.00 & 0.0 & 0.0 & 0.00 & 0.00 \\
\hline 2-METHYL-2-BUTENE & 0.1 & 0.04 & 0.4 & 0.0 & 0.00 & 0.0 & 0.1 & 0.17 & 0.3 & 0.0 & 0.06 & 0.18 \\
\hline CYCLOPENTADIENE & 0.0 & 0.00 & 0.0 & 0.0 & 0.00 & 0.0 & 0.0 & 0.00 & 0.0 & 0.0 & 0.00 & 0.00 \\
\hline 2,2-DIMETHYLBUTANE & 0.0 & 0.00 & 0.0 & 0.0 & 0.00 & 0.0 & 0.0 & 0.00 & 0.0 & 0.0 & 0.00 & 0.00 \\
\hline CYCLOPENTENE & $\overline{0.0}$ & 0.00 & 0.0 & 0.0 & 0.00 & 0.0 & 0.0 & 0.00 & 0.0 & 0.0 & 0.00 & 0.00 \\
\hline 4-METHYL-1-PENTENE & 0.0 & 0.00 & 0.0 & 0.0 & 0.00 & 0.0 & 0.0 & 0.00 & 0.0 & 0.0 & 0.00 & 0.00 \\
\hline 3-METHYL-1-PENTENE & 0.0 & 0.00 & 0.0 & 0.0 & 0.00 & 0.0 & 0.0 & 0.00 & 0.0 & 0.0 & 0.00 & 0.00 \\
\hline CYCLOPENTANE & 0.0 & 0.00 & 0.0 & 0.0 & 0.00 & 0.0 & 0.0 & 0.00 & 0.0 & 0.0 & 0.00 & 0.00 \\
\hline 2,3-DIMETHYLBUTANE & 0.1 & 0.07 & 0.1 & 0.0 & 0.00 & 0.0 & 0.0 & 0.00 & 0.0 & 0.0 & 0.06 & 0.03 \\
\hline MTBE & 0.0 & 0.00 & 0.0 & 0.0 & 0.00 & 0.0 & 0.0 & 0.00 & 0.0 & 0.0 & 0.00 & 0.00 \\
\hline
\end{tabular}




\begin{tabular}{|c|c|c|c|c|c|c|c|c|c|c|c|c|}
\hline \multirow[b]{2}{*}{ COMPOUND } & \multicolumn{3}{|c|}{ BAG 1} & \multicolumn{3}{|c|}{ BAG 2 } & \multicolumn{3}{|c|}{ BAG 3} & \multicolumn{3}{|c|}{ FTP } \\
\hline & MG/MI & $\begin{array}{l}\text { WEIGHT } \\
\text { NMOG \% }\end{array}$ & $\begin{array}{l}\text { OZONE, } \\
\text { MG/MI }\end{array}$ & MG/MI & $\begin{array}{l}\text { WEIGHT } \\
\text { NMOG } \%\end{array}$ & $\begin{array}{l}\text { OZONE } \\
\text { MG/MI }\end{array}$ & MaMI & $\begin{array}{l}\text { WEIGHT } \\
\text { NMOG \% }\end{array}$ & $\begin{array}{l}\text { OZONE } \\
\text { MG/MI }\end{array}$ & MG/MI & $\begin{array}{l}\text { WEIGHT } \\
\text { NMOG \% }\end{array}$ & $\begin{array}{l}\text { OZONE } \\
\text { MGMI }\end{array}$ \\
\hline 2,3-DIMETHYL-1-BUTENE & 0.0 & 0.00 & 0.0 & 0.0 & 0.00 & 0.0 & 0.0 & 0.00 & 0.0 & 0.0 & 0.00 & 0.00 \\
\hline 4-METHYL-CIS-2-PENTENE & $\overline{0.0}$ & 0.00 & 0.0 & 0.0 & 0.00 & 0.0 & 0.0 & 0.00 & 0.0 & 0.0 & 0.00 & 0.00 \\
\hline 2-METHYLPENTANE & $\overline{0.1}$ & 0.07 & 0.2 & 0.0 & 0.00 & 0.0 & 0.0 & 0.00 & $\overline{0.0}$ & 0.0 & 0.06 & 0.04 \\
\hline 4-METHYL-TRANS-2-PENTENE & 0.0 & 0.00 & 0.0 & 0.0 & 0.00 & 0.0 & 0.0 & 0.00 & 0.0 & 0.0 & 0.00 & 0.00 \\
\hline 3-METHYLPENTANE & 0.1 & 0.04 & 0.1 & 0.0 & 0.00 & 0.0 & 0.0 & 0.00 & 0.0 & 0.0 & 0.03 & 0.02 \\
\hline 2-METHYL-1-PENTENE & 0.0 & 0.00 & 0.0 & 0.0 & 0.00 & 0.0 & 0.0 & 0.00 & 0.0 & 0.0 & 0.00 & 0.00 \\
\hline 1-HEXENE & $\overline{0.0}$ & 0.00 & 0.0 & 0.0 & 0.00 & 0.0 & 0.0 & 0.00 & 0.0 & 0.0 & 0.00 & 0.00 \\
\hline HEXANE & 0.0 & 0.00 & 0.0 & 0.0 & 0.00 & 0.0 & 0.0 & 0.00 & 0.0 & 0.0 & 0.00 & 0.00 \\
\hline UNIDENTIFIED C6 OLEFINS & 0.0 & 0.00 & 0.0 & 0.0 & 0.00 & 0.0 & 0.0 & 0.00 & 0.0 & 0.0 & 0.00 & $\overline{0.00}$ \\
\hline TRANS-3-HEXENE & 0.1 & 0.05 & 0.7 & 0.0 & 0.00 & 0.0 & 0.0 & 0.00 & 0.0 & 0.0 & 0.04 & 0.14 \\
\hline CIS-3-HEXENE & 0.0 & 0.00 & 0.0 & 0.0 & 0.00 & 0.0 & 0.0 & 0.00 & 0.0 & 0.0 & 0.00 & 0.00 \\
\hline TRANS-2-HEXENE & 0.0 & 0.00 & 0.0 & 0.0 & 0.00 & 0.0 & 0.0 & 0.00 & 0.0 & 0.0 & 0.00 & 0.00 \\
\hline 3-METHYL-TRANS-2-PENTENE & 0.0 & 0.00 & 0.0 & 0.0 & 0.00 & 0.0 & 0.0 & 0.00 & 0.0 & 0.0 & 0.00 & 0.00 \\
\hline 2-METHYL-2-PENTENE & 0.0 & 0.00 & 0.0 & 0.0 & 0.00 & 0.0 & 0.0 & 0.00 & 0.0 & 0.0 & 0.00 & 0.00 \\
\hline 3-METHYLCYCLOPENTENE & 0.0 & 0.00 & 0.0 & 0.0 & 0.00 & 0.0 & 0.0 & 0.00 & 0.0 & 0.0 & 0.00 & $\overline{0.00}$ \\
\hline CIS-2-HEXENE & $\overline{0.0}$ & 0.00 & 0.0 & 0.0 & 0.00 & 0.0 & 0.0 & 0.00 & 0.0 & 0.0 & 0.00 & 0.00 \\
\hline ETBE & 0.0 & 0.00 & 0.0 & 0.0 & 0.00 & 0.0 & 0.0 & 0.00 & 0.0 & 0.0 & 0.00 & 0.00 \\
\hline 3-METHYL-CIS-2-PENTENE & 0.0 & 0.00 & 0.0 & 0.0 & 0.00 & 0.0 & 0.0 & 0.00 & 0.0 & 0.0 & 0.00 & 0.00 \\
\hline 2,2-DIMETHYLPENTANE & 0.0 & 0.02 & 0.0 & 0.0 & 0.00 & 0.0 & 0.0 & 0.00 & 0.0 & 0.0 & 0.02 & 0.01 \\
\hline METHYLCYCLOPENTANE & 0.0 & 0.00 & 0.0 & 0.0 & 0.00 & 0.0 & 0.0 & 0.00 & 0.0 & 0.0 & 0.00 & 0.00 \\
\hline 2,4-DIMETHYLPENTANE & 0.2 & 0.12 & 0.4 & 0.0 & 2.95 & 0.0 & 0.0 & 0.00 & $\overline{0.0}$ & $\overline{0.1}$ & 0.12 & 0.10 \\
\hline 2,3,3-TRIMETHYL-1-BUTENE & 0.0 & 0.00 & 0.0 & 0.0 & 0.00 & 0.0 & 0.0 & 0.00 & 0.0 & 0.0 & 0.00 & 0.00 \\
\hline 2,2,3-TRIMETHYLBUTANE & $\overline{0.0}$ & 0.00 & 0.0 & 0.1 & 18.73 & 0.2 & 0.0 & 0.00 & 0.0 & 0.1 & 0.16 & 0.09 \\
\hline 3,4-DIMETHYL-1-PENTENE & $\overline{0.0}$ & 0.00 & 0.0 & 0.0 & 0.00 & 0.0 & 0.0 & 0.00 & 0.0 & 0.0 & 0.00 & 0.00 \\
\hline 1-METHYLOYCLOPENTENE & 0.0 & 0.00 & 0.0 & 0.0 & 0.00 & 0.0 & 0.0 & 0.00 & 0.0 & $\mathbf{0 . 0}$ & 0.00 & 0.00 \\
\hline BENZENE & $\overline{0.2}$ & 0.09 & 0.1 & 0.0 & 0.00 & 0.0 & 0.0 & 0.00 & 0.0 & 0.0 & 0.07 & 0.01 \\
\hline 3-METHYL-1-HEXENE & 0.0 & 0.00 & 0.0 & 0.0 & 0.00 & 0.0 & 0.0 & 0.00 & 0.0 & 0.0 & 0.00 & 0.00 \\
\hline 3,3-DIMETHYLPENTANE & 0.0 & 0.00 & 0.0 & 0.0 & 0.00 & 0.0 & 0.0 & 0.00 & 0.0 & 0.0 & 0.00 & 0.00 \\
\hline CYCLOHEXANE & 0.0 & 0.00 & 0.0 & 0.0 & 0.00 & 0.0 & 0.0 & 0.00 & 0.0 & 0.0 & 0.00 & 0.00 \\
\hline 2-METHYLHEXANE & 0.0 & 0.00 & 0.0 & 0.0 & 0.00 & 0.0 & 0.0 & 0.00 & 0.0 & 0.0 & 0.00 & 0.00 \\
\hline 2,3-DIMETHYLPENTANE & 0.3 & 0.18 & 0.5 & 0.0 & 0.00 & 0.0 & 0.0 & 0.00 & 0.0 & 0.1 & 0.15 & 0.10 \\
\hline 1,1-DIMETHYLCYCLOPENTANE & 0.0 & 0.00 & 0.0 & 0.0 & 0.00 & 0.0 & 0.0 & 0.00 & 0.0 & 0.0 & 0.00 & 0.00 \\
\hline CYCLOHEXENE & 0.0 & 0.00 & 0.0 & 0.0 & 0.00 & 0.0 & 0.0 & 0.00 & 0.0 & 0.0 & 0.00 & 0.00 \\
\hline 3-METHYLHEXANE & 0.0 & 0.00 & 0.0 & 0.0 & 0.00 & 0.0 & 0.0 & 0.00 & 0.0 & 0.0 & 0.00 & 0.00 \\
\hline CIS-1,3-DIMETHYLCYCLOPENTANE & 0.0 & 0.00 & 0.0 & 0.0 & 0.00 & 0.0 & 0.0 & 0.00 & 0.0 & 0.0 & 0.00 & 0.00 \\
\hline 3-ETHYLPENTANE & 0.0 & 0.00 & 0.0 & 0.0 & 0.00 & 0.0 & 0.0 & 0.00 & 0.0 & 0.0 & 0.00 & 0.00 \\
\hline TAANS-1,2-DIMETHYLCYCLOPENTANE & 0.0 & 0.00 & 0.0 & 0.0 & 0.00 & 0.0 & 0.0 & 0.00 & 0.0 & 0.0 & 0.00 & 0.00 \\
\hline TRANS-1,3-DIMETHYLCYCLOPENTANE & 0.0 & 0.00 & 0.0 & 0.0 & 0.00 & 0.0 & 0.0 & 0.00 & 0.0 & 0.0 & 0.00 & 0.00 \\
\hline 1-HEPTENE & 0.0 & 0.00 & 0.0 & 0.0 & 0.00 & 0.0 & 0.0 & 0.00 & 0.0 & 0.0 & 0.00 & 0.00 \\
\hline 2,2,4-TRIMETHYLPENTANE & 0.8 & 0.43 & 0.7 & 0.0 & 0.00 & 0.0 & 0.0 & 0.05 & 0.0 & 0.2 & 0.36 & 0.10 \\
\hline 2-METHYL-1-HEXENE & 0.0 & 0.00 & 0.0 & 0.0 & 0.00 & 0.0 & 0.0 & 0.00 & 0.0 & 0.0 & 0.00 & 0.00 \\
\hline TRANS-3-HEPTENE & 0.0 & 0.00 & 0.0 & 0.0 & 0.00 & 0.0 & 0.0 & 0.00 & 0.0 & 0.0 & 0.00 & 0.00 \\
\hline HEPTANE & 0.0 & 0.00 & 0.0 & 0.0 & 0.00 & 0.0 & 0.0 & 0.00 & 0.0 & 0.0 & 0.00 & 0.00 \\
\hline UNIDENTIFIED C7 & 0.0 & 0.00 & 0.0 & 0.0 & 0.00 & 0.0 & 0.0 & 0.00 & 0.0 & 0.0 & 0.00 & 0.00 \\
\hline
\end{tabular}




\begin{tabular}{|c|c|c|c|c|c|c|c|c|c|c|c|c|}
\hline \multirow[b]{2}{*}{ COMPOUND } & \multicolumn{3}{|c|}{ BAG 1} & \multicolumn{3}{|c|}{ BAG 2} & \multicolumn{3}{|c|}{$\overline{B A G} 3$} & \multicolumn{3}{|c|}{ FTP } \\
\hline & MG/MI & $\begin{array}{l}\text { WEIGHT } \\
\text { NMOQ } \%\end{array}$ & $\begin{array}{l}\text { OZONE, } \\
\text { MG/MI }\end{array}$ & MG/MI & $\begin{array}{l}\text { WEIGHT } \\
\text { NMOG \% }\end{array}$ & $\begin{array}{l}\text { OZONE } \\
\text { MGMMI }\end{array}$ & MG/MI & $\begin{array}{l}\text { WEIGHT } \\
\text { NMOQ \% }\end{array}$ & $\begin{array}{l}\text { OZONE } \\
\text { MG/MI }\end{array}$ & MG/MI & $\begin{array}{l}\text { WEIGHT } \\
\text { NMOG } \%\end{array}$ & $\begin{array}{l}\text { OZONE } \\
\text { MG/MI }\end{array}$ \\
\hline 2-METHYL-2-HEXENE & 0.0 & 0.00 & 0.0 & 0.0 & 0.00 & 0.0 & 0.0 & 0.00 & 0.0 & 0.0 & 0.00 & 0.00 \\
\hline 3-METHYL-TRANS-3-HEXENE & 0.0 & 0.00 & 0.0 & 0.0 & 0.00 & 0.0 & 0.0 & 0.00 & 0.0 & 0.0 & 0.00 & 0.00 \\
\hline TAANS-2-HEPTENE & 0.0 & 0.00 & 0.0 & 0.0 & 0.00 & 0.0 & 0.0 & 0.00 & 0.0 & 0.0 & 0.00 & 0.00 \\
\hline 3-ETHYL-CIS-2-PENTENE & 0.0 & 0.00 & 0.0 & 0.0 & 0.00 & 0.0 & 0.0 & 0.00 & 0.0 & 0.0 & 0.00 & 0.00 \\
\hline 2,4,4-TRIMETHYL-1-PENTENE & 0.0 & 0.00 & 0.0 & 0.0 & 0.00 & 0.0 & 0.0 & 0.00 & 0.0 & 0.0 & 0.00 & 0.00 \\
\hline 2,2,4-TRIMETHYL-1-PENTENE & 0.0 & 0.00 & 0.0 & 0.0 & 0.00 & 0.0 & 0.0 & 0.00 & 0.0 & 0.0 & 0.00 & 0.00 \\
\hline 2,3-DIMETHYL-2-PENTENE & 0.0 & 0.00 & 0.0 & 0.0 & 0.00 & 0.0 & 0.0 & 0.00 & 0.0 & 0.0 & 0.00 & 0.00 \\
\hline CIS-2-HEPTENE & 0.0 & 0.00 & 0.0 & 0.0 & 0.00 & 0.0 & 0.0 & 0.00 & 0.0 & 0.0 & 0.00 & 0.00 \\
\hline METHYLCYCLOHEXANE & 0.0 & 0.00 & 0.0 & $\overline{0.0}$ & 0.00 & 0.0 & 0.0 & 0.00 & 0.0 & 0.0 & 0.00 & 0.00 \\
\hline CIS-1,2-DIMETHYLCYCLOPENTANE & 0.0 & 0.00 & 0.0 & 0.0 & 0.00 & 0.0 & 0.0 & 0.00 & 0.0 & 0.0 & 0.00 & 0.00 \\
\hline 2,2-DIMETHYLHEXANE & 0.1 & 0.06 & 0.1 & 0.0 & 0.00 & 0.0 & 0.0 & 0.00 & 0.0 & 0.0 & 0.05 & 0.03 \\
\hline 1,1,3-TRIMETHYLCYCLOPENTANE & 0.0 & 0.00 & 0.0 & 0.0 & 0.00 & 0.0 & 0.0 & 0.00 & 0.0 & 0.0 & 0.00 & 0.00 \\
\hline 2,4,4-TRIMETHYL-2-PENTENE & 0.0 & 0.00 & 0.0 & 0.0 & 0.00 & 0.0 & 0.0 & 0.00 & 0.0 & 0.0 & 0.00 & 0.00 \\
\hline 2,2,3-TRIMETHYLPENTANE & 0.0 & 0.00 & 0.0 & 0.0 & 0.00 & 0.0 & 0.0 & 0.00 & 0.0 & 0.0 & 0.00 & 0.00 \\
\hline 2,5-DIMETHYLHEXANE & 0.0 & 0.00 & 0.0 & 0.0 & 0.00 & 0.0 & 0.0 & 0.00 & 0.0 & 0.0 & 0.00 & 0.00 \\
\hline ETHYLCYCLOPENTANE & 0.0 & 0.00 & 0.0 & 0.0 & 0.00 & 0.0 & 0.0 & 0.00 & 0.0 & 0.0 & 0.00 & 0.00 \\
\hline 2,4-DIMETHYLHEXANE & 0.1 & 0.08 & 0.2 & 0.0 & 0.00 & 0.0 & 0.0 & 0.00 & 0.0 & 0.0 & 0.07 & 0.05 \\
\hline 1-TRANS-2-CIS-4-TRIMETHYLCYCLOPENTANE & 0.0 & 0.00 & 0.0 & 0.0 & 0.00 & 0.0 & 0.0 & 0.00 & 0.0 & 0.0 & 0.00 & 0.00 \\
\hline 3,3-DIMETHYLHEXANE & 0.0 & 0.00 & 0.0 & 0.0 & 0.00 & 0.0 & 0.0 & 0.00 & 0.0 & 0.0 & 0.00 & 0.00 \\
\hline 1-TAANS-2-CIS-3-TRIMETHYLCYCLOPENTANE & 0.0 & 0.00 & 0.0 & 0.0 & 0.00 & 0.0 & 0.0 & 0.00 & 0.0 & 0.0 & 0.00 & 0.00 \\
\hline 2,3,4-TRIMETHYLPENTANE & 0.0 & 0.00 & 0.0 & 0.0 & 0.00 & 0.0 & 0.0 & 0.00 & 0.0 & 0.0 & 0.00 & 0.00 \\
\hline 2,3,3-TRIMETHYLPENTANE & 0.2 & 0.12 & 0.3 & 0.0 & 0.00 & 0.0 & 0.0 & 0.00 & 0.0 & 0.0 & 0.09 & 0.05 \\
\hline TOLUENE & 0.7 & 0.39 & 2.0 & 0.0 & 0.00 & 0.0 & 0.0 & 0.00 & 0.0 & 0.2 & 0.32 & 0.41 \\
\hline 2,3-DIMETHYLHEXANE & 0.0 & 0.00 & 0.0 & 0.0 & 0.00 & 0.0 & 0.0 & 0.00 & 0.0 & 0.0 & 0.00 & 0.00 \\
\hline 1,1,2-TRIMETHYLCYCLOPENTANE & 0.0 & 0.00 & 0.0 & 0.0 & 0.00 & 0.0 & 0.0 & 0.00 & 0.0 & 0.0 & 0.00 & 0.00 \\
\hline 2-METHYLHEPTANE & 0.0 & 0.01 & 0.0 & 0.0 & 0.00 & 0.0 & 0.0 & 0.00 & 0.0 & 0.0 & 0.01 & 0.00 \\
\hline 3,4-DIMETHYLHEXANE & 0.0 & 0.00 & 0.0 & 0.0 & 0.00 & 0.0 & 0.0 & 0.00 & 0.0 & 0.0 & 0.00 & 0.00 \\
\hline 2,2,4,4-TETRAMETHYLPENTANE & 0.0 & 0.00 & 0.0 & 0.0 & 0.00 & 0.0 & 0.0 & 0.00 & 0.0 & 0.0 & 0.00 & 0.00 \\
\hline 4-METHYLHEPTANE & 0.0 & 0.00 & 0.0 & 0.0 & 0.00 & 0.0 & 0.0 & 0.00 & 0.0 & 0.0 & 0.00 & 0.00 \\
\hline 2-METHYL-3-ETHYLPENTANE & 0.0 & 0.00 & 0.0 & 0.0 & 0.00 & 0.0 & 0.0 & 0.00 & 0.0 & 0.0 & 0.00 & 0.00 \\
\hline 2,6-DIMETHYLHEPTANE & 0.0 & 0.00 & 0.0 & 0.0 & 0.00 & 0.0 & 0.0 & 0.00 & 0.0 & 0.0 & 0.00 & 0.00 \\
\hline 3-METHYLHEPTANE & 0.0 & 0.02 & 0.0 & 0.0 & 0.00 & 0.0 & 0.0 & 0.00 & 0.0 & 0.0 & 0.01 & 0.01 \\
\hline 1-CIS,2-TRANS,3-TRIMETHYLCYCLOPENTANE & 0.0 & 0.00 & 0.0 & 0.0 & 0.00 & 0.0 & 0.0 & 0.00 & 0.0 & 0.0 & 0.00 & 0.00 \\
\hline CIS-1,3-DIMETHYLCYCLOHEXANE & 0.0 & 0.00 & 0.0 & 0.0 & 0.00 & 0.0 & 0.0 & 0.00 & 0.0 & 0.0 & 0.00 & 0.00 \\
\hline TAANS-1,4-DIMETHYLCYCLOHEXANE & 0.0 & 0.00 & 0.0 & 0.0 & 0.00 & 0.0 & 0.0 & 0.00 & 0.0 & 0.0 & 0.00 & 0.00 \\
\hline 3-ETHYLHEXANE & 0.0 & 0.00 & 0.0 & 0.0 & 0.00 & 0.0 & 0.0 & 0.00 & 0.0 & 0.0 & 0.00 & 0.00 \\
\hline 2,2,5-TRIMETHYLHEXANE & 0.0 & 0.00 & 0.0 & 0.0 & 0.00 & 0.0 & 0.0 & 0.00 & 0.0 & 0.0 & 0.00 & 0.00 \\
\hline CIS-1-METHYL-3-ETHYLCYCLOPENTANE & 0.0 & 0.00 & 0.0 & 0.0 & 0.00 & 0.0 & 0.0 & 0.00 & 0.0 & 0.0 & 0.00 & 0.00 \\
\hline 1,1-DIMETHYLCYCLOHEXANE & 0.0 & 0.00 & 0.0 & 0.0 & 0.00 & 0.0 & 0.0 & 0.00 & 0.0 & 0.0 & 0.00 & 0.00 \\
\hline TAANS-1-METHYL-2-ETHYLCYCLOPENTANE & 0.0 & 0.00 & 0.0 & 0.0 & 0.00 & 0.0 & 0.0 & 0.00 & 0.0 & 0.0 & 0.00 & 0.00 \\
\hline 1-METHYL-1-ETHYL-CYCLOPENTANE & 0.0 & 0.00 & 0.0 & 0.0 & 0.00 & 0.0 & 0.0 & 0.00 & 0.0 & 0.0 & 0.00 & 0.00 \\
\hline 2,4,4-TAIMETHYLHEXANE & 0.0 & 0.00 & 0.0 & 0.0 & 0.00 & 0.0 & 0.0 & 0.00 & 0.0 & 0.0 & 0.00 & 0.00 \\
\hline 2,2,4-TRIMETHYLHEXANE & 0.0 & 0.00 & 0.0 & 0.0 & 0.00 & 0.0 & 0.0 & 0.00 & 0.0 & 0.0 & 0.00 & 0.00 \\
\hline TRANS-1,2-DIMETHYLCYCLOHEXANE & 0.0 & 0.00 & 0.0 & 0.0 & 0.00 & 0.0 & 0.0 & 0.00 & 0.0 & 0.0 & 0.00 & 0.00 \\
\hline
\end{tabular}




\begin{tabular}{|c|c|c|c|c|c|c|c|c|c|c|c|c|}
\hline \multirow[b]{2}{*}{ COMPOUND } & \multicolumn{3}{|c|}{ BAG 1} & \multicolumn{3}{|c|}{$B A G 2$} & \multicolumn{3}{|c|}{ BAG 3} & \multicolumn{3}{|c|}{ FTP } \\
\hline & MG/MI & $\begin{array}{l}\text { WEIGHT } \\
\text { NMOG } \%\end{array}$ & $\begin{array}{l}\text { OZONE, } \\
\text { MG/MI }\end{array}$ & MG/MI & $\begin{array}{l}\text { WEIGHT } \\
\text { NMOG \% }\end{array}$ & $\begin{array}{l}\text { OZONE } \\
\text { MG/MI }\end{array}$ & MG/MI & $\begin{array}{l}\text { WEIGHT } \\
\text { NMOG } \%\end{array}$ & $\begin{array}{l}\text { OZONE } \\
\text { MGIMI }\end{array}$ & ME/MI & $\begin{array}{l}\text { WEIGHT } \\
\text { NMOQ \% }\end{array}$ & $\begin{array}{l}\text { OZONE } \\
\text { MG/MI }\end{array}$ \\
\hline 1-OCTENE & 0.0 & 0.00 & 0.0 & 0.0 & 0.00 & 0.0 & 0.0 & 0.00 & 0.0 & 0.0 & 0.00 & 0.00 \\
\hline TRANS-4-OCTENE & 0.0 & 0.00 & 0.0 & 0.0 & 0.00 & 0.0 & 0.0 & 0.00 & 0.0 & 0.0 & 0.00 & 0.00 \\
\hline OCTANE & 0.0 & 0.00 & 0.0 & 0.0 & 0.00 & 0.0 & 0.0 & 0.00 & 0.0 & 0.0 & 0.00 & 0.00 \\
\hline UNIDENTIFIED C8 & 0.0 & 0.02 & 0.1 & 0.0 & 0.00 & 0.0 & 0.0 & 0.01 & 0.0 & 0.0 & 0.02 & 0.03 \\
\hline TRANS-2-OCTENE & 0.0 & 0.00 & 0.0 & 0.0 & 0.00 & 0.0 & 0.0 & 0.00 & 0.0 & 0.0 & 0.00 & 0.00 \\
\hline TAANS-1,3-DIMETHYLCYCLOHEXANE & 0.0 & 0.00 & 0.0 & 0.0 & 0.00 & 0.0 & 0.0 & 0.00 & 0.0 & 0.0 & 0.00 & 0.00 \\
\hline CIS-1,4-DIMETHYLCYCLOHEXANE & 0.0 & 0.00 & 0.0 & 0.0 & 0.00 & 0.0 & 0.0 & 0.00 & 0.0 & 0.0 & 0.00 & 0.00 \\
\hline CIS-2-OCTENE & 0.0 & 0.00 & 0.0 & 0.0 & 0.00 & 0.0 & 0.0 & 0.00 & 0.0 & $\overline{0.0}$ & 0.00 & 0.00 \\
\hline 2,3,6-TAIMETHYLHEXANE & 0.0 & 0.00 & 0.0 & 0.0 & 0.00 & 0.0 & 0.0 & 0.00 & 0.0 & 0.0 & 0.00 & 0.00 \\
\hline CIS-1-METHYL-2-ETHYLCYCLOPENTANE & 0.0 & 0.00 & 0.0 & 0.0 & 0.00 & 0.0 & 0.0 & 0.00 & 0.0 & 0.0 & 0.00 & 0.00 \\
\hline 2-METHYL-2-ETHYLHEPTANE & 0.0 & 0.00 & 0.0 & 0.0 & 0.00 & 0.0 & 0.0 & 0.00 & 0.0 & 0.0 & 0.00 & 0.00 \\
\hline 2,4-DIMETHYLHEPTANE & 0.0 & 0.00 & 0.0 & 0.1 & 18.78 & 0.2 & 0.0 & 0.00 & 0.0 & 0.1 & 0.15 & 0.09 \\
\hline 4,4-DIMETHYLHEPTANE & 0.0 & 0.00 & 0.0 & 0.0 & 0.00 & 0.0 & 0.0 & 0.00 & 0.0 & 0.0 & 0.00 & 0.00 \\
\hline CIS-1,2-DIMETHYLCYCLOHEXANE & 0.0 & 0.00 & 0.0 & 0.0 & 0.00 & 0.0 & 0.0 & 0.00 & 0.0 & 0.0 & 0.00 & 0.00 \\
\hline ETHYLCYCLOHEXANE & 0.0 & 0.00 & 0.0 & 0.0 & 0.00 & 0.0 & 0.0 & 0.00 & 0.0 & 0.0 & 0.00 & 0.00 \\
\hline PROPYLCYCLOHEXANE & 0.0 & 0.00 & 0.0 & 0.0 & 0.00 & 0.0 & 0.0 & 0.00 & 0.0 & 0.0 & 0.00 & 0.00 \\
\hline 2-METHYL-4-ETHYLHEXANE & 0.0 & 0.00 & 0.0 & 0.0 & 0.00 & 0.0 & 0.0 & 0.00 & 0.0 & 0.0 & 0.00 & 0.00 \\
\hline 2,6-DIMETHYLHEPTANE & 0.0 & 0.00 & 0.0 & 0.0 & 0.00 & 0.0 & 0.0 & 0.00 & 0.0 & 0.0 & 0.00 & 0.00 \\
\hline 1,1,3-TRIMETHYLCYCLOHEXANE & 0.0 & 0.00 & 0.0 & 0.0 & 0.00 & 0.0 & 0.0 & 0.00 & 0.0 & 0.0 & 0.00 & 0.00 \\
\hline 2,6-DIMETHYLHEPTANE & 0.0 & 0.00 & 0.0 & 0.0 & 0.00 & 0.0 & 0.0 & 0.00 & 0.0 & 0.0 & 0.00 & 0.00 \\
\hline 3,3-DIMETHYLHEPTANE & 0.0 & 0.00 & 0.0 & 0.0 & 0.00 & 0.0 & 0.0 & 0.00 & 0.0 & 0.0 & 0.00 & 0.00 \\
\hline 3,5-DIMETHYLHEPTANE & 0.0 & 0.00 & 0.0 & 0.0 & 0.00 & 0.0 & 0.0 & 0.00 & 0.0 & 0.0 & 0.00 & 0.00 \\
\hline ETHYLBENZENE & 0.1 & 0.05 & 0.2 & 0.0 & 0.00 & 0.0 & 0.0 & 0.00 & 0.0 & 0.0 & 0.04 & 0.05 \\
\hline 2,3-DIMETHYLHEPTANE & 0.0 & 0.00 & 0.0 & 0.0 & 0.00 & 0.0 & 0.0 & 0.00 & 0.0 & 0.0 & 0.00 & 0.00 \\
\hline m-\&p-XYLENE & 0.1 & 0.07 & 1.0 & 0.0 & 0.00 & 0.0 & 0.0 & 0.00 & 0.0 & 0.0 & 0.06 & 0.20 \\
\hline 4-METHYLOCTANE & 0.0 & 0.00 & 0.0 & 0.0 & 0.00 & 0.0 & 0.0 & 0.00 & 0.0 & 0.0 & 0.00 & 0.00 \\
\hline 2-METHYLOCTANE & 0.0 & 0.00 & 0.0 & 0.0 & 0.00 & 0.0 & 0.0 & 0.00 & 0.0 & 0.0 & 0.00 & 0.00 \\
\hline 3-METHYLOCTANE & 0.0 & 0.00 & 0.0 & 0.0 & 0.00 & 0.0 & 0.0 & 0.00 & 0.0 & 0.0 & 0.00 & 0.00 \\
\hline STYRENE & 0.0 & 0.00 & 0.0 & 0.0 & 0.00 & 0.0 & 0.0 & 0.00 & 0.0 & 0.0 & 0.00 & 0.00 \\
\hline O-XYLENE & 0.1 & 0.03 & 0.3 & 0.0 & 0.00 & 0.0 & 0.0 & 0.00 & 0.0 & 0.0 & 0.02 & 0.07 \\
\hline 2,4,6-TAIMETHYLHEXANE & 0.0 & 0.00 & 0.0 & 0.0 & 0.00 & 0.0 & 0.0 & 0.00 & 0.0 & 0.0 & 0.00 & 0.00 \\
\hline 1-NONENE & 0.0 & 0.00 & 0.0 & 0.0 & 0.00 & 0.0 & 0.0 & 10.00 & 0.0 & 0.0 & 0.00 & 0.00 \\
\hline NONANE & 0.0 & 0.00 & 0.0 & 0.0 & 0.00 & 0.0 & 0.0 & 0.00 & 0.0 & 0.0 & 0.00 & 0.00 \\
\hline ISOPROPYLBENZENE (CUMENE) & 0.0 & 0.00 & 0.0 & 0.0 & 0.00 & 0.0 & 0.0 & 0.00 & 0.0 & 0.0 & 0.00 & 0.00 \\
\hline 2,2-DIMETHYLOCTANE & 0.0 & 0.00 & 0.0 & 0.0 & 0.00 & 0.0 & 0.0 & 0.00 & 0.0 & 0.0 & 0.00 & 0.00 \\
\hline 2,4-DIMETHYLOCTANE & 0.0 & 0.00 & 0.0 & 0.0 & 0.00 & 0.0 & 0.0 & 0.00 & 0.0 & 0.0 & 0.00 & 0.00 \\
\hline n-PROPYLBENZENE & 0.0 & 0.00 & 0.0 & 0.0 & 0.00 & 0.0 & 0.0 & 0.00 & 0.0 & 0.0 & 0.00 & 0.00 \\
\hline 1-METHYL-3-ETHYLBENZENE & 0.1 & 0.06 & 0.8 & 0.0 & 0.00 & 0.0 & 0.0 & 0.00 & 0.0 & 0.0 & 0.05 & 0.16 \\
\hline 1-METHYL-4-ETHYLBENZENE & 0.0 & 0.00 & 0.0 & 0.0 & 0.00 & 0.0 & 0.0 & 0.00 & 0.0 & 0.0 & 0.00 & 0.00 \\
\hline 1,3,6-TRIMETHYLBENZENE & 0.0 & 0.00 & 0.0 & 0.0 & 0.00 & 0.0 & 0.0 & 0.00 & 0.0 & 0.0 & 0.00 & 0.00 \\
\hline 1-METHYL-2-ETHYLBENZENE & 0.0 & 0.00 & 0.0 & 0.0 & 0.00 & 0.0 & 0.0 & 0.00 & 0.0 & 0.0 & 0.00 & 0.00 \\
\hline 1,2,4-TRIMETHYLBENZENE & 0.1 & 0.07 & 1.1 & 0.0 & 0.00 & 0.0 & 0.0 & 0.00 & 0.0 & 0.0 & 0.05 & 0.22 \\
\hline DECANE & 0.0 & 0.01 & 0.0 & 0.1 & 9.79 & 0.0 & 0.0 & 0.06 & 0.0 & 0.0 & 0.09 & 0.02 \\
\hline ISOBUTYLBENZENE & 0.0 & 0.01 & 0.0 & 0.1 & 9.24 & 0.1 & 0.0 & 0.06 & 0.0 & 0.0 & 0.09 & 0.08 \\
\hline
\end{tabular}


崖

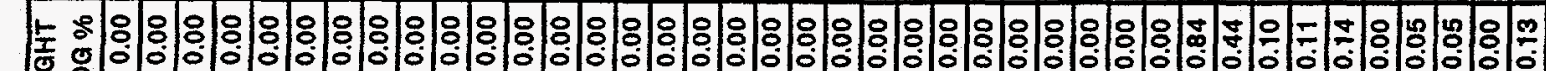

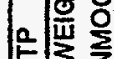

I4 32

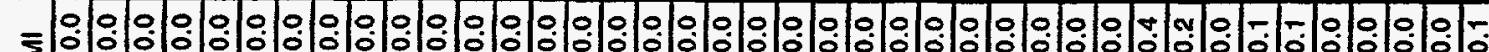
$\stackrel{\odot}{2}$

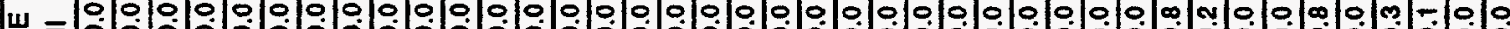
ż 总 올

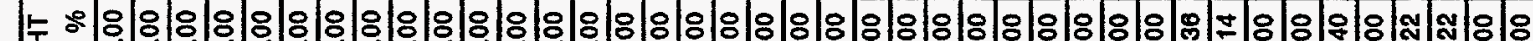
- 志

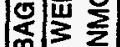

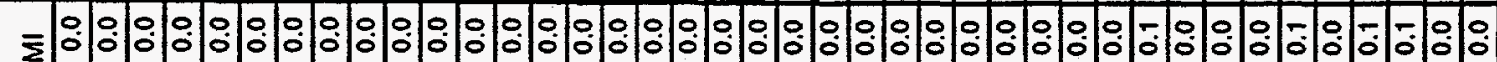
产 u No

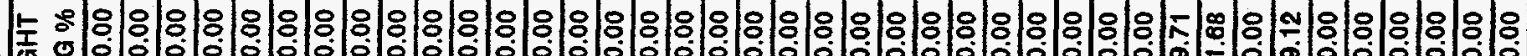

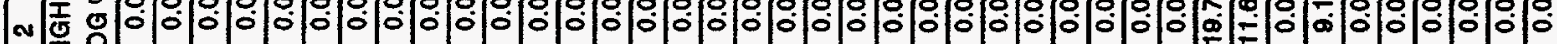
道要

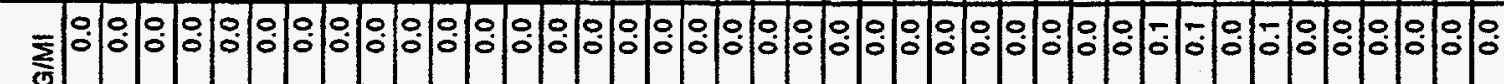
$\frac{5}{\Sigma}$

出 苋

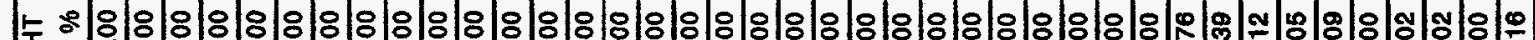
- 壬

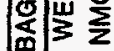

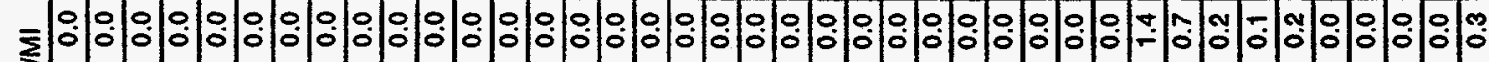

$$
\begin{aligned}
& \text { 을 }
\end{aligned}
$$

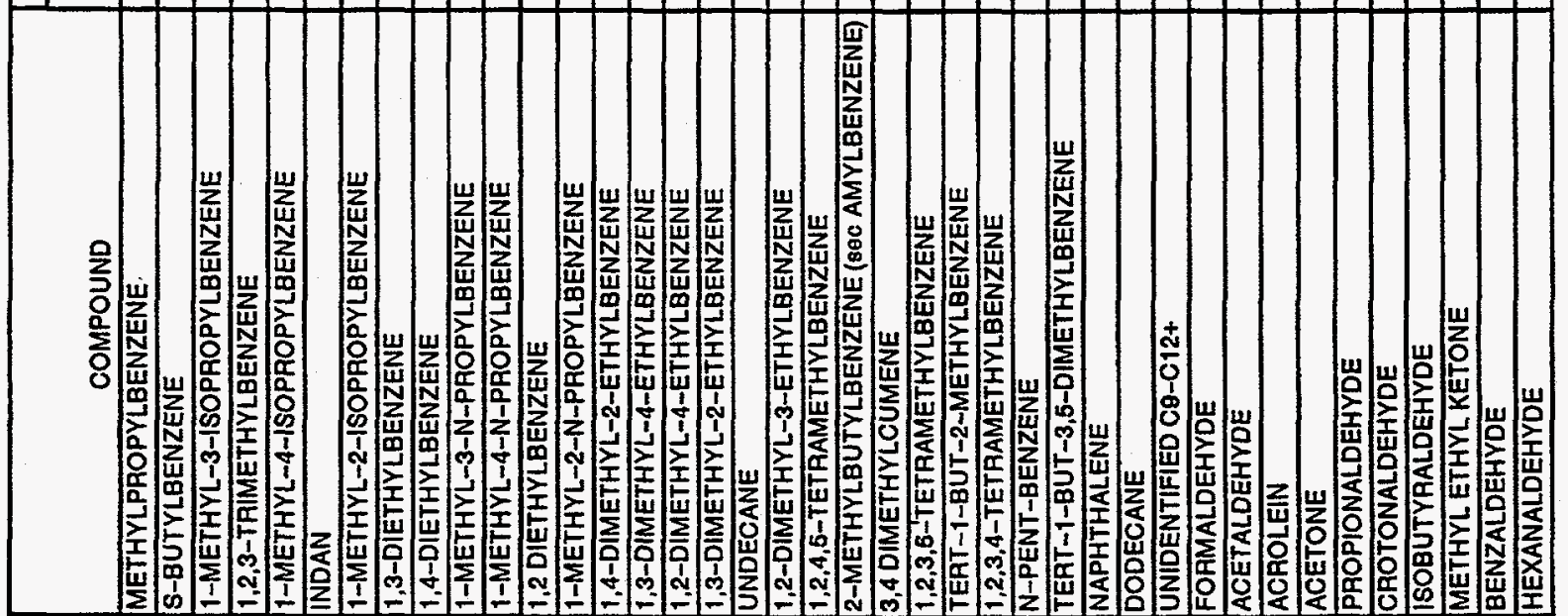


TEST NO: BLB-CP2-04S

TEST CAR: 1994 PONTIAC GRANDAM

TEST DATE: $10 / 2 / 95$

TEST FUEL: EM-1968-F

\begin{tabular}{|c|c|c|c|c|c|c|c|c|c|c|c|c|}
\hline \multirow[b]{2}{*}{ COMPOUND } & \multicolumn{3}{|c|}{ BAG1 } & \multicolumn{3}{|c|}{ BAG 2} & \multicolumn{3}{|c|}{$\overline{B A G 3}$} & \multicolumn{3}{|c|}{ FTP } \\
\hline & MG/MI & \begin{tabular}{|l|} 
WEIGHT \\
NMOG $\%$ \\
\end{tabular} & $\begin{array}{l}\text { OZONE, } \\
\text { MGMI }\end{array}$ & MG/MI & $\begin{array}{l}\text { WEIGHT } \\
\text { NMOQ } \%\end{array}$ & $\begin{array}{l}\text { OZONE } \\
\text { MG/MI }\end{array}$ & MG/MI & $\begin{array}{l}\text { WEIGHT } \\
\text { NMOG } \%\end{array}$ & $\begin{array}{l}\text { OZONE } \\
\text { MGMMI }\end{array}$ & MG/MI & $\begin{array}{l}\text { WEIGHT } \\
\text { NMOQ } \%\end{array}$ & $\begin{array}{l}\text { OZONE } \\
\text { MG/MI }\end{array}$ \\
\hline METHANE & 26.8 & & 0.4 & 0.0 & & 0.0 & 9.7 & & 0.1 & 8.3 & & 0.12 \\
\hline ETHANE & 5.0 & 2.66 & 1.3 & 0.0 & 0.04 & 0.0 & 1.0 & 4.46 & 0.3 & 1.3 & 2.71 & 0.33 \\
\hline ETHYLENE & 12.6 & 6.46 & 92.1 & 0.0 & 0.46 & 0.1 & 0.9 & 3.71 & 6.2 & 2.9 & 6.87 & 20.84 \\
\hline PROPANE & 0.1 & 0.06 & 0.1 & 0.0 & 0.00 & 0.0 & 0.0 & 0.16 & 0.0 & 0.0 & 0.07 & 0.02 \\
\hline PROPYLENE & $\overline{10.3}$ & 5.25 & 96.4 & 0.0 & 0.27 & 0.1 & 0.7 & 3.23 & 7.0 & 2.3 & 4.79 & 22.04 \\
\hline ACETYLENE & 6.6 & 3.35 & 3.3 & 0.0 & 0.00 & 0.0 & 0.0 & 0.00 & 0.0 & 1.4 & 2.79 & 0.68 \\
\hline PROPADIENE & 0.0 & 0.00 & 0.0 & 0.0 & 0.00 & 0.0 & 0.0 & 0.00 & 0.0 & 0.0 & 0.00 & 0.00 \\
\hline BUTANE & 1.7 & 0.88 & 1.8 & 0.0 & 0.86 & 0.0 & 0.1 & 0.20 & 0.1 & 0.4 & 0.81 & 0.40 \\
\hline TRANS-2-BUTENE & 0.8 & 0.39 & 7.6 & 0.0 & 0.00 & 0.0 & 0.1 & 0.44 & 1.0 & 0.2 & 0.38 & 1.87 \\
\hline 1-BUTENE & 1.0 & 0.53 & 9.2 & 0.0 & 0.00 & 0.0 & 0.1 & 0.41 & 0.8 & 0.2 & 0.49 & 2.15 \\
\hline 2-METHYLPROPENE (ISOBUTYLENE) & 9.0 & 4.61 & 47.8 & 0.0 & 0.00 & 0.0 & 1.1 & 4.64 & 5.7 & 2.2 & 4.43 & 11.51 \\
\hline 2,2-DIMETHYLPROPANE (NEOPENTANE) & 0.1 & 0.05 & 0.0 & 0.0 & 0.00 & 0.0 & 0.0 & 0.00 & 0.0 & 0.0 & 0.04 & 0.01 \\
\hline PROPYNE & 0.0 & 0.00 & 0.0 & 0.0 & 0.00 & 0.0 & 0.0 & 0.00 & 0.0 & 0.0 & 0.00 & 0.00 \\
\hline 1,3-8UTADIENE & 1.1 & 0.57 & 12.2 & 0.0 & 0.00 & 0.0 & 0.1 & 0.41 & 1.0 & 0.3 & 0.63 & 2.82 \\
\hline 2-METHYLPROPANE (ISOBUTANE) & 0.1 & 0.07 & 0.2 & 0.0 & 0.44 & 0.0 & 0.0 & 0.00 & 0.0 & 0.0 & 0.07 & 0.04 \\
\hline 1-BUTYNE & 0.0 & 0.00 & 0.0 & 0.0 & 0.00 & 0.0 & 0.0 & 0.00 & 0.0 & 0.0 & 0.00 & 0.00 \\
\hline METHANOL & 0.0 & 0.00 & 0.0 & 0.0 & 0.00 & 0.0 & 0.0 & 0.00 & 0.0 & 0.0 & 0.00 & 0.00 \\
\hline CIS-2-BUTENE & 0.6 & 0.30 & 5.8 & 0.0 & 0.00 & 0.0 & 0.0 & 0.00 & 0.0 & 0.1 & 0.25 & 1.22 \\
\hline 3-METHYL-1-BUTENE & 0.0 & 0.00 & 0.0 & 0.0 & 0.00 & 0.0 & 0.0 & 0.00 & 0.0 & 0.0 & 0.00 & 0.00 \\
\hline ETHANOL & 0.0 & 0.00 & 0.0 & 0.0 & 0.00 & 0.0 & 0.0 & 0.00 & 0.0 & 0.0 & 0.00 & 0.00 \\
\hline 2-METHYLBUTANE (ISOPENTANE) & 12.8 & 6.54 & 17.6 & 0.3 & 6.98 & 0.4 & 1.8 & 7.67 & 2.4 & 3.3 & 6.71 & 4.63 \\
\hline 2-BUTYNE & 0.0 & 0.00 & 0.0 & 0.0 & 0.00 & 0.0 & 0.0 & 0.00 & 0.0 & 0.0 & 0.00 & 0.00 \\
\hline 1-PENTENE & 0.3 & 0.14 & 1.7 & 0.0 & 0.00 & 0.0 & 0.0 & 0.00 & 0.0 & 0.1 & 0.11 & 0.35 \\
\hline 2-METHYL-1-BUTENE & 0.6 & 0.33 & 3.1 & 0.0 & 0.00 & 0.0 & 0.1 & 0.55 & 0.6 & 0.2 & 0.34 & 0.82 \\
\hline PENTANE & 1.8 & 0.96 & 2.0 & 0.0 & 0.00 & 0.0 & 0.5 & 2.36 & 0.6 & 0.5 & 1.11 & 0.56 \\
\hline UNIDENTIFIED C5 OLEFINS & 0.0 & 0.00 & 0.0 & 0.0 & 0.00 & 0.0 & 0.0 & 0.00 & 0.0 & 0.0 & 0.00 & 0.00 \\
\hline 2-METHYL-1,3-BUTADIENE & 1.0 & 0.49 & 8.8 & 0.0 & 0.00 & 0.0 & 0.1 & 0.39 & 0.8 & 0.2 & 0.46 & 2.05 \\
\hline TRANS-2-PENTENE & 0.5 & 0.24 & 4.1 & 0.0 & 0.00 & 0.0 & 0.1 & 0.44 & 0.9 & 0.1 & 0.26 & 1.10 \\
\hline 3,3-DIMETHYL-1-BUTENE & 0.0 & 0.00 & 0.0 & 0.0 & 0.00 & 0.0 & 0.0 & 0.00 & 0.0 & 0.0 & 0.00 & 0.00 \\
\hline CIS-2-PENTENE & 0.2 & 0.11 & 1.9 & 0.0 & 0.00 & 0.0 & 0.0 & 0.00 & 0.0 & 0.0 & 0.09 & 0.39 \\
\hline 2-METHYL-2-BUTENE & 1.4 & 0.70 & 8.8 & 0.0 & 0.00 & 0.0 & 0.2 & 0.79 & 1.2 & 0.3 & 0.68 & 2.14 \\
\hline CYCLOPENTADIENE & 0.6 & 0.25 & 3.8 & 0.0 & 0.00 & 0.0 & 0.0 & 0.00 & 0.0 & $\overline{0.1}$ & 0.21 & 0.79 \\
\hline 2,2-DIMETHYLBUTANE & 0.2 & 0.11 & 0.2 & 0.0 & 0.00 & 0.0 & 0.1 & 0.38 & 0.1 & 0.1 & 0.14 & 0.08 \\
\hline CYCLOPENTENE & 0.1 & 0.08 & 0.9 & 0.0 & 0.00 & 0.0 & 0.0 & 0.00 & 0.0 & 0.0 & 0.05 & 0.18 \\
\hline 4-METHYL-1-PENTENE & 0.1 & 0.06 & 0.5 & 0.0 & 0.00 & 0.0 & 0.0 & 0.00 & 0.0 & 0.0 & 0.06 & 0.11 \\
\hline 3-METHYL-1-PENTENE & 0.1 & 0.05 & 0.5 & 0.0 & 0.00 & 0.0 & 0.0 & 0.00 & 0.0 & 0.0 & 0.04 & 0.00 \\
\hline CYCLOPENTANE & 0.0 & 0.00 & 0.0 & 0.0 & 0.00 & 0.0 & 0.0 & 0.00 & 0.0 & 0.0 & 0.00 & 0.00 \\
\hline 2,3-DIMETHYLBUTANE & 1.5 & 0.75 & 1.6 & 0.0 & 1.23 & 0.0 & 0.3 & 1.47 & 0.4 & 0.4 & 0.86 & 0.45 \\
\hline MTBE & 7.1 & 3.64 & 4.4 & 0.0 & 0.00 & 0.0 & 0.0 & 0.00 & 0.0 & 1.5 & 3.02 & 0.92 \\
\hline
\end{tabular}




\begin{tabular}{|c|c|c|c|c|c|c|c|c|c|c|c|c|}
\hline \multirow[b]{2}{*}{ COMPOUND } & \multicolumn{3}{|c|}{$\overline{B A G 1}$} & \multicolumn{3}{|c|}{ 8AG2 } & \multicolumn{3}{|c|}{ BAG 3} & \multicolumn{3}{|c|}{ FTP } \\
\hline & MG/MI & $\begin{array}{l}\text { WEIGHT } \\
\text { NMOG \% }\end{array}$ & $\begin{array}{l}\text { OZONE, } \\
\text { MG/MI }\end{array}$ & MG/MI & $\begin{array}{l}\text { WEIGHT } \\
\text { NMOG \% }\end{array}$ & $\begin{array}{l}\text { OZONE } \\
\mathrm{MG/MI}\end{array}$ & MG/MI & $\begin{array}{l}\text { WEIGHT } \\
\text { NMOG \% }\end{array}$ & $\begin{array}{l}\text { OZONE } \\
\text { MG/MI }\end{array}$ & MG/MI & $\begin{array}{l}\text { WEIGHT } \\
\text { NMOG \% }\end{array}$ & $\begin{array}{l}\text { OZONE } \\
\text { MG/MI }\end{array}$ \\
\hline 2,3-DIMETHYL-1-BUTENE & 0.0 & 0.00 & 0.0 & 0.0 & 0.00 & 0.0 & 0.0 & 0.00 & 0.0 & 0.0 & 0.00 & 0.00 \\
\hline 4-METHYL-CIS-2-PENTENE & 0.0 & 0.00 & 0.0 & 0.0 & 0.00 & 0.0 & 0.0 & 0.00 & 0.0 & 0.0 & 0.00 & 0.00 \\
\hline 2-METHYLPENTANE & 2.3 & 1.19 & 3.6 & 0.2 & 5.89 & 0.3 & 0.6 & 2.64 & 0.9 & 0.8 & 1.56 & 1.17 \\
\hline 4-METHYL-TAANS-2-PENTENE & 0.0 & 0.00 & 0.0 & 0.0 & 0.00 & 0.0 & 0.0 & 0.00 & 0.0 & 0.0 & 0.00 & 0.00 \\
\hline 3-METHYLPENTANE & $1 . \overline{6}$ & 0.76 & 2.2 & 0.1 & 2.77 & 0.2 & 0.4 & 1.65 & 0.6 & 0.5 & 0.95 & $\overline{0.71}$ \\
\hline 2-METHYL-1-PENTENE & 0.1 & 0.07 & 0.6 & 0.0 & 0.00 & 0.0 & 0.0 & 0.00 & 0.0 & 0.0 & 0.06 & 0.13 \\
\hline 1-HEXENE & 0.1 & 0.06 & 0.5 & 0.0 & 0.00 & 0.0 & 0.0 & 0.00 & 0.0 & 0.0 & 0.05 & 0.10 \\
\hline HEXANE & 1.1 & 0.58 & 1.1 & 0.2 & 4.19 & 0.1 & 0.3 & 1.38 & 0.3 & 0.4 & 0.82 & 0.39 \\
\hline UNIDENTIFIED C6 OLEFINS & 0.2 & 0.12 & 1.6 & 0.0 & 0.00 & 0.0 & 0.0 & 0.00 & 0.0 & 0.0 & 0.10 & 0.33 \\
\hline TRANS-3-HEXENE & 0.2 & 0.09 & 1.2 & 0.0 & 0.00 & 0.0 & 0.0 & 0.00 & 0.0 & 0.0 & 0.08 & 0.26 \\
\hline CIS-3-HEXENE & 0.3 & 0.13 & 1.8 & 0.0 & 0.00 & 0.0 & 0.1 & 0.40 & 0.6 & $\overline{0.1}$ & 0.16 & 0.64 \\
\hline TRANS-2-HEXENE & 0.0 & 0.00 & 0.0 & 0.0 & 0.00 & 0.0 & 0.0 & 0.00 & 0.0 & 0.0 & 0.00 & 0.00 \\
\hline 3-METHYL-TRANS-2-PENTENE & 0.4 & 0.18 & 2.4 & 0.0 & 0.00 & 0.0 & 0.0 & 0.00 & 0.0 & 0.1 & 0.16 & 0.60 \\
\hline 2-METHYL-2-PENTENE & 0.3 & 0.13 & 1.7 & 0.0 & 0.00 & 0.0 & 0.0 & 0.00 & 0.0 & 0.1 & 0.11 & 0.36 \\
\hline 3-METHYLCYCLOPENTENE & 0.0 & 0.00 & 0.0 & 0.0 & 0.00 & 0.0 & 0.0 & 0.00 & 0.0 & 0.0 & 0.00 & 0.00 \\
\hline CIS-2-HEXENE & 0.2 & 0.08 & 1.0 & 0.0 & 0.00 & 0.0 & 0.0 & 0.00 & 0.0 & 0.0 & 0.07 & 0.21 \\
\hline ETBE & 0.0 & 0.00 & 0.0 & 0.0 & 0.00 & 0.0 & 0.0 & 0.00 & 0.0 & 0.0 & 0.00 & 0.00 \\
\hline 3-METHYL-CIS-2-PENTENE & 0.3 & 0.14 & 1.9 & 0.0 & 0.00 & 0.0 & 0.0 & 0.00 & 0.0 & 0.1 & 0.12 & 0.39 \\
\hline 2,2-DIMETHYLPENTANE & 0.7 & 0.38 & 1.0 & 0.1 & 2.47 & 0.1 & 0.2 & 0.83 & 0.3 & 0.3 & 0.52 & 0.36 \\
\hline METHYLCYCLOPENTANE & 0.1 & 0.05 & 0.3 & 0.0 & 0.00 & 0.0 & 0.0 & 0.00 & 0.0 & $\overline{0.0}$ & 0.04 & 0.06 \\
\hline 2,4-DIMETHYLPENTANE & 3.9 & 2.00 & 7.0 & 0.1 & 2.63 & 0.2 & 0.7 & 3.09 & 1.3 & 1.1 & 2.17 & 1.89 \\
\hline 2,3,3-TRIMETHYL-1-BUTENE & 0.0 & 0.00 & 0.0 & 0.0 & 0.00 & 0.0 & 0.0 & 0.00 & 0.0 & 0.0 & 0.00 & 0.00 \\
\hline 2,2,3-TAIMETHYLBUTANE & 0.3 & 0.16 & 0.4 & 0.2 & 4.14 & 0.2 & 0.1 & 0.55 & 0.2 & 0.2 & 0.36 & 0.23 \\
\hline 3,4-DIMETHYL-1-PENTENE & 0.0 & 0.00 & 0.0 & 0.0 & 0.00 & 0.0 & 0.0 & 0.00 & 0.0 & 0.0 & 0.00 & 0.00 \\
\hline 1-METHYLCYCLOPENTENE & 0.6 & 0.23 & 2.6 & 0.3 & 7.03 & 1.5 & 0.0 & 0.00 & 0.0 & 0.2 & 0.46 & 1.29 \\
\hline BENZENE & 8.6 & 4.42 & 3.6 & 0.2 & 6.19 & 0.1 & 2.1 & 9.15 & 0.9 & $\overline{2.5}$ & 5.11 & 1.05 \\
\hline 3-METHYL-1-HEXENE & 0.0 & 0.00 & 0.0 & 0.0 & 0.00 & 0.0 & 0.0 & 0.00 & 0.0 & 0.0 & 0.00 & 0.00 \\
\hline 3,3-DIMETHYLPENTANE & 0.2 & 0.11 & 0.2 & 0.0 & 0.00 & 0.0 & 0.0 & 0.00 & 0.0 & 0.0 & 0.09 & 0.03 \\
\hline CYCLOHEXANE & 0.1 & 0.07 & 0.2 & 0.0 & 0.00 & 0.0 & 0.0 & 0.00 & 0.0 & 0.0 & 0.06 & 0.04 \\
\hline 2-METHYLHEXANE & 0.0 & 0.00 & 0.0 & 0.0 & 0.00 & 0.0 & 0.0 & 0.00 & 0.0 & 0.0 & 0.00 & 0.00 \\
\hline 2,3-DIMETHYLPENTANE & 7.2 & 3.71 & 10.9 & 0.2 & 4.75 & 0.3 & 1.4 & 6.16 & 2.1 & 2.0 & 4.06 & 3.00 \\
\hline 1.1-DIMETHYLCYCLOPENTANE & 0.0 & 0.00 & 0.0 & 0.0 & 0.00 & 0.0 & 0.0 & 0.00 & 0.0 & 0.0 & 0.00 & 0.00 \\
\hline CYCLOHEXENE & 0.0 & 0.00 & 0.0 & 0.0 & 0.00 & 0.0 & 0.0 & 0.00 & 0.0 & 0.0 & 0.00 & 0.00 \\
\hline 3-METHYLHEXANE & 1.5 & 0.74 & 2.0 & 0.0 & 1.20 & 0.1 & 0.3 & 1.37 & 0.4 & 0.4 & 0.84 & 0.58 \\
\hline CIS-1,3-DIMETHYLCYCLOPENTANE & 0.2 & 0.10 & 0.5 & 0.0 & 0.00 & 0.0 & 0.1 & 0.35 & 0.2 & 0.1 & 0.13 & 0.16 \\
\hline 3-ETHYLPENTANE & 0.0 & 0.00 & 0.0 & 0.0 & 0.00 & 0.0 & 0.0 & 0.00 & 0.0 & 0.0 & 0.00 & 0.00 \\
\hline TRANS-1,2-DIMETHYLCYCLOPENTANE & 0.0 & 0.00 & 0.0 & 0.0 & 0.00 & 0.0 & 0.0 & 0.00 & 0.0 & 0.0 & 0.00 & 0.00 \\
\hline TRANS-1,3-DIMETHYLCYCLOPENTANE & 0.0 & 0.00 & 0.0 & 0.0 & 0.00 & 0.0 & 0.0 & 0.00 & 0.0 & 0.0 & 0.00 & 0.00 \\
\hline 1-HEPTENE & 0.0 & 0.00 & 0.0 & 0.0 & 0.00 & 0.0 & 0.0 & 0.00 & 0.0 & 0.0 & 0.00 & 0.00 \\
\hline 2,2,4-TRIMETHYLPENTANE & 13.5 & 6.89 & 12.5 & 0.1 & 3.16 & 0.1 & 2.9 & 12.48 & 2.7 & 3.7 & 7.47 & 3.40 \\
\hline 2-METHYL-1-HEXENE & 0.0 & 0.00 & 0.0 & 0.0 & 0.00 & 0.0 & 0.0 & 0.00 & 0.0 & 0.0 & 0.00 & 0.00 \\
\hline TRANS-3-HEPTENE & 0.0 & 0.00 & 0.0 & 0.0 & 0.00 & 0.0 & 0.0 & 0.00 & 0.0 & 0.0 & 0.00 & 0.00 \\
\hline HEPTANE & 1.0 & 0.49 & 0.8 & 0.0 & 1.02 & 0.0 & 0.1 & 0.56 & 0.1 & 0.3 & 0.52 & 0.21 \\
\hline UNIDENTIFIED C7 & 1.1 & 0.58 & 5.1 & 0.0 & 0.36 & 0.1 & 0.2 & 0.84 & 0.9 & 0.3 & 0.61 & 1.34 \\
\hline
\end{tabular}




\begin{tabular}{|c|c|c|c|c|c|c|c|c|c|c|c|c|}
\hline \multirow[b]{2}{*}{ COMPOUND } & \multicolumn{3}{|c|}{ BAG 1} & \multicolumn{3}{|c|}{ BAG 2} & \multicolumn{3}{|c|}{ BAG 3} & \multicolumn{3}{|c|}{ FTP } \\
\hline & MG/MI & $\begin{array}{l}\text { WEIGHT } \\
\text { NMOG } \%\end{array}$ & $\begin{array}{l}\text { OZONE, } \\
\text { MG/MI }\end{array}$ & MG/MI & $\begin{array}{l}\text { WEIGHT } \\
\text { NMOQ } \%\end{array}$ & $\begin{array}{l}\text { OZONE } \\
\text { MG/MI }\end{array}$ & MG/MI & $\begin{array}{l}\text { WEIGHT } \\
\text { NMOG \% }\end{array}$ & $\begin{array}{l}\text { OZONE } \\
\text { MGMII }\end{array}$ & MG/MI & $\begin{array}{l}\text { WEIGHT } \\
\text { NMOG } \%\end{array}$ & $\begin{array}{l}\text { OZONE } \\
\text { MG/MI }\end{array}$ \\
\hline 2-METHYL-2-HEXENE & 0.2 & 0.08 & 0.9 & 0.0 & 0.00 & 0.0 & 0.0 & 0.00 & 0.0 & 0.0 & 0.07 & 0.19 \\
\hline 3-METHYL-TAANS-3-HEXENE & 0.0 & 0.00 & 0.0 & 0.0 & 0.00 & 0.0 & 0.0 & 0.00 & 0.0 & 0.0 & 0.00 & 0.00 \\
\hline TRANS-2-HEPTENE & 0.1 & 0.05 & 0.6 & 0.0 & 0.00 & 0.0 & 0.0 & 0.00 & 0.0 & 0.0 & 0.04 & 0.13 \\
\hline 3-ETHYL-CIS-2-PENTENE & 0.0 & 0.00 & 0.0 & 0.0 & 0.00 & $\overline{0.0}$ & $\overline{0.0}$ & 0.00 & 0.0 & 0.0 & 0.00 & 0.00 \\
\hline 2,4,4-TRIMETHYL-1-PENTENE & 0.1 & 0.08 & 0.4 & 0.0 & 0.00 & 0.0 & 0.0 & 0.00 & 0.0 & 0.0 & 0.06 & 0.08 \\
\hline 2,2,4-TRIMETHYL-1-PENTENE & 0.0 & 0.00 & 0.0 & 0.0 & 0.00 & 0.0 & 0.0 & 0.00 & 0.0 & 0.0 & 0.00 & 0.00 \\
\hline 2,3-DIMETHYL-2-PENTENE & 0.0 & 0.00 & 0.0 & 0.0 & 0.00 & 0.0 & 0.0 & 0.00 & 0.0 & 0.0 & 0.00 & 0.00 \\
\hline CIS-2-HEPTENE & 0.1 & 0.06 & 0.6 & 0.0 & 0.00 & 0.0 & 0.0 & 0.00 & 0.0 & 0.0 & 0.05 & 0.13 \\
\hline METHYLCYCLOHEXANE & 0.2 & 0.08 & 0.3 & 0.0 & 0.00 & 0.0 & 0.0 & 0.00 & 0.0 & 0.0 & 0.07 & 0.06 \\
\hline CIS-1,2-DIMETHYLCYCLOPENTANE & 0.0 & 0.00 & 0.0 & 0.0 & 0.00 & 0.0 & 0.0 & 0.00 & 0.0 & 0.0 & 0.00 & 0.00 \\
\hline 2,2-DIMETHYLHEXANE & 1.4 & 0.74 & 1.7 & 0.0 & 0.00 & 0.0 & 0.3 & 1.19 & 0.3 & 0.4 & 0.77 & 0.45 \\
\hline 1,1,3-TAIMETHYLCYCLOPENTANE & 0.0 & 0.00 & 0.0 & 0.0 & 0.00 & 0.0 & 0.0 & 0.00 & 0.0 & 0.0 & 0.00 & 0.00 \\
\hline 2,4,4-TAIMETHYL-2-PENTENE & 0.0 & 0.00 & 0.0 & 0.0 & 0.00 & 0.0 & 0.0 & 0.00 & 0.0 & 0.0 & 0.00 & 0.00 \\
\hline 2,2,3-TRIMETHYLPENTANE & $\overline{0.0}$ & 0.00 & 0.0 & 0.0 & 0.00 & 0.0 & 0.0 & 0.00 & 0.0 & 0.0 & 0.00 & 0.00 \\
\hline 2,5-DIMETHYLHEXANE & 0.0 & 0.00 & 0.0 & 0.0 & 0.00 & 0.0 & 0.0 & 0.00 & 0.0 & 0.0 & 0.00 & 0.00 \\
\hline ETHYLCYCLOPENTANE & 0.0 & 0.00 & 0.0 & 0.0 & 0.00 & 0.0 & 0.0 & 0.00 & 0.0 & 0.0 & 0.00 & 0.00 \\
\hline 2,4-DIMETHYLHEXANE & 1.6 & 0.82 & 2.4 & 0.0 & 0.00 & 0.0 & 0.3 & 1.44 & 0.5 & 0.4 & 0.87 & $\overline{0.63}$ \\
\hline 1-TRANS-2-CIS-4-TRIMETHYLCYCLOPENTANE & 0.0 & 0.00 & 0.0 & 0.0 & 0.00 & 0.0 & 0.0 & 0.00 & 0.0 & 0.0 & 0.00 & 0.00 \\
\hline 3,3-DIMETHYLHEXANE & 0.1 & 0.04 & 0.1 & 0.0 & 0.00 & 0.0 & 0.0 & 0.00 & 0.0 & 0.0 & 0.04 & 0.02 \\
\hline 1-TRANS-2-CIS-3-TRIMETHYLCYCLOPENTANE & 0.0 & 0.00 & 0.0 & 0.0 & 0.00 & 0.0 & 0.0 & 0.00 & 0.0 & 0.0 & 0.00 & 0.00 \\
\hline 2,3,4-TRIMETHYLPENTANE & 0.0 & 0.00 & 0.0 & 0.0 & 0.00 & 0.0 & 0.0 & 0.00 & 0.0 & 0.0 & 0.00 & 0.00 \\
\hline 2,3,3-TAIMETHYLPENTANE & $\overline{3.1}$ & 1.59 & 3.7 & 0.1 & 3.57 & 0.2 & 0.7 & 2.88 & 0.8 & 0.9 & 1.84 & 1.08 \\
\hline TOLUENE & 16.7 & 8.66 & 46.7 & 0.7 & 20.21 & 2.0 & 1.8 & 7.84 & 5.0 & $\overline{4.4}$ & 8.93 & 11.92 \\
\hline 2,3-DIMETHYLHEXANE & 2.1 & 1.09 & 2.8 & 0.0 & 0.00 & 0.0 & 0.0 & 0.00 & 0.0 & 0.4 & 0.81 & 0.59 \\
\hline 1,1,2-TRIMETHYLCYCLOPENTANE & 0.0 & 0.00 & 0.0 & 0.0 & 0.00 & 0.0 & 0.0 & 0.00 & 0.0 & 0.0 & 0.00 & 0.00 \\
\hline 2-METHYLHEPTANE & 1.0 & 0.51 & 0.8 & 0.0 & 0.00 & 0.0 & 0.2 & 0.72 & 0.2 & 0.3 & $\overline{0.61}$ & 0.24 \\
\hline 3,4-DIMETHYLHEXANE & 0.0 & 0.00 & 0.0 & 0.0 & 0.00 & 0.0 & 0.0 & 0.00 & 0.0 & 0.0 & 0.00 & 0.00 \\
\hline $2,2,4,4-T E T R A M E T H Y L P E N T A N E$ & 0.0 & 0.00 & 0.0 & 0.0 & 0.00 & 0.0 & 0.0 & 0.00 & 0.0 & 0.0 & 0.00 & 0.00 \\
\hline 4-METHYLHEPTANE & 0.3 & 0.17 & 0.4 & 0.0 & 0.00 & 0.0 & 0.0 & 0.00 & 0.0 & 0.1 & $\overline{0.14}$ & 0.08 \\
\hline 2-METHYL-3-ETHYLPENTANE & 0.0 & 0.00 & 0.0 & 0.0 & 0.00 & 0.0 & 0.0 & 0.00 & 0.0 & 0.0 & 0.00 & 0.00 \\
\hline 2,6-DIMETHYLHEPTANE & 0.0 & 0.00 & 0.0 & 0.0 & 0.00 & 0.0 & 0.0 & 0.00 & 0.0 & 0.0 & 0.00 & 0.00 \\
\hline 3-METHYLHEPTANE & 0.3 & 0.14 & 0.3 & 0.0 & 0.00 & 0.0 & 0.2 & 0.82 & 0.2 & 0.1 & 0.22 & 0.11 \\
\hline 1-CIS,2-TRANS,3-TRIMETHYLCYCLOPENTANE & 0.0 & 0.00 & 0.0 & 0.0 & 0.00 & 0.0 & 0.0 & 0.00 & 0.0 & 0.0 & 0.00 & 0.00 \\
\hline CIS-1,3-DIMETHYLCYCLOHEXANE & 0.0 & 0.00 & 0.0 & 0.0 & 0.00 & 0.0 & 0.0 & 0.00 & 0.0 & 0.0 & 0.00 & 0.00 \\
\hline TRANS-1,4-DIMETHYLCYCLOHEXANE & 1.3 & 0.67 & 2.5 & 0.0 & 0.00 & 0.0 & 0.3 & 1.14 & 0.5 & 0.3 & 0.70 & 0.67 \\
\hline 3-ETHYLHEXANE & 0.0 & 0.00 & 0.0 & 0.0 & 0.00 & 0.0 & 0.0 & 0.00 & 0.0 & 0.0 & 0.00 & 0.00 \\
\hline 2,2,6-TAIMETHYLHEXANE & 0.1 & 0.05 & 0.1 & 0.0 & 0.00 & 0.0 & 0.0 & 0.00 & 0.0 & 0.0 & 0.04 & 0.02 \\
\hline CIS-1-METHYL-3-ETHYLCYCLOPENTANE & 0.0 & 0.00 & 0.0 & 0.0 & 0.00 & 0.0 & 0.0 & 0.00 & 0.0 & 0.0 & 0.00 & 0.00 \\
\hline 1,1-DIMETHYLCYCLOHEXANE & 0.0 & 0.00 & 0.0 & 0.0 & 0.00 & 0.0 & 0.0 & 0.00 & 0.0 & 0.0 & 0.00 & 0.00 \\
\hline TRANS-1-METHYL-2-ETHYLCYCLOPENTANE & 0.0 & 0.00 & 0.0 & 0.0 & 0.00 & 0.0 & 0.0 & 0.00 & 0.0 & 0.0 & 0.00 & 0.00 \\
\hline 1-METHYL-1-ETHYL-CYCLOPENTANE & 0.0 & 0.00 & 0.0 & 0.0 & 0.00 & 0.0 & 0.0 & 0.00 & 0.0 & 0.0 & 0.00 & 0.00 \\
\hline 2,4,4-TRIMETHYLHEXANE & 0.0 & 0.00 & 0.0 & 0.0 & 0.00 & 0.0 & 0.0 & 0.00 & 0.0 & 0.0 & 0.00 & 0.00 \\
\hline 2,2,4-TAIMETHYLHEXANE & 0.0 & 0.00 & 0.0 & 0.0 & 0.00 & 0.0 & 0.0 & 0.00 & 0.0 & 0.0 & 0.00 & 0.00 \\
\hline TRANS-1,2-DIMETHYLCYCLOHEXANE & 0.0 & 0.00 & 0.0 & 0.0 & 0.00 & 0.0 & 0.0 & 0.00 & 0.0 & 0.0 & 0.00 & 0.00 \\
\hline
\end{tabular}




\begin{tabular}{|c|c|c|c|c|c|c|c|c|c|c|c|c|}
\hline \multirow[b]{2}{*}{ COMPOUND } & \multicolumn{3}{|c|}{ BAG 1} & \multicolumn{3}{|c|}{ BAG 2} & \multicolumn{3}{|c|}{$\overline{B A G 3}$} & \multicolumn{3}{|c|}{ FTP } \\
\hline & MG/MI & $\begin{array}{l}\text { WEIGHT } \\
\text { NMOG } \%\end{array}$ & $\begin{array}{l}\text { OZONE, } \\
\text { MG/MI }\end{array}$ & MG/MI & $\begin{array}{l}\text { WEIGHT } \\
\text { NMOG } \%\end{array}$ & $\begin{array}{l}\text { OZONE } \\
\text { MG/MI }\end{array}$ & MG/MI & $\begin{array}{l}\text { WEIGHT } \\
\text { NMOG \% }\end{array}$ & $\begin{array}{l}\text { OZONE } \\
\text { MG/MI }\end{array}$ & Ma/MI & $\begin{array}{l}\text { WEIGHT } \\
\text { NMOG \% }\end{array}$ & $\begin{array}{l}\text { OZONE } \\
\text { MG/MI }\end{array}$ \\
\hline 1-OCTENE & 0.1 & 0.06 & 0.3 & 0.0 & $\overline{0.00}$ & 0.0 & 0.0 & 0.00 & 0.0 & 0.0 & 0.05 & 0.06 \\
\hline TRANS-4-OCTENE & 0.1 & 0.04 & 0.4 & 0.0 & 0.00 & 0.0 & 0.0 & 0.00 & 0.0 & 0.0 & 0.03 & 0.09 \\
\hline OCTANE & 0.7 & $\overline{0.34}$ & $\overline{0.4}$ & 0.0 & 0.00 & 0.0 & 0.1 & 0.48 & $\overline{0.1}$ & 0.2 & 0.34 & 0.10 \\
\hline UNIDENTIFIED C8 & 1.8 & 0.92 & 6.1 & 0.0 & 1.32 & 0.2 & 0.1 & 0.44 & 0.3 & 0.4 & 0.87 & $\overline{1.45}$ \\
\hline TRANS-2-OCTENE & 0.1 & 0.05 & 0.6 & 0.0 & 0.00 & 0.0 & 0.0 & 0.00 & 0.0 & 0.0 & 0.04 & 0.11 \\
\hline TRANS-1,3-DIMETHYLCYCLOHEXANE & 0.0 & 0.00 & 0.0 & 0.0 & 0.00 & 0.0 & 0.0 & 0.00 & 0.0 & 0.0 & 0.00 & 0.00 \\
\hline CIS-1,4-DIMETHYLCYCLOHEXANE & $\overline{0.0}$ & 0.00 & 0.0 & 0.0 & 0.00 & 0.0 & 0.0 & 0.00 & 0.0 & 0.0 & 0.00 & $\overline{0.00}$ \\
\hline CIS-2-OCTENE & 0.0 & 0.00 & 0.0 & 0.0 & 0.00 & 0.0 & 0.0 & 0.00 & 0.0 & 0.0 & 0.00 & 0.00 \\
\hline 2,3,6-TRIMETHYLHEXANE & $\overline{0.2}$ & 0.10 & 0.2 & 0.0 & 0.00 & 0.0 & 0.0 & 0.00 & 0.0 & 0.0 & 0.08 & 0.05 \\
\hline CIS-1-METHYL-2-ETHYLCYCLOPENTANE & $\overline{0.0}$ & 0.00 & 0.0 & 0.0 & 0.00 & 0.0 & 0.0 & 0.00 & 0.0 & 0.0 & 0.00 & 0.00 \\
\hline 2-METHYL-2-ETHYLHEPTANE & 0.0 & 0.00 & $\overline{0.0}$ & 0.0 & 0.00 & 0.0 & $\overline{0.0}$ & 0.00 & 0.0 & 0.0 & 0.00 & 0.00 \\
\hline 2,4-DIMETHYLHEPTANE & 0.1 & 0.07 & 0.2 & 0.2 & 6.55 & 0.3 & 0.0 & 0.00 & 0.0 & 0.2 & 0.31 & 0.21 \\
\hline 4,4-DIMETHYLHEPTANE & 0.0 & 0.00 & 0.0 & 0.0 & 0.00 & 0.0 & 0.0 & 0.00 & 0.0 & 0.0 & 0.00 & 0.00 \\
\hline CIS-1,2-DIMETHYLCYCLOHEXANE & $\overline{0.2}$ & 0.08 & $\overline{0.3}$ & 0.0 & 0.00 & 0.0 & 0.0 & 0.00 & 0.0 & 0.0 & $\overline{0.07}$ & 0.06 \\
\hline ETHYLCYCLOHEXANE & $\overline{0.3}$ & 0.14 & $\overline{0.5}$ & 0.0 & $\overline{0.00}$ & 0.0 & 0.0 & 0.00 & 0.0 & 0.1 & 0.12 & 0.11 \\
\hline PROPYLCYCLOHEXANE & 0.0 & 0.00 & 0.0 & 0.0 & 0.00 & 0.0 & 0.0 & 0.00 & 0.0 & 0.0 & 0.00 & 0.00 \\
\hline 2-METHYL-4-ETHYLHEXANE & 0.0 & 0.00 & 0.0 & 0.0 & 0.00 & 0.0 & 0.0 & 0.00 & $\overline{0.0}$ & 0.0 & 0.00 & 0.00 \\
\hline 2,6-DIMETHYLHEPTANE & 0.0 & 0.00 & 0.0 & 0.0 & 0.00 & 0.0 & 0.0 & 0.00 & 0.0 & 0.0 & 0.00 & 0.00 \\
\hline 1,1,3-TRIMETHYLCYCLOHEXANE & 0.0 & 0.00 & 0.0 & 0.0 & 0.00 & 0.0 & 0.0 & 0.00 & 0.0 & 0.0 & 0.00 & 0.00 \\
\hline 2,5-DIMETHYLHEPTANE & 0.0 & 0.00 & 0.0 & 0.0 & 0.00 & 0.0 & 0.0 & 0.00 & 0.0 & 0.0 & 0.00 & 0.00 \\
\hline 3,3-DIMETHYLHEPTANE & $\overline{0.0}$ & 0.00 & 0.0 & 0.0 & 0.00 & 0.0 & 0.0 & 0.00 & 0.0 & 0.0 & 0.00 & 0.00 \\
\hline 3,5-DIMETHYLHEPTANE & 0.0 & 0.00 & 0.0 & 0.0 & 0.00 & 0.0 & 0.0 & 0.00 & 0.0 & 0.0 & 0.00 & 0.00 \\
\hline ETHYLBENZENE & 5.0 & 2.55 & 13.5 & 0.0 & 0.00 & 0.0 & 0.5 & 2.20 & 1.4 & 1.2 & 2.41 & 3.18 \\
\hline 2,3-DIMETHYLHEPTANE & 0.0 & 0.00 & 0.0 & 0.0 & 0.00 & 0.0 & 0.0 & 0.00 & 0.0 & 0.0 & 0.00 & 0.00 \\
\hline m-\&p-XYLENE & 11.2 & 5.72 & 82.5 & 0.0 & 1.07 & 0.3 & 0.8 & 3.34 & 6.7 & 2.6 & 5.23 & 18.88 \\
\hline 4-METHYLOCTANE & 0.0 & 0.00 & 0.0 & 0.0 & 0.00 & 0.0 & 0.0 & 0.00 & 0.0 & 0.0 & 0.00 & 0.00 \\
\hline 2-METHYLOCTANE & 0.6 & 0.28 & 0.6 & 0.0 & 0.00 & 0.0 & 0.1 & 0.50 & 0.1 & 0.1 & 0.30 & 0.17 \\
\hline 3-METHYLOCTANE & 0.3 & 0.16 & 0.4 & 0.0 & 0.00 & 0.0 & 0.0 & 0.00 & 0.0 & 0.1 & 0.13 & 0.07 \\
\hline STYRENE & 0.0 & 0.00 & 0.0 & 0.0 & 0.00 & 0.0 & 0.0 & 0.00 & 0.0 & 0.0 & 0.00 & 0.00 \\
\hline O-XYLENE & 3.8 & 1.85 & 24.6 & 0.2 & 4.86 & 1.1 & 0.3 & 1.32 & 2.0 & 1.0 & 1.08 & 6.25 \\
\hline 2,4,6-TRIMETHYLHEXANE & 0.0 & 0.00 & 0.0 & 0.0 & 0.00 & 0.0 & 0.0 & 0.00 & 0.0 & 0.0 & 0.00 & 0.00 \\
\hline 1-NONENE & 0.3 & 0.17 & 0.7 & 0.0 & 0.00 & 0.0 & 0.0 & 0.00 & 0.0 & 0.1 & $\overline{0.14}$ & 0.16 \\
\hline NONANE & 0.2 & 0.12 & 0.1 & 0.0 & 0.00 & 0.0 & 0.0 & 0.00 & 0.0 & 0.0 & 0.10 & 0.03 \\
\hline ISOPROPYLBENZENE (CUMENE) & 0.2 & 0.09 & 0.4 & 0.0 & 0.00 & 0.0 & 0.0 & 0.00 & 0.0 & 0.0 & 0.08 & 0.08 \\
\hline 2,2-DIMETHYLOCTANE & 0.2 & 0.09 & 0.2 & 0.0 & 0.00 & 0.0 & 0.0 & 0.00 & 0.0 & 0.0 & 0.07 & 0.04 \\
\hline 2,4-DIMETHYLOCTANE & 0.0 & 0.00 & 0.0 & 0.0 & 0.00 & 0.0 & 0.0 & 0.00 & 0.0 & 0.0 & 0.00 & 0.00 \\
\hline n-PROPYLBENZENE & 0.6 & 0.31 & 1.3 & 0.0 & 0.00 & 0.0 & 0.0 & 0.00 & 0.0 & 0.1 & $\overline{0.26}$ & 0.27 \\
\hline 1-METHYL-3-ETHYLBENZENE & 2.4 & 1.25 & $\overline{17.6}$ & 0.0 & 0.00 & 0.0 & 0.2 & 0.82 & 1.4 & $\overline{0.6}$ & 1.15 & 4.03 \\
\hline 1-METHYL-4-ETHYLBENZENE & 1.1 & 0.54 & 7.7 & 0.0 & 0.00 & 0.0 & 0.0 & 0.00 & 0.0 & 0.2 & 0.45 & 1.59 \\
\hline 1,3,5-TRIMETHYLBENZENE & 1.0 & 0.51 & 10.1 & 0.0 & 0.00 & 0.0 & 0.1 & 0.34 & 0.8 & 0.2 & $\overline{0.47}$ & 2.32 \\
\hline 1-METHYL-2-ETHYLBENZENE & 0.8 & 0.40 & 6.6 & 0.0 & 0.00 & 0.0 & 0.0 & 0.00 & 0.0 & 0.2 & 0.33 & 1.17 \\
\hline 1,2,4-TRIMETHYLBENZENE & 2.8 & 1.42 & 24.6 & 0.0 & 0.00 & 0.0 & 0.2 & 0.73 & 1.5 & 0.6 & 1.28 & 5.52 \\
\hline DECANE & 0.0 & 0.02 & 0.0 & 0.0 & 1.29 & 0.0 & 0.0 & 0.11 & 0.0 & 0.0 & 0.08 & 0.02 \\
\hline ISOBUTYLBENZENE & $\overline{0.0}$ & 0.02 & 0.1 & 0.0 & 1.22 & 0.1 & 0.0 & 0.11 & 0.0 & 0.0 & 0.08 & 0.07 \\
\hline
\end{tabular}




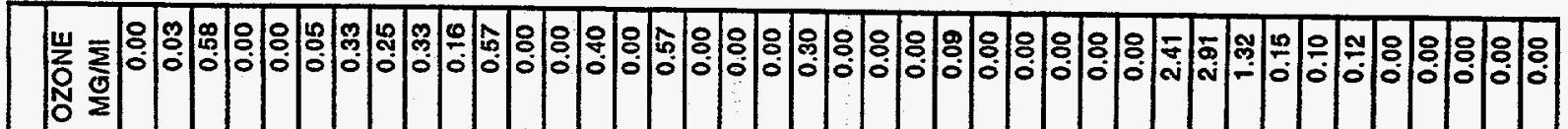

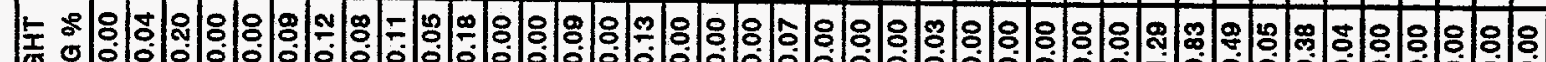
歨崖

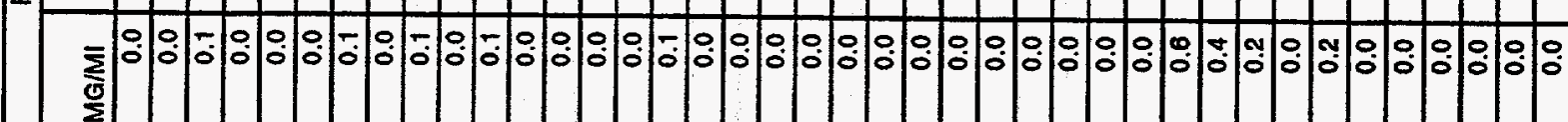

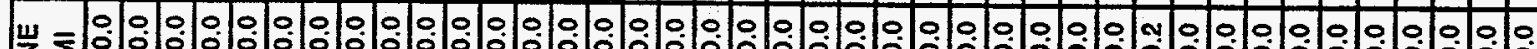

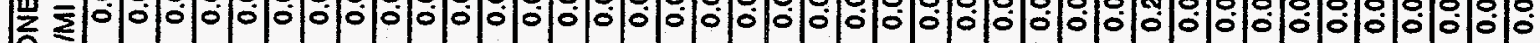
雚

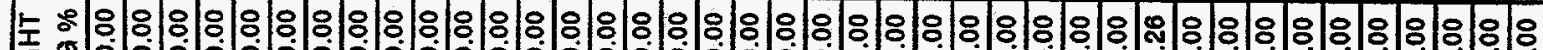

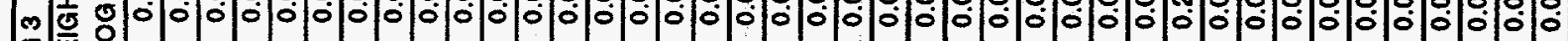
高紊

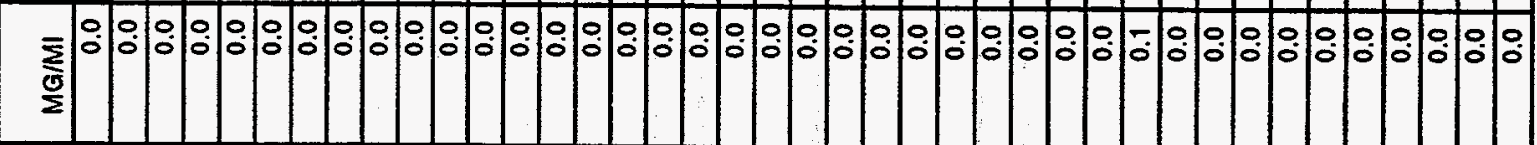

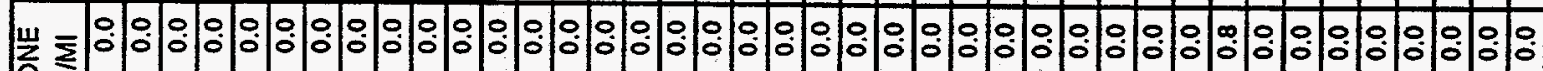
通 올

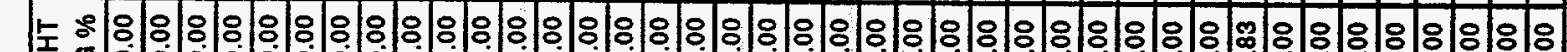
ฯ

曽岁

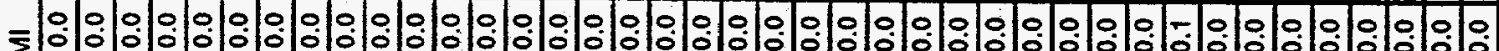
$\frac{0}{2}$

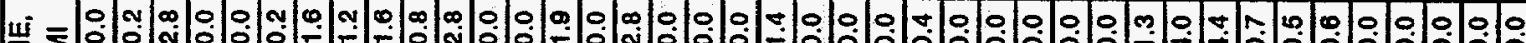

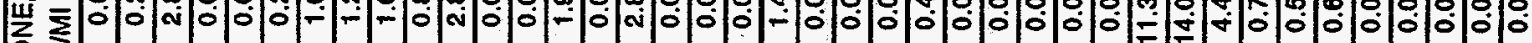

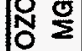
- 声 客岁

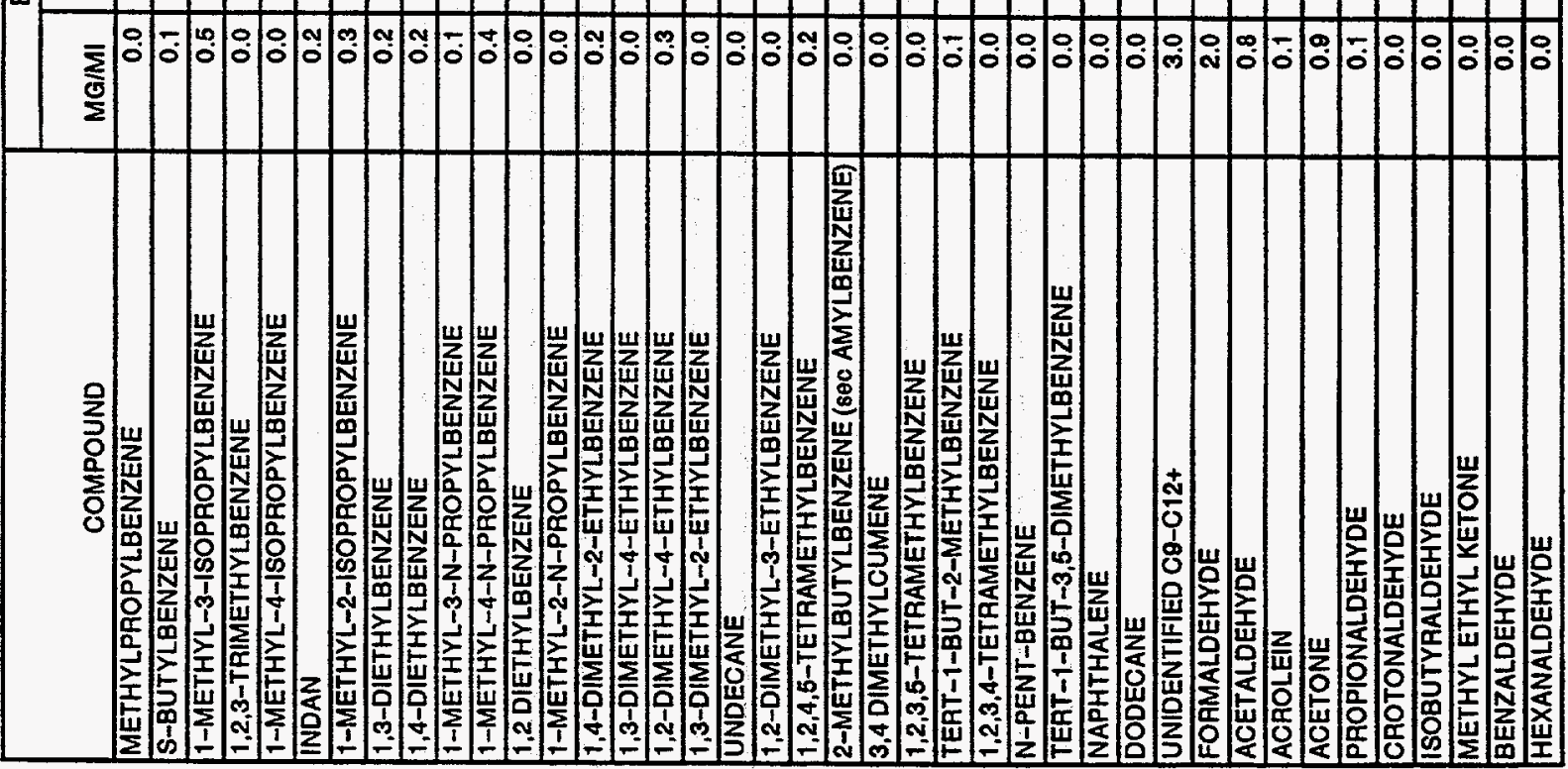


TEST NUMBER: $100 \mathrm{NB}$ (NC)-02S

TEST CAR: 1994 PONTIAC GRANDAM

TEST DATE: 11/20/95

TEST FUEL: $100 \%$ BUTANE

\begin{tabular}{|c|c|c|c|c|c|c|c|c|c|c|c|c|}
\hline \multirow[b]{2}{*}{ COMPOUND } & \multicolumn{3}{|c|}{ BAG 1} & \multicolumn{3}{|c|}{ BAG 2} & \multicolumn{3}{|c|}{ BAG 3 } & \multicolumn{3}{|c|}{ FTP } \\
\hline & MG/MI & \begin{tabular}{|l|} 
WEIGHT \\
NMOG \%
\end{tabular} & $\begin{array}{l}\text { OZONE, } \\
\text { MG/MI }\end{array}$ & MG/MI & \begin{tabular}{|l|} 
WEIGHT \\
NMOQ $\%$ \\
\end{tabular} & $\begin{array}{l}\text { OZONE } \\
\text { MG/MI }\end{array}$ & MG/MI & $\begin{array}{l}\text { WEIGHT } \\
\text { NMOG } \%\end{array}$ & $\begin{array}{l}\text { OZONE } \\
\text { MGMI }\end{array}$ & MG/MI & \begin{tabular}{|l|} 
WEIGHT \\
NMOG $\%$
\end{tabular} & $\begin{array}{l}\text { OZONE } \\
\text { MGIMI }\end{array}$ \\
\hline METHANE & 55.5 & & 0.8 & 46.6 & & 0.7 & 53.0 & & 0.8 & 50.3 & & 0.74 \\
\hline ETHANE & 17.6 & 1.38 & 4.4 & 17.6 & 1.64 & 4.4 & 0.0 & 0.00 & 0.0 & 12.7 & 1.18 & 3.17 \\
\hline ETHYLENE & 209.1 & 16.41 & 1524.1 & 199.2 & 18.69 & 1452.5 & 154.4 & 16.39 & 1125.4 & 188.9 & 17.57 & 1376.95 \\
\hline PROPANE & 3.8 & 0.30 & 1.8 & 2.8 & 0.26 & 1.3 & 1.8 & 0.19 & 0.9 & 2.7 & 0.25 & 1.30 \\
\hline PROPYLENE & 104.9 & 8.24 & 986.5 & 101.7 & 9.54 & 955.7 & 76.2 & 8.09 & 716.4 & 95.3 & 8.86 & 895.91 \\
\hline ACETYLENE & 74.8 & 6.87 & 37.4 & 63.4 & 5.95 & 31.7 & 59.9 & 6.36 & 29.9 & 64.8 & 6.03 & 32.41 \\
\hline PROPADIENE & 0.0 & 0.00 & 0.0 & 0.0 & 0.00 & 0.0 & 0.0 & 0.00 & 0.0 & 0.0 & 0.00 & 0.00 \\
\hline BUTANE & 783.0 & 61.48 & 798.7 & 600.2 & 56.29 & 612.2 & 573.4 & 60.87 & 584.9 & 630.9 & 58.68 & 643.54 \\
\hline TRANS-2-BUTENE & 3.0 & 0.23 & 29.6 & 2.8 & 0.26 & 27.8 & 2.4 & 0.26 & 23.8 & 2.7 & 0.25 & 27.12 \\
\hline 1-BUTENE & 8.1 & 0.64 & 72.3 & 7.7 & 0.73 & 68.9 & 6.1 & 0.65 & 54.4 & 7.4 & 0.68 & 65.58 \\
\hline 2-METHYLPROPENE (ISOBUTYLENE) & 0.5 & 0.04 & 2.7 & 0.5 & 0.05 & 2.6 & 1.5 & 0.16 & 8.1 & 0.8 & 0.07 & 4.12 \\
\hline 2,2-DIMETHYLPROPANE (NEOPENTANE) & 0.0 & 0.00 & 0.0 & 0.0 & 0.00 & 0.0 & 0.0 & 0.00 & 0.0 & 0.0 & 0.00 & 0.00 \\
\hline PAOPYNE & 0.0 & 0.00 & 0.0 & 0.0 & 0.00 & 0.0 & 0.0 & 0.00 & 0.0 & 0.0 & 0.00 & 0.00 \\
\hline 1,3-BUTADIENE & 1.9 & 0.15 & 20.2 & 2.0 & 0.19 & 21.9 & 1.4 & 0.15 & 15.2 & 1.8 & 0.17 & 19.66 \\
\hline 2-METHYLPROPANE (ISOBUTANE) & 0.7 & 0.05 & 0.8 & 0.5 & 0.04 & 0.6 & 0.5 & 0.05 & 0.6 & 0.5 & 0.05 & 0.63 \\
\hline 1-BUTYNE & 0.0 & 0.00 & 0.0 & 0.0 & 0.00 & 0.0 & 0.0 & 0.00 & 0.0 & 0.0 & 0.00 & 0.00 \\
\hline METHANOL & 0.0 & 0.00 & 0.0 & 0.0 & 0.00 & 0.0 & 0.0 & 0.00 & 0.0 & 0.0 & 0.00 & 0.00 \\
\hline CIS-2-BUTENE & 2.1 & 0.17 & 21.1 & 2.0 & 0.19 & 20.3 & 1.8 & 0.19 & 17.5 & 2.0 & 0.18 & 10.68 \\
\hline 3-METHYL-1-BUTENE & 0.0 & 0.00 & 0.0 & 0.0 & 0.00 & 0.0 & 0.0 & 0.00 & 0.0 & 0.0 & 0.00 & 0.00 \\
\hline ETHANOL & 0.0 & 0.00 & 0.0 & 0.0 & 0.00 & 0.0 & 0.0 & 0.00 & 0.0 & 0.0 & 0.00 & 0.00 \\
\hline 2-METHYLBUTANE (ISOPENTANE) & 2.7 & 0.21 & 3.7 & 0.5 & 0.05 & 0.8 & 4.8 & 0.50 & 6.6 & 2.2 & 0,20 & 2.97 \\
\hline 2-BUTYNE & 0.0 & 0.00 & 0.0 & 0.0 & 0.00 & 0.0 & 0.0 & 0.00 & 0.0 & 0.0 & 0.00 & 0.00 \\
\hline 1-PENTENE & 0.1 & 0.01 & 0.6 & 0.3 & 0.03 & 2.1 & 0.1 & 0.01 & 0.6 & 0.2 & 0.02 & 1.39 \\
\hline 2-METHYL-1-BUTENE & 0.3 & 0.02 & 1.3 & 0.2 & 0.02 & 1.0 & 0.3 & 0.04 & 1.7 & 0.3 & 0.02 & 1.25 \\
\hline PENTANE & 0.3 & 0.03 & 0.3 & 0.2 & 0.02 & 0.2 & 0.6 & 0.06 & 0.6 & 0.3 & 0.03 & 0.36 \\
\hline UNIDENTIFIED C5 OLEFINS & 0.0 & 0.00 & 0.0 & 0.0 & 0.00 & 0.0 & 0.0 & 0.00 & 0.0 & 0.0 & 0.00 & 0.00 \\
\hline 2-METHYL-1,3-BUTADIENE & 1.2 & 0.09 & 10.7 & 0.6 & 0.05 & 5.1 & 1.0 & 0.11 & 9.0 & 0.8 & 0.08 & 7.33 \\
\hline TRANS-2-PENTENE & 0.3 & 0.02 & 2.3 & 0.3 & 0.03 & 2.4 & 0.3 & 0.03 & 2.7 & 0.3 & 0.03 & 2.47 \\
\hline 3,3-DIMETHYL-1-BUTENE & 0.0 & 0.00 & 0.0 & 0.0 & 0.00 & 0.0 & 0.0 & 0.00 & 0.0 & 0.0 & 0.00 & 0.00 \\
\hline CIS-2-PENTENE & 0.1 & 0.01 & 1.0 & 0.1 & 0.01 & 1.2 & 0.2 & 0.02 & 1.6 & 0.1 & 0.01 & 1.27 \\
\hline 2-METHYL-2-BUTENE & 0.1 & 0.01 & 0.8 & 0.2 & 0.02 & 1.1 & 0.2 & 0.02 & 1.0 & 0.2 & 0.01 & 1.02 \\
\hline CYCLOPENTADIENE & 0.0 & 0.00 & 0.0 & 0.0 & 0.00 & 0.0 & 0.0 & 0.00 & 0.0 & 0.0 & 0.00 & 0.00 \\
\hline 2,2-DIMETHYLBUTANE & 0.0 & 0.00 & 0.0 & 0.0 & 0.00 & 0.0 & 0.1 & 0.01 & 0.1 & 0.0 & 0.00 & 0.02 \\
\hline CYCLOPENTENE & 0.0 & 0.00 & 0.0 & 0.0 & 0.00 & 0.0 & 0.0 & 0.00 & 0.0 & 0.0 & 0.00 & 0.00 \\
\hline 4-METHYL-1-PENTENE & 0.0 & 0.00 & 0.0 & 0.0 & 0.00 & 0.0 & 0.0 & 0.00 & 0.0 & 0.0 & 0.00 & 0.00 \\
\hline 3-METHYL-1-PENTENE & 0.0 & 0.00 & 0.0 & 0.0 & 0.00 & 0.0 & 0.0 & 0.00 & 0.0 & 0.0 & 0.00 & 0.00 \\
\hline CYCLOPENTANE & 0.0 & 0.00 & 0.0 & 0.0 & 0.00 & 0.0 & 0.0 & 0.00 & 0.0 & 0.0 & 0.00 & 0.00 \\
\hline 2,3-DIMETHYLBUTANE & 0.1 & 0.01 & 0.1 & 0.0 & 0.00 & 0.0 & 0.3 & 0.04 & 0.4 & 0.1 & 0.01 & 0.12 \\
\hline MTBE & 0.0 & 0.00 & 0.0 & 0.0 & 0.00 & 0.0 & 0.0 & 0.00 & 0.0 & 0.0 & 0.00 & 0.00 \\
\hline
\end{tabular}




\begin{tabular}{|c|c|c|c|c|c|c|c|c|c|c|c|c|}
\hline \multirow[b]{2}{*}{ COMPOUND } & \multicolumn{3}{|c|}{ BAG 1} & \multicolumn{3}{|c|}{ BAG 2} & \multicolumn{3}{|c|}{$\overline{B A G ~} 3$} & \multicolumn{3}{|c|}{ FTP } \\
\hline & MG/MI & $\begin{array}{l}\text { WEIGHT } \\
\text { NMOG \% }\end{array}$ & $\begin{array}{l}\text { OZONE, } \\
\text { MG/MI }\end{array}$ & MGMI & $\begin{array}{l}\text { WEIGHT } \\
\text { NMOG \% }\end{array}$ & $\begin{array}{l}\text { OZONE } \\
\text { MG/MI }\end{array}$ & MG/MI & $\begin{array}{l}\text { WEIGHT } \\
\text { NMOG } \%\end{array}$ & $\begin{array}{l}\text { OZONE } \\
\text { MG/MI }\end{array}$ & MG/MI & $\begin{array}{l}\text { WEIGHT } \\
\text { NMOG } \%\end{array}$ & $\begin{array}{l}\text { OZONE } \\
\text { MG/MI }\end{array}$ \\
\hline 2,3-DIMETHYL-1-BUTENE & 0.0 & 0.00 & 0.0 & 0.0 & 0.00 & 0.0 & 0.0 & 0.00 & 0.0 & 0.0 & 0.00 & 0.00 \\
\hline 4-METHYL-CIS-2-PENTENE & 0.0 & 0.00 & 0.0 & 0.0 & 0.00 & 0.0 & 0.0 & 0.00 & 0.0 & 0.0 & 0.00 & 0.00 \\
\hline 2-METHYLPENTANE & 0.1 & 0.01 & 0.2 & 0.2 & 0.02 & 0.3 & $\overline{0.5}$ & 0.05 & $\overline{0.7}$ & 0.3 & 0.02 & 0.39 \\
\hline 4-METHYL-TRANS-2-PENTENE & 0.0 & 0.00 & 0.0 & 0.0 & 0.00 & 0.0 & 0.0 & 0.00 & 0.0 & 0.0 & 0.00 & 0.00 \\
\hline 3-METHYLPENTANE & $\overline{0.0}$ & 0.00 & 0.0 & 0.0 & 0.00 & 0.0 & 0.3 & 0.03 & 0.4 & $\overline{0.1}$ & 0.01 & 0.12 \\
\hline 2-METHYL-1-PENTENE & 0.1 & 0.00 & 0.3 & 0.0 & 0.00 & 0.0 & $\overline{0.1}$ & 0.01 & 0.3 & 0.0 & 0.00 & 0.13 \\
\hline 1-HEXENE & 0.1 & 0.00 & $\overline{0.3}$ & 0.0 & 0.00 & 0.0 & $\overline{0.1}$ & 0.01 & $\overline{0.3}$ & 0.0 & 0.00 & 0.13 \\
\hline HEXANE & 0.1 & 0.00 & 0.1 & 0.1 & 0.01 & 0.1 & 0.2 & $\overline{0.03}$ & 0.2 & 0.1 & 0.01 & 0.14 \\
\hline UNIDENTIFIED C6 OLEFINS & 0.6 & 0.05 & 4.2 & 0.6 & 0.06 & 4.2 & $\overline{0.5}$ & 0.06 & 3.0 & 0.6 & 0.05 & 3.86 \\
\hline TRANS-3-HEXENE & 0.0 & 0.00 & 0.0 & 0.0 & 0.00 & 0.0 & 0.0 & 0.00 & 0.0 & 0.0 & 0.00 & 0.00 \\
\hline CIS-3-HEXENE & 0.0 & 0.00 & 0.0 & 0.0 & 0.00 & 0.0 & 0.0 & 0.00 & 0.0 & 0.0 & 0.00 & 0.00 \\
\hline TRANS-2-HEXENE & 0.0 & 0.00 & 0.0 & 0.0 & 0.00 & 0.0 & 0.0 & 0.00 & 0.0 & 0.0 & 0.00 & 0.00 \\
\hline 3-METHYL-TRANS-2-PENTENE & $\overline{0.0}$ & 0.00 & 0.0 & 0.0 & 0.00 & 0.0 & 0.0 & 0.00 & 0.0 & 0.0 & 0.00 & 0.00 \\
\hline 2-METHYL-2-PENTENE & 0.0 & 0.00 & $\overline{0.0}$ & 0.0 & 0.00 & 0.0 & 0.0 & 0.00 & 0.0 & 0.0 & 0.00 & 0.00 \\
\hline 3-METHYLCYCLOPENTENE & 0.0 & 0.00 & 0.0 & 0.0 & 0.00 & 0.0 & 0.0 & 0.00 & 0.0 & 0.0 & 0.00 & 0.00 \\
\hline CIS-2-HEXENE & 0.0 & 0.00 & 0.0 & 0.0 & 0.00 & 0.0 & 0.0 & 0.00 & 0.0 & 0.0 & 0.00 & 0.00 \\
\hline ETBE & 0.0 & 0.00 & 0.0 & 0.0 & 0.00 & 0.0 & 0.0 & 0.00 & 0.0 & 0.0 & 0.00 & 0.00 \\
\hline 3-METHYL-CIS-2-PENTENE & 0.0 & 0.00 & 0.0 & 0.0 & 0.00 & 0.0 & 0.0 & 0.00 & 0.0 & 0.0 & 0.00 & 0.00 \\
\hline 2,2-DIMETHYLPENTANE & 0.0 & 0.00 & 0.0 & 0.0 & 0.00 & 0.0 & $\overline{0.6}$ & 0.07 & 0.9 & 0.2 & 0.02 & 0.25 \\
\hline METHYLCYCLOPENTANE & 0.2 & 0.01 & 0.5 & 0.0 & 0.00 & 0.0 & 0.0 & 0.00 & 0.0 & 0.0 & 0.00 & 0.10 \\
\hline 2,4-DIMETHYLPENTANE & 0.1 & 0.01 & 0.1 & 0.0 & 0.00 & 0.0 & 0.3 & 0.03 & 0.6 & 0.1 & 0.01 & 0.18 \\
\hline 2,3,3-TAIMETHYL-1-BUTENE & 0.0 & 0.00 & 0.0 & 0,0 & 0.00 & 0.0 & 0.0 & 0.00 & 0.0 & 0.0 & 0.00 & 0.00 \\
\hline 2,2,3-TRIMETHYLBUTANE & 0.1 & 0.01 & 0.1 & 0.1 & 0.01 & 0.2 & 0.1 & 0.01 & 0.1 & 0.1 & 0.01 & 0.17 \\
\hline 3,4-DIMETHYL-1-PENTENE & 0.0 & 0.00 & 0.0 & 0.0 & 0.00 & 0.0 & 0.0 & 0.00 & 0.0 & 0.0 & 0.00 & 0.00 \\
\hline 1-METHYLCYCLOPENTENE & 0.0 & 0.00 & 0.0 & 0.0 & 0.00 & 0.0 & 0.0 & 0.00 & 0.0 & 0.0 & 0.00 & 0.00 \\
\hline BENZENE & 0.7 & 0.08 & 0.3 & 0.6 & 0.08 & 0.3 & 1.2 & 0.13 & 0.5 & 0.8 & 0.08 & 0.35 \\
\hline 3-METHYL-1-HEXENE & 0.0 & 0.00 & 0.0 & 0.0 & 0.00 & 0.0 & 0.0 & 0.00 & $\overline{0.0}$ & 0.0 & 0.00 & 0.00 \\
\hline 3,3-DIMETHYLPENTANE & 0.0 & 0.00 & 0.0 & 0.0 & 0.00 & 0.0 & 0.0 & 0.00 & $\overline{0.0}$ & 0.0 & 0.00 & 0.00 \\
\hline CYCLOHEXANE & 0.0 & 0.00 & 0.0 & 0.0 & 0.00 & 0.0 & 0.0 & 0.00 & 0.0 & 0.0 & 0.00 & 0.00 \\
\hline 2-METHYLHEXANE & 0.0 & 0.00 & 0.0 & 0.0 & 0.00 & 0.0 & 0.0 & 0.00 & 0.0 & 0.0 & 0.00 & 0.00 \\
\hline 2,3-DIMETHYLPENTANE & 0.0 & 0.00 & 0.0 & 0.0 & 0.00 & 0.1 & 0.3 & 0.03 & 0.5 & 0.1 & 0.01 & 0.17 \\
\hline 1,1-DIMETHYLCYCLOPENTANE & 0.0 & 0.00 & 0.0 & 0.0 & 0.00 & 0.0 & 0.0 & 0.00 & $\overline{0.0}$ & 0.0 & 0.00 & 0.00 \\
\hline CYCLOHEXENE & 0.0 & 0.00 & 0.0 & 0.0 & 0.00 & 0.0 & 0.0 & 0.00 & 0.0 & 0.0 & 0.00 & 0.00 \\
\hline 3-METHYLHEXANE & 0.0 & 0.00 & 0.0 & 0.0 & 0.00 & 0.0 & 0.1 & 0.01 & 0.1 & 0.0 & 0.00 & 0.03 \\
\hline CIS-1,3-DIMETHYLCYCLOPENTANE & 0.0 & 0.00 & 0.0 & 0.0 & 0.00 & 0.0 & 0.0 & 0.00 & 0.0 & 0.0 & 0.00 & 0.00 \\
\hline 3-ETHYLPENTANE & 0.0 & 0.00 & 0.0 & 0.0 & 0.00 & 0.0 & 0.0 & 0.00 & 0.0 & 0.0 & 0.00 & 0.00 \\
\hline TRANS-1,2-DIMETHYLCYCLOPENTANE & 0.0 & 0.00 & 0.0 & 0.0 & 0.00 & 0.0 & 0.0 & 0.00 & 0.0 & 0.0 & 0.00 & 0.00 \\
\hline TRANS-1,3-DIMETHYLCYCLOPENTANE & 0.0 & 0.00 & 0.0 & 0.0 & 0.00 & 0.0 & 0.0 & 0.00 & 0.0 & 0.0 & 0.00 & 0.00 \\
\hline 1-HEPTENE & 0.0 & 0.00 & 0.0 & 0.0 & 0.00 & 0.0 & 0.0 & 0.00 & 0.0 & 0.0 & 0.00 & 0.00 \\
\hline 2,2,4-TRIMETHYLPENTANE & 0.3 & 0.03 & 0.3 & 0.1 & 0.01 & 0.1 & 0.3 & 0.04 & 0.3 & 0.2 & 0.02 & 0.22 \\
\hline 2-METHYL-1-HEXENE & 0.0 & 0.00 & 0.0 & 0.0 & 0.00 & 0.0 & 0.0 & 0.00 & 0.0 & 0.0 & 0.00 & 0.00 \\
\hline TAANS-3-HEPTENE & 0.0 & 0.00 & 0.0 & 0.0 & 0.00 & 0.0 & 0.0 & 0.00 & 0.0 & 0.0 & 0.00 & 0.00 \\
\hline HEPTANE & 0.0 & 0.00 & 0.0 & 0.0 & 0.00 & 0.0 & 0.1 & 0.01 & 0.1 & 0.0 & 0.00 & 0.03 \\
\hline UNNIDENTIFIED C7 & 0.0 & 0.00 & 0.0 & 0.0 & 0.00 & 0.0 & 0.0 & 0.00 & 0.0 & 0.0 & 0.00 & 0.00 \\
\hline
\end{tabular}


w

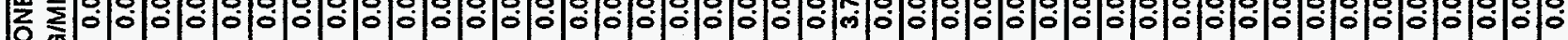
贶 $\frac{0}{2}$

恋 道窟

三

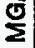

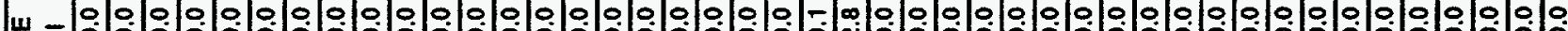

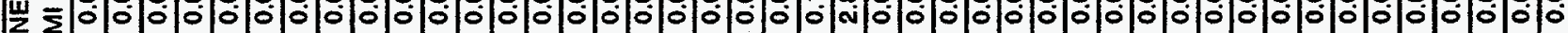
象 $\frac{0}{\Sigma}$

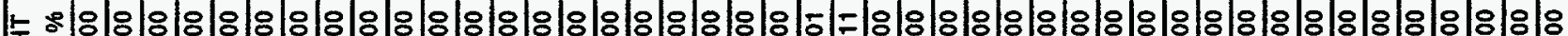
o 浐商

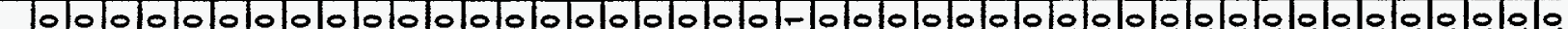
三 $\stackrel{0}{\Sigma}$

岸 三 品

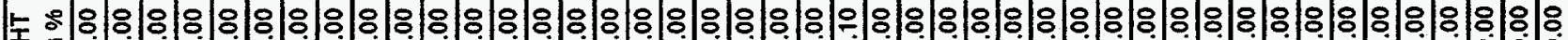
焉

家

z $\stackrel{\mathrm{S}}{\Sigma}$

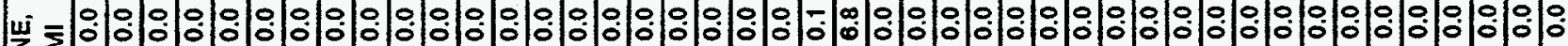
兽 雚

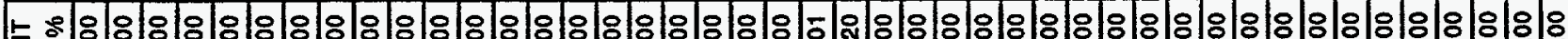
浐㟧

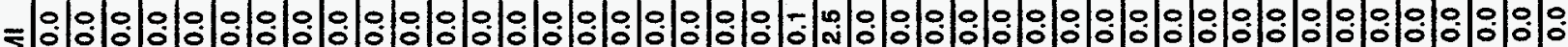
离

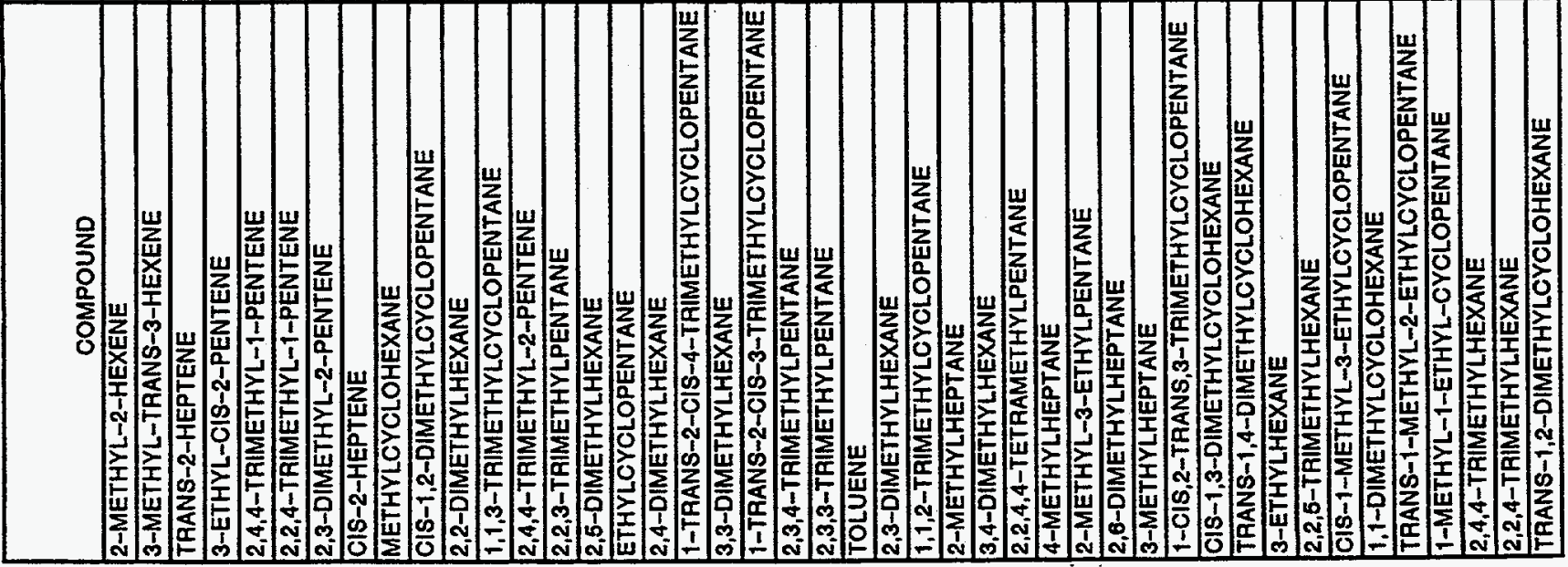




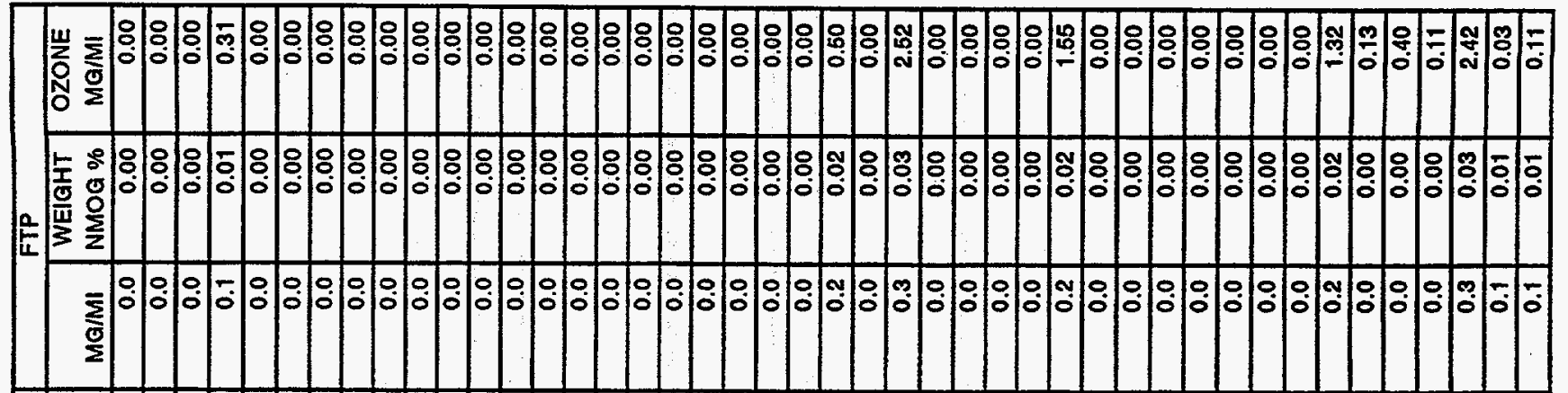

崖 过

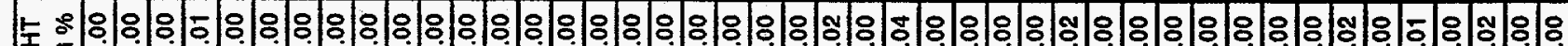
m 这崖产 表

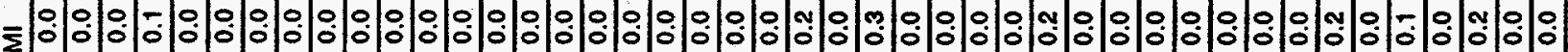
$\frac{\mathrm{O}}{\Sigma}$

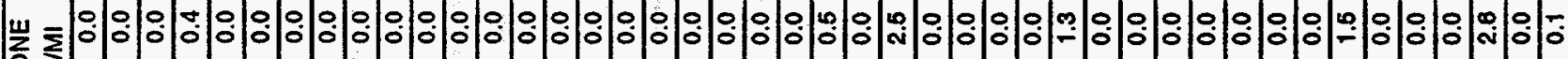
융 일

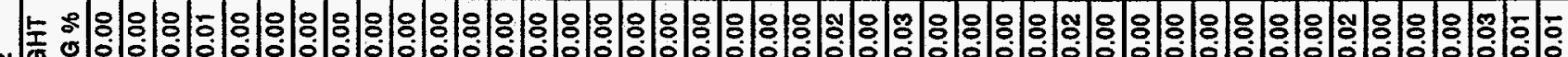

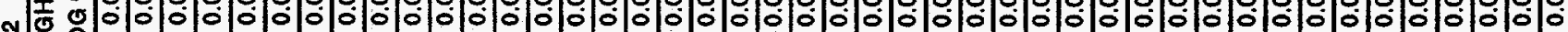
这迹高

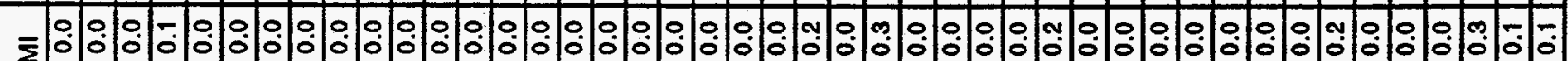

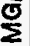

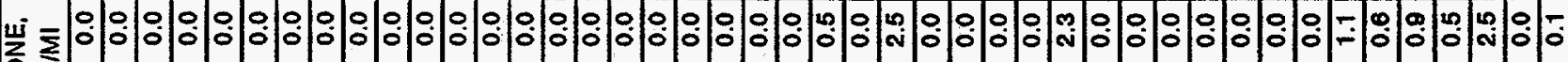
通 产

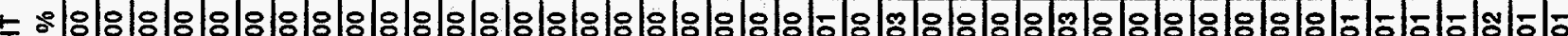
隹

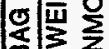

这

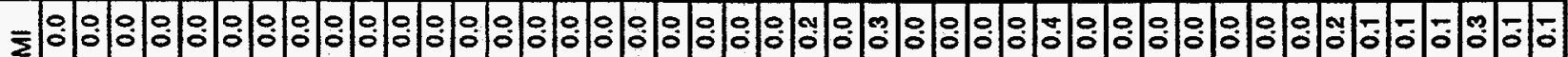
$\stackrel{0}{\Sigma}$

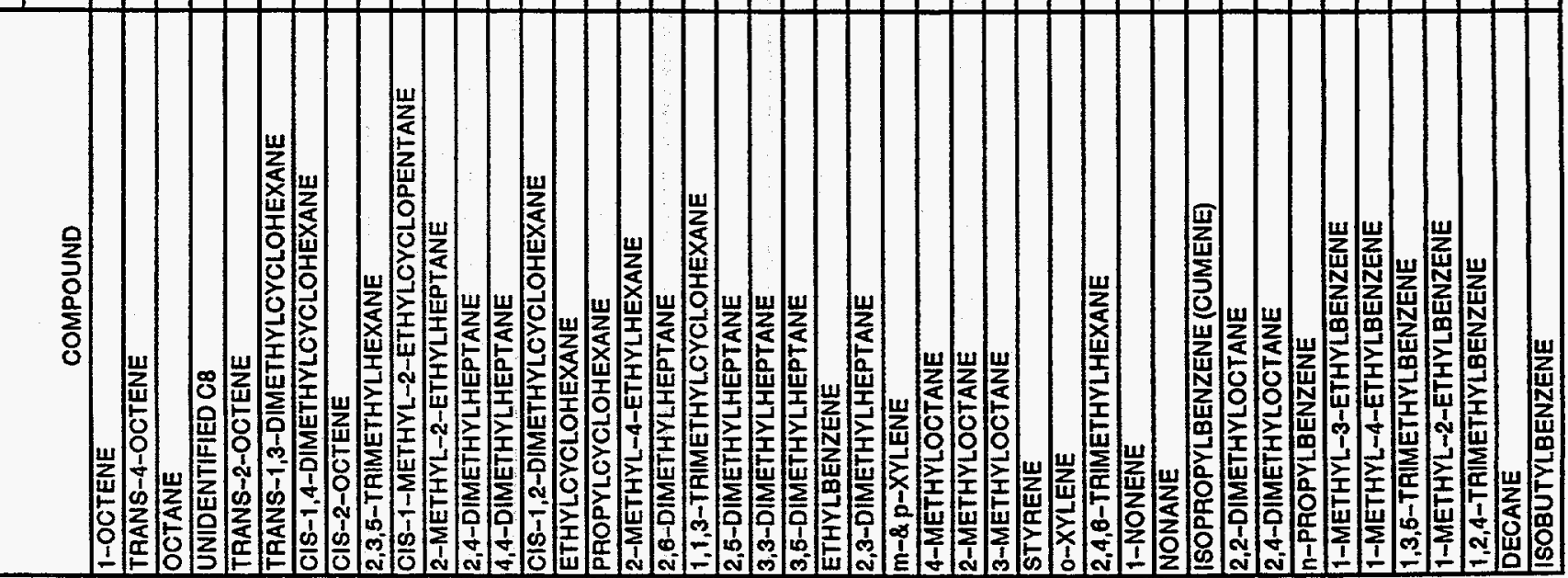




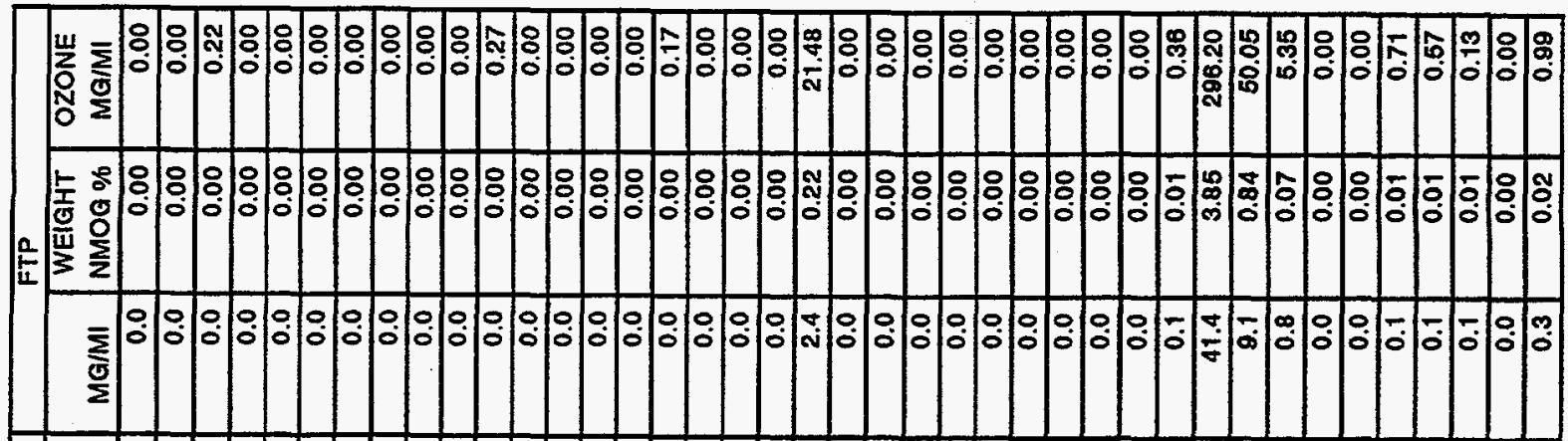

崖 居

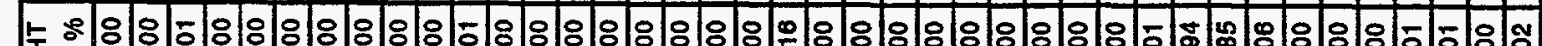

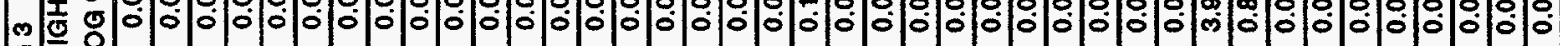
要崖

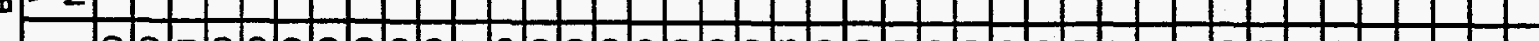

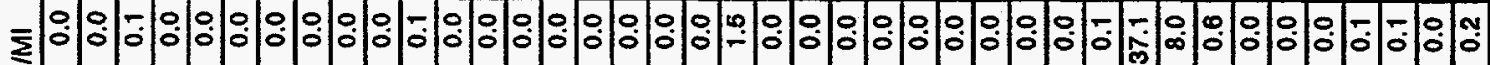

율

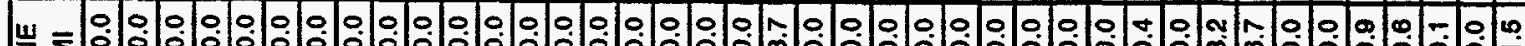

崖 通 $\frac{0}{\Sigma}$

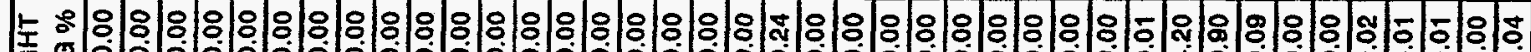
N 年

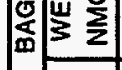

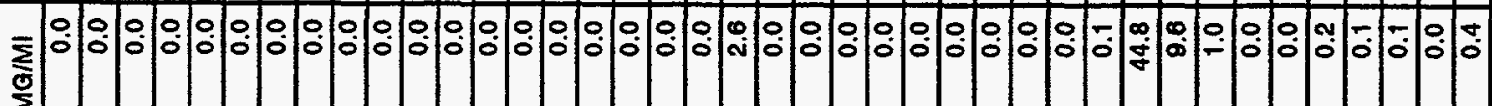

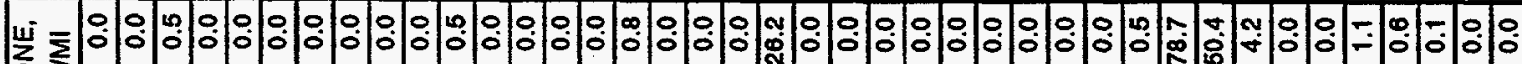
疍

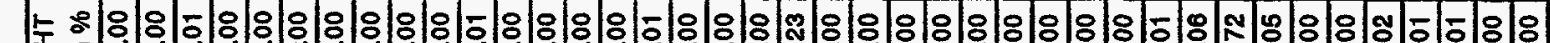
- 秋 象商

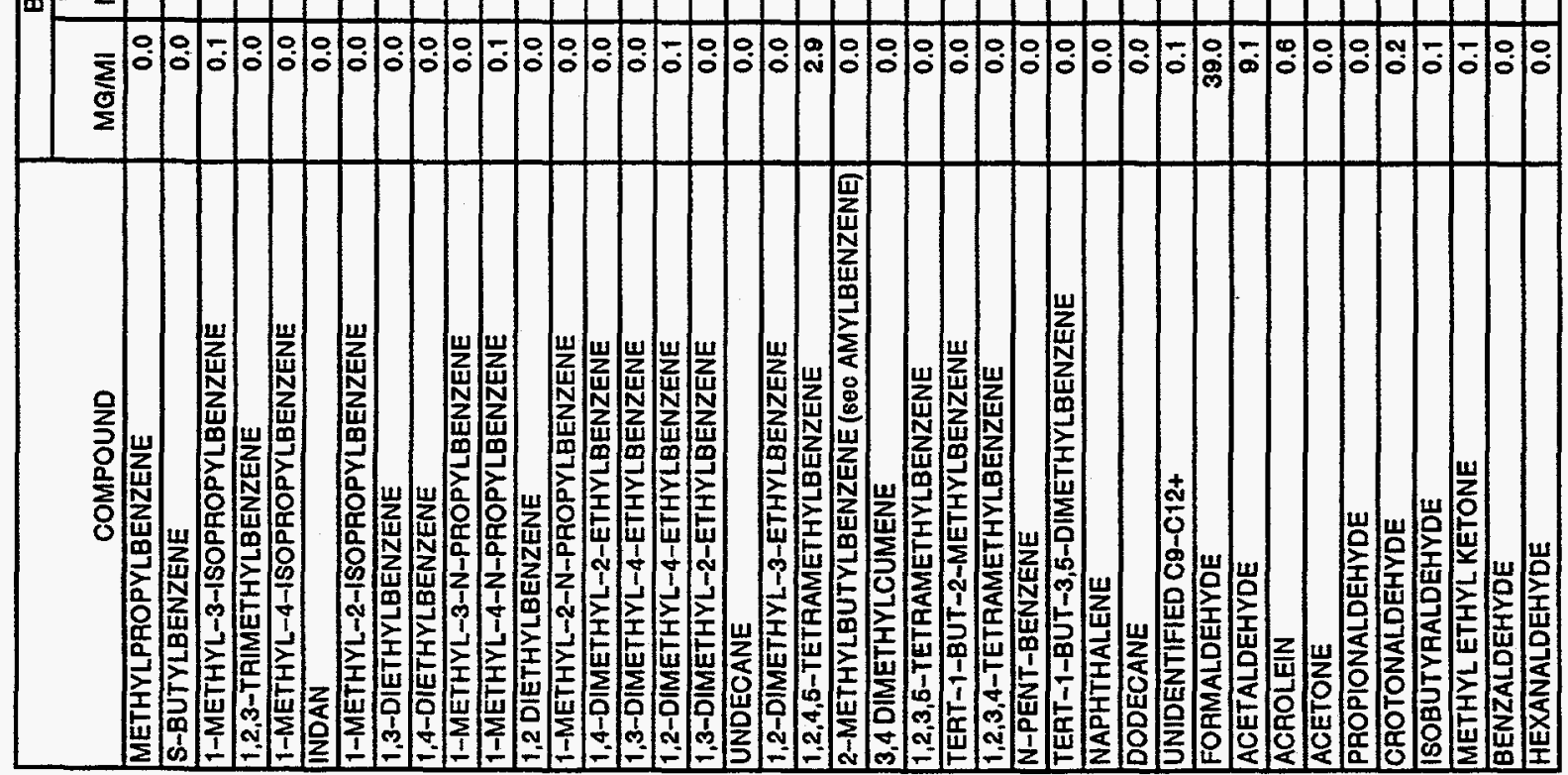


TEST NUMBER: 100P(NC)-02S

TEST CAR: 1994 PONTIAC GRANDAM

TEST DATE: $11 / 30 / 95$

TEST FUEL: $100 \%$ PROPANE

\begin{tabular}{|c|c|c|c|c|c|c|c|c|c|c|c|c|}
\hline \multirow[b]{2}{*}{ COMPOUND } & \multicolumn{3}{|c|}{ BAG 1} & \multicolumn{3}{|c|}{ BAG 2} & \multicolumn{3}{|c|}{ BAG 3} & \multicolumn{3}{|c|}{ FTP } \\
\hline & MG/MI & $\begin{array}{l}\text { WEIGHT } \\
\text { NMOG \% }\end{array}$ & $\begin{array}{l}\text { OZONE, } \\
\text { MG/MI }\end{array}$ & MG/MI & $\begin{array}{l}\text { WEIGHT } \\
\text { NMOG \% }\end{array}$ & $\begin{array}{l}\text { OZONE } \\
\text { MG/MI }\end{array}$ & MG/MI & $\begin{array}{l}\text { WEIGHT } \\
\text { NMOQ } \%\end{array}$ & $\begin{array}{l}\text { OZONE } \\
\text { MG/MI }\end{array}$ & MG/MI & $\begin{array}{l}\text { WEIGHT } \\
\text { NMOG } \%\end{array}$ & $\begin{array}{l}\text { OZONE } \\
\text { MGMI }\end{array}$ \\
\hline METHANE & 37.1 & & 0.5 & 42.1 & & 0.6 & 34.7 & & 0.5 & 39.0 & & 0.58 \\
\hline ETHANE & 13.6 & 1.40 & 3.4 & 15.4 & 1.68 & 3.9 & 0.0 & 0.00 & 0.0 & 10.8 & 1.22 & 2.69 \\
\hline ETHYLENE & 137.6 & 14.20 & 1003.2 & 151.3 & 16.63 & 1103.0 & 118.7 & 15.61 & 865.6 & 139.4 & 15.78 & 1016.54 \\
\hline PROPANE & 659.5 & 68.07 & 316.6 & 580.3 & 63.39 & 278.6 & 493.4 & 64.87 & 236.8 & 572.8 & 64.81 & 274.93 \\
\hline PROPYLENE & 68.1 & 7.03 & 640.1 & 74.5 & 8.14 & 700.5 & 58.3 & 7.67 & 548.1 & 68.7 & 7.77 & 646.77 \\
\hline ACETYLENE & 46.2 & 4.77 & 23.1 & 49.0 & 5.35 & 24.5 & 41.9 & 6.51 & 21.0 & 46.4 & 6.26 & 23.22 \\
\hline PROPADIENE & 0.0 & 0.00 & 0.0 & 0.0 & 0.00 & 0.0 & 0.0 & 0.00 & 0.0 & 0.0 & 0.00 & 0.00 \\
\hline BUTANE & 2.1 & 0.21 & 2.1 & 0.3 & 0.03 & 0.3 & 1.6 & 0.21 & 1.6 & 1.0 & 0.12 & 1.04 \\
\hline TRANS-2-BUTENE & 0.1 & 0.01 & 1.4 & 0.1 & 0.02 & 1.4 & 0.2 & 0.03 & 2.4 & 0.2 & 0.02 & 1.69 \\
\hline 1-BUTENE & 2.5 & 0.26 & 22.5 & 2.7 & 0.29 & 23.9 & 2.2 & 0.29 & 19.6 & 2.5 & 0.28 & 22,43 \\
\hline 2-METHYLPROPENE (ISOBUTYLENE) & 0.4 & 0.04 & 2.0 & 0.4 & 0.04 & 2.0 & 1.4 & 0.18 & 7.2 & 0.6 & 0.07 & 3.42 \\
\hline 2,2-DIMETHYLPROPANE (NEOPENTANE) & 0.0 & 0.00 & 0.0 & 0.0 & 0.00 & 0.0 & 0.0 & 0.00 & 0.0 & 0.0 & 0.00 & 0.00 \\
\hline PROPYNE & 0.0 & 0.00 & 0.0 & 0.0 & 0.00 & 0.0 & 0.0 & 0.00 & 0.0 & 0.0 & 0.00 & 0.00 \\
\hline 1,3-BUTADIENE & 0.9 & 0.09 & 9.8 & 0.8 & 0.09 & 8.8 & 0.8 & 0.11 & 9.3 & 0.8 & 0.10 & 9.21 \\
\hline 2-METHYLPROPANE (ISOBUTANE) & 0.4 & 0.04 & 0.6 & 0.5 & 0.05 & 0.6 & 0.5 & 0.08 & 0.6 & 0.5 & 0.05 & 0.55 \\
\hline 1-BUTYNE & 0.0 & 0.00 & 0.0 & 0.0 & 0.00 & 0.0 & 0.0 & 0.00 & 0.0 & 0.0 & 0.00 & 0.00 \\
\hline METHANOL & 0.0 & 0.00 & 0.0 & 0.0 & 0.00 & 0.0 & 0.0 & 0.00 & 0.0 & 0.0 & 0.00 & 0.00 \\
\hline CIS-2-BUTENE & 0.1 & 0.01 & 1.0 & 0.1 & 0.01 & 1.1 & 0.2 & 0.02 & 1.8 & 0.1 & 0.01 & 1.26 \\
\hline 3-METHYL-1-BUTENE & 0.0 & 0.00 & 0.0 & 0.0 & 0.00 & 0.0 & 0.0 & 0.00 & 0.0 & 0.0 & 0.00 & 0.00 \\
\hline ETHANOL & 0.0 & 0.00 & 0.0 & 0.0 & 0.00 & 0.0 & 0.0 & 0.00 & 0.0 & 0.0 & 0.00 & 0.00 \\
\hline 2-METHYLBUTANE (ISOPENTANE) & 1.1 & 0.11 & 1.5 & 0.0 & 0.00 & 0.0 & 3.5 & 0.48 & 4.8 & 1.2 & 0.13 & 1.64 \\
\hline 2-BUTYNE & 0.0 & 0.00 & 0.0 & 0.0 & 0.00 & 0.0 & 0.0 & 0.00 & 0.0 & 0.0 & 0.00 & 0.00 \\
\hline 1-PENTENE & 0.1 & 0.02 & 0.8 & 0.2 & 0.02 & 1.4 & 0.2 & 0.02 & 1.2 & 0.2 & 0.02 & 1.23 \\
\hline 2-METHYL-1-BUTENE & 0.2 & 0.02 & 0.0 & 0.0 & 0.00 & 0.0 & 0.2 & 0.03 & 1.1 & 0.1 & 0.01 & 0.50 \\
\hline PENTANE & 0.2 & 0.02 & 0.2 & 0.1 & 0.01 & 0.1 & 0.4 & 0.05 & 0.4 & 0.2 & 0.02 & 0.19 \\
\hline UNIDENTIFIED C5 OLEFINS & 0.1 & 0.01 & 1.1 & 0.0 & 0.00 & 0.0 & 0.2 & 0.02 & 1.4 & 0.1 & 0.01 & 0.62 \\
\hline 2-METHYL-1,3-BUTADIENE & 1.0 & 0.11 & 9.4 & 0.8 & 0.08 & 7.1 & 0.9 & 0.12 & 8.6 & 0.8 & 0.10 & 7.89 \\
\hline TRANS-2-PENTENE & 0.1 & 0.01 & 1.2 & 0.2 & 0.02 & 1.7 & 0.2 & 0.03 & 2.0 & 0.2 & 0.02 & 1.69 \\
\hline 3,3-DIMETHYL-1-BUTENE & 0.0 & 0.00 & 0.0 & 0.0 & 0.00 & 0.0 & 0.0 & 0.00 & 0.0 & 0.0 & 0.00 & 0.00 \\
\hline CIS-2-PENTENE & 0.0 & 0.00 & 0.0 & 0.0 & 0.00 & 0.0 & 0.0 & 0.00 & 0.0 & 0.0 & 0.00 & 0.00 \\
\hline 2-METHYL-2-BUTENE & 0.1 & 0.01 & 0.5 & 0.0 & 0.00 & 0.1 & 0.1 & 0.01 & 0.7 & 0.1 & 0.01 & 0.37 \\
\hline CYCLOPENTADIENE & 0.0 & 0.00 & 0.0 & 0.0 & 0.00 & 0.0 & 0.0 & 0.00 & 0.0 & 0.0 & 0.00 & 0.00 \\
\hline 2,2-DIMETHYLBUTANE & 0.0 & 0.00 & 0.0 & 0.0 & 0.00 & 0.0 & 0.0 & 0.00 & 0.0 & 0.0 & 0.00 & 0.00 \\
\hline CYCLOPENTENE & 0.0 & 0.00 & 0.0 & 0.0 & 0.00 & 0.0 & 0.0 & 0.00 & 0.0 & 0.0 & 0.00 & 0.00 \\
\hline 4-METHYL-1-PENTENE & 0.0 & 0.00 & 0.0 & 0.0 & 0.00 & 0.0 & 0.0 & 0.00 & 0.0 & 0.0 & 0.00 & 0.00 \\
\hline 3-METHYL-1-PENTENE & 0.0 & 0.00 & 0.0 & 0.0 & 0.00 & 0.0 & 0.0 & 0.00 & 0.0 & 0.0 & 0.00 & 0.00 \\
\hline CYCLOPENTANE & 0.0 & 0.00 & 0.0 & 0.0 & 0.00 & 0.0 & 0.0 & 0.00 & 0.0 & 0.0 & 0.00 & 0.00 \\
\hline 2,3-DIMETHYLBUTANE & 0.0 & 0.00 & 0.0 & 0.0 & 0.00 & 0.0 & $\overline{0.2}$ & 0.03 & 0.2 & 0.1 & 0.01 & 0.06 \\
\hline MTBE & 0.0 & 0.00 & 0.0 & 0.0 & 0.00 & 0.0 & 0.0 & 0.00 & 0.0 & 0.0 & 0.00 & 0.00 \\
\hline
\end{tabular}




\begin{tabular}{|c|c|c|c|c|c|c|c|c|c|c|c|c|}
\hline \multirow[b]{2}{*}{ COMPOUND } & \multicolumn{3}{|c|}{ BAG 1} & \multicolumn{3}{|c|}{ BAG 2} & \multicolumn{3}{|c|}{$\mathrm{BAG} 3$} & \multicolumn{3}{|c|}{ FTP } \\
\hline & MG/MI & $\begin{array}{l}\text { WEIGHT } \\
\text { NMOQ \% }\end{array}$ & $\begin{array}{l}\text { OZONE, } \\
\text { MG/MI }\end{array}$ & MG/MI & $\begin{array}{l}\text { WEIGHT } \\
\text { NMOG \% }\end{array}$ & $\begin{array}{l}\text { OZONE } \\
\text { MG/MI }\end{array}$ & MG/MI & $\begin{array}{l}\text { WEIGHT } \\
\text { NMOG \% }\end{array}$ & $\begin{array}{l}\text { OZONE } \\
\text { MG/MI }\end{array}$ & MG/MI & $\begin{array}{l}\text { WEIGHT } \\
\text { NMOG \% }\end{array}$ & $\begin{array}{l}\text { OZONE } \\
\text { MG/MI }\end{array}$ \\
\hline 2,3-DIMETHYL-1-BUTENE & 0.0 & 0.00 & 0.0 & 0.0 & 0.00 & 0.0 & 0.0 & 0.00 & 0.0 & 0.0 & 0.00 & 0.00 \\
\hline 4-METHYL-CIS-2-PENTENE & 0.0 & 0.00 & 0.0 & 0.0 & 0.00 & 0.0 & 0.0 & 0.00 & 0.0 & 0.0 & 0.00 & 0.00 \\
\hline 2-METHYLPENTANE & 0.0 & 0.00 & 0.0 & 0.0 & 0.00 & 0.0 & 0.2 & 0.03 & 0.3 & 0.1 & 0.01 & 0.00 \\
\hline 4-METHYL-TRANS-2-PENTENE & 0.0 & 0.00 & 0.0 & 0.0 & 0.00 & 0.0 & 0.0 & 0.00 & 0.0 & 0.0 & 0.00 & 0.00 \\
\hline 3-METHYLPENTANE & 0.0 & 0.00 & 0.0 & 0.0 & 0.00 & 0.0 & 0.1 & 0.01 & 0.1 & 0.0 & 0.00 & 0.04 \\
\hline 2-METHYL-1-PENTENE & 0.1 & 0.01 & 0.3 & 0.0 & 0.00 & 0.0 & 0.1 & 0.01 & 0.2 & 0.0 & 0.00 & 0.12 \\
\hline 1-HEXENE & 0.1 & 0.01 & 0.3 & 0.0 & 0.00 & 0.0 & 0.1 & 0.01 & 0.2 & 0.0 & 0.00 & 0.12 \\
\hline HEXANE & 0.0 & 0.00 & 0.0 & 0.1 & 0.01 & 0.1 & 0.1 & 0.01 & 0.1 & 0.1 & 0.01 & 0.08 \\
\hline UNIDENTIFIED C6 OLEFINS & 0.5 & 0.05 & 3.3 & 0.6 & 0.06 & 4.0 & 0.5 & 0.06 & 3.1 & 0.5 & 0.06 & 3.59 \\
\hline TRANS-3 HEXENE & 0.0 & 0.00 & 0.0 & 0.0 & 0.00 & 0.0 & 0.0 & 0.00 & 0.0 & 0.0 & 0.00 & 0.00 \\
\hline CIS-3-HEXENE & 0.0 & 0.00 & 0.0 & 0,0 & 0.00 & 0.0 & 0.0 & 0.00 & 0.0 & 0.0 & 0.00 & 0.00 \\
\hline TRANS-2-HEXENE & 0.0 & 0.00 & 0.0 & 0.0 & 0.00 & 0.0 & 0.0 & 0.00 & 0.0 & 0.0 & 0.00 & 0.00 \\
\hline 3-METHYL-TRANS-2-PENTENE & 0.0 & 0.00 & 0.0 & 0.0 & 0.00 & 0.0 & 0.0 & 0.00 & 0.0 & 0.0 & 0.00 & 0.00 \\
\hline 2-METHYL-2-PENTENE & 0.0 & 0.00 & 0.0 & 0.0 & 0.00 & 0.0 & 0.0 & 0.00 & 0.0 & 0.0 & 0.00 & 0.00 \\
\hline 3-METHYLCYCLOPENTENE & 0.0 & 0.00 & 0.0 & 0.0 & 0.00 & 0.0 & 0.0 & 0.00 & 0.0 & 0.0 & 0.00 & 0.00 \\
\hline CIS-2-HEXENE & 0.0 & 0.00 & 0.0 & 0.0 & 0.00 & 0.0 & 0.0 & 0.00 & 0.0 & 0.0 & 0.00 & 0.00 \\
\hline ETBE & 0.0 & 0.00 & 0.0 & 0.0 & 0.00 & 0.0 & 0.0 & 0.00 & 0.0 & 0,0 & 0.00 & 0.00 \\
\hline 3-METHYL-CIS-2-PENTENE & 0.0 & 0.00 & 0.0 & 0.0 & 0.00 & 0.0 & 0.0 & 0.00 & 0.0 & 0.0 & 0.00 & 0.00 \\
\hline 2,2-DIMETHYLPENTANE & 0.0 & 0.00 & 0.0 & 0.0 & 0.00 & 0.0 & 0.1 & 0.01 & 0.1 & 0.0 & 0.00 & 0.03 \\
\hline METHYLCYCLOPENTANE & 0.0 & 0.00 & 0.0 & 0.0 & 0.00 & 0.0 & 0.0 & 0.00 & 0.0 & 0.0 & 0.00 & 0.00 \\
\hline 2,4-DIMETHYLPENTANE & 0.0 & 0.00 & 0.0 & 0.0 & 0.00 & 0.0 & 0.2 & 0.02 & 0.3 & 0.0 & 0.01 & 0.09 \\
\hline 2,3,3-TAIMETHYL-1-BUTENE & 0.0 & 0.00 & 0.0 & 0.0 & 0.00 & 0.0 & 0.0 & 0.00 & 0.0 & 0.0 & 0.00 & 0.00 \\
\hline 2,2,3-TAIMETHYLBUTANE & 0.0 & 0.00 & 0.0 & 0.0 & 0.00 & 0.0 & 0.0 & 0.00 & 0.0 & 0.0 & 0.00 & 0.00 \\
\hline 3,4-DIMETHYL-1-PENTENE & 0.0 & 0.00 & 0.0 & 0.0 & 0.00 & 0.0 & 0.0 & 0.00 & 0.0 & 0.0 & 0.00 & 0.00 \\
\hline 1-METHYLCYCLOPENTENE & 0.0 & 0.00 & 0.0 & 0.0 & 0.00 & 0.0 & 0.0 & 0.00 & 0.0 & 0.0 & 0.00 & 0.00 \\
\hline BENZENE & 0.3 & 0.03 & 0.1 & 0.6 & 0.08 & 0.2 & 0.3 & 0.04 & 0.1 & 0.4 & 0.05 & 0.18 \\
\hline 3-METHYL-1-HEXENE & 0.0 & 0.00 & 0.0 & 0.0 & 0.00 & 0.0 & 0.0 & 0.00 & 0.0 & 0.0 & 0.00 & 0.00 \\
\hline 3,3-DIMETHYLPENTANE & 0.0 & 0.00 & 0.0 & 0.0 & 0.00 & 0.0 & 0.0 & 0.00 & 0.0 & 0.0 & 0.00 & 0.00 \\
\hline CYCLOHEXANE & 0.0 & 0.00 & 0.0 & 0.0 & 0.00 & 0.0 & 0.0 & 0.00 & 0.0 & 0.0 & 0.00 & 0.00 \\
\hline 2-METHYLHEXANE & 0.0 & 0.00 & 0.0 & 0.0 & 0.00 & 0.0 & 0.0 & 0.00 & 0.0 & 0.0 & 0.00 & 0.00 \\
\hline 2,3-DIMETHYLPENTANE & 0.0 & 0.00 & 0.0 & 0.0 & 0.00 & 0.0 & 0.2 & 0.03 & 0.3 & 0.1 & 0.01 & 0.10 \\
\hline 1,1-DIMETHYLCYCLOPENTANE & 0.0 & 0.00 & 0.0 & 0.0 & 0.00 & 0.0 & 0.0 & 0.00 & 0.0 & 0.0 & 0.00 & 0.00 \\
\hline CYCLOHEXENE & 0.0 & 0.00 & 0.0 & 0.0 & 0.00 & 0.0 & 0.0 & 0.00 & 0.0 & 0,0 & 0.00 & 0.00 \\
\hline 3-METHYLHEXANE & 0.0 & 0.00 & 0.0 & 0.0 & 0.00 & 0.0 & 0.1 & 0.02 & 0.2 & 0.0 & 0.00 & 0.06 \\
\hline CIS-1,3-DIMETHYLCYCLOPENTANE & 0.0 & 0.00 & 0.0 & 0.0 & 0.00 & 0.0 & 0.0 & 0.00 & 0.0 & 0.0 & 0.00 & 0.00 \\
\hline 3-ETHYLPENTANE & 0.0 & 0.00 & 0.0 & 0.0 & 0.00 & 0.0 & 0,0 & 0.00 & 0.0 & 0.0 & 0.00 & 0.00 \\
\hline TRANS-1,2-DIMETHYLCYCLOPENTANE & 0.0 & 0.00 & 0.0 & 0.0 & 0.00 & 0.0 & 0.0 & 0.00 & 0.0 & 0.0 & 0.00 & 0.00 \\
\hline TRANS-1,3-DIMETHYLCYCLOPENTANE & 0.0 & 0.00 & 0.0 & 0.0 & 0.00 & 0.0 & 0.0 & 0.00 & 0.0 & 0.0 & 0.00 & 0.00 \\
\hline 1-HEPTENE & 0.0 & 0.00 & 0.0 & 0.0 & 0.00 & 0.0 & 0.0 & 0.00 & 0.0 & 0.0 & 0.00 & 0.00 \\
\hline 2,2,4-TRIMETHYLPENTANE & 0.2 & 0.02 & 0.2 & 0.0 & 0.00 & 0.0 & 0.2 & 0.03 & 0.2 & 0.1 & 0.01 & 0.09 \\
\hline 2-METHYL-1-HEXENE & 0.0 & 0.00 & 0.0 & 0.0 & 0.00 & 0.0 & 0.0 & 0.00 & 0.0 & 0.0 & 0.00 & 0.00 \\
\hline TRANS-3-HEPTENE & 0.0 & 0.00 & 0.0 & 0.0 & 0.00 & 0.0 & 0.0 & 0.00 & 0.0 & 0.0 & 0.00 & 0.00 \\
\hline HEPTANE & 0.0 & 0.00 & 0.0 & 0.1 & 0.01 & 0.1 & 0.1 & 0.01 & 0.1 & 0.1 & 0.01 & 0.04 \\
\hline UNIDENTIFIED C7 & 0.0 & 0.00 & 0.0 & 0.0 & 0.00 & 0.0 & 0.0 & 0.00 & 0.0 & 0.0 & 0.00 & 0.00 \\
\hline
\end{tabular}




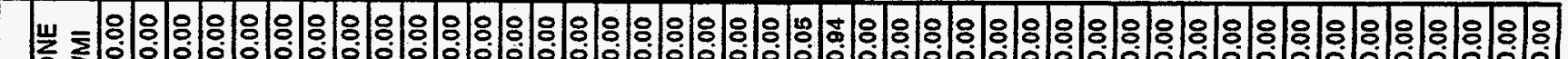
Z 垈

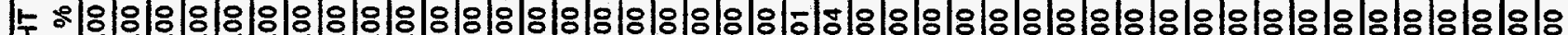

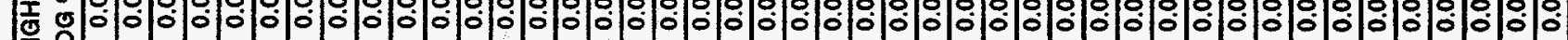

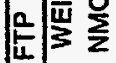

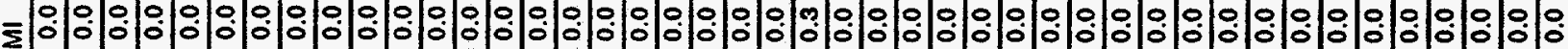
$\frac{0}{2}$

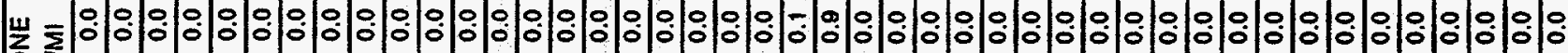
尽

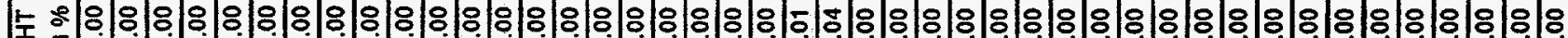

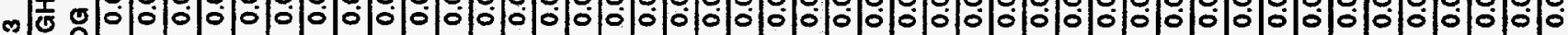
(0)

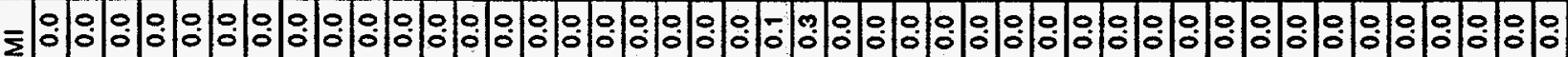
$\frac{\mathrm{O}}{2}$

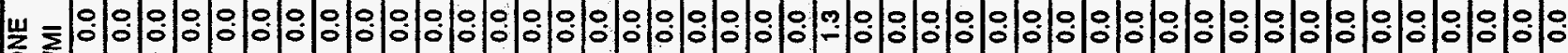
空 $\frac{\mathrm{O}}{2}$

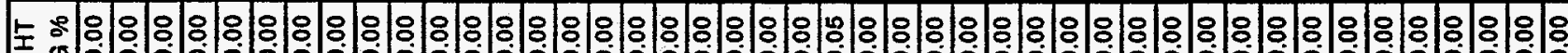
o

要这

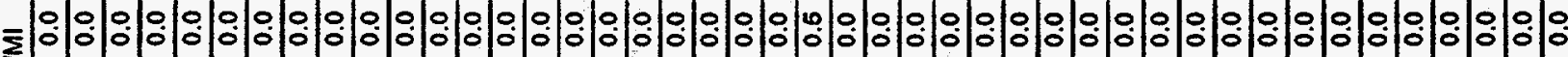

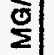

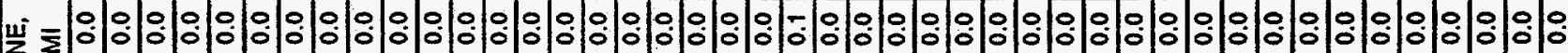
兽

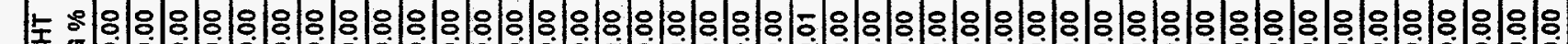
焉迹

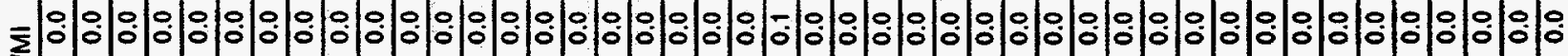
ङ

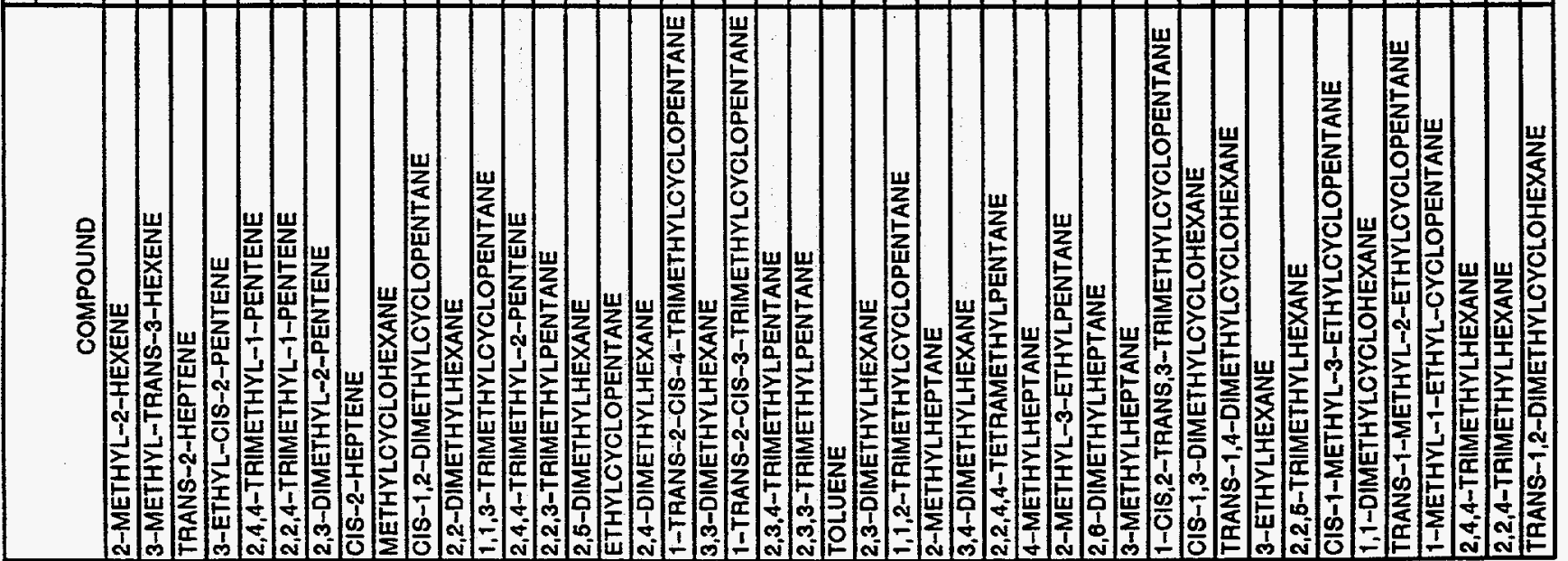




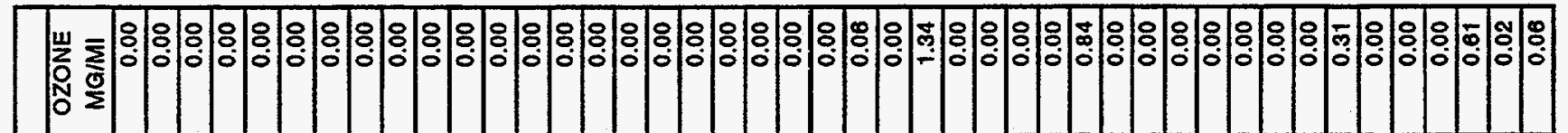

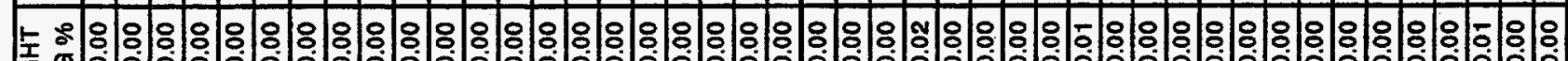
a

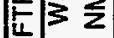

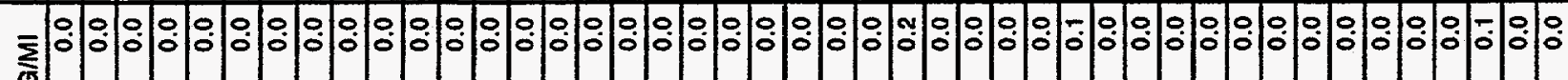

$\sum$

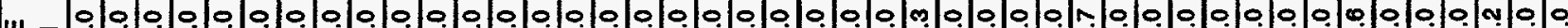

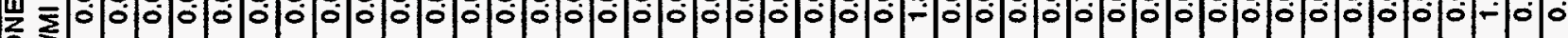
N

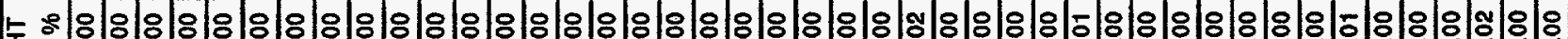

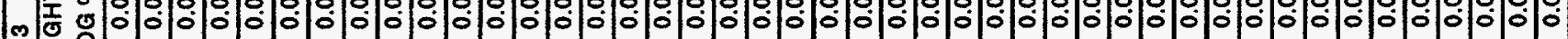

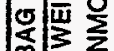

$\infty$

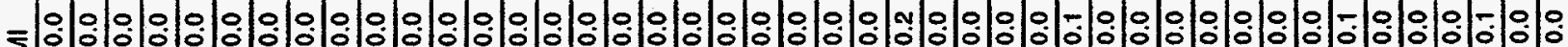

$\stackrel{\sum}{\Sigma}$

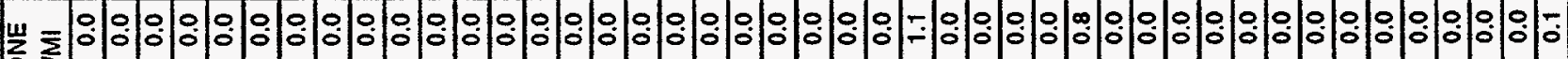
空

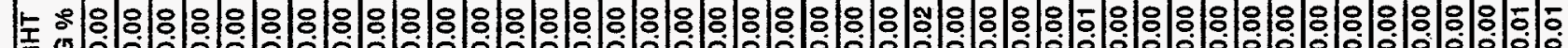
N

家崖

乏

高

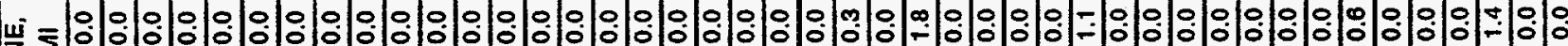

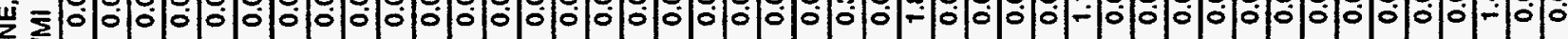
No

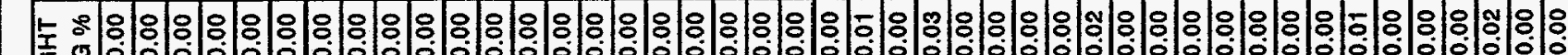

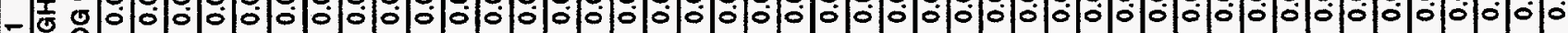

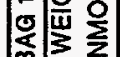

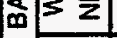

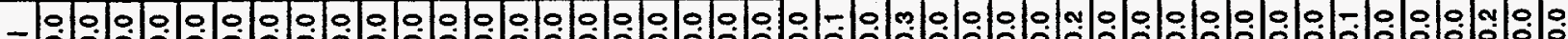

इ 을

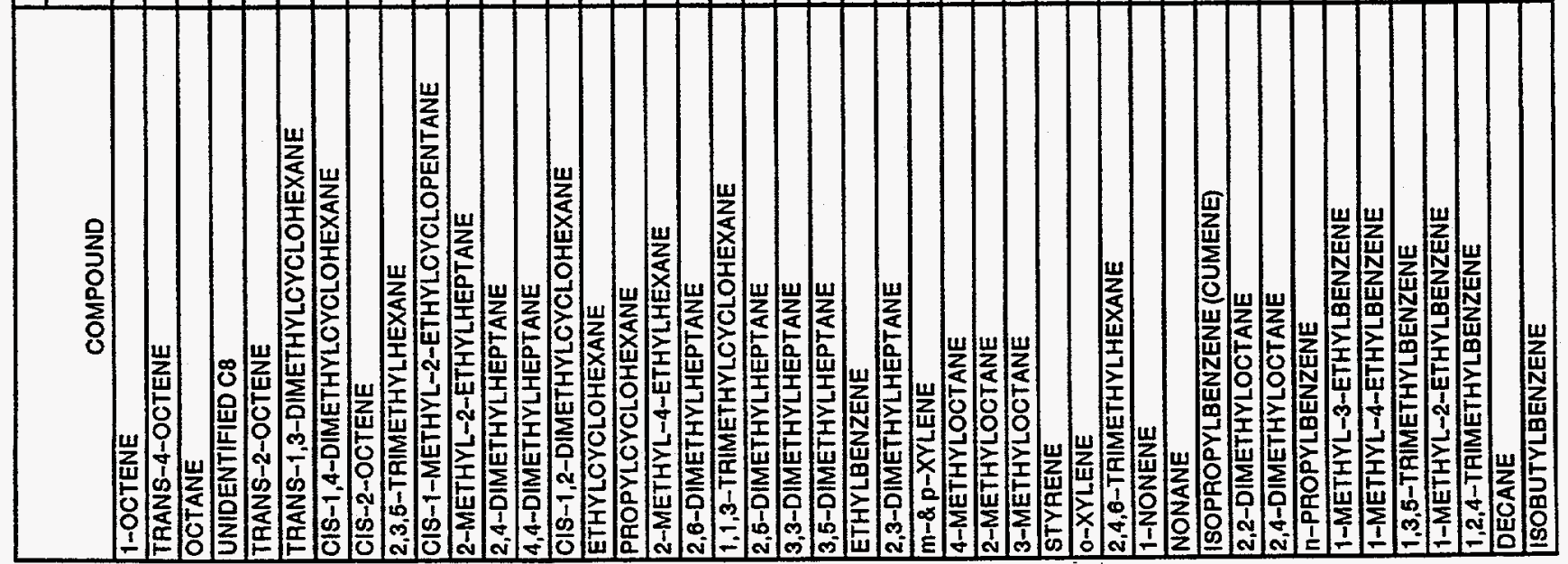




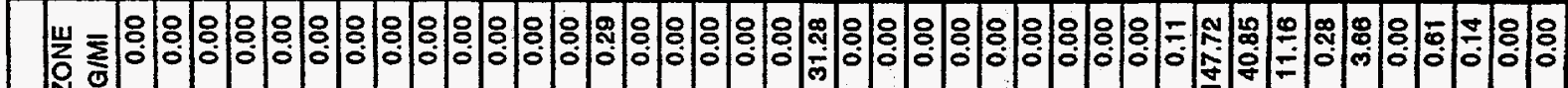
空 올

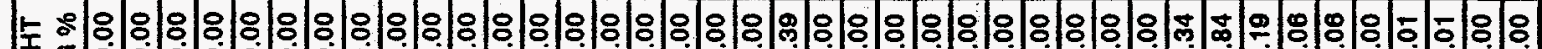
일 울

点运要

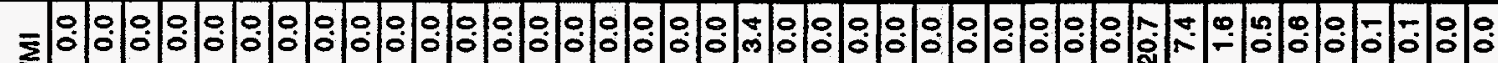

$$
\begin{aligned}
& \text { 这 }
\end{aligned}
$$

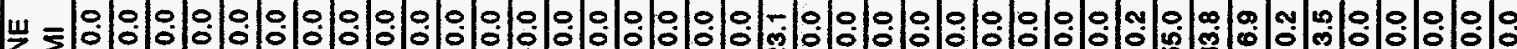
范 N

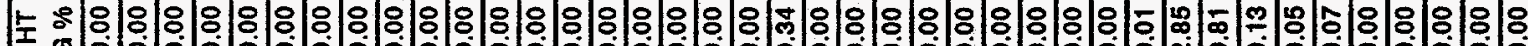

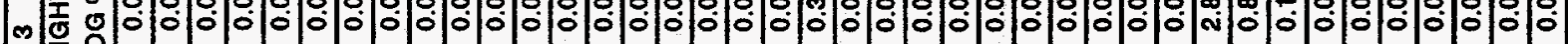
要零

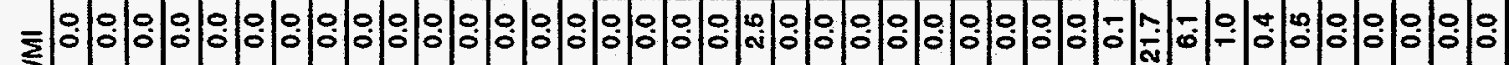
$\frac{\Phi}{\Sigma}$

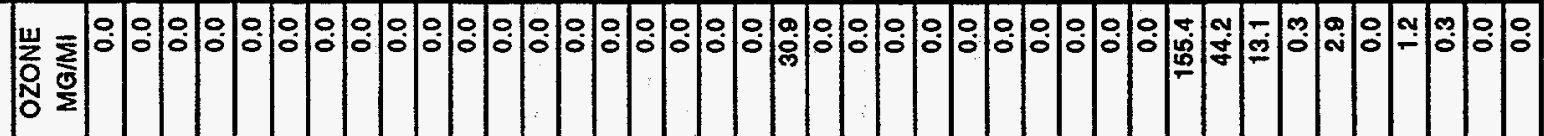

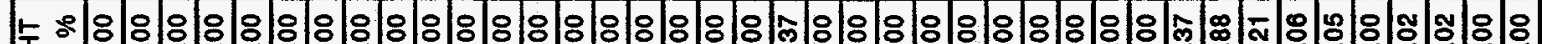
N

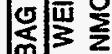

$$
\begin{aligned}
& \text { = } \\
& \stackrel{2}{2}
\end{aligned}
$$

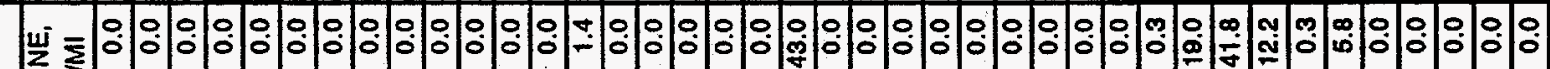
疍

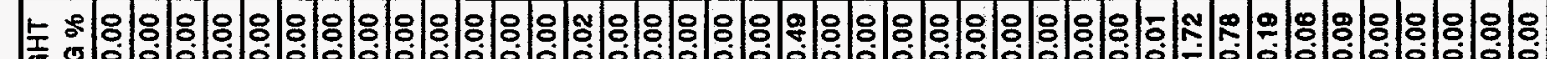
要

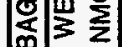

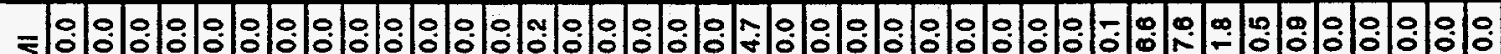
$\stackrel{\$}{\mathrm{~S}}$

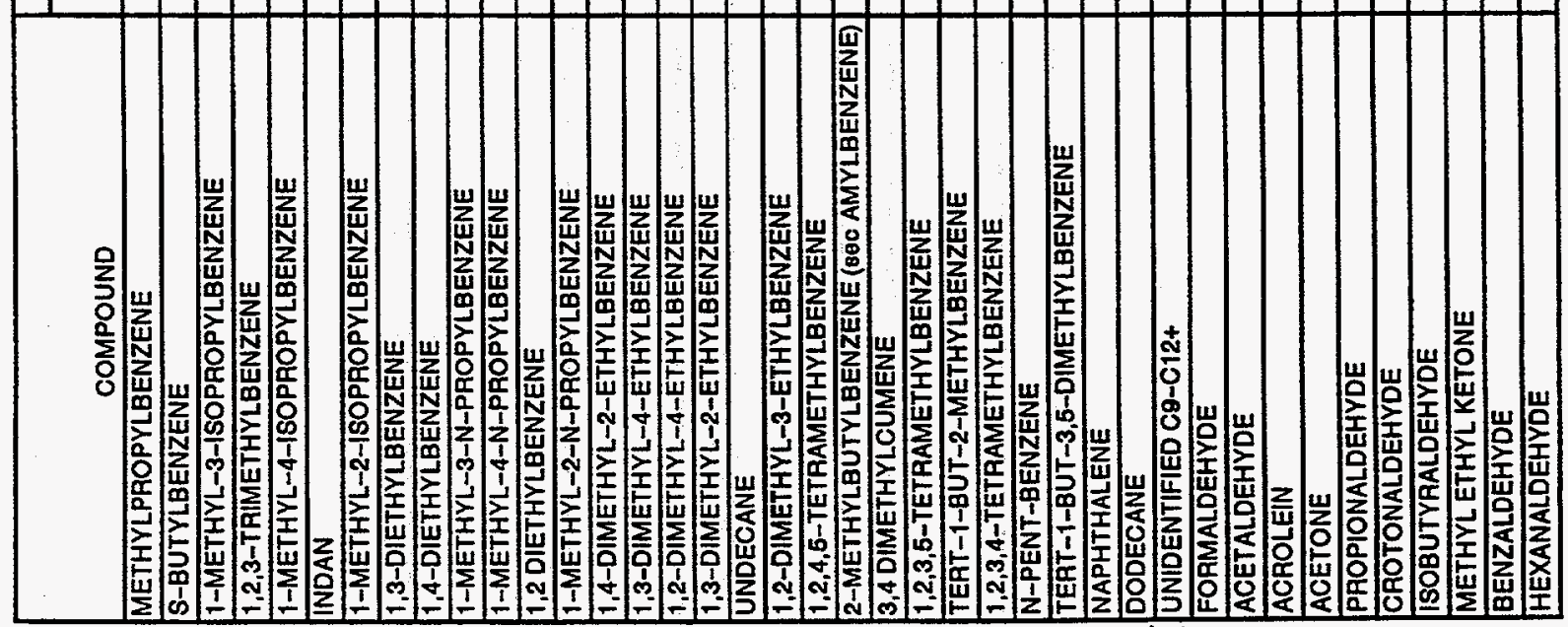


TEST NUMBER: 50NB/50P(NC)-02S

TEST CAR: 1994 PONTIAC GRANDAM

TEST DATE: $12 / 4 / 95$

TEST FUEL: $\quad 60 \%$ BUTANE/60\% PROPANE

\begin{tabular}{|c|c|c|c|c|c|c|c|c|c|c|c|c|}
\hline \multirow[b]{2}{*}{ COMPOUND } & \multicolumn{3}{|c|}{ BAG 1} & \multicolumn{3}{|c|}{ BAG 2} & \multicolumn{3}{|c|}{ BAG 3} & \multicolumn{3}{|c|}{ FTP } \\
\hline & MG/MI & $\begin{array}{l}\text { WEIGHT } \\
\text { NMOG } \%\end{array}$ & $\begin{array}{l}\text { OZONE, } \\
\text { MG/MI }\end{array}$ & MG/MI & $\begin{array}{l}\text { WEIGHT } \\
\text { NMOG } \%\end{array}$ & $\begin{array}{l}\text { OZONE } \\
\text { MG/MI }\end{array}$ & MG/MI & $\begin{array}{l}\text { WEIGHT } \\
\text { NMOG } \%\end{array}$ & $\begin{array}{l}\text { OZONE } \\
\text { MG/MI }\end{array}$ & MG/MI & $\begin{array}{l}\text { WEIGHT } \\
\text { NMOG } \%\end{array}$ & $\begin{array}{l}\text { OZONE } \\
\text { MGMII }\end{array}$ \\
\hline METHANE & 39.9 & & 0.6 & 43.4 & & 0.6 & 41.3 & & 0.6 & 42.1 & & 0.62 \\
\hline ETHANE & 14.4 & 1.35 & 3.6 & 15.8 & 1.62 & 3.9 & 0.0 & 0.00 & 0.0 & 11.1 & 1.17 & 2.79 \\
\hline ETHYLENE & 152.2 & 14.22 & 1109.2 & 163.5 & 16.84 & 1191.9 & 134.4 & 16.11 & 979.7 & 163.1 & 16.05 & 1116.33 \\
\hline PROPANE & 451.5 & 42.18 & 216.7 & 364.7 & 37.56 & 175.1 & 317.8 & 38.10 & 152.6 & 369.9 & 38.77 & 177.63 \\
\hline PAOPYLENE & 77.4 & 7.23 & 727.8 & 82.6 & 8.50 & 776.2 & 66.6 & 7.98 & 625.6 & 77.1 & 8.08 & 724.68 \\
\hline ACETYLENE & 61.2 & 4.78 & 25.6 & 53.4 & 5.50 & 26.7 & 49.2 & 5.89 & 24.6 & 51.8 & 5.43 & 25.90 \\
\hline PROPADIENE & 0.0 & 0.00 & 0.0 & 0.0 & 0.00 & 0.0 & 0.0 & 0.00 & 0.0 & 0.0 & 0.00 & 0.00 \\
\hline BUTANE & 258.3 & 24.13 & 263.4 & 222.4 & 22.90 & 226.9 & 202.7 & 24.30 & 206.7 & 224.5 & 23.53 & 228.94 \\
\hline TRANS-2-BUTENE & 1.0 & 0.09 & 9.5 & 1.0 & 0.10 & 10.1 & 1.0 & 0.12 & 9.7 & 1.0 & 0.10 & 9.88 \\
\hline 1-BUTENE & 4.1 & 0.38 & 36.3 & 4.3 & 0.44 & 38.2 & 3.6 & $0.4 \overline{3}$ & 31.6 & 4.0 & 0.42 & 35.99 \\
\hline 2-METHYLPROPENE (ISOBUTYLENE) & 0.4 & 0.04 & 2.4 & 0.5 & 0.05 & 2.5 & 1.4 & 0.17 & 7.4 & 0.7 & 0.08 & 3.80 \\
\hline 2,2-DIMETHYLPROPANE (NEOPENTANE) & 0.0 & 0.00 & 0.0 & 0.0 & 0.00 & 0.0 & 0.0 & 0.00 & 0.0 & 0.0 & 0.00 & 0.00 \\
\hline PROPYNE & 0.0 & 0.00 & 0.0 & 0.0 & 0.00 & 0.0 & 0.0 & 0.00 & 0.0 & 0.0 & 0.00 & 0.00 \\
\hline 1,3-BUTADIENE & 1.4 & 0.13 & 15.0 & 1.3 & 0.14 & 14.5 & 1.2 & 0.15 & 13.4 & 1.3 & 0.14 & 14.28 \\
\hline 2-METHYLPROPANE (ISOBUTANE) & 1.1 & 0.10 & 1.3 & 0.9 & 0.09 & 1.1 & 0.7 & 0.09 & 0.9 & 0.9 & 0.10 & 1.10 \\
\hline 1-BUTYNE & 0.0 & 0.00 & 0.0 & 0.0 & 0.00 & 0.0 & 0.0 & 0.00 & 0.0 & 0.0 & 0.00 & 0.00 \\
\hline METHANOL & 0.0 & 0.00 & 0.0 & 0.0 & 0.00 & 0.0 & 0.0 & 0.00 & 0.0 & 0.0 & 0.00 & 0.00 \\
\hline CIS-2-BUTENE & 0.7 & 0.07 & 7.1 & 0.8 & 0.08 & 7.6 & 0.7 & 0.09 & 7.2 & 0.7 & 0.08 & 7.34 \\
\hline 3-METHYL-1-BUTENE & 0.0 & 0.00 & 0.0 & 0.0 & 0.00 & 0.0 & 0.0 & 0.00 & 0.0 & 0.0 & 0.00 & 0.00 \\
\hline ETHANOL & 0.0 & 0.00 & 0.0 & 0.0 & 0.00 & 0.0 & 0.0 & 0.00 & 0.0 & 0.0 & 0.00 & 0.00 \\
\hline 2-METHYLBUTANE (ISOPENTANE) & 2.9 & 0.27 & 4.0 & 0.7 & 0.07 & 1.0 & 3.9 & 0.47 & $\overline{5.4}$ & 2.1 & 0.21 & 2.83 \\
\hline 2-BUTYNE & 0.0 & 0.00 & 0.0 & 0.0 & 0.00 & 0.0 & 0.0 & 0.00 & 0.0 & 0.0 & 0.00 & 0.00 \\
\hline 1-PENTENE & 0.1 & 0.01 & 0.8 & 0.3 & 0.03 & 2.0 & 0.2 & 0.02 & 1.0 & 0.2 & 0.03 & 1.51 \\
\hline 2-METHYL-1-BUTENE & 0.1 & 0.01 & 0.7 & 0.0 & 0.00 & 0.0 & 0.3 & 0.03 & 1.2 & 0.1 & 0.01 & 0.49 \\
\hline PENTANE & 0.2 & 0.02 & 0.2 & 0.2 & 0.02 & 0.2 & 0.4 & 0.05 & 0.4 & 0.3 & 0.03 & 0.27 \\
\hline UNIDENTIFIED C5 OLEFINS & 0.0 & 0.00 & 0.0 & 0.0 & 0.00 & 0.0 & 0.1 & 0.01 & 1.0 & 0.0 & 0.00 & 0.27 \\
\hline 2-METHYL-1,3-BUTADIENE & 1.1 & 0.11 & 10.2 & 0.8 & 0.08 & 7.2 & 1.0 & 0.12 & 8.9 & 0.9 & 0.10 & 8.32 \\
\hline TRANS-2-PENTENE & 0.2 & 0.02 & 2.0 & 0.2 & 0.02 & 1.9 & 0.2 & 0.02 & 1.7 & 0.2 & 0.02 & 1.86 \\
\hline 3,3-DIMETHYL-1-BUTENE & 0.0 & 0.00 & 0.0 & 0.0 & 0.00 & 0.0 & 0.0 & 0.00 & 0.0 & 0.0 & 0.00 & 0.00 \\
\hline CIS-2-PENTENE & 0.0 & 0.00 & 0.0 & 0.0 & 0.00 & 0.0 & 0.0 & 0.00 & 0.0 & 0.0 & 0.00 & 0.00 \\
\hline 2-METHYL-2-BUTENE & 0.1 & 0.01 & 0.7 & 0.1 & 0.01 & 0.4 & 0.2 & 0.02 & 1.0 & 0.1 & 0.01 & 0.60 \\
\hline CYCLOPENTADIENE & 0.0 & 0.00 & 0.0 & 0.0 & 0.00 & 0.0 & 0.0 & 0.00 & 0.0 & 0.0 & 0.00 & 0.00 \\
\hline 2,2-DIMETHYLBUTANE & 0.0 & 0.00 & 0.0 & 0.0 & 0.00 & 0.0 & 0.0 & 0.00 & 0.0 & 0.0 & 0.00 & 0.00 \\
\hline CYCLOPENTENE & 0.0 & 0.00 & 0.0 & 0.0 & 0.00 & 0.0 & 0.0 & 0.00 & 0.0 & 0.0 & 0.00 & 0.00 \\
\hline 4-METHYL-1-PENTENE & 0.0 & 0.00 & 0.0 & 0.0 & 0.00 & 0.0 & 0.1 & 0.01 & 0.5 & 0.0 & 0.00 & 0.13 \\
\hline 3-METHYL-1-PENTENE & 0.0 & 0.00 & 0.0 & 0.0 & 0.00 & 0.0 & 0.0 & 0.00 & 0.0 & 0.0 & 0.00 & 0.00 \\
\hline CYCLOPENTANE & 0.0 & 0.00 & 0.0 & 0.0 & 0.00 & 0.0 & 0.0 & 0.00 & 0.0 & 0.0 & 0.00 & 0.00 \\
\hline 2,3-DIMETHYLBUTANE & 0.1 & 0.01 & 0.1 & 0.2 & 0.02 & 0.2 & 0.1 & 0.02 & 0.1 & 0.2 & 0.02 & 0.18 \\
\hline MTBE & 0.0 & 0.00 & 0.0 & 0.0 & 0.00 & 0.0 & 0.0 & 0.00 & 0.0 & 0.0 & 0.00 & 0.00 \\
\hline
\end{tabular}




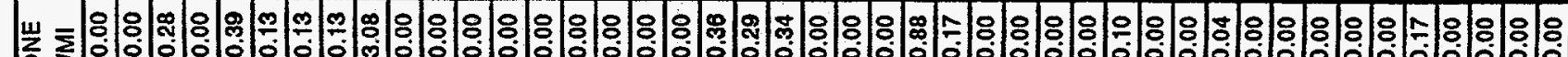
Z No

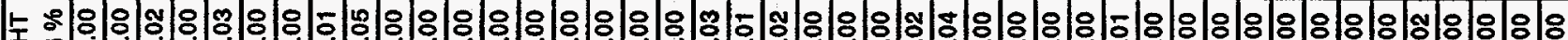

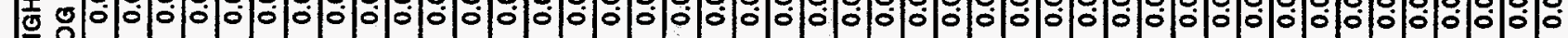
은

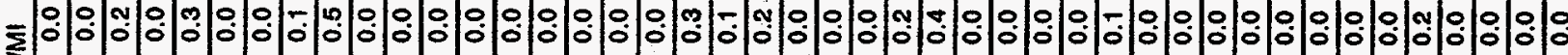
$\frac{\mathrm{s}}{2}$

出 倠

F 응ㅇㅇ응 -

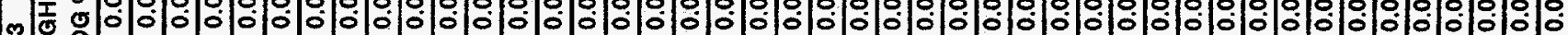
震

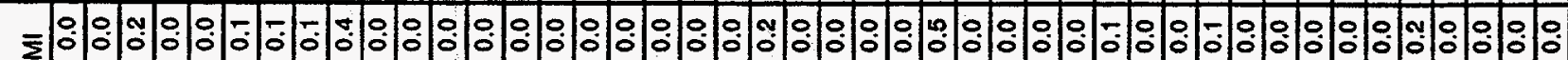
$\stackrel{0}{\Sigma}$

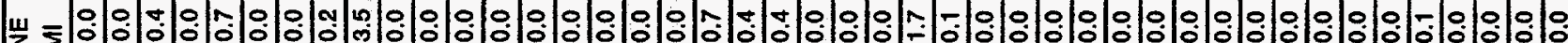

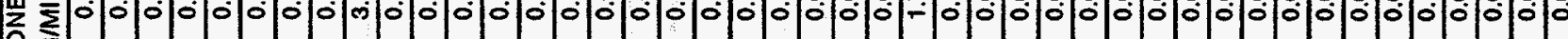
忟 $\frac{\text { O }}{2}$

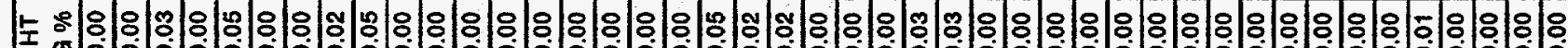

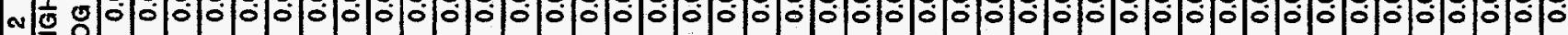
震

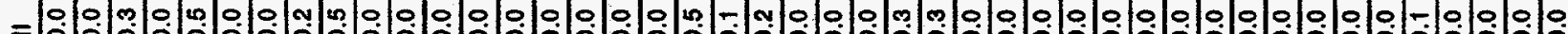

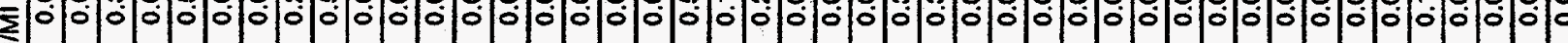
일

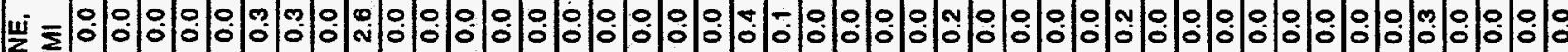
옹 을

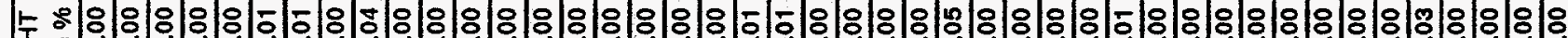

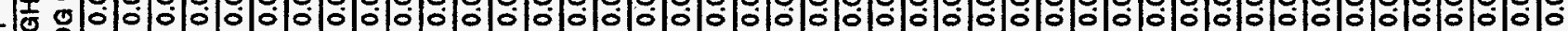
要恶

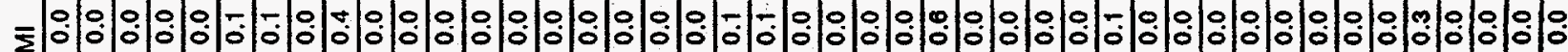
श

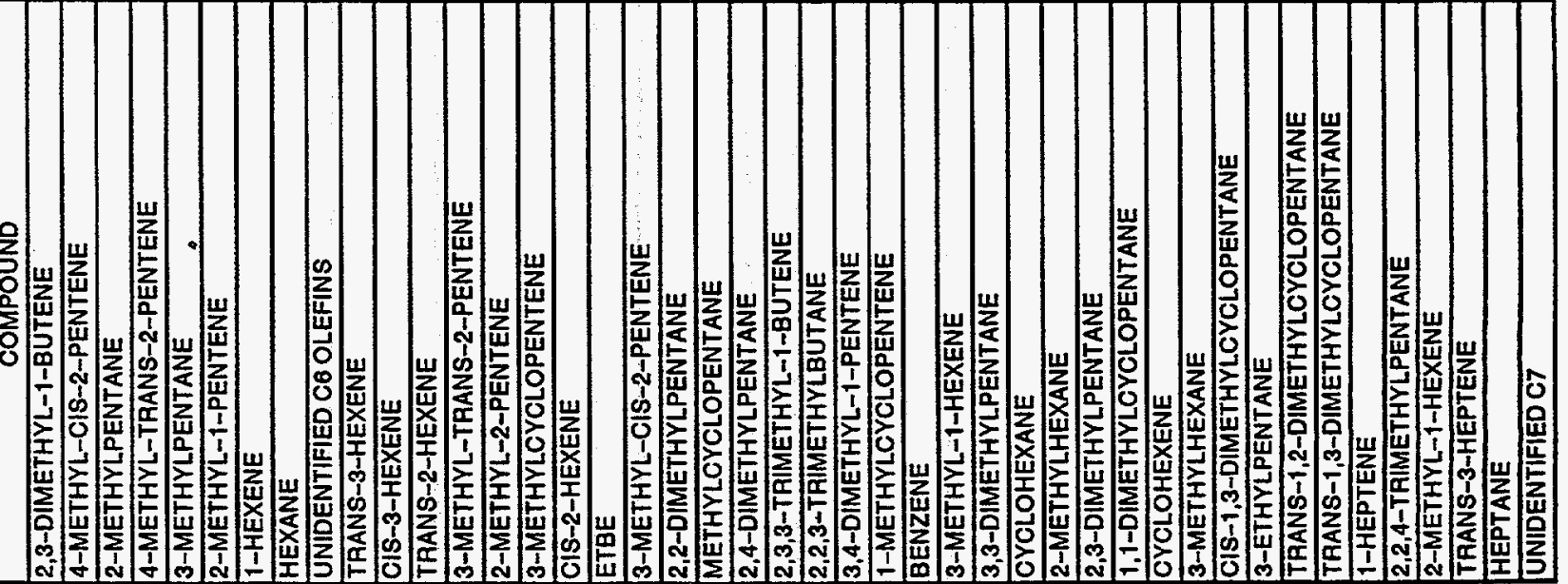




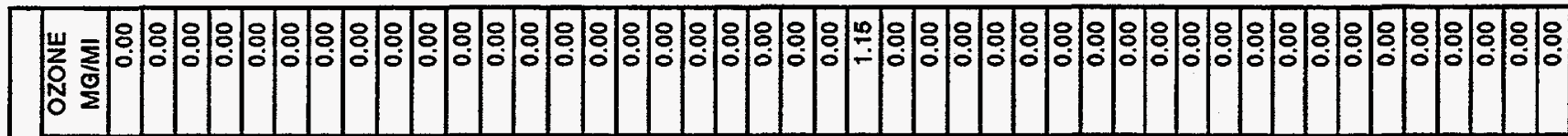

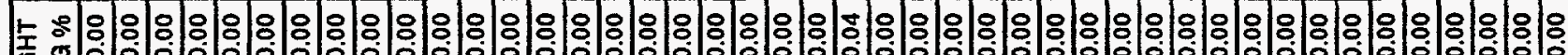
产 恶

至 3

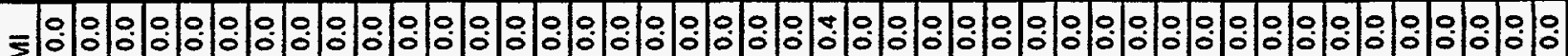

울

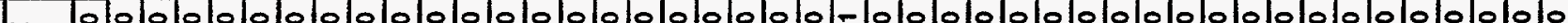

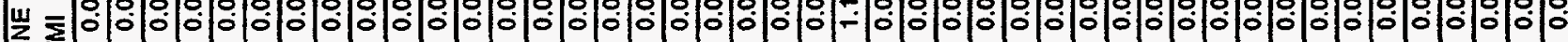
范

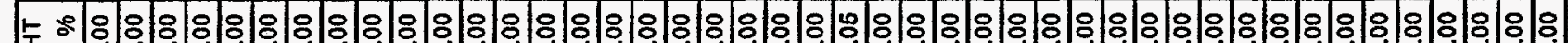
- I

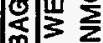

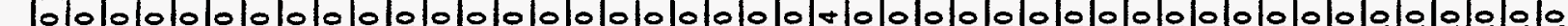
三 $\stackrel{\mathrm{O}}{\Sigma}$

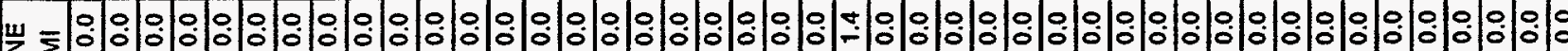

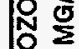

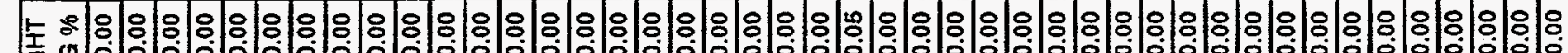
象

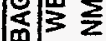

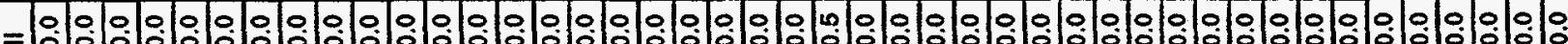
$\sum_{\Sigma}$

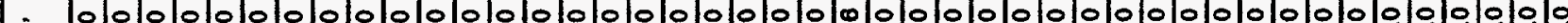

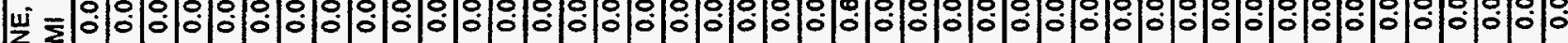
目 $\stackrel{0}{\Sigma}$

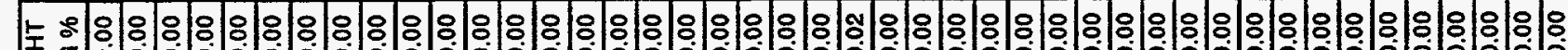
- 西 0

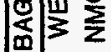

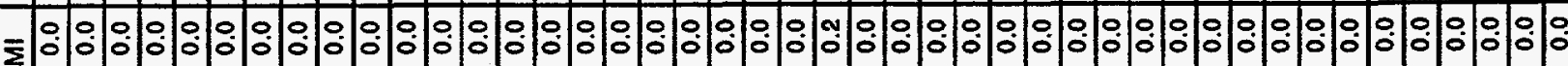
$\sum$

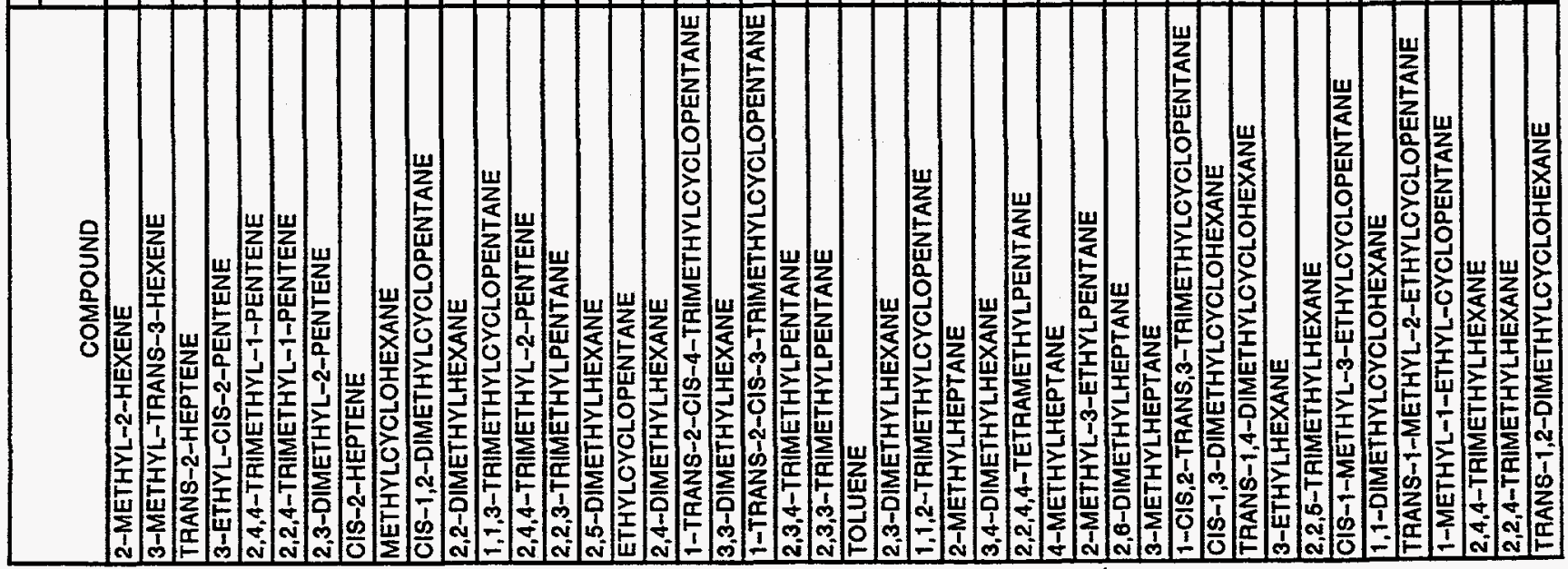




\begin{tabular}{|c|c|c|c|c|c|c|c|c|c|c|c|c|}
\hline \multirow[b]{2}{*}{ COMPOUND } & \multicolumn{3}{|c|}{ BAG 1} & \multicolumn{3}{|c|}{ BAG 2} & \multicolumn{3}{|c|}{$\overline{B A G} 3$} & \multicolumn{3}{|c|}{ FTP } \\
\hline & MG/MI & $\begin{array}{l}\text { WEIGHT } \\
\text { NMOG } \%\end{array}$ & $\begin{array}{l}\text { OZONE, } \\
\text { MG/MI }\end{array}$ & MG/MI & $\begin{array}{l}\text { WEIGHT } \\
\text { NMOG \% }\end{array}$ & $\begin{array}{l}\text { OZONE } \\
\text { MG/MI }\end{array}$ & MG/MI & $\begin{array}{l}\text { WEIGHT } \\
\text { NMOG } \%\end{array}$ & $\begin{array}{l}\text { OZONE } \\
\text { MG/MI }\end{array}$ & MG/MI & $\begin{array}{l}\text { WEIGHT } \\
\text { NMOQ \% }\end{array}$ & $\begin{array}{l}\text { OZONE } \\
\text { MG/MI }\end{array}$ \\
\hline 1-OCTENE & 0.0 & 0.00 & 0.0 & 0.0 & 0.00 & 0.0 & 0.0 & 0.00 & 0.0 & 0.0 & 0.00 & 0.00 \\
\hline TRANS-4-OCTENE & 0.0 & 0.00 & 0.0 & 0.0 & 0.00 & 0.0 & 0.0 & 0.00 & 0.0 & 0.0 & 0.00 & 0.00 \\
\hline OCTANE & 0.0 & 0.00 & 0.0 & 0.0 & 0.00 & 0.0 & 0.0 & .0 .00 & 0.0 & 0.0 & 0.00 & 0.00 \\
\hline UNIDENTIFIED C8 & 0.0 & 0.00 & 0.0 & 0.0 & 0.00 & 0.0 & 0.0 & 0.00 & 0.0 & 0.0 & 0.00 & 0.00 \\
\hline TRANS-2-OCTENE & 0.0 & 0.00 & 0.0 & 0.0 & 0.00 & 0.0 & 0.0 & 0.00 & 0.0 & 0.0 & 0.00 & 0.00 \\
\hline TRANS-1,3-DIMETHYLCYCLOHEXANE & 0.0 & 0.00 & 0.0 & 0.0 & 0.00 & 0.0 & 0.0 & 0.00 & 0.0 & 0.0 & 0.00 & 0.00 \\
\hline CIS-1,4-DIMETHYLCYCLOHEXANE & 0.0 & 0.00 & 0.0 & 0.0 & 0.00 & 0.0 & 0.0 & 0.00 & 0.0 & 0.0 & 0.00 & 0.00 \\
\hline CIS-2-OCTENE & 0.0 & 0.00 & 0.0 & 0.0 & 0.00 & 0.0 & 0.0 & 0.00 & 0.0 & 0.0 & 0.00 & 0.00 \\
\hline 2,3,5-TRIMETHYLHEXANE & 0.0 & 0.00 & 0.0 & 0.0 & 0.00 & 0.0 & 0.0 & 0.00 & 0.0 & 0.0 & 0.00 & 0.00 \\
\hline CIS-1-METHYL-2-ETHYLCYCLOPENTANE & 0.0 & 0.00 & 0.0 & 0.0 & 0.00 & 0.0 & $\overline{0.0}$ & 0.00 & 0.0 & 0.0 & 0.00 & 0.00 \\
\hline 2-METHYL-2-ETHYLHEPTANE & 0.0 & 0.00 & 0.0 & 0.0 & 0.00 & 0.0 & 0.0 & 0.00 & 0.0 & 0.0 & 0.00 & 0.00 \\
\hline 2,4-DIMETHYLLEPTANE & 0.0 & 0.00 & 0.0 & 0.0 & 0.00 & 0.0 & 0.0 & 0.00 & 0.0 & 0.0 & 0.00 & 0.00 \\
\hline 4,4-DIMETHYLHEPTANE & 0.0 & 0.00 & 0.0 & 0.0 & 0.00 & 0.0 & 0.0 & 0.00 & 0.0 & 0.0 & 0.00 & 0.00 \\
\hline CIS-1,2-DIMETHYLCYCLOHEXANE & 0.0 & 0.00 & 0.0 & 0.0 & 0.00 & 0.0 & 0.0 & 0.00 & 0.0 & 0.0 & 0.00 & 0.00 \\
\hline ETHYLCYCLOHEXANE & 0.0 & 0.00 & 0.0 & 0.0 & 0.00 & 0.0 & 0.0 & 0.00 & 0.0 & 0.0 & 0.00 & 0.00 \\
\hline PAOPYLCYCLOHEXANE & 0.0 & 0.00 & 0.0 & 0.0 & 0.00 & 0.0 & 0.0 & 0.00 & 0.0 & 0.0 & 0.00 & 0.00 \\
\hline 2-METHYL-4-ETHYLHEXANE & 0.0 & 0.00 & 0.0 & 0.0 & 0.00 & 0.0 & 0.0 & 0.00 & 0.0 & 0.0 & 0.00 & 0.00 \\
\hline 2,6-DIMETHYLHEPTANE & 0.0 & 0.00 & 0.0 & 0.0 & 0.00 & 0.0 & 0.0 & 0.00 & 0.0 & 0.0 & 0.00 & 0.00 \\
\hline 1,1,3-TAIMETHYLCYCLOHEXANE & 0.0 & 0.00 & 0.0 & 0.0 & 0.00 & 0.0 & 0.0 & 0.00 & 0.0 & 0.0 & 0.00 & 0.00 \\
\hline 2,5-DIMETHYLHEPTANE & 0.0 & 0.00 & 0.0 & 0.0 & 0.00 & 0.0 & 0.0 & 0.00 & 0.0 & 0.0 & 0.00 & 0.00 \\
\hline 3,3-DIMETHYLHEPTANE & 0.0 & 0.00 & 0.0 & 0.0 & 0.00 & 0.0 & 0.0 & 0.00 & 0.0 & 0.0 & 0.00 & 0.00 \\
\hline 3,5-DIMETHYLHEPTANE & 0.0 & 0.00 & 0.0 & 0.0 & 0.00 & 0.0 & 0.0 & 0.00 & 0.0 & 0.0 & 0.00 & 0.00 \\
\hline ETHYLBENZENE & 0.0 & 0.00 & 0.0 & 0.0 & 0.00 & 0.0 & 0.1 & 0.01 & 0.2 & 0.0 & 0.00 & 0.07 \\
\hline 2,3-DIMETHYLHEPTANE & 0.0 & 0.00 & 0.0 & 0.0 & 0.00 & 0.0 & 0.0 & 0.00 & 0.0 & 0.0 & 0.00 & 0.00 \\
\hline$m-\& p-X Y L E N E$ & 0.2 & 0.02 & 1.2 & 0.2 & 0.02 & 1.2 & 0.1 & 0.02 & 1.0 & 0.2 & 0.02 & 1.18 \\
\hline 4-METHYLOCTANE & 0.0 & 0.00 & 0.0 & 0.0 & 0.00 & 0.0 & 0.0 & 0.00 & 0.0 & 0.0 & 0.00 & 0.00 \\
\hline 2-METHYLOCTANE & 0.0 & 0.00 & 0.0 & 0.0 & 0.00 & 0.0 & 0.0 & 0.00 & 0.0 & 0.0 & 0.00 & 0.00 \\
\hline 3-METHYLOCTANE & 0.0 & 0.00 & 0.0 & 0.0 & 0.00 & 0.0 & 0.0 & 0.00 & 0.0 & 0.0 & 0.00 & 0.00 \\
\hline STYRENE & 0.0 & 0.00 & 0.0 & 0.0 & 0.00 & 0.0 & 0.0 & 0.00 & 0.0 & 0.0 & 0.00 & 0.00 \\
\hline O-XYLENE & 0.1 & 0.01 & 0.8 & 0.0 & 0.00 & 0.0 & 0.2 & 0.02 & 1.3 & 0.1 & 0.01 & 0.50 \\
\hline 2,4,6-TAIMETHYLLEXANE & 0.0 & 0.00 & 0.0 & 0.0 & 0.00 & 0.0 & 0.0 & 0.00 & 0.0 & 0.0 & 0.00 & 0.00 \\
\hline 1-NONENE & 0.0 & 0.00 & 0.0 & 0.0 & 0.00 & 0.0 & 0.0 & 0.00 & 0.0 & 0.0 & 0.00 & 0.00 \\
\hline NONANE & 0.0 & 0.00 & 0.0 & 0.0 & 0.00 & 0.0 & 0.0 & 0.00 & 0.0 & 0.0 & 0.00 & 0.00 \\
\hline ISOPROPYLBENZENE (CUMENE) & 0.0 & 0.00 & 0.0 & 0.0 & 0.00 & 0.0 & 0.0 & 0.00 & 0.0 & 0.0 & 0.00 & 0.00 \\
\hline 2,2-DIMETHYLOCTANE & 0.0 & 0.00 & 0.0 & 0.0 & 0.00 & 0.0 & 0.0 & 0.00 & 0.0 & 0.0 & 0.00 & 0.00 \\
\hline 2,4-DIMETHYLOCTANE & 0.0 & 0.00 & 0.0 & 0.0 & 0.00 & 0.0 & 0.0 & 0.00 & 0.0 & 0.0 & 0.00 & 0.00 \\
\hline n-PROPYLBENZENE & 0.0 & 0.00 & 0.0 & 0.0 & 0.00 & 0.0 & 0.0 & 0.00 & 0.0 & 0.0 & 0.00 & 0.00 \\
\hline 1-METHYL-3-ETHYLBENZENE & 0.0 & 0.00 & 0.0 & 0.0 & 0.00 & 0.0 & 0.0 & 0.00 & 0.0 & 0.0 & 0.00 & 0.00 \\
\hline 1-METHYL-4-ETHYLBENZENE & 0.0 & 0.00 & 0.0 & 0.0 & 0.00 & 0.0 & 0.0 & 0.00 & 0.0 & 0.0 & 0.00 & 0.00 \\
\hline 1,3,6-TRIMETHYLBENZENE & 0.0 & 0.00 & 0.0 & 0.0 & 0.00 & 0.0 & 0.0 & 0.00 & 0.0 & 0.0 & 0.00 & 0.00 \\
\hline 1-METHYL-2-ETHYLBENZENE & 0.0 & 0.00 & 0.0 & 0.0 & 0.00 & 0.0 & 0.0 & 0.00 & 0.0 & 0.0 & 0.00 & 0.00 \\
\hline 1,2,4-TRIMETHYLBENZENE & 0.0 & 0.00 & 0.0 & 0.0 & 0.00 & 0.0 & 0.0 & 0.00 & 0.0 & 0.0 & 0.00 & 0.00 \\
\hline DECANE & 0.1 & 0.01 & 0.0 & 0.0 & 0.00 & 0.0 & 0.0 & 0.00 & 0.0 & 0.0 & 0.00 & 0.01 \\
\hline ISOBUTYLBENZENE & 0.1 & 0.01 & 0.1 & 0.0 & 0.00 & 0.0 & 0.0 & 0.00 & 0.0 & 0.0 & 0.00 & 0.02 \\
\hline
\end{tabular}




\begin{tabular}{|c|c|c|c|c|c|c|c|c|c|c|c|c|}
\hline \multirow[b]{2}{*}{ COMPOUND } & \multicolumn{3}{|c|}{ BAG 1} & \multicolumn{3}{|c|}{ BAG 2} & \multicolumn{3}{|c|}{ BAG 3} & \multicolumn{3}{|c|}{ FTP } \\
\hline & $M G / M I$ & $\begin{array}{l}\text { WEIGHT } \\
\text { NMOG \% }\end{array}$ & $\begin{array}{l}\text { OZONE, } \\
\text { MGMI }\end{array}$ & MG/MI & \begin{tabular}{|l|} 
WEIGHT \\
NMOG \%
\end{tabular} & $\begin{array}{l}\text { OZONE } \\
\text { MG/MI }\end{array}$ & MG/MI & $\begin{array}{l}\text { WEIGHT } \\
\text { NMOG } \%\end{array}$ & $\begin{array}{l}\text { OZONE } \\
\text { MGMII }\end{array}$ & MG/MI & $\begin{array}{l}\text { WEIGHT } \\
\text { NMOG } \%\end{array}$ & $\begin{array}{l}\text { OZONE } \\
\text { MGMMI }\end{array}$ \\
\hline METHYLPROPYLBENZENE & 0.0 & 0.00 & 0.0 & 0.0 & 0.00 & 0.0 & 0.0 & 0.00 & 0.0 & 0.0 & 0.00 & 0.00 \\
\hline S-BUTYLBENZENE & 0.0 & 0.00 & 0.0 & 0.0 & 0.00 & 0.0 & 0.0 & 0.00 & 0.0 & 0.0 & 0.00 & 0.00 \\
\hline 1-METHYL-3-ISOPROPYLBENZENE & 0.0 & 0.00 & 0.0 & 0.0 & 0.00 & 0.0 & 0.0 & 0.00 & 0.0 & 0.0 & 0.00 & 0.00 \\
\hline 1,2,3-TRIMETHYLBENZENE & 0.0 & 0.00 & 0.0 & 0.0 & 0.00 & 0.0 & 0.0 & 0.00 & 0.0 & 0.0 & 0.00 & 0.00 \\
\hline 1-METHYL-4-ISOPROPYLBENZENE & 0.0 & 0.00 & 0.0 & 0.0 & 0.00 & 0.0 & 0.0 & 0.00 & 0.0 & 0.0 & 0.00 & 0.00 \\
\hline INDAN & 0.0 & 0.00 & 0.0 & 0.0 & 0.00 & 0.0 & 0.0 & 0.00 & 0.0 & 0.0 & 0.00 & 0.00 \\
\hline 1-METHYL-2-18OPROPYLBENZENE & 0.0 & 0.00 & 0.0 & 0.0 & 0.00 & 0.0 & 0.0 & 0.00 & 0.0 & 0.0 & 0.00 & 0.00 \\
\hline 1,3-DIETHYLBENZENE & 0.0 & 0.00 & 0.0 & 0.0 & 0.00 & 0.0 & 0.0 & 0.00 & 0.0 & 0.0 & 0.00 & 0.00 \\
\hline 1,4-DIETHYLBENZENE & 0.0 & 0.00 & 0.0 & 0.0 & 0.00 & 0.0 & 0.0 & 0.00 & 0.0 & 0.0 & 0.00 & 0.00 \\
\hline 1-METHYL-3-N-PAOPYLBENZENE & 0.0 & 0.00 & 0.0 & 0.0 & 0.00 & 0.0 & 0.0 & 0.00 & 0.0 & 0.0 & 0.00 & 0.00 \\
\hline 1-METHYL-4-N-PROPYLBENZENE & 0.0 & 0.00 & 0.0 & 0.0 & 0.00 & 0.0 & 0.0 & 0.00 & 0.0 & 0.0 & 0.00 & 0.00 \\
\hline 1.2 DIETHYLBENZENE & 0.0 & 0.00 & 0.0 & 0.0 & 0.00 & 0.0 & 0.0 & 0.00 & 0.0 & 0.0 & 0.00 & 0.00 \\
\hline 1-METHYL-2-N-PROPYLBENZENE & 0.0 & 0.00 & 0.0 & 0.0 & 0.00 & 0.0 & 0.0 & 0.00 & 0.0 & 0.0 & 0.00 & 0.00 \\
\hline 1,4-DIMETHYL-2-ETHYLBENZENE & 0.0 & 0.00 & 0.0 & 0.0 & 0.00 & 0.0 & 0.0 & 0.00 & 0.0 & 0.0 & 0.00 & 0.00 \\
\hline 1,3-DIMETHYL-4-ETHYLBENZENE & 0.0 & 0.00 & 0.0 & 0.0 & 0.00 & 0.0 & 0.0 & 0.00 & 0.0 & 0.0 & 0.00 & 0.00 \\
\hline 1,2-DIMETHYL-4-ETHYLBENZENE & 0.0 & 0.00 & 0.0 & 0.0 & 0.00 & 0.0 & 0.0 & 0.00 & 0.0 & 0.0 & 0.00 & 0.00 \\
\hline 1,3-DIMETHYL-2-ETHYLBENZENE & 0.0 & 0.00 & 0.0 & 0.0 & 0.00 & 0.0 & 0.0 & 0.00 & 0.0 & 0.0 & 0.00 & 0.00 \\
\hline UNDECANE & 0.0 & 0.00 & 0.0 & 0.0 & 0.00 & 0.0 & 0.0 & 0.00 & 0.0 & 0.0 & 0.00 & 0.00 \\
\hline 1,2-DIMETHYL-3-ETHYLBENZENE & 0.0 & 0.00 & 0.0 & 0.0 & 0.00 & 0.0 & 0.0 & 0.00 & 0.0 & 0.0 & 0.00 & 0.00 \\
\hline 1,2,4,5-TETRAMETHYLBENZENE & 1.3 & 0.12 & 12.1 & 1.0 & 0.10 & 8.7 & 3.2 & 0.38 & 28.9 & 1.6 & 0.17 & 14.96 \\
\hline 2-METHYLBUTYLBENZENE (SOC AMYLBENZENE) & 0.0 & 0.00 & 0.0 & 0.0 & 0.00 & 0.0 & 0.0 & 0.00 & 0.0 & 0.0 & 0.00 & 0.00 \\
\hline 3,4 DIMETHYLCUMENE & 0.0 & 0.00 & 0.0 & 0.0 & 0.00 & 0.0 & 0.0 & 0.00 & 0.0 & 0.0 & 0.00 & 0.00 \\
\hline 1,2,3,5-TETAAMETHYLBENZENE & 0.0 & 0.00 & 0.0 & 0.0 & 0.00 & 0.0 & 0.0 & 0.00 & 0.0 & 0.0 & 0.00 & 0.00 \\
\hline TERT-1-BUT-2-METHYLBENZENE & 0.0 & 0.00 & 0.0 & 0.0 & 0.00 & 0.0 & 0.0 & 0.00 & 0.0 & 0.0 & 0.00 & 0.00 \\
\hline 1,2,3,4-TETRAMETHYLBENZENE & 0.0 & 0.00 & 0.0 & 0.0 & 0.00 & 0.0 & 0.0 & 0.00 & 0.0 & 0.0 & 0.00 & 0.00 \\
\hline N-PENT-BENZENE & 0.0 & 0.00 & 0.0 & 0.0 & 0.00 & 0.0 & 0.0 & 0.00 & 0.0 & 0.0 & 0.00 & 0.00 \\
\hline TERT-1-BUT-3,6-DIMETHYLBENZENE & 0.0 & 0.00 & 0.0 & 0.0 & 0.00 & 0.0 & 0.0 & 0.00 & 0.0 & 0.0 & 0.00 & 0.00 \\
\hline NAPHTHALENE & 0.0 & 0.00 & 0.0 & 0.0 & 0.00 & 0.0 & 0.0 & 0.00 & 0.0 & 0.0 & 0.00 & 0.00 \\
\hline DODECANE & 0.0 & 0.00 & 0.0 & 0.0 & 0.00 & 0.0 & 0.0 & 0.00 & 0.0 & 0.0 & 0.00 & 0.00 \\
\hline UNIDENTIFIED C9-C12+ & 0.1 & 0.01 & 0.3 & 0.0 & 0.00 & 0.0 & 0.0 & 0.01 & 0.2 & 0.0 & 0.00 & 0.11 \\
\hline FORMALDEHYDE & 36.6 & 3.42 & 262.0 & 41.8 & 4.31 & 299.0 & 33.2 & 3.97 & 237.1 & 38.4 & 4.02 & 274.24 \\
\hline ACETALDEHYDE & 8.2 & 0.77 & 45.4 & 8.3 & 0.86 & 45.9 & 6.7 & 0.81 & 37.2 & 7.9 & 0.82 & 43.39 \\
\hline ACROLEIN & 2.1 & 0.20 & 14.1 & 2.1 & 0.22 & 14.2 & 1.5 & 0.18 & 10.2 & 1.9 & 0.20 & 13.08 \\
\hline ACETONE & 0.3 & 0.03 & 0.2 & 0.1 & 0.02 & 0.1 & 0.4 & 0.05 & 0.2 & 0.3 & 0.03 & 0.14 \\
\hline PROPIONALOEHYDE & 0.6 & 0.06 & 4.2 & 0.3 & 0.03 & 1.7 & 0.8 & 0.10 & 5.4 & 0.5 & 0.05 & 3.22 \\
\hline CROTONALDEHYDE & 0.0 & 0.00 & 0.0 & 0.0 & 0.00 & 0.0 & 0.0 & 0.00 & 0.0 & 0.0 & 0.00 & 0.00 \\
\hline ISOBUTYRALDEHYDE & 0.0 & 0.00 & 0.0 & 0.0 & 0.00 & 0.0 & 0.0 & 0.00 & 0.0 & 0.0 & 0.00 & 0.00 \\
\hline METHYLETHYL KETONE & 0.0 & 0.00 & 0.0 & 0.0 & 0.00 & 0.0 & 0.0 & 0.00 & 0.0 & 0.0 & 0.00 & 0.00 \\
\hline BENZALDEHYDE & 0.0 & 0.00 & 0.0 & 0.0 & 0.00 & 0.0 & 0.0 & 0.00 & 0.0 & 0.0 & 0.00 & 0.00 \\
\hline HEXANALDEHYDE & 0.0 & 0.00 & 0.0 & 0.0 & 0.00 & 0.0 & 0,0 & 0.00 & 0.0 & 0.0 & 0.00 & 0.00 \\
\hline
\end{tabular}




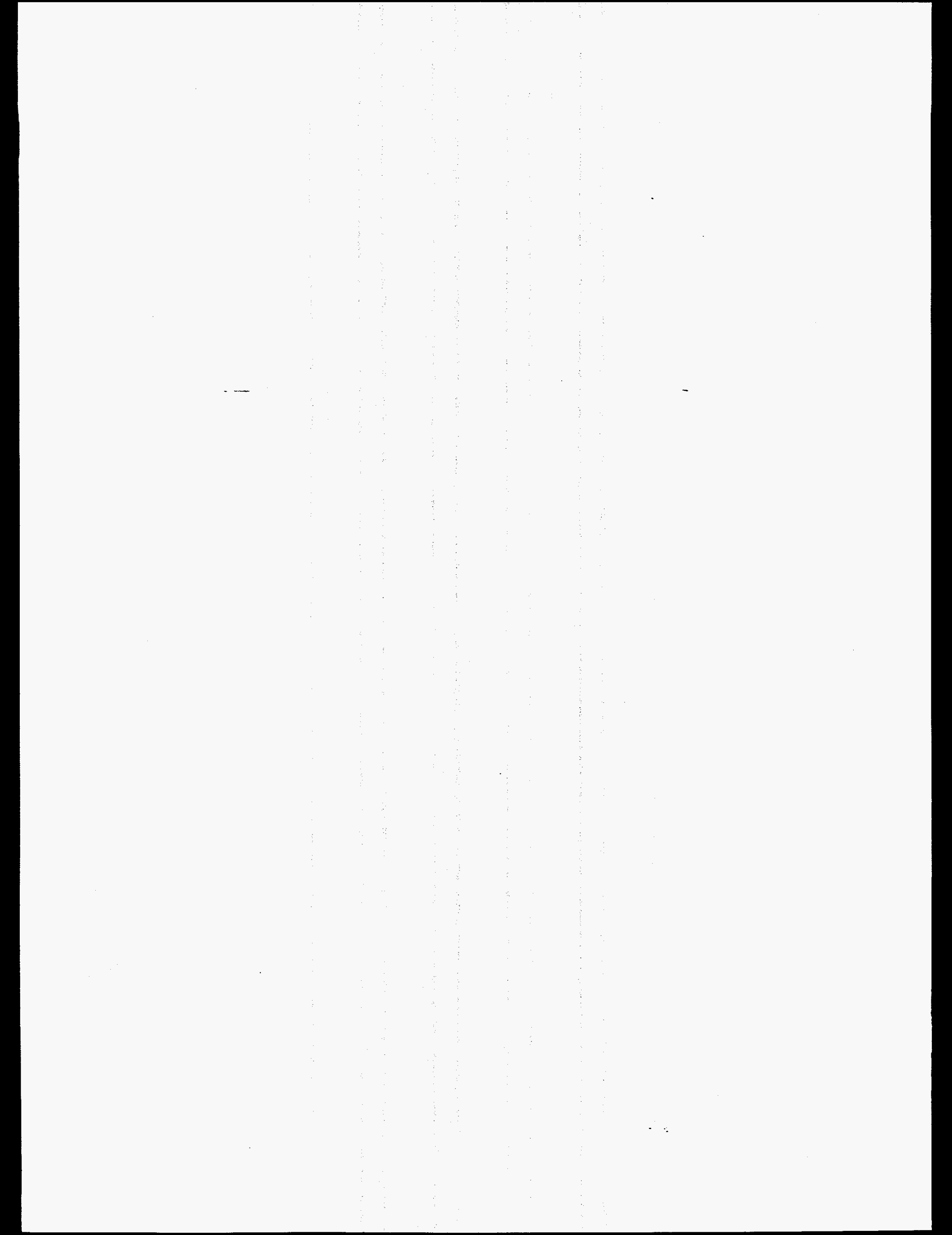




\section{Appendix C}

Detailed Computer Printouts of the FTP Regulated Emission Tests Results from Task 2

\begin{tabular}{|c|c|c|c||}
\hline Test No. & $\begin{array}{c}\text { Soak Time, } \\
\text { hours }\end{array}$ & Vehicle Prep & Page No. \\
\hline \hline TA4-E85-BASE & 0 & Standard & C-1 \\
\hline TA4-E85-18HR & 18 & Extended & C-3 \\
\hline TA4-E85-24HR & 24 & Extended & C-5 \\
\hline TA4-E85-36HR & 36 & Extended & C-7 \\
\hline TA4-E85-36HRC & 36 & Standard & C-9 \\
\hline
\end{tabular}




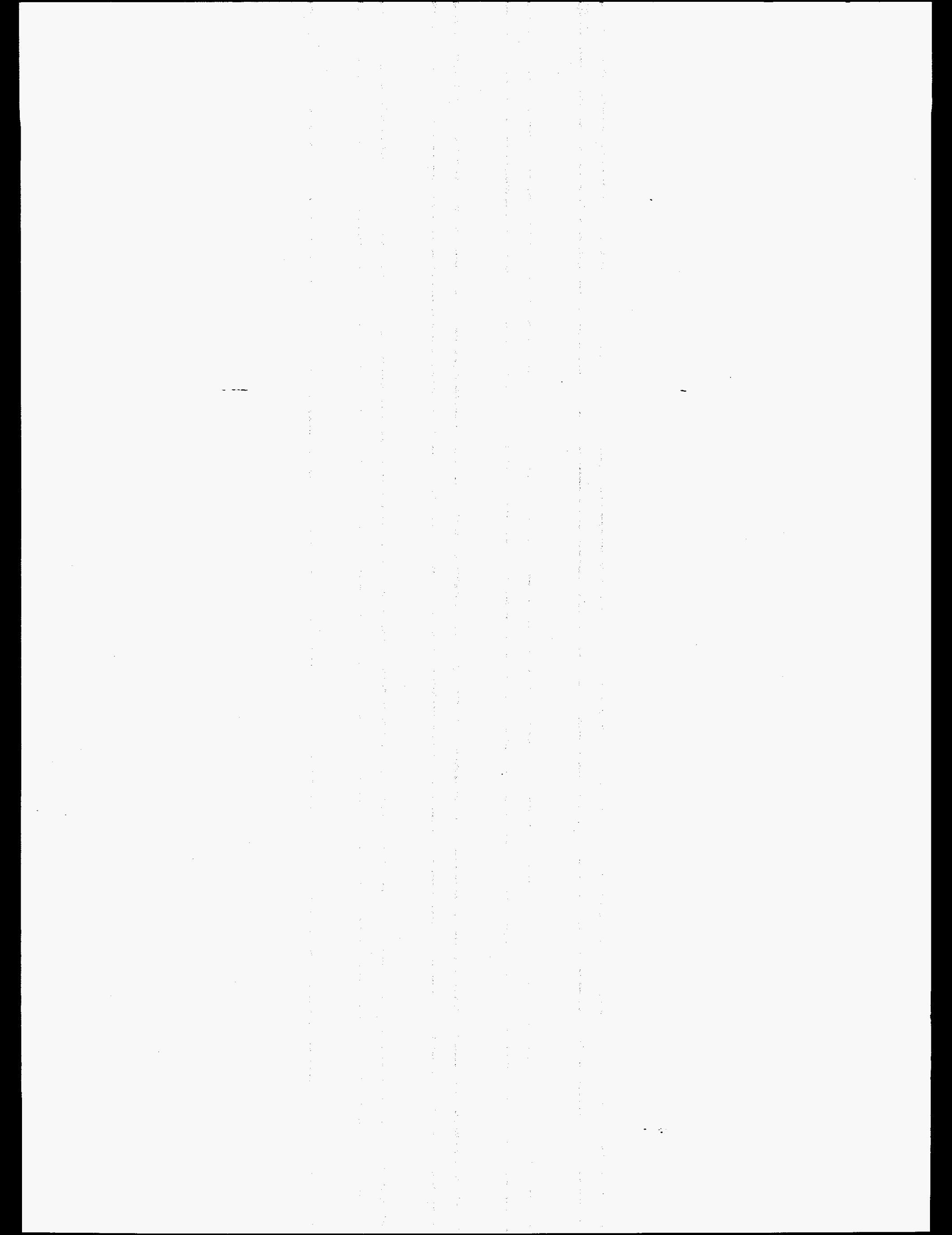


SOUTHWEST RESEARCH IRSPITUTE - DEPARTYENT OF EMISSIONS RESEARCH

COHPUTER PROGRAH LDT 1.5-R

VEHICLE NULBER E85

VEHICLE HODEL 93 FORD TAURUS

ENGINE $3.0 \mathrm{~L}$ (183 CID)- 76

TRAFSUISSION $\quad$ A4

ODOHETER 4671 MILES ( 7515 KM)

BARONETER 29.34 IN HG (745.2 HA BG)

RELATIVE HOYIDITY 35.1 PCT.

BAG NOYBRR
BAG DESCRIPITON

ROI TIME SECONDS

DRY/WET CORRECTION FACIOR, SAIP/BACR

WEASURED DISTAHCE UILES (RM)

BLONER FLON RATE SCFU (SCHH)

GAS METER FLOW RATE SCFY (SCOR)

TOTAL FLOW SCF (SCM)

HC SAMPLE HETER/RANGE/PPH (BAG)

BC BCKGRD HETER/RANGE/PPH

CO SAMPLE METER/RANGE/PPH

CO BCKGRD HETER/RANGE/PPH

CO2 SAMPLE METER/RANGE/PCT

CO2 BCKGRD HETER/RANGE/PCT

NOX SAHPLE METER/RANGE/PPN (BAG) (D)

HOX BCKGRD HETER/RANGE/PPH

CH4 SAMPLE PPU (1.150)

CH4 BCKGRD PPH

DILOTION FACTOR

HC CONCEATRRATTON PPM

CO CONCEATRATION PPM

$\mathrm{CO} 2$ CONCENTRATION PCT

HOX CONCERIRATTON PPI

CH4 CONCENTRATION PPH

MNHC CONCENTRATTON PPH

THC HASS GRAHS

CO MASS GRAHS

$\mathrm{CO} 2$ HASS GRAMS

MOX MRSS GRAHS

CH4 MASS GRAHS

MBC HASS GRAMS (FID)

FUER HASS KG

FOEL ECOHOHY UPG (L/100KM)
4-BAG CARB FTP VEHICLE EUISSION RESOLTS

TEST TA4-E85-BASE

DATE 11/16/95 RON

DYHO 2 BAG CART 2

ACTOAL ROAD LOAD $6.80 \mathrm{EP}(5.07 \mathrm{KW})$

TEST WEIGET 3500 LBS ( $1587 \mathrm{kG}$ )

PROIECT NO. 08-6068-200

DRY BULB TEMPRRATURE $72.0^{\circ} \mathrm{F}\left(22.2^{\circ} \mathrm{C}\right)$

NOX HUIDITY C.F. $\quad .865$

BTHANOL $\quad 85 \% \quad$ EH $-2154-\mathrm{P}$

FUEL DERSITY $6.480 \mathrm{LB} / \mathrm{GAL}$

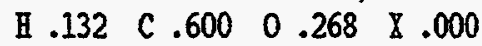

\begin{tabular}{|c|c|c|c|}
\hline $1 \mathrm{~A}$ & IB & 2 & 3 \\
\hline COLD TRANSTEITT & COLD TRAHSIEHT & STABILIZED & HOT TRANSIEAT \\
\hline 0-140 SEC.) & $(140-505$ SBC. $)$ & (505-1372 SEC.) & $(0-505$ SEC. $)$ \\
\hline 139.8 & 365.6 & 866.7 & 506.0 \\
\hline .981/.990 & $.976 / .990$ & $.981 / .990$ & $.979 / .990$ \\
\hline $.68(1.09)$ & $2.96(4.76)$ & $3.84(6.18)$ & $3.64\langle 5.86\}$ \\
\hline $322.7(9.14)$ & $322.5(9.13)$ & $326.8(9.25)$ & $325.6(9.22)$ \\
\hline $.28(.01)$ & $.29(.01)$ & $.28(.01)$ & $.28(.01)$ \\
\hline 752. ( 21.3) & 1967. ( & 4724. (133.8) & 2748. ( 77.8) \\
\hline
\end{tabular}

$22.9 / \quad 3 / 228.49 \quad 34.1 / 2 / 34.08$

$\begin{array}{llllll}.8 / & 3 / & 7.98 & 7.6 / & 2 / & 7.60\end{array}$

$\begin{array}{lll}10.8 / 2 / 2 & 10.79\end{array}$

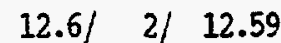

$88.8 / \quad 1 / 914.16 \quad 59.4 / 12 / 58.54$

$7.3 / 2 / \quad 7.30$

$7.1 / 2 / \quad 7.10$

$\begin{array}{llllll}1.5 / & 1 / & 10.57 & 1.5 / & 12 / & 1.45\end{array}$

$83.9 / \quad 14 / \quad .7306 \quad 97.9 / 14 / 1.0918$

$10.0 / 12 / \quad 9.66$

$12.3 / 12 / 11.89$

$1.2 / 12 / \quad 1.16$

1.1/ $12 / 1.06$

$11.4 / 14 / .0362$

13.5/ $14 / \quad .0441$

$81.9 / 14 / \quad .6909$

$91.7 / \quad 14 / \quad .9104$

$33.9 / 2 / 34.02$

$10.3 /$ i) 2.66

$13.3 / \quad 14 / \quad .0433$

13.4/ $14 / \quad .0437$

$.5 / \quad 2 / \quad .50$

15.32

2.18

1.1) $1 / \quad .29$

$3.8 / \quad 1 / \quad .99$

$5.7 /$ 1/ 1.48

$\begin{array}{lll}.8 / \quad 1 / & .21\end{array}$

4.87

2.12

.7) $1 / \quad .18$

6.29

2.12

15.09

11.50

221.04

878.62

27.15

55.09

.6968

33.55

13.29

114.38

1.0516

2.40

8.37

13.04

4.479

21.798

271.87

1.183

.189

1.406

.144

13.83 (17.01)

18.26

3.90

13.86

6.01

8.31

10.53

.6500

.8699

1.31

4.31

2.86

.60

1.06

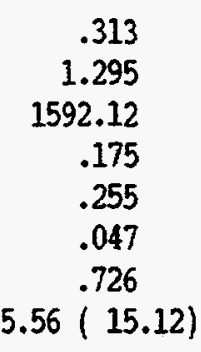

.290

.954

1239.59

.169

.223

.047

.565

$17.67(13.31)$

$15.56(15.12)$

$18.94(12.42)$

4-BAG COMPOSITE RESULTS

$\begin{array}{llr}\text { THC } & \text { G/MI } & .393 \\ \mathrm{CO} & \text { G/MI } & 1.706 \\ \mathrm{HOX} & \text { G/MI } & .117\end{array}$

FUEL BCONONY UPG (L/100RM) 16.65 (14.13)

$\begin{array}{lrl}\text { CH4 } & \text { G/MI } & .080 \\ \text { INHC } & \text { G/MI } & .115 \\ \text { CARBONIL G/MI } & .045 \\ \text { ALCOBOL } & \text { G/MI } & .153 \\ \text { IMNOG G/MI. } & .314 \quad(\text { RAF }=1.00)\end{array}$


COIPUTER PROGRAM LDT 1.5-R

VEHICLE NOUBER E85

VEHICLE HODEL 93 FORD TAUROS

ENGINE 3.0 L $(183 \mathrm{CID})-\nabla 6$

TRAYSUISSTON A4

ODOUETER

SOUTHWEST RESEARCH IISTTTUTE - DEPARTMETT OF EMISSIONS RESEARCH

4-BAG CARB FTP VEHICLE EIISSIOH RESULTS

PROJECT 10. 08-6068-200

TEST TA4-E85-BASE

DATE $11 / 16 / 95$ RON

DHO 2 BAG CART 2

ACTUAL ROAD LOAD $6.80 \mathrm{HP}(5.07 \mathrm{KW})$

TEST WEIGHT' 3500 LBS ( 1587 RG)

BAPONETER 29.34 IN HG (745.2 HM HG) DRY BULB TEMPERATURE $72.0^{\circ} \mathrm{F}\left(22.2^{\circ} \mathrm{C}\right)$ RELATTVE HUYIDITY 35.1 PCT.

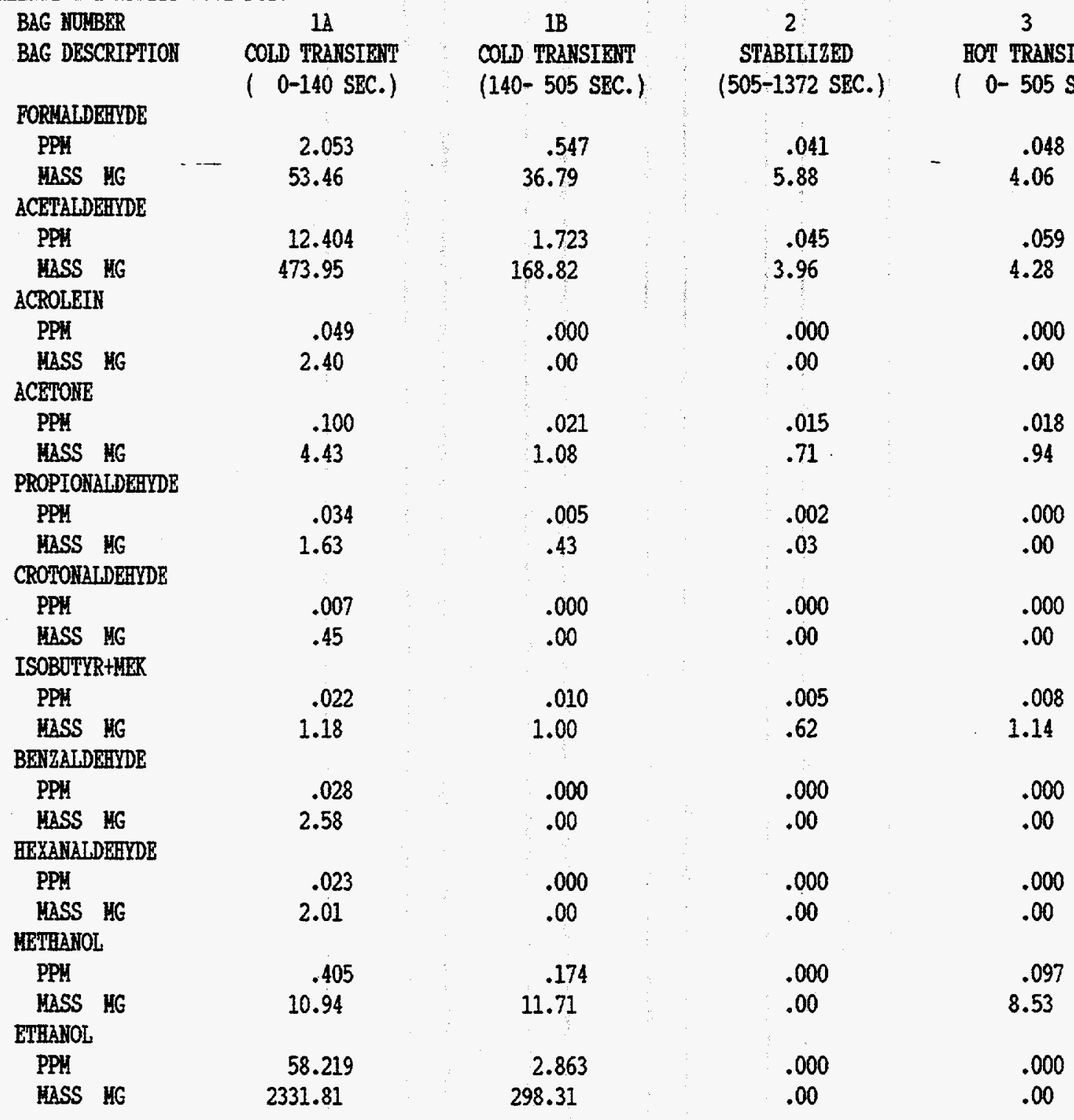

ETHANOL $85 \% \quad$ EM-2154-F

FOEL DEISITY $6.480 \mathrm{LB} /$ GAL

В $.132 \quad$ C $.600 \quad 0.268 \quad \& .000$

MOK HOMIDITY C.F. .865

3

.048

.06

.059

28

.000

.00

.018

.94

.000

(1)

.000

.008

1.14

.000

.00

.000

.097

8.53

.006

.030

.000

.013

.002

.000

.004

.000

.000

.013

.000

.00

4-BAG CONPOSITE RESULTS

$\begin{array}{llr}\text { FORUALDEHYDE } & \text { HG/MI } & 6.288 \\ \text { ACETALDEHYDE } & \text { HG/MI } & 37.835 \\ \text { ACROLEIH } & \text { HG/MI } & .138 \\ \text { ACETOHE } & \text { HG/MI } & .484 \\ \text { PROPIONALD. } & \text { HG/MI } & .122\end{array}$

CROTOIRLD. MG/MI

.026

ISOBUTYR+HER HG/HI

.295

BENZALDBHYDE HG/MI

.148

HEXATALLDEHYDE MG/MI

.116

HETHANOL HG/MI

1.953

ETHANOL MG/MI

151.315 
$\begin{array}{lcl}\text { SOUTHWEST RESEARCH INSTITUTE - DEPARTMENT OF EMTSSTONS RESEARCH } \\ \text { COHPOTER PROGRAM LDT 1.5-R } & \text { 4-BAG CARB FTP VEHICLE EMISSION RESULTS PROJECT N0. 08-6068-200 }\end{array}$
VEHICLE NUIBER E85

VEHICLE HODEL 93 FORD TIOROS

ENGTHE $3.0 \mathrm{~L}$ (183 CID)- 76

TRANSHISSION 24

ODONETER $\quad 4711$ MILES ( $7579 \mathrm{KM})$
TEST TA4-E85-18RR

DATE $11 / 17 / 95$ RON

DYNO 2 BAG CART 2

ACTUAL ROAD LOAD $6.80 \mathrm{HP}$ ( $5.07 \mathrm{KW})$

TEST WEIGHT 3500 LBS ( $1587 \mathrm{KG}$ )
BAROUETER 29.18 IN HG (741.2 HM BG) DRY BULB TEMPERATURE $73.0^{\circ} \mathrm{F}\left(22.8^{\circ} \mathrm{C}\right)$ RELATIVE HUHIDITY 53.8 PCT.

BAG IUURER

BAG DESCRIPTION

RON TINE SBCONDS

DRY/WET CORRECTION FACTOR, SAYP/BACK

MEASURED DISTANCE MILBS (RM)

BLOWER FLON RRTE SCFM (SCMH)

GAS YETTER FLOT RATE SCFH (SCYM)

TOTAL FLON SCF (SCM)

HC SAIPLE HETER/RATGE/PPN (BAG)

HC BCKGRD HETER/RANGE/PPH

CO SAMPLE HETER/RAMGE/PPH

CO BCRGRD METER/RANGE/PPH

CO2 SAMPLE UETER/RAFGE/PCT

CO2 BCKGRD METER/RANGR/PCT

HOX SAYPLE BETER/RAHGE/PPM (BAG) (D)

MOX BCRGRD METER/RANGE/PPH

CH4 SAPPLE PPH (1.160)

CH4 BCKGRD PPM

DILOTION FACTOR

HC CONCENIRATION PPH

CO CONCERPRATION PPH

$\mathrm{CO} 2$ CONCENTRATION PCT

HOX CONCEMTRATION PPH

CH4 CONCENTRATION PPH

HMHC CONCEYTRATION PPH

$\begin{array}{ll}\text { THC } & \text { MASS GRAHS } \\ \text { CO } & \text { HASS GRAHS } \\ \text { CO2 } & \text { MASS GRAHS } \\ \text { HOX } & \text { HASS GRAMS } \\ \text { CH4 } & \text { HASS GRAHS } \\ \text { MHHC } & \text { HASS GRAHS (PID) } \\ \text { FORL HASS KG } \\ \text { FOEL ECOHOHY MPG (L/10ORM) }\end{array}$

THC MASS GRAMS

CO MASS GRAHS

$\mathrm{CO} 2$ MASS GRAYS

NOX MASS GRAMS

CH4 MASS GRAMS

MHHC HASS GRAMS (PID)

FURL ECONOHY MPG (L/10ORM)

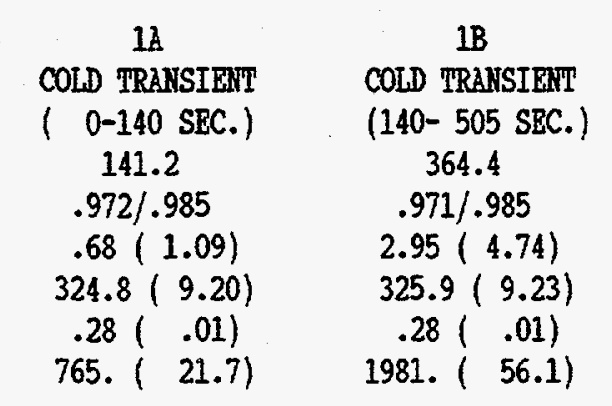

$20.6 / 2 / \quad 20.59$

$4.5 / \quad 2 / \quad 4.50$

$\begin{array}{llll}9.7 / & 12 / & 9.37\end{array}$

$\begin{array}{llll}.8 / & 12 / & .77\end{array}$

$93.3 / \quad 14 / \quad .9534$

$12.8 / 14 / .0414$

$63.6 / 1 / 16.09$

$.3 / 1 / \quad .08$

14.47

2.26

13.23

16.43

8.30

.9151

16.02

12.38

2.07

.212

.209

363.04

.640

.179

.026

.166

$12.01(19.58)$
$92.0 / \quad 1 / \quad 9.26$

$42.6 / \quad 1 / 4.29$

$12.2 / 12 / 11.79$

$.7 / \quad 12 / \quad .68$

$58.8 / 1 / 1.0816$

$2.6 / 1 / \quad .0482$

$.5 / 1 / \quad .13$

$.41 / 10$

5.39

11.68

5.34

10.68

1.0375

.04

3.37

1.43

.182

.698

1065.53

.004

.126

.046

.485

$17.84(13.19)$
2.21
GASOLIME $85 \%$ D-2154-F

POBL DEHSITY $6.480 \mathrm{LB} / \mathrm{GAL}$

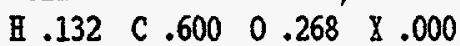

IOX HUIIDITY C.F. $\quad .964$

$\begin{array}{cc}2 & 3 \\ \text { STABILIZED } & \text { HOT TRAMSIEMT } \\ (505-1372 \text { SEC. }) & (0-505 \text { SEC. }) \\ 867.0 & 505.1 \\ .976 / .985 & .973 / .985 \\ 3.84(6.18) & 3.63(5.85) \\ 326.2(9.24) & 323.7(9.17) \\ .28(.01) & .28(.01) \\ 4717 .(133.6) & 2727 .(77.2)\end{array}$

$\begin{array}{rrrrrr}51.2 / & 1 / & 5.15 & 55.0 / & 1 / & 5.54 \\ 43.5 / & 1 / & 4.38 & 43.5 / & 1 / & 4.38 \\ 3.4 / & 12 / & 3.28 & 4.5 / & 12 / & 4.34 \\ .8 / & 12 / & .77 & 1.2 / & 12 / & 1.16 \\ 81.4 / & 14 / & .6813 & 91.5 / & 14 / & .9052 \\ 13.1 / & 14 / & .0425 & 13.3 / & 14 / & .0433 \\ .3 / & 1 / & .08 & 2.5 / & 1 / & .65 \\ .3 / & 1 / & .08 & .3 / & 1 / & .08 \\ 2.95 & & & 3.15 & \\ 2.25 & & & 2.25 & \end{array}$

13.96

1.47

3.12

.8651

.58

1.06

.25

.072

.281

1223.13

.082

.054

.011

.557

$15.81(14.88)$

$19.18(12.27)$

4-BAG CONPOSITE RESULTS

$\begin{array}{lll}\text { THC } & \text { G/MI } & .040 \\ \text { CO } & \text { G/MI } & .125 \\ \text { HOX } & \text { G/MI } & .044\end{array}$

FUEL ECONOHI MPG (L/100RM) $16.76(14.03)$
CH4

MAEC

G/MI

G/MI

CARBOINYL G/MI

ALCOHOL G/MI

IBHOG

G/MI .
.032
.006
.003
.000
$.009 \quad(R A P=1.00\}$ 
SOUTHWEST RESEARCH INSPTTOIR - DEPARTHETY OF EMISSIOHS RESEARCH

VEHICLE NUNBER B85

VEHICLE HODEL 93 FORD TAUROS

ENGTNE

TRANSHISSIOI

ODOHEIER

$3.0 \mathrm{~L}(183 \mathrm{CID})-\nabla 6$

A4

4711 MILES ( $7579 \mathrm{KH})$
TEST TA4-E85-18ER

DATE $11 / 17 / 95$ ROR

DHY 2 BAG CART 2

ACIUAL ROAD LOAD 6.80 HP ( 5.07 KTI)

TEST WEIGHI 3500 LBS ( 1587 RG)
BAROHETER 29.18 IN HG (741.2 HM HG) RBLATIVE HUHIDTTY 53.8 PCT.

BAG NOIBER

BAG DESCRIPIION

COLD TRAHSIEITT

FORULLDEHYDE

PPH $\quad .192$

HASS HG

ACETALDEHYDE

PPH

HASS HG

BCROLEIX

PPI

HASS HG

ACEIONE

PPH

MASS HG

PROPIONALDEHYDE

PPH

HASS HG

CROTONALDEHYDE

PPH

HASS HG

ISOBUTYR+LER

PPH

HASS HG

BEHZALDERYDE

PPH

HASS HG

HEXANATDERYDE

PPI

MASS HG

METHAMOL

PPH

HASS HG

ETHAYOL

PPH

HASS HG
5.05

.020

.55

.000

.00

.023

.97

.000

.00

.000

.00

.000

.00

.000

.00

.000

.00

.000

.00

.040

.81

DRY BULB TEAPERATURE $73.0^{\circ} \mathrm{P}\left(22.8^{\circ} \mathrm{C}\right)$

$1 B$

COLD TRANSIEHT

(140- 505 SEC.)

.088

6.01

.012

.62

.000

.00

.014

1.33

.000

.00

.000

.00

.000

.02

.000

.00

.000

.00

.000

.00

.034

1.47
2

STABILIZED

(505-1372 SEC.)
GASOLIIE $85 \%$

EHI-2154-F

FUEL DERSITY $6.480 \mathrm{LB} / \mathrm{GAL}$

H $.132 \quad$ C . .600 $0.268 \quad \& .000$

HOX HUIIDITY C.P. .964

3

HOT TRARSIERT

( 0- 505 SEC.)

.059

5.58

.056

9.08

.009

.48

.000

.00

.005

.36

.003

.38

.000

.00

.000

.00

.000

.00

.000

.00

.000

.00

.014

.00
BACRGRODD

.000

.006

.000

.004

.001

.000

.000

.000

.000

.021
.021

4-BAG COIPOSITE RESOLTS

FORHALDERYDE HG/MI

2.279

.159

.000

ACROLETN

HG/MI

ACETONE

HG/MI

PROPIONAID. HG/MI

CROTONALD. HG/MI

ISOBUTYR+1IER HG/MI

BEIZALDEHYDE HG/MI

HEXANLLDEHYDE HG/MI

.000

.058

HETHAHOL HG/MI

.120

ETHANOL : HG/MI

.169 
SOUTHWEST RESEARCH INSTITUTE - DEPARTHENT OF EUISSTONS RESEARCH

COHPUTER PROGRAH LDT 1.5-R 4-BAG CARB FTP VEHICLE BHISSION RESULTS PROJECI NO. 08-6068-200

\begin{tabular}{|c|c|c|}
\hline VERICLE NOEBER & E85 & TEST TA4-E85-24:RR \\
\hline VEHICLE HODEL & 93 FORD TAUROS & DATE $11 / 20 / 95$ \\
\hline ENGINR & $3.0 \mathrm{~L}(183 \mathrm{CID})-\nabla 6$ & BAG CART 2 \\
\hline TRAIISHISS] & & ACTUAL ROAD LOAD $6.80 \mathrm{H}$ \\
\hline ODOHETER & 4767 KIIES ( $7670 \mathrm{KM})$ & TEST WEIGHT 3500 IBS \\
\hline
\end{tabular}

BARONETER 29.35 IN EG (745.5 WW HG) DRY BOLB TEMPERATURR $74.0^{\circ} \mathrm{F}\left(23.3^{\circ} \mathrm{C}\right)$ RELATTVE BUHIDTTY 50.7 PCT.

BAG NOHBRR
BAG DESCRIPIION

RON TIME SBCONDS

DRY/WET CORRECTION FACTOR, SAYP/BACR

HEASURED DISTANCE MILES (RM)

BLONER FLOW RATE SCFH (SCHA)

GAS HETER FLOW RATE SCFH (SCHA)

TOTAL FLOW SCF (SCH)

HC SAMPLE METER/RANGE/PPH (BAG)

HC BCKGRD HETER/RAYGE/PPH

CO SAMPLE HETER/RAHGE/PPH

CO BCKGRD METER/RANGE/PPH

CO2 SAMPLE METER/RANGE/PCT

CO2 BCRGRD HETER/RANGE/PCT

NOX SAMPLE METER/RAMGE/PPN (BAG) (D)

NOX BCRERD METER/RANGE/PPY

CH4 SAMPLE PPM (1.160)

CH4 BCRGRD PPH

DILUTTON FACTOR

HC CONCEITRATION PPH

CO CONCEITRATION PPH

CO2 CONCENTRATTON PCT

HOX CONCENTRATION PPH

CH4 CONCENTRRATION PPH

WHEC CONCENTRATTON PPH

THC HASS GRAHS

CO HASS GRAKS

$\mathrm{CO} 2$ MASS GRAMS

NOX HASS GRAMS

CH4 MASS GRAMS

MAHC MASS GRAHS (FID)

FUEL HASS RG

FUEL ECONOHY UPG (L/100RN)

$\begin{array}{ccc}1 A & \text { IB } & 2 \\ \text { COLD TRANSIRRT } & \text { COLD TRAFSIENT } & \text { STABILIZED } \\ (0-140 \text { SEC. }) & (140-505 \text { SEC. }) & (505-1372 \text { SEC. }) \\ 140.0 & 365.0 & 867.4 \\ .972 / .985 & .971 / .985 & .976 / .985 \\ .68(1.09) & 2.96(4.75) & 3.86(6.22) \\ 321.6(9.11) & 321.6(9.11) & 324.9(9.20) \\ .28(.01) & .29(.01) & .28(.01) \\ 751 .(21.3) & 1958 .(55.5) & 4701 .(133.1)\end{array}$

$24.5 / 2 / 24.49$

$6.4 / 2 / \quad 6.40$

$27.2 / \quad 12 / \quad 26.41$

2.2) $12 / 2.12$

$54.4 / \quad 1 / \quad .9992$

$2.8 / 1 / \quad 0519$

$63.7 /$ 1/ 16.12

$.611 / \quad .16$

14.83

2.43

12.60

18.60

23.44

.9514

15.97

12.59

4.02

.233

.581

370.48

.620

.179

.049

.169

$11.74(20.04)$

$9.9 / 2 / \quad 9.89$

$6.01 \quad 2 / \quad 6.00$

$6.5 / 12 / 6.28$

1.4/ $12 / 1.35$

$60.1 / 1 / 1.1060$

2.7) $1 / \quad .0500$

$.7 / 1 / \quad .18$

$.5) \quad 1 / \quad .13$

5.10

2.36

11.42

4.42

4.80

1.0603

.06

2.95

.99

.148

.310

1076.61

.006

.109

.032

.490

$17.72(13.28)$

$15.76(14.92)$
BTHAHOL $85 \% \quad$ BH-2154-F

FULL DENSTTY $6.480 \mathrm{LB} / \mathrm{GAL}$

स $.132 \quad$ C $.600 \quad 0 \quad .268 \quad$ X .000
HOX BOMIDITY C.F. .954

$\begin{array}{rrr}58.0 / & 1 / & 5.84 \\ 56.7 / & 1 / & 5.71 \\ 4.0 / & 12 / & 3.86 \\ 1.3 / & 12 / & 1.25 \\ 81.9 / & 14 / & .6909 \\ 13.5 / & 14 / & .0441 \\ .4 / & 1 / & .10 \\ .4 / & 1 / & .10 \\ 2.57 & \\ 2.35 & \end{array}$

$69.4 / \quad 1 / 6.99$

$55.7 / \quad 1 / 5.61$

$4.4 / 12 / 4.25$

$1.3 / 12 / 1.25$

$91.6 / 14 / .9078$

$13.6 / 14 / \quad .0444$

$3.1 / 1 / \quad .81$

$.4 / \quad 1 / \quad .10$

3.57

2.28

13.92

1.78

2.94

.8666

.71

1.45

.08

.087

.265

1228.13

.100

.075

.003

.559

.720

$19.04(12.36)$

4-BAG COIPOSITE RESULIS

$\begin{array}{lll}\text { THC } & \text { G/MI } & .034 \\ \text { CO } & \text { G/MI } & .124 \\ \text { HOY } & \text { G/NI } & .044\end{array}$

FUEL BCONOHY MPG (L/100KM) 16.67 (14.12)

\begin{tabular}{|c|c|c|}
\hline $\mathrm{CH} 4$ & G/HI & .026 \\
\hline NHEC & G/HI & .005 \\
\hline CARBOMLL & G/MI & .002 \\
\hline ALCOHOL & G/MI & .000 \\
\hline NHOG & G/MI & $.008 \quad(\mathrm{RAP}=1.00)$ \\
\hline
\end{tabular}


SOUTHWEST RESEARCH INSTITUTE - DEPARTHETT OF MISSTONS RESEARCH

COAPOTER PROGRAH LDT 1.5-R

4-BAG CARB FTP VEHICLE EIISSION RESULTS

PROJECT 10. 08-6068-200

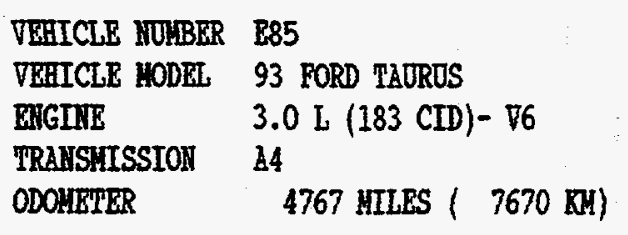

BARONETER 29.35 IN HG (745.5 NM BG) RBLATIVE BUHIDITY 50.7 PCT.

BAG NOHBER

BAG DESCRIPTION

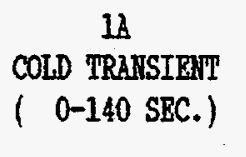

FORNAIDEHYDE

PPM

.157

HASS HG

ACETALDEHYDE

PPH

HBSS HG

ACROLEII

PPH

MASS HG

ACETONE

PPH

HASS HG

PROPIONALDEHYDE

PPV

HASS HG

CROTONALDEHYDE

PPH

UIASS HG

ISOBUTYR+MEK

PPH

MaSS HG

BENZALDEHYDE

PPH

MASS HG

HEZANALDEHYDE

PPH

HASS HG

METHANOL

PPH $\quad .000$

HASS HG $\quad .00$

BTHANOL

PPM

MASS HG
TEST T24-B85-24BR

DATE 11/20/95 ROI

DHO 2 BAG CART 2

ACTOAL ROAD LOAD 6.80 HP ( $5.07 \mathrm{kNN}$ )

TEST WEIGHT 3500 LBS ( 1587 KG)
BTHANOL 85\% BH-2154-F

FOEL DEISSITY $6.480 \mathrm{LB} / \mathrm{GAL}$

H $.132 \quad$ C $.600 \quad 0.268 \quad$ \.000

NOX HOHIDITY C.F. .954

DRY BULB TERPERAIURE $74.0^{\circ} \mathrm{F}\left(23.3^{\circ} \mathrm{C}\right)$

IB

COLD TRANSIENT

(140- 505 SEC.)

.062

3.79

.023

1.58

.003

.42

.007

.67

.000

.00

.000

.00

.002

.22

.000

.00

.000

.00

.000

.00

.025

.85
2

STABILIZED

(505-1372 SBC.)

.050

7.13

.008

.31

.000

.00

.002

.03

.001

.17

.000

.00

.000

.00

.000

.00

.000

.00

.000

.00

.015

.00
3

HOT TRANSIENT

( 0- 505 SEC.)

.052

4.37

.009

.32

.000

.00

.002

.00

.000

.00

.000

.00

.000

.06

.000

.00

.000

.00

.037

1.96

.032

2.08
BACKEROORD

.006

.007

.000

.003

.000

.000

.000

.000

.000

.018

.018

4-BAG COUPOSITE RESULTS

FORMALDEHYDE HG/NI

.024

ACROLEIN HG/MI

.024
.042

ACETONE

HG/MI

.042
.023

CROTONALD. MG/MI

ISOBUTYR+HER HG/MI

.000

BENZALDEHYDE HG/MI

.017

HEXANAL.DEHYDE WG/MI

.000

HETHANOL HG/MI

PROPIONALD. HG/MI

BTHANOL

MG/II 


\section{COHPOTER PROGRAH LDT 1.5-R 4-BAG CARB FTP VEHICLE EHISSTON RESULTS PROJECT HO. 08-6068-200}

VEHICLE NOUBER E85

\begin{tabular}{|c|c|}
\hline VEHICLE HODEI & 93 FORD TAUROS \\
\hline EHGINE & $3.0 \mathrm{~L}(183 \mathrm{CID})-\mathrm{V} 6$ \\
\hline TRAHSHISSION & A4 \\
\hline ODOHETER & $7750 \mathrm{KM})$ \\
\hline
\end{tabular}

BARONETER $29.30 \mathrm{IN}$ HG (744.2 $\mathrm{MH}$ HG) RELATIVE HORIDITY 53.2 PCT,

BAG NOMBER

BAG DESCRIPITON

RON TIHE SECONDS

DRY/WET CORRECTION FACTOR, SAMP/BACK

HEASURED DISTANCE HILES (RA)

BLONER FLON RATE SCFH (SCNM)

GAS NETER FLOW RATE SCFY (SCH)

TOTAL FLON SCF (SCM)

BC SAYPLE UETER/RAYGE/PPH (BAG)

HC BCKGRD HETER/RANGE/PPH

CO SAYPLE HETER/RANGE/PPH

CO BCRGRD HETER/RANGE/PPH

CO2 SAHPLE HETER/RANGE/PCT

CO2 BCRGRD HETER/RANGE/PCT

HOX SAHPLE WETER/RANGE/PPH (BAG) (D)

NOX BCRGRD VETER/RANGE/PPH

CH4 SAMPLE PPH (1.150)

CH4 BCKGRD PPM

DILOTION FACTOR

HC CONCENTRATION PPH

CO CONCEMTRATTON PPM

CO2 CONCENTRATTON PCT

NOX CONCENTRATION PPH

CH4 CONCENTRATTON PPH

WHE CONCENTRATION PPN

THC MASS GRAHS

CO MASS GRAHS

$\mathrm{CO} 2$ MASS GRAMS

HOX MASS GRAMS

CH4 MASS GRAHS

NWHC MASS GRAMS (FID)

FOEL MASS KG

FOEL ECONOHY UPG (L/100RM)
TEST TA4-E85-36BR

DATE $11 / 22 / 95$ RON

DWNO 2 BAG CART 2

ACTUAL ROAD LOAD $6.80 \mathrm{KP}$ ( $5.07 \mathrm{KN}$ )

TEST WEIGHT 3500 LBS ( 1587 KG)
BTHANOL $85 \% \quad$ BN-2154-F

FUEL DEISITY $6.480 \mathrm{LB} / \mathrm{GAL}$

$\begin{array}{lllllllll}\text { H } & .132 & \mathrm{C} & .600 & 0 & .268 & \mathrm{X} & .000\end{array}$
HOX HOMTDITY C.F. .949

DRY BULB PEYPERATURE $72.0^{\circ} \mathrm{F}\left(22.2^{\circ} \mathrm{C}\right)$

$$
\begin{array}{cc}
1 \mathrm{~A} & \text { IB } \\
\text { COLD TRANSIERT } & \text { COLD TRAISIENT } \\
(0-140 \text { SEC. }) & (140-505 \text { SEC. }) \\
139.8 & 365.6 \\
.973 / .986 & .971 / .986 \\
.67(1.08) & 2.94(4.74) \\
321.3(9.10) & 325.3(9.21) \\
.28(.01) & .29(.01) \\
749 .(21.2) & 1984 .(56.2)
\end{array}
$$

$\begin{array}{rrrrrr}55.5 / / & 2 / & 55.47 & 17.0 / & 2 / & 16.99 \\ 5.7 / & 2 / & 5.70 & 5.5 / & 2 / & 5.50 \\ 51.8 / & 14 / & 231.27 & 24.4 / & 12 / & 23.67 \\ .5 / & 14 / & 2.02 & 1.5 / & 12 / & 1.45 \\ 93.8 / & 14 / & .9673 & 97.7 / & 14 / & 1.0852 \\ 13.9 / & 14 / & .0456 & 14.1 / & 14 / & .0464 \\ 64.5 / & 1 / & 16.32 & 2.4 / & 1 / & .63 \\ 1.1 / & 1 / & .29 & .9 / & 1 / & .24 \\ & 15.88 & & & 6.81 & \\ & 2.71 & & & 2.66 & \end{array}$

12.72

50.22

220.29

.9253

16.05

13.37

29.88

.813

5.442

359.48

.618

.189

.366

.168

$11.74(20.03)$
$17.0 / 2 / \quad 16.99$

$5.5 / 2 / \quad 5.50$

$24.4 / \quad 12 / 23.67$

1.5/ $12 / 1.45$

97.7) $14 / 1.0852$

14.1/ $14 / \quad .0464$

2.4) $1 / \quad .63$

2.66

11.62

11.97

21.37

1.0428

.41

4.37

6.33

.468

1.398

1072.76

.042

.164

.205

.490

17.67 (13.31)
2

STABILIZED

(505-1372 SEC.)

866.9

$.977 / .986$

$3.84(6.17)$

$326.3(9.24)$

$.28(.01)$

4718. ( 133.6)

$52.0 / 1 / 5.23$

$49.9 /$ 1/ 5.02

$3.6 / 12 / 3.47$

1.3/ $12 / 1.25$

81.7/ 14/ .6871

14.2/ $14 / \quad .0468$

1.4/ $1 / \quad .37$

$.8 / \quad 1 / \quad .21$

2.63

2.59

18.39

.48

2.19

.6428

.17

.18

.31

.051

.341

1572.50

.041

.016

.024

.716

$15.75(14.93)$
3

BOT TRARSIERT

( 0 - 505 SEC.) 505.4

$.974 / .986$

$3.63(5.85)$

$325.6(9.22)$

$.28(.01)$

2745. ( 77.7)

$64.6 / 1 / \quad 6.50$

$47.8 /$ 1/ 4.81

$5.0 / \quad 12 / 4.83$

$1.3 / 12 / 1.25$

$91.0 / 14 / .8924$

14.1/ 14/ .0464

$2.8 / \quad 1 / \quad .73$

$.8 / \quad 1 / \quad .21$

3.85

2.46

14.16

2.03

3.50

.8493

.54

1.56

.28

.097

.317

1208.59

.076

.081

.012

.550

$19.42(12.12)$

4-BAG COAPOSITE RESULTS

$\begin{array}{ccc}\text { THC } & \text { G/MI } & .088 \\ \text { CO } & \text { G/MI } & .465 \\ \text { HOX } & \text { G/MI } & .049\end{array}$

FUEL ECONONY APG (L/100KN) 16.74 (14.05)

$\begin{array}{lrl}\text { CH4 } & \text { G/MI } & .029 \\ \text { NMEC } & \text { G/MI } & .037 \\ \text { CARBOML G/MI } & .013 \\ \text { ALCOHOL G/MI } & .010 \\ \text { NMOG G/MI . } & .059 \quad \text { (RAP }=1.00)\end{array}$


SOUTHWEST RESEARCH INSTITUTE - DEPARTHERT OF EMISSIOHS RESEARCH

CONPUTER PROGRAM LDT 1.5-R 4-BAG CARB FTP VEHICLE EMISSION RESULTS

PROJECT NO. 08-6068-200

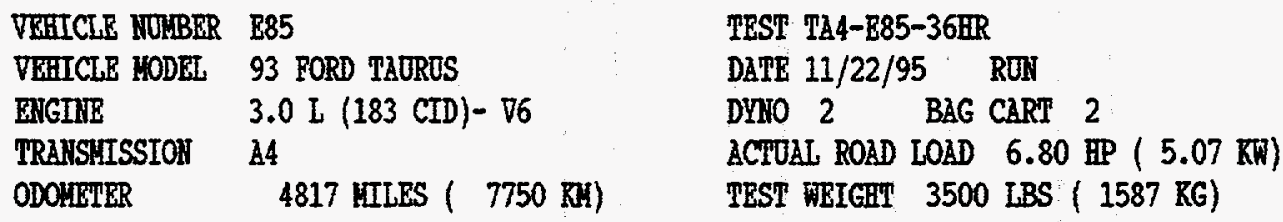

TEST TA4-E85-36HR

DaTE $11 / 22 / 95$ RUN

DYITO 2 BAG CART 2

ACTUAL ROAD LOAD $6.80 \mathrm{HP}(5.07 \mathrm{KW})$

TEST WEIGHT 3500 LBS ( $1587 \mathrm{KG}$ )

125.92

4-BAG CONPOSITE RESULTS
ETHANOL 85\% BH-2154-F

PURL DEISITY $6.480 \mathrm{LB} / \mathrm{GAL}$

$\begin{array}{lllllll}\text { II } .132 & \mathrm{C} .600 & 0 & 0.268 & \mathbb{1} .000\end{array}$

HOX BOHIDITY C.P. .949

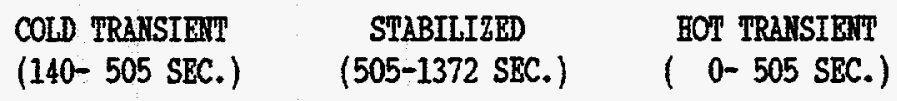

COLD TRATSIEIT'

(140- 505 SEC.)

STABILIZED

(505-1372 SBC.)

HOT TRANSIEHT

( 0- 505 SEC.)

1B

.079

5.27

.536

53.12

.000

.00

.000

.00

.000

.00

.000

.00

.000

.00

.000

.00

.000

.00

.000

.00

.416

40.94
2
3

.030

2.75

.009

.52

.000

.00

.002

.03

.000

.00

.000

.00

.000

.00

.000

.00

.000

.00

.000

.00

.000

.00
BACKGROORD

.001

.005

.000

.002

.000

.000

.000

.000

.000

.014

.027

CROTONALD, HG/MI

ACROLEIN HG/MI

BENZALDEHYDE HG/MI 
COAPOTER PROGRBM LDT 1.5-R

VEBICLE NOKBER E85

VEHICLE HODEL 93 FORD TAURUS

ENGINE $\quad 3.0 \mathrm{~L}(183 \mathrm{CID})-\nabla 6$

TRANSHISSTON 14

ODOHETER 4874 MILES ( 7842 KM)

BARONETER 29.17 IN HG (740.9 WH BG)

RELATIVE HOHIDITY 53.8 PCT.

BAG NOLBER

BAG DESCRIPPION

RON TIME SECONDS

DRY/WET CORRECTIOI FACTOR, SAMP/BICR

UEASURED DISTANCE MILBS (RA)

BLOWER FLOF RATE SCFY (SCHA)

GAS WETER FLOW RaTE SCFK (SCMM)

TOPAL FLON SCF (SCH)

HC SAMPLE UETER/RANGE/PPM (BAG)

HC BCKERD HETER/RANGE/PPH

CO SAMPLE UETER/RANGE/PPH

CO BCRGRD METER/RANGE/PPH

CO2 SAEPLE HETER/RAIGE/PCT

C02 BCKGRD HETER/RANGE/PCT

NOX SAYPLE HETER/RANGE/PPH (BBG) (D)

NOY BCKGRD METER/RANGE/PPH

CH4 SAIPLE PPI (1.150)

CH4 BCRERD PPH

DILUTION FACTOR

HC COHCENTRATION PPM

CO COYCENTRATION PPM

$\mathrm{CO} 2$ CONCENTRRAPION PCT

HOX CONCERTRATION PPH

CB4 CONCERTRATION PPH

MAHC CONCENTRATTON PPH

THC MASS GRAHS

CO HASS GRAVS

$\mathrm{CO} 2$ HASS GRAHS

HOX HRSS GRABS

CH4 HASS GRALS

IIHC HASS GRAHS (FID)

FUEL HASS KG

POEL ECONOHY MPG (L/10OKN)
SOUTHWEST RESEBRCH INSTITUPE - DEPARTYIETI OF EUISSTOHS RESEARCH

4-BAG CARB FTP VEHICLE EHISSTON RESULTS

PROTECT 110. 08-6068-200
TEST TA4-E85-36HRC

DATE $12 / 1 / 95 \quad$ RUN

DNO 2 BAG CART 2

ACTOAL ROAD LOAD $6.80 \mathrm{BP}$ ( $5.07 \mathrm{KW}$ )

TEST WEICHT 3500 LBS ( $1587 \mathrm{KG}$ )
DRY BULB TERPERATURR $73.0^{\circ} \mathrm{F}\left(22.8^{\circ} \mathrm{C}\right)$

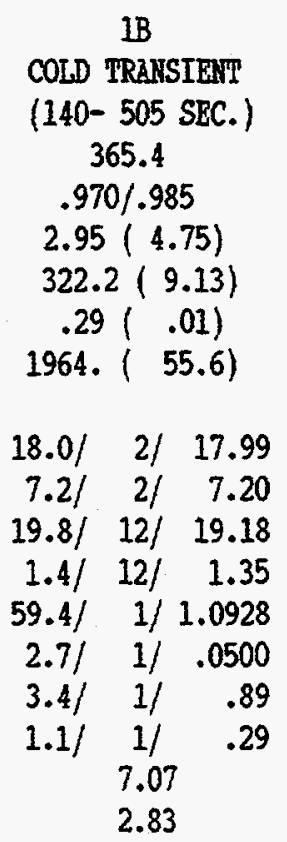

12.62

191.21

881.41

.8562

29.23

14.96

99.80

3.820

21.685

331.28

1.139

.211

1.216

.170

$11.73(20.05)$
11.54

11.42

17.15

1.0471

.62

4.49

5.31

.456

1.110

1066.38

.064

.166

.170

.486

$17.85(13.18)$
ETHANOL $85 \%$ BI-2154-P

FOEL DEHSITY $6.480 \mathrm{LB} / \mathrm{GAL}$

H $.132 \quad$ C $.600 \quad 0.268 \quad \searrow .000$
HOX HOHIDITY C.F. .964

$\begin{array}{cc}2 & 3 \\ \text { STABILIZED } & \text { HOT TRANSIENT } \\ (505-1372 \text { SEC. }) & (0-505 \text { SEC. }) \\ 866.8 & 505.3 \\ .976 / .985 & .973 / .985 \\ 3.85(6.20) & 3.64(5.86) \\ 323.9(9.17) & 322.3(9.13) \\ .28(.01) & .27(.01) \\ 4684 .(132.6) & 2716 .(76.9)\end{array}$

$\begin{array}{rrrrrr}59.0 / & 1 / & 5.94 & 67.9 / & 1 / & 6.84 \\ 58.2 / & 1 / & 5.86 & 55.2 / & 1 / & 5.56 \\ 3.4 / & 12 / & 3.28 & 5.2 / & 12 / & 5.02 \\ 1.1 / & 12 / & 1.06 & 1.2 / & 12 / & 1.16 \\ 81.6 / & 14 / & .6851 & 91.5 / & 14 / & .9052 \\ 14.0 / & 14 / & .0460 & 14.1 / & 14 / & .0464 \\ .9 / & 1 / & .24 & 3.7 / & 1 / & .96 \\ .9 / & 1 / & .24 & .8 / & 1 / & .21 \\ 2.92 & & 4.05 & \\ 2.71 & & & 2.81 & \end{array}$

13.96

1.68

3.77

.8622

.6416

.77

1.44

.35

$-.01$

$-.01$

.049

.080

.338

1558.15

.003

.031

.000

.709

1214.25

.109

.074

.000

.553

$15.97(14.73)$

$19.36(12.15)$

4-BAG COMPOSITE RESULTS

$\begin{array}{llr}\text { THC } & \text { G/MI } & .258 \\ C O & \text { G/MI } & 1.380 \\ \text { HOX } & \text { G/MI } & .078\end{array}$

FOEL ECONOHY MPG (L/100XM) 16.88 (13.94)

$\begin{array}{lrl}\text { CH4 } & \text { G/MI } & .031 \\ \text { MHHC G/MI } & .080 \\ \text { CARBONYL G/MI } & .034 \\ \text { ALCOHOL G/MI } & .113 \quad \\ \text { MHOG G/MI. } & .227 \quad(R A F=1.00)\end{array}$


SOUTHWEST RESEARCH IISTITUTE - DEPARTHENT OF EMISSIONS RESEARCH

COMPUTER PROGRAM LDT $1.5-\mathrm{R}$

4-BAG CARB FIP VEHICLE EMISSION RESULTS

PROJECT $10.08-6068-200$

TEHICLE NTDBER E85

$\begin{array}{ll}\text { VEHICLE HODEL } & 93 \text { FORD TAURUS } \\ \text { ENGIWE } & 3.0 \mathrm{~L} \text { (183 CID)- } 76 \\ \text { TRAHSHISSTON } & \text { A4 } \\ \text { ODOHETER } & 4874 \text { MILES ( } 7842 \mathrm{KM})\end{array}$

BAROMETER 29.17 IN HG (740.9 $\mathrm{WM}$ HG) DRY BULB TEMPERATURE $73.0^{\circ} \mathrm{F}\left(22.8^{\circ} \mathrm{C}\right)$ RELLTIVE HDIIDITY 53.8 PCT.

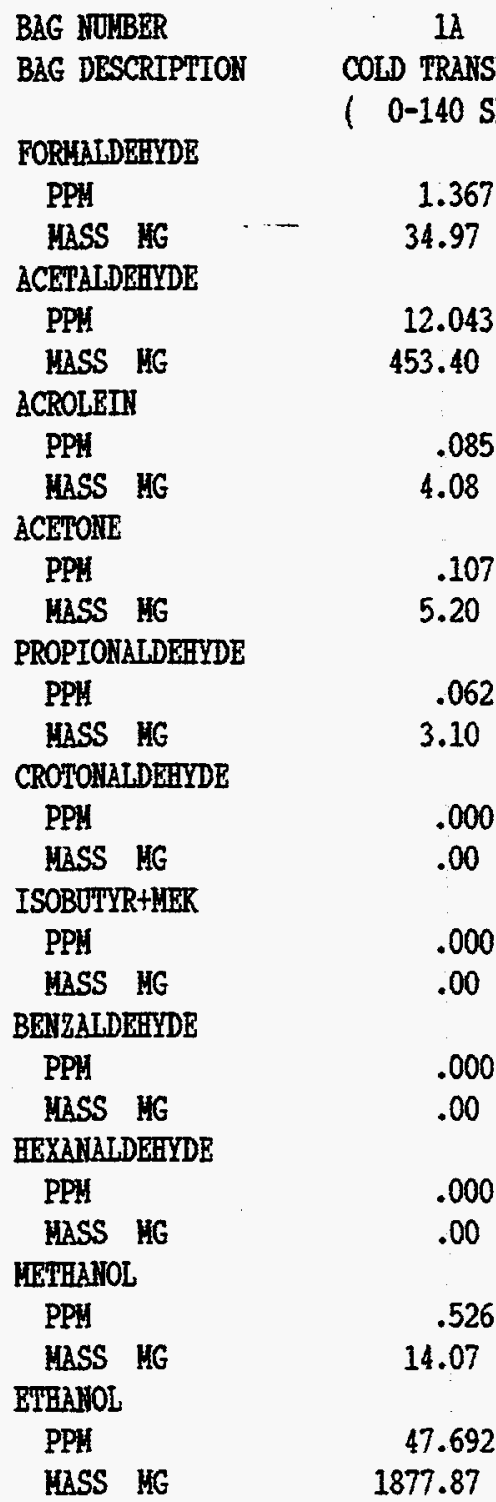

4-BAG CONPOSITE RESULTS

FORMALDEHYDE MG/MI

ACETALDEHYDE HG/MI

ACROLEIN HG/MI

ACETONE MG/MI

PROPTONALD. HG/MI
TEST TA4-585-36RRC

DATE $12 / 1 / 95$ RON

DHFO 2 BAG CART 2

ACTUAE ROAD LOAD $6.80 \mathrm{HP}$ ( $5.07 \mathrm{KW}$ )
TEST WEIGHT 3500 LBS ( 1587 KG)

BIHAMOL $85 \% \quad$ BR-21.54-F

FORL DENSITY $6.480 \mathrm{LB} / \mathrm{GAL}$

H $.132 \quad C \quad .600 \quad 0.268 \quad \searrow .000$

HOX HUHIDITY C.F. .964

3

COLD TRANSIENT : STABILIZED GOT TRAHSIBNT

(140- 505 SEC.) (505-1372 SBC.)

.112

.481

46.86

.000

.00

.006

.48

11.51

.000

.00

.000

.00

.000

.00

.000

.00

.000

.00

.035

1.66

.636

63.09

3.311

28.842

.235

1.863

.178
.011

.52

.000

.00

.000

.00

.000

.00

.000

.00

.000

.00

.000

.00

.000

.00

.023

1.09

.046

2.77
( 0- 505 SEC.)

.028

2.09

.039

5.46

.009

.50

.000

.00

.040

.000

.00

.000

.00

.000

.00

BACRGROUND .006

.007

.000

.003

.000

.000

.000

.000

.000

.013

.032

1.10
CROTONALD. HG/MI

ISOBOTYR+HER HG/MI

BENZALDRFYDE HG/KI

HEXAMALDWHYDE KG/MI

METHANOL HG/MI

BTHAHOL HG/MI
.000

.000

.000

.000

.987

111.852 


\section{Appendix D}

Detailed Computer Printouts of the FTP

Regulated Emission Test Results from Task 4

\begin{tabular}{|c|c|c|}
\hline Fuel & Test No. & Page \\
\hline \hline RFG & FT-RFG-BASE & D-1 \\
\hline RFG & FT-RFG-3A & D-2 \\
\hline M85 & FT-M85-1 & D-4 \\
\hline E85 & FT-E85D-1 & D6 \\
\hline CNG & FT-CNG-1 & D-8 \\
\hline LPG & FT-LPG-1 & D-10 \\
\hline
\end{tabular}



SOUTHIEST RESEARCH INSTIPUTE - DEPRRTHENT OF EMISSIONS RESEARCH

COMPOTER PROGRAH LDT 1.4-R 3-BAG EPA PTP VBHICLE BMISSTON RESULTS

PROJECT HO. $08-6068-400$

VEHICLE NOWBER 90X

VEHICLE HODEL 94 FORD TARUROS

EMGINE $\quad 3.0 \mathrm{~L}(181 \mathrm{CID})-\mathrm{A4}$

TRANSMISSION A4

ODOMETER $\quad 19420$ HILES ( $31246 \mathrm{KH}$ )

BARONETER $29.28 \mathrm{IN} \mathrm{BG}\left(743.7 \mathrm{MH}\right.$ HG) DRY BULB TEMPERATURE $73.0^{\circ} \mathrm{F}\left(22.8^{\circ} \mathrm{C}\right)$ RELATIVE EUHIDIPY 57.6 PCT.

BAG NOMBER

BAG DESCRIPTIOH

RON TIHE SECONDS

DRY/WET CORRECTTON FACTOR, SAMP/BACK

MEASURED DISTLANCE MILES (RM)

BLOWER FLOW RATE SCFY (SCEM)

GAS HETER FLOW RATE SCFH (SCWN)

TOTAL FLOA SCF (SCM)

HC SAHPLE HETER/RAMGE/PPM (BAG)

HC BCKGRD METER/RANGE/PPH

CO SAHPLE HETER/RANGE/PPH

CO BCKERD METER/RANGE/PPM

CO2 SALPLE HETER/RANGE/PCT

CO2 BCKGRD METER/RaRGE/PCT

NOX SAMPLE HETER/RAHGE/PPY (BAG) (D)

NOX BCKGRD METER/RAHGE/PPM

CH4 SAMPLE PPH (1.150)

CH4 BCRGRD PPH

DILUTION PACTOR

HC CONCENTRATIOI PPN

CO CONCEITTRATION PPH

$\mathrm{CO} 2$ COACENPIRATION PCT

HOX CONCENTRATION PPH

CH4 CONCENTRATION PPH

BNHC CONCENTRATION PPH

HC MASS GRAHS

$C O$ MASS GRAHS

$\mathrm{CO} 2$ HASS GRAMS

HOX MASS GRAHS

CH4 MASS GRAMS

MNHC MASS GRAYS (FID)

FUEL MASS KG

FUEL ECOHOHY HPG (L/10OKM)
TEST FT-RFG-BASE

DATE 10/12/95 RUN

DHHO 3 BAG CART 2

ACTUAL ROAD LOAD $6.80 \mathrm{HP}(5.07 \mathrm{~kW})$

TEST HEIGRT 3500 LBS ( 1587 RG)

$$
\begin{gathered}
1 \\
\text { COLD TRANSIENT } \\
(0-505 \text { SEC. }) \\
\vdots 05.2 \\
\therefore: 3 / .984 \\
3.60(5.80) \\
308.9(8.75) \\
.28(.01) \\
2603 .(73.7)
\end{gathered}
$$

$\begin{array}{rrrrrr}46.7 / & 2 / & 46.67 & 62.5 / & 1 / & 6.29 \\ 5.4 / & 2 / & 5.40 & 49.7 / & 1 / & 5.00 \\ 73.8 / & 13 / & 176.83 & 22.3 / & 12 / & 21.61 \\ .5 / & 13 / & 1.10 & .8 / & 12 / & .77 \\ 61.4 / & 1 / & 1.1304 & 85.1 / & 14 / & .7555 \\ 2.8 / & 1 / & .0519 & 13.7 / & 14 / & .0448 \\ 38.7 / & 1 / & 9.70 & 7.5 / & 1 / & 1.88 \\ .8 / & 1 / & .20 & .7 / & 1 / & .18 \\ & 5.11 & & & 2.98 & \\ & 2.29 & & & 2.14 & \end{array}$

11.63

41.74

168.66

1.0830

9.52

3.02

38.27

1.809
14.476
1461.81
1.320
.148
1.627
.479
$21.45(10.97)$

17.67

1.57

20.18

.7132

1.72

.96

.47

.118

2.995

1664.39

.411

.082

.034

.537

$20.37(11.55)$

GASOIINE RFG ERH-2050-F

FOEL DENSITY $6.291 \mathrm{LB} / \mathrm{GAL}$

स $.135 \quad C .849 \quad 0.016 \quad \& \quad 000$

NOX HEMIDITY C.F. .984

3

HOT TRANSIENT

( 0- 505 SEC.)

505.1

$.974 / .984$

$3.59(5.78)$

$310.5(8.79)$

$.27(.01)$

2616. (74.1)

$88.9 /$ I/ 8.95

$46.3 / \quad 1 / 4.66$

$33.2 / \quad 12 / 32.31$

.5/ $12 / \quad .48$

$94.5 / \quad 14 / \quad .9872$

13.5/ 14/ .0441

$16.8 / \mathrm{l} / \mathrm{H} 4.21$

$.5 / \quad 1 / \quad .13$

3.35

2.25

13.51

4.63

30.65

.9464

4.10

1.26

3.18

.202

2.643

1283.66

.571

.062

.136

.414

$24.74(9.51)$

3-BAG COMPOSITE RESULTS

$\begin{array}{llr}\text { HC } & \text { G/MI } & .136 \\ \text { CO } & \text { G/MI } & 1.443 \\ \text { HOY } & \text { G/MI } & .176\end{array}$

FOEI ECONONY HPG (L/10OKM) $21.67(10.85)$
CH4 G/MI $\quad .024$

SWHC G/HI $\quad .109$ 
SOUTHAEST RRSEARCH IISTITUTE - DEPARTHENT OF RHISSIOIS RESEARCH

CONPUTRR PROGRAM IDT 1.5-R 3-BAG EPA FIP VEHICLE EISISSION RESULTS

PROJECT 10. 08-6068-400

VEHTCLE HULBER $90 \mathrm{X}$

VERICLE YODEL 94 FORD TAURUS

BHGIHE $\quad 3.0 \mathrm{~L}(181 \mathrm{CID})-\mathrm{A4}$

TRANSISSSION 24

ODONEIER 19533 YILES ( 31428 KM)

BAROUETER 29.18 IN HG (741.2 IM HG)

RELATIVE HUIIDITY 54.4 PCT.

BAG NOVBER

BAG DESCRIPIION

RON TIHE SECONDS

DRI/WET CORRECTION FACTOR, SAMP/BACK

HERSURED DISTARCE YILES (RM)

BLONER FLON RATE SCFH (SCHN)

GAS IETER FLOH RAYE SCFH (SCHM)

TOTAL FLON SCF (SCH)

HC SAMPLE HETER/RANGE/PPH (BAG)

HC BCRGRD METER/RANGE/PPI

CO SAYPLE METER/RANGE/PPH

CO BCRGRD HETER/RANGE/PPH

CO2 SAYPLE AELER/RAIGE/PCT

CO2 BCKGRD HETER/RANGE/PCT

HOZ SAMPLE HETER/RAHGE/PPH (BAG) (D)

10Y BCKGRD HETER/RAHGE/PPI

CH4 SAMPLE PPH (1.160)

CH4 BCRGRD PPH

DILOTION FACTOR

BC CONCERTRRATIOII PPH

CO CONCWHTRATTOH PPH

$\mathrm{CO} 2$ CONCERTRATION PCT

MOX COHCENTRRTTON PPH

CB4 CONCERTRATIOII PPH

HIHC CONCEHTRATION PPI

HC YASS GRHMS

CO MASS GRAHS

$\mathrm{CO} 2$ HASS GRAMS

HOZ MASS GRAWS

CH4 HASS GRAHS

MHHC MASS GRAMS (FID)

FUEL MASS RG

FOEL ECONONY UPG (L/10OKH)
TEST FT-RFG-3A

DATE 10/30/95 RON

DHF 3 BAG CARP 2

ACTOAL ROAD LOAD $6.80 \mathrm{HP}$ ( $5.07 \mathrm{KM}$ )

TRST WEIEHT 3500 LBS ( 1587 KG)
DRY BULB TEIPERATURE $74.0^{\circ} \mathrm{P}\left(23.3^{\circ} \mathrm{C}\right)$

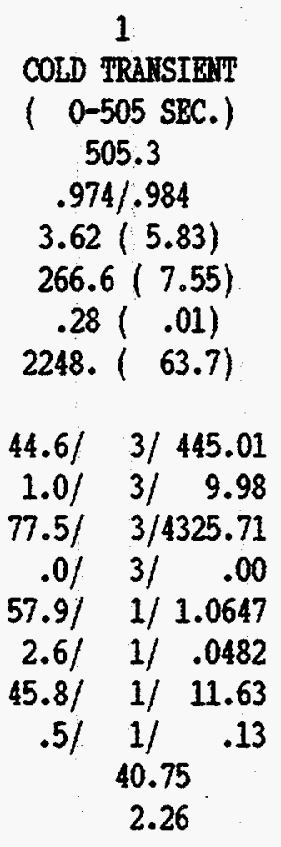

8.79

436.17

4159.94

1.0220

11.51

38.75

391.22

16.322

308.279

1191.06

1.370

1.644

14.360

.555

$18.61(12.64)$
2

STABILIZED

(505-1372 SBC.)

867.1

$.977 / .984$

$3.83(6.17)$

$263.2(7.45)$

$.29(.01)$

3808. (107.8)

$20.7 / 3 / 206.54$

$1.4 / 3 / 13.97$

$71.9 / 2 / 1803.88$

$.0 / 2 / \quad .00$

$85.5 / 14 / \quad .7640$

$13.7 / 14 / .0448$

$40.9 / 1 / 10.40$

$.6 / 1 / \quad .16$

14.07

2.25

13.97

193.57

1745.33

.7224

10.25

11.98

179.67

12.272

219.121

1426.25

2.067

.861

11.173

.582

18.81 ( 12.51$)$
GASOLIHE RFG ERI-2060-E

PUEL DEISITY $6.291 \mathrm{LB} /$ GAL

H $.135 \quad C \quad .849 \quad 0 \quad .016 \quad \& \quad .000$

HOX HOWIDITY C.P. .977

3

HOT TRANSIERT

( 0 - 505 SEC.)

505.4

$.975 / .984$

$3.64(5.85)$

$261.5(7.41)$

$.28(.01)$

2205. ( 62.4)

$36.0 / 3 / 359.20$

$1.5 / 3 / 14.97$

$76.3 / \quad 3 / 4214.38$

$.01 \quad 3 / \quad .00$

93.5/ $14 / \quad .9589$

$13.3 / 14 / \quad .0433$

$52.2 / 1 / 13.24$

$.6) \quad 1 / .16$

33.53

2.24

9.56

345.80

4061.56

.9202

13.10

31.53

309.22

12.695

295.266

1052.01

1.529

1.313

11.134

.500

$20.74(11.34)$

3-BAG CONPOSITE RESULTS

$$
\begin{array}{llr}
\text { HC } & \text { G/MI } & 3.554 \\
\text { CO } & \text { G/MI } & 69.668 \\
\text { HOX } & \text { G/MI } & .473
\end{array}
$$

NOX G/HI $\quad .473$
$19.28(12.20)$

$\begin{array}{llr}\text { CH4 } & \text { G/HI } & .310 \\ \text { INHC } & \text { G/HI } & 3.175\end{array}$


COUPOTER PROGRAH LDT 1.5-R

SOUTHERST RESEARCH IISTITUTE - DEPARTYERT OF EIISSTORS RESEARCH

3-BAG EPA FPP VEHICLE EHISSION RESDLTS

PROJBCT HO. 08-6068-400

\begin{tabular}{|c|c|}
\hline & \\
\hline VसHTCLE PODET & OA POPD TARPTIC \\
\hline BHGINE & $3.0 \mathrm{~L}(181 \mathrm{CID})-14$ \\
\hline TRARSHISSIOH & A4 \\
\hline ODCHETER & 19533 HILES ( $31428 \mathrm{RM})$ \\
\hline
\end{tabular}

BAROUETER 29.18 IN HG (741.2 NM HG) RELATIVE GDWIDETY 54.4 PCT.
BAG HOIBER
BAG DESCRIPTI
FORULLDEHYDE

PPH

HASS HG

ACETALDEHYDE

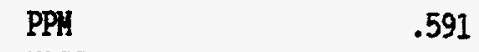

MASS HG 66.69

ACROLEIN

$\begin{array}{ll}\text { PPH } & .028\end{array}$

MASS HG $\quad 4.06$

ACETONE

PPH

HASS HG

PROPIONALDEHYDE

PPY!

MASS MG

CROTOMALDEHYDE

PPH $\quad .046$

HASS HG

ISOBUTYR+YIER

PPH

HASS HG

BEIYZALDBHHDE

PPH

HASS HG

HEXANALDEHYDE

PPI

HASS HG

UETTHANOL

PPH $\quad 3.377$

MASS HG

ETPEANOL

PPH

MASS HG

.296

44.27

.099

14.72

8.26

.047

8.81

.072

19.73

.007

1.68

279.00

.050

5.98
TRST FT-RPG-3A

DAPE 10/30/95 ROH

DHNO 3 BAG CART 2

ACTOAI ROAD LOAD 6.80 IP ( $5.07 \mathrm{KW}$ )

TEST WEIGHT 3500 LBS ( 1587 KG)

DRY BULB TEYPERATURE $74.0^{\circ} \mathrm{P}\left(23.3^{\circ} \mathrm{C}\right)$
2

STABILIZED

(505-1372 SEC.)
3-BAG COMPOSITE RESULTS

FORUALDEHYDE HG/MI

ACETALDEHYDE HG/MI

ACROLEIN HG/MI

ACETONE HG/MI

PROPIONALD. HG/MI
.871

113.99

.697

133.70

.028

6.78

.53

1

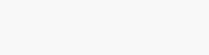

.06

6

99

.087
21.97

21.97

.043

13.09

.039

12.39

.082

37.73

.005

2.26

1.695

237.97

.024

4.90

18.141

23.826

1.255

13.038

4.183
3

HOT TRANSIERT

( 0- 505 SEC.)

.068

4.88

.247

27.08

.010

1.47

.084

12.24

.035

5.11

.008

1.38

.003

.54

.001

.13

.000

.00

2.979

241.65

.035

4.03

CROTOHALD. MG/MI

ISOBUTYR+HER HG/MI

BENZALDEHYDE HG/MI

HEXAMTLDEHYDE MG/MI

METHAYYLL MG/HI

ETHEANOL HG/MI
GASOLINE RFG BH-2060-P

FOE[ DETSITY $6.291 \mathrm{LB} / \mathrm{GAL}$

$\begin{array}{lllll}\text { \# } .135 & \mathrm{C} .849 & 0 & 0.016 \quad \& .000\end{array}$

HOX HUMIDITY C.P. .977

BACKGROTID

.004

.004

.000

.001

.001

.000

.000

.000

.000

.000

.000

2.335

2.208

6.202

.400

66.413

1.309 
SOUTHTEST RESEARCH IHSTTTOTE - DEPARTHETT OF RMISSIOIS RRSEARCH

CONPJTER PROERAY LDT 1.5-R 3-BAG BPA FIP PEHICLE EIISSION RESULTS

PROJECT M0. 08-6068-400

VEHTCLB HOUBER $90 \mathrm{X}$

VRHICLE HODEL 94 FORD TAORUS

EHGIFE $\quad 3.0 \mathrm{~L}$ (181 CID)- 14

TRAMSIISSION $\$ 4$

ODONETER 19581 VILLS ( 31505 KM)

BAROHETER 29.10 IN HG (739.1 NA BG)

RELLATIVE HU:IDITY $52.6 \mathrm{PCT}$.

BAG IJIEBRR

BAG DESCRIPIION

RON TINE SECOMDS

DRY/WET CORRECTION FACIOR, SAMP/BACK

URASURED DISTAHCE YILES (RM)

BLOWER FLOW RATE SCFH (SCAN)

GAS UETER FLON RATE SCFH (SCHN)

TOFAL FLOW SCF (SCH)

BC SAIPLE METER/RAHGE/PPN (BAG)

HC BCKGRD HETER/RAMGE/PPH

CO SAYPLE METER/RAHGE/PPH

$C O$ BCKGRD HETER/RANGE/PPH

CO2 SAYPLE HETER/RANGE/PCT

CO2 BCKGRD HETER/RAHGE/PCT

HOX SAMPLE HETER/RAHGE/PPH (BAG) (D)

MOX BCKERD HETER/RAHGR/PPH

CH4 SAIPLE PPH (1.160)

CH4 BCRGRD PPH

DILUTTON FACTOR

HC CONCBMTRATION PPH

CO CONCENTRATION PPN

$\mathrm{CO} 2$ COHCEHTRATION PCT

HOX CONCANTRATTON PPH

CH4 CONCEITRATTON PPH

RHC CONCEHTRATION PPH

OAHCE MASS GRAMS

CO MASS GRAHS

$\mathrm{CO} 2$ HASS GRAHS

MOX MASS GRAYS

CH4 MASS GRAMS

RHC MRSS GRAHS (FID)

FOEL MASS RG

FUEL ECOHONY WPG (L/100RN)
TEST FT-1185-1

DAIE 11/2/95 ROH

DHFO 3 BAG CART 2

ACIUAL ROAD LOAD $6.80 \mathrm{IP}$ ( $5.07 \mathrm{kM})$

TEST NEIEHP 3500 LBS ( 1587 KG)
DRY BULB TEIPERATURE $77.0^{\circ} \mathrm{F}\left(25.0^{\circ} \mathrm{C}\right)$

1

COLD TRAHSIERT

( 0-505 SBC.)

505.2

$.964 / .983$

$3.62(5.82)$

$261.6(7.41)$

$.27(.01)$

2205. ( 62.4)

$24.2 / 3 / 241.46$

$\begin{array}{lll}1.0 / & 3 / & 9.98\end{array}$

$62.8 / 3 / 3061.88$

$.01 \quad 3 / \quad .00$

$58.3 / \quad 1 / 1.0722$

2.6/ $\quad 1 / \quad .0482$

$11.0 / \quad 1 / 2.84$

.1) $1 / \quad .03$

14.05

2.15

8.58

232.65

2919.26

1.0296

2.82

12.15

112.45

9.648

212.184

1176.92

.337

.506

4.048

.977

11.13 ( 21.13)
2

STABILIZED

(505-1372 SEC.)

866.5

$.970 / .983$

$3.82(6.15)$

$259.6(7.35)$

$.28(.01)$

3753. (106.3)

$11.2 / 3 / 111.75$

$.9 / 3 / 8.98$

64.7/ $2 / 1543.31$

$.2 / \quad 2 / \quad 2.96$

$83.3 / 14 / .7184$

$13.0 / 14 / \quad .0421$

$.9 / \quad 1 / \quad .24$

$.1 / 1 / \quad .03$

8.34

2.09

13.57

103.43

1483.80

.6794

.21

6.40

63.12

7.076

183.584

1321.92

.043

.454

3.868

1.035

11.10 ( 21.19)
HETHAMOL EN-2062-P

FUEL DEISITY $6.623 \mathrm{LB} / \mathrm{GAL}$

$\begin{array}{lllllll}\text { H } .127 & \mathrm{C} & .431 & 0.442 & \mathrm{X} .000\end{array}$

HOX HUIIDITI C.P. 1.001

3

HOT TRAHSIERT

( 0- 505 SEC.)

505.9

$.966 / .983$

$3.61(5.81)$

$259.6(7.35)$

$.28(.01)$

2191. ( 62.1)

$13.6 / 3 / 135.70$

$.8 / 3 / \quad 7.98$

$91.1 / 2 / 2596.80$

$.21 \quad 2 / \quad 2.96$

$93.2 / 14 / .9506$

$12.8 / 14 / \quad .0414$

$2.9 / \quad 1 / \quad .76$

$.1 / 1 / \quad .03$

11.16

2.07

9.83

128.53

2481.94

.9135

.73

9.30

69.44

5.300

179.291

1037.74

.087

.385

2.484

.847

$12.81(18.36)$

3-BAG COIPOSITE RESULIS

$$
\begin{array}{llr}
\text { OUHCT } & \text { G/RI } & 1.914 \\
C D & \text { G/MI } & 50.667 \\
\text { HOX } & \text { G/MI } & .032
\end{array}
$$

FOEL ECONOHY UPG (L/100RH) $11.55(20.37)$
CH4 G/MI $\quad .120$

HIHC G/MI .944 
SODTHINEST RESEARCH ITSTITUTE - DEPARTHENT OF HUISSIOHS RESEARCH

COIPUTER PROGRAH LDT $1.5-R$

3-BAG BPB FIP VEHICLR EHISSTON RESULTS

PROJECT 10. 08-6068-400

VEHICLE IUTBER 90X

VBHICLE HODEL 94 PORD TAURUS

EMGINE $\quad 3.0 \mathrm{~L}$ (181 CID)- 14

TRAISHISSTON 24

ODOHIRTER 19581 MILES ( 31505 KU)

BAROHETER 29.10 III HG (739.1 $\mathrm{MH}$ HG) RELATIVE RUIIDITY 52.6 PCT.

BAG WDUBRR

BAG DESCRIPTIOH

FORHALDEHDE

PPH $\quad 5.369$

HaSS HG

ACETALDEHYDE

PPH $\quad .185$

HASS HG

ACROLEII

PPY

MASS KG

ACETONE

PPH

MASS HG

PROPTONALDEEHYDE

PPH $\quad .028$

HESS HG

CROTONALDEHYDE

PPII

HaSS HG

ISOBOTYR+MER

PPH

HASS HG

BETZALDEHYDE

PPH $\quad .025$

MASS HG

HEXANALDEEHYDE

PPN

MASS HG

HETHAAYOL

PPH $\quad 155.860$

HASS HG

EITHAMOL

PPY

MASS HG

TRST FT-I85-1

DATE $11 / 2 / 95$ ROII

DYHO 3 BAG CARI 2

ACTUAL ROAD LOAD $6.80 \mathrm{HP}$ ( $5.07 \mathrm{KW}$ )

TEST WETGHT 3500 LBS ( 1587 RG)

DRY BULB TEYPERATURE $77.0^{\circ} \mathrm{F}\left(25.0^{\circ} \mathrm{C}\right)$

2

STABILIZED

(505-1372 SEC.)

1.986

254.93

.084

15.16

.000

.00

.028

6.74

.010

2.24

.000

.00

.008

2.45

.007

3.09

.000

.00

51.945

7136.53

.000

.00
.000

.00
3

HOP TRAYSIENT

( 0 - 505 SEC. $)$

4.939

369.37

.143

15.34

.000

.00

.040

5.70

.021

2.95

.006

1.00

.015

2.59

.016

4.24

.000

.00

76.458

6107.49

.000

.00
UETHAMOL BI?-2062-P

FORL DENSITY $6.623 \mathrm{LB} / \mathrm{GAL}$

$\begin{array}{lllllllll}\text { H } .127 & \mathrm{C} & .431 & 0 & .442 & \mathrm{X} .000\end{array}$

HOX HJMIDITY C.F. 1.001

3-BAG COIPOSITE RESULTS

FORHALDEHYDE WG/AI

CCEPALDERTOE HG/HI

LCROLBII HG/HI

ACETONE HG/MI

PROPIOHALD. HG/MI

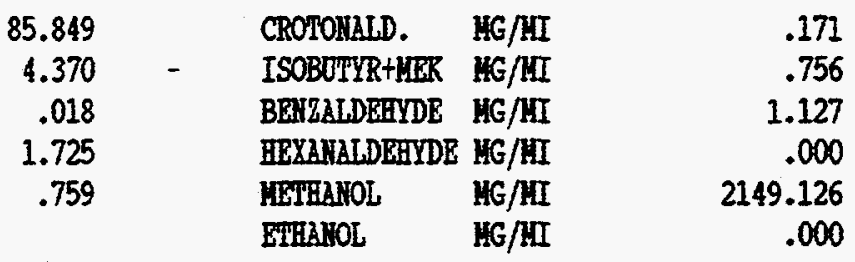

D-5 


\begin{abstract}
SOUTHWEST RESEARCH IISTITUTE - DEPARTMENT OF EHISSIOHS RESEARCH
COIPOTER PROGRAM LDT 1.5-R

3-BAG EPA FTP VEHICLE EUISSTON RESULTS

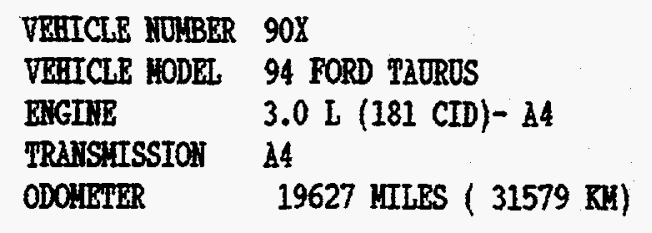

TEST FT-E85D-1

DATE 11/ 7/95 RUTI

DYHO 3 BAG CART 2

ACTUAL ROAD LOAD 6.80 BP ( $5.07 \mathrm{KN}$ )

TEST WEIGIFT 3500 LBS ( 1587 RG)
\end{abstract}

PROJECT 10. 08-6068-400
BAROUETER 29.15 II HG (740.4 WH HG) RRLATIVE HOYIDITY 49.5 PCT.

BAG ITUIBER

BAG DRSCRIPTIOH

RUI TTHE SECONDS

DRY/WET CORRECTION FACTOR, SAMP/BACR

HEASURED DISTAHCE HILBS (XW)

BLOFER FLOF RAIE SCFH (SCHN)

GAS HETER PLOW RATE SCFH (SCHN)

TOTAL FLOW SCP (SCH)

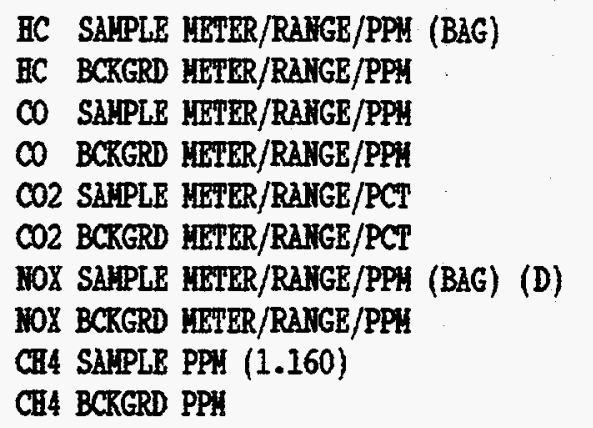

DILUTTOI FACTOR

HC COHCENTRAPTON PPH

CO COICERTRATION PPH

CO2 COHCEITIRATION PCT

HOY COHCEITRATION PPH

CH4 COHCEYTRATTON PPH

REC COMCENTRATTON PPH

\section{OHHCR MRSS GRAYS}

$C O$ HASS GRAMS

$\mathrm{CO} 2$ MASS GRAMS

HOX HASS GRALS

CH4 MASS GRHWS

RHC HASS GRAHS (PID)

FUEL MASS RG

FOEL ECOHOAY UPG (L/100KM)
DRY BULB TEYPERATURE $72.0^{\circ} \mathrm{P}\left(22.2^{\circ} \mathrm{C}\right)$

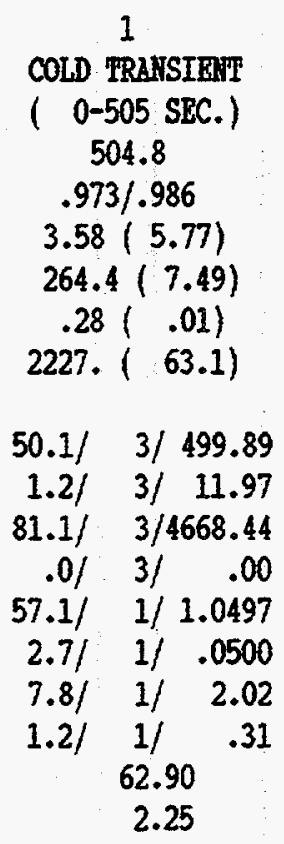

8.24

489.37

4481.79

1.0057

1.75

60.92

329.39

\subsection{4}

329.099

1161.43

.196

2.562

11.980

.790

$13.38(17.58)$

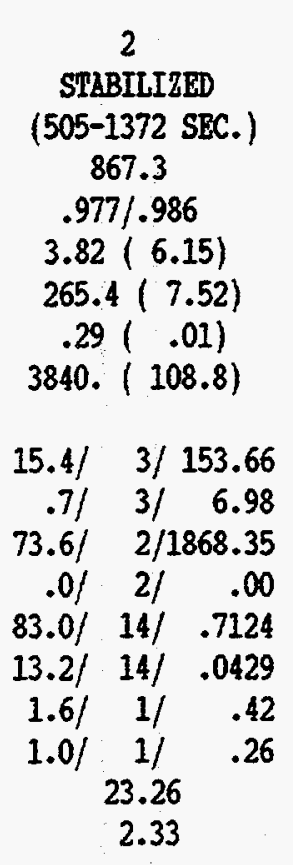

13.99

147.17

1808.06

.6726

.18

21.09

132.33

10.566

228.924

1339.25

.034

1.529

8.299

.779

$14.49(16.24)$
BTHAYOL E85D BH-2061-F

FUELL DEMSITY $6.506 \mathrm{LB} / \mathrm{GAL}$

$\begin{array}{llllllll}\text { H } .132 & \mathrm{C} & .612 & 0.256 & \mathrm{X} .000\end{array}$

HOX BOIIDITY C.F. .932
HOT TRANSIEITI

( 0 - 505 SEC.)

505.3

$.975 / .986$

$3.59(5.78)$

$263.4(7.46)$

$.28(.01)$

2220. ( 62.9)

21.7/ 3/ 216.52

$.9 / 3 / \quad 8.98$

$64.2 / \quad 3 / 3172.84$

$.0 / \quad 3 / \quad .00$

$92.0 / 14 / .9183$

$13.3 / 14 / .0433$

2.5/ $1 / \quad .65$

$.91 \quad 1 / \quad .24$

29.42

2.27

10.21

208.42

3055.52

.8793

.44

27.37

172.26

8.744

223.673

1012.23

.049

1.147

6.246

.626

$16.94(13.89)$
3-BAG COMPOSITE RRSULTS

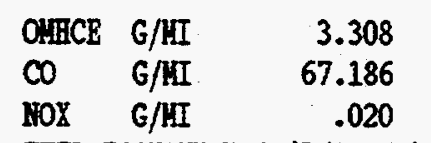

FOEL BCOHCNY MPG (L/100RM)
$14.87(15.82)$ 
SOUTHWEST RESEARCH INSTITUTE - DEPARTIETTI OF EIISSIOIS RESEARCH

COIPUTER PROGRAH LDT 1.5-R 3-BAG EPA FIP VRHICLE EMISSION RESOLIS

PROJECI HO. 08-6068-400

$\begin{array}{ll}\text { VBHICLE NULBER } & 90 \mathrm{X} \\ \text { VEHICLR HODEL } & 94 \text { FORD TAURUS } \\ \text { BIGINE } & 3.0 \text { L (181 CID)- } 14 \\ \text { TRAFSWISSIOH } & \text { A4 } \\ \text { ODOHETRR } & 19627 \text { MILES ( } 31579 \text { KA) }\end{array}$

BAROHETER 29.15 IN HE (740.4 MH HG) RELATIVE HDYIDITY 49.5 PCT.

BAG WOEBER
BAG DRSCRIPITON

1
COLD TRANSIEITI
$(0-505$ SEC. $)$

FORUALDERYDE

PPW
HASS HG
CEPTALDEHYDE

PPH $\quad 21.693$

HASS MG

ACROLEIN

PPH

HBSS HG

ACETOIE

PPH

HASS HG

PROPTOHALDEHYDE

PPN

HASS HG

CROTONALDEHYDE

PPHI $\quad .046$

HASS HG

ISOBUTYR+ HER

PPPI

MASS HG

BENZALDERYDE

PPH

HASS HG

HEXAHALDERTOE

PPY

HASS HE

METHANOL

PPH
HASS HG
MHAHOL

PPN

HASS HG

$$
\begin{gathered}
1.407 \\
107.43
\end{gathered}
$$

2438.24

.041

5.84

.287

42.48

.106

15.66

8.27

.045

8.34

.026

7.14

.000

.00

8.357

682.69

102.783

12083.60
TRST FT-E85D-1

DATE 11/ 7/95 RUH

DHO 3 BAG CART 2

ACTJAL ROAD LOLD $6.80 \mathrm{BP}$ ( $5.07 \mathrm{~kW}$ )

TEST WEIGHT 3500 LBS ( 1587 RE)

DRY BULB TETPERATURE $72.0^{\circ} \mathrm{F}\left(22.2^{\circ} \mathrm{C}\right)$

EHHABOL R85D EH-2061-P

FOEL DERSITY $6.506 \mathrm{LB} / \mathrm{GAL}$

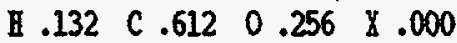

HOX HIHIDITY C.F. $\quad .932$

BACKGROOND

(505-1372 SBC. $) \quad(0-505$ SEC. $)$

$\begin{array}{ccc}.312 & .551 & .006 \\ 40.64 & 41.70 & \\ 8.845 & 11.863 & .007\end{array}$

1721.15

1331.19

$.000 \quad .001$

.000

$.06 \quad .19$

.101

.197

29.13

.018

4.63

.010

1.46

.006

2.00

.010

1.82

.011

.018

3.24

.006

.014

3.70

.000

.00

.000

.00

.000

.000

.000

.000

2.071

292.10

3.081

250.90

9.508

23.204

2722.67

3-BAG COIPOSITT RESULTS

$$
\begin{array}{ll}
\text { FORUALDERYDE HG/MI } \\
\text { ACETALDEHYYDE HG/MI } \\
\text { ACROLEIN } & \text { HG/MI } \\
\text { ACETONE } & \text { HG/MI } \\
\text { PROPIONHID. HG/MI }
\end{array}
$$

1932.55

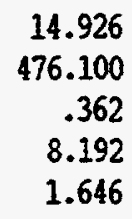

CROTONALD. MG/MI

ISOBUTYR+NER MG/II

BWZALDEEYDE HG/MI

HEXAMTLLDEHYDE MG/MI

VETHAROL KG/MI

ETHBHOL HG/MI

.000

.000

.009

.019 
SOUTHWEST RESEARCH IRSTITOTE - DEPARTHENT OF EMISSIONS RESEARCH

CONPUTER PROGRA IDT 1.4-R 3-BAG EPA FTP VEHICLE EMISSION RESOLTS PROTECT NO. 08-6068-400

VEETCLE NUMBER $90 \%$

VEHICLE HODEL 94 FORD TAURUS

BNGINE $\quad 3.0 \mathrm{~L}$ (181 CID $)-6$

TRANSUISSION A-4

ODOHETER 19673 MILES ( 31653 KM)

BAROHETER 29.01 IN EG (736.9 HG) RELATTVE HOHIDITY 42.3 PCT.

BAG NUUBER

BAG DESCRIPTION

RUA TIME SECONDS

DRY/WET CORRECTION FACTOR, SAMP/BACK

MEASORED DISTANCE MILES (KM)

BLOWER FLON RATE SCFN (SCHR)

GAS METER FLOW RATE SCFM (SCMH)

TOTAL FLOW SCF (SCN)

HC SAMPLE METER/RANGE/PPH (BAG)
HC BCKGRD METER/RANGE/PPH
CO SAMPLE METER/RANGE/PPH
CO BCKGRD METER/RANGE/PPH
CO2 SAMPLE METER/RANGE/PCT
C02 BCKGRD METER/RAHGE/PCT
NOX SAMPLE METER/RANGE/PPM (BAG) (D)
NOX BCRGRD HETER/RANGE/PPH
CH4 SAMPLE PPH (1.160)
CH4 BCKGRD PPH

DILUTION FACTOR

HC CONCENTRATION PPH

CO CONCBNTRATION PPH

CO2 CONCENTRATION PCT

NOX CONCENTRETION PPH

CH4 CONCENTRATION PPM

IMHC CONCENTRATION PPH

FIDHC MASS GRAMS

CO MASS GRAMS

$\mathrm{CO2}$ MASS GRAMS

HOX MASS GRAYS

CH4 MASS GRAMS

MHE MASS GRAYS (FID)

FOEL MASS RG

FUEL ECONONY MPG (L/10OKM)

\section{TEST FT-CNG-1}

DATE $12 / 18 / 95$ RON

DHO 3 BAG CART 2

ACTOAL ROAD LOAD $6.80 \mathrm{KP}$ ( $5.07 \mathrm{~kW})$

TEST WEICHP 3500 LBS ( 1587 RG)
DRY BULB TFYPERATURE $72.0^{\circ} \mathrm{F}\left(22.2^{\circ} \mathrm{C}\right)$

$$
\begin{gathered}
1 \\
\text { COLD TRAHSIEHT } \\
(0-505 \text { SEC. }) \\
505.3 \\
.969 / .988 \\
3.63(5.84) \\
263.0(7.45) \\
.00(.00) \\
2215 .(62.7)
\end{gathered}
$$

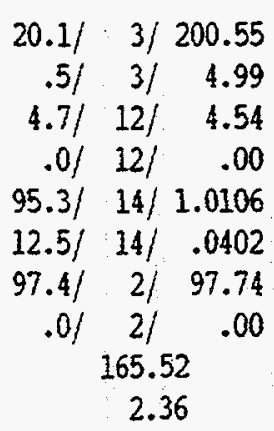

9.46

196.09

4.34

.9746

97.74

163.41

6.54

8.624

.317

1119.35

10.539

6.834

.236

.412

$21.42(10.98)$
2

STABILIZED

(505-1372 SEC.)

867.0

$.975 / .988$

$3.90(6.28)$

$260.9(7.39)$

$.00(.00)$

3771. $(106.8)$
$11.7 / 3 / 116.74$
$.5 / 3 / \quad 4.99$
$.0 / 12 / \quad .00$
$.0 / 12 / \quad .00$
$82.0 /$ 14/ .6928
$12.2 / 14 / .0391$
$31.9 / 2 / 32.01$
$.01 \quad 21 \quad .00$
98.34
2.36

13.84

112.11

.00

.6565

32.01

96.15

.57

8.393

.000

1283.56

5.876

6.845

.035

.471

$20.15(11.67)$
NAT GAS

FOEL DEHSITY $5.360 \mathrm{~EB} / \mathrm{GAL}$

$\begin{array}{lllllll}\text { В } .229 & \mathrm{C} .712 & 0 & 0.000 & \mathrm{X} .059\end{array}$

HOX HEMIDITY C.F. $\quad .899$

$$
\begin{gathered}
3 \\
\text { HOT TRANSIENT } \\
(0-505 \text { SEC. }) \\
505.4 \\
.971 / .988 \\
3.63(5.84) \\
260.8(7.39) \\
.00(.00) \\
2197 .(62.2)
\end{gathered}
$$

$13.2 / 3 / 131.71$

.5) $3 / \quad 4.99$

$.2 / \quad 12 / \quad .19$

$.0 / \quad 12 / \quad .00$

91.6/ $14 / \quad .9078$

$12.3 / 14 / \quad .0395$

$94.4 / 2 / \quad 94.73$

$.5 / \quad 2 / \quad .50$

111.50

2.31

10.58

127.19

.19

.8721

94.28

109.41

.27

\subsection{8}

.013

993.34

10.082

4.538

.010

.364

$24.28(9.69)$

3-BAG COUPOSITE RESULTS

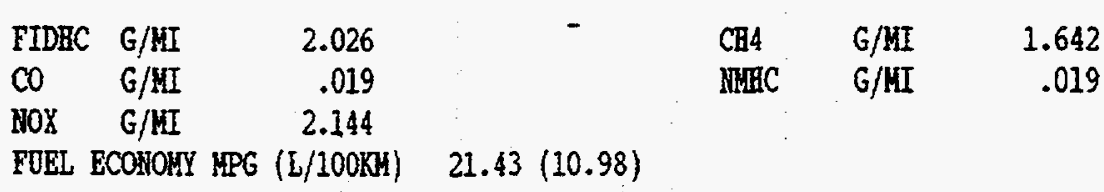


$\begin{array}{lcl}\text { SOUTHURST RESEARCH INSTITUTE - DEPARTHENT OF ENISSIONS RESEARCH } \\ \text { COMPUTER PROGRAM LDT 1.4-R } & 3-\text { BAG EPA FTP VEHICLE EMISSION RESULTS } & \text { PROJECT NO. 08-6068-400 }\end{array}$

VEBICLE NOEBER $90 \mathrm{X}$

VEHICLE HODEL 94 FORD TAUROS

BNGINE $\quad 3.0 \mathrm{~L}$ (181 CID) -6

TRANSHISSION A-4

ODOHETER $\quad 19805$ HILES ( 31866 KM)

BAROHETER 29.15 IN HG (740.4 MH BG)

RELLTTVE HOMTDITY 34.5 PCT.

BAG IUUBER

BAG DESCRIPTION

RON TINE SECONDS

DRY/WET CORRECTION FACTOR, SAMP/BACK

MEASORED DISTANCE MILES (RN)

BLONER FLOW RATE SCFH (SCW

GAS HEPER FLON RATE SCFH (SCRM)

TOTAL FLOW SCF (SCM)

BC SAMPLE METER/RANGE/PPM (BAG)

BC BCRGRD NETER/RANGE/PPH

CO SAMPLE HETER/RANGE/PPM

CO BCKGRD METER/RANGE/PPY

CO2 SAMPLR HETER/RANGE/PCP

CO2 BCRGRD HETER/RHNGE/PCT

NOX SAYPLE HETER/RAMGE/PPM (BAG) (D)

NOX BCKGRD HETER/RANGE/PPH

CH4 SAPPLE PPH (1.160)

CH4 BCKGRD PPH

DILOTION FACTOR

HC COACEHTRATTON PPH

CO CONCENTRATION PPH

$\mathrm{CO} 2$ CONCEITRRATION PCT

MOX CONCENTRATION PPM

CE4 CONCEATPRATION PPH

WHAC CONCENTRATION PPM

FIDEC MASS GRAHS

$C O$ HASS GRAHS

CO2 MASS GRAMS

HOX MASS GRAMS

CH4 HaSS GRAMS

NAIEC MASS GRAHS (FID)

FOEL MASS KG

FOBL ECONOHY MPG (L/100KM)
TEST FT-CTC-1

DATE $1 / 25 / 96$ RON

DYNO 3 BAG CARP 2

ACPUAL ROAD IOAD $6.80 \mathrm{HP}$ ( $5.07 \mathrm{KN}$ )

TEST WEIGHT 3500 LBS ( 1587 KG)
DRY BOLB TEMPERATURE $75.0^{\circ} \mathrm{F}\left(23.9^{\circ} \mathrm{C}\right)$

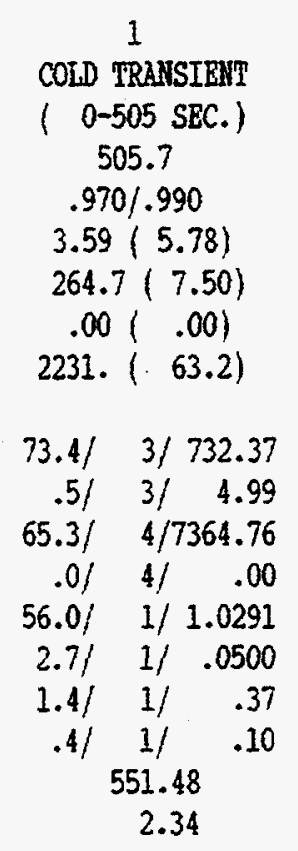
$73.4 / 3 / 732.37$
$.5 / 3 / \quad 4.99$
$65.3 / 4 / 7364.76$
$.01 \quad 4 / \quad .00$
$56.01 \quad 1 / 1.0291$
$2.7 / 1 / \quad .0500$
1.4/ $1 / \quad .37$
.4) $1 / \quad .10$
551.48
2.34

5.41

728.30

7061.76

.9883

.28

549.57

90.80

32.260

519.411

1143.22

.030

23.148

3.308

.737

$11.85(19.85)$

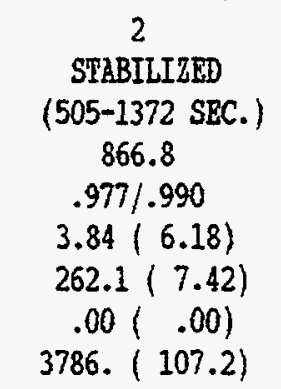

$24.7 / 3 / 246.45$

$.5 / 3 / \quad 4.99$

$98.5 / 2 / 2938.89$

$.01 \quad 21 \quad .00$

$\begin{array}{llll}79.0 / & 14 / & .6372\end{array}$

$13.8 / 14 / .0452$

$.5 / 1 / \quad .13$

.4) $1 / \quad .10$

172.35

2.39

10.30

241.95

2851.56

.5964

.04

170.19

44.53

18.188

355.956

1170.75

.007

12.165

2.753

.641

$14.56(16.15)$
NAT GAS

FUEL DEHSITY $5.360 \mathrm{LB} / \mathrm{GAL}$

B .229 C.712 $0.000 \times .059$

NOX HONIDITY C.F. .879

3

HOT TRANSIENT

( 0- 505 SEC.)

505.9

$.974 / .990$

$3.62(5.82)$

$260.3(7.37)$

$.00(.00)$

2195. ( 62.2$)$

$32.3 / 3 / 322.28$

$.6 / 3 / \quad 5.99$

$51.5 / \quad 4 / 4857.09$

$.01 \quad 4 / \quad .00$

$\begin{array}{lll}87.6 / & 14 / \quad .8104\end{array}$

$14.0 / 14 / \quad .0460$

$.8 /$ If .21

$.7 / \quad 1 / \quad .18$

234.39

2.62

7.44

317.10

4688.23

.7706

.05

232.12

47.84

13.820

339.286

877.01

.005

9.619

1.715

.522

$16.86(13.95)$

3-BAG COMPOSITE RESULTS

$\begin{array}{llr}\text { FIDHC } & \text { G/MI } & 5.363 \\ \text { CO } & \text { G/MI } & 103.738 \\ \text { HOX } & \text { G/MI } & .003\end{array}$

FU:LL ECOHONY IPG (L/100RN) 14.53 (16.19)

$\begin{array}{llr}\text { CH4 } & \text { G/NI } & 3.706 \\ \text { NHEC } & \text { G/NI } & .692\end{array}$




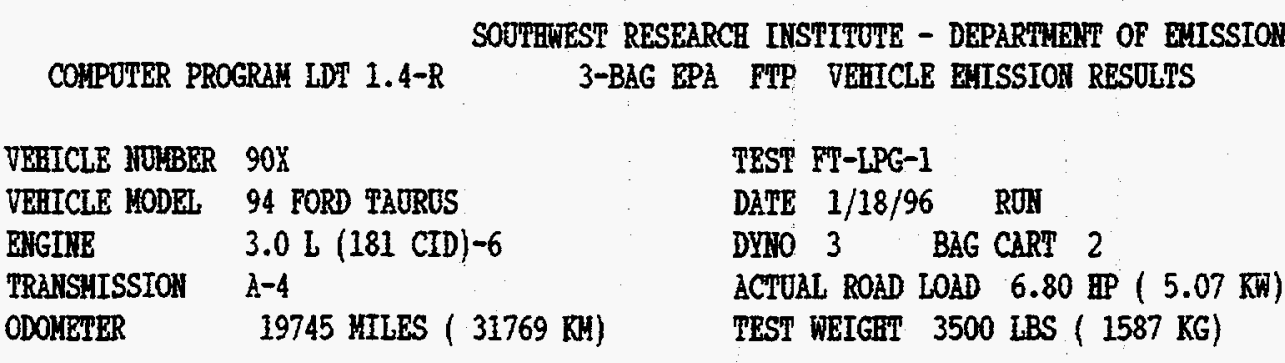

BARONETER 29.07 IN EG (738.4 MM HG) RELATIVE HOUIDITY 13.6 PCT.

BAG ITUIBER

BAG DESCRIPTION

RUN TTHE SECONDS

DRY/WET CORRECTION_FACTOR, SAHP/BRCK

UEASURED DISTANCE MILES (RM)

BLONER FLON RATE SCFH (SCWN)

GAS HETER FLON RATE SCFM (SCMH)

TOTAL FLON SCF (SCH)

BC SAMPLE HETER/RANGE/PPM (BAG)

HC BCKGRD HETER/RANGE/PPH

CO SAYPLE HETER/RaPGE/PPN

CO BCRGRD HETER/RAHGE/PPH

CO2 SAIPLE HETER/RANGE/PCT

CO2 BCKGRD HETER/RANGE/PCT

NOX SAYPLE YETER/RAMGE/PPH (BAG) (D)

NOY BCKGRD HETER/RATGE/PPH

CH4 SAYPLE PPM (1.160)

CH4 BCKERD PPY

DILOTION FACTOR

HC COHCENTRATION PPH

CO COHCENTPRATTON PPH

$\mathrm{CO2}$ CONCEATIRATTON PCT

HOK CONCEYYRATION PPH

CH4 CONCENTRATTON PPH

BRE CONCERTRATTON PPH

FIDHC MASS GRAMS

CO MASS GRAMS

$\mathrm{CO2}$ MASS GRAMS

WOY MASS GRAMS

CH4 HASS GRAMS

MNHC MASS GRAMS (FID)

FOEL MASS KG

FEEL ECOHOHY MPG (L/100RH)
DRY BULB TEYPERATURE $70.0^{\circ} \mathrm{P}\left(21.1^{\circ} \mathrm{C}\right)$

$$
\begin{aligned}
& 1 \\
& \text { COLD TRAISIENT } \\
& (0-505 \text { SEC. }) \\
& 505.7 \\
& .985 / .997 \\
& 3.68(5.92) \\
& 265.8(7.53) \\
& .00(.00) \\
& 2241 .(63.5)
\end{aligned}
$$

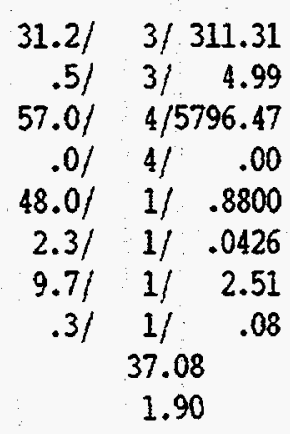

7.88

306.95

5651.98

.8428

2.44

35.42

265.86

11.901
417.536
979.16
.231
1.498
9.728
.558
$17.12(13.74)$
2

STABILIZED

(505-1372 SBC.)

866.8

$.988 / .997$

$3.85(6.20)$

$267.2(7.57)$

$.00(.00)$

3860. (109.3)

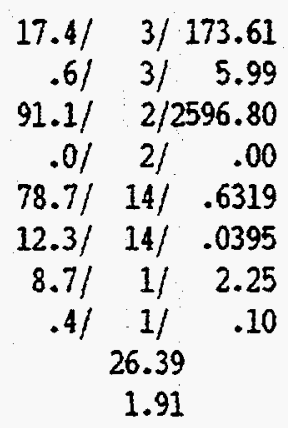

12.86

168.09

2547.11

.5955

2.16

24.62

139.53

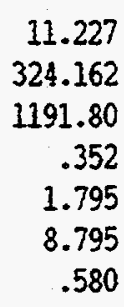

$17.28(13.62)$
PROPAITE

FURL DERSITY $5.729 \mathrm{LB} / \mathrm{GAL}$

स $.183 \quad C .817 \quad 0.000 \quad \& .000$

NOX HUUIDITY C.F. $\quad .780$

3

HOT TRANSIEITI

( 0-505 SBC.)

505.8

$.986 / .997$

$3.63(5.84)$

$263.9(7.47)$

$.00(.00)$

2224. (63.0)

$22.4 / 3 / 223.50$

.5) $3 / 4.99$

$73.2 / 3 / 3933.48$

$.0 / \quad 3 / \quad .00$

$88.0 /$ 14/ .8196

$12.6 / 14 / .0406$

$7.0 / 1 / 1.82$

$.4 / 1 / \quad .10$

19.98

1.89

9.48

219.04

3840.98

.7832

1.72

18.30

197.82

8.431

281.691

903.35

.162

.768

7.186

.458

$20.59(11.43)$

3-BAG COMPOSITE RESELTS

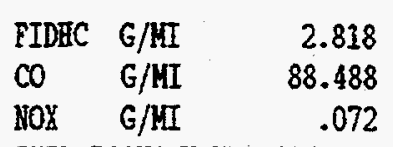

FUEL ECONONY MPG (L/10OKN)

$\begin{array}{llr}\text { CH4 } & \text { G/HI } & .383 \\ \text { INHC } & \text { G/MI } & 2.275\end{array}$


ublic reporting burden for this collection of information is estimated to average 1 hour per response, including the time for reviewing instructions, searching existing data ources, gathering and maintaining the data needed, and completing and reviewing the collection of information. Send comments regarding this burden estimate or any other spect of this collection of information, including suggestions for reducing this burden, to Washington Headquarters Services, Directorate for Information Operations and seports, 1215 Jefferson Davis Highway, Suite 1204, Artington, VA 22202-4302, and to the Office of Management and Budget, Paperwork Reduction Project (0704-0188), Vashington, DC 20503.

\begin{tabular}{|l|l|l|}
\hline AGENCY USE ONLY (Leave blank) & $\begin{array}{l}\text { 2. REPORT DATE } \\
\text { June } 1997\end{array}$ & $\begin{array}{l}\text { 3. REPORT TYPE AND DATES COVERED } \\
\text { Subcontract report }\end{array}$ \\
\hline
\end{tabular}

TITLE AND SUBTITLE

5. FUNDING NUMBERS

(C) YAW-3-13253-01

(TA) FU703610

AUTHOR(S)

Kevin A. Whitney

PERFORMING ORGANIZATION NAME(S) AND ADDRESS(ES)

Southwest Research Institute

220 Culebra Road

O Drawer 28510

San Antonio, TX 78228-0501

SPONSORING/MONITORING AGENCY NAME(S) AND ADDRESS(ES)

lational Renewable Energy Laboratory

617 Cole Boulevard

solden, CO 80401-3393

\section{SUPPLEMENTARY NOTES}

2a. DISTRIBUTION/AVAILABILITY STATEMENT

lational Technical Information Service

I.S. Department of Commerce

285 Port Royal Road

pringfield, VA 22161

3. ABSTRACT (Maximum 200 words) This report, which covers the second year of this effort, describes the laboratory work to accomplish four independent tasks 1) speciation of hydrocarbon exhaust emissions from a light-duty vehicle operated over the chassis dynamometer portion of the light-duty FTP after modifications or operation on butane and butane blends; (2) evaluation of NREL's Variable Conductance Vacuum Insulated Catalytic Converter Test Article 4 for the reduction of old-start FTP exhaust emissions after extended soak periods for a Ford FFV Taurus operating on E85; (3) support of the University of Dayton Research Institute UDRI) in an attempt to define correlations between engine-out combustion products identified by SwRI during chassis dynamometer testing, and those found uring flow tube reactor experiments conducted by UDRl; and (4) characterization of small-diameter particulate matter from a Ford Taurus FFV operating in a imulated fuel-rich failure mode on CNG, LPG, M85, E85, and reformulated gasoline.

\section{SUBJECT TERMS}

Itemative fuels, transportation fuels, air quality, emissions

7. SECURITY CLASSIFICATION OF REPORT
18. SECURITY CLASSIFICATION OF THIS PAGE
19. SECURITY CLASSIFICATION OF ABSTRACT
8. PERFORMING ORGANIZATION REPORT NUMBER

DE97050813

10. SPONSORING/MONITORING AGENCY REPORT NUMBER

NRELSR-540-23143

12b. DISTRIBUTION CODE

UC-1504 
